\title{
Projecting contact matrices in 177 geographical regions: an update and comparison with empirical data for the COVID-19 era Supplementary Material
}

\author{
Kiesha Prem, Kevin van Zandvoort, Petra Klepac, Rosalind M Eggo, Nicholas G Davies, \\ Centre for the Mathematical Modelling of Infectious Diseases COVID-19 Working Group, \\ Alex R Cook, Mark Jit ${ }^{1}$
}

Contents

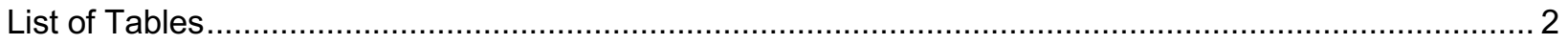

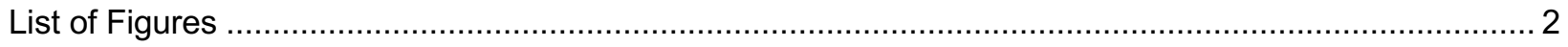

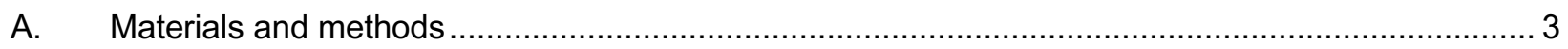

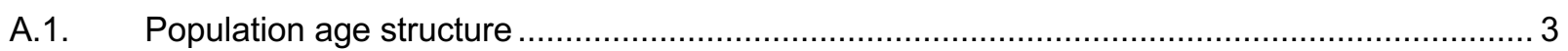

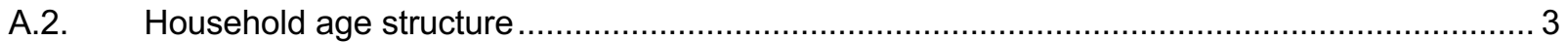

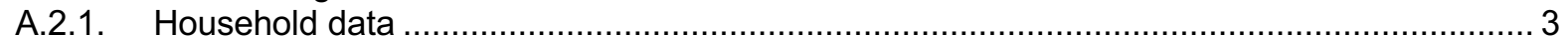

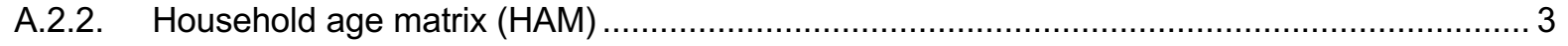

A.2.3. Household age matrix (HAM) validation for POLYMOD and DHS countries ...................... 5

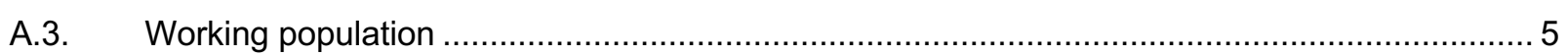

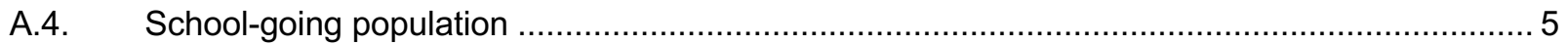

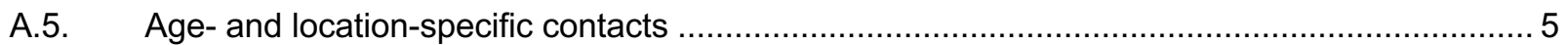

A.6. Age- and location-specific contacts by rural and urban areas .......................................... 9

A.7. Age-stratified compartmental model of the physical distancing interventions for COVID-19 ..... 9

A.8. Hierarchical model of POLYMOD contact data .............................................................. 11

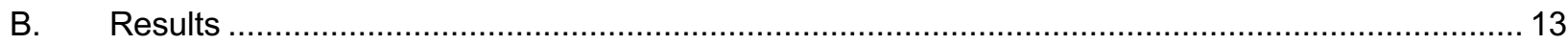

B.1. Household age matrix (HAM) validation for POLYMOD and DHS countries......................... 13

B.2. Household age matrix (HAM) urban and rural comparison ................................................. 31

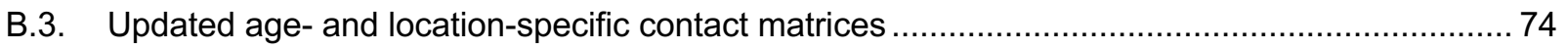

B.4. Comparison of the empirical and 2017 synthetic age-specific contact matrices in ten geographical regions

B.5. Age-specific infection attack rate of COVID-19 and comparison of the empirical and updated synthetic age-specific contact matrices in ten geographical regions........................................... 255

B.6. Degree of symmetry for empirical and synthetic matrices............................................... 259

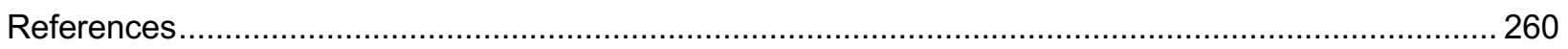

${ }^{1}$ Correspondence to mark.jit@Ishtm.ac.uk. 


\section{List of Tables}

A Description of Demographic Household Surveys from 43 countries............................................. 4

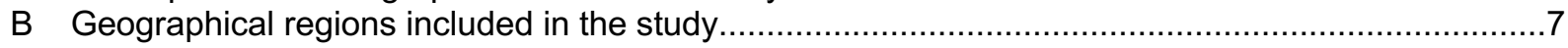

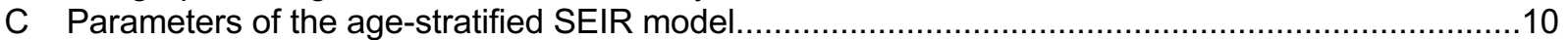

D Possible reason for discrepancies in age-specific infection attack rates..................................257

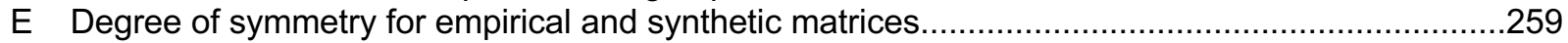

\section{List of Figures}

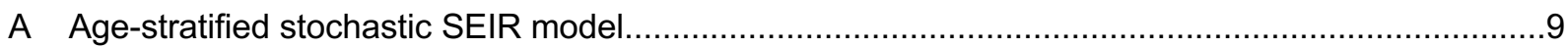

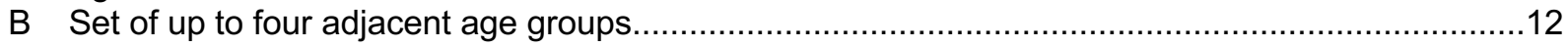

C Smoothness between successive age groups on the prior distribution......................................13

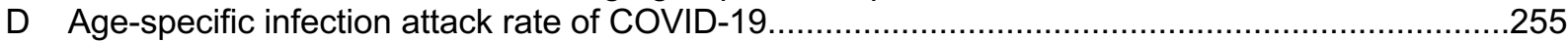

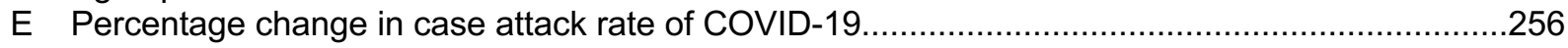




\section{A. Materials and methods}

\section{A.1. Population age structure}

The United Nations Population Division provides the population age composition in five-year age intervals $P_{a}$ (i.e., number of individuals in age group $a$ ) for the 177 geographical regions in this study [1]. They also present the estimation of rural and urban population age compositions for the 177 geographical regions [2].

\section{A.2. Household age structure}

\section{A.2.1. Household data}

We extracted the household data from POLYMOD contact study [3] — for the eight POLYMOD countries: Belgium, Germany, Finland, the United Kingdom, Italy, Luxembourg, the Netherlands, and Poland. The Demographic Household Surveys (DHS) provides nationally-representative household surveys of the whole population for several low- and middle-income countries [4]. Table A lists the $43 \mathrm{DHS}$ countries included in the study. The largest survey conducted in India in 2015 included $\sim 3$ million individuals from $\sim 600,000$ households. The DHS also provides data for rural and urban areas, allowing us to derive rural-urban household age matrices.

\section{A.2.2. Household age matrix (HAM)}

To estimate the age-specific contacts at home for county $c$, we need to first construct the household age matrix (HAM) for country $c$. The HAM of country $c, \mathbf{h}_{a, \alpha}^{c}$, represents the mean number of household members of age $\alpha$ of an individual aged $a$. Although this can be easily derived for the eight POLYMOD and 43 DHS countries, it is not straightforward for other countries or geographical regions without available household structure data. In Prem et al. [5], we provide detailed steps to project a country's household age structure.

1. Population ratio matrices: For the 51 POLYMOD and DHS countries, we first derive their population ratio matrices $\Pi_{a, \alpha}^{c}$ by dividing the elements of $\operatorname{HAM}\left(\mathbf{h}_{a, \alpha}^{c}\right)$ by the proportion of the population of country $c$ aged $a, P_{a}^{c}$. The population ratio matrix of a country measures the propensity of having a household member aged $\alpha$ for an individual aged $a$ after adjusting for the population age structure. Because we know the population age composition for the 177 geographical regions, we can project their HAM by extrapolating the relationship between $P_{a}^{c}$ and $\mathbf{h}_{a, \alpha}^{c}$ as estimated from the POLYMOD and DHS countries, and applying that to the country's population age profile. We could simply use the mean population ratio matrix of the POLYMOD and DHS countries to derive the HAM of country $c, \mathbf{h}_{a, \alpha}^{c}$, as follows $P_{a}^{c} \overline{\Pi_{a, \alpha}^{c}}$.

2. HAM weights: However, as household age structures vary across countries in different stages of development and with different demographics, we use 14 country characteristics from the World Bank and United Nations Educational, Scientific and Cultural Organization Institute for Statistics (UIS) databases [6] to quantify the similarity of these 126 geographical regions (with no available household data). The 14 country characteristics include gross domestic product per capita, total fertility rate and adolescent fertility rate, population density, population growth rate, under-five mortality rate, the life expectancy of males and females, mortality rates of males, risk of maternal death, mortality from road traffic injury, the incidence of tuberculosis, as proxies for overall health, internet penetration rate, and secondary school education attainment levels in the country. We then calculate the pairwise Euclidean distances of each of the 14 standardized variables of these 126 geographical regions with the 51 POLYMOD and DHS countries. We generated 10000 bootstrap samples of the pairwise distances between countries (between indicators) and selected the combination that maximized the correlation between $P_{a}^{c}$ and $\sum_{\alpha} P_{\alpha}^{c} \mathbf{h}_{a, \alpha}^{c}$. For each of the 126 geographical regions, the reciprocal of the bootstrapped pairwise distances provide the weights which we then use to derive the weighted mean of the population ratio matrices of the 51 POLYMOD and DHS countries.

3. HAM projection: Using the derived weighted mean of the population ratio matrices, we project the $\mathrm{HAM} \mathbf{h}_{a, \alpha}^{c}$ for geographical region $c$. 
Table A. Description of Demographic Household Surveys from 43 countries.

\begin{tabular}{|c|c|c|c|c|c|}
\hline Country & $\begin{array}{c}\text { Country } \\
\text { code }\end{array}$ & $\begin{array}{c}\text { Survey } \\
\text { year }\end{array}$ & Survey Type & $\begin{array}{c}\text { Number of } \\
\text { households }\end{array}$ & $\begin{array}{l}\text { Number of } \\
\text { individuals }\end{array}$ \\
\hline Afghanistan & AFG & 2015 & Standard DHS & 24395 & 203708 \\
\hline Angola & AGO & 2015 & Standard DHS & 16109 & 74902 \\
\hline Bangladesh & BGD & 2014 & Standard DHS & 17300 & 81624 \\
\hline Benin & BEN & 2017 & Standard DHS & 14156 & 74673 \\
\hline Bolivia (Plurinational State of) & $\mathrm{BOL}$ & 2008 & Standard DHS & 19564 & 77757 \\
\hline Cambodia & KHM & 2014 & Standard DHS & 15825 & 74122 \\
\hline Cameroon & CMR & 2011 & Standard DHS & 14214 & 72622 \\
\hline Chad & TCD & 2014 & Standard DHS & 17233 & 99620 \\
\hline Colombia & $\mathrm{COL}$ & 2015 & Standard DHS & 44614 & 162459 \\
\hline Congo & COG & 2011 & Standard DHS & 11632 & 51449 \\
\hline Democratic Republic of the Congo & COD & 2013 & Standard DHS & 18171 & 95949 \\
\hline Dominican Republic & DOM & 2013 & Standard DHS & 11464 & 41267 \\
\hline Ethiopia & ETH & 2016 & Standard DHS & 16650 & 75224 \\
\hline Ghana & GHA & 2017 & Standard DHS & 11835 & 43945 \\
\hline Guatemala & GTM & 2014 & Standard DHS & 21383 & 102510 \\
\hline Guinea & GIN & 2018 & Standard DHS & 7912 & 49543 \\
\hline Guyana & GUY & 2009 & Standard DHS & 5632 & 22845 \\
\hline Haiti & $\mathrm{HTI}$ & 2016 & Standard DHS & 13405 & 59547 \\
\hline Honduras & HND & 2011 & Standard DHS & 21362 & 100555 \\
\hline India & IND & 2015 & Standard DHS & 601509 & 2869043 \\
\hline Indonesia & IDN & 2017 & Standard DHS & 47963 & 197723 \\
\hline Kenya & KEN & 2014 & Standard DHS & 36430 & 153840 \\
\hline Kyrgyzstan & KGZ & 2012 & Standard DHS & 8040 & 35805 \\
\hline Lesotho & LSO & 2014 & Standard DHS & 9402 & 40197 \\
\hline Liberia & LBR & 2013 & Standard DHS & 9333 & 48219 \\
\hline Malawi & $\mathrm{MWI}$ & 2015 & Standard DHS & 26361 & 120492 \\
\hline Maldives & MDV & 2016 & Standard DHS & 6050 & 32656 \\
\hline Mali & MLI & 2018 & Standard DHS & 9510 & 54571 \\
\hline Nepal & NPL & 2016 & Standard DHS & 11040 & 49064 \\
\hline Niger & NER & 2012 & Standard DHS & 10750 & 64011 \\
\hline Nigeria & NGA & 2018 & Standard DHS & 40427 & 188010 \\
\hline Pakistan & PAK & 2017 & Standard DHS & 14540 & 100869 \\
\hline Peru & PER & 2012 & Continuous DHS & 27218 & 103211 \\
\hline Philippines & $\mathrm{PHL}$ & 2017 & Standard DHS & 27496 & 120273 \\
\hline Senegal & SEN & 2017 & Continuous DHS & 8380 & 78950 \\
\hline Sierra Leone & SLE & 2013 & Standard DHS & 12629 & 75299 \\
\hline South Africa & ZAF & 2016 & Standard DHS & 11083 & 38850 \\
\hline Timor-Leste & TLS & 2016 & Standard DHS & 11502 & 61496 \\
\hline Togo & TGO & 2013 & Standard DHS & 9549 & 46577 \\
\hline Uganda & UGA & 2016 & Standard DHS & 19588 & 91167 \\
\hline Viet Nam & VNM & 2005 & Standard AIS & 6337 & 26833 \\
\hline Zambia & ZMB & 2018 & Standard DHS & 12831 & 65454 \\
\hline Zimbabwe & ZWE & 2015 & Standard DHS & 10534 & 43706 \\
\hline
\end{tabular}




\section{A.2.3. Household age matrix (HAM) validation for POLYMOD and DHS countries}

In section A.2.2, we describe the methods used to project the HAM of a country.

We perform internal validation using leave-one-out validation to verify that the HAM describing household structure could be reverse-engineered for the POLYMOD and DHS countries for which empirical household age matrices were available. The steps involve projecting that country's household age structure as if it were unknown (i.e., following steps 1-3 described in section A.2.2) and then comparing against the empirical household data to assess the method's performance.

\section{A.3. Working population}

We allow the number of age-specific contacts made at the workplace to depend on the age structure of the workforce. To derive the working population matrices for each geographical location, we used the 2019 labour force participation rate by sex and 5-year age groups $w_{a}$ for the 177 geographical regions from the International Labour Organization (ILO) [7] to compute a joint distribution of the working population, $\mathbf{W}$. The working population, $\mathbf{W}^{c}$, a square matrix with elements describing the probability of encounters between two ages- $a$ and $\alpha$-in the workforce of country/geographical region $c$, is given by

$$
\mathbf{W}_{a, \alpha}^{c}=w_{a}^{c} \times w_{\alpha}^{c} .
$$

After constructing the working population distribution of ages $\mathbf{W}^{c}$, we project the age-specific contact patterns in the workplace for non-POLYMOD countries.

\section{A.4. School-going population}

Similarly, for age-specific contact patterns at school, we construct the age-specific school-going population, including teachers, before projecting the contact patterns in schools. Information on enrolment rates, age ranges of students, pupil-to-teacher ratios by education levels (i.e., pre-primary, primary, secondary, and tertiary) were obtained from UIS [6] and the distribution of teachers by age from Organisation for Economic Co-operation and Development (OECD) [8].

To project the school population, we perform the following steps:

1. Number of students estimation: We first estimate the proportion of students in each age interval using the country-specific enrolment rates and the starting and ending ages of students by education level. Together with the population age structure $P_{a}^{c}$, we then estimate the number of students $s_{a}^{c}$ aged $a$ in country $c$. We also estimate the number of students in each education level $l, s_{l}^{c}$, for the education levels pre-primary, primary, secondary, and tertiary.

2. Number of teachers estimation: After estimating the number of students for each education level in country $c, s_{l}^{c}$, we use the country-specific pupil-to-teacher ratio to estimate the number of teachers for each education level, $l, t_{l}^{c}$. Together with labour force participation rates by aged and the distribution of teachers by ages, we then estimate the number of teachers $t_{a}^{c}$ aged $a$ in country $c$.

3. School-going population construction: We project the school population distribution of ages, as follows:

$$
\mathbf{S}_{a, \alpha}^{c}=\left(\frac{s_{a}^{c}}{\sum_{i} s_{i}^{c}}+\frac{t_{a}^{c}}{\sum_{i} t_{i}^{c}}\right) \times\left(\frac{s_{\alpha}^{c}}{\sum_{i} s_{i}^{c}}+\frac{t_{\alpha}^{c}}{\sum_{i} t_{i}^{c}}\right) .
$$

4. The elements of $\mathbf{S}_{a, \alpha}^{c}$ present the probability of encounters between two ages within schools.

\section{A.5. Age- and location-specific contacts}

In Prem et al. (2017) [5], we employed a Bayesian hierarchical modelling framework to estimate modelling to estimate the proclivity of age- and location-specific contact patterns in the POLYMOD countries. The framework estimates both individual-level and population-level parameters and addresses the multi-level 
structure of the data, accounting for repeat measurements of contacts made in different settings by the same individual in the data. The parameter $\lambda_{a, \alpha}^{L}$ is the key estimand in the model, and it quantifies typical contact rates between individuals of age groups $a$ and $\alpha$ at location $L$. With the projected populations at home, work and school available for the 177 geographical regions (listed in Table B), we can now deduce the possible age- and location-specific contact matrices:

- $\quad$ Age-specific contacts at home: Given the projected HAM $\mathbf{h}_{a, \alpha}^{c}$ for country $c$, we can now deduce the possible age-specific contact patterns at home for country $\mathrm{c}$ to be:

$$
\mu_{a, \alpha}^{H, c}=\lambda_{a, \alpha}^{H} \times \mathbf{h}_{a, \alpha}^{c} .
$$

- Age-specific contacts in the workplace: Having projected the age-specific working populations for the 177 geographical regions $\mathbf{W}_{a, \alpha}^{c}$, we can infer the age-specific contact in the workplace for country $c$ by the following expression:

$$
\mu_{a, \alpha}^{W, c}=\lambda_{a, \alpha}^{W} \times \mathbf{W}_{a, \alpha}^{c} \times \frac{P_{\alpha}^{c}}{P_{\alpha}^{P O L Y M O D}} .
$$

- $\quad$ Age-specific contact in school: With the projected school-going population available for the 177 geographical regions $\mathbf{S}_{a, \alpha}^{c}$, we can now deduce the possible age-specific contact patterns in school for country c, as follows:

$$
\mu_{a, \alpha}^{S, c}=\lambda_{a, \alpha}^{S} \times \mathbf{S}_{a, \alpha}^{c} \times \frac{P_{\alpha}^{c}}{P_{\alpha}^{P O L Y M O D}} .
$$

- Age-specific contact at other locations: We estimate the possible age-specific contacts at other locations (i.e., non-home, work, or school) for country c, as follows:

$$
\mu_{a, \alpha}^{O, c}=\lambda_{a, \alpha}^{o} \times \frac{P_{\alpha}^{c}}{P_{\alpha}^{P O L Y M O D} .}
$$


Table B. Geographical regions included in the study.

\begin{tabular}{|c|c|c|c|}
\hline Regions & County code & Regions & County code \\
\hline Afghanistan & AFG & Ecuador & ECU \\
\hline Albania & ALB & Egypt & EGY \\
\hline Algeria & DZA & El Salvador & SLV \\
\hline Angola & AGO & Equatorial Guinea & GNQ \\
\hline Argentina & ARG & Eritrea & ERI \\
\hline Armenia & ARM & Estonia & EST \\
\hline Austria & AUT & Eswatini & SWZ \\
\hline Azerbaijan & AZE & Ethiopia & ETH \\
\hline Bahamas & BHS & Fiji & FJI \\
\hline Bahrain & BHR & Finland & FIN \\
\hline Bangladesh & BGD & France & FRA \\
\hline Barbados & BRB & Gabon & GAB \\
\hline Belarus & BLR & Gambia & GMB \\
\hline Belgium & BEL & Georgia & GEO \\
\hline Belize & BLZ & Germany & DEU \\
\hline Benin & BEN & Ghana & GHA \\
\hline Bhutan & BTN & Greece & GRC \\
\hline Bolivia (Plurinational State of) & BOL & Guatemala & GTM \\
\hline Bosnia and Herzegovina & $\mathrm{BIH}$ & Guinea & GIN \\
\hline Botswana & BWA & Guinea-Bissau & GNB \\
\hline Brazil & BRA & Guyana & GUY \\
\hline Brunei Darussalam & BRN & Honduras & HND \\
\hline Bulgaria & BGR & Hungary & HUN \\
\hline Burkina Faso & BFA & Iceland & ISL \\
\hline Burundi & $\mathrm{BDI}$ & India & IND \\
\hline Cabo Verde & CPV & Indonesia & IDN \\
\hline Cambodia & KHM & Iran (Islamic Republic of) & IRN \\
\hline Cameroon & CMR & Iraq & $\mathrm{IRQ}$ \\
\hline Canada & CAN & Ireland & IRL \\
\hline Central African Republic & CAF & Israel & ISR \\
\hline Chad & TCD & Italy & ITA \\
\hline Chile & $\mathrm{CHL}$ & Jamaica & JAM \\
\hline China & $\mathrm{CHN}$ & Jordan & JOR \\
\hline China, Hong Kong SAR & HKG & Kazakhstan & KAZ \\
\hline China, Macao SAR & MAC & Kenya & KEN \\
\hline Colombia & $\mathrm{COL}$ & Kuwait & KWT \\
\hline Comoros & COM & Kyrgyzstan & KGZ \\
\hline Congo & COG & Lao People's Democratic Republic & LAO \\
\hline Costa Rica & CRI & Latvia & LVA \\
\hline$C \sqrt{ } ¥$ te d'Ivoire & $\mathrm{CIV}$ & Lesotho & LSO \\
\hline Croatia & HRV & Liberia & LBR \\
\hline Cuba & CUB & Libya & LBY \\
\hline Cyprus & CYP & Lithuania & LTU \\
\hline Czechia & CZE & Luxembourg & LUX \\
\hline Dem. People's Republic of Korea & PRK & Madagascar & MDG \\
\hline Democratic Republic of the Congo & COD & Malawi & MWI \\
\hline Denmark & DNK & Malaysia & MYS \\
\hline Djibouti & DJI & Maldives & MDV \\
\hline Dominican Republic & DOM & Mali & MLI \\
\hline
\end{tabular}




\begin{tabular}{|c|c|c|c|}
\hline Regions & County code & Regions & County code \\
\hline Malta & MLT & Serbia & SRB \\
\hline Mauritania & MRT & Sierra Leone & SLE \\
\hline Mauritius & MUS & Singapore & SGP \\
\hline Mexico & MEX & Slovakia & SVK \\
\hline Mongolia & MNG & Slovenia & SVN \\
\hline Montenegro & MNE & Solomon Islands & SLB \\
\hline Morocco & MAR & South Africa & ZAF \\
\hline Mozambique & MOZ & South Sudan & SSD \\
\hline Myanmar & MMR & Spain & ESP \\
\hline Namibia & NAM & Sri Lanka & LKA \\
\hline Nepal & NPL & State of Palestine & PSE \\
\hline Netherlands & NLD & Sudan & SDN \\
\hline New Zealand & NZL & Suriname & SUR \\
\hline Nicaragua & $\mathrm{NIC}$ & Sweden & SWE \\
\hline Niger & NER & Switzerland & $\mathrm{CHE}$ \\
\hline Nigeria & NGA & Syrian Arab Republic & SYR \\
\hline North Macedonia & MKD & Tajikistan & TJK \\
\hline Norway & NOR & Thailand & THA \\
\hline Oman & OMN & Timor-Leste & TLS \\
\hline Pakistan & PAK & Togo & TGO \\
\hline Panama & PAN & Tonga & TON \\
\hline Papua New Guinea & PNG & Trinidad and Tobago & TTO \\
\hline Paraguay & PRY & Tunisia & TUN \\
\hline Peru & PER & Turkey & TUR \\
\hline Philippines & $\mathrm{PHL}$ & Turkmenistan & TKM \\
\hline Poland & POL & Uganda & UGA \\
\hline Portugal & PRT & Ukraine & UKR \\
\hline Puerto Rico & PRI & United Arab Emirates & ARE \\
\hline Qatar & QAT & United Kingdom & GBR \\
\hline Republic of Korea & KOR & United Republic of Tanzania & TZA \\
\hline Republic of Moldova & MDA & United States of America & USA \\
\hline Romania & ROU & Uruguay & URY \\
\hline Russian Federation & RUS & Uzbekistan & UZB \\
\hline Rwanda & RWA & Vanuatu & VUT \\
\hline Saint Lucia & LCA & Venezuela (Bolivarian Republic of) & VEN \\
\hline Saint Vincent and the Grenadines & VCT & Viet Nam & VNM \\
\hline Samoa & WSM & Yemen & YEM \\
\hline Sao Tome and Principe & STP & Zambia & ZMB \\
\hline Saudi Arabia & SAU & Zimbabwe & ZWE \\
\hline Senegal & SEN & & \\
\hline
\end{tabular}




\section{A.6. Age- and location-specific contacts by rural and urban areas}

The population age structure at the various locations-in the household, workplaces, schools, general community - varies by rural and urban subregions of country. Hence, we would expect the contact patterns within a country to also vary by rural and urban subregions. To account for these differences, we also stratified the age- and location-specific contact matrices by rural-urban areas.

The United Nations Population Division provides the estimation of rural and urban population age compositions for the 177 geographical regions [2]. The nationally-representative DHS household surveys provide data for rural and urban areas, allowing us to derive rural-urban HAM and project rural-urban HAM for the countries with no available household data (following steps 1-3 described in section A.2.2). ILO provides the age-specific labour force participation rates by rural and urban regions [9], allowing us to determine the working population age structure, $\mathbf{W}_{a, \alpha}^{c}$, for country $c$ by rural-urban subregions. Using the differences in rural and urban schools' pupil-to-teacher from the OECD [10], we construct rural and urban school-going population age structures, $\mathbf{S}_{a, \alpha}^{c}$.

After projecting the populations at home, work and school for rural and urban subregions, we can then deduce the possible age- and location-specific contact matrices following the steps detailed in section A.5.

\section{A.7. Age-stratified compartmental model of the physical distancing interventions for COVID-19}

We adapt a discrete-time Susceptible-Exposed-Infectious-Recovered (SEIR) compartmental model [11,12] presented in Figure A. The model population is closed, and it ignores all demographic changes in the population (i.e., births, deaths, and ageing). The model stratifies the population into (i) disease states: susceptible, exposed (infected but not yet infectious), infectious (i.e., preclinical, clinical, or subclinical), and recovered (or died/removed) states; and (ii) 5-year age groups until age 70 years and a single category aged $\geq 75$ years, resulting in 16 age bands.

We implement the model stochastically and allow it to select random values of parameters from the set of uncertainty distributions presented in Table C , adapted from $[11,12]$.

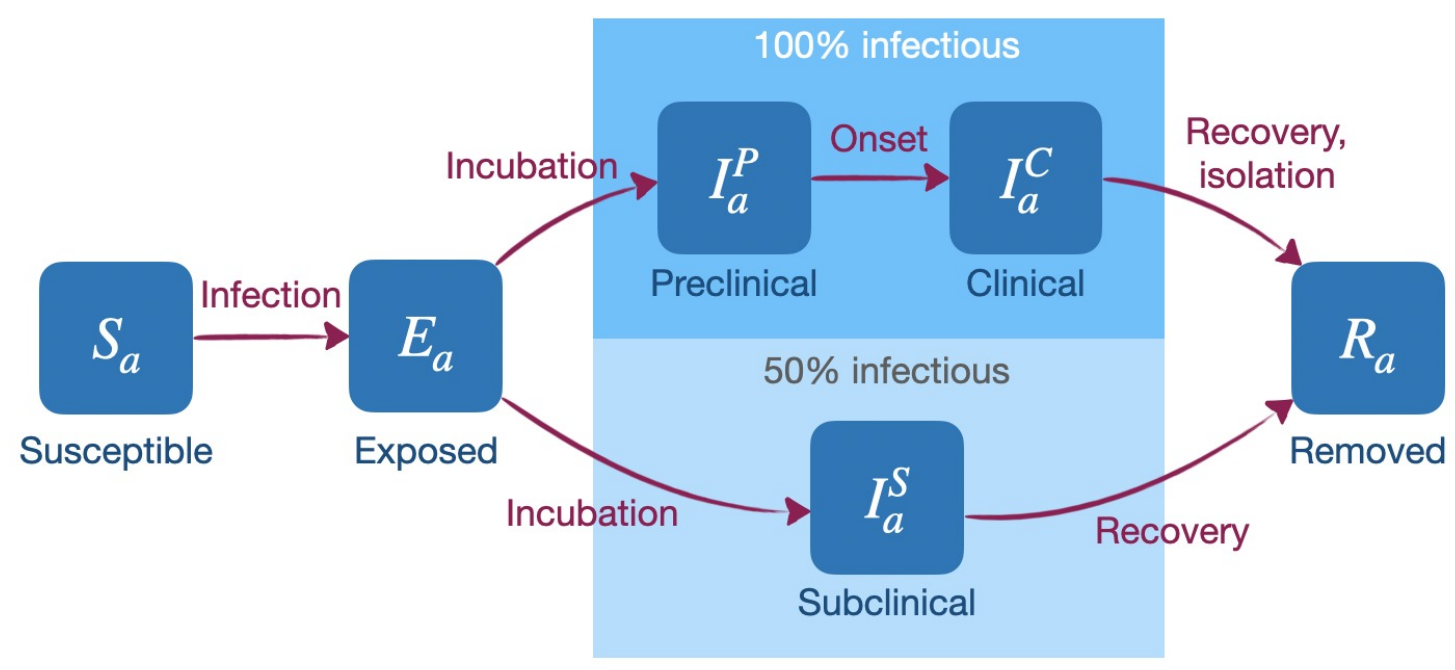

Figure A. Age-stratified stochastic SEIR model. Adapted from Davies et al. (2020) [11]. 
Table C. Parameters of the age-stratified SEIR model.

\begin{tabular}{|c|c|c|c|}
\hline & Description & Values & References \\
\hline$\Delta t$ & Time step for simulation & 0.25 days or 6 hours & \\
\hline \multirow[t]{2}{*}{$d_{E}$} & Latent period in days & $d_{E} \sim \operatorname{Gamma}(4,4)$ & {$[14-16]$} \\
\hline & Duration of infectiousness in days & & \\
\hline$d_{P}$ & (i) during the preclinical phase & $d_{P} \sim \operatorname{Gamma}(4,4)$ & {$[17]$} \\
\hline$d_{C}$ & (ii) during the clinical phase & $d_{C} \sim \operatorname{Gamma}(4,4)$ & {$[14-16]$} \\
\hline$d_{S}$ & (iii) during the subclinical phase & $d_{S} \sim \operatorname{Gamma}(4,4)$ & $\begin{array}{l}\text { Assumed to be the same as } \\
\text { the total duration of } \\
\text { infectiousness for clinical } \\
\text { cases (i.e., } d_{P}+d_{C} \text { ) }\end{array}$ \\
\hline$y_{a}$ & $\begin{array}{l}\text { Probability of clinical symptoms on infection for } \\
\text { individuals aged } a\end{array}$ & $\begin{array}{l}\text { Age-dependent, as } \\
\text { estimated in }[18 \%]\end{array}$ & [18] \\
\hline$f$ & Relative infectiousness of subclinical cases & $50 \%$ & Assumed \\
\hline$u$ & $\begin{array}{l}\text { Probability of transmission per contact with an } \\
\text { infectious individual }\end{array}$ & Refer to text & Derived \\
\hline$N_{a}$ & Number of individuals aged $a$ & Demographic data & [1] \\
\hline$c_{a, \alpha}$ & $\begin{array}{l}\text { Number of contacts per day with individuals } \\
\text { aged } \alpha \text { by an individual age } a\end{array}$ & $\begin{array}{l}\text { Empirical and synthetic } \\
\text { contact matrices }\end{array}$ & \\
\hline \multirow[t]{7}{*}{$R_{0}$} & Basic reproduction number & $R_{0} \sim \operatorname{Normal}\left(2.6,0.5^{2}\right)$ & [13] \\
\hline & $\begin{array}{l}\text { Proportion of hospitalised cases requiring critical } \\
\text { care } \\
\text { Duration of disease in days }\end{array}$ & $30 \%$ & [20] \\
\hline & (i) severe, non-critical disease & & NHS Digit \\
\hline & (ii) severe, critical disease & $\sim \operatorname{Gamma}(10,10)$ & {$[20]$} \\
\hline & Delay from symptom onset & & \\
\hline & (i) to becoming a severe case (days) & $\sim \operatorname{Gamma}(7,7)$ & {$[19,20]$} \\
\hline & (ii) to death (days) & $\sim \operatorname{Gamma}(22,22)$ & {$[19,21]$} \\
\hline
\end{tabular}

As described in [11,12], susceptible individuals might acquire the infection when they come in contact with an infectious person and enter the exposed disease state before they become infectious and later either recover or die. The time step of the model is 6 hours. We assume age-specific mixing patterns of individuals alter their likelihood of being exposed to the virus given a certain number of infectious people in the population. The force of infection defined as the rate at which susceptible individuals become exposed $t$, and is given by for any age group $a$ and time increment $t$ as

$$
\lambda_{a, t}=u \times \sum_{\alpha} c_{a, \alpha} \times \frac{I_{\alpha}^{P}+I_{\alpha}^{C}+f \times I_{\alpha}^{S}}{N_{\alpha}}
$$

where $u$ is the probability of transmission per contact with an infectious person, $c_{a, \alpha}$ denotes the number of contacts per day with individuals aged $\alpha$ by an individual in age group $a$ per time increment (drawn from the contact matrix), and $\frac{\left(I_{\alpha}^{P}+I_{\alpha}^{C}+f I_{\alpha}^{S}\right)}{N_{\alpha}}$ is the probability that any age $\alpha$ individual contacted is infectious, with $f$ denoting the relative infectiousness of subclinical cases, compared to clinical cases. The basic reproduction number $R_{0}$ is defined as the average number of secondary infections generated by an infectious individual in a fully susceptible population, we calculated it as the absolute value of the dominant eigenvalue of the next generation matrix, which was derived by linearising the system at epidemic equilibrium [13]. For any stochastic run, $u$ is derived from the ratio of this eigenvalue and the $R_{0}$ value selected for that run. We considered six contact matrices when modelling the interventions to the COVID-19 pandemic: the empirically-constructed contact matrices at the study-year and adjusted for the 2020 population, the 2017 synthetic matrices, and the updated synthetic matrices at the national, rural, or urban settings.

The duration (in days) an individual spends in states $E, I P, I C$, or $I S$ is drawn from distributions $d_{E}, d_{P}, d_{C}$ and $d_{S}$, respectively (Table C) [14-17]. After the latency period, infected individuals are divided into clinical (symptomatic) and sub-clinical (asymptomatic) states with probability $y_{a}$ and $1-y_{a}$, respectively [18]. Clinical individuals will first experience a preclinical phase where they are still infectious (denoted as $I^{P}$ in Figure A), followed by a clinical and infectious phase (denoted as $I^{C}$ in Figure A). We assume that 
subclinical individuals, represented as $I^{S}$ in Figure A, are half as infectious as clinical cases. Clinical and subclinical individuals have the same duration of infectiousness, and we assume that their clinical severity does not affect their infectiousness. These individuals will later recover or die, and we assume that all individuals who have recovered (or have left the infectious phase) are immune until the end of the simulations. We account for delays in the transitions to different clinical disease states (such as symptom onset to severe case, critical or not; and from onset of severe symptoms to recovery or death) [19-21]. These delays are drawn from distributions reported in the literature; however, they do not change the force of infection or transmissibility.

\section{A.8. Hierarchical model of POLYMOD contact data}

To address the multi-level structure of the data, with repeat measurements of contacts made in different settings by the same individual, we employed Bayesian hierarchical modelling to estimate the proclivity of age-specific and location specific contact patterns in each of the POLYMOD countries, as this provided a flexible framework to estimate both individual-level and population-level parameters.

The number of contacts made by individual $i$ at a particular location $L$ with someone in age group $\alpha, X_{i, \alpha}^{L}$, is modelled to be Poisson with mean $\mu_{i, \alpha}^{L}$,

$$
X_{i, \alpha}^{L} \sim \operatorname{Po}\left(\mu_{i, \alpha}^{L}\right)
$$

where

- $\quad$ the ages of individual $i, a_{i}$, and his contact, $\alpha$, are categorised into 5 -year age intervals, $\{1,2, \ldots, 16\}$; and

- $\quad L$ indicates the location of the contact namely home $(\mathrm{L}=\mathrm{H})$, work $(\mathrm{W})$, school $(\mathrm{S})$ and other $(\mathrm{O})$.

The Poisson mean parameter has the general form $\mu_{i, \alpha}^{L}=\sigma_{i} \lambda_{a_{i}, \alpha}^{L}$ and varies across locations. In this model, $\sigma_{i}$ is a random effect belonging to individual $i$ which characterises differences in social activity levels across locations and allows for greater than Poisson variability in the number of contacts.

The mean was $\mu_{i, \alpha}^{H}=\sigma_{i} \lambda_{a_{i}, \alpha}^{H}\left(v_{i, \alpha}+\delta_{H}\right)$ for home contacts, $\mu_{i, \alpha}^{W}=\sigma_{i} \lambda_{a_{i}, \alpha}^{W}\left(w_{i}+\delta_{W}\right)$ for work contacts, $\mu_{i, \alpha}^{S}=$ $\sigma_{i} \lambda_{a_{i}, \alpha}^{S}\left(s_{i}+\delta_{S}\right)$ for school contacts and $\mu_{i, \alpha}^{O}=\sigma_{i} \lambda_{a_{i}, \alpha}^{O}$ for contacts at all other locations. The number of cohabitants of $i$ of age $\alpha, v_{i, \alpha}$, represents household age structure, while $w_{i}$ and $s_{i}$ indicate if $i$ went to work or school on the day of the survey. Contact with visitors at home, workplace or school is allowed via background contact parameters, $\delta_{H}, \delta_{W}$ and $\delta_{S}$. The parameter $\lambda_{a_{i}, \alpha}^{L}$ quantifies typical contact rates between individuals of age groups $a_{i}$ and $\alpha$ at location $L$ and is the key estimand in the model.

Prior distribution of parameters: The $\lambda_{a_{i}, \alpha}^{L}$ parameter was given a hierarchical prior to impose smoothness between successive age groups, i.e.:

$$
\log \lambda_{a_{i}, \alpha}^{L}=\sum_{\mathcal{A}, A: \mathcal{A}, A \in \mathcal{N}_{a_{i}, \alpha}} \frac{\epsilon_{\mathcal{A}, A}^{L}}{\left|\mathcal{N}_{a_{i}, \alpha}\right|}
$$

where $\epsilon_{\mathcal{A}, A}^{L}$ is a hyperparameter of $\lambda_{a_{i, \alpha},}^{L}$ and $\mathcal{N}_{a_{i}, \alpha}$ is the set of (up to) four adjacent age groups together with $\left(a_{i}, \alpha\right)$ itself (presented in Figure $\mathbf{B}$ ). The adjacent age groups (light blue regions) together with $\left(a_{i}, \alpha\right)$ (dark blue regions i.e., $\mathrm{A}, \mathrm{B}$, and $\mathrm{C}$ ) are elements of $\mathcal{N}_{a_{i}, \alpha}$. 


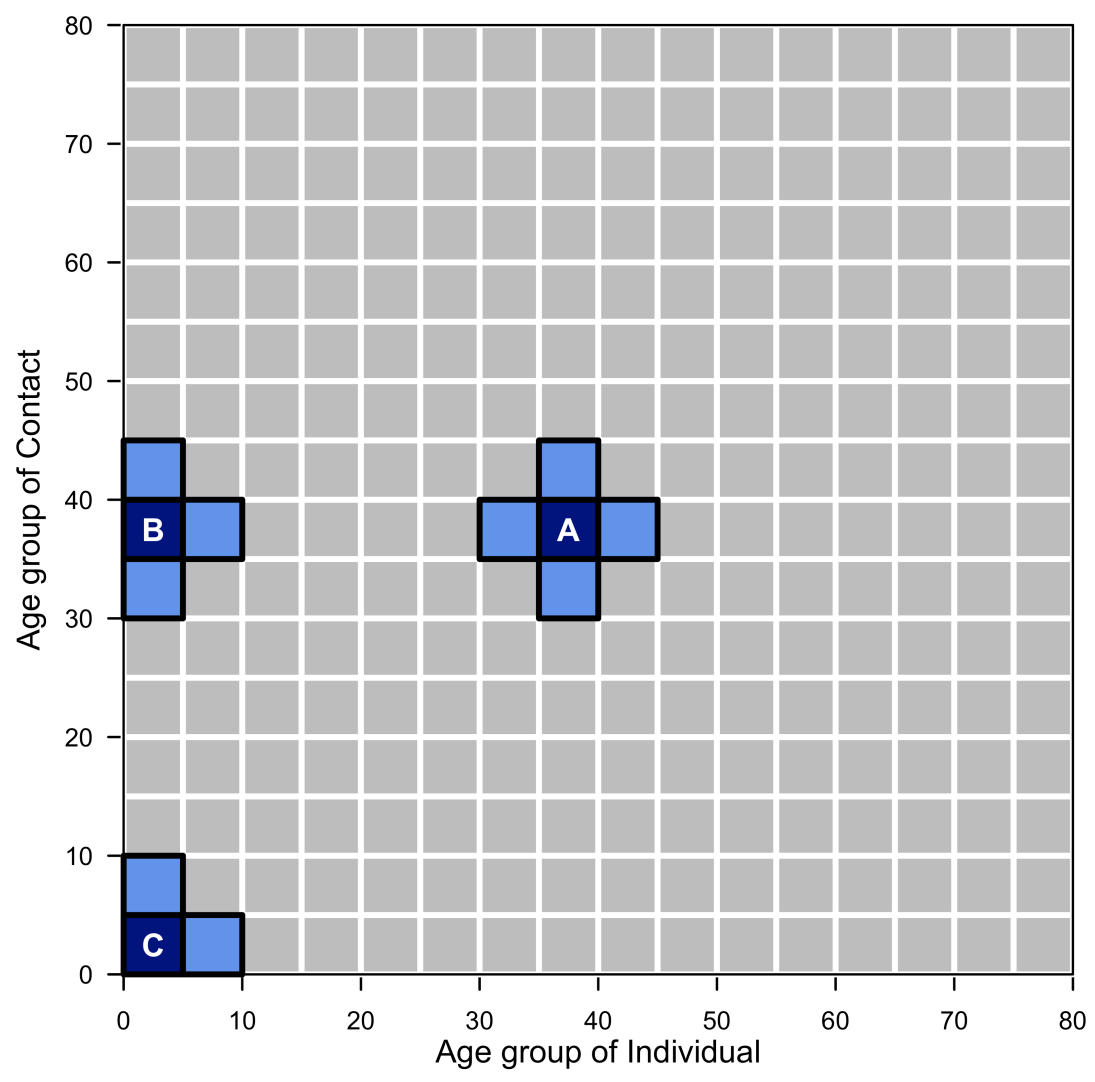

Figure B. Set of up to four adjacent age groups.

Contact surveys conducted in several countries [3] indicate strong assortativity of social contacts with age. Hence, to allow the number of contacts to be comparable for individuals of similar ages, the hierarchical prior was designed to impose smoothness between successive age groups (right panel of Figure $\mathbf{C}$ ). This smoothness on the prior distribution of the parameter $\lambda_{a_{i}, \alpha}^{L}$ to allow the number of contacts to be comparable for individuals of similar ages (Figure $\mathbf{C}$ ). The hyperparameter $\epsilon_{\mathcal{A}, A}^{L}$ has a non-informative prior distribution of Normal $\sim\left(0,100^{2}\right)$ as represented on the left of Figure $\mathbf{C}$. 

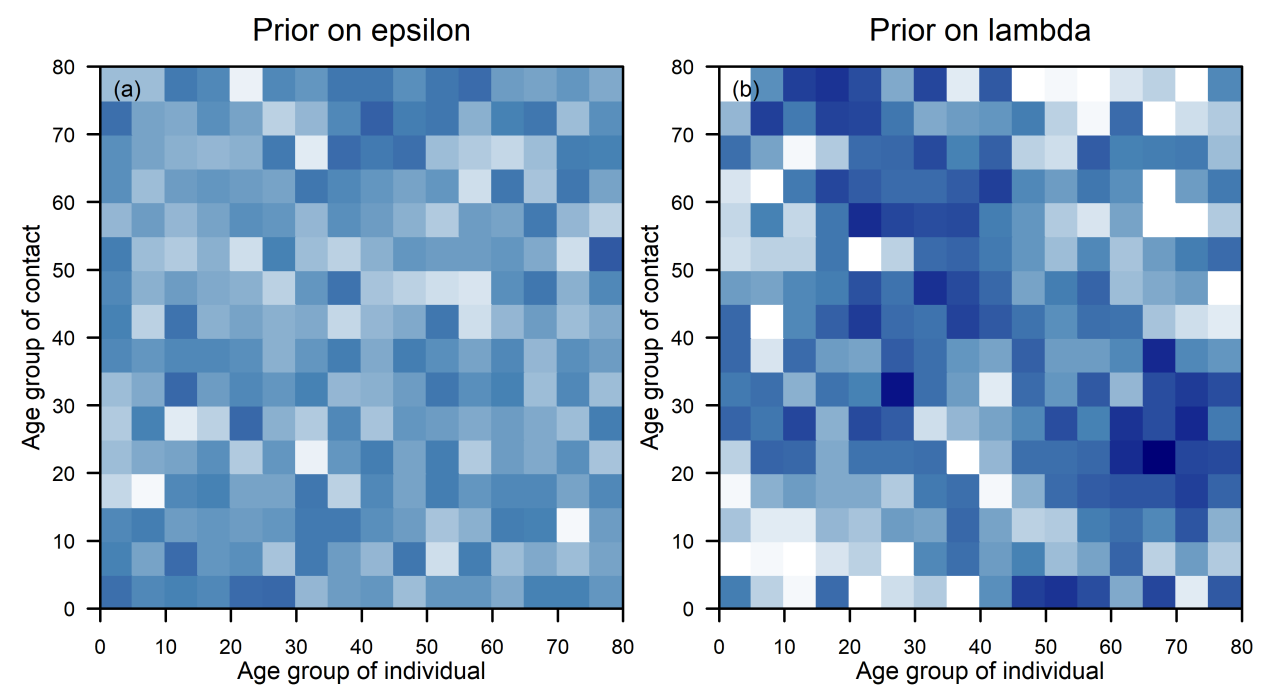

Figure C. Smoothness between successive age groups on the prior distribution.

Throughout, we used non-informative prior distributions for all parameters and hyperparameters in the model, presented in Prem et al. (2017) [5], unless otherwise noted.

\section{B. Results}

\section{B.1. Household age matrix (HAM) validation for POLYMOD and DHS countries}

The empirical household age structures (i.e., from the data) and the modelled household age structures (i.e., the leave-one-out validation, assuming no available household data) for each POLYMOD and DHS countries are represented in the first and second panels respectively of the figures in this section. In the third panel, a scatter plot of the entries in the observed (x-axis) and modelled (y-axis) HAM. Using the methods described in section A.2.2, we reconstructed the empirical household age structures for the POLYMOD and DHS countries with high fidelity (median correlation between the observed and modelled HAM 0.92, with an interquartile range 0.85-0.95). 
HAM of Belgium
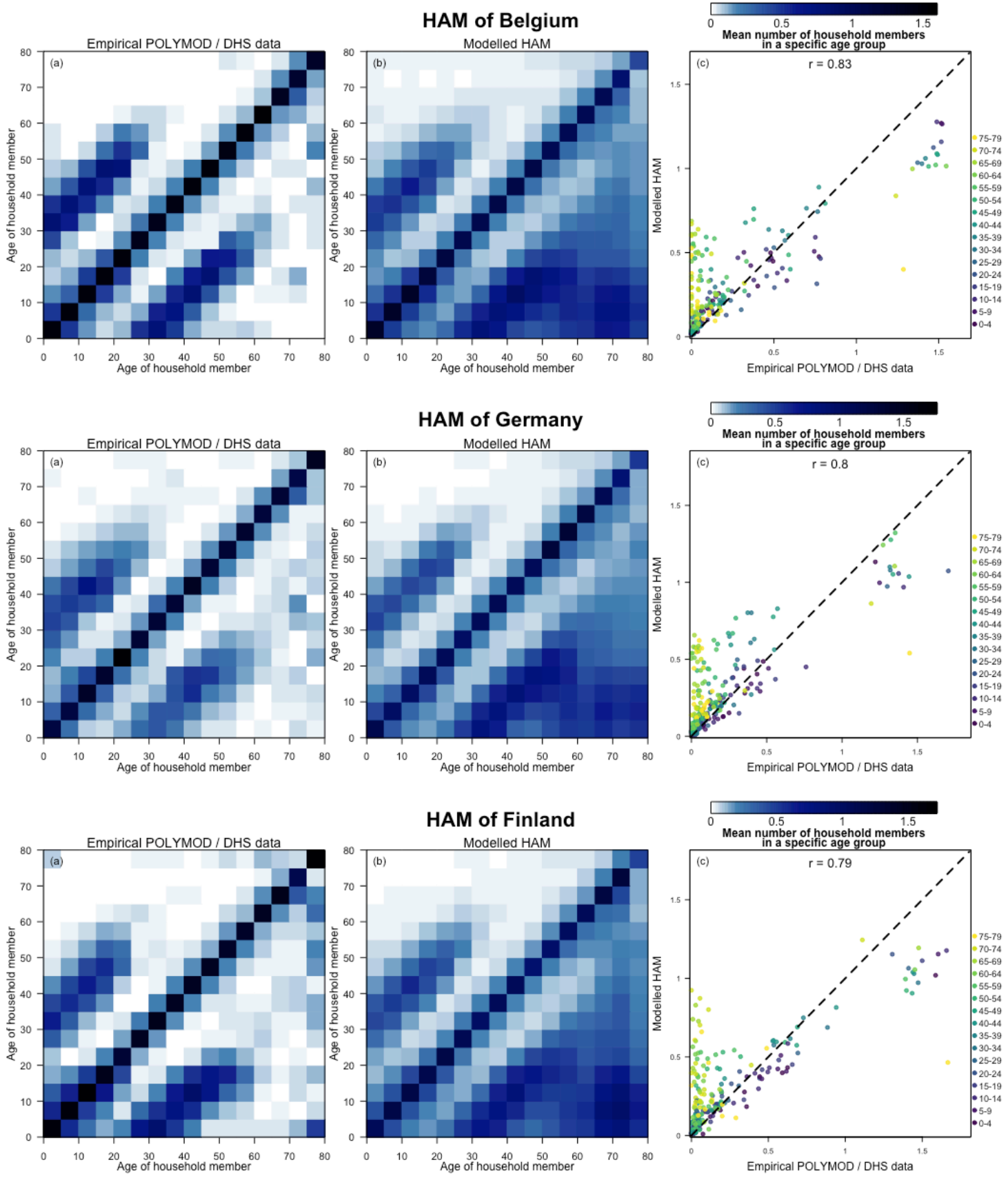
HAM of United Kingdom
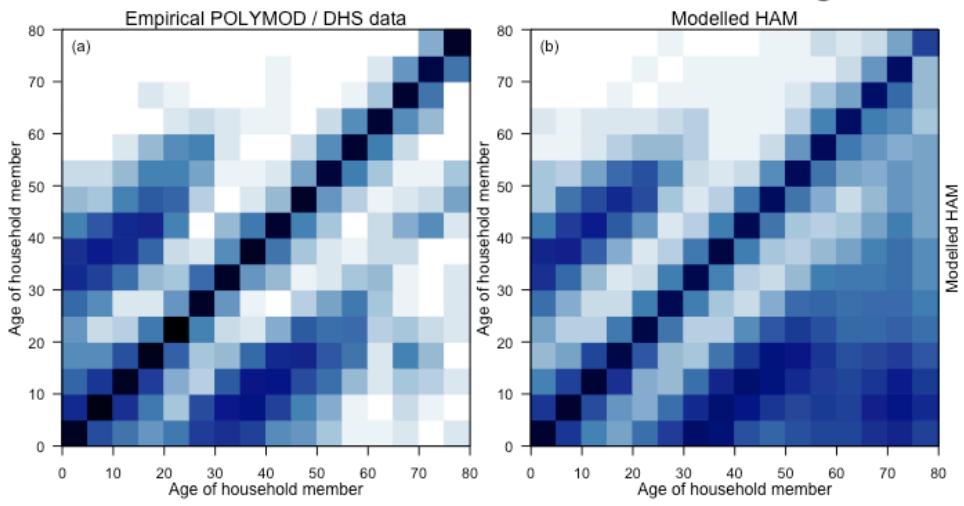

HAM of Italy
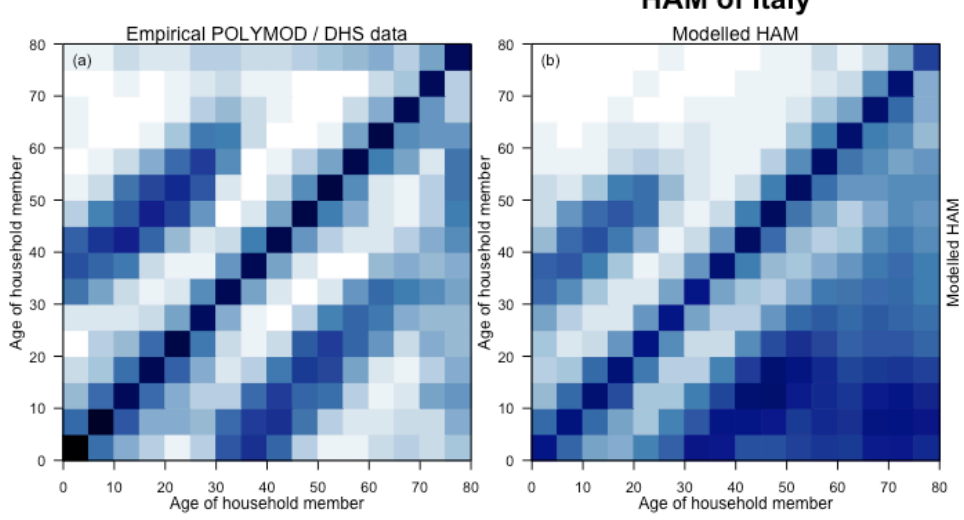

HAM of Luxembourg

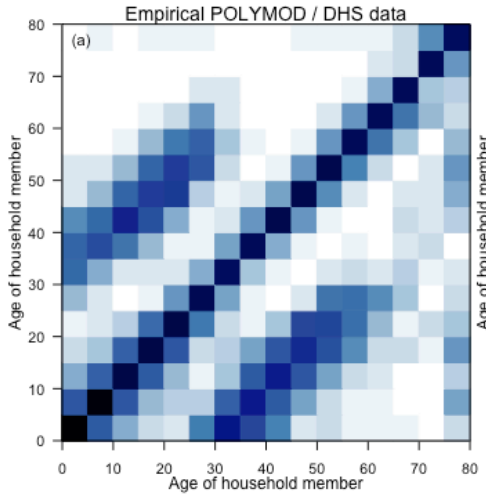

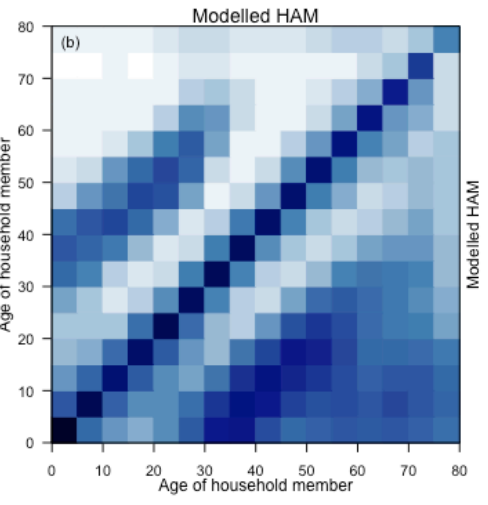
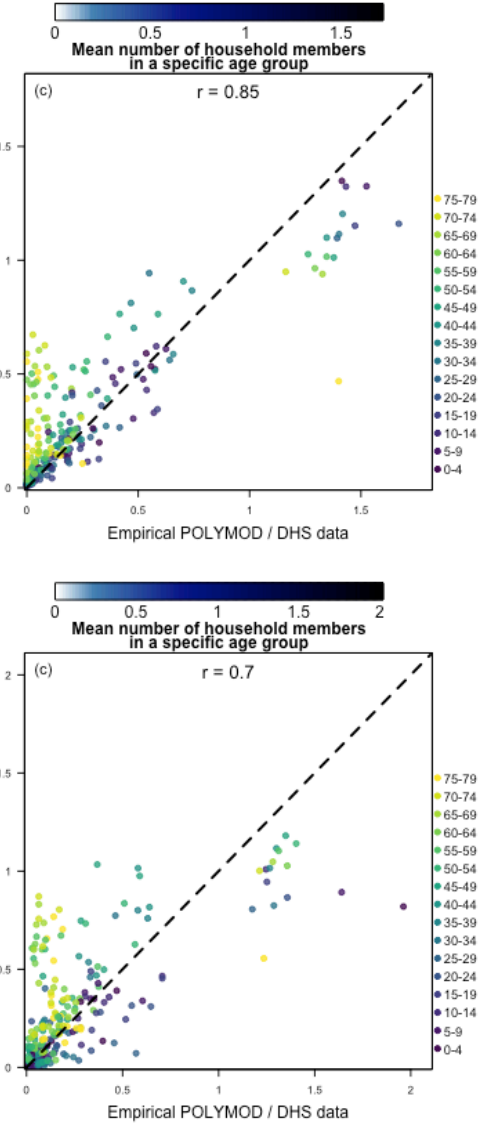

Empirical POLYMOD / DHS data

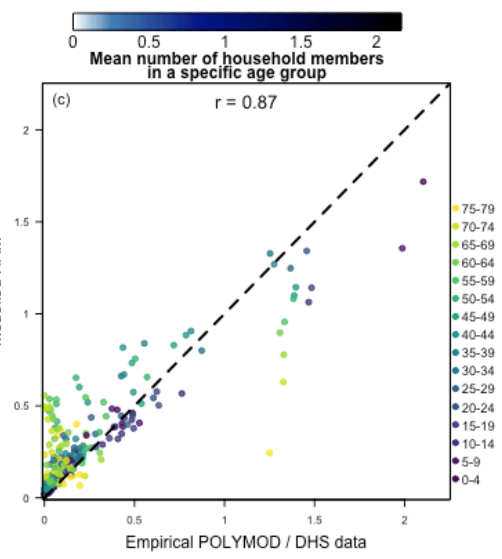


HAM of Netherlands
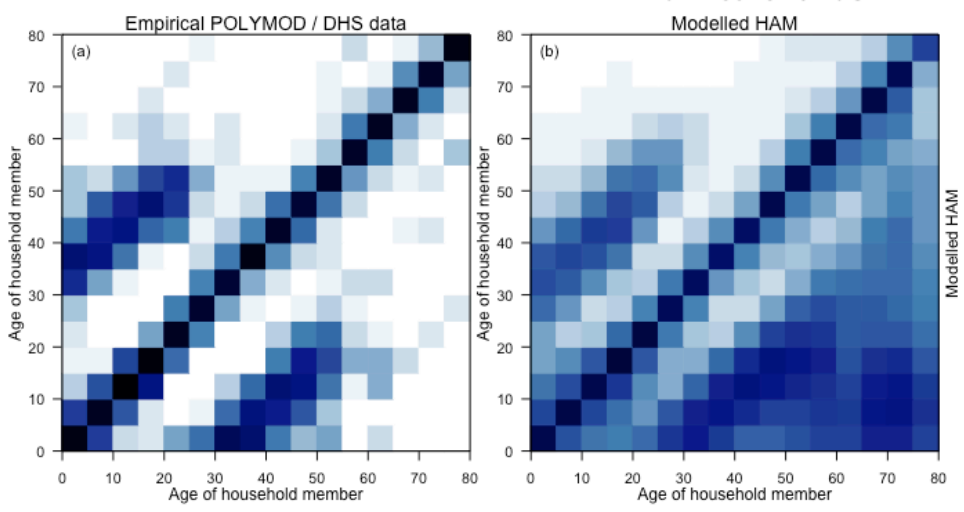

HAM of Poland
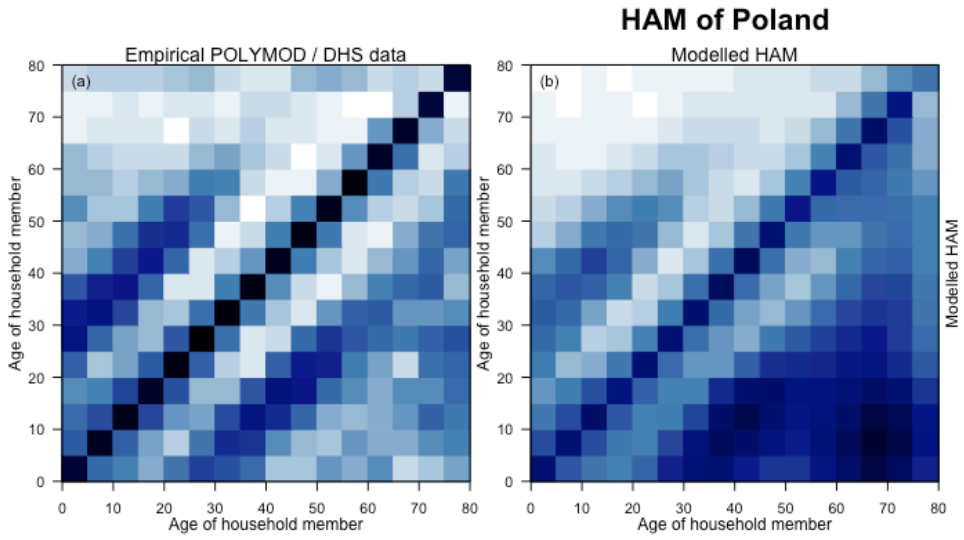

HAM of Afghanistan

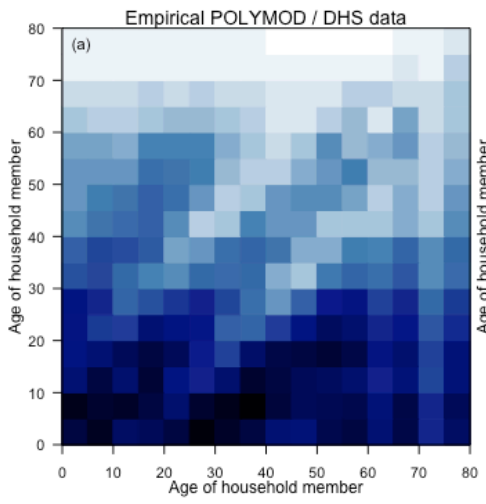
Modelled HAM

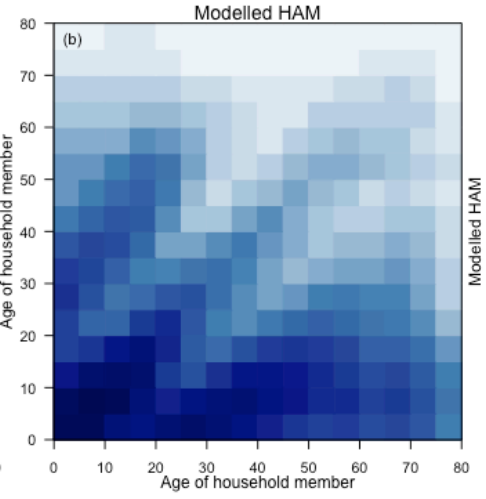

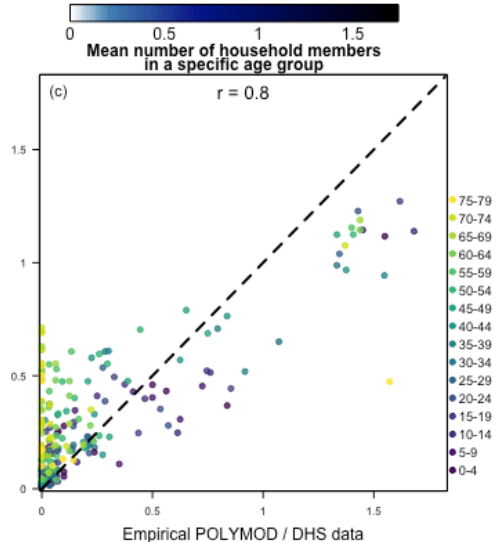
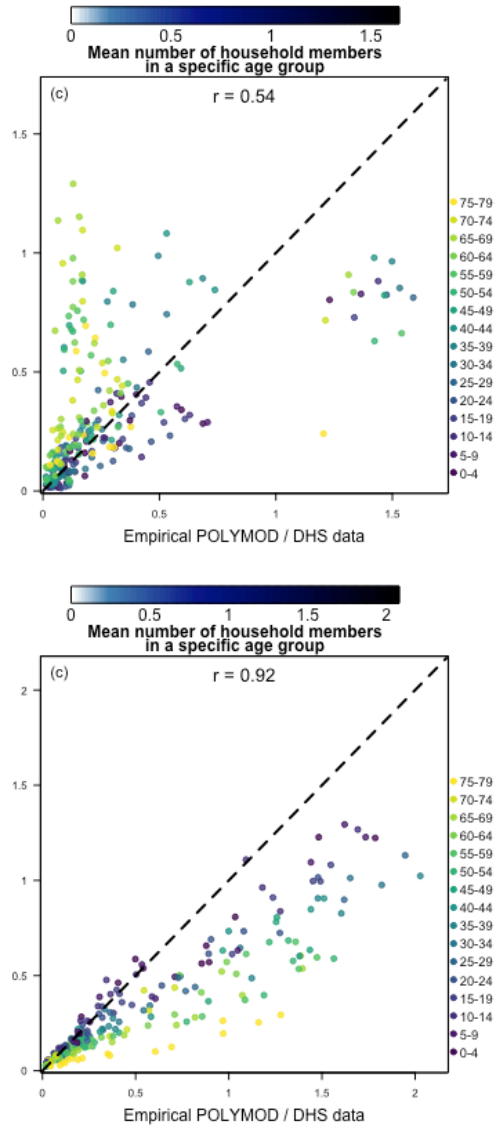

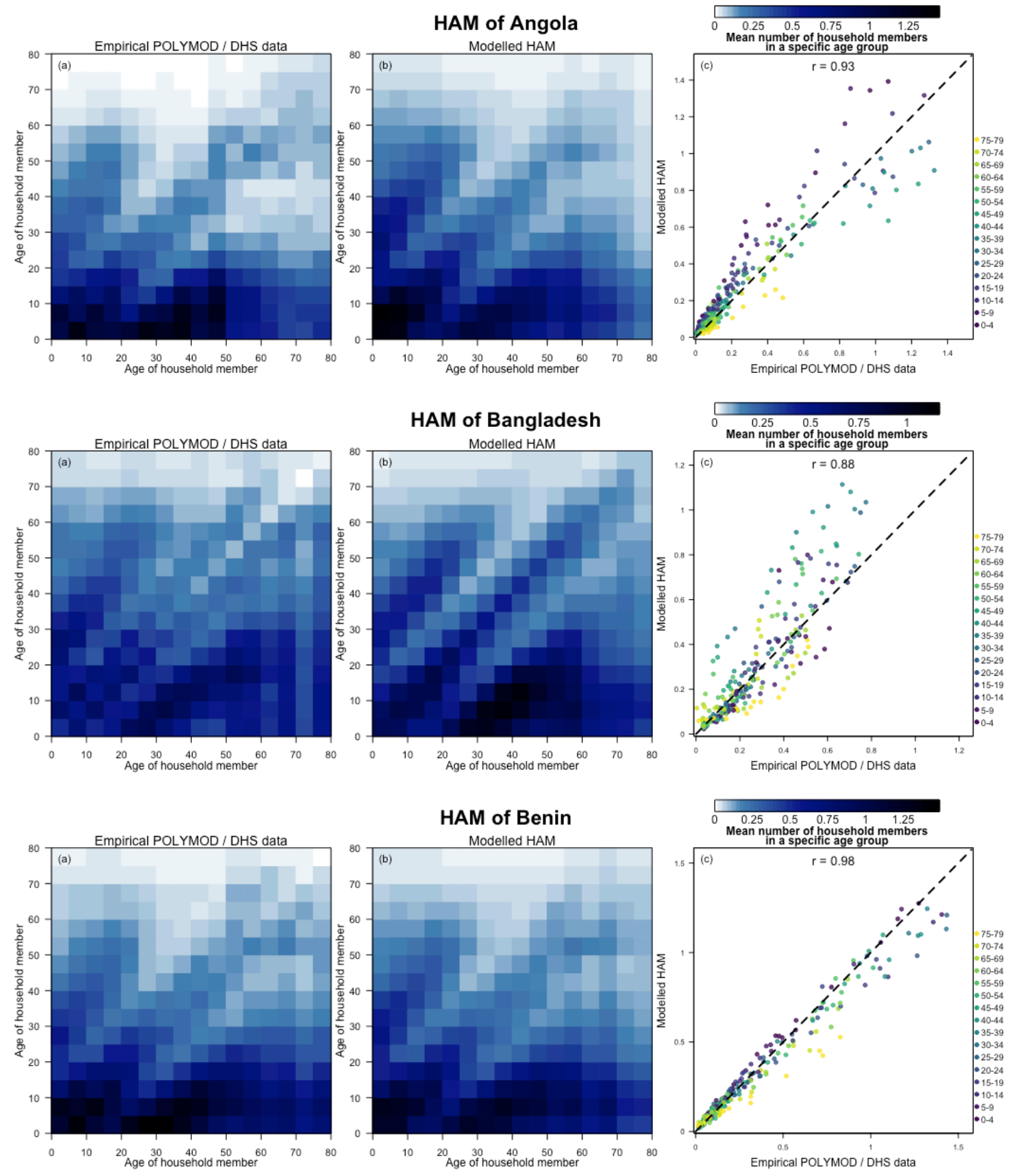


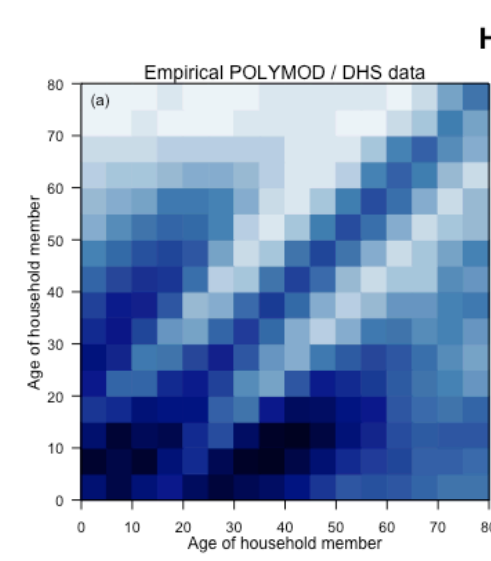

HAM of Bolivia (Plurinational State of)
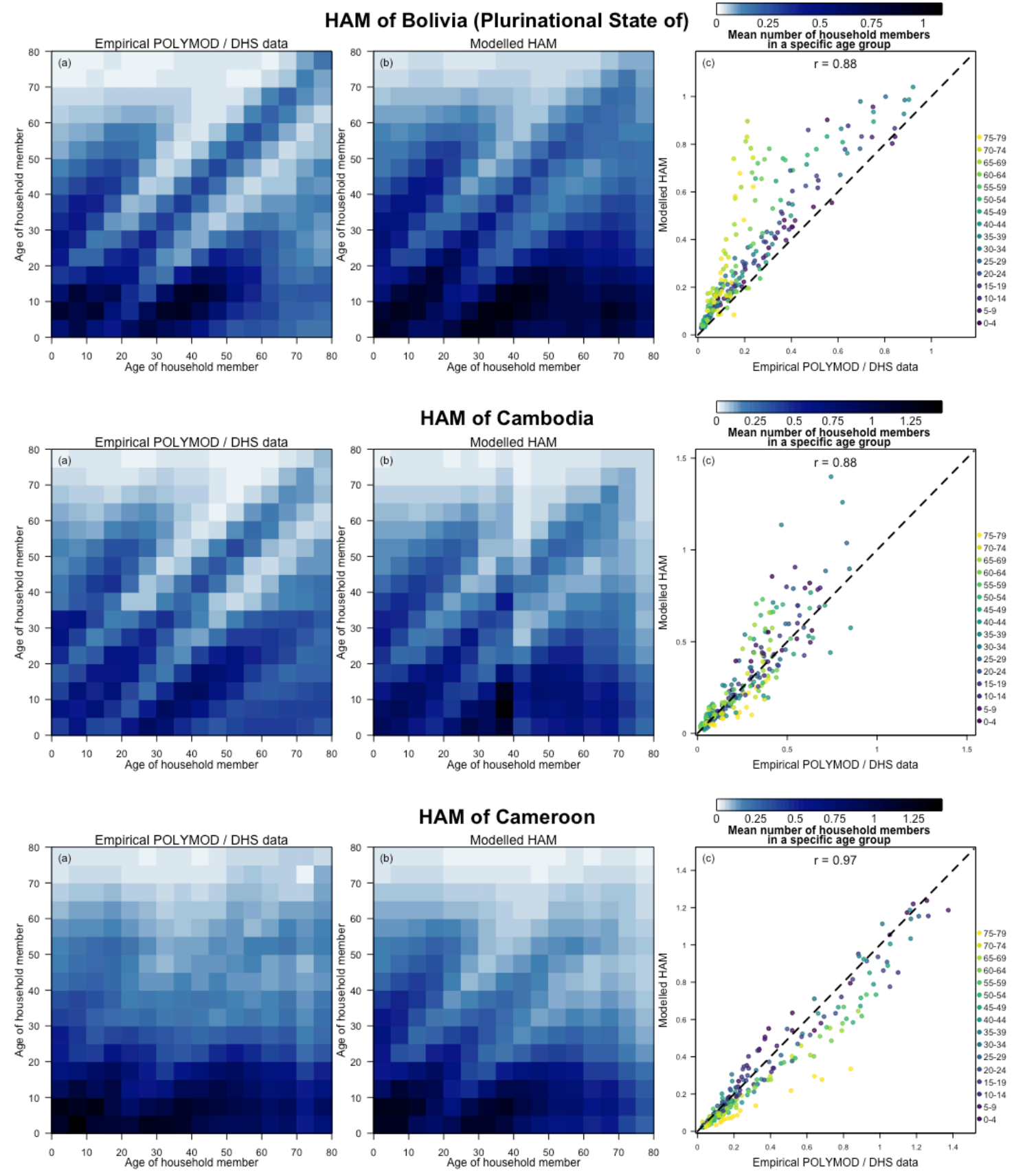

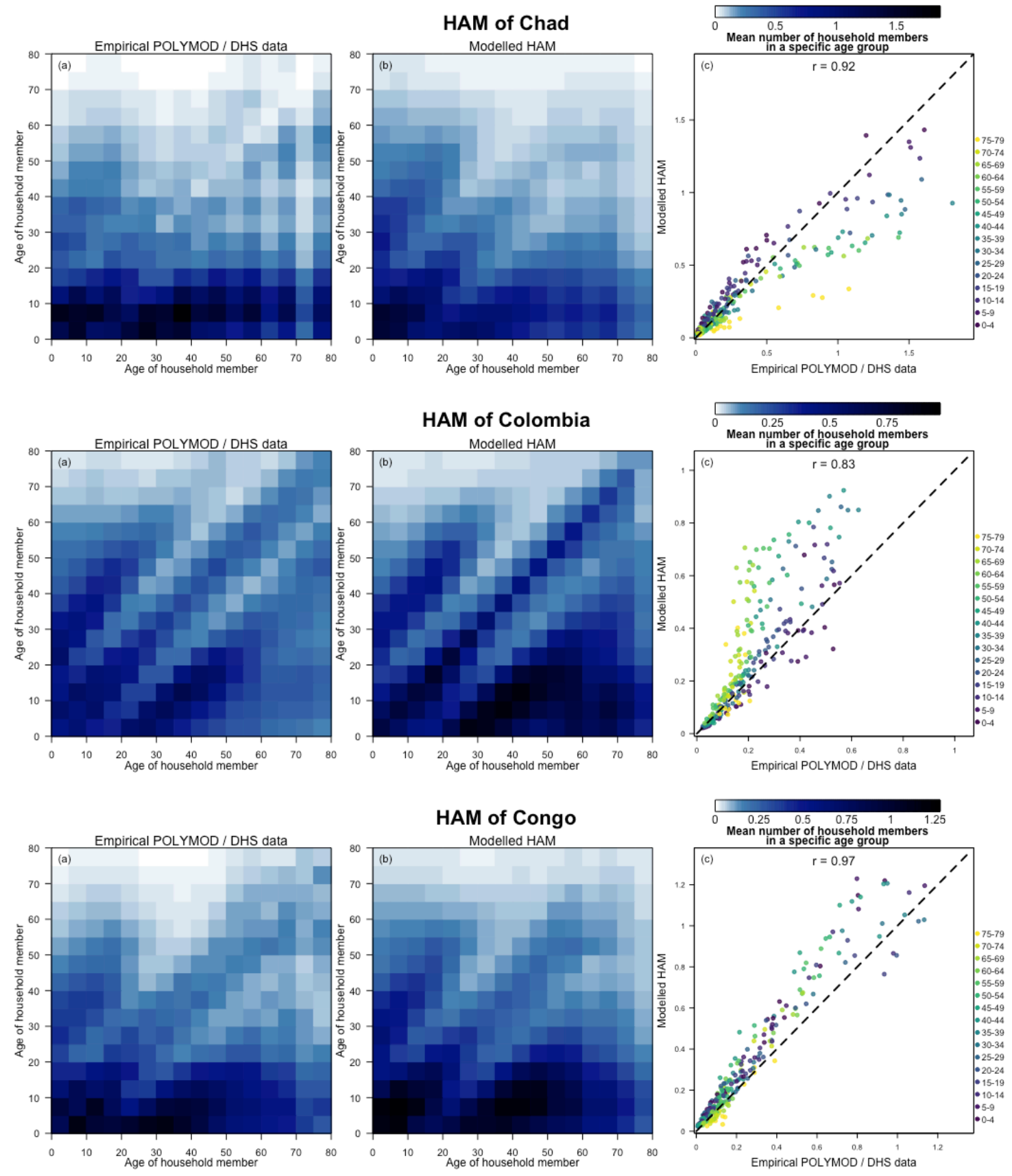


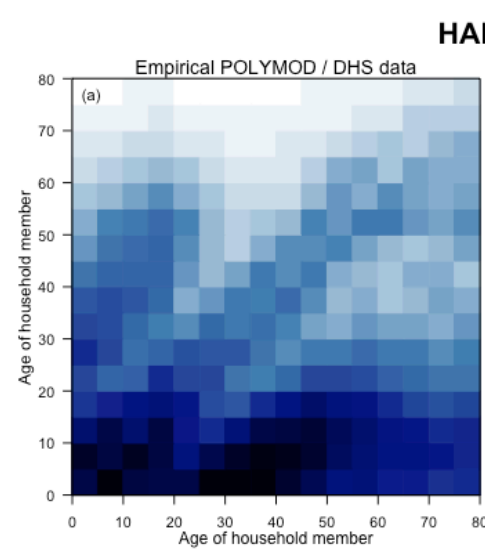

HAM of Democratic Republic of the Congo
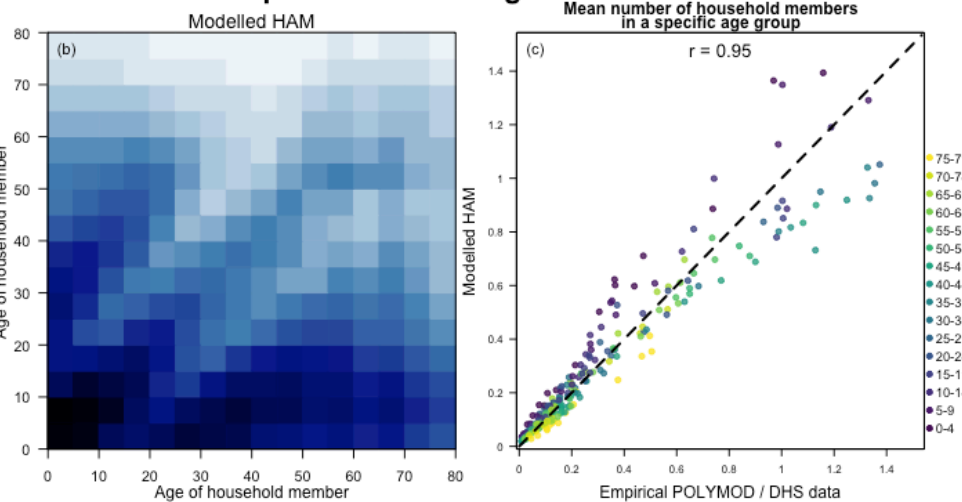

HAM of Dominican Republic
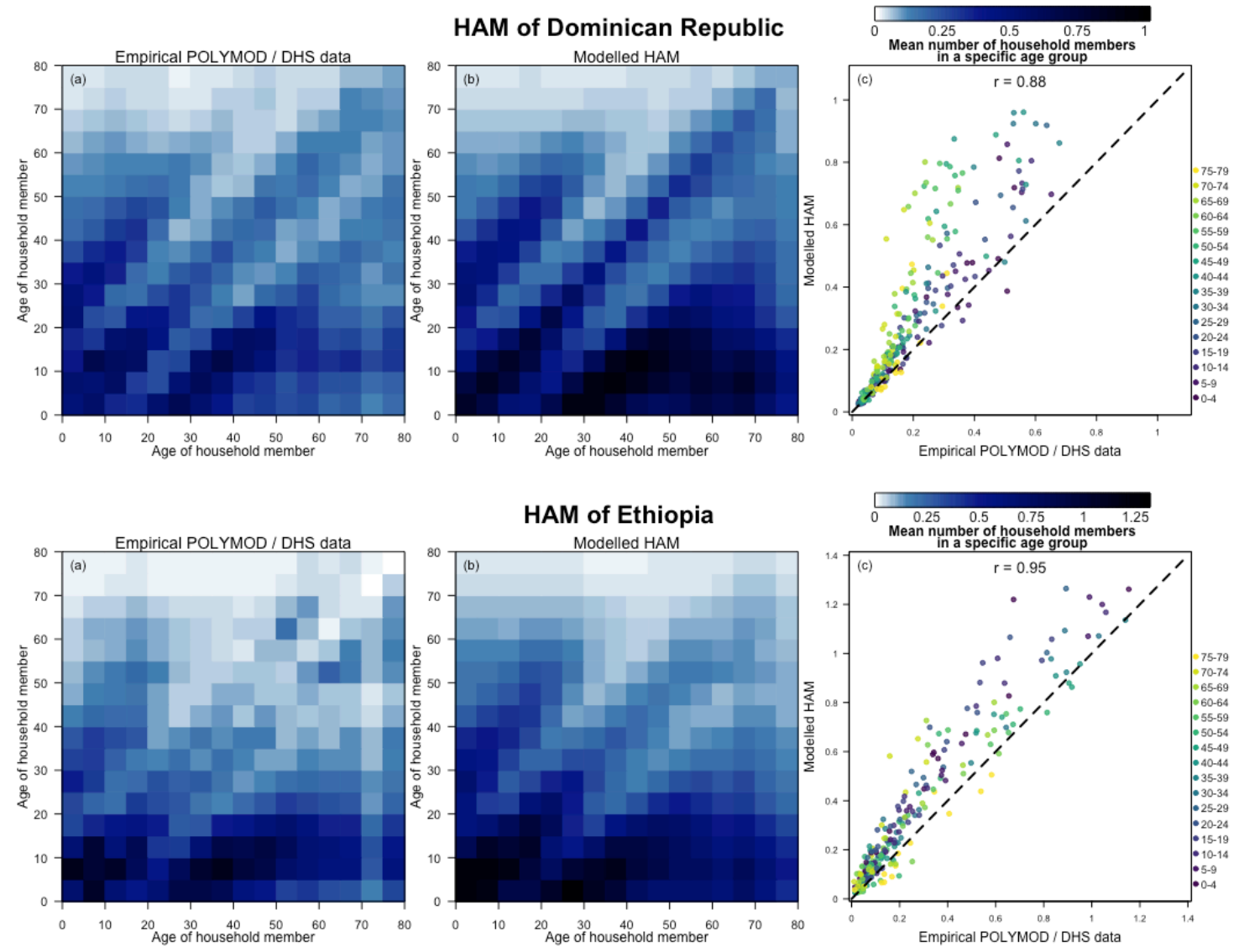

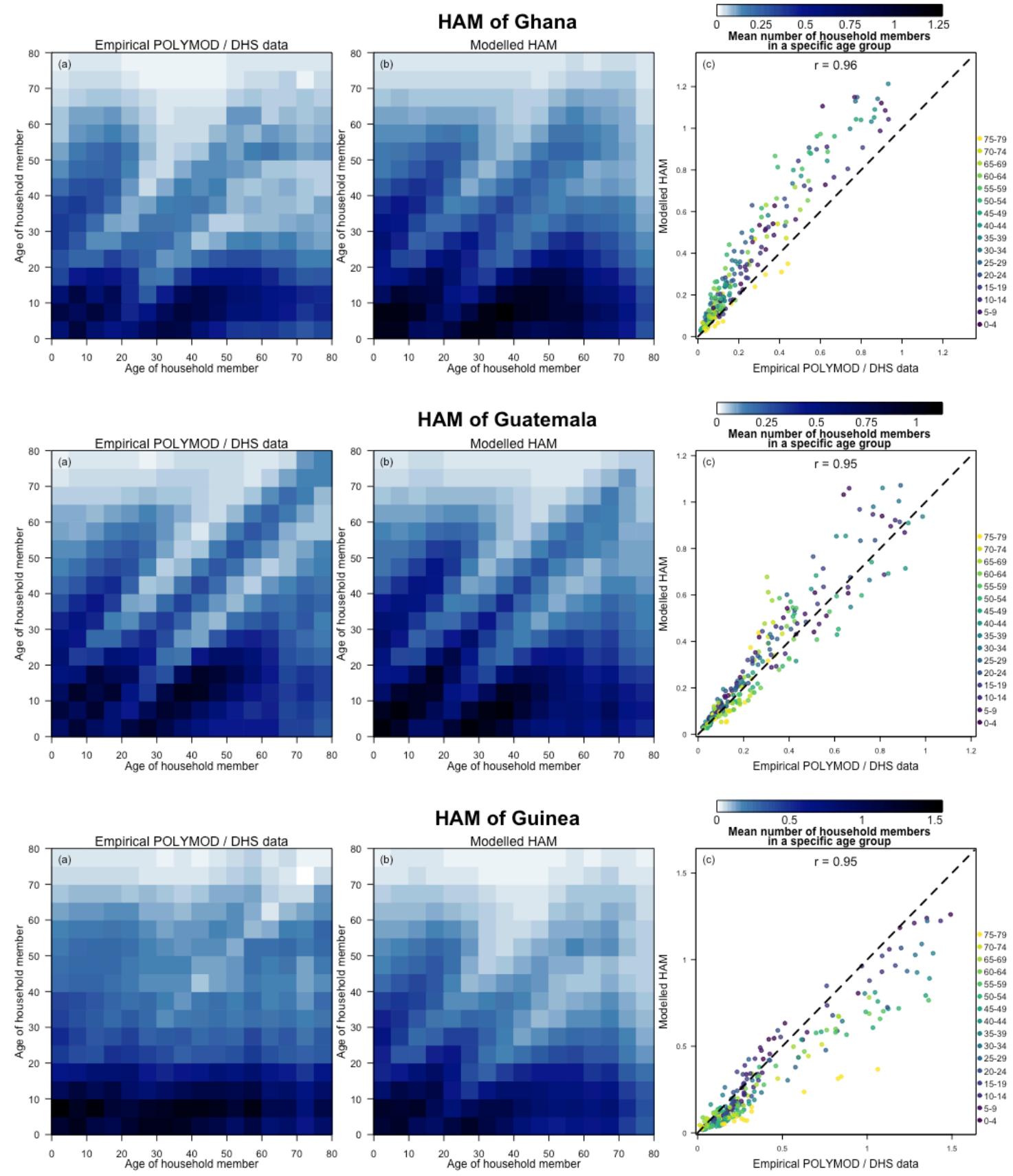

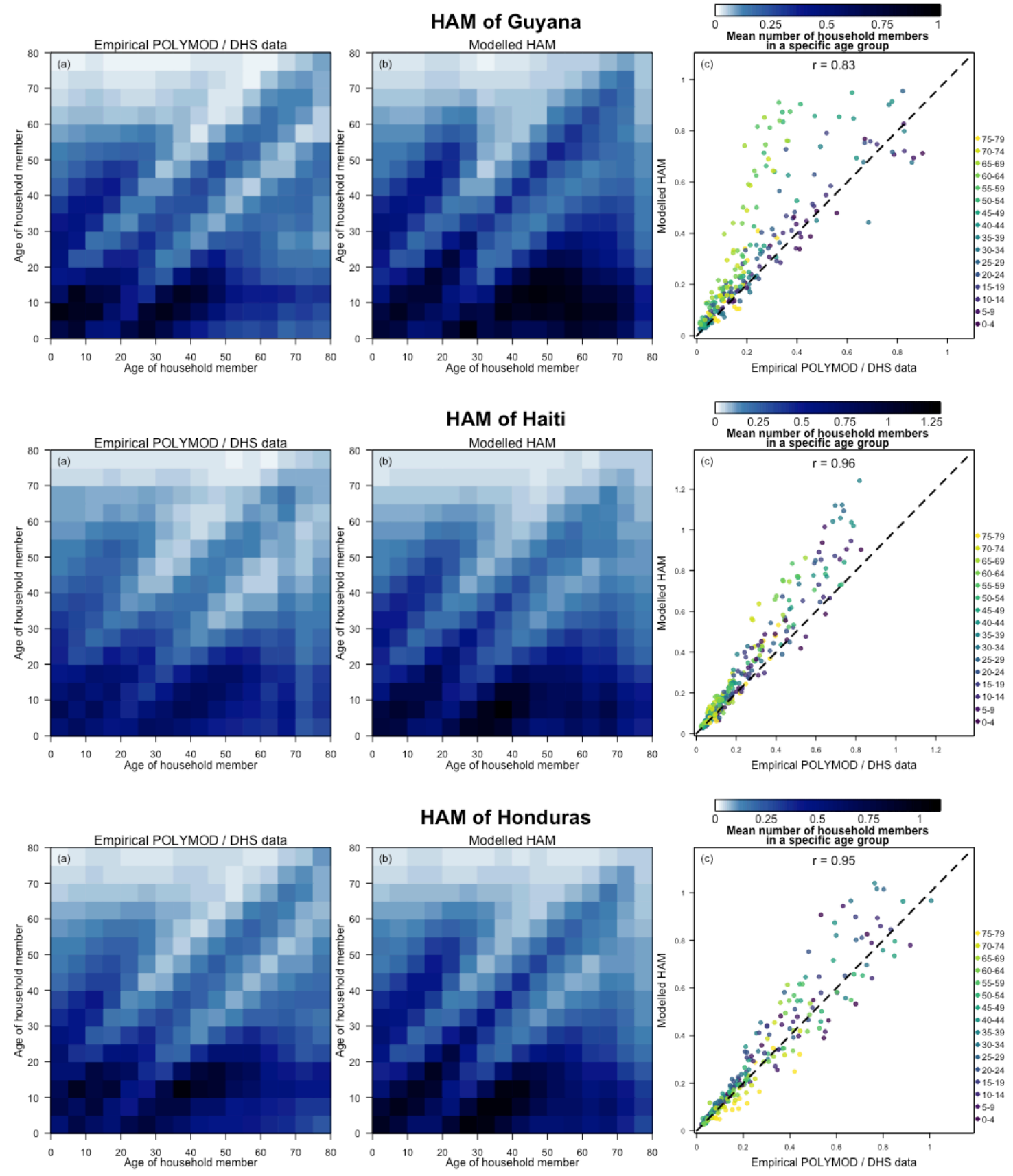

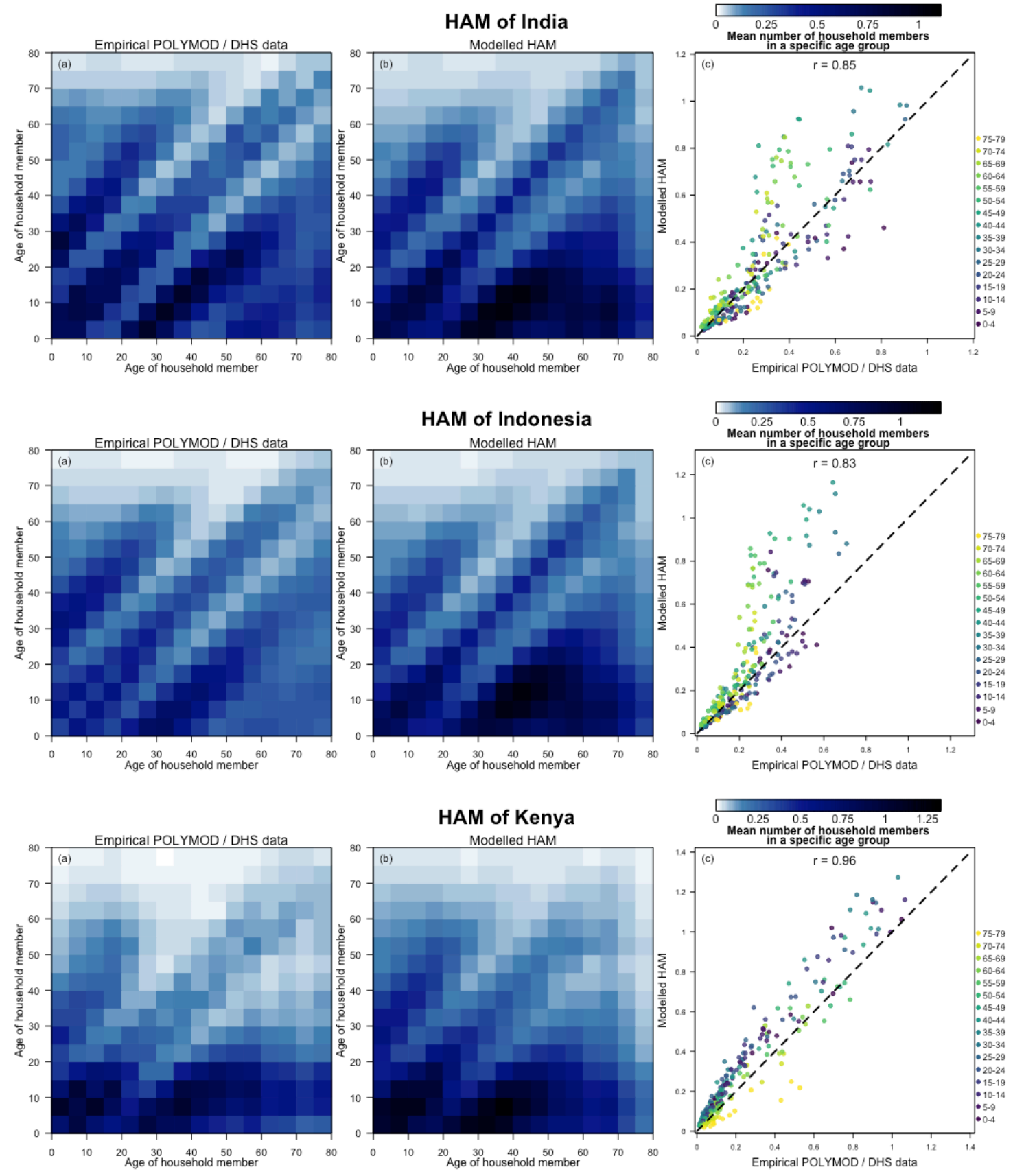

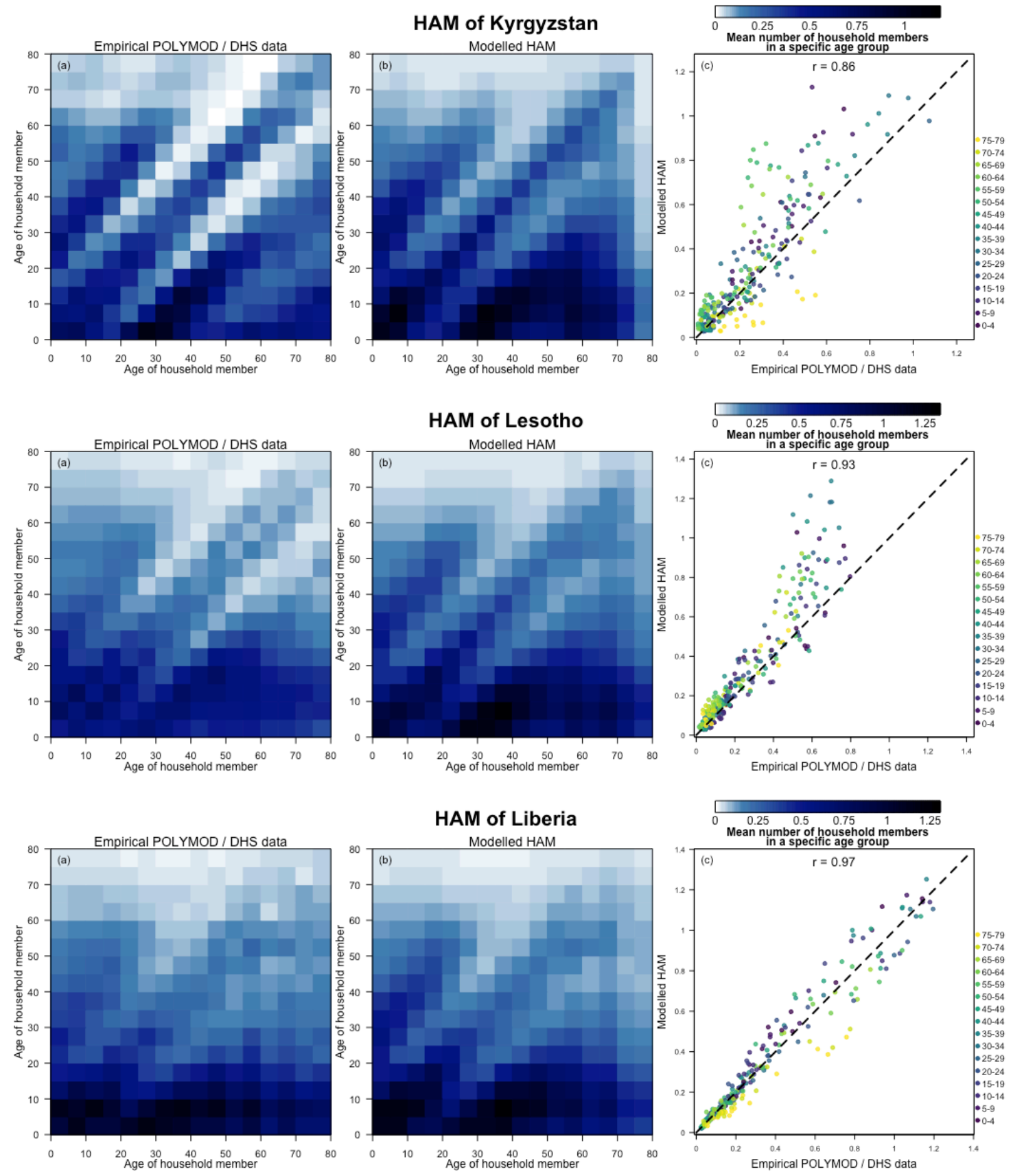

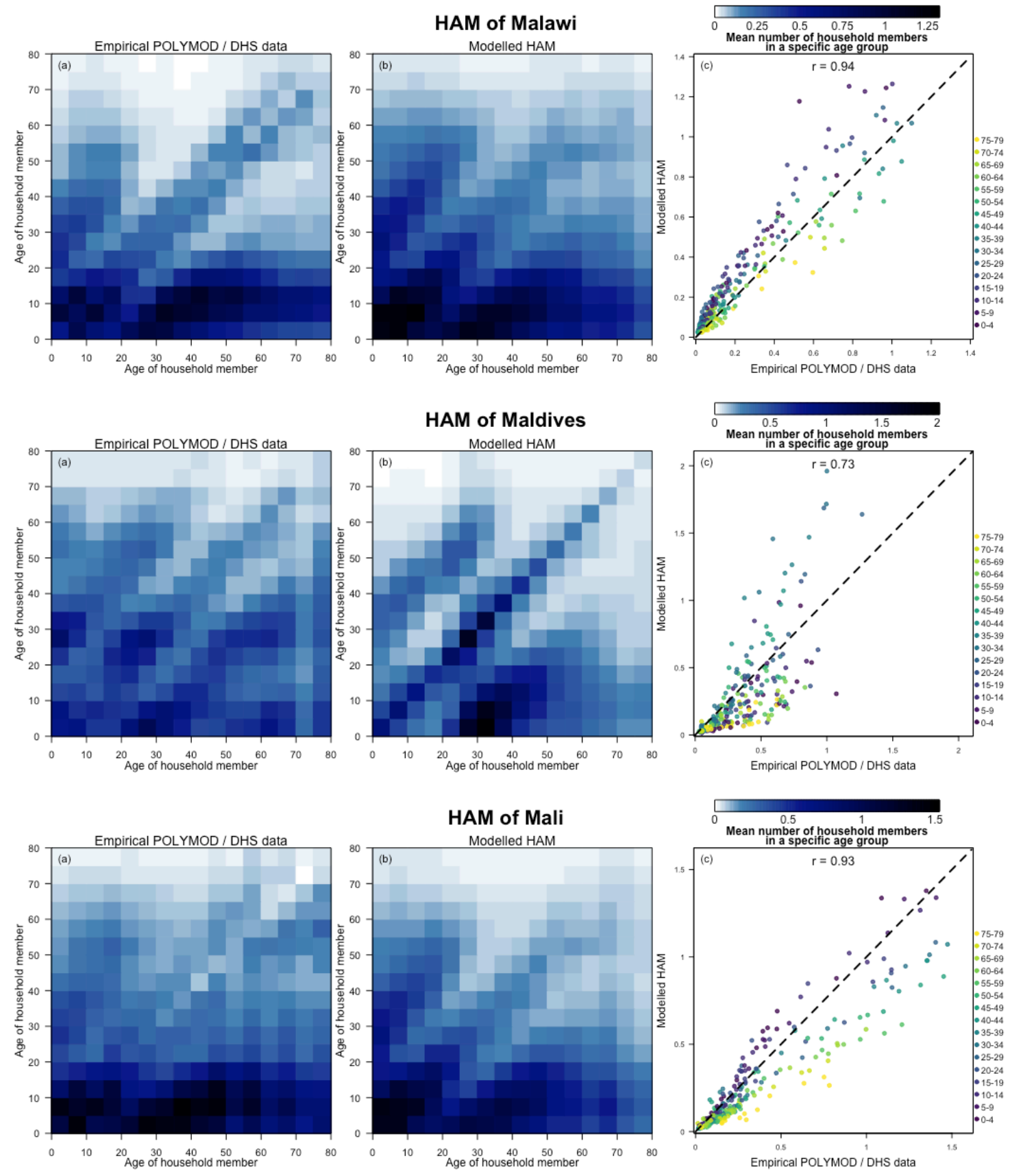

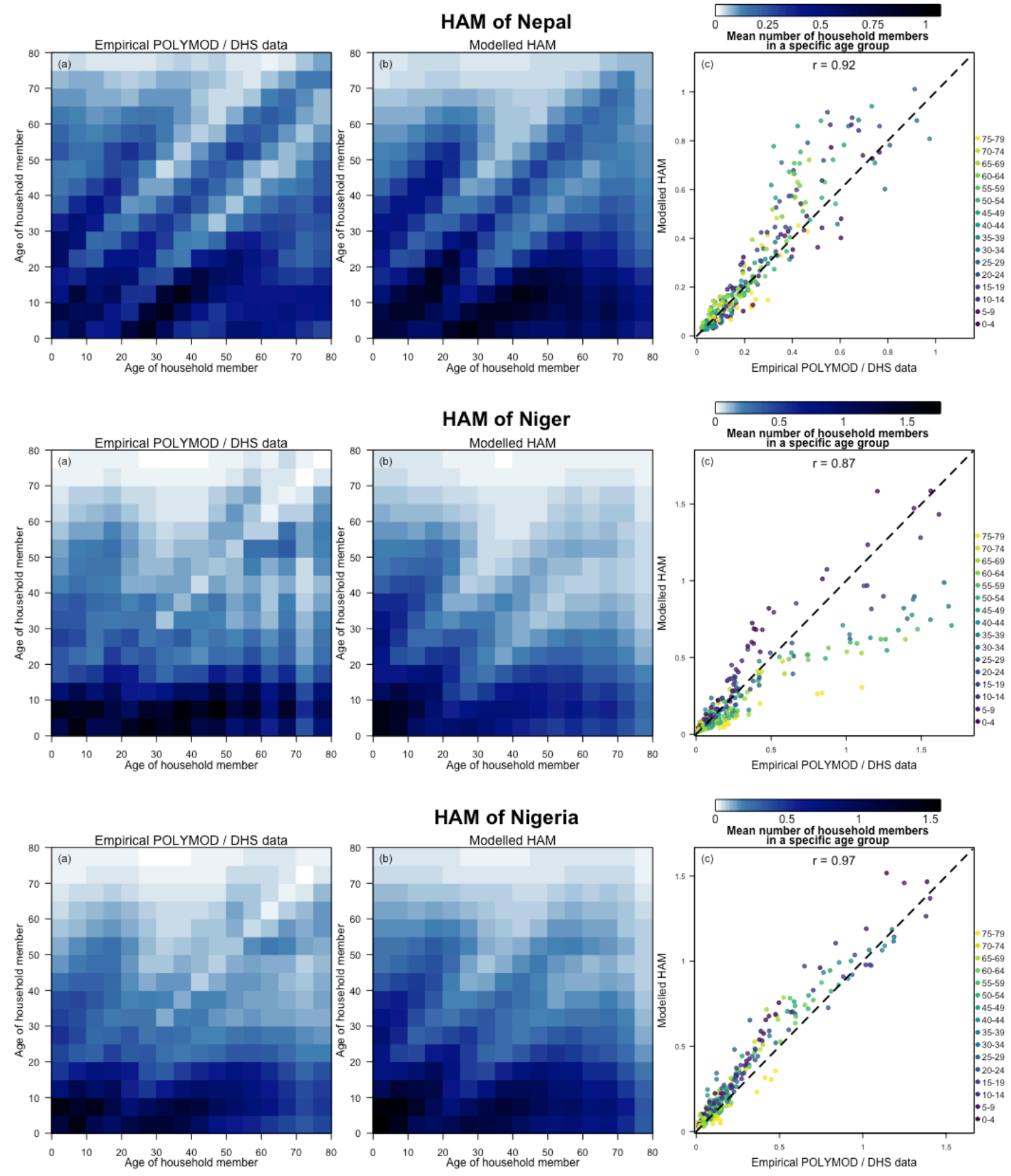

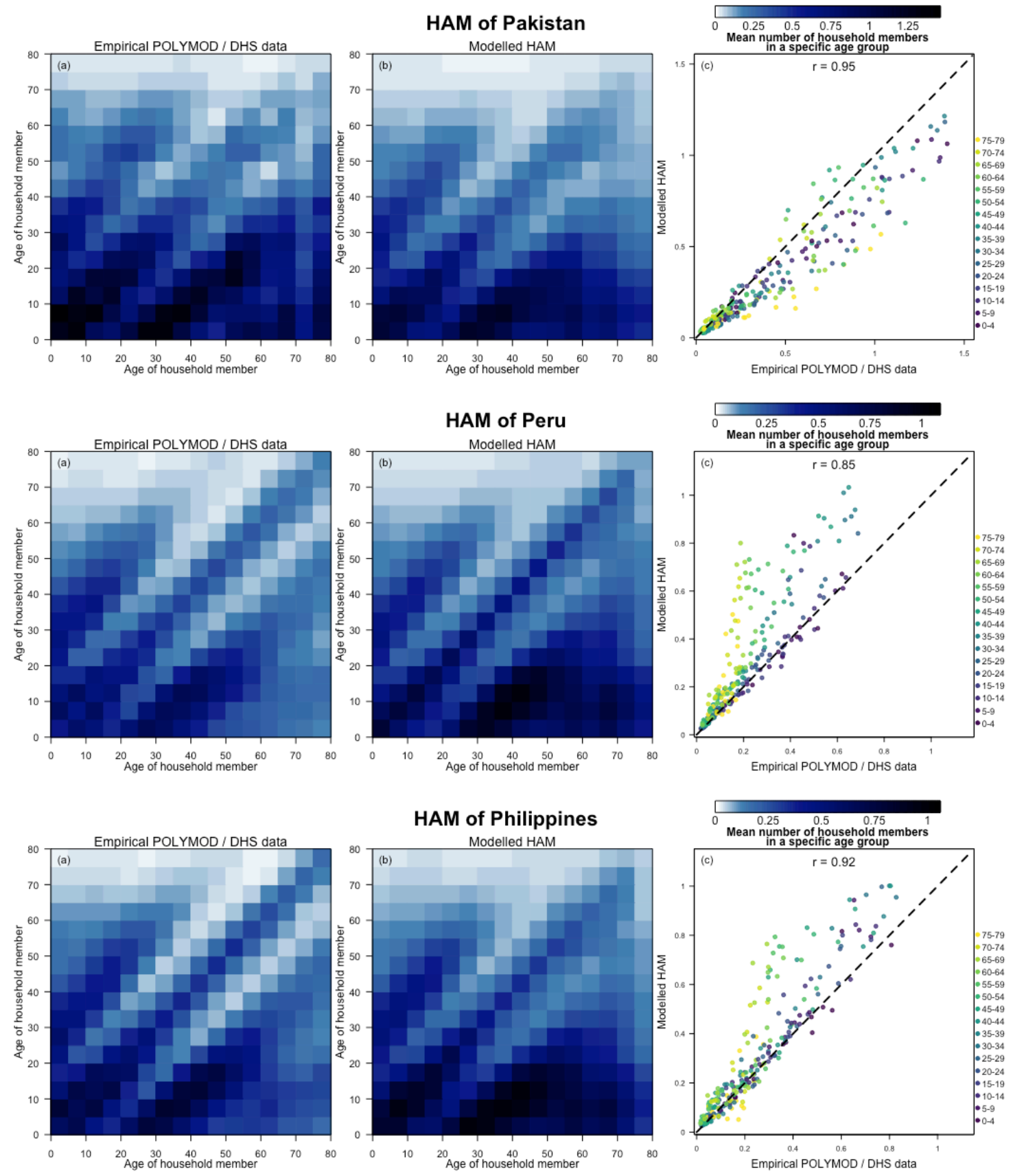

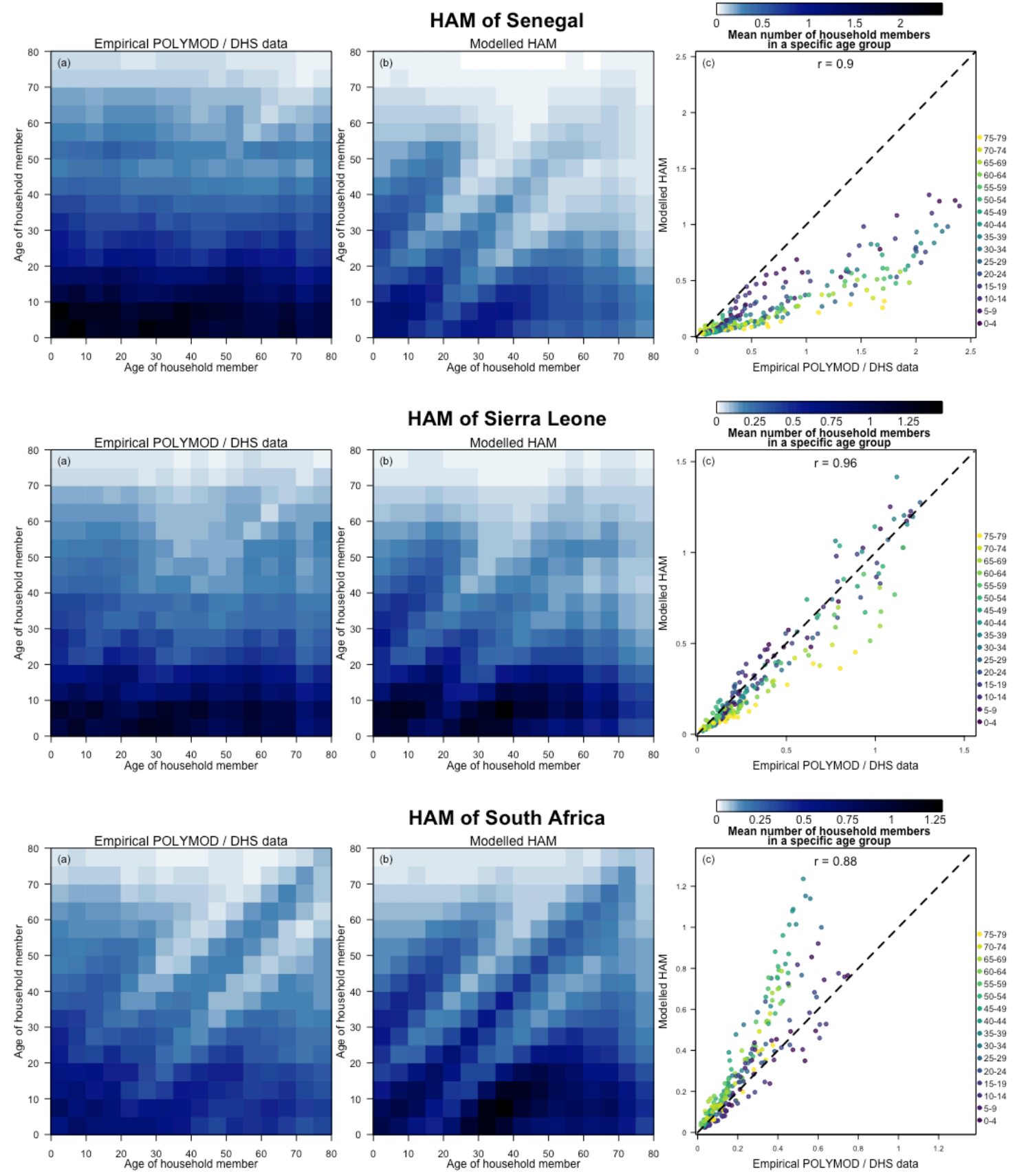
HAM of Timor-Leste
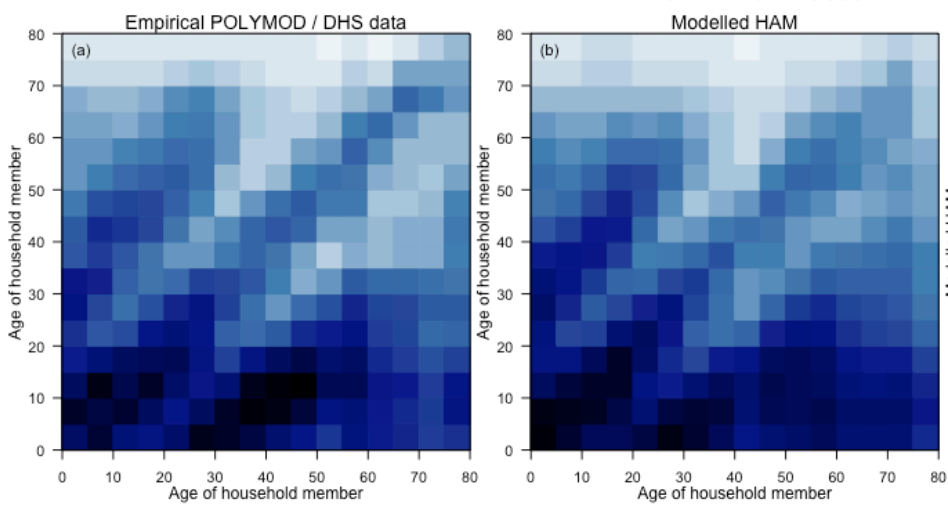

HAM of Togo
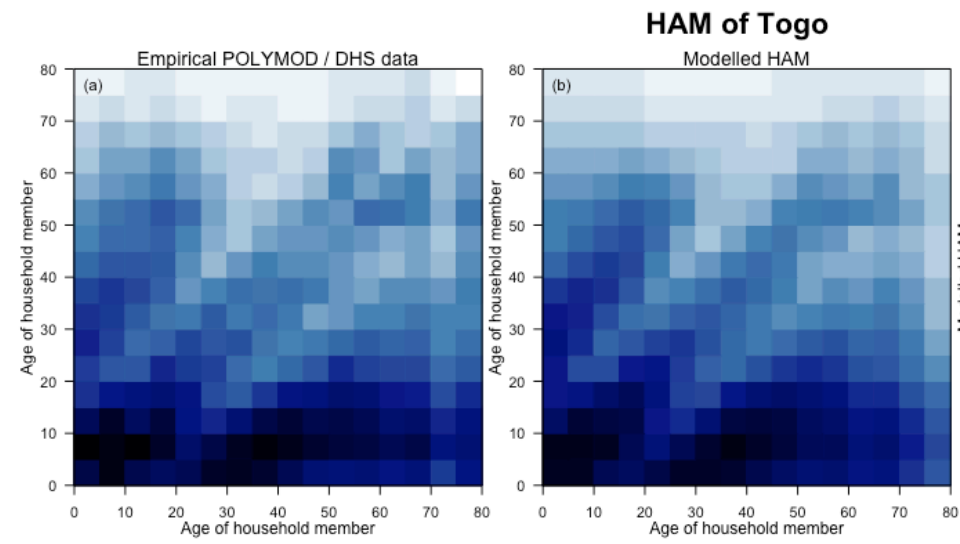

HAM of Uganda

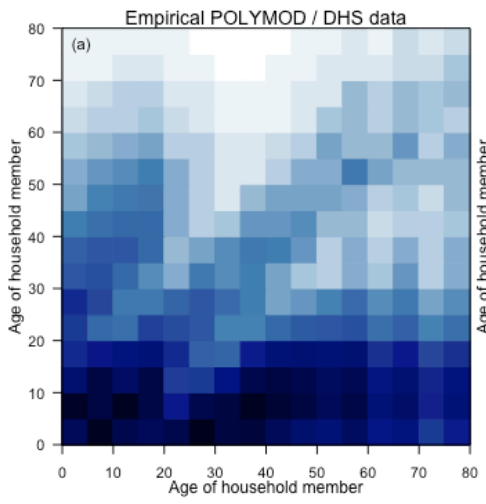

Modelled HAM

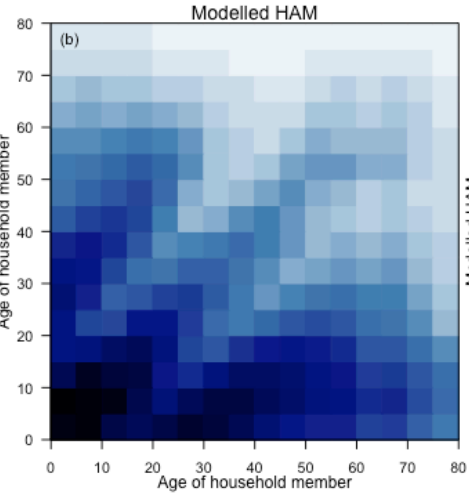

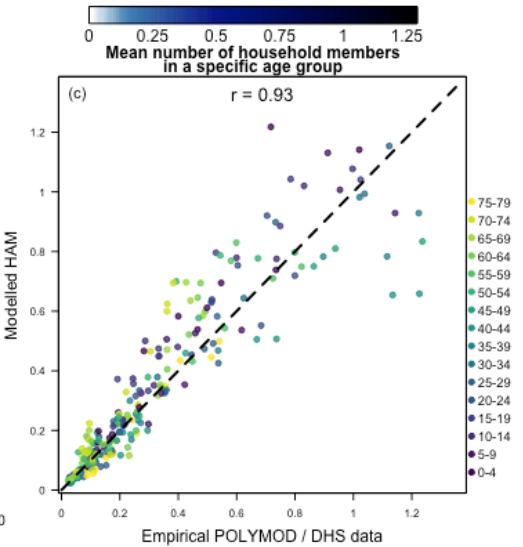

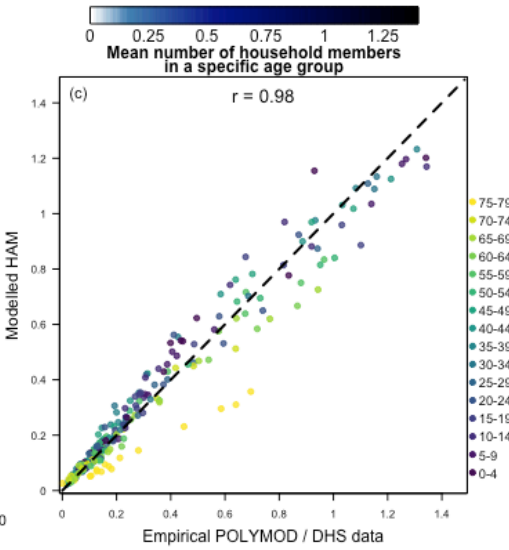

Empirical POLYMOD / DHS data

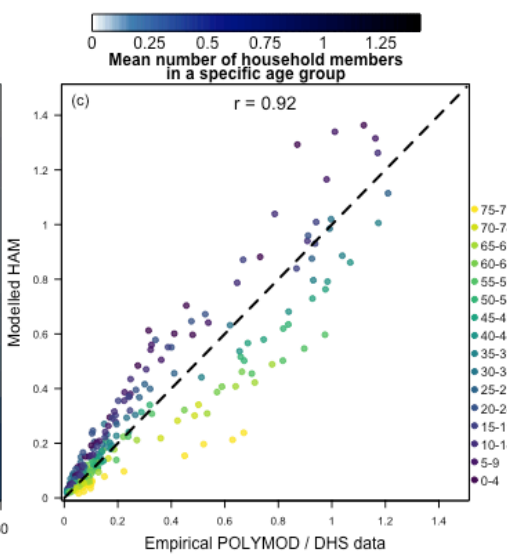



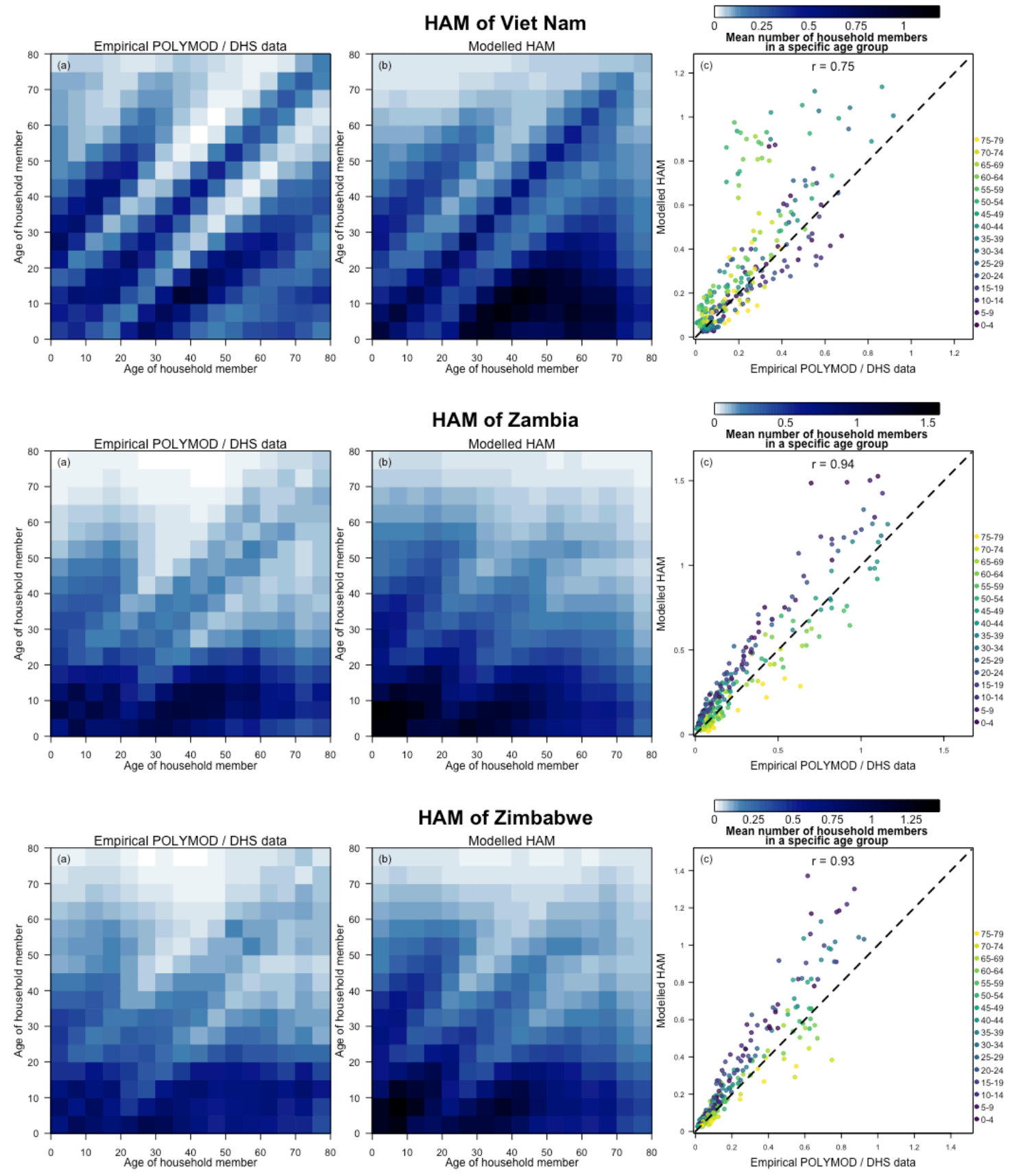


\section{B.2. Household age matrix (HAM) urban and rural comparison}

Using rural-urban stratified population and household data, we present the population age compositions and household age matrices for rural and urban areas by the rural and urban subregions for countries with recent DHS household surveys.

Empirical DHS household data: Afghanistan
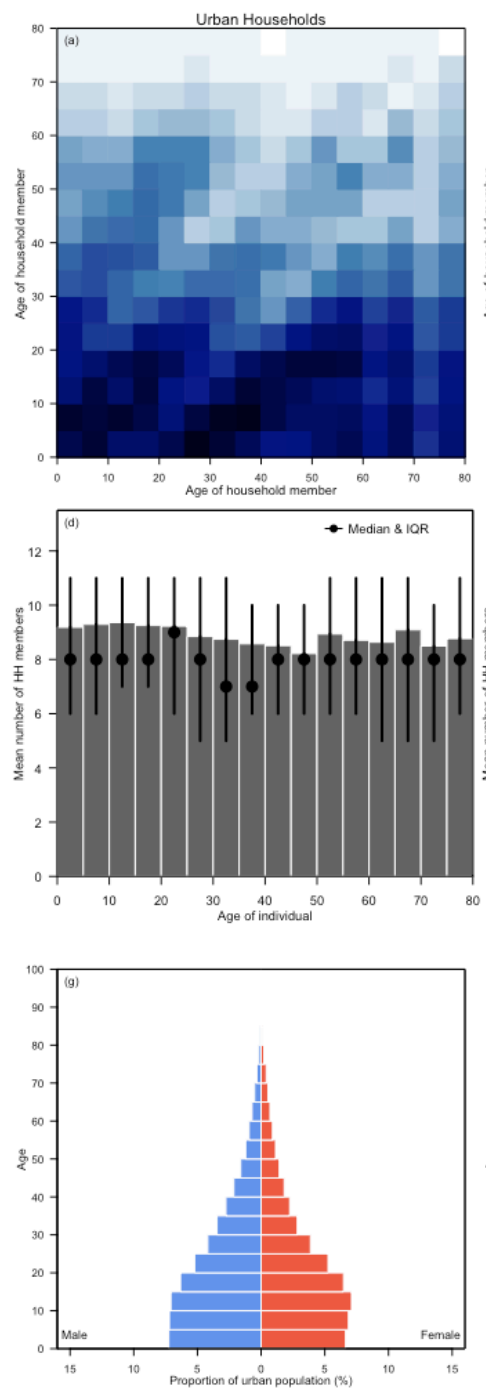
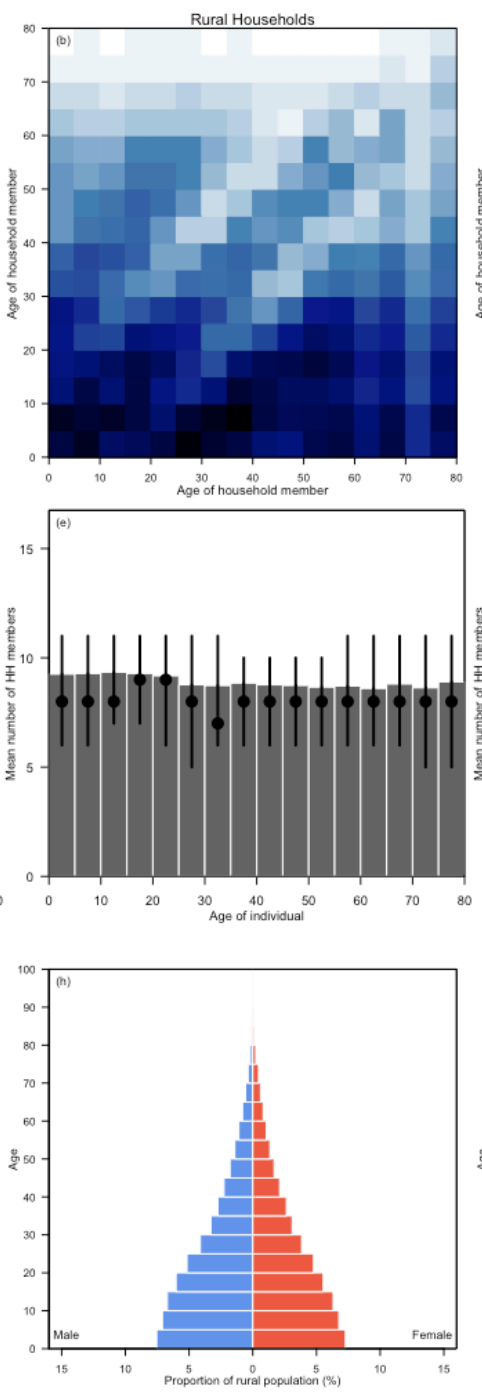

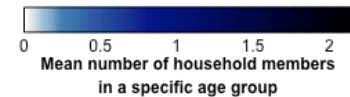

in a specific age group
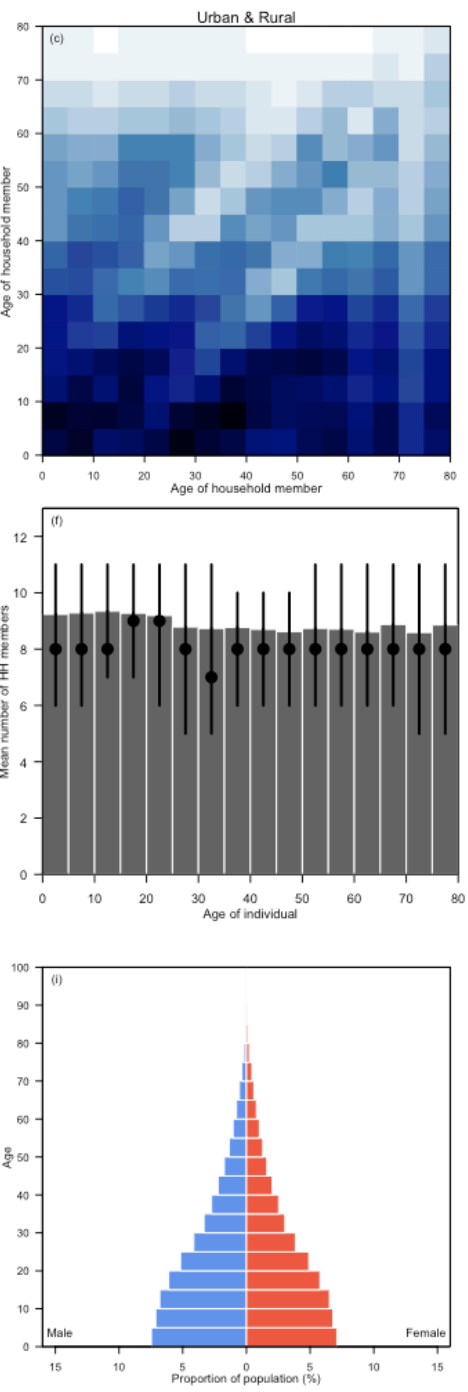
Empirical DHS household data: Angola
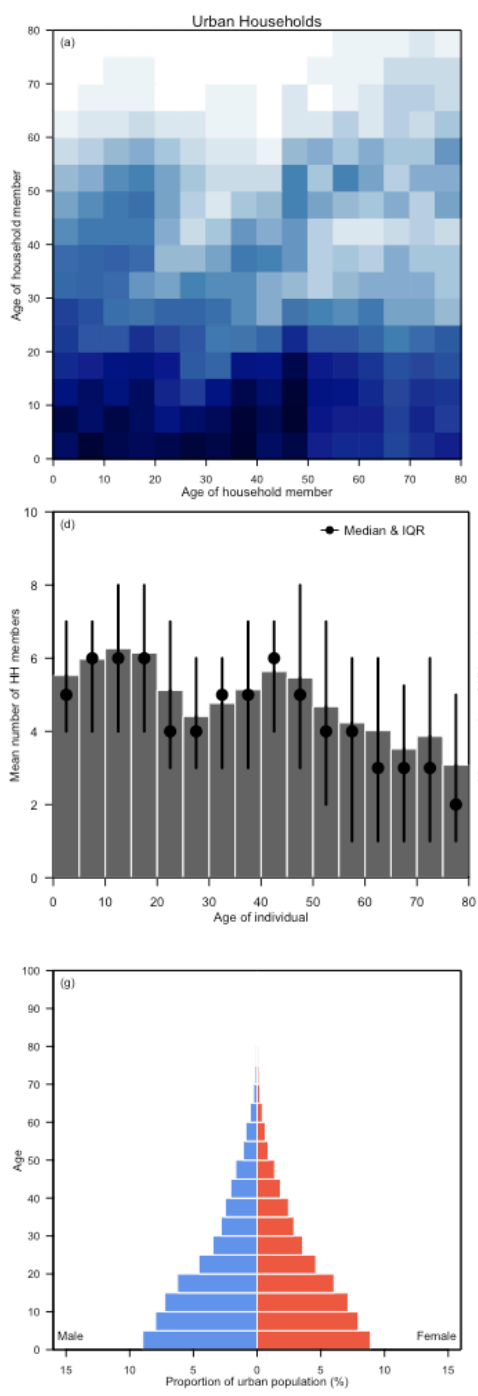
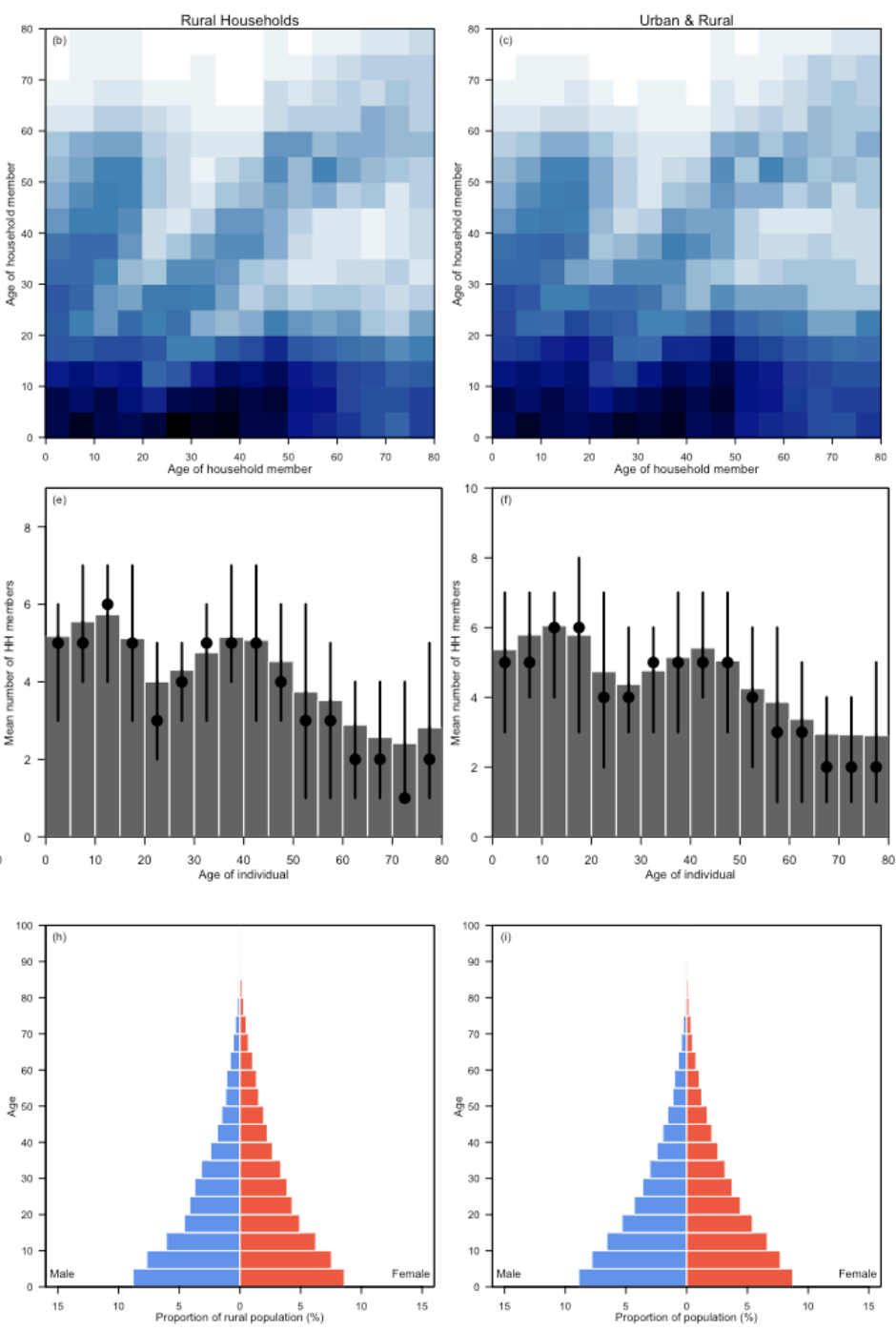
Empirical DHS household data: Bangladesh
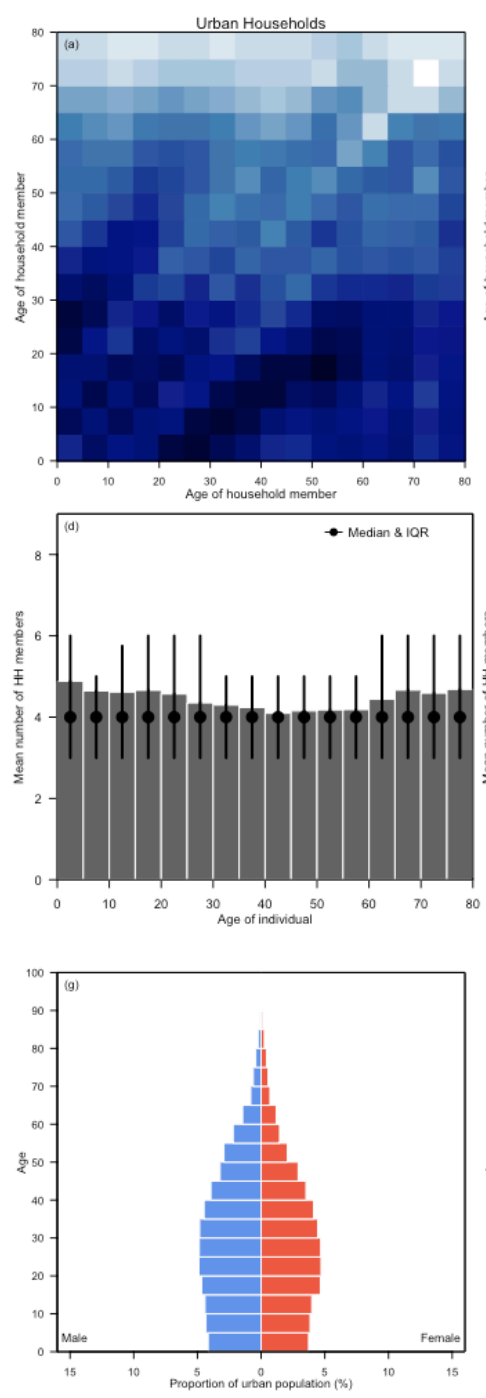
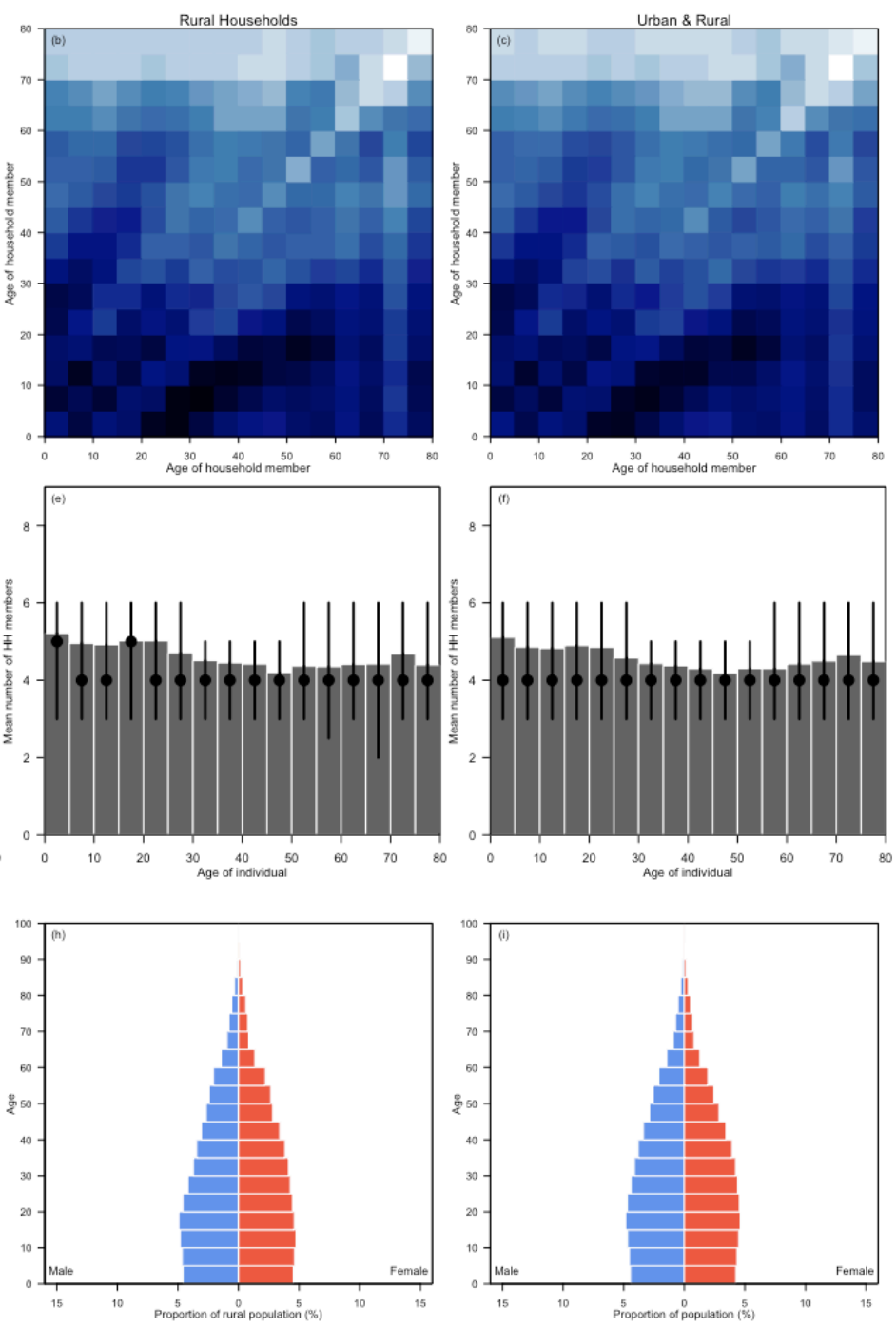
Empirical DHS household data: Benin
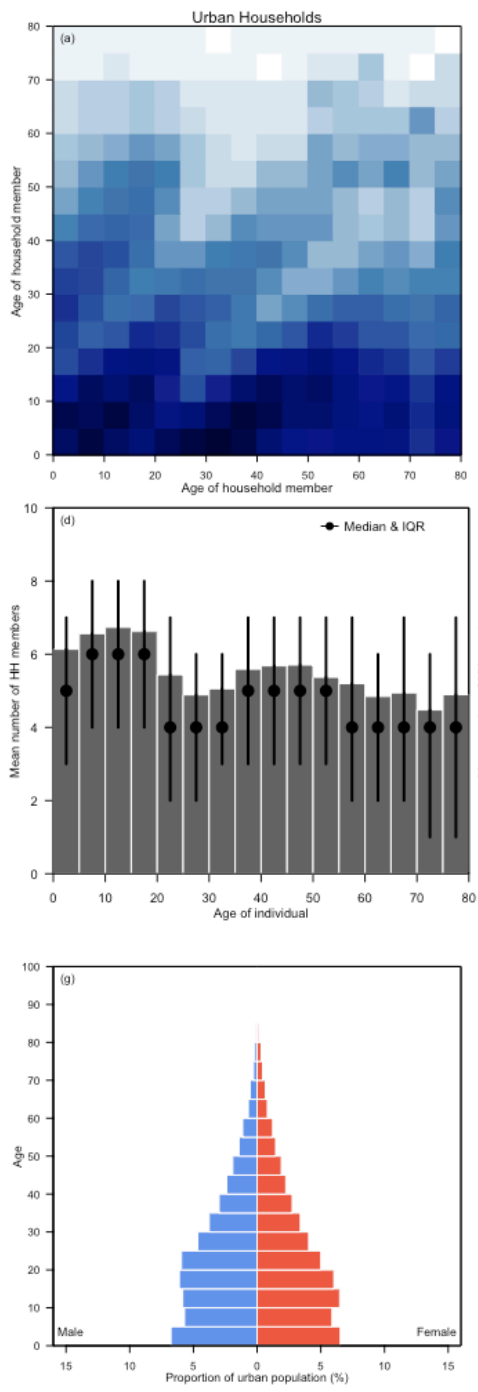
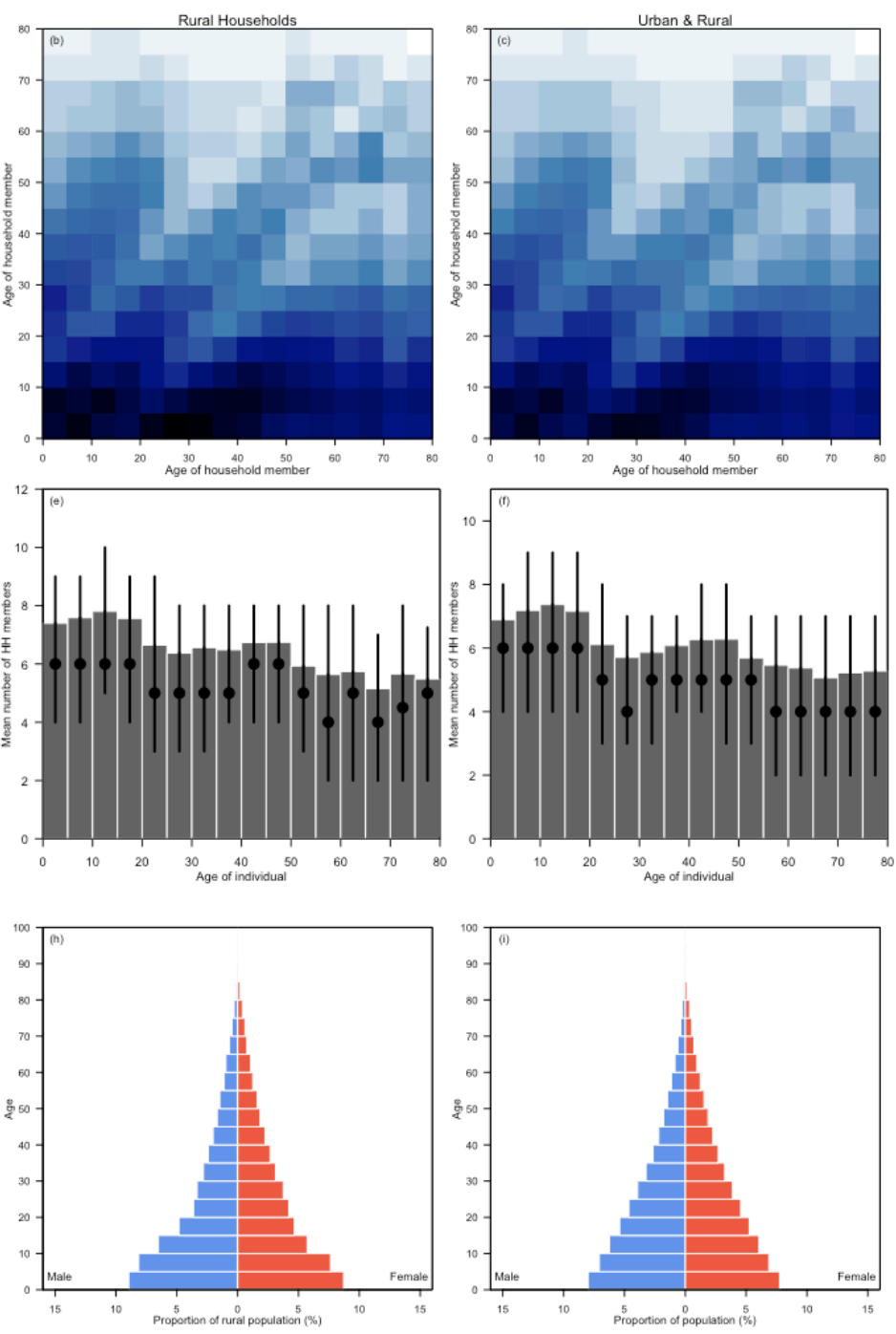
Empirical DHS household data: Bolivia (Plurinational State of)
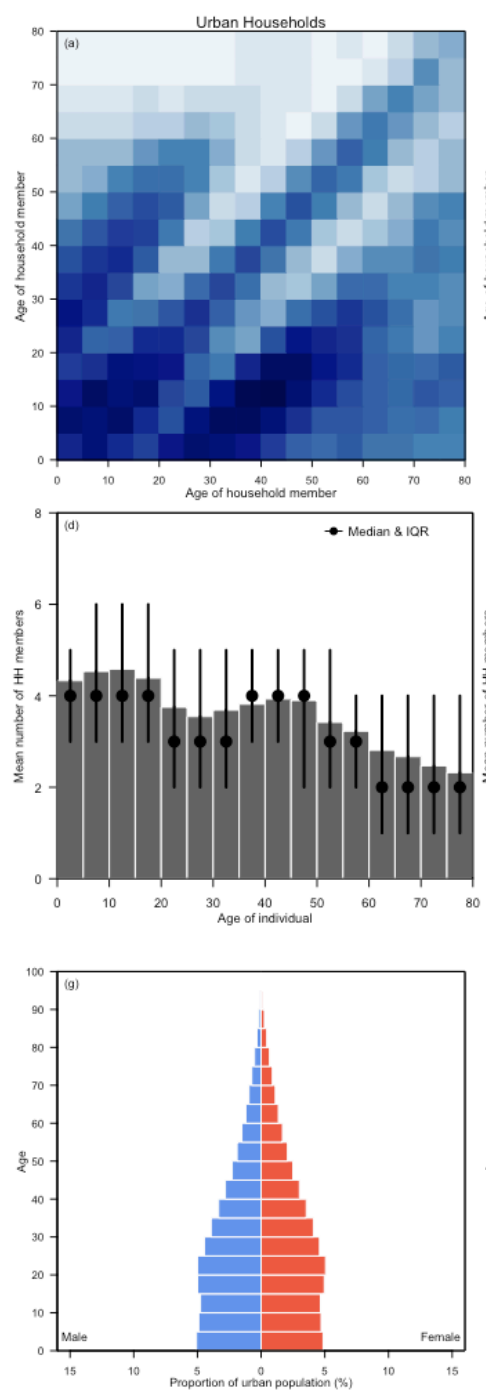
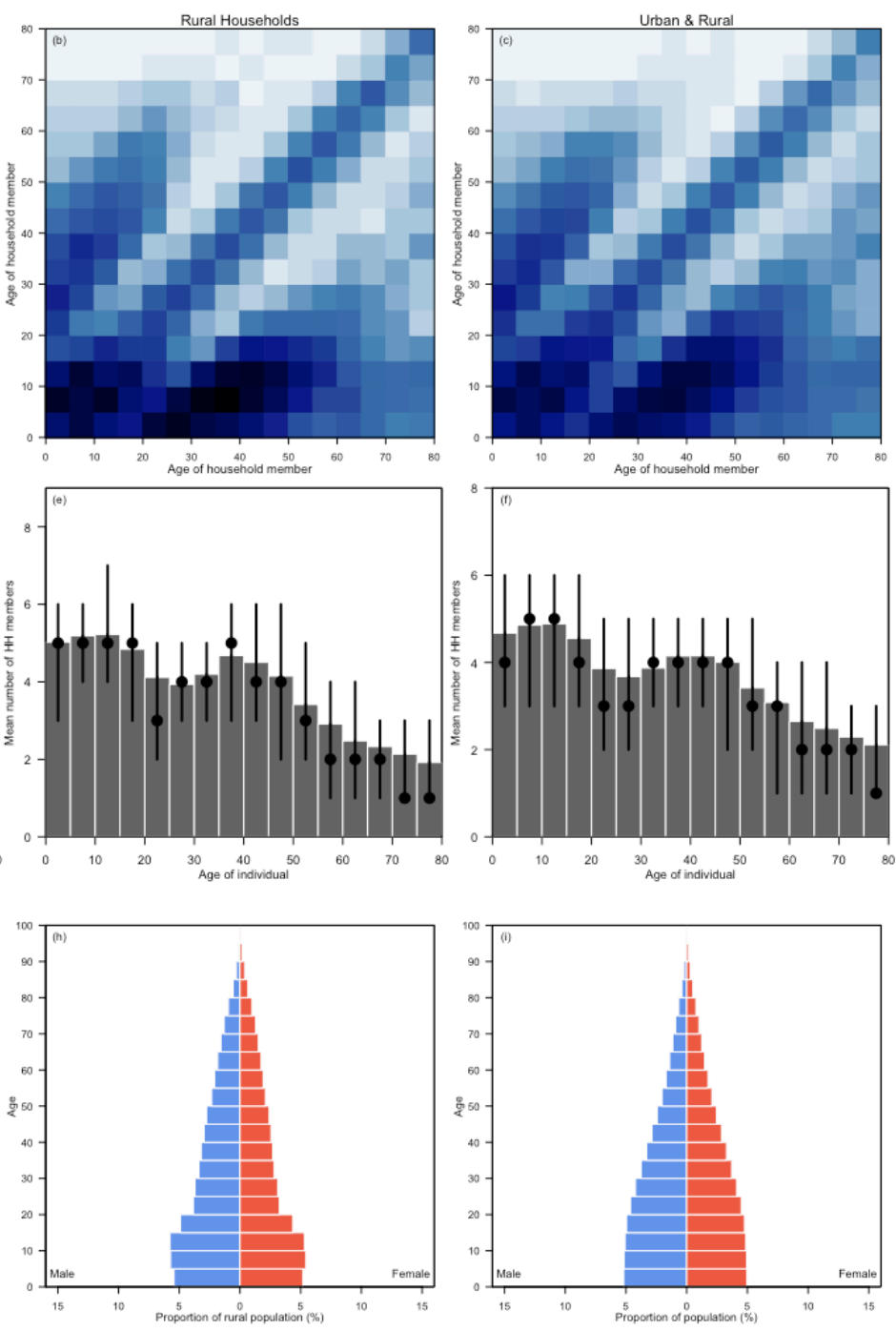
Empirical DHS household data: Cambodia
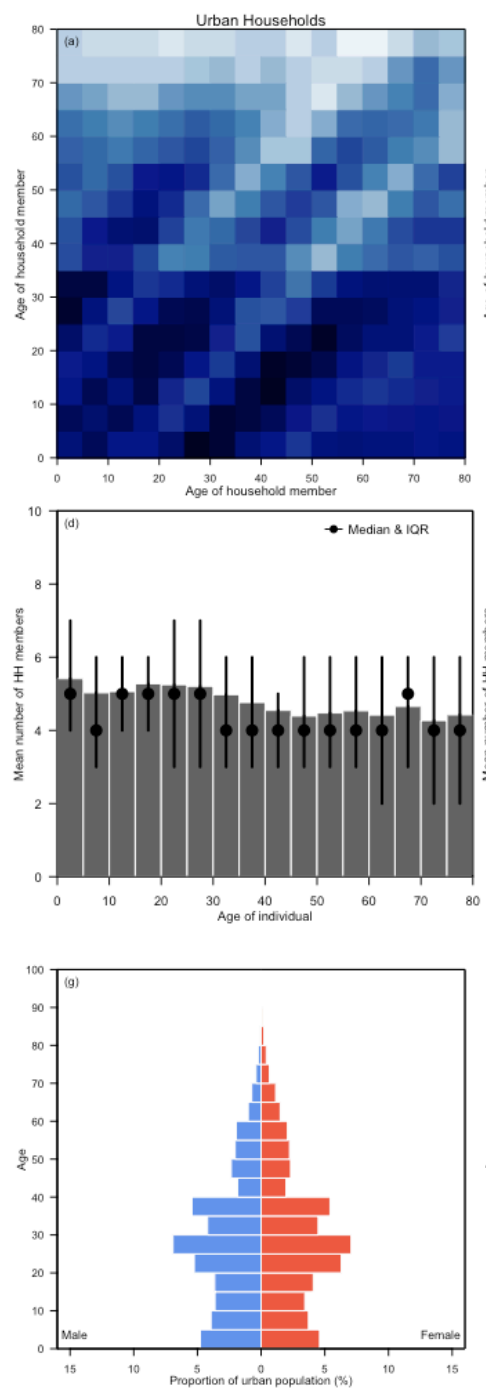
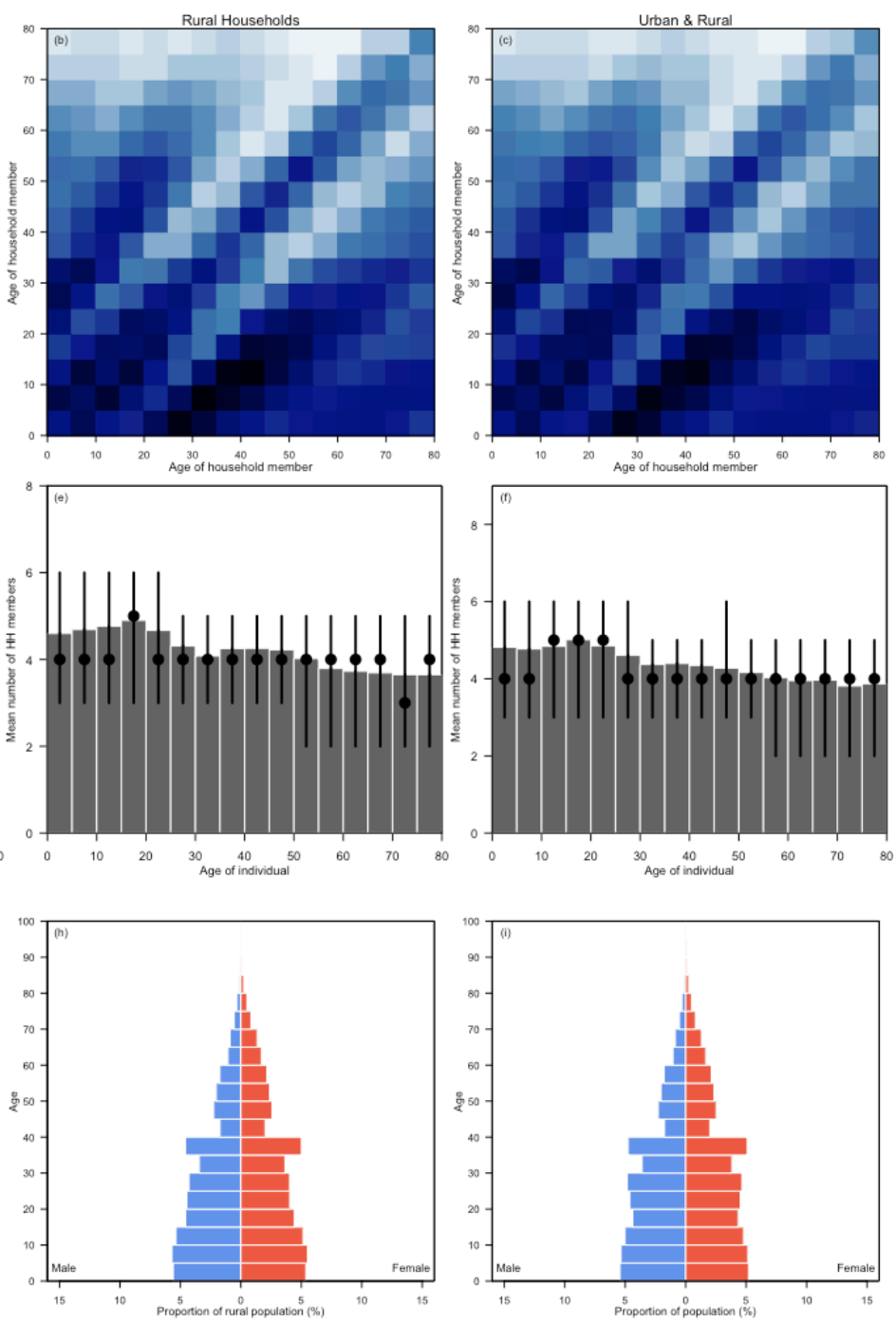
Empirical DHS household data: Cameroon
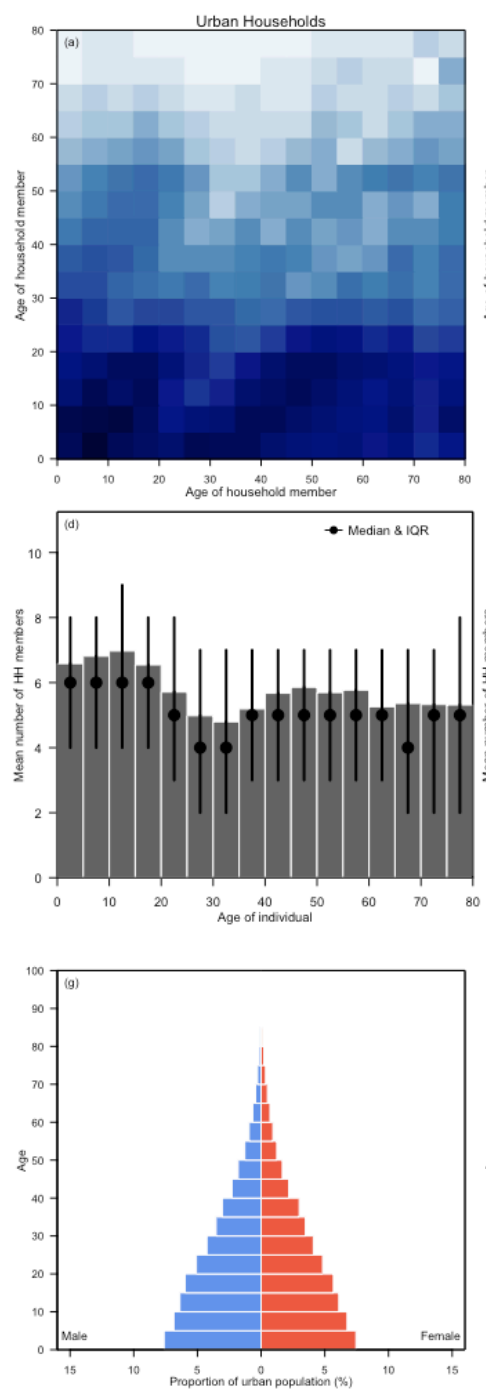
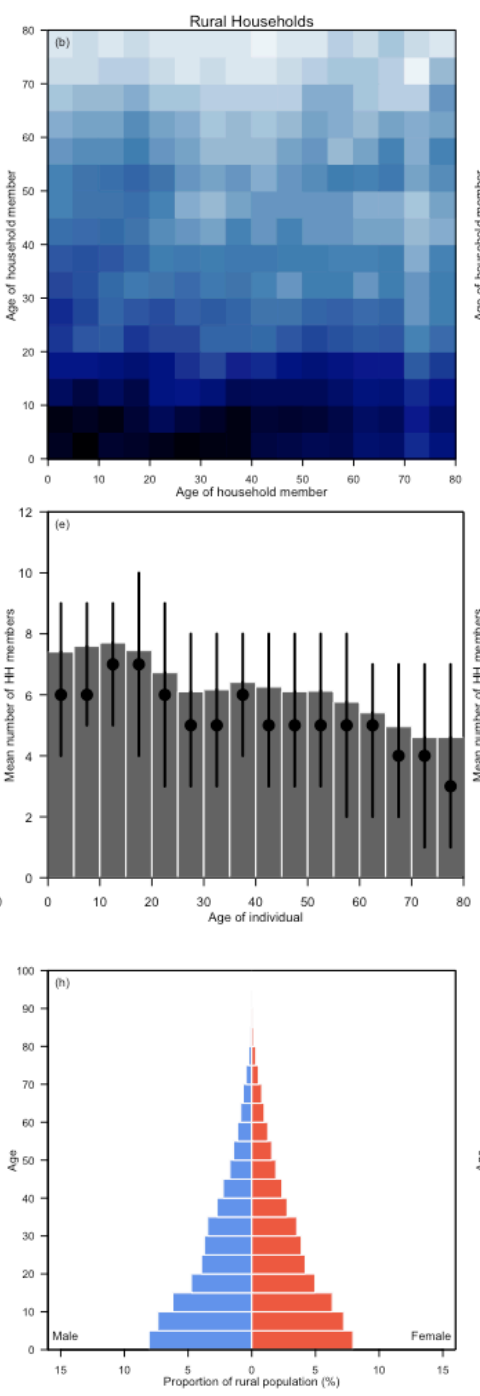

\begin{tabular}{lcc}
\hline 0.5 & 1 & 1.5 \\
& & 0
\end{tabular}

in a specific age group
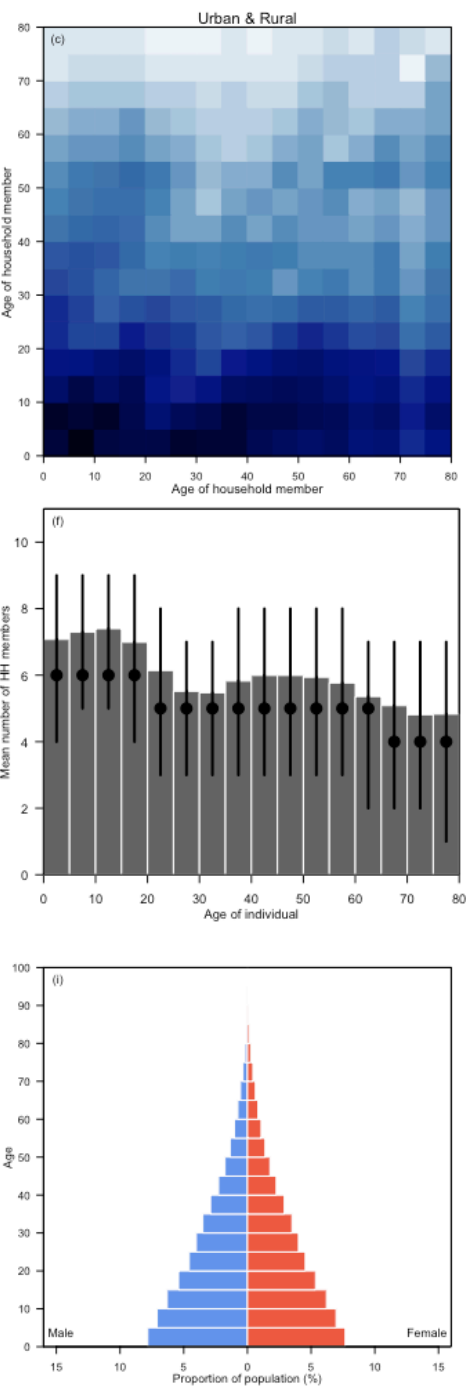
Empirical DHS household data: Chad
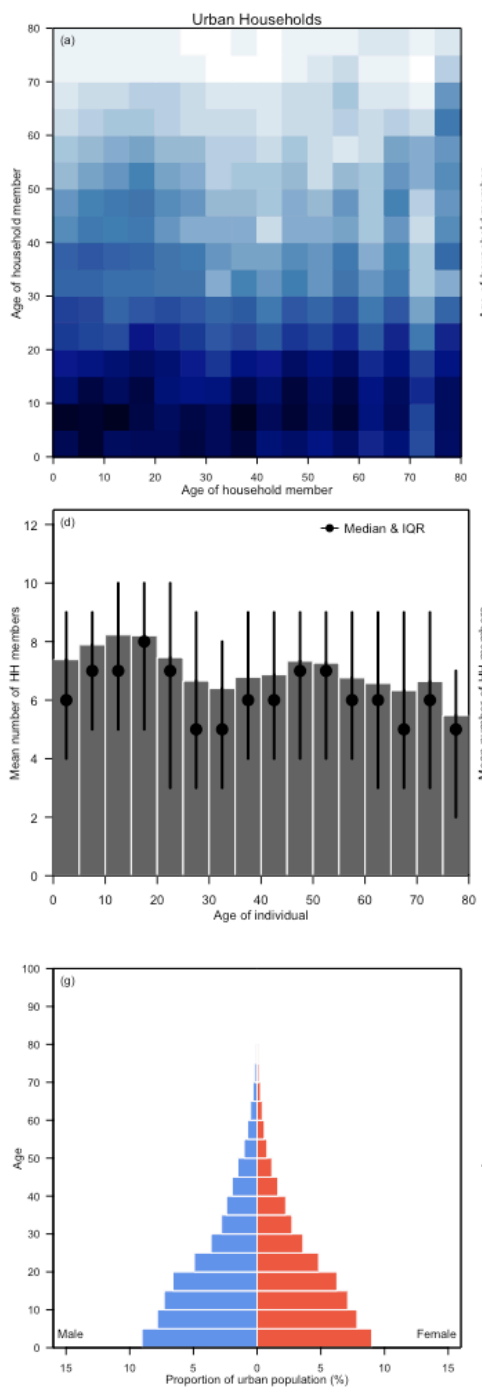
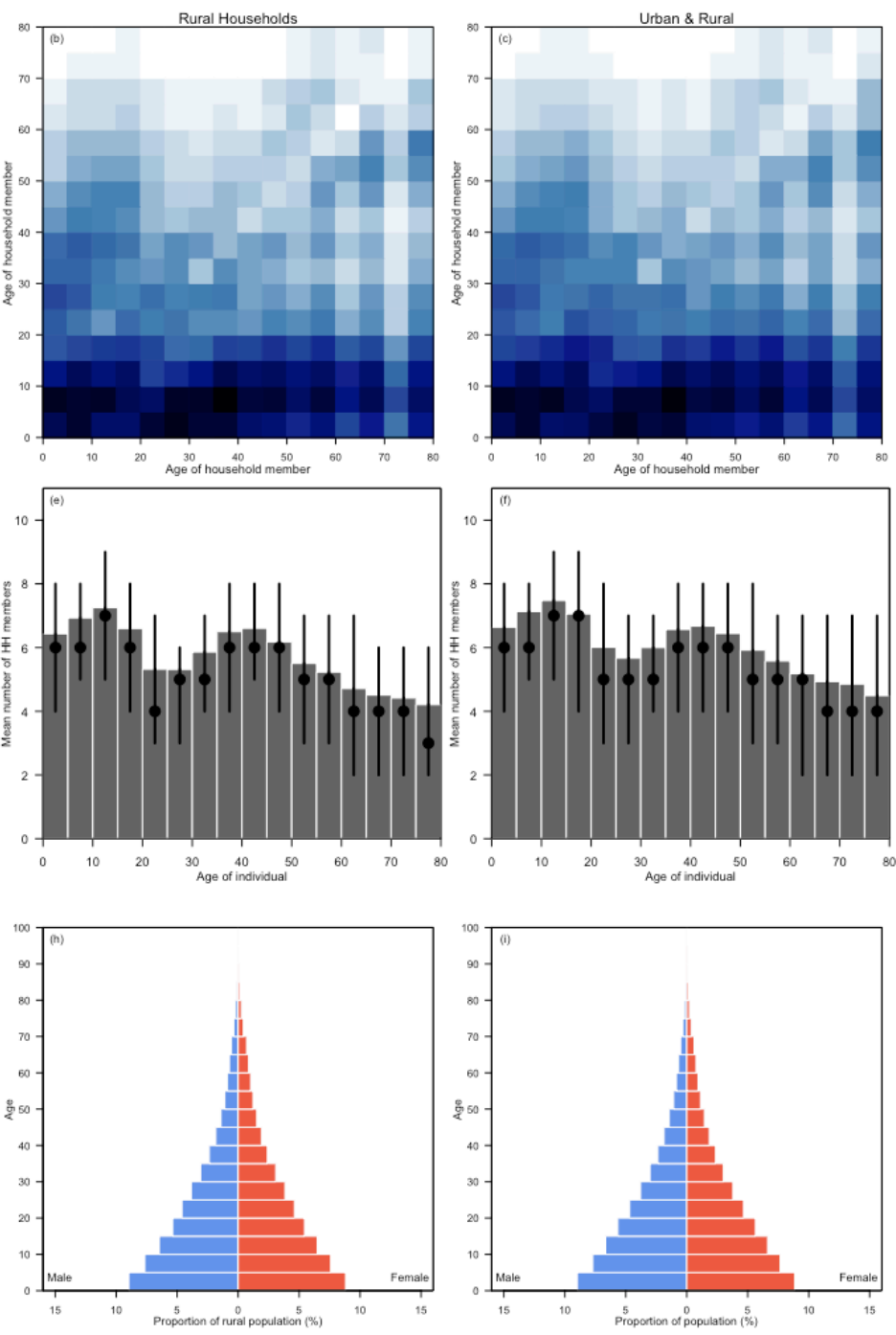
Empirical DHS household data: Colombia
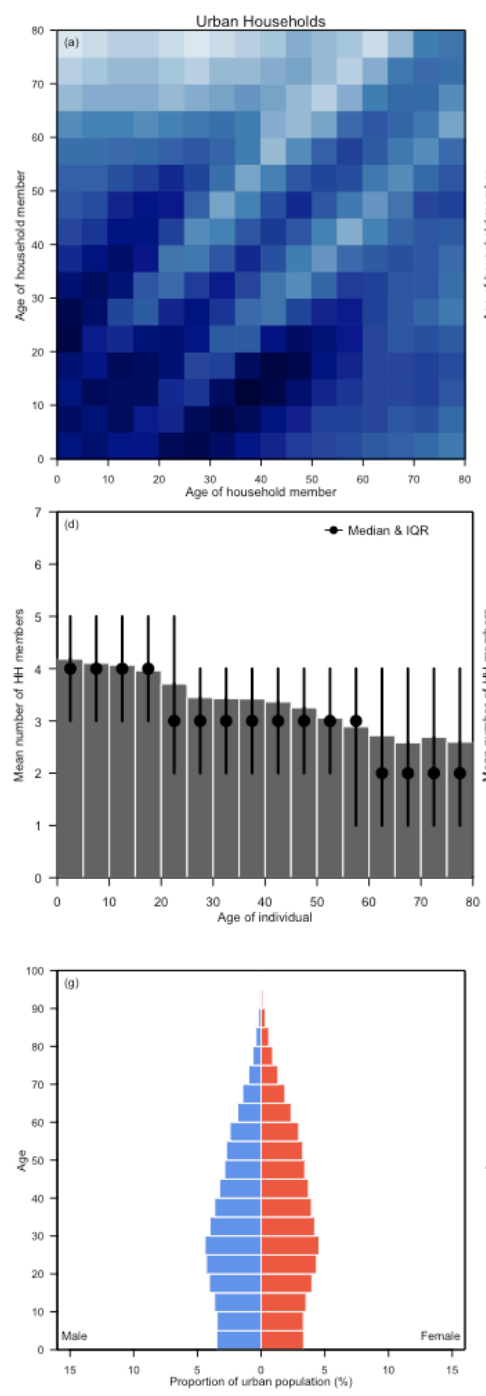
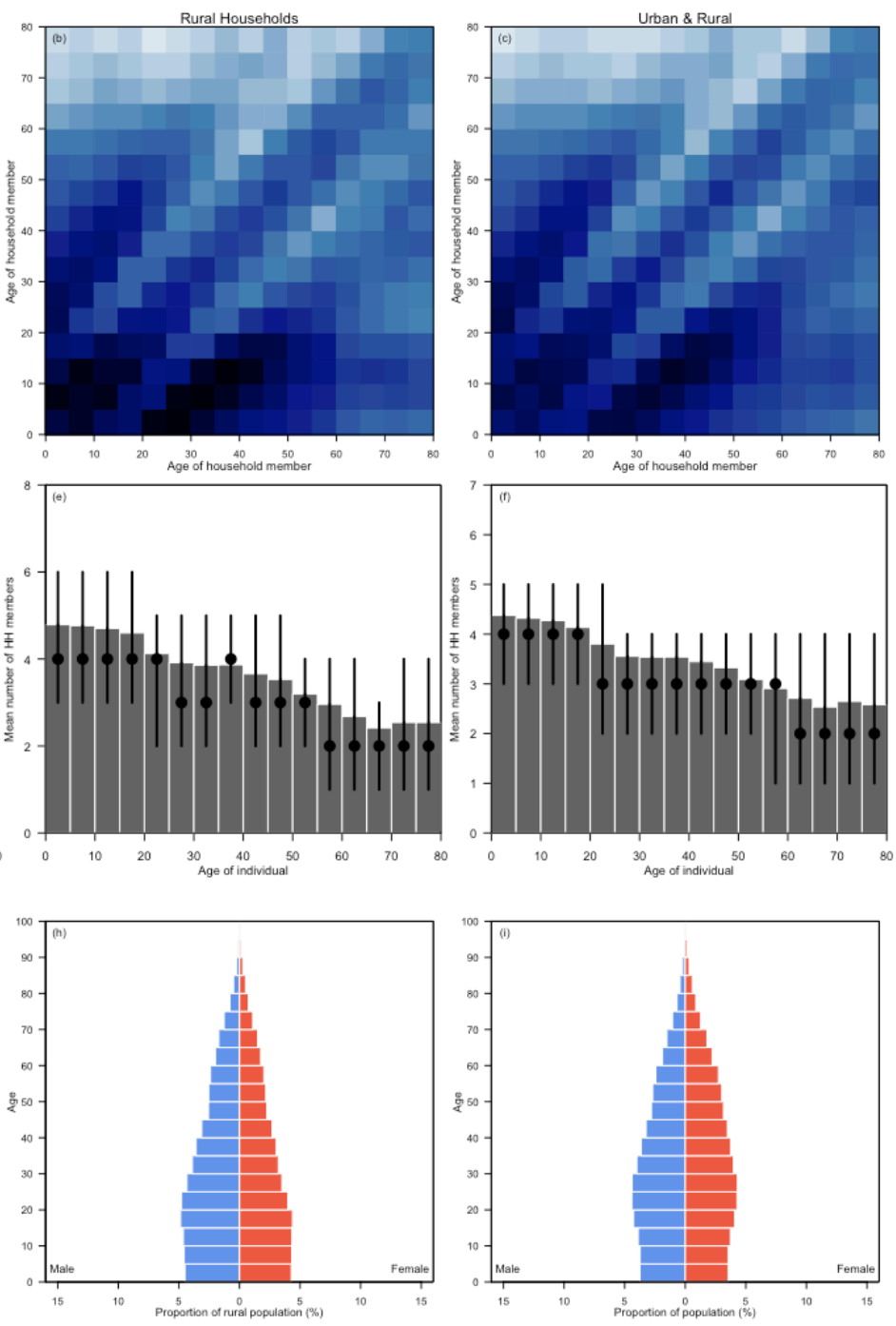
Empirical DHS household data: Congo
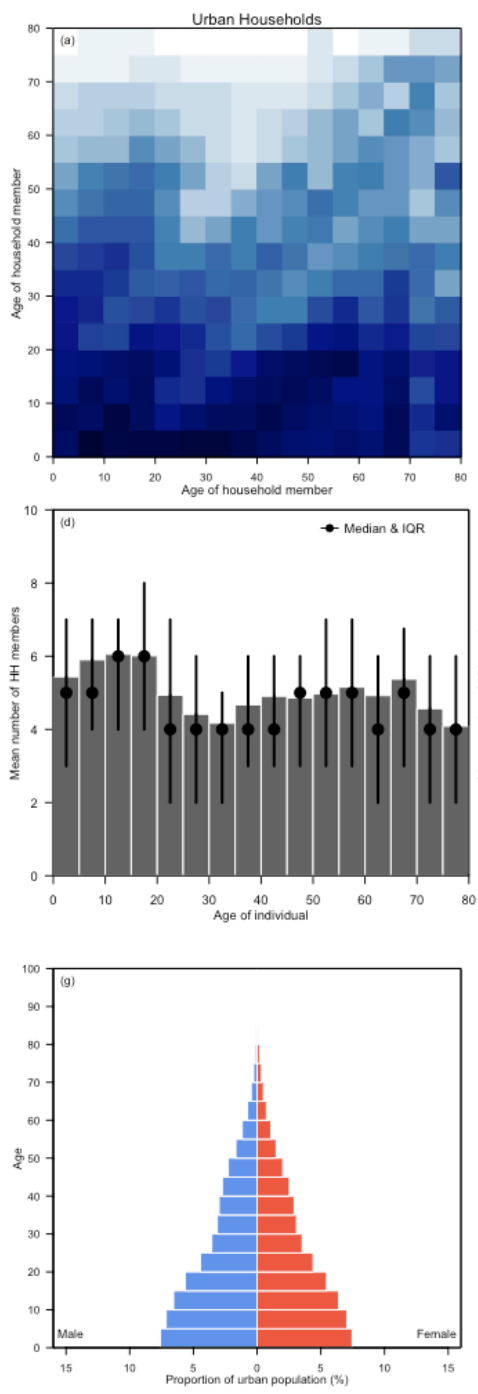
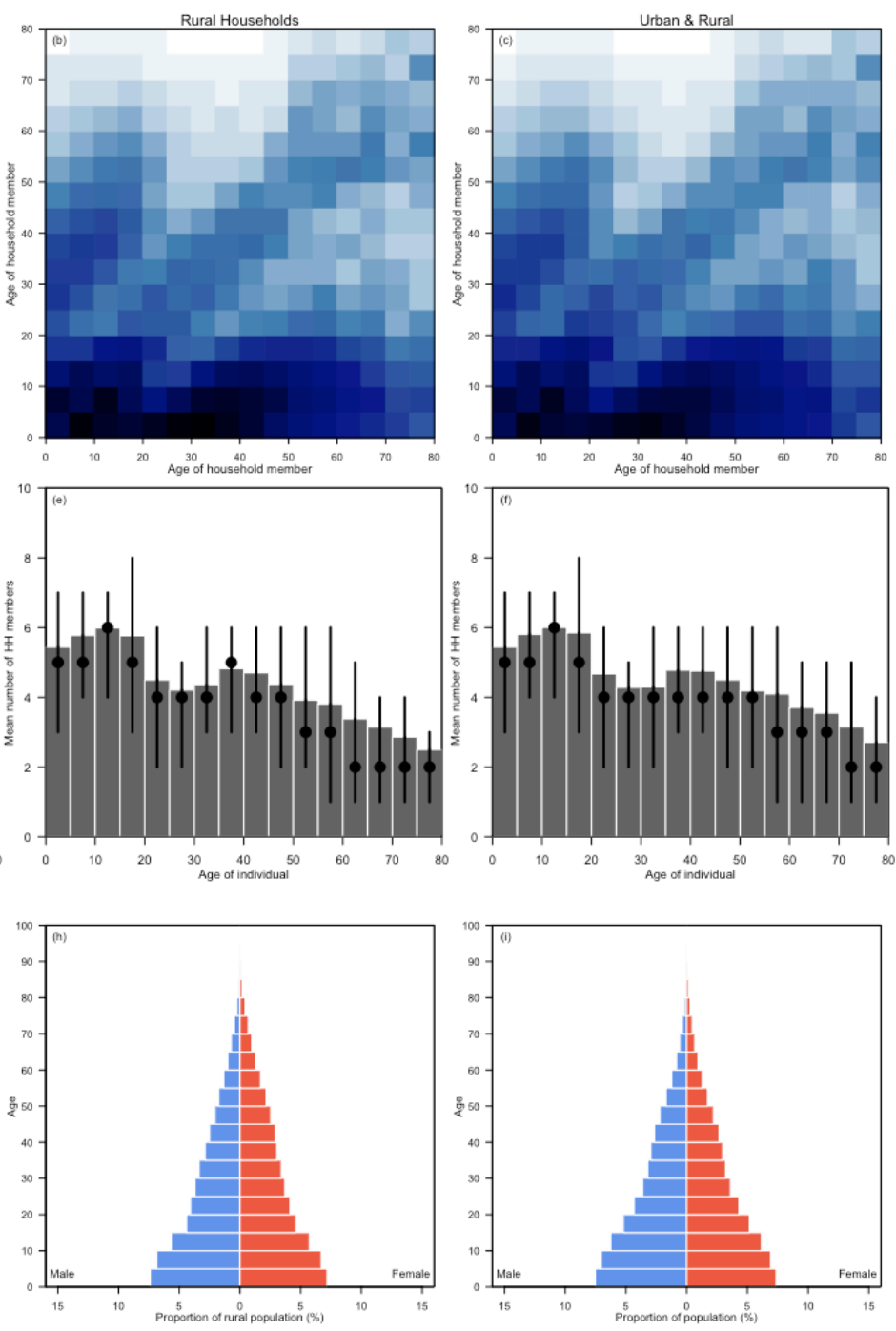
Empirical DHS household data: Democratic Republic of the Congo

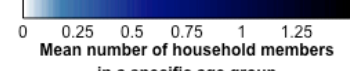
in a specific age group
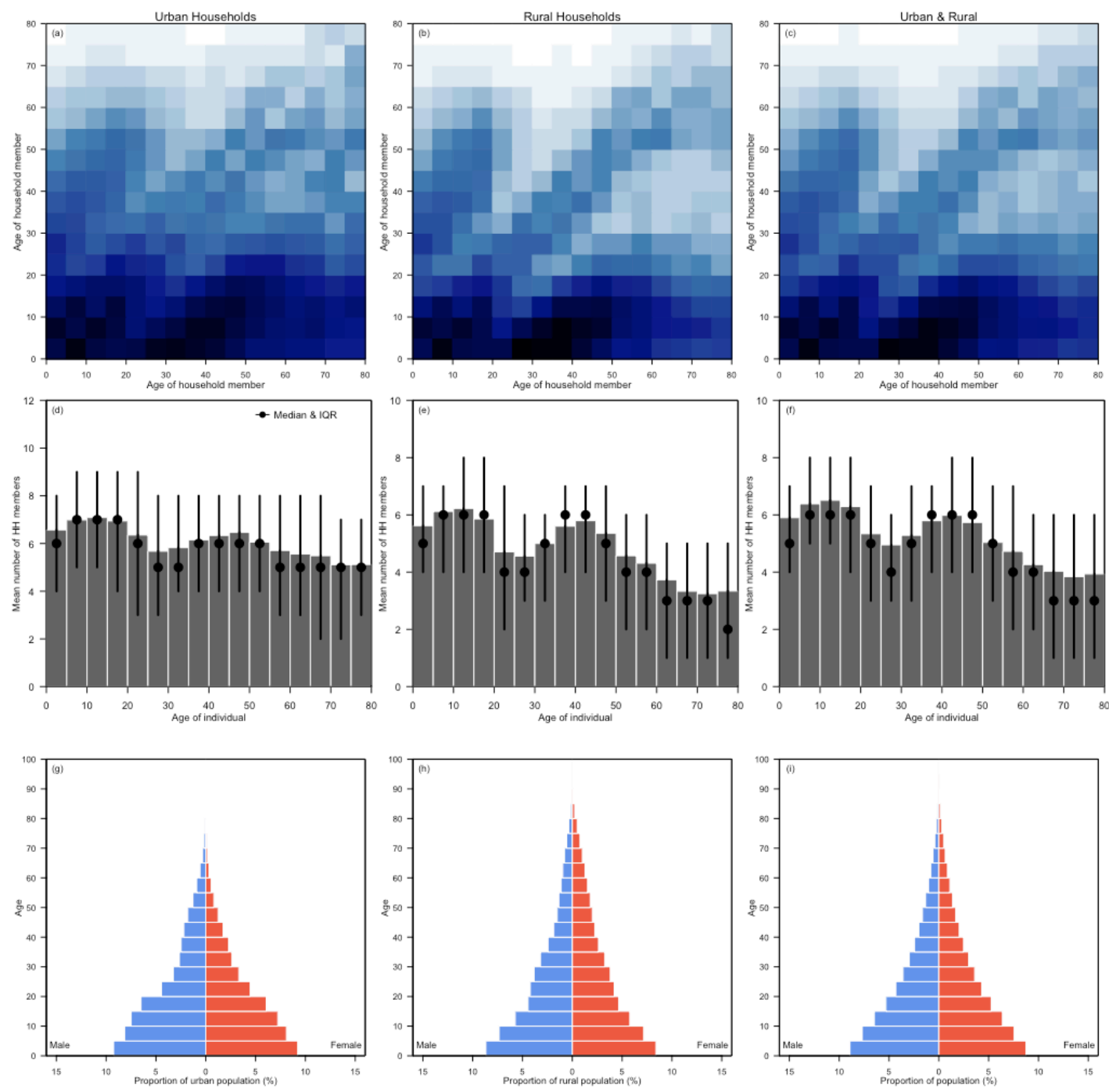
Empirical DHS household data: Dominican Republic
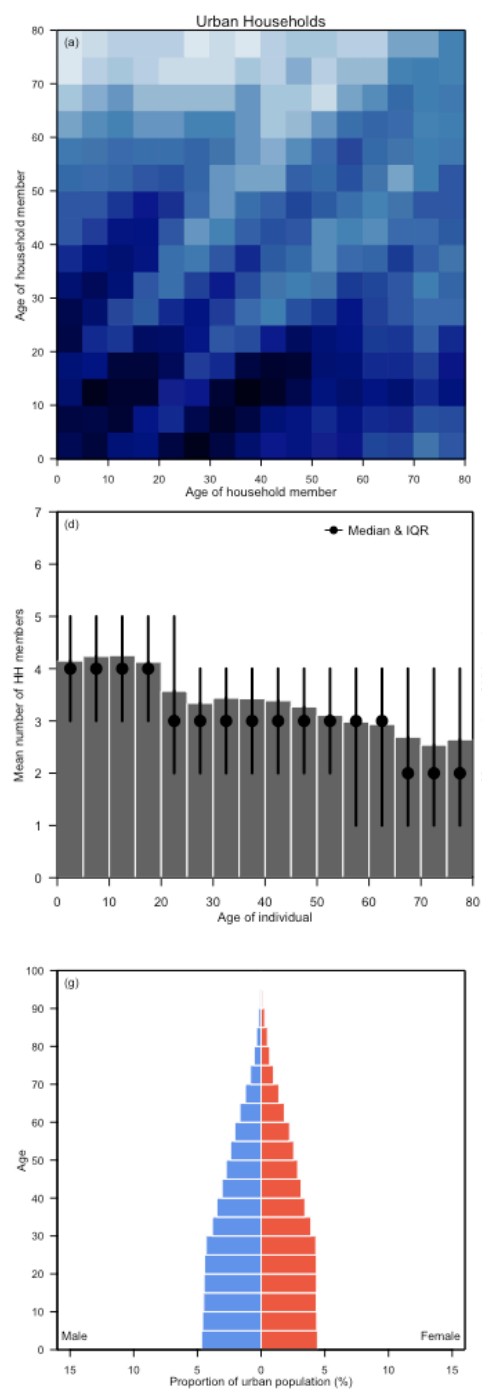
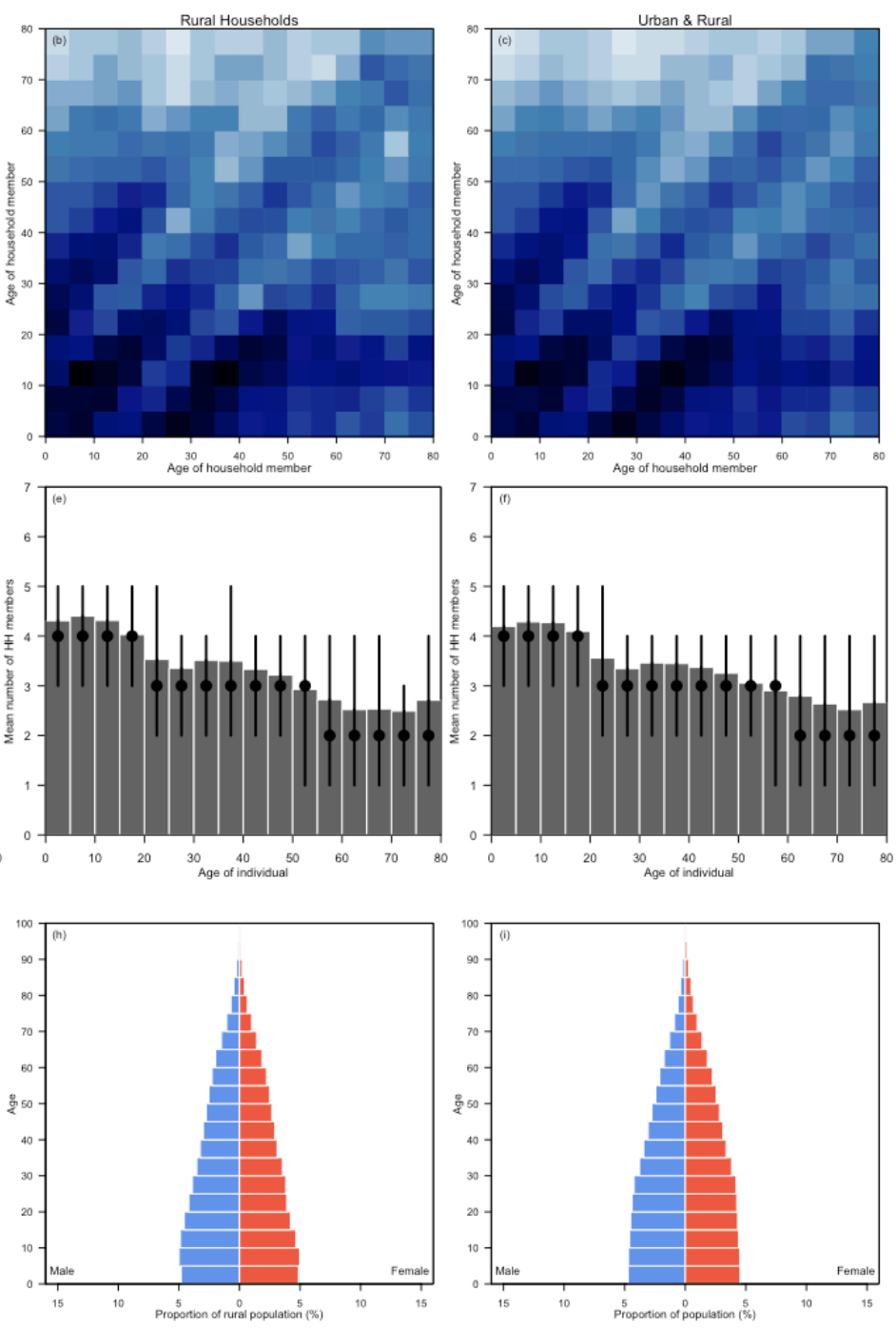
Empirical DHS household data: Ethiopia
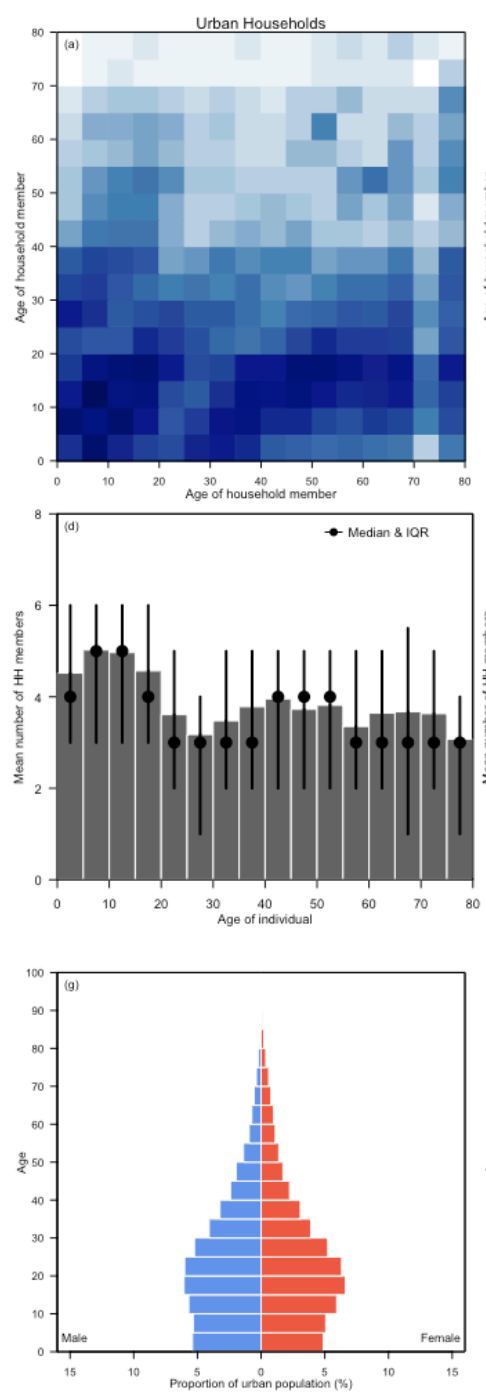
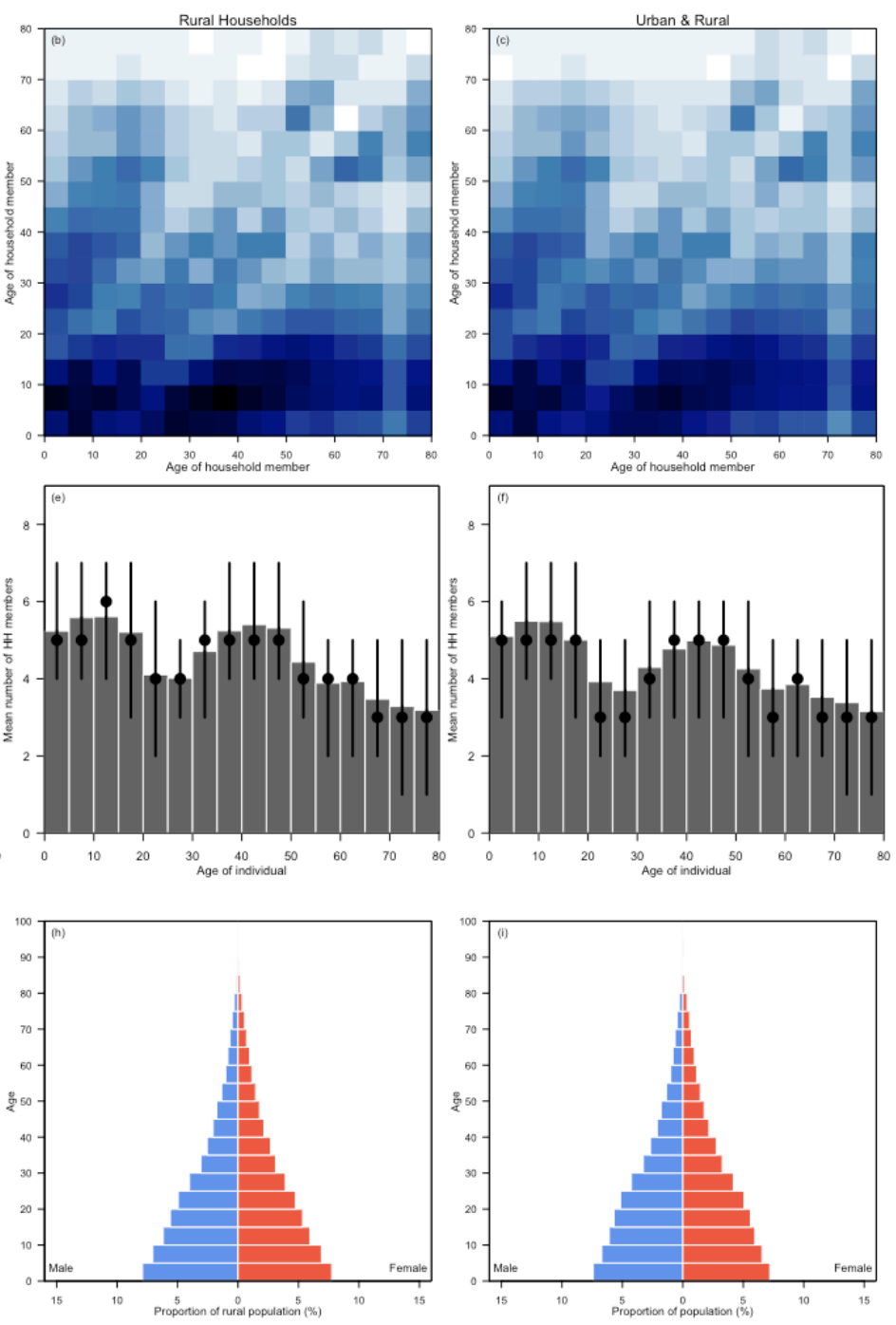
Empirical DHS household data: Ghana
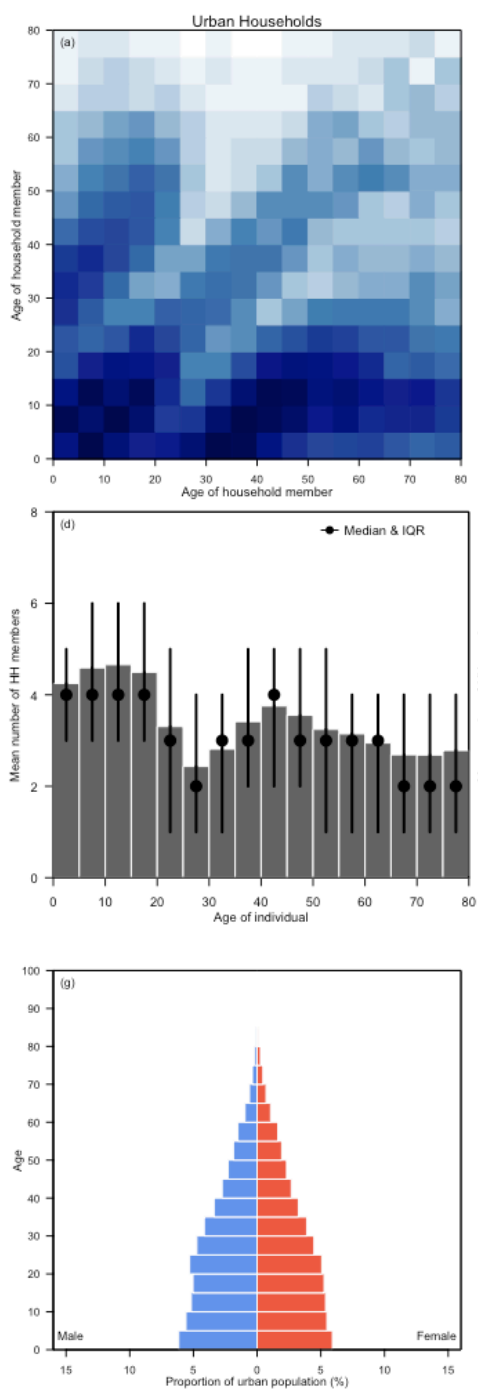
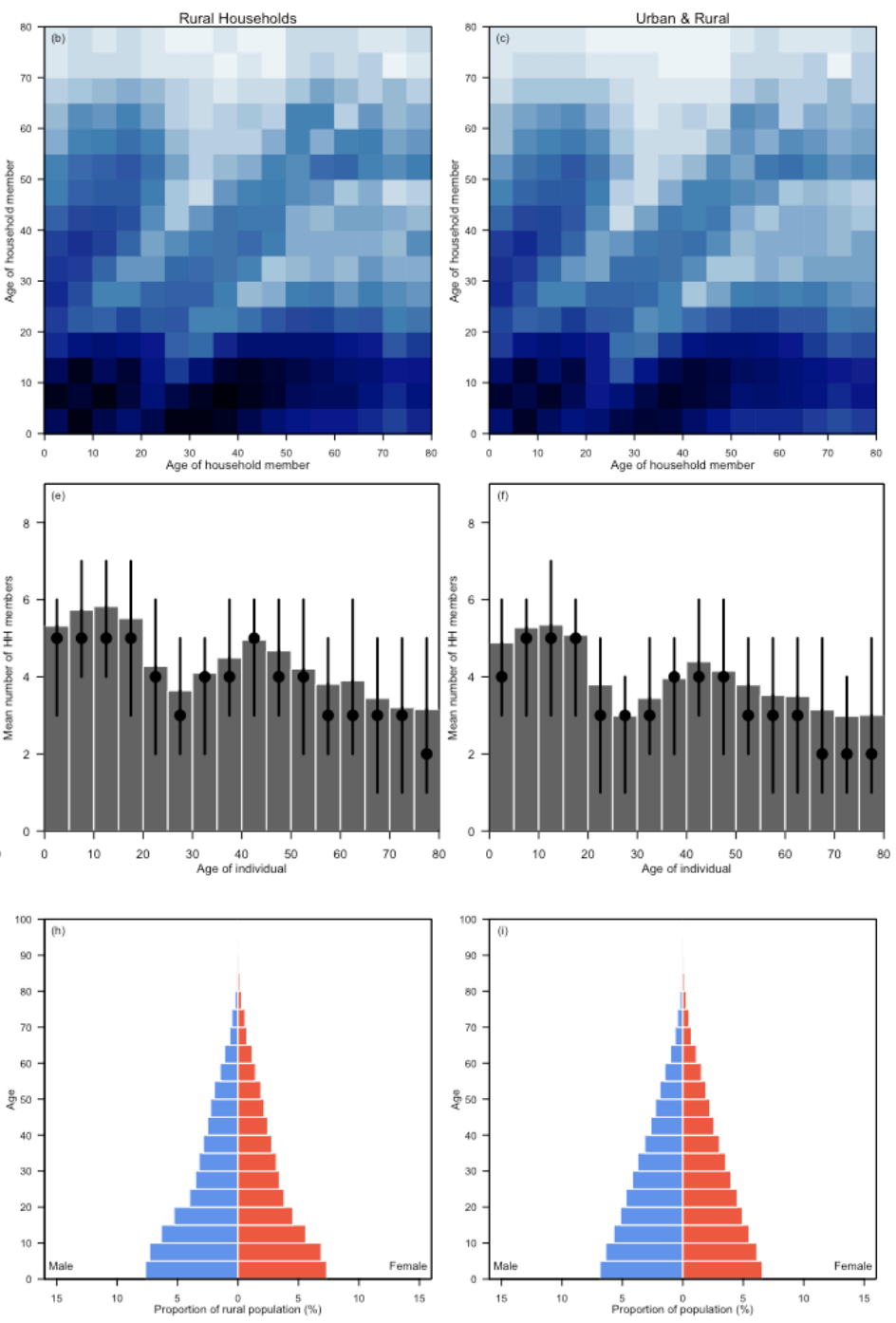
Empirical DHS household data: Guatemala
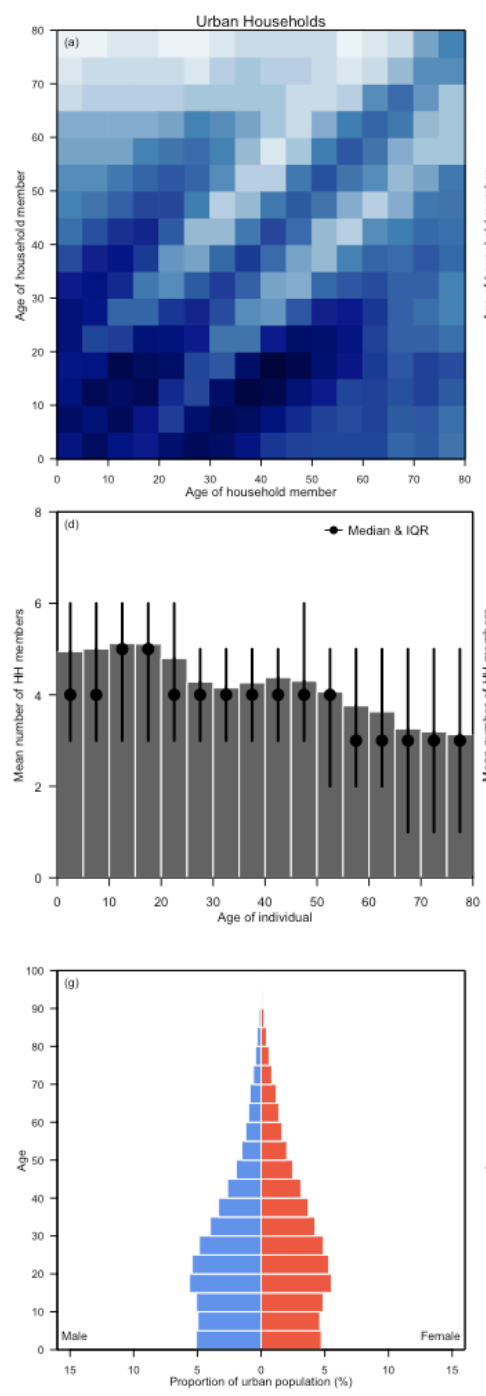
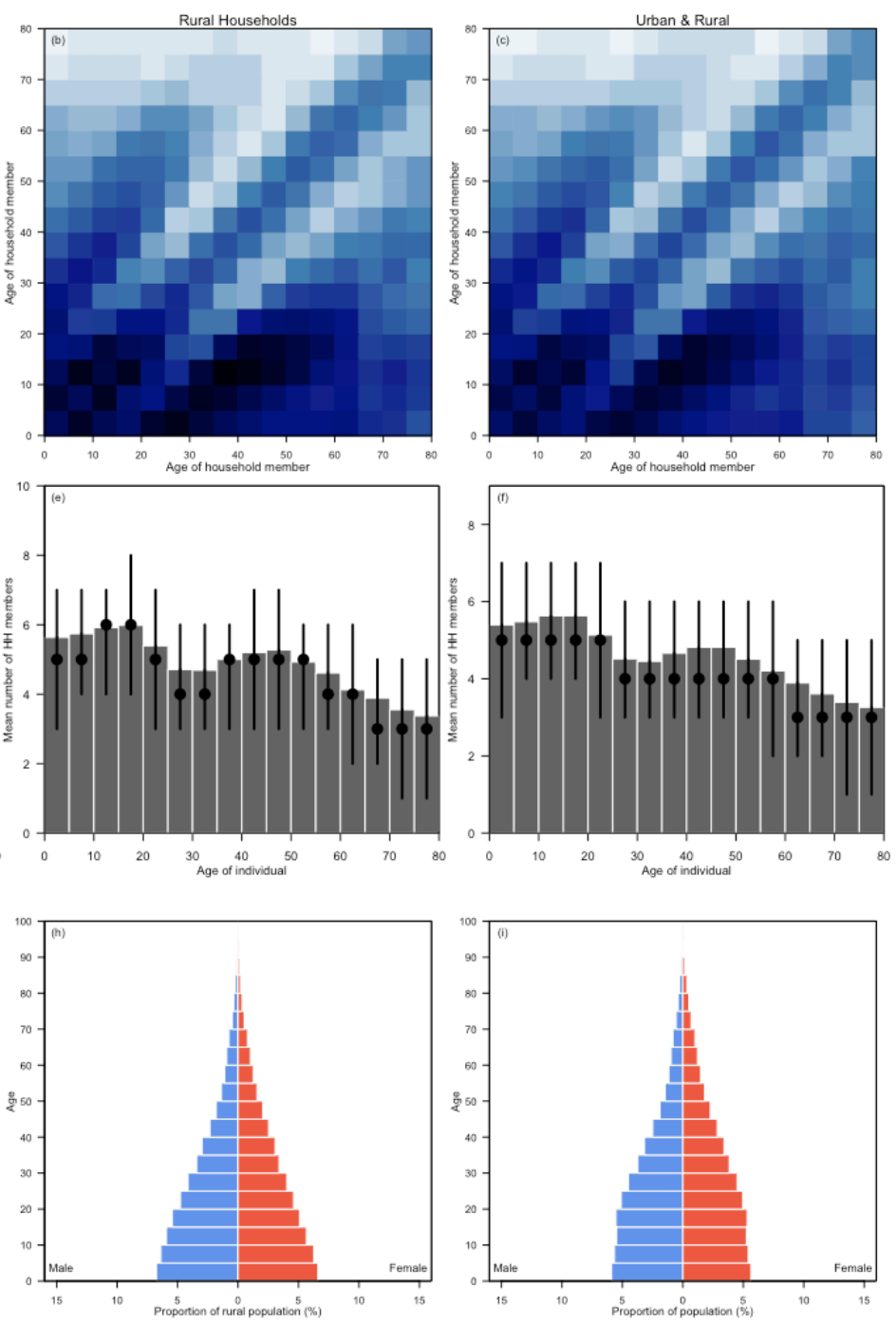
Empirical DHS household data: Guinea
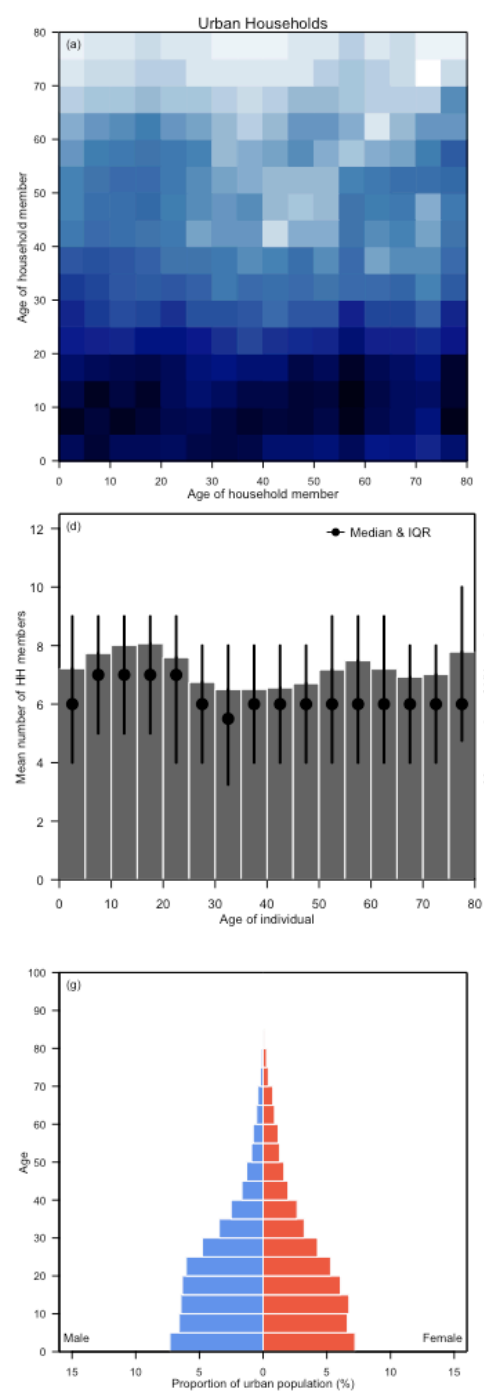
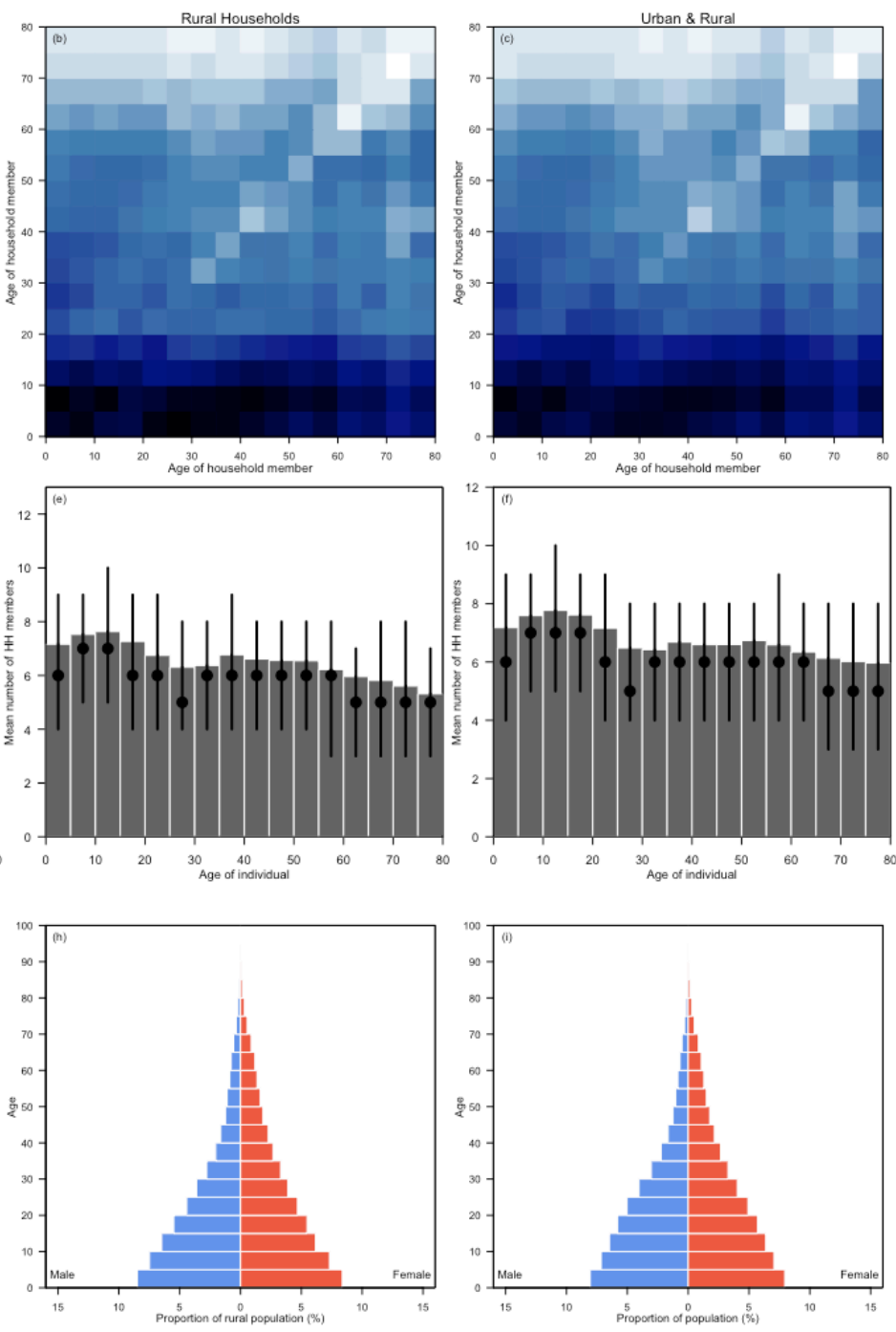
Empirical DHS household data: Guyana
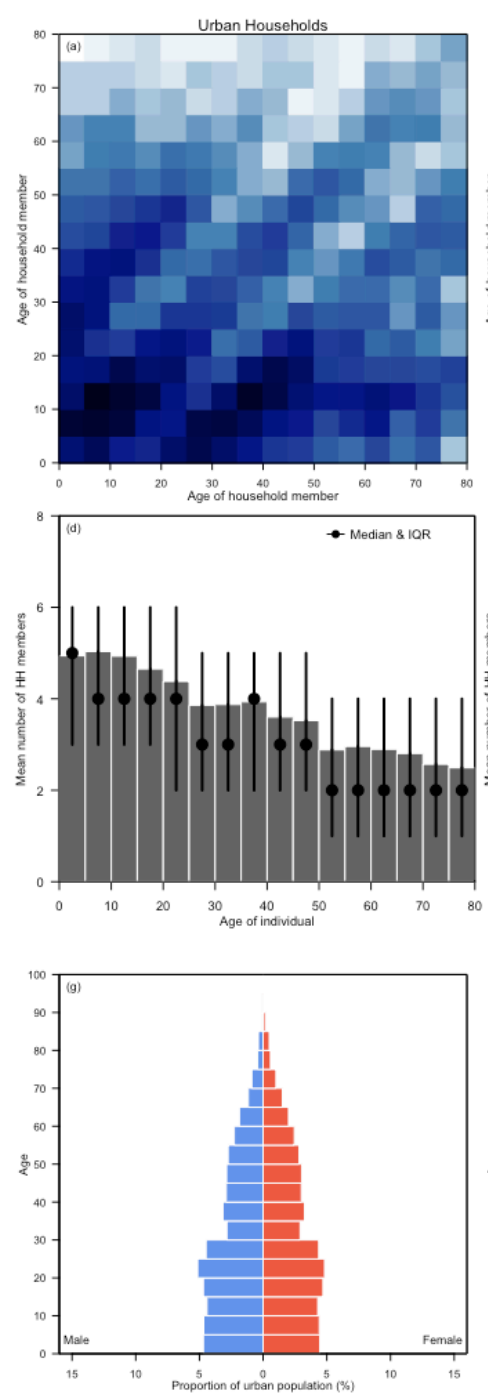
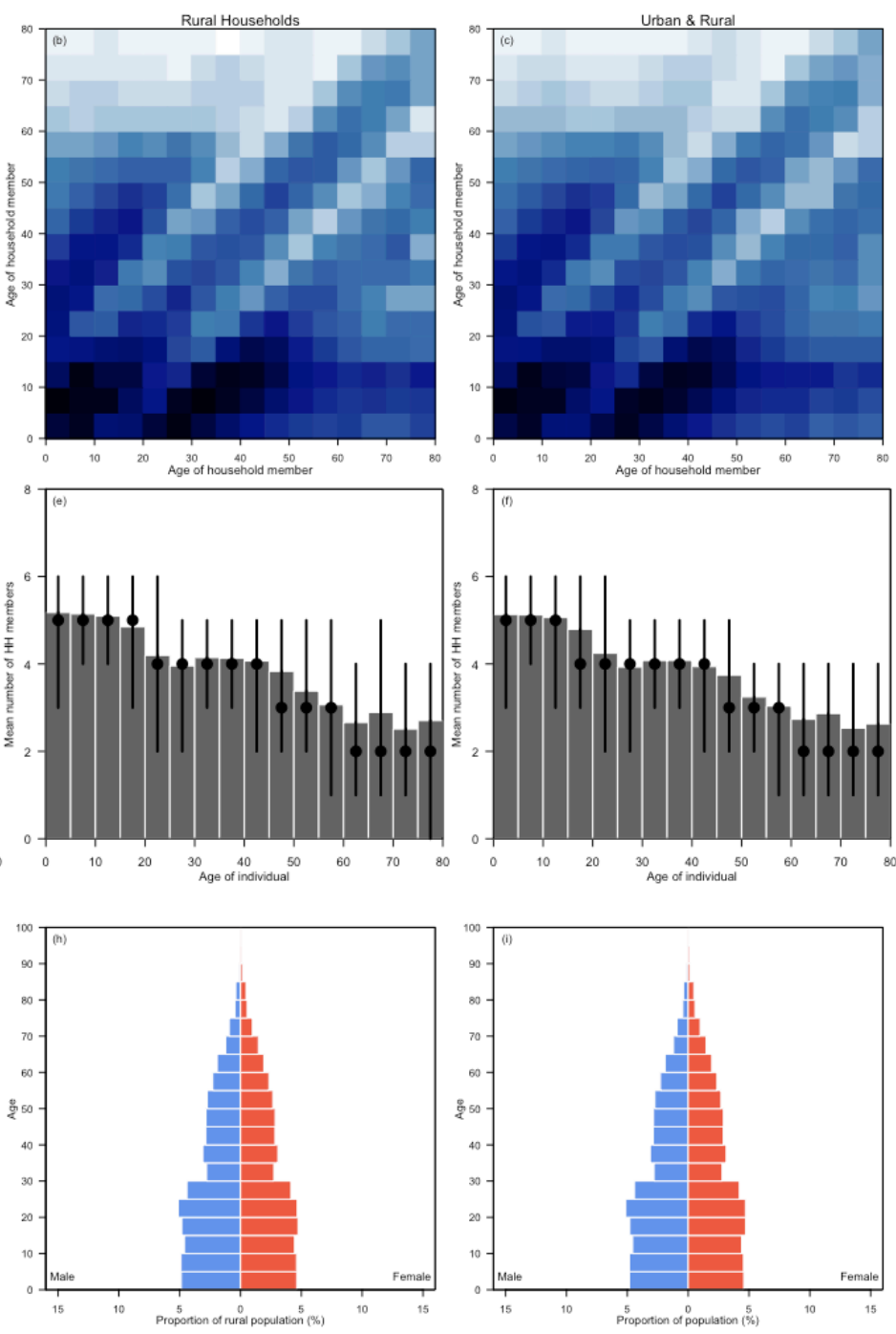
Empirical DHS household data: Haiti

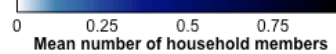
in a specific age group
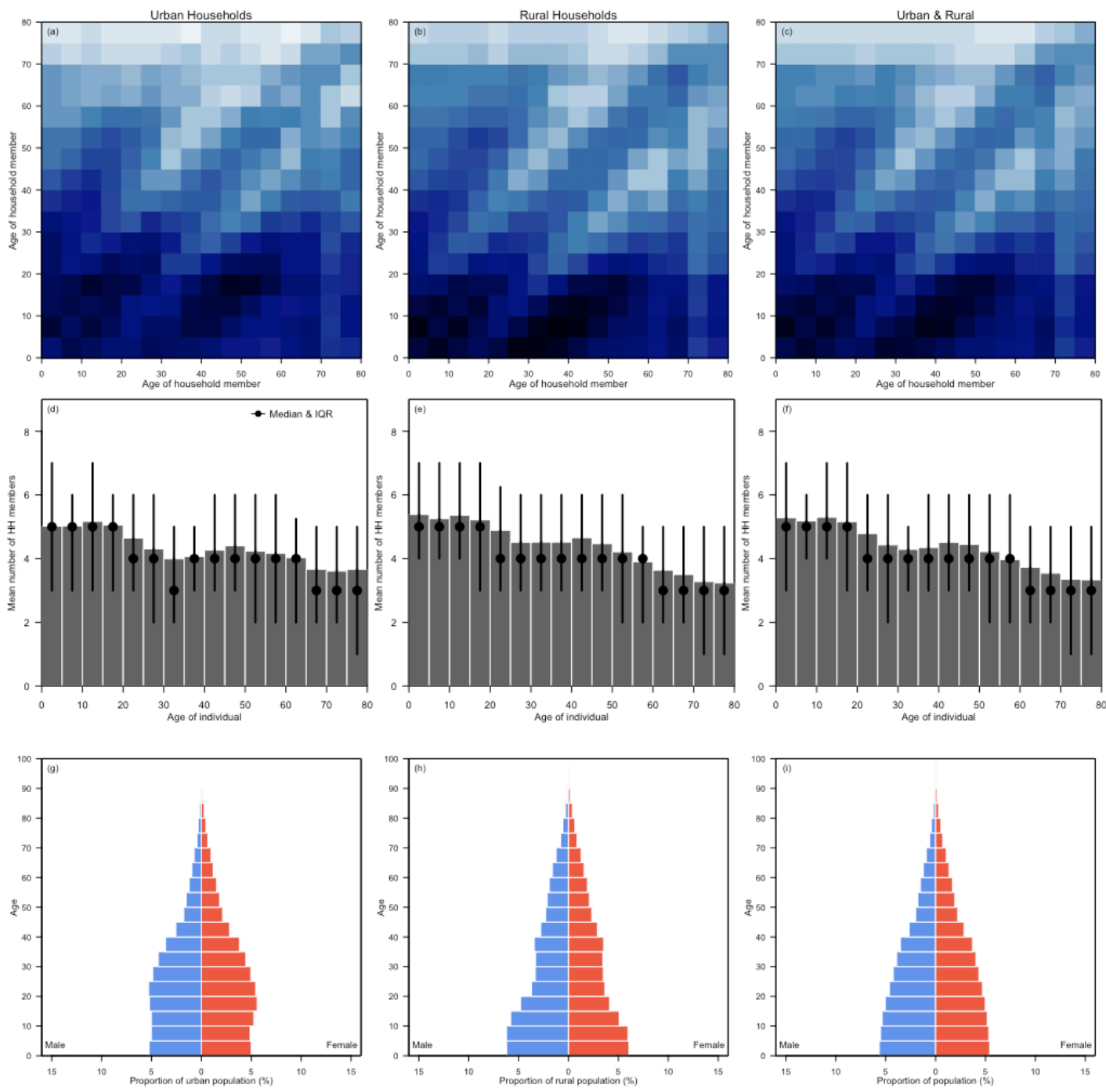
Empirical DHS household data: Honduras
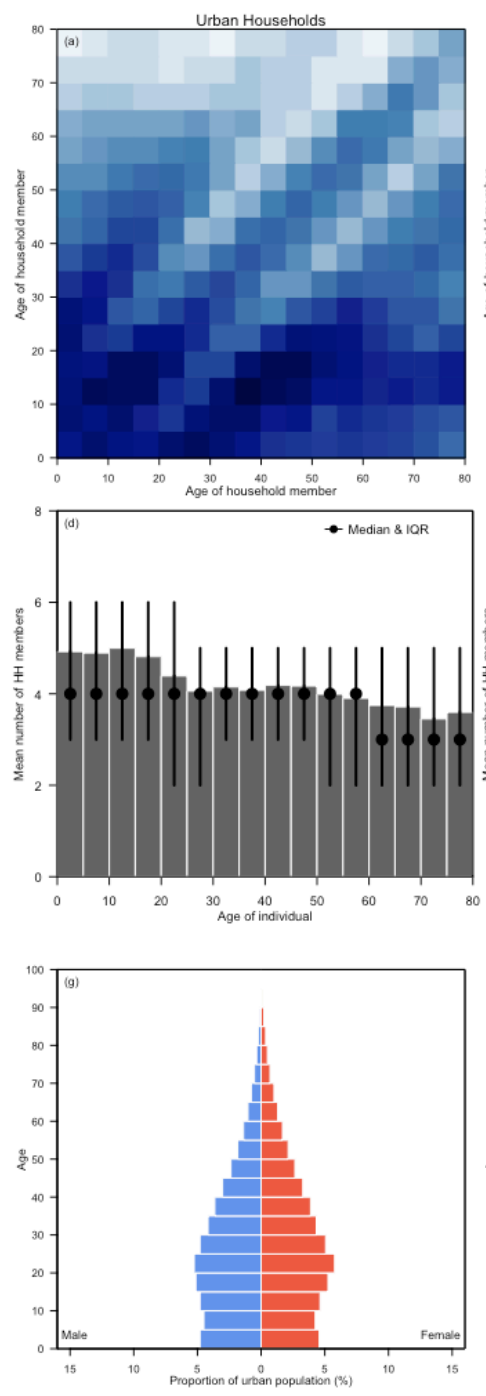
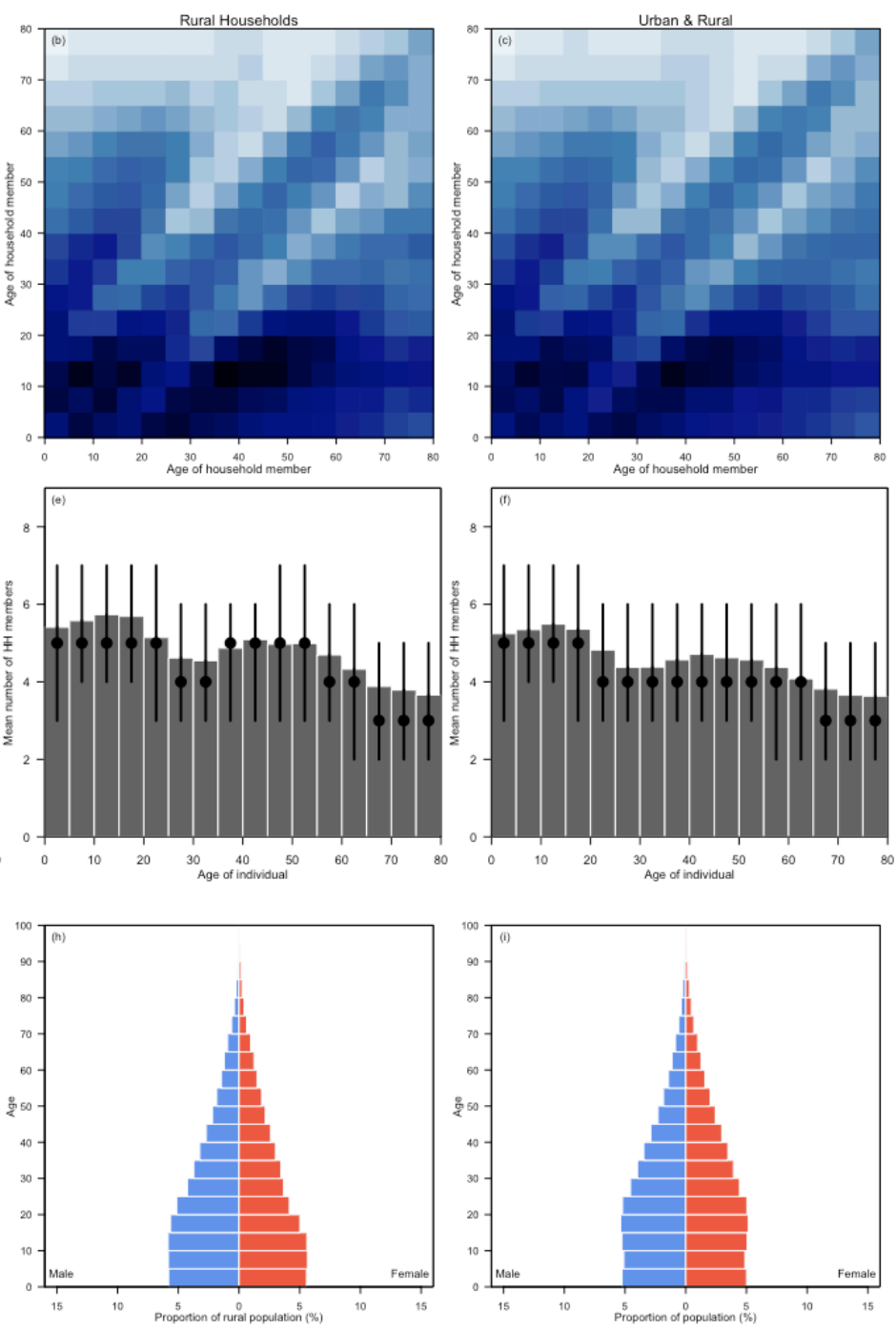
Empirical DHS household data: India
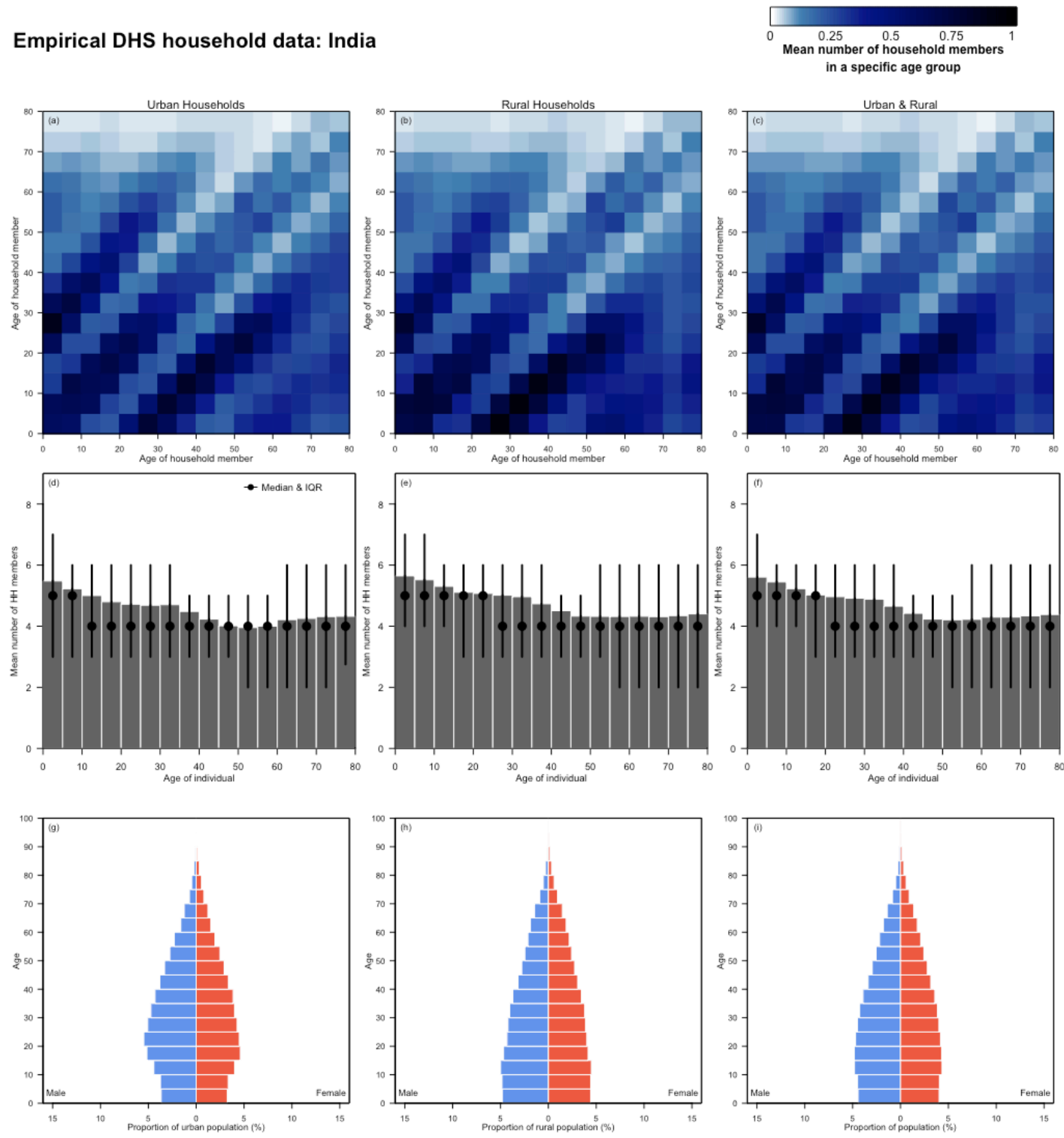
Empirical DHS household data: Indonesia
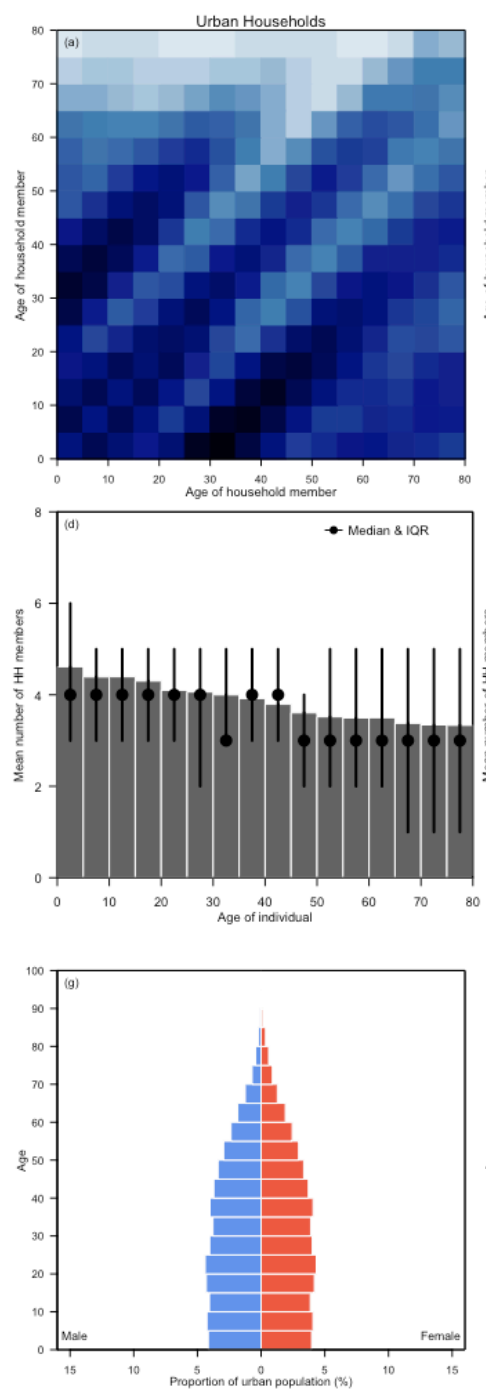
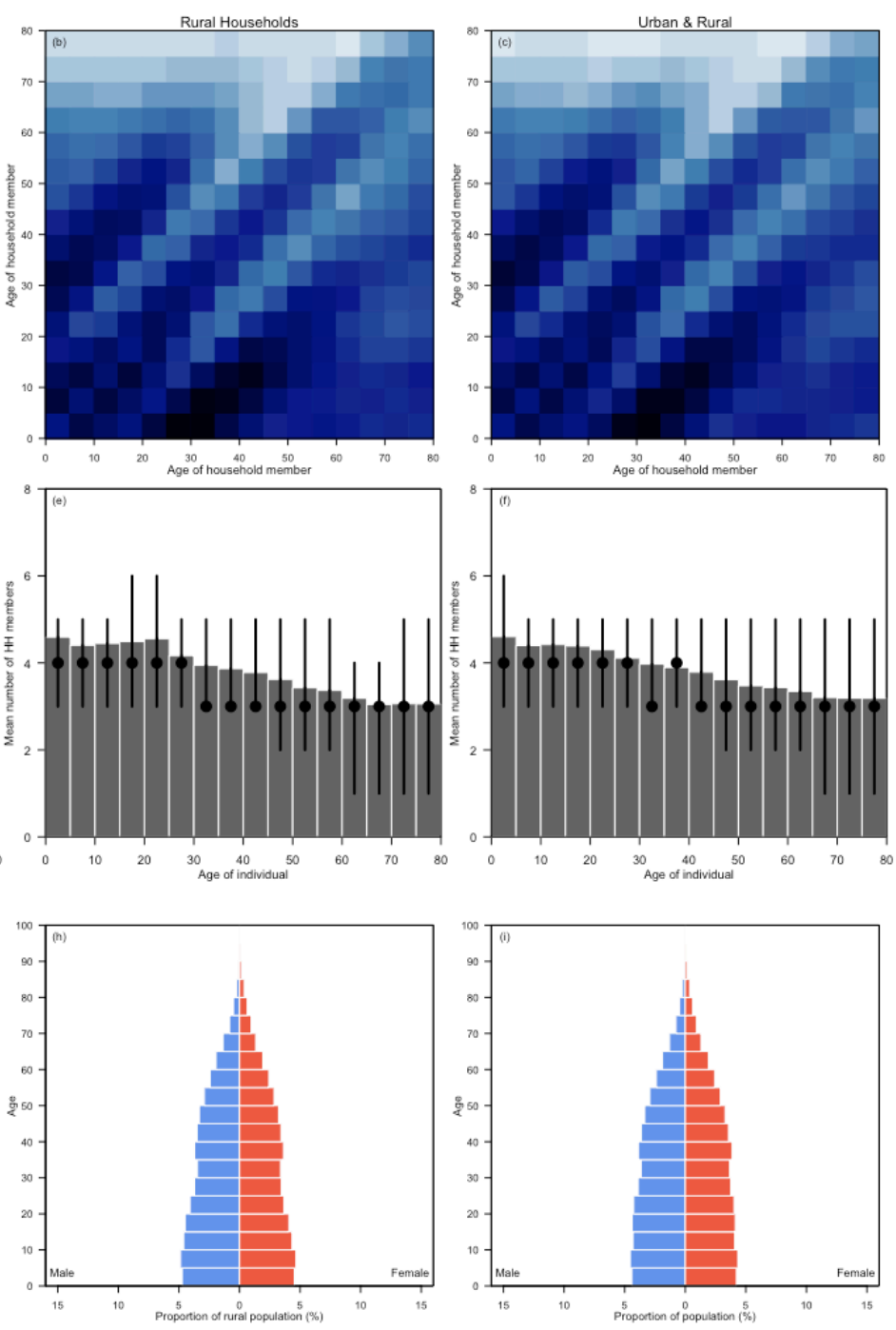
Empirical DHS household data: Kenya
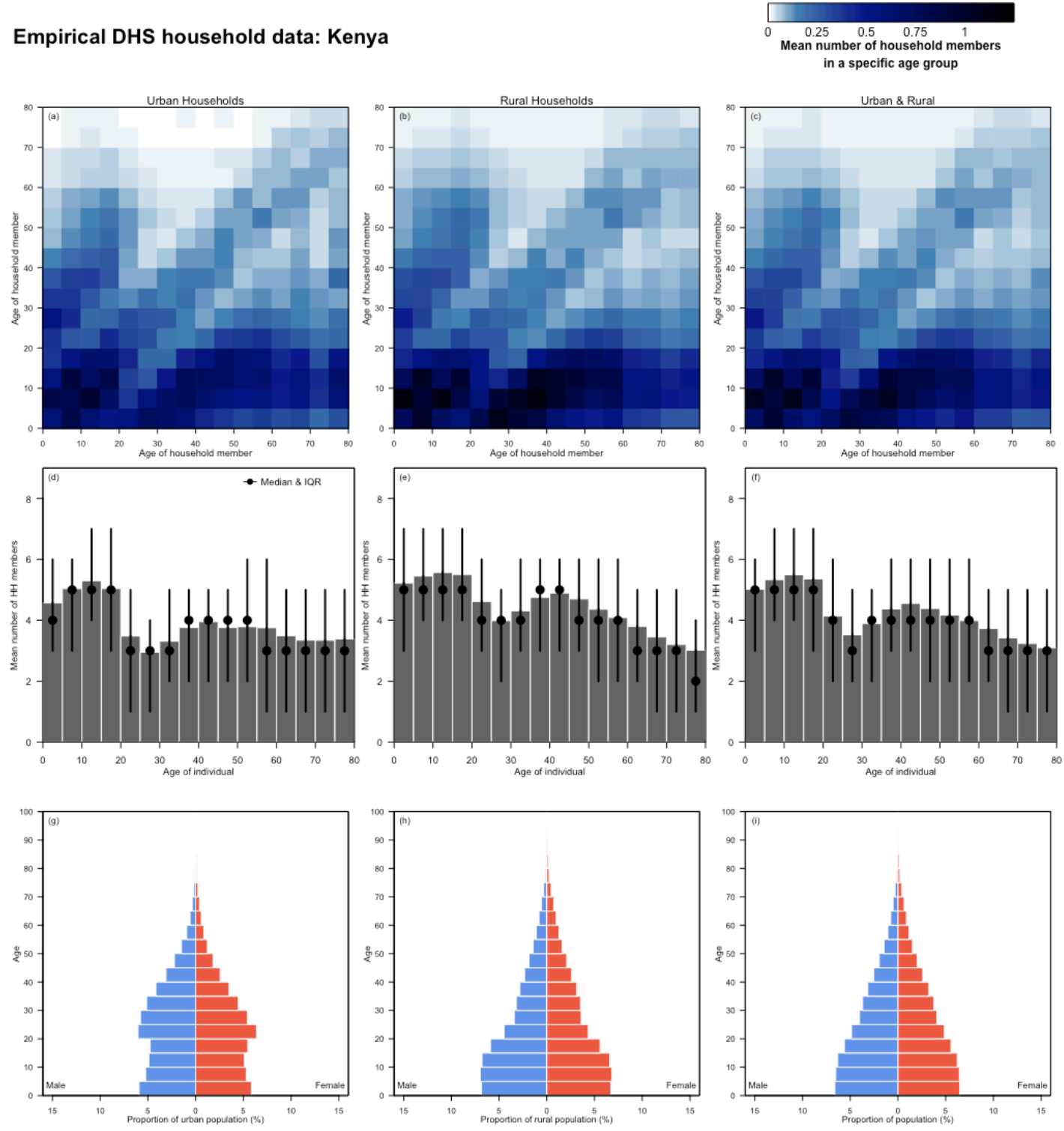
Empirical DHS household data: Kyrgyzstan
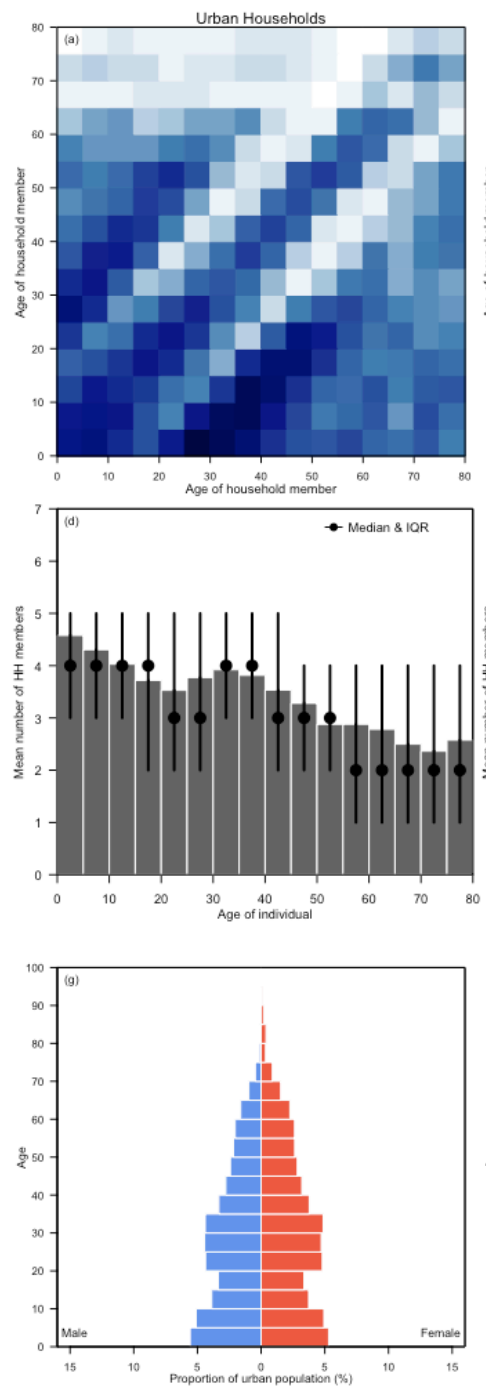
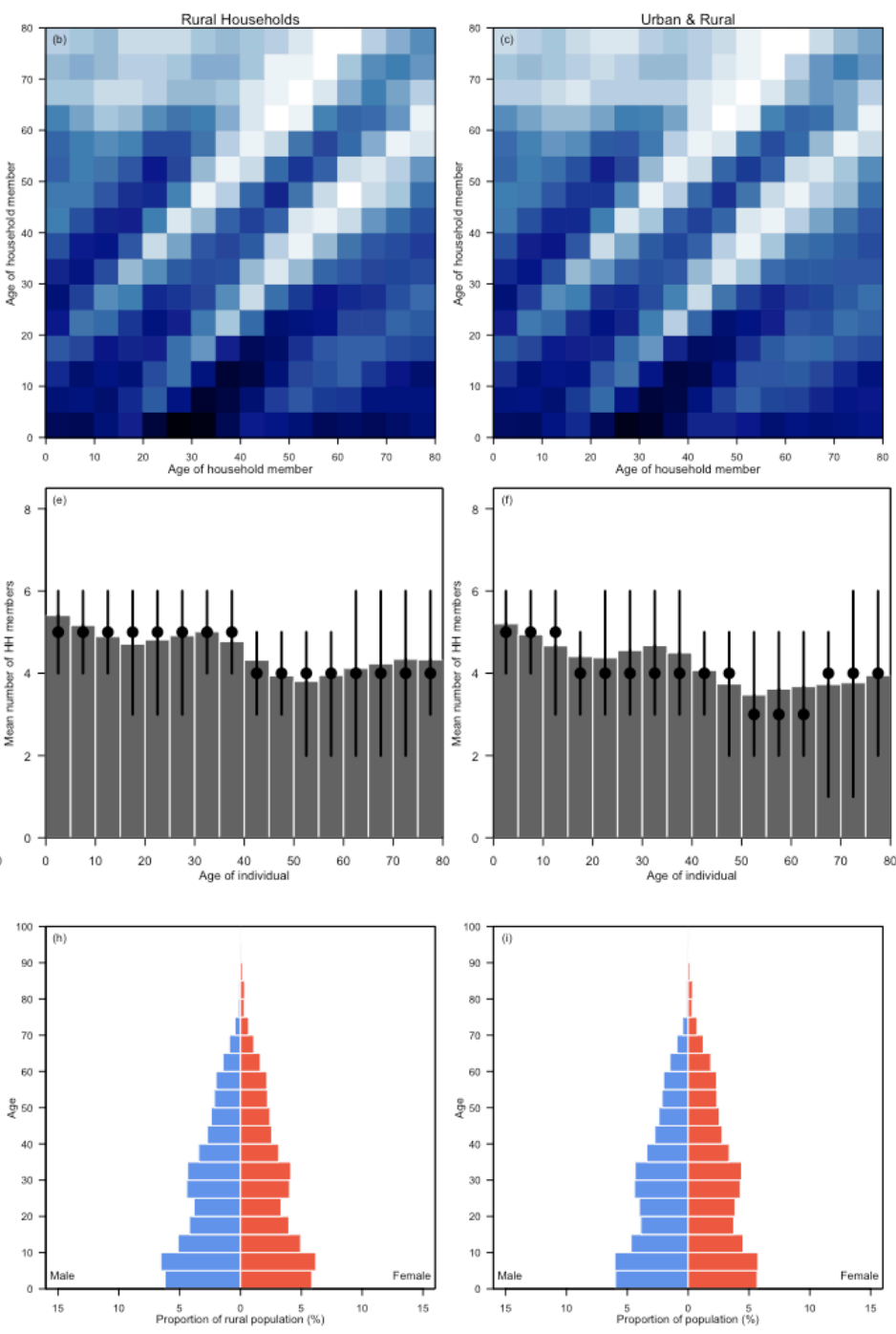
Empirical DHS household data: Lesotho
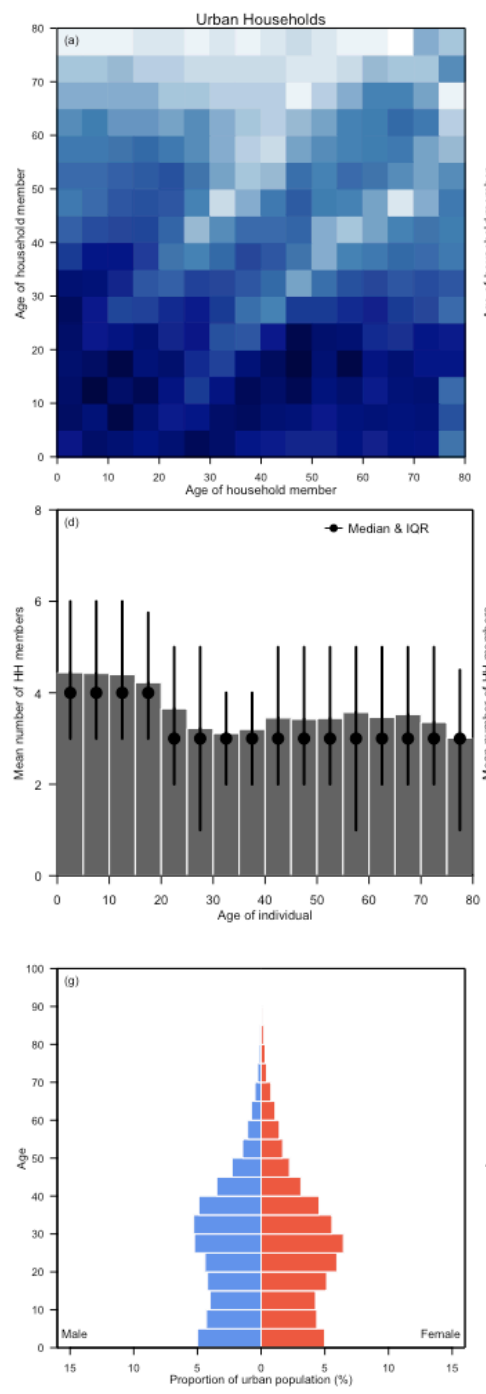
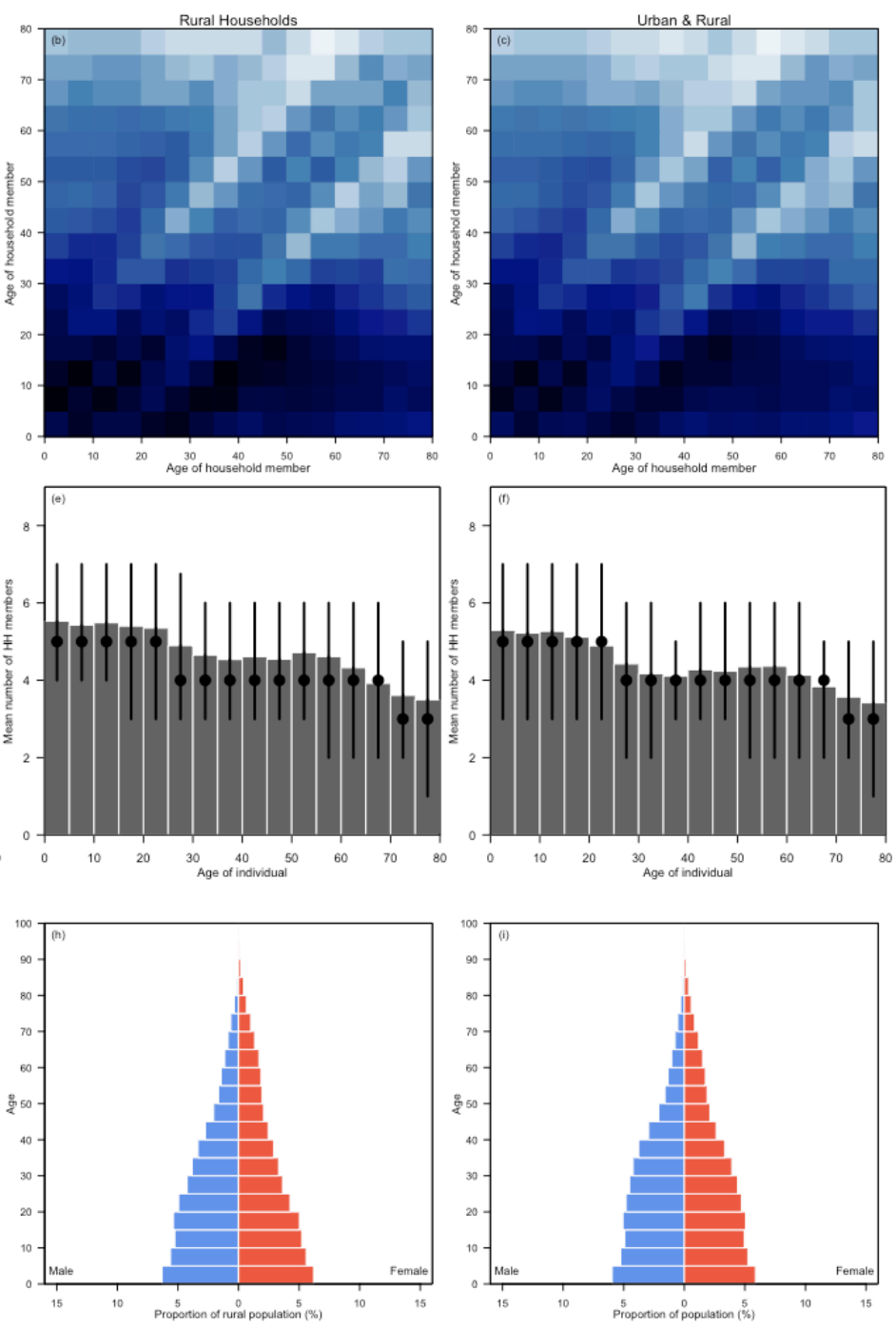
Empirical DHS household data: Liberia
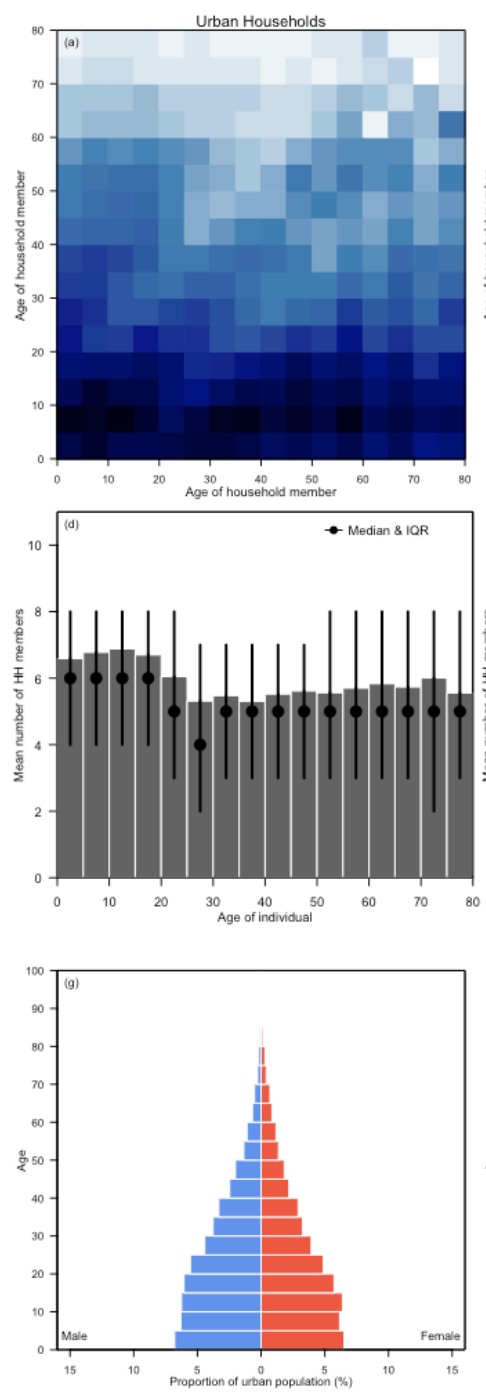
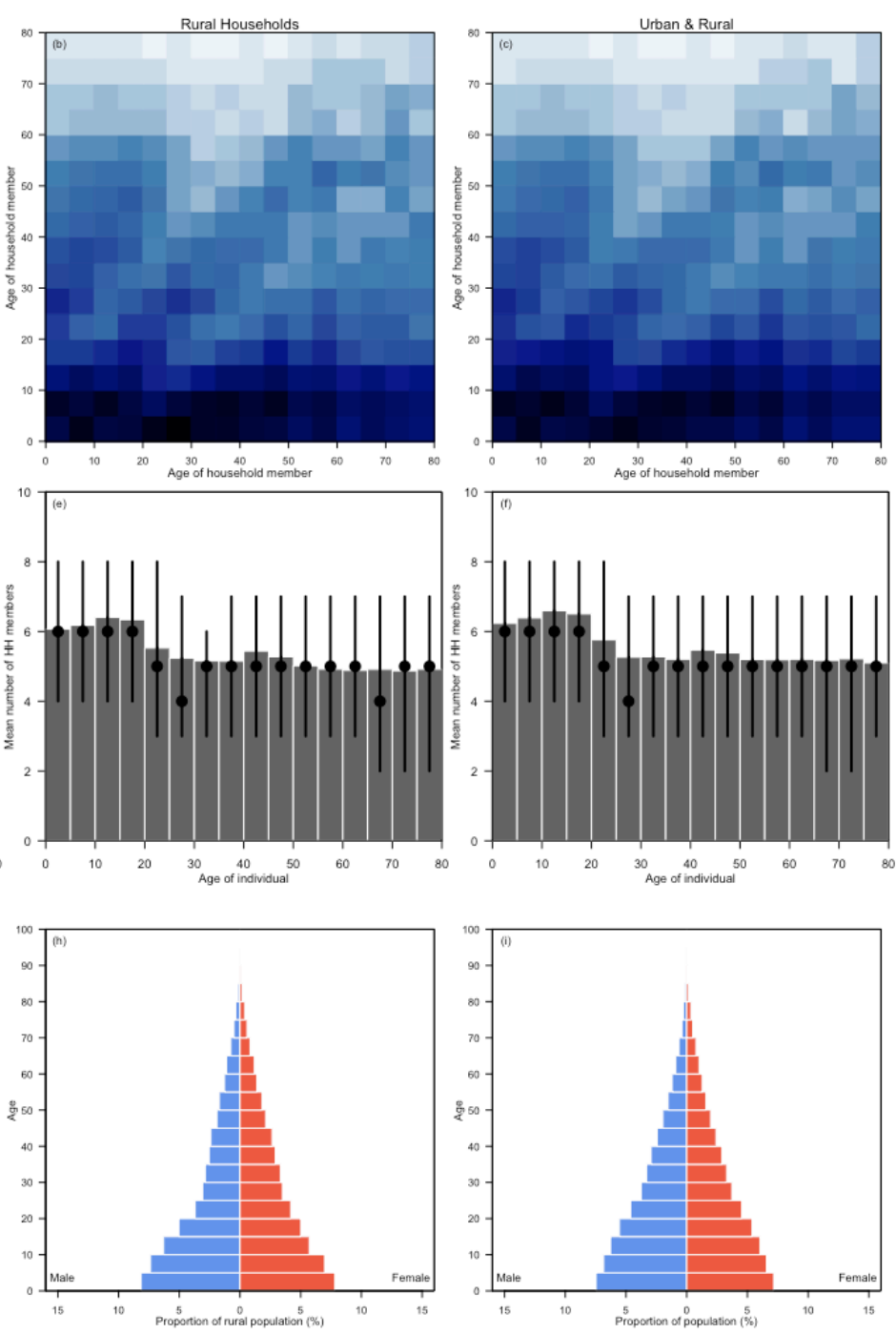
Empirical DHS household data: Malawi
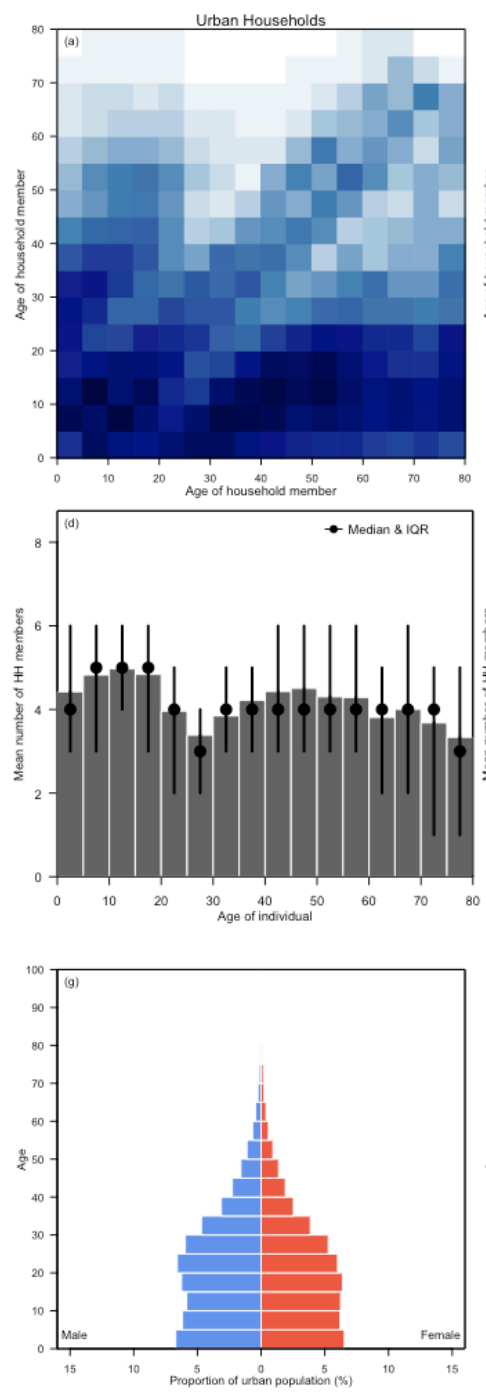
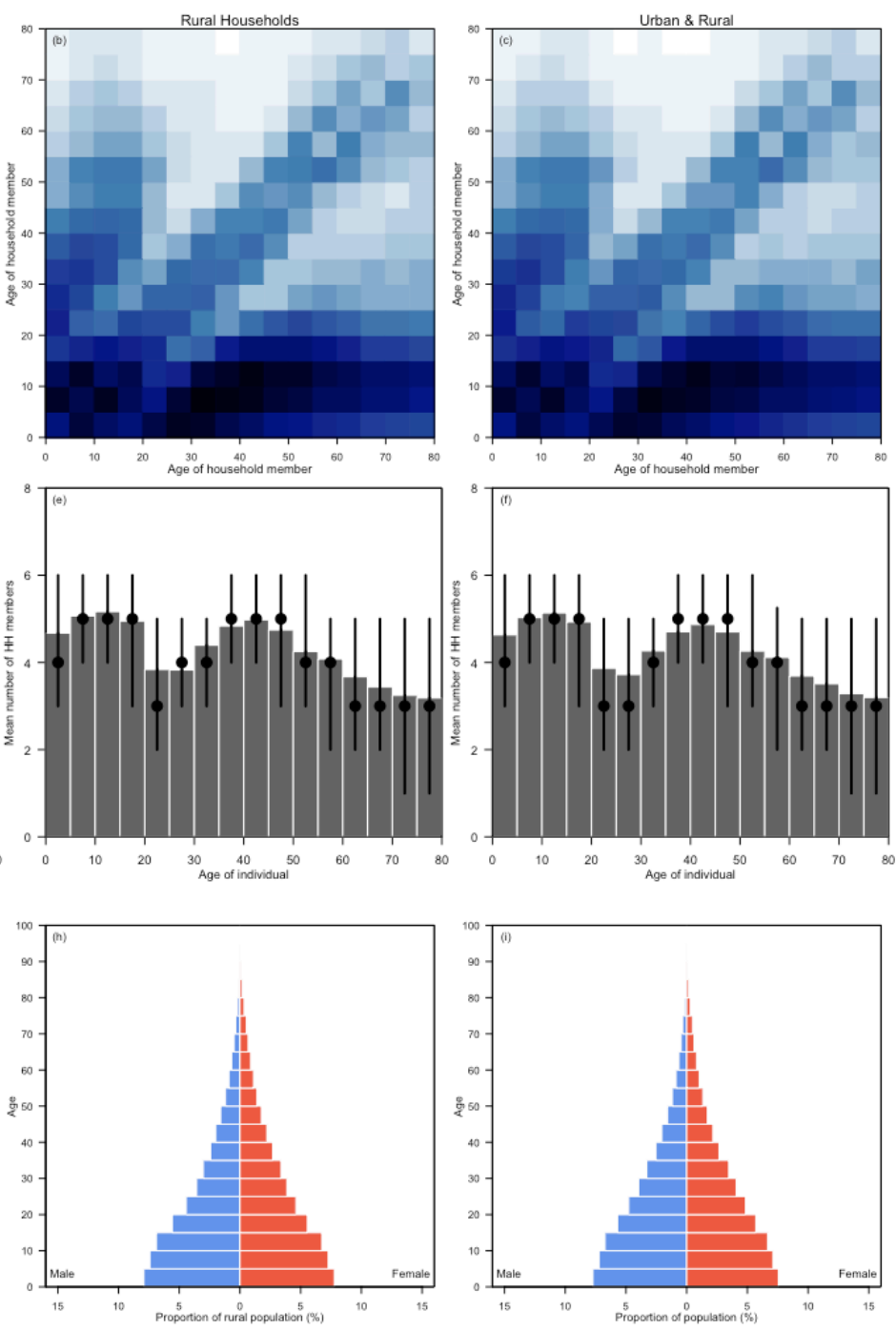
Empirical DHS household data: Maldives
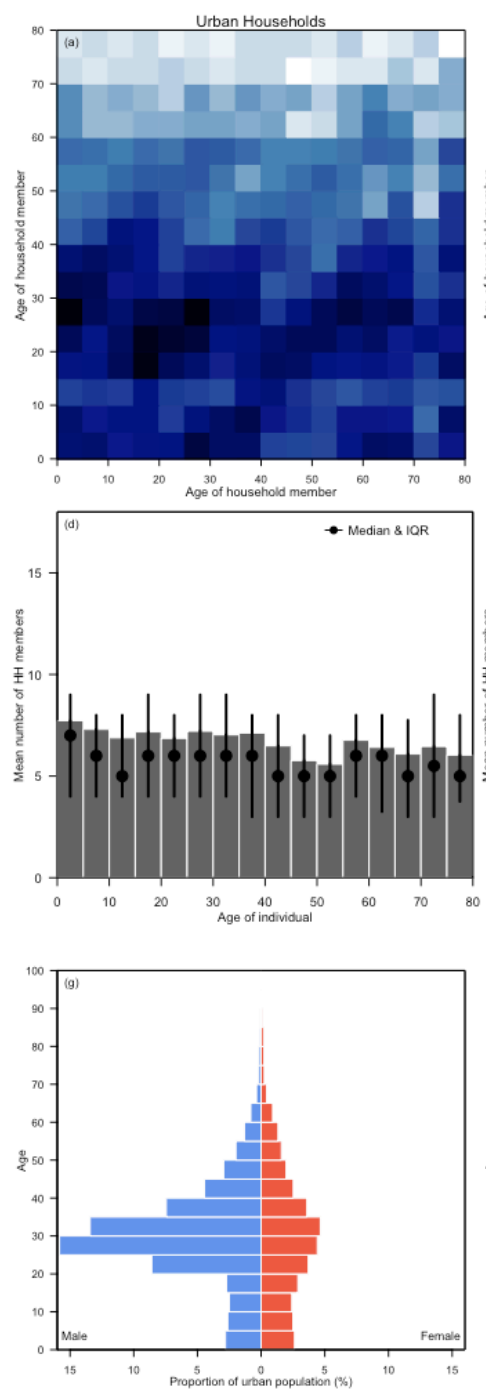
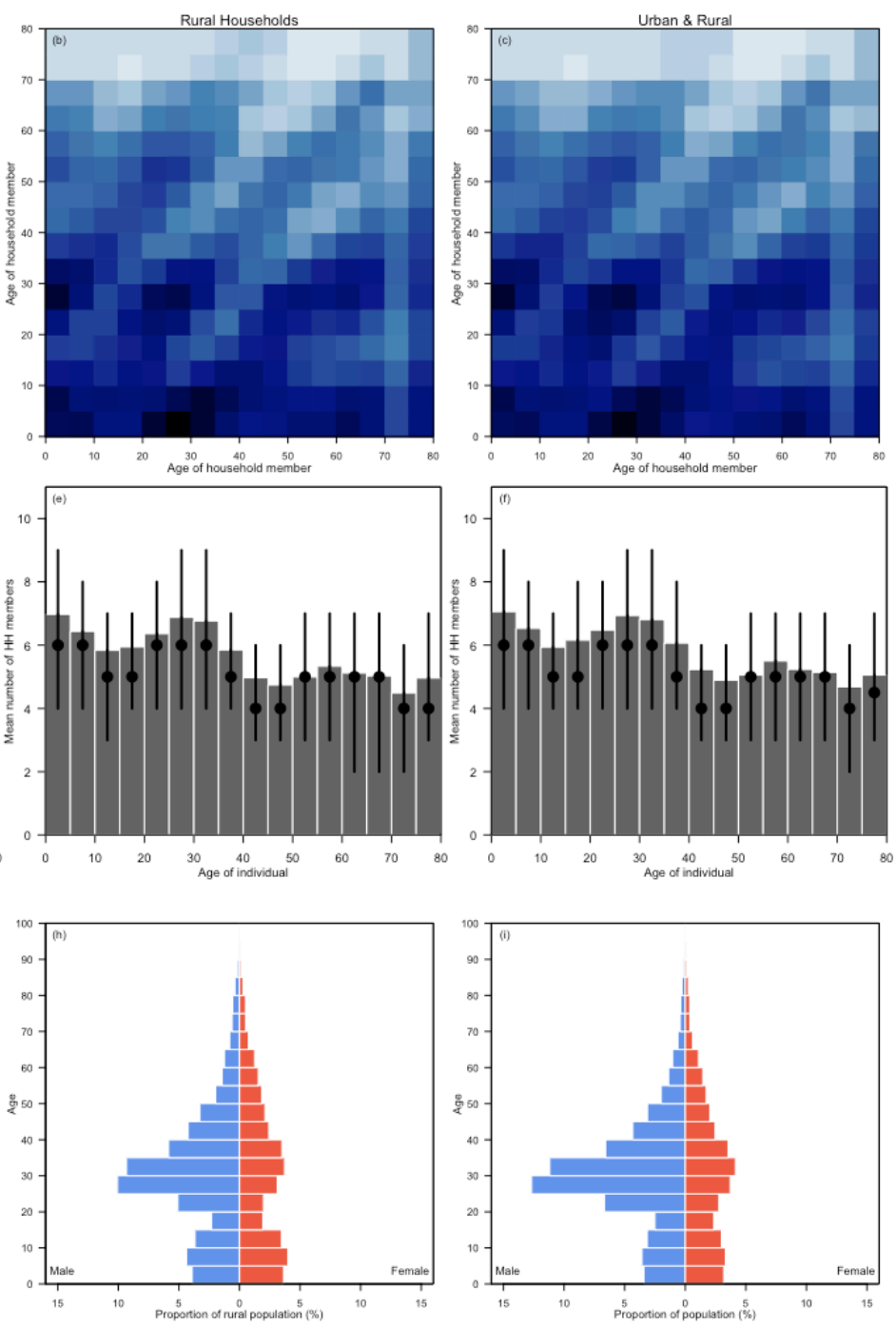
Empirical DHS household data: Mali
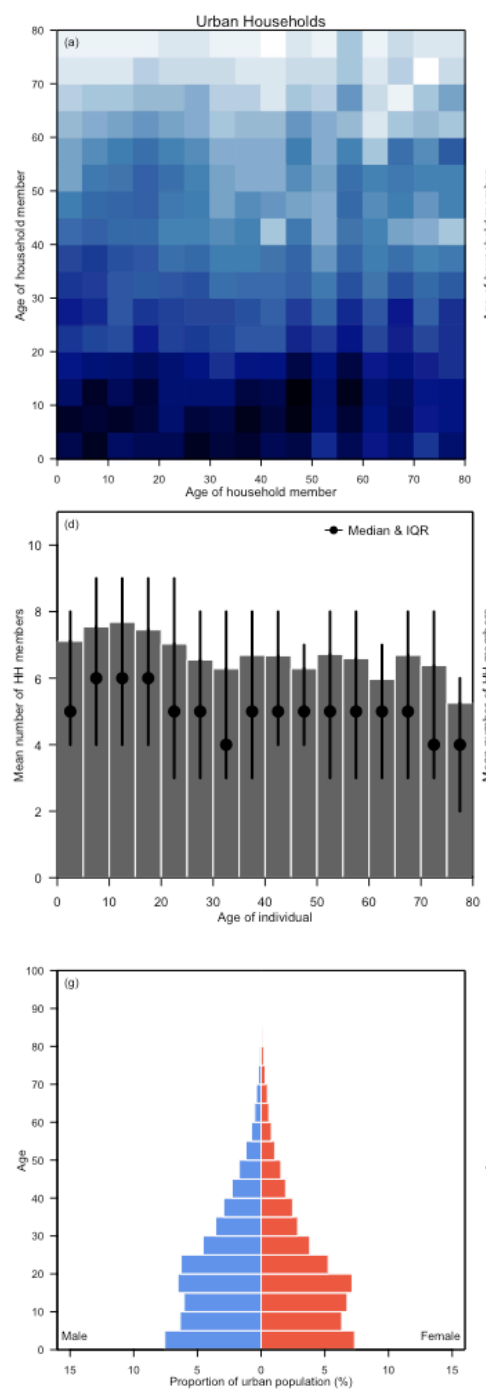
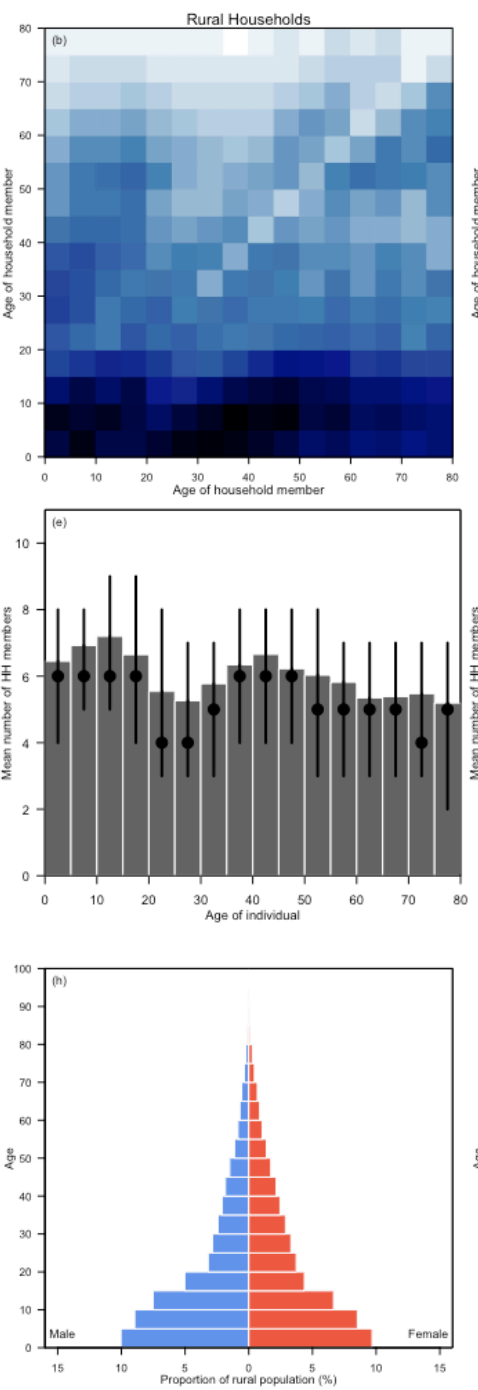

\begin{tabular}{lll}
\hline 0.5 & 1 & 1.5 \\
& 1 & 1
\end{tabular} in a specific age group
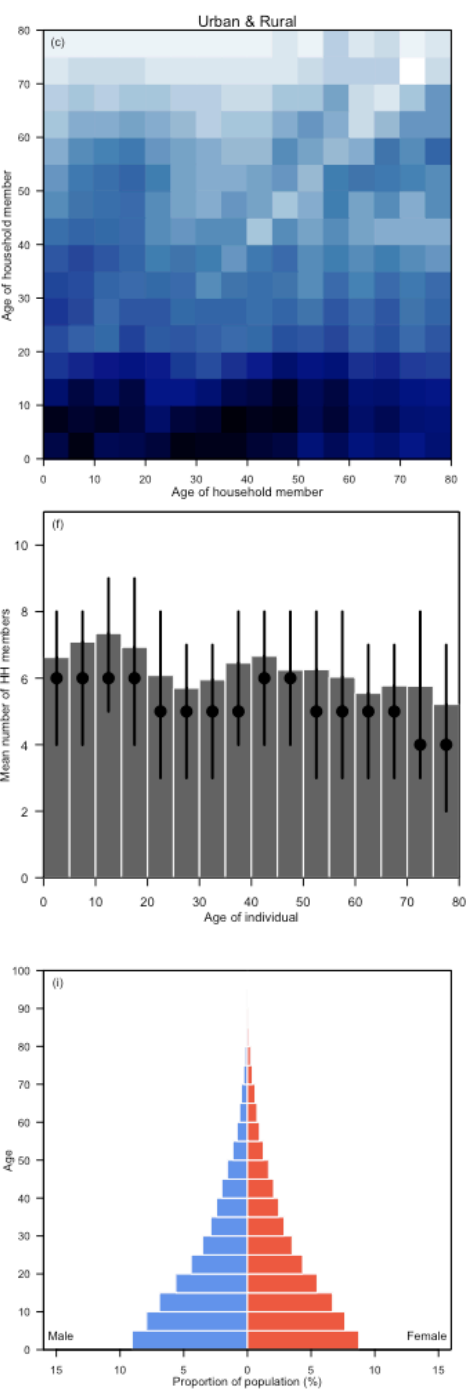
Empirical DHS household data: Nepal
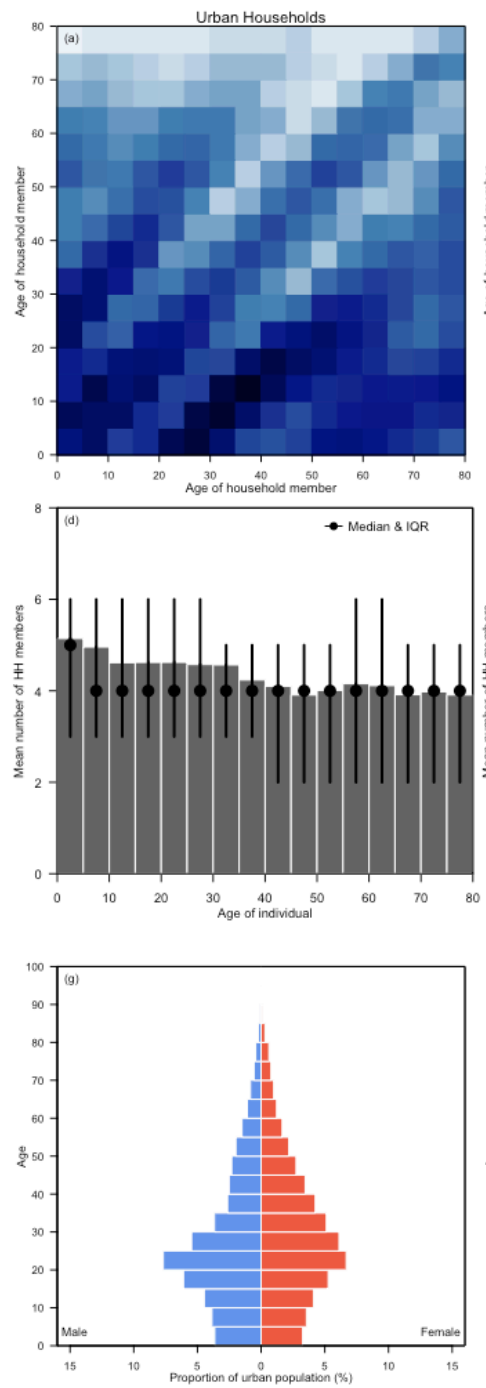
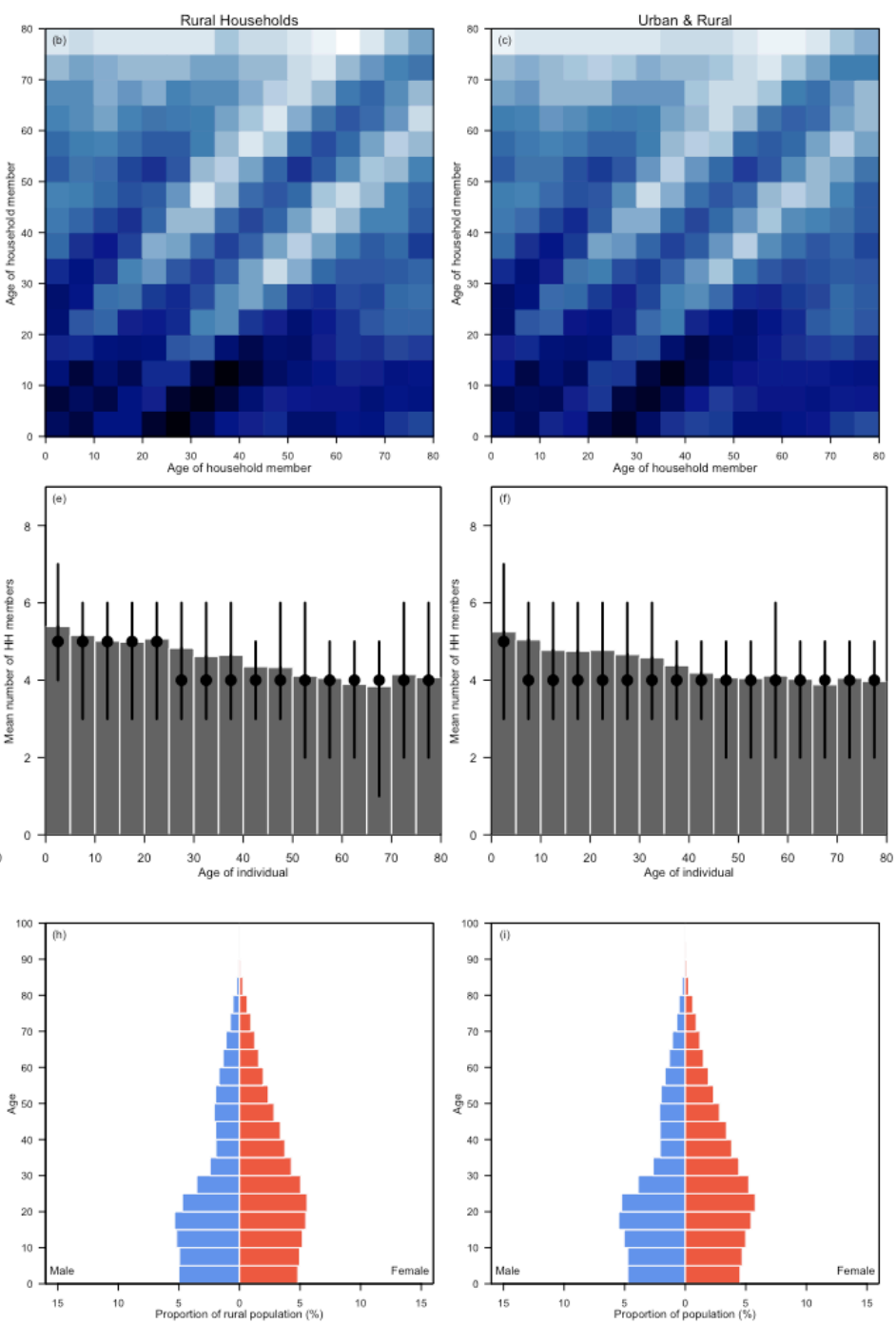
Empirical DHS household data: Niger
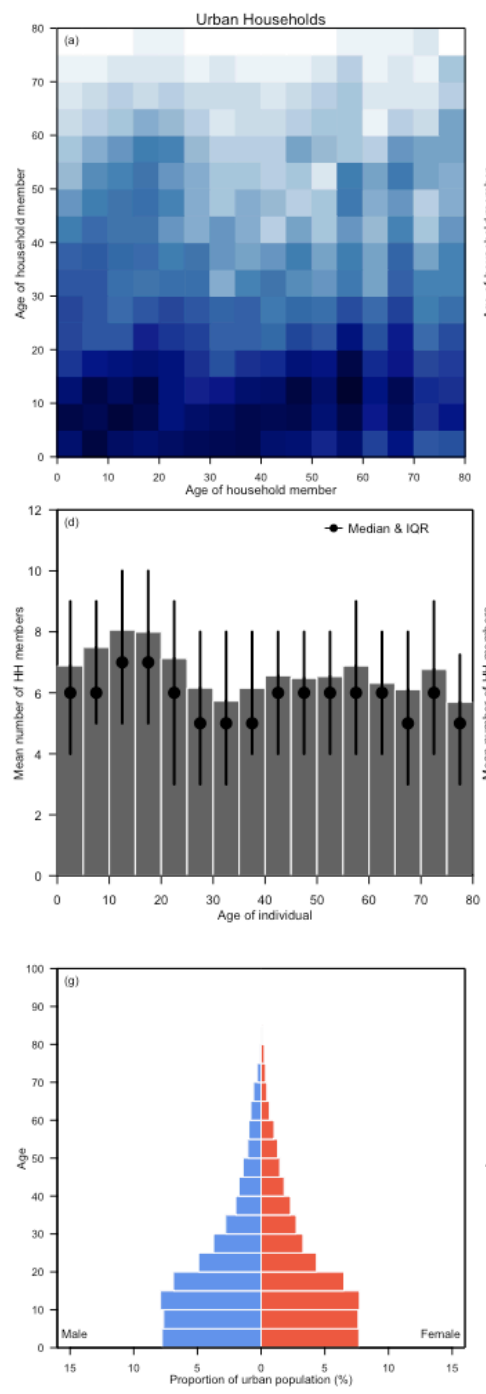
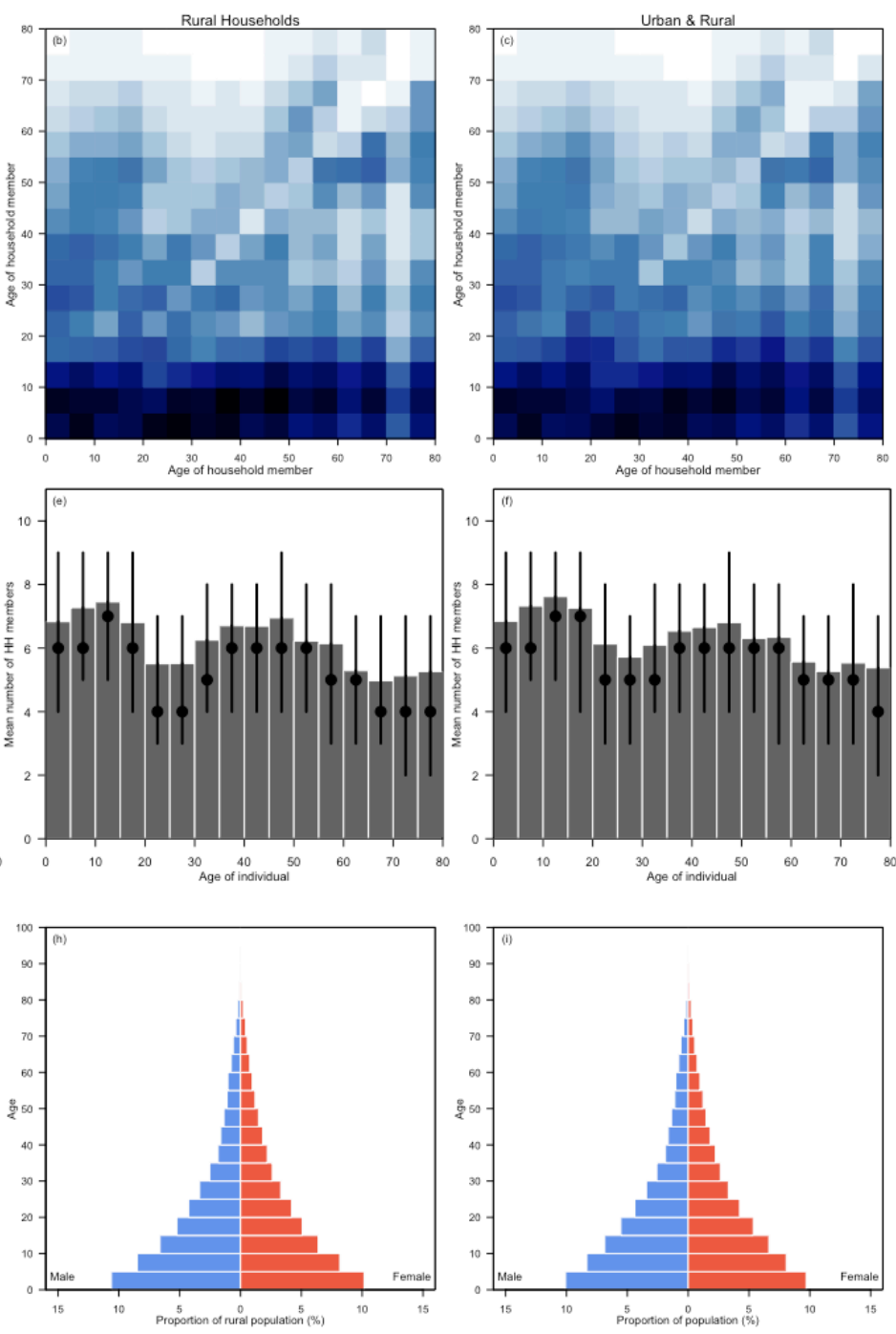
Empirical DHS household data: Nigeria
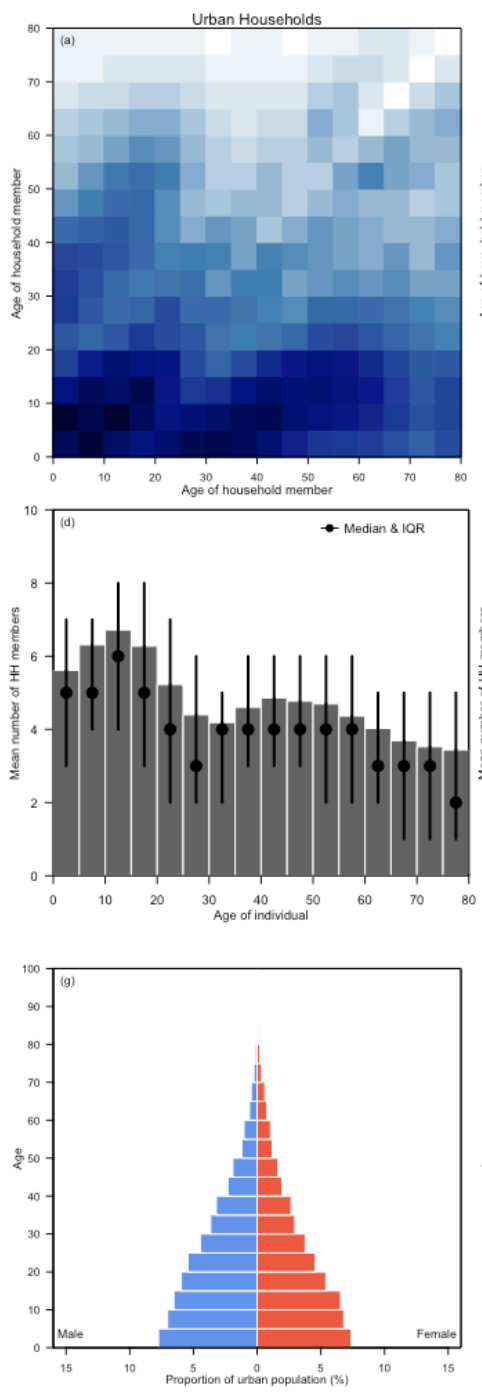
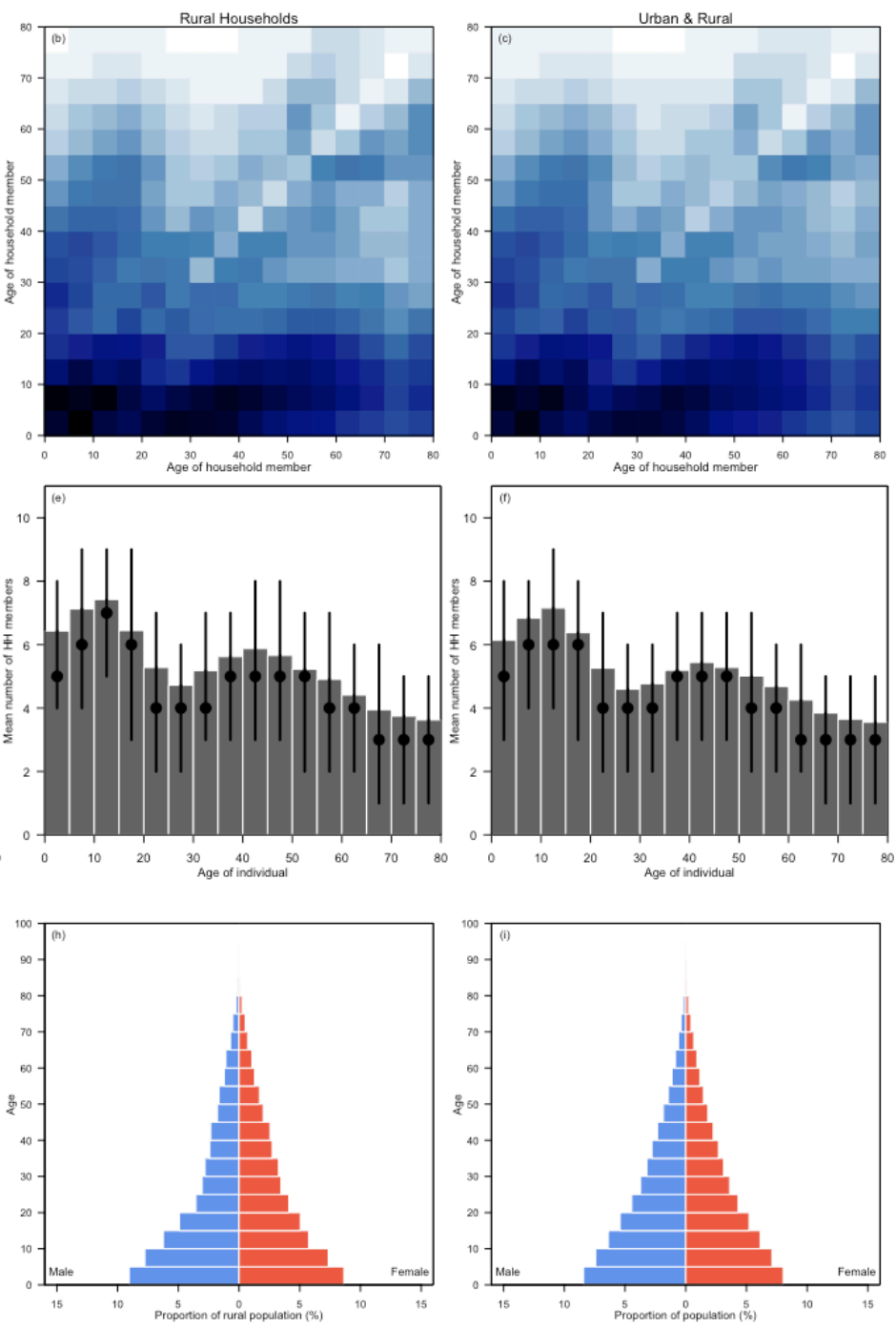
Empirical DHS household data: Pakistan
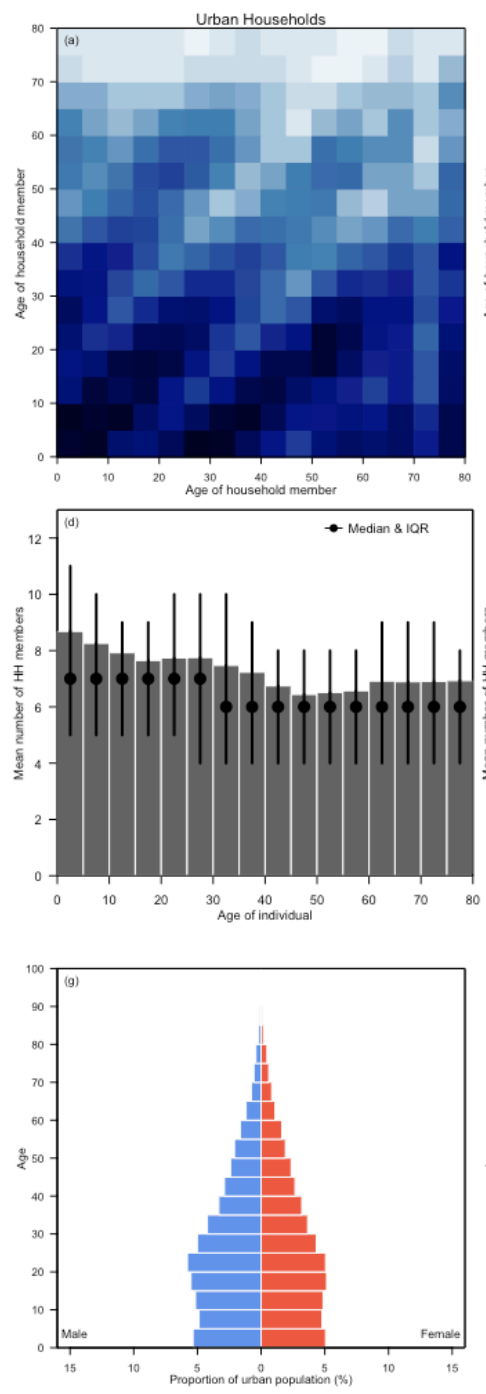
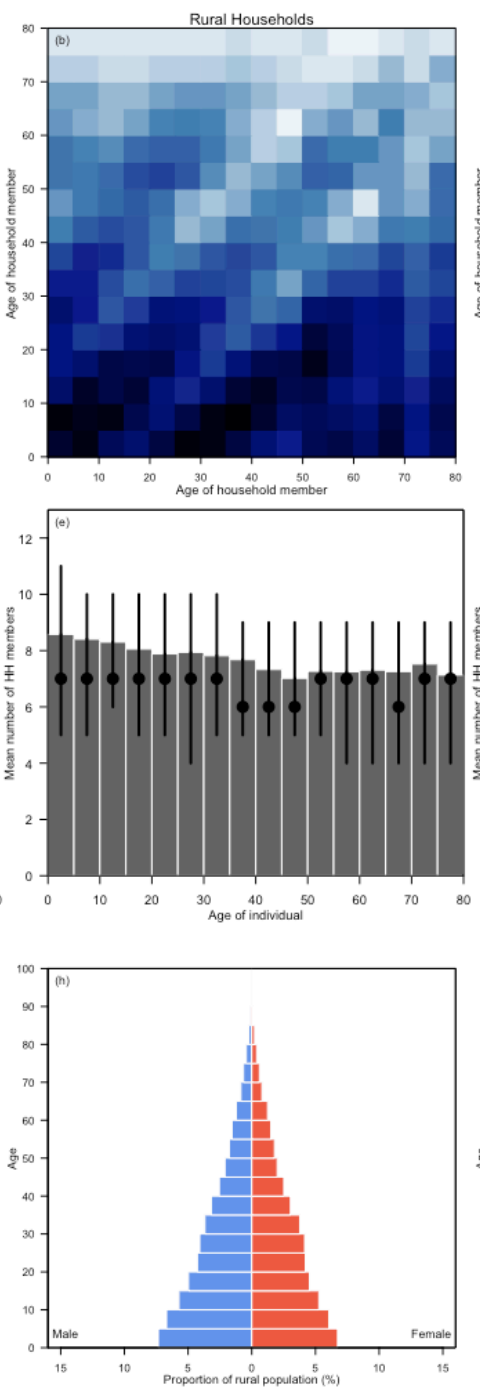

$\begin{array}{lll}0.5 & 1 & 1.5 \\ & 1 & \end{array}$ in a specific age group
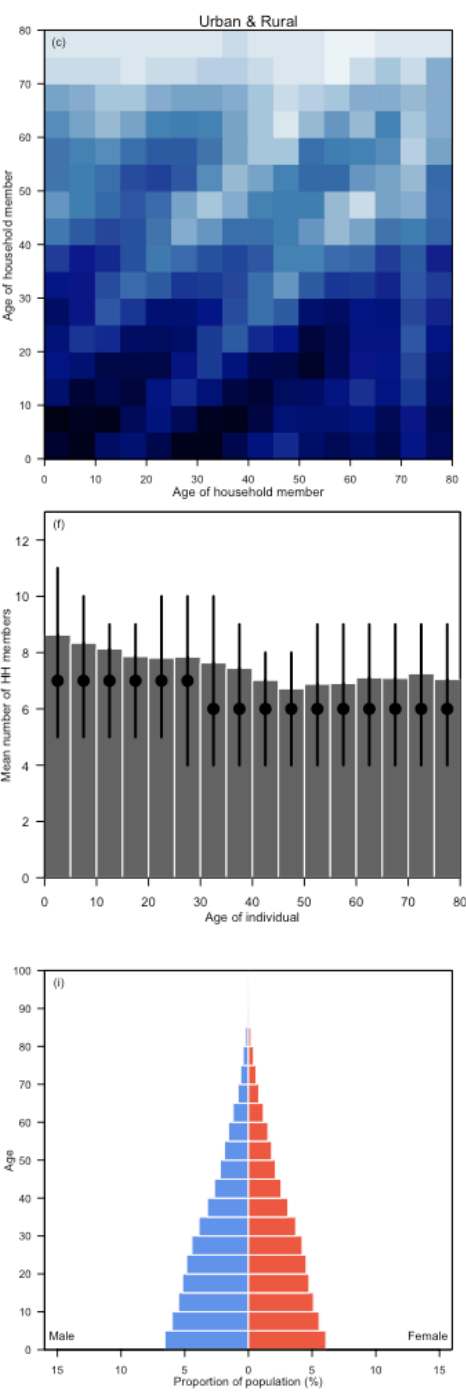
Empirical DHS household data: Peru
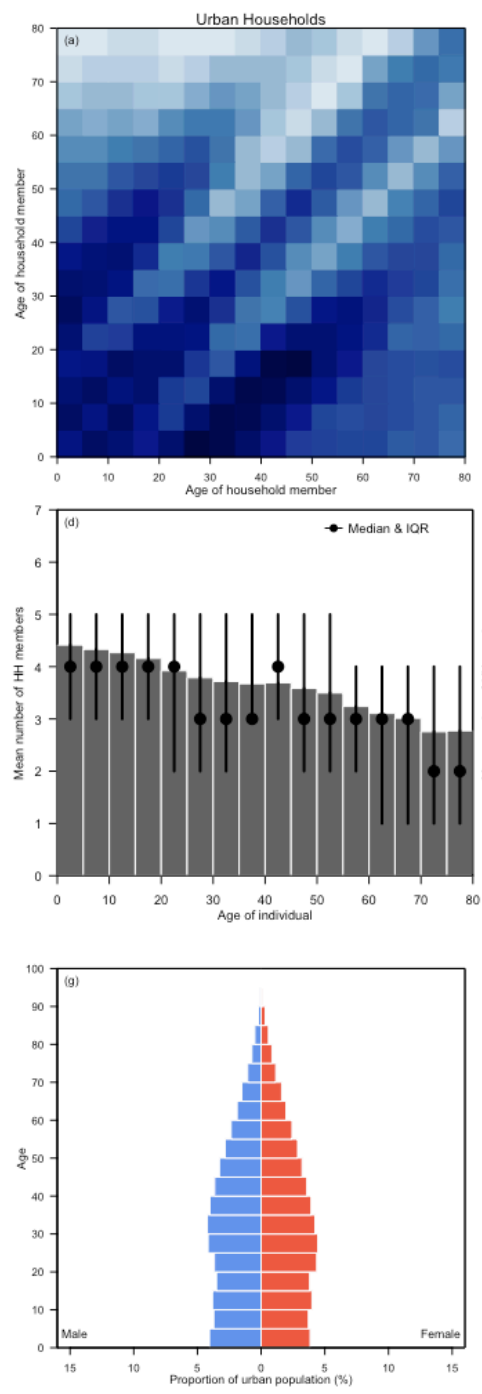
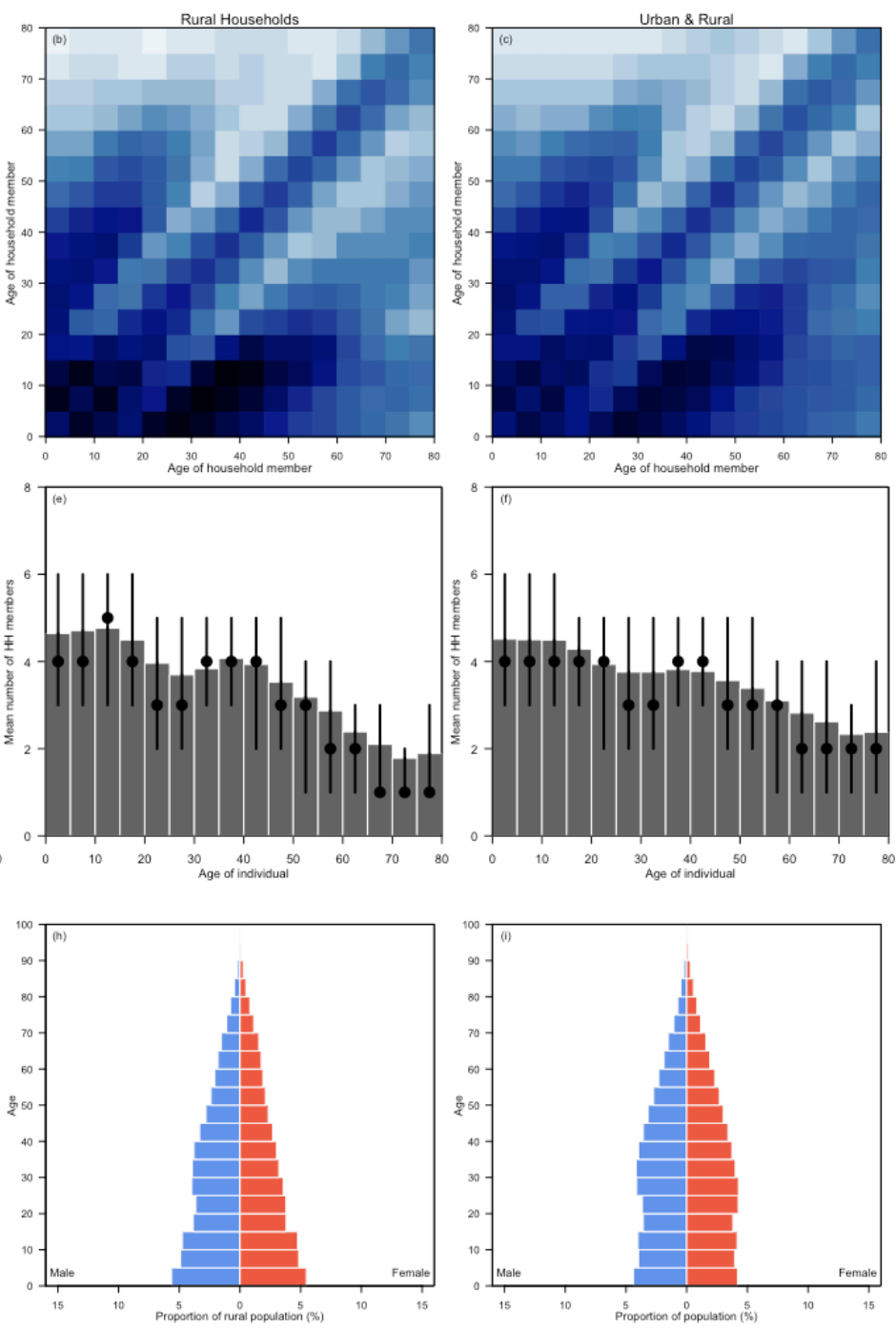
Empirical DHS household data: Philippines
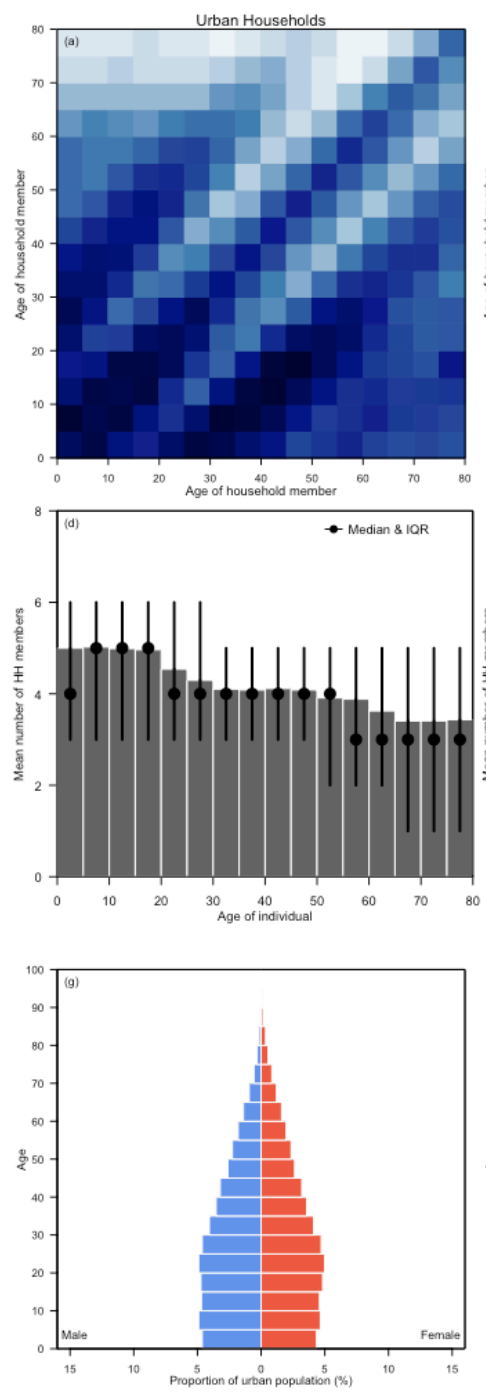
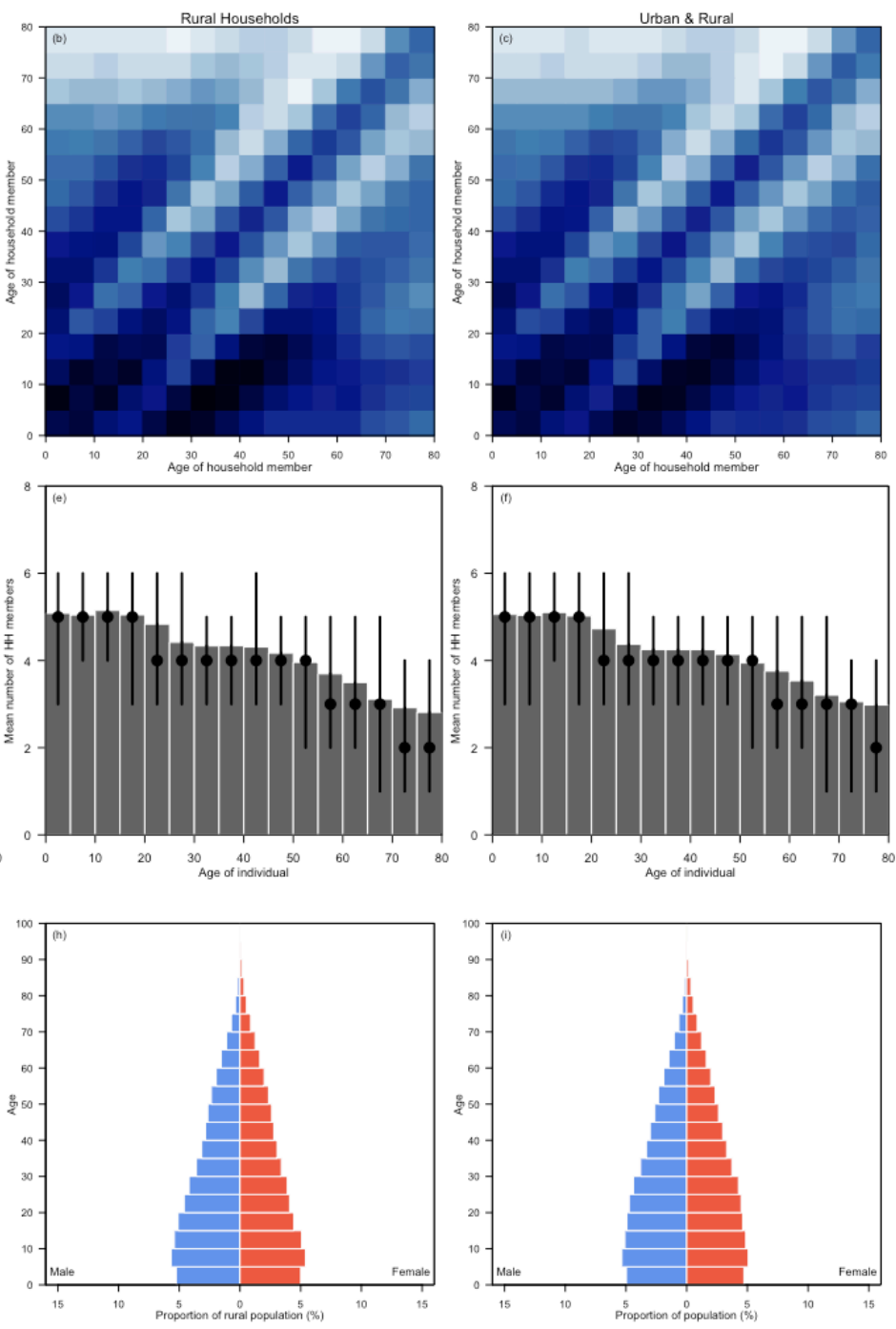
Empirical DHS household data: Senegal
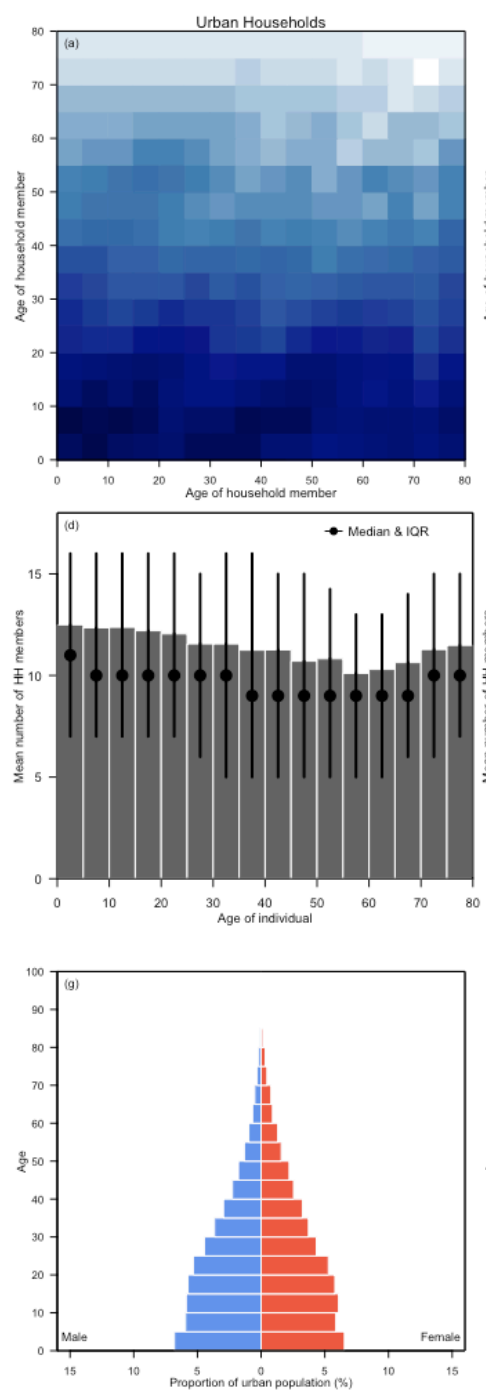
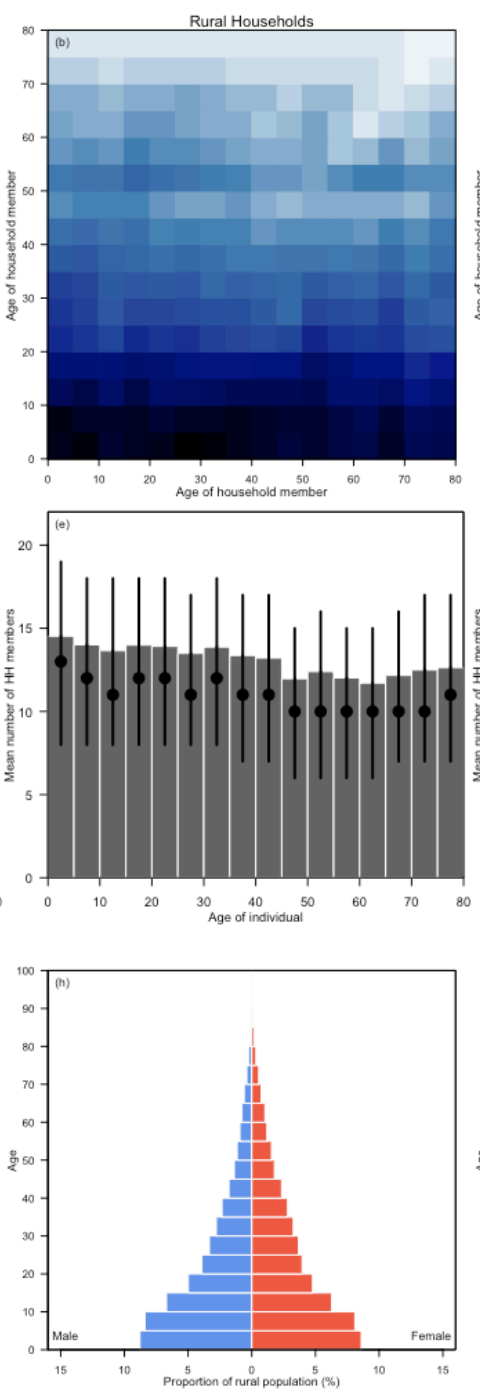

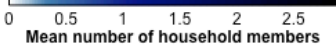

in a specific age group
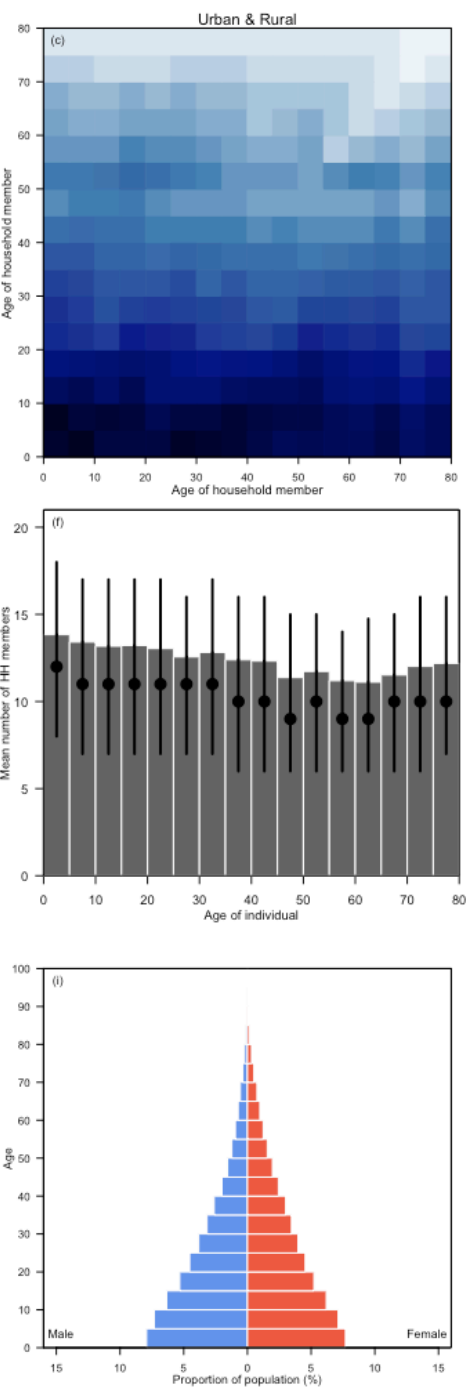
Empirical DHS household data: Sierra Leone
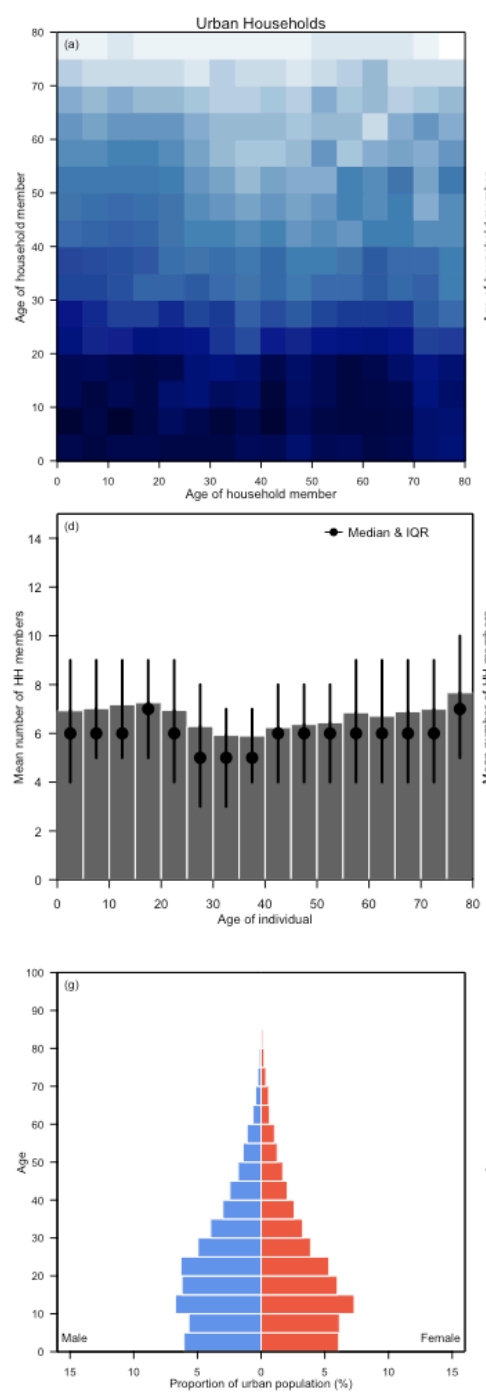
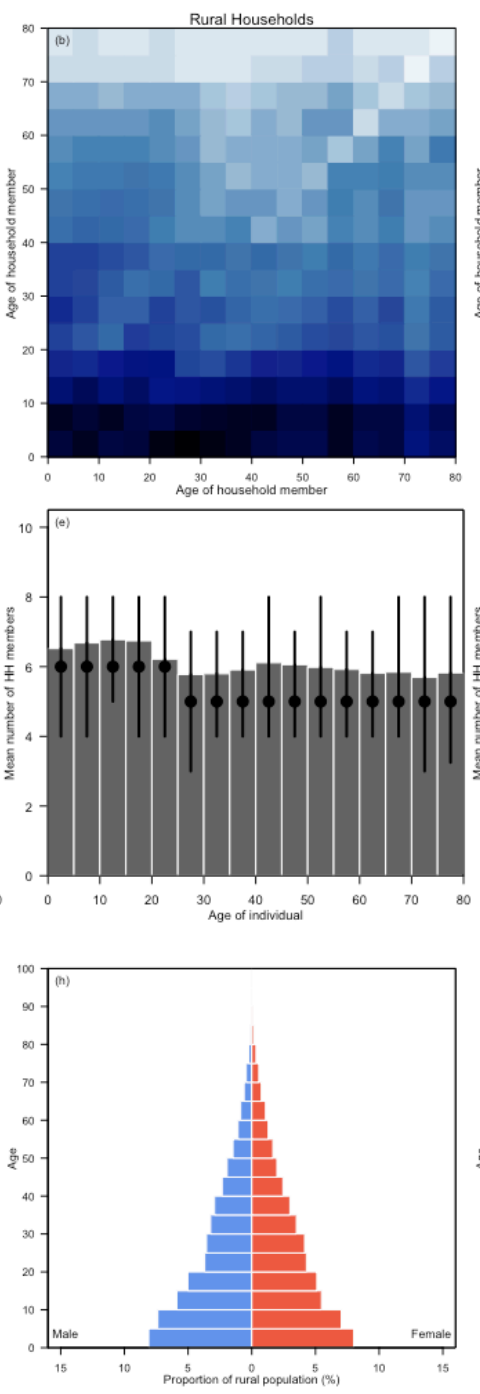

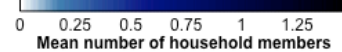

in a specific age group
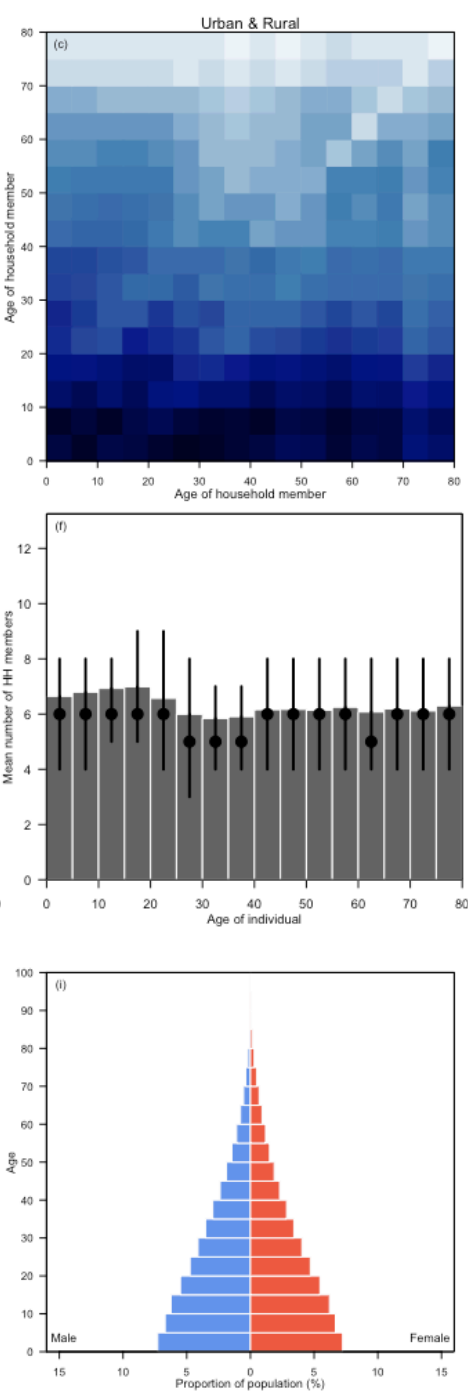
Empirical DHS household data: South Africa
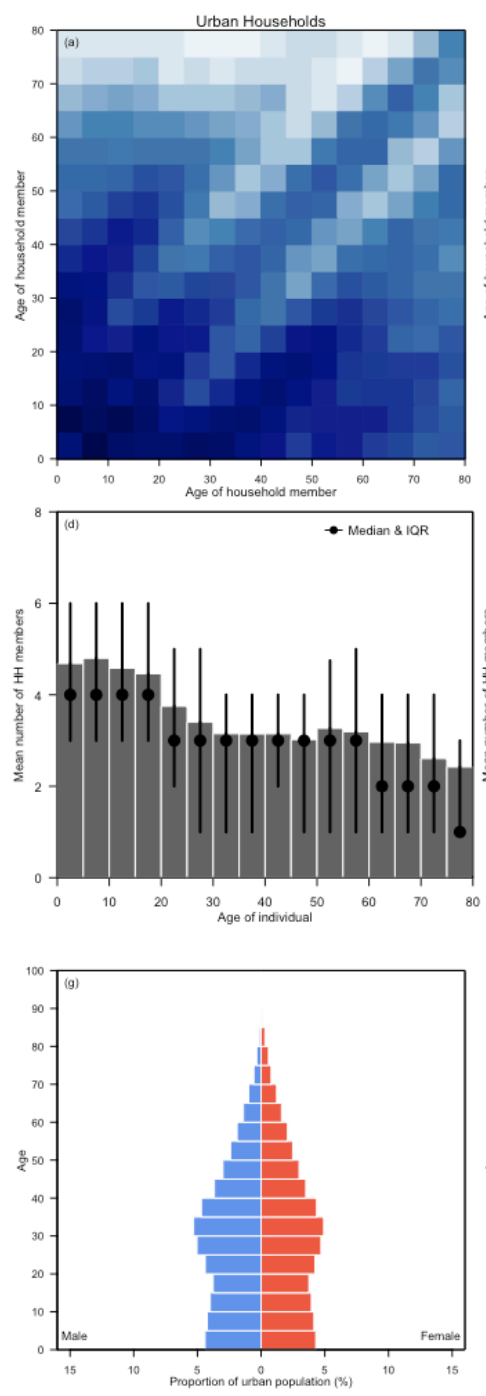
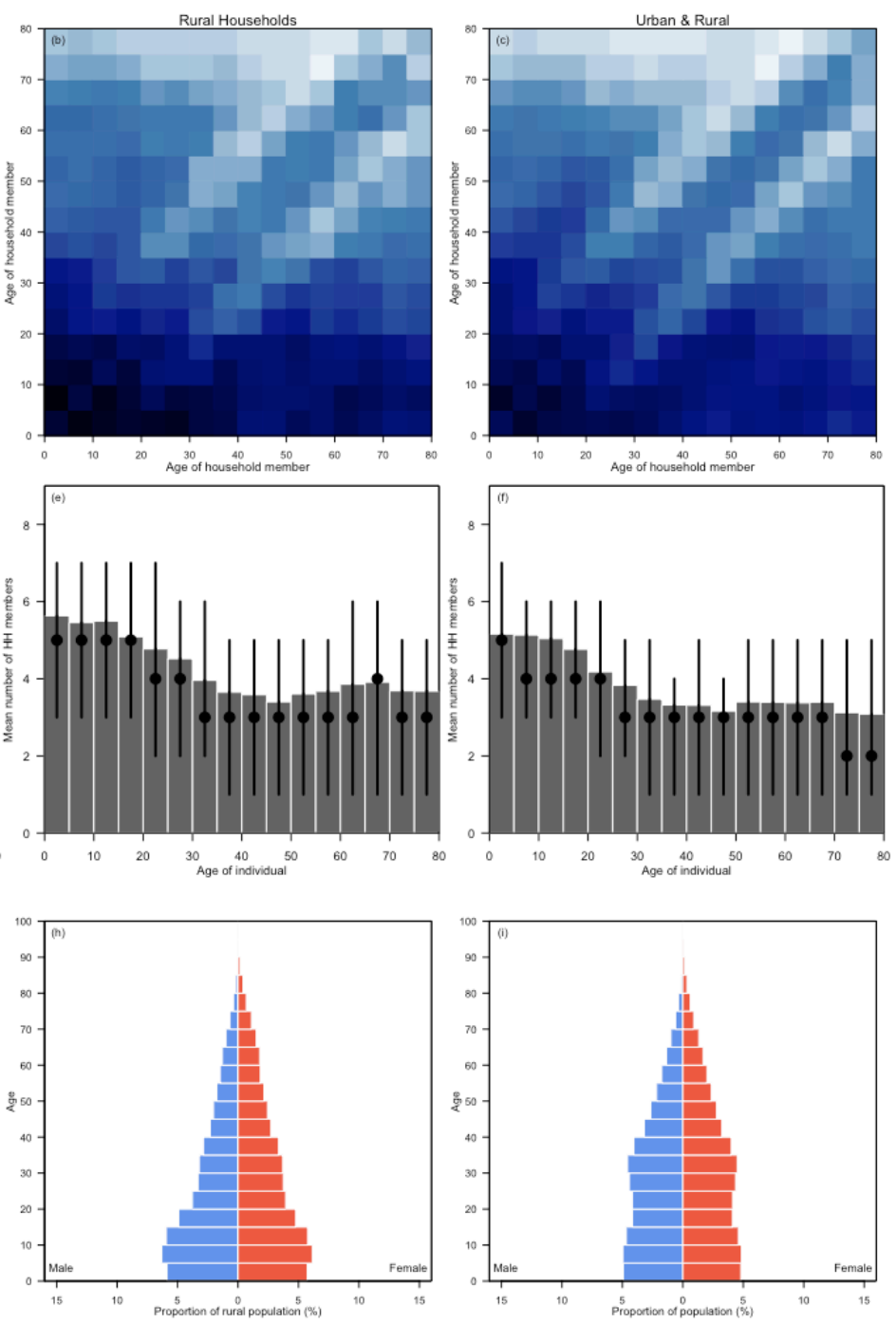
Empirical DHS household data: Timor-Leste
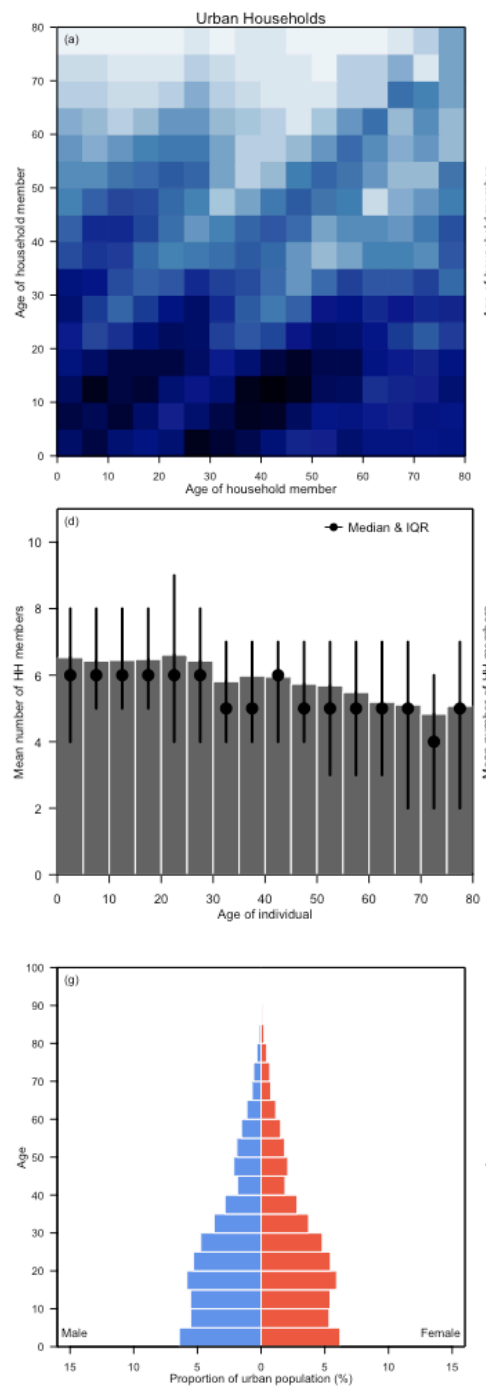
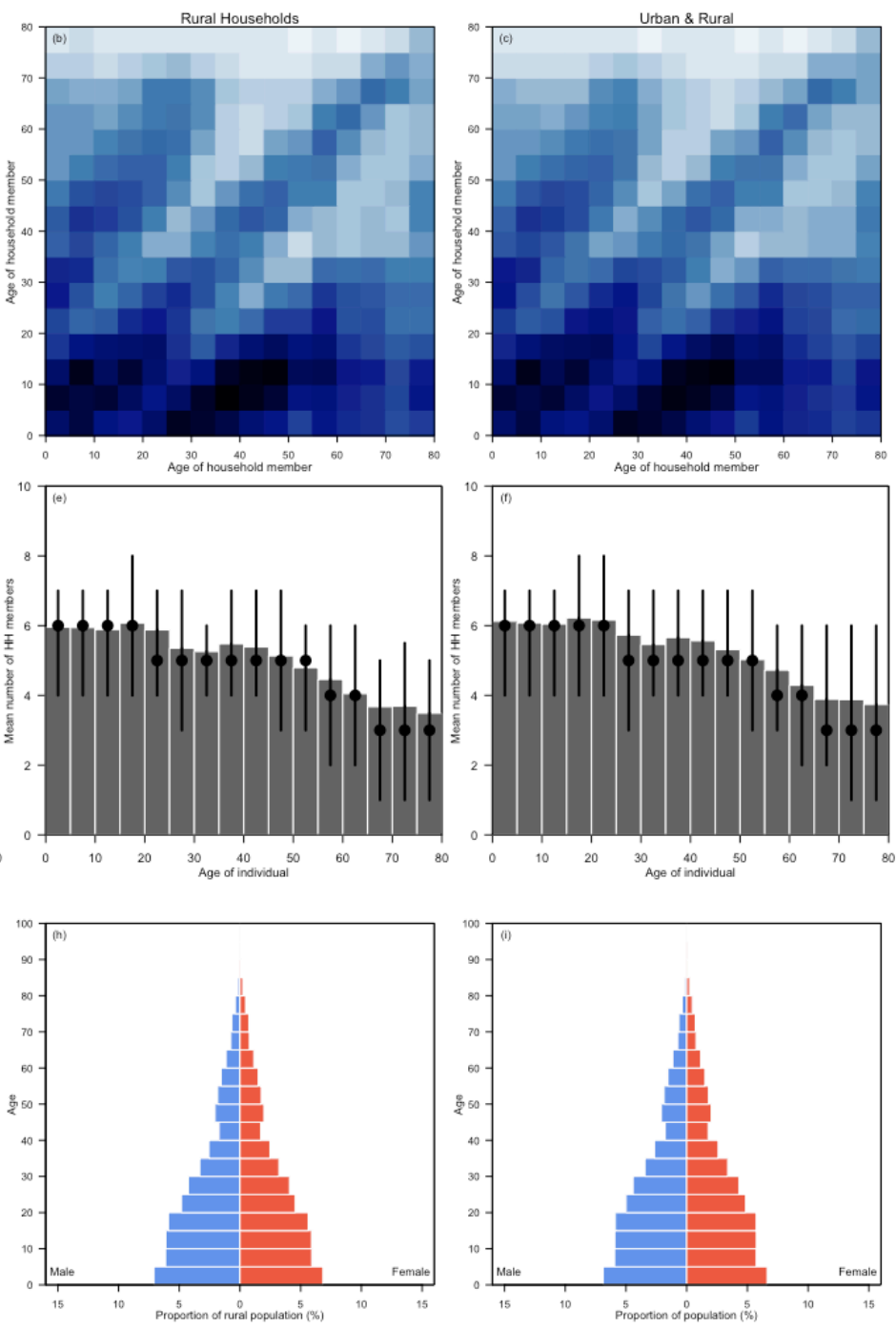
Empirical DHS household data: Togo
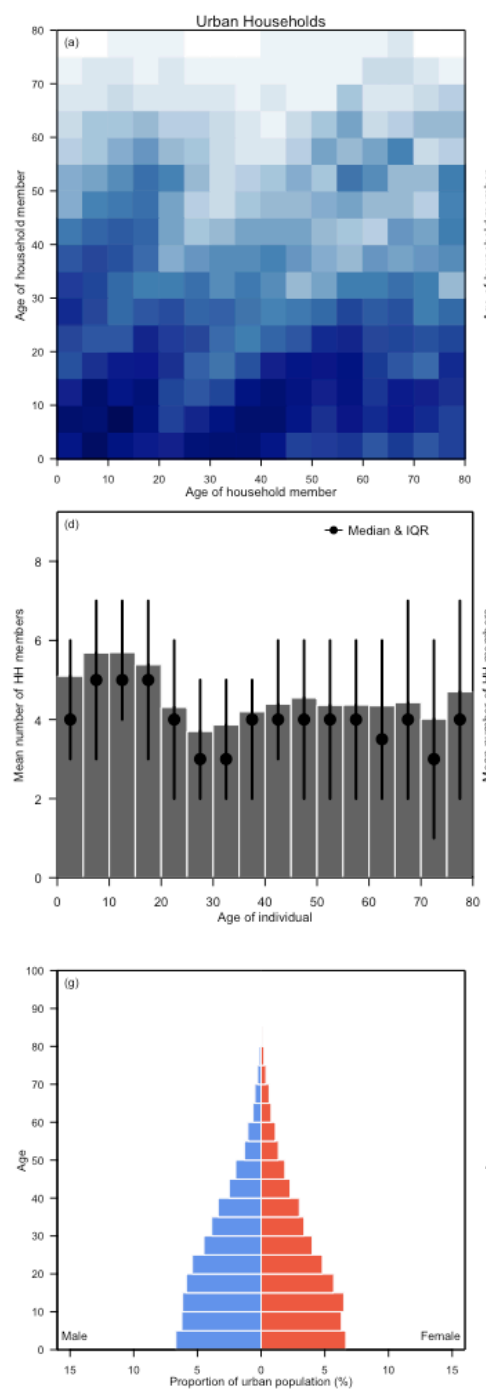
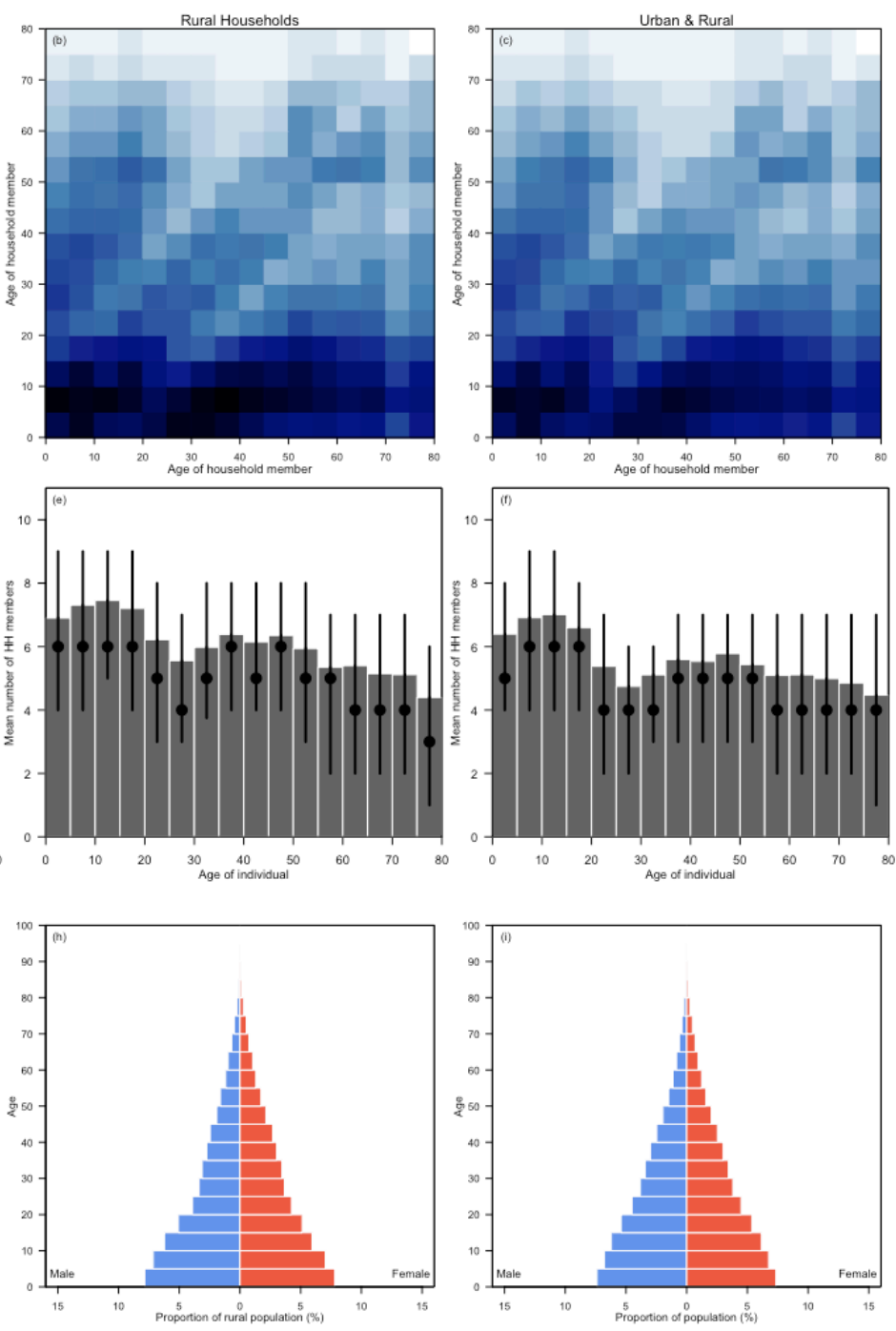
Empirical DHS household data: Uganda
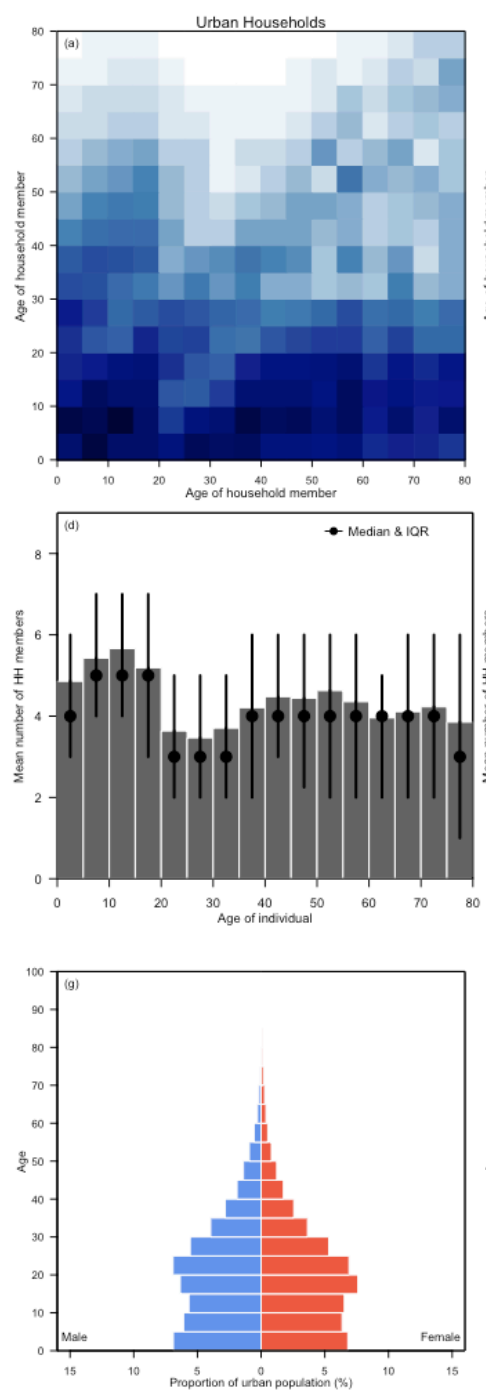
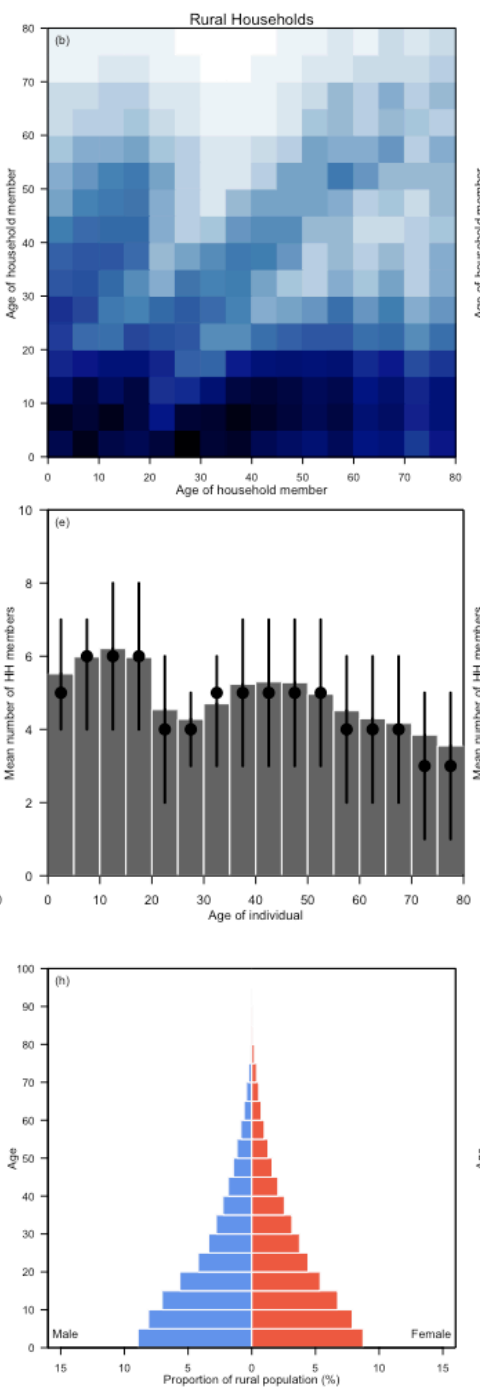

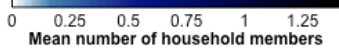

in a specific age group
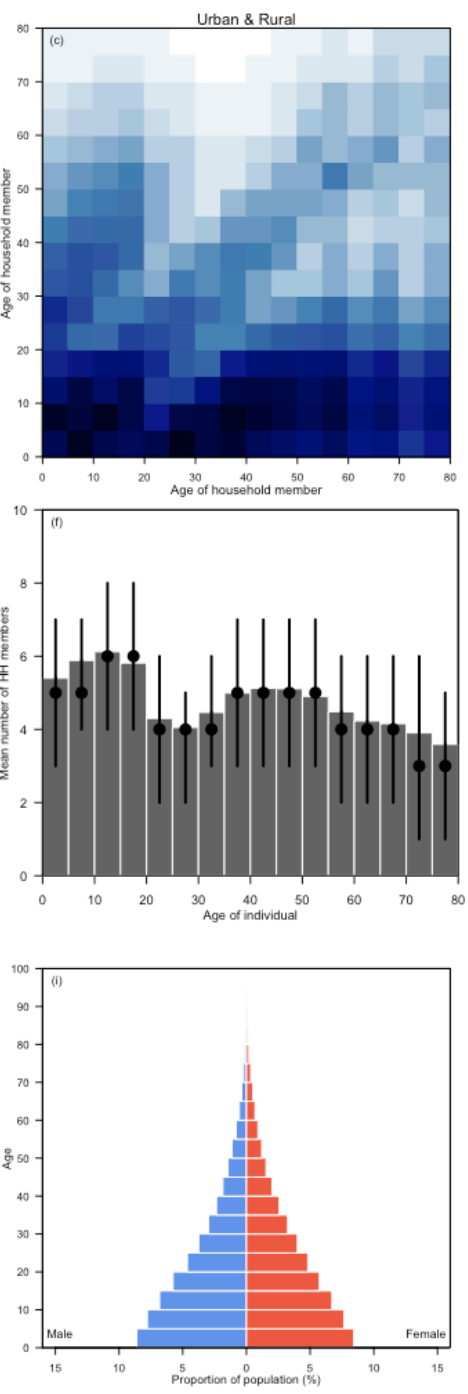
Empirical DHS household data: Viet Nam
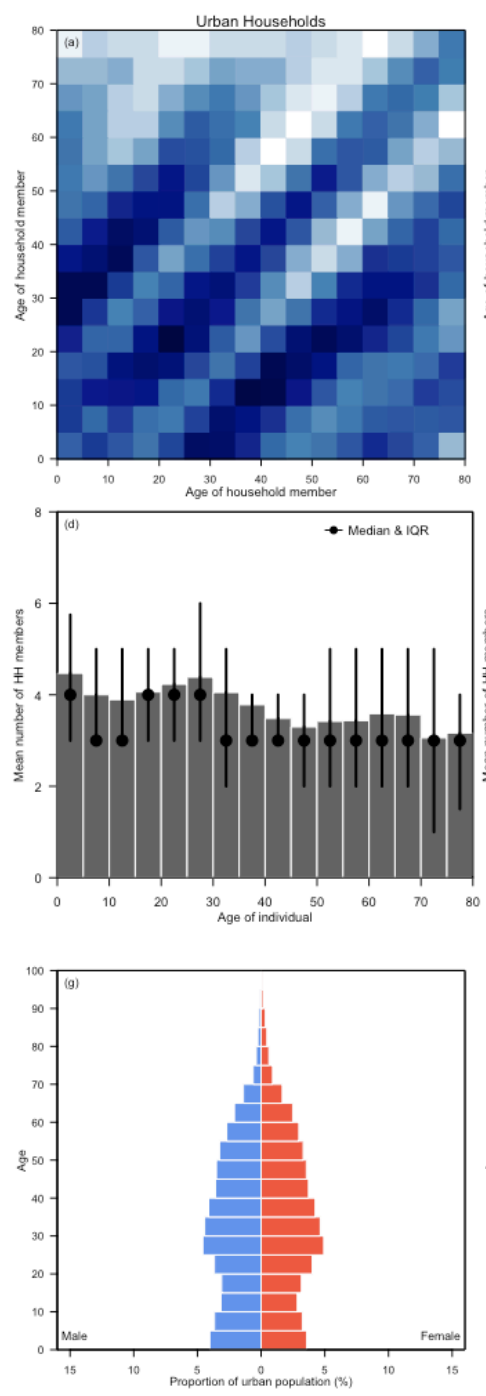
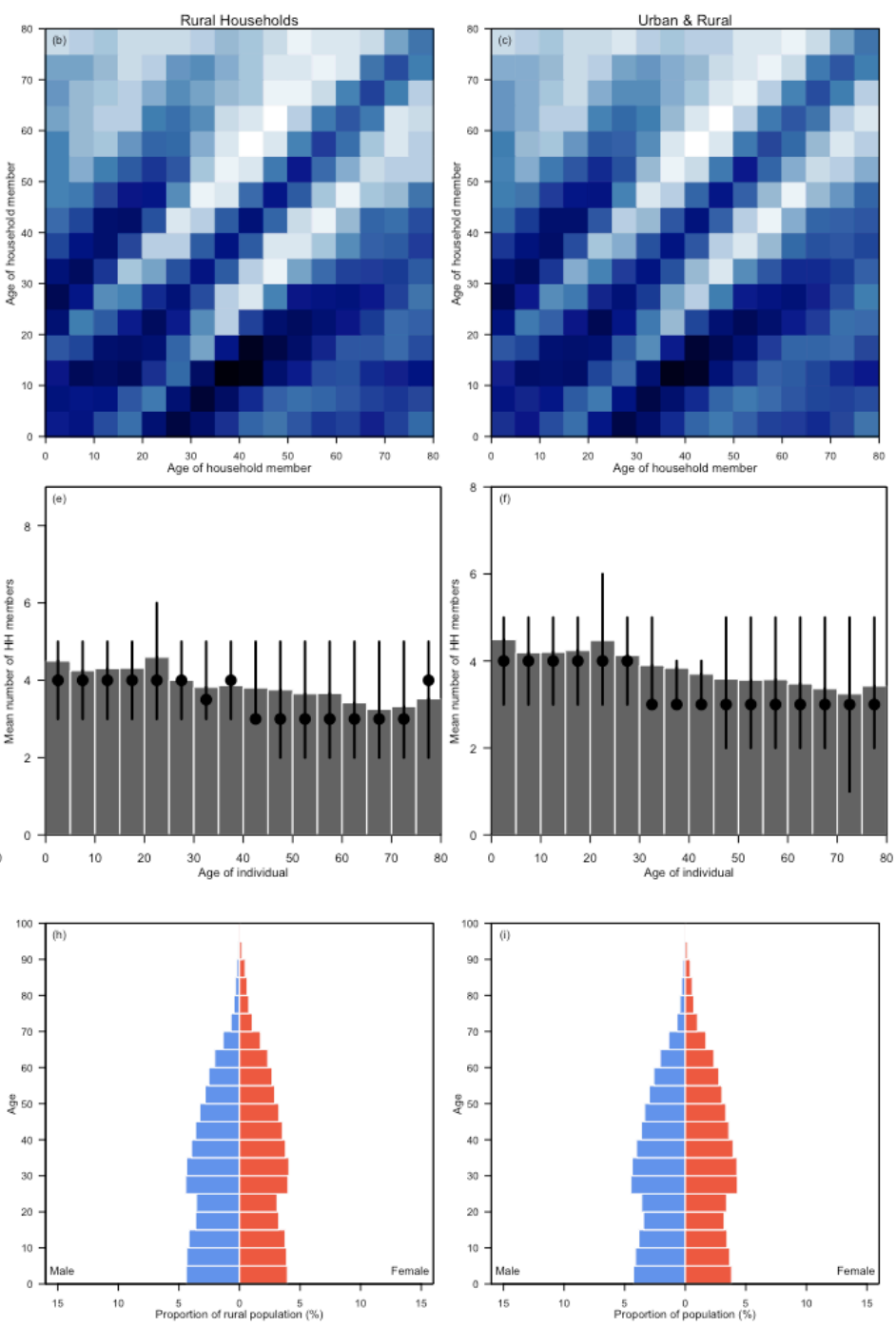
Empirical DHS household data: Zambia
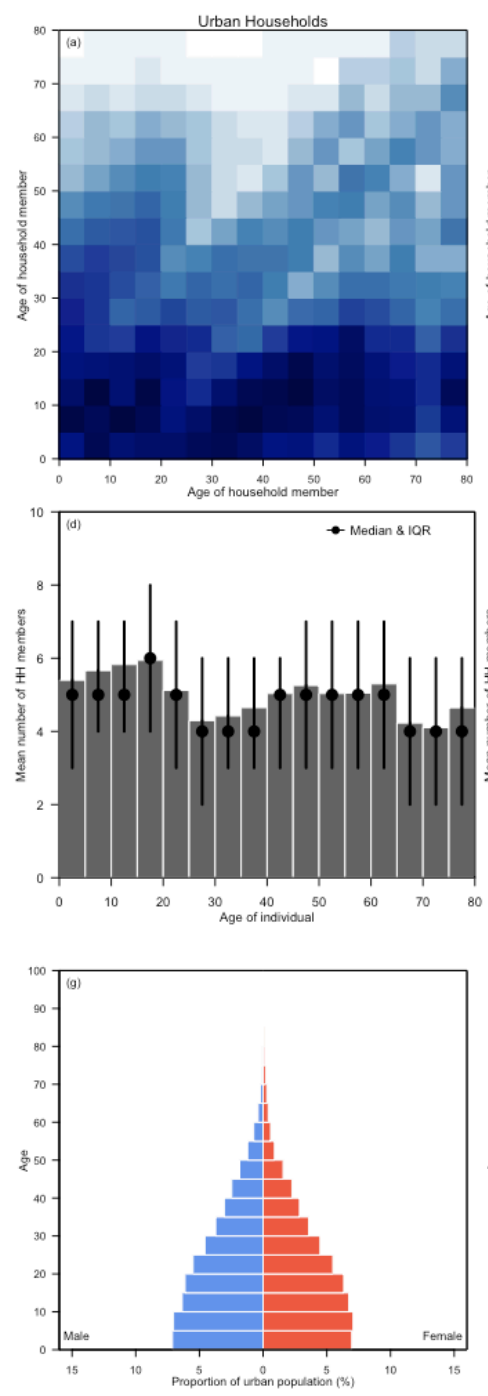
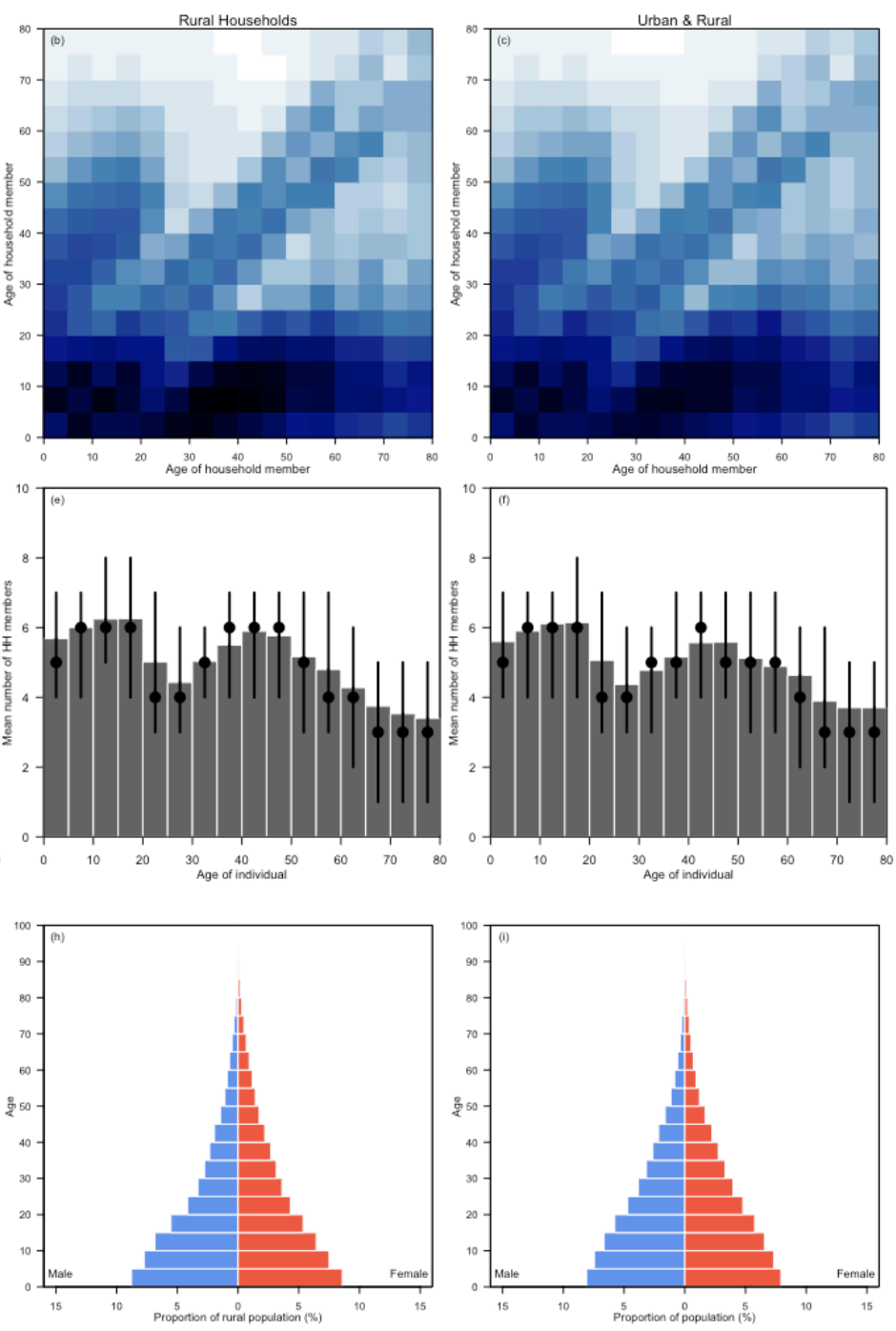
Empirical DHS household data: Zimbabwe
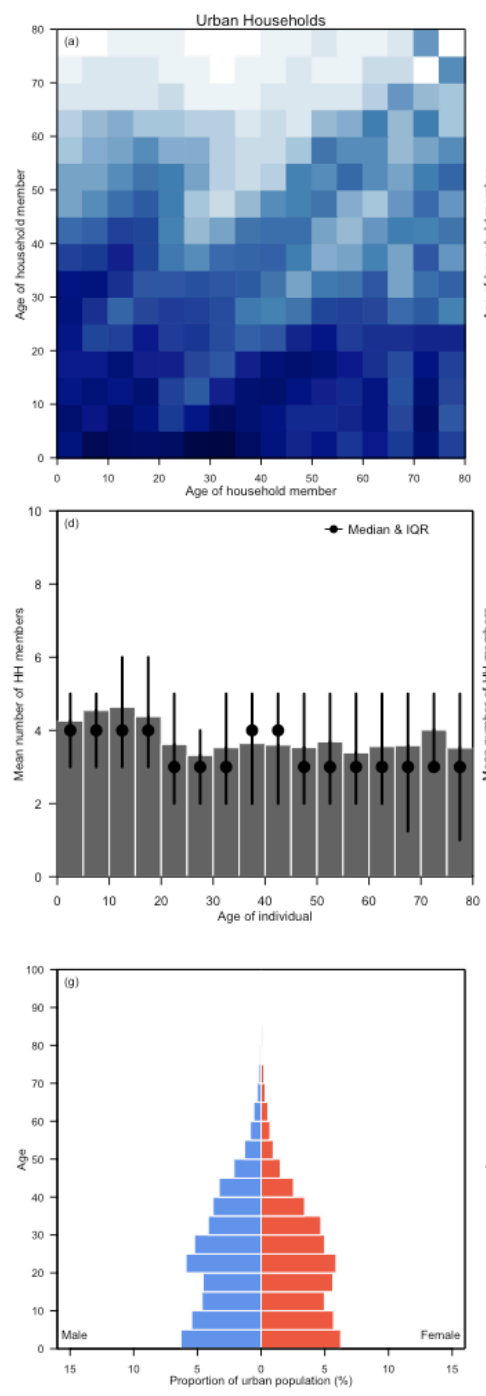
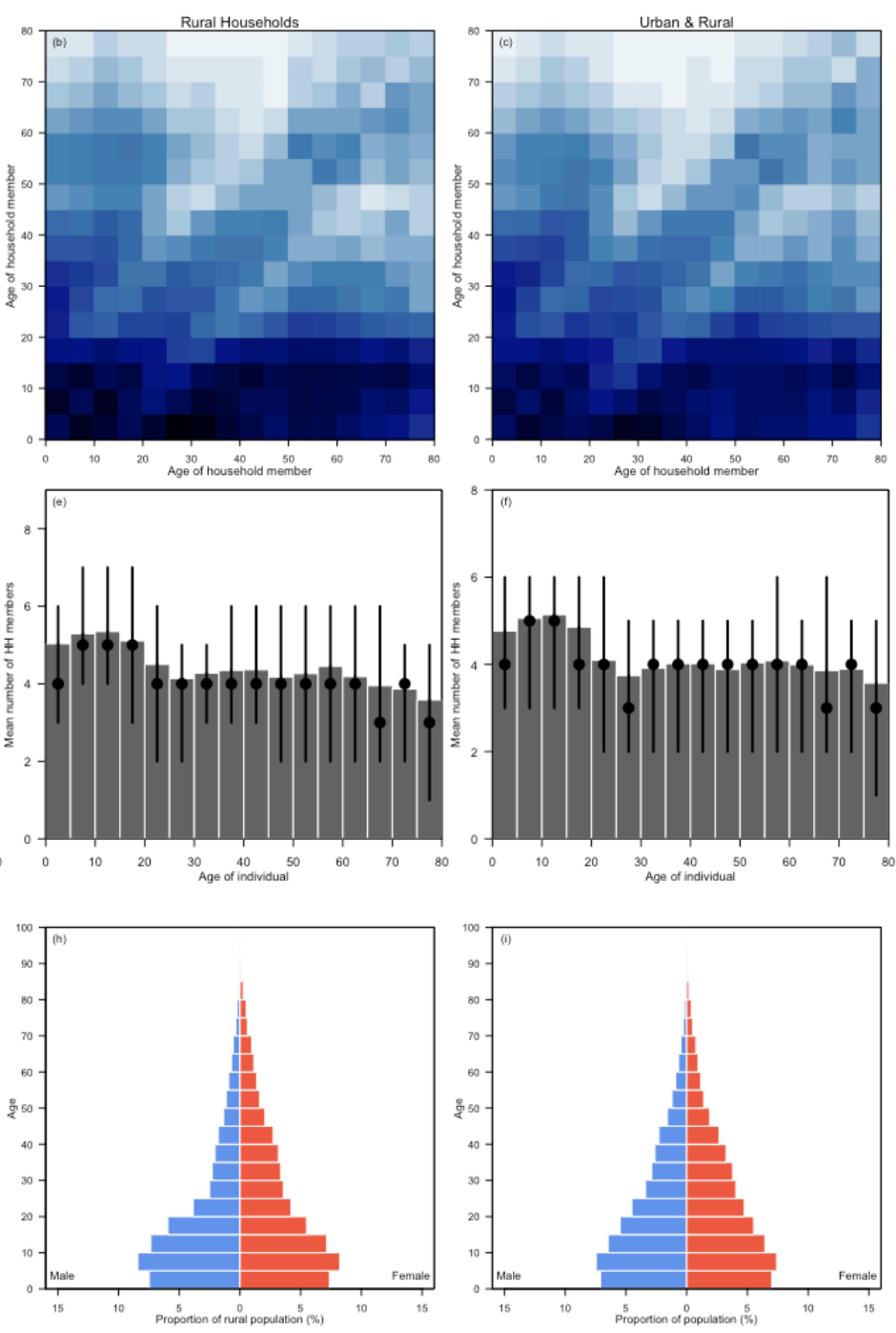


\section{B.3. Updated age- and location-specific contact matrices}

The 2017 synthetic matrices provide validated approximations to age-and-location-specific contact matrices for 152 geographical regions. We have therefore updated these matrices with the most recent data (Demographic Household Surveys, World Bank and UN Population Division) extending the coverage to 177 geographical locations, covering $97.2 \%$ of the world's population. 
Afghanistan
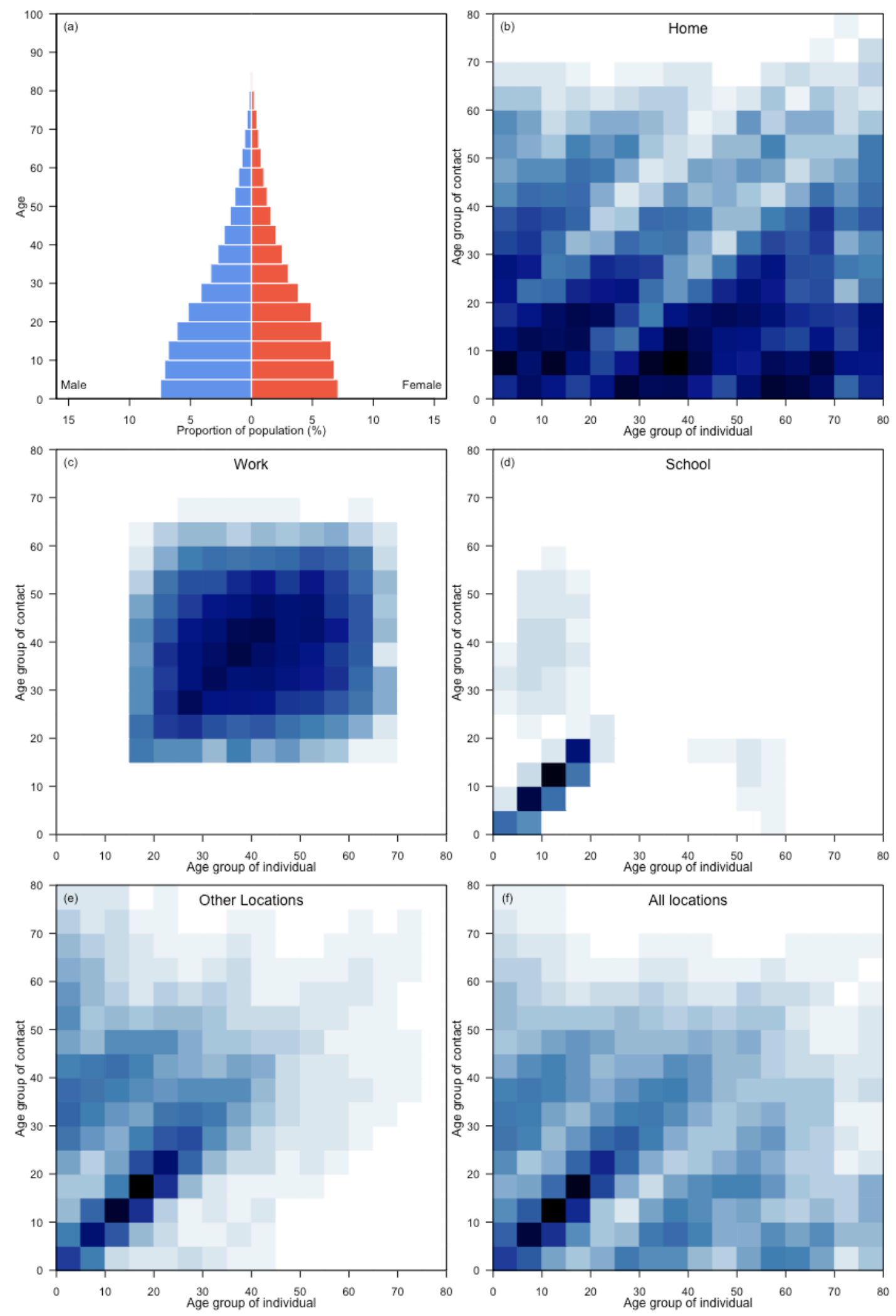


\section{Albania}
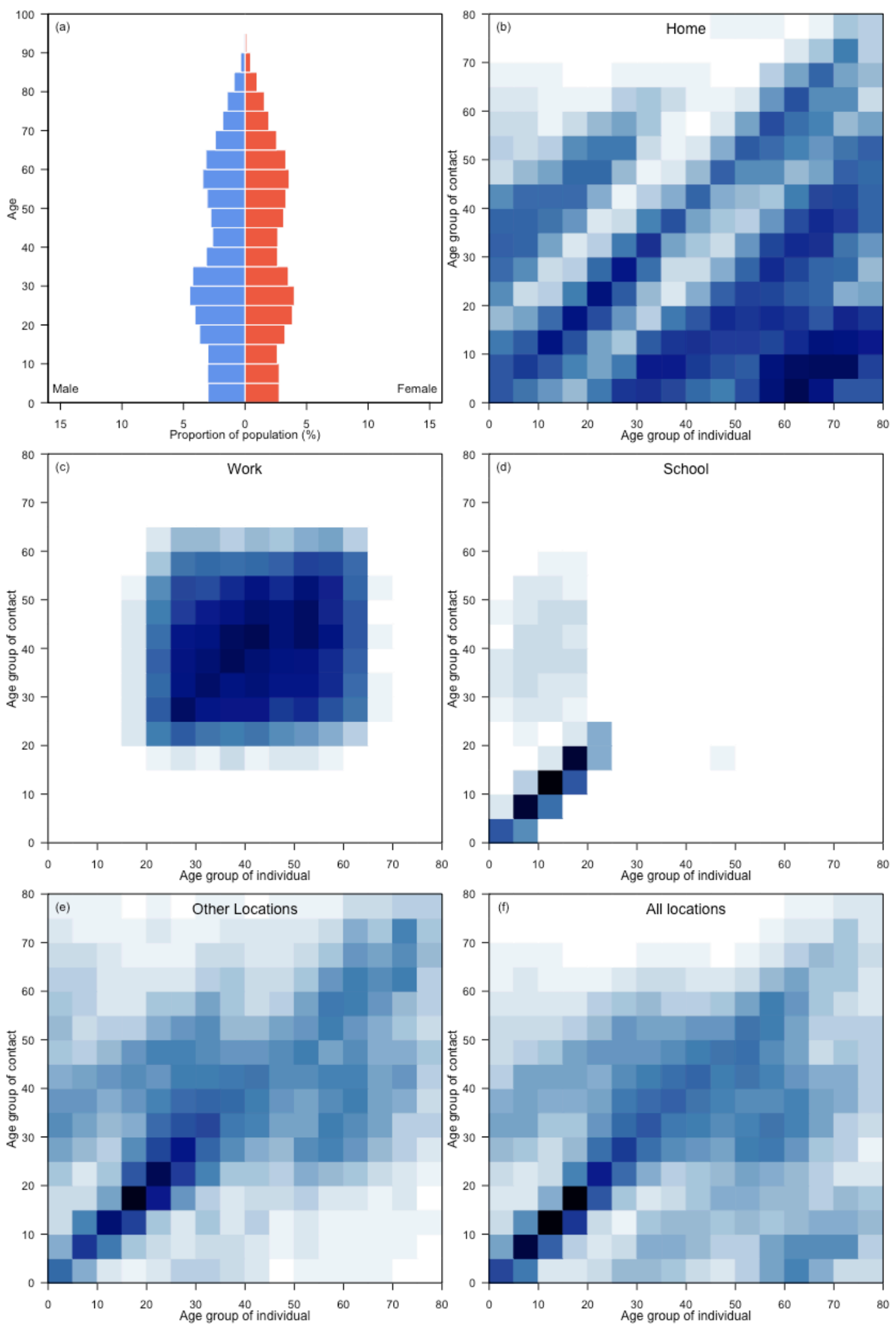


\section{Algeria}
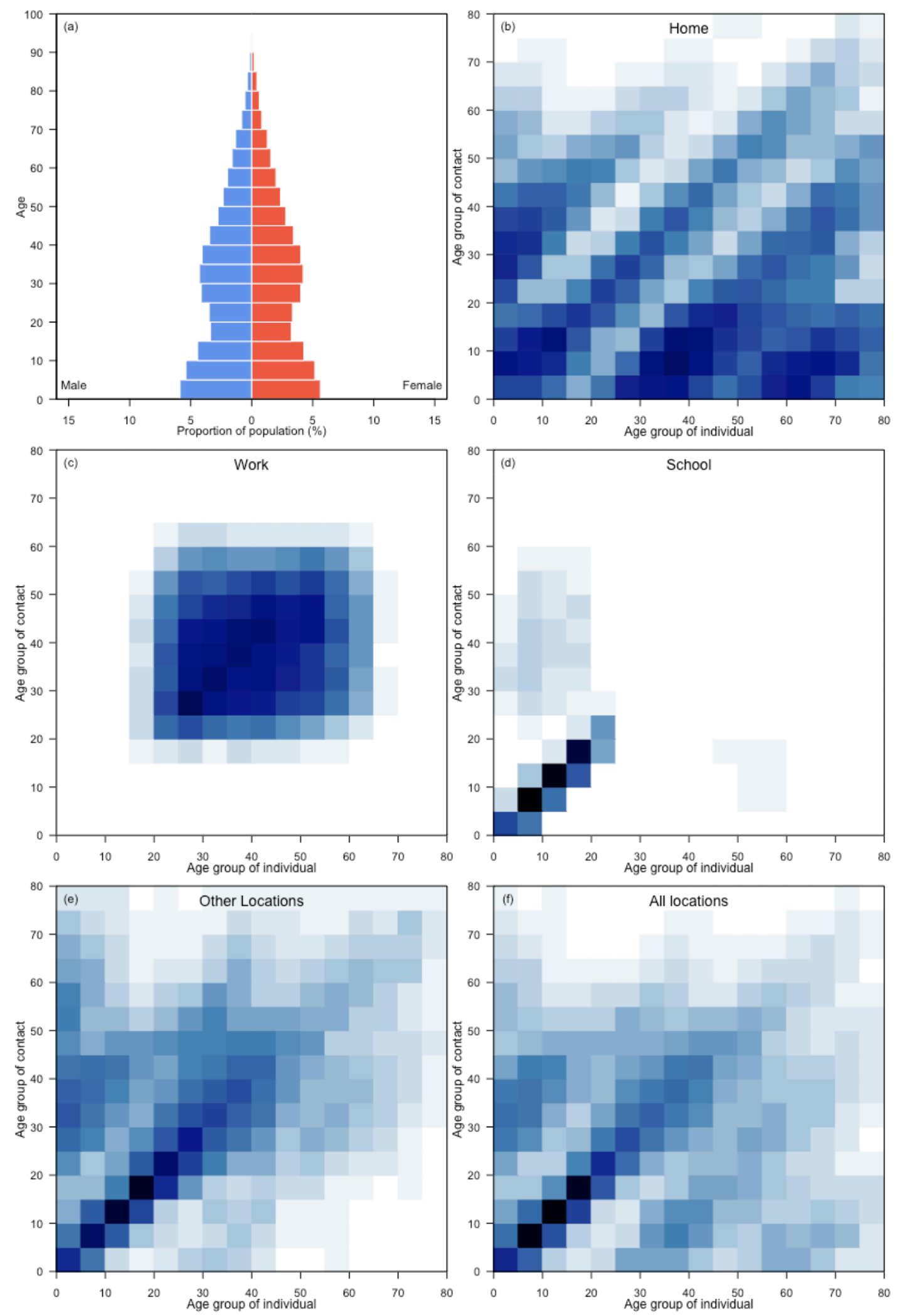


\section{Angola}
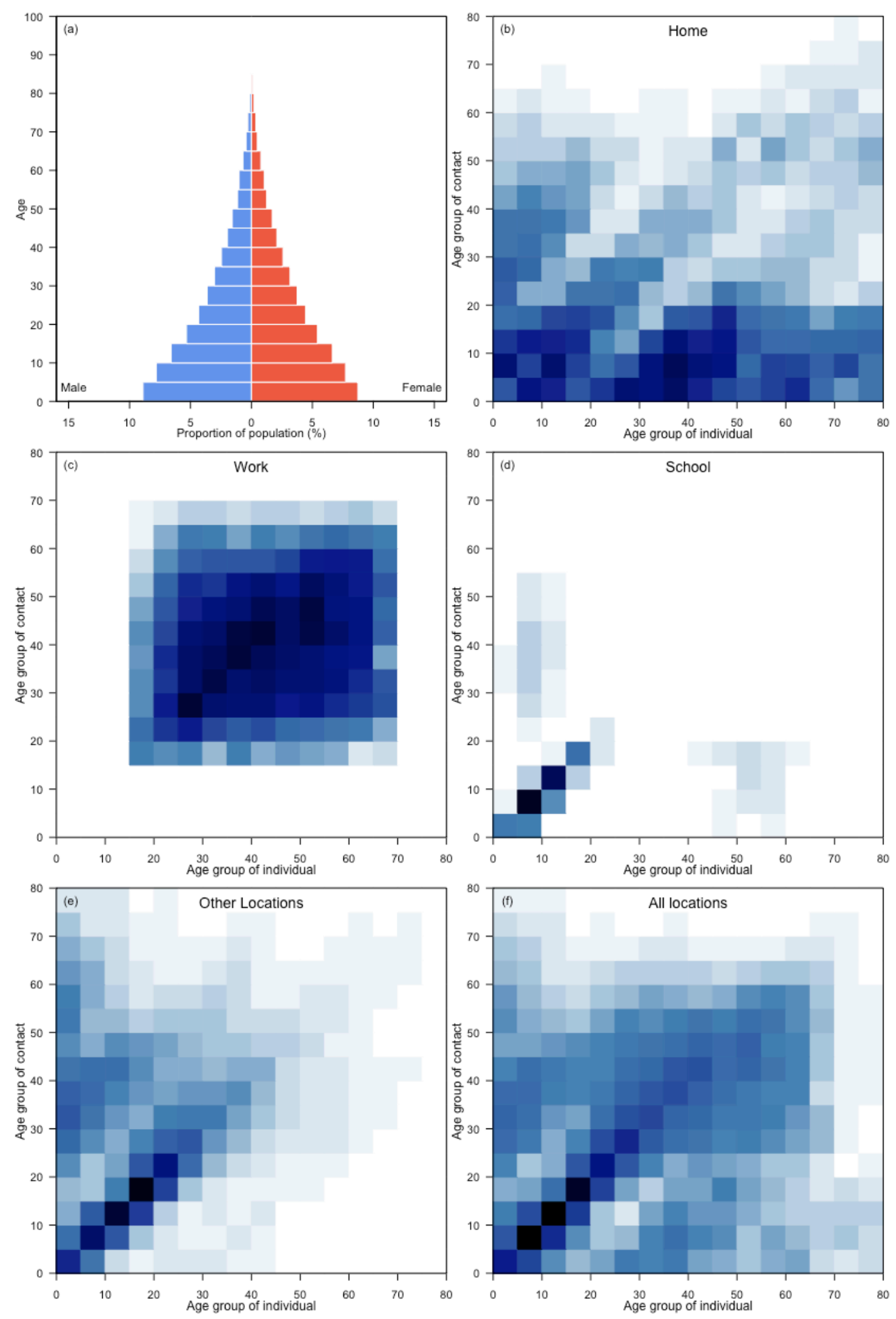


\section{Argentina}
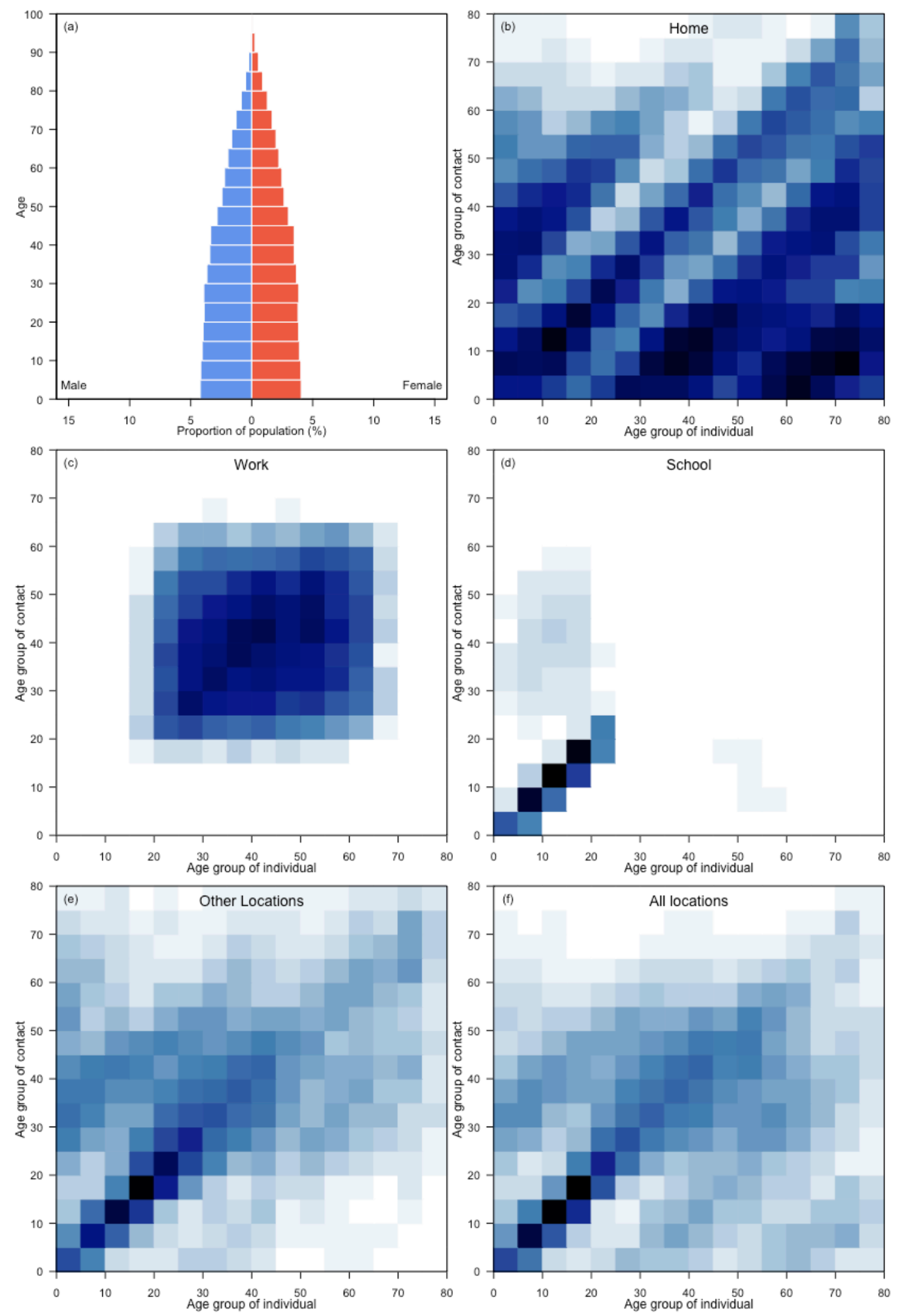


\section{Armenia}
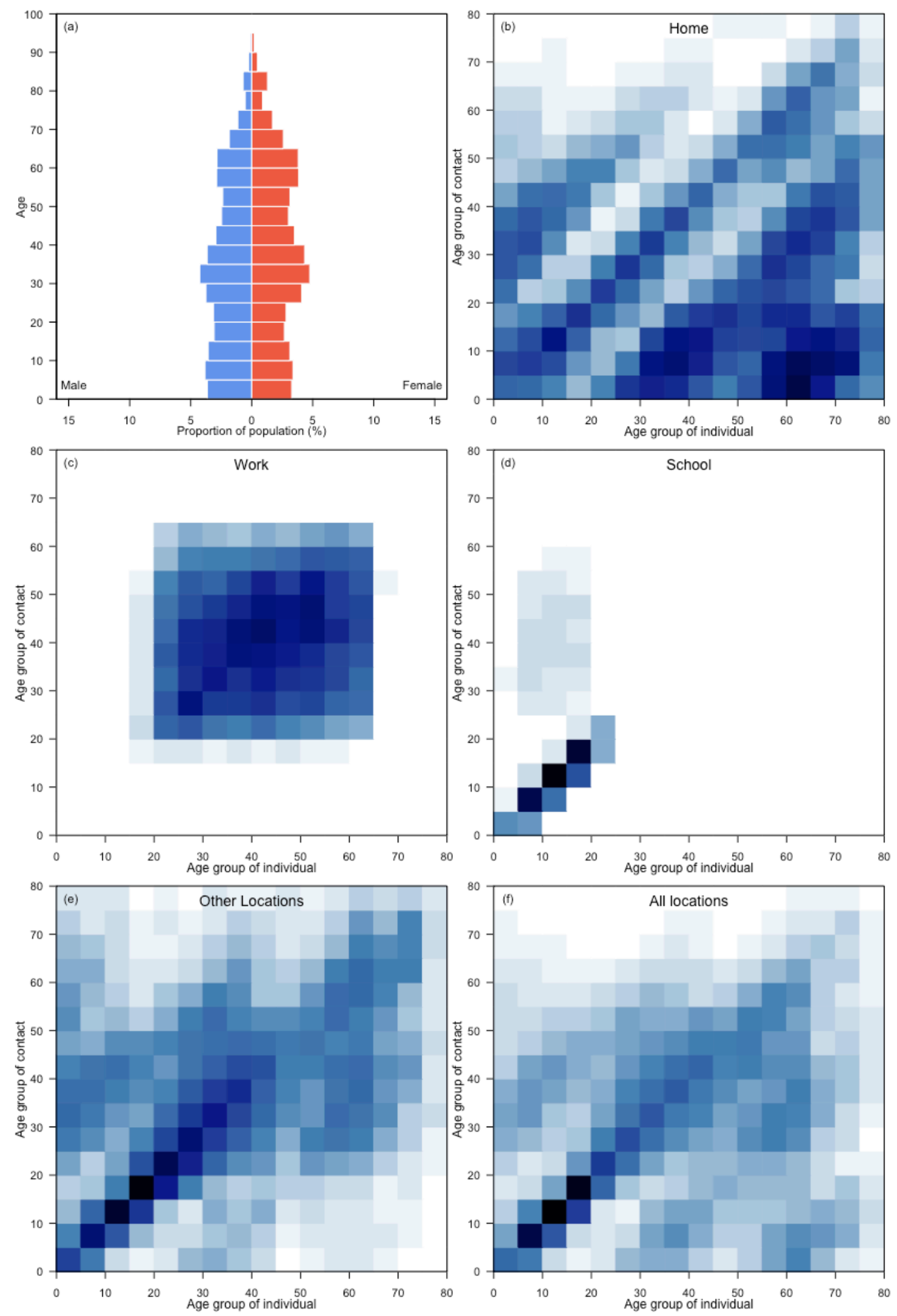


\section{Austria}
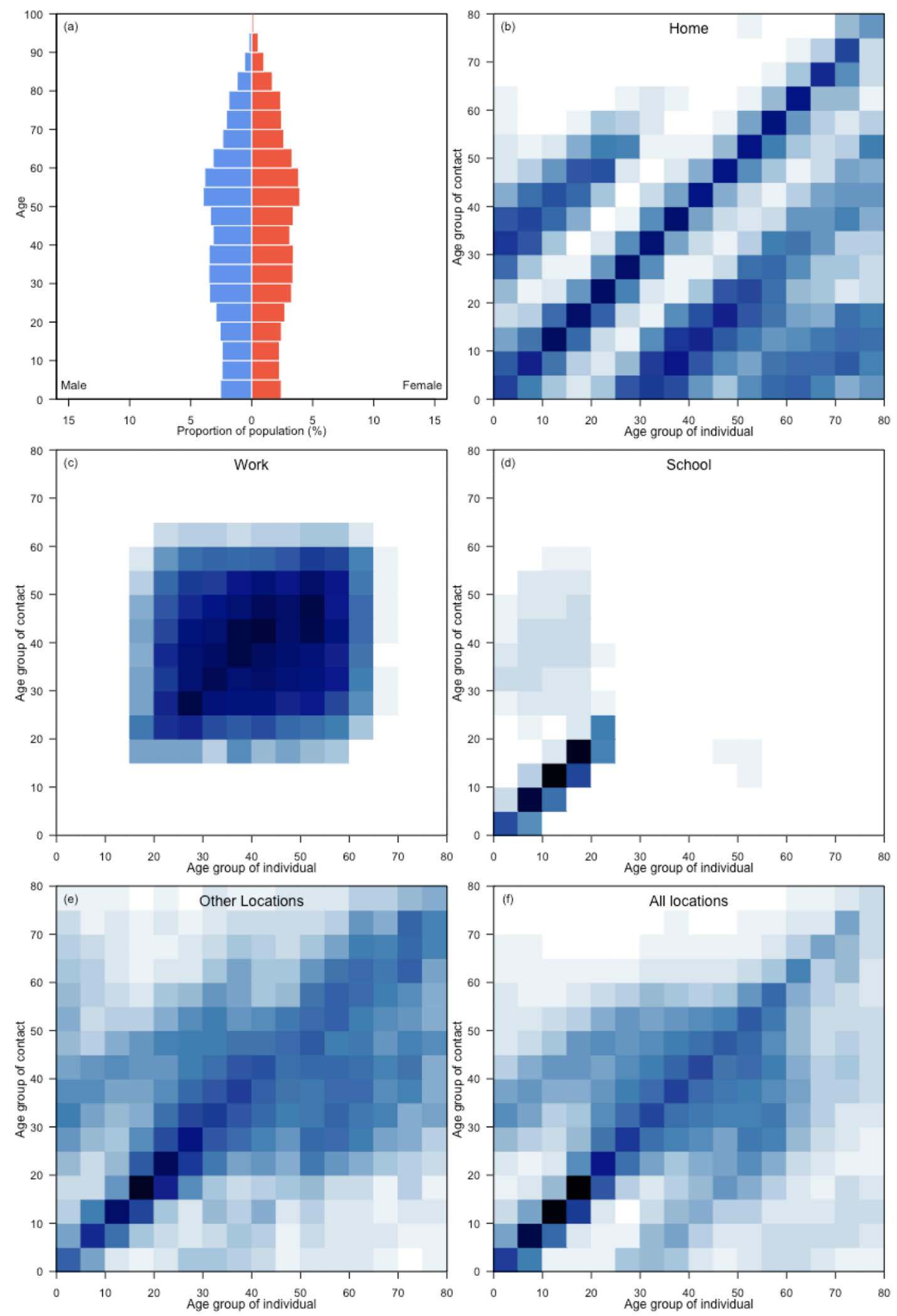
Azerbaijan
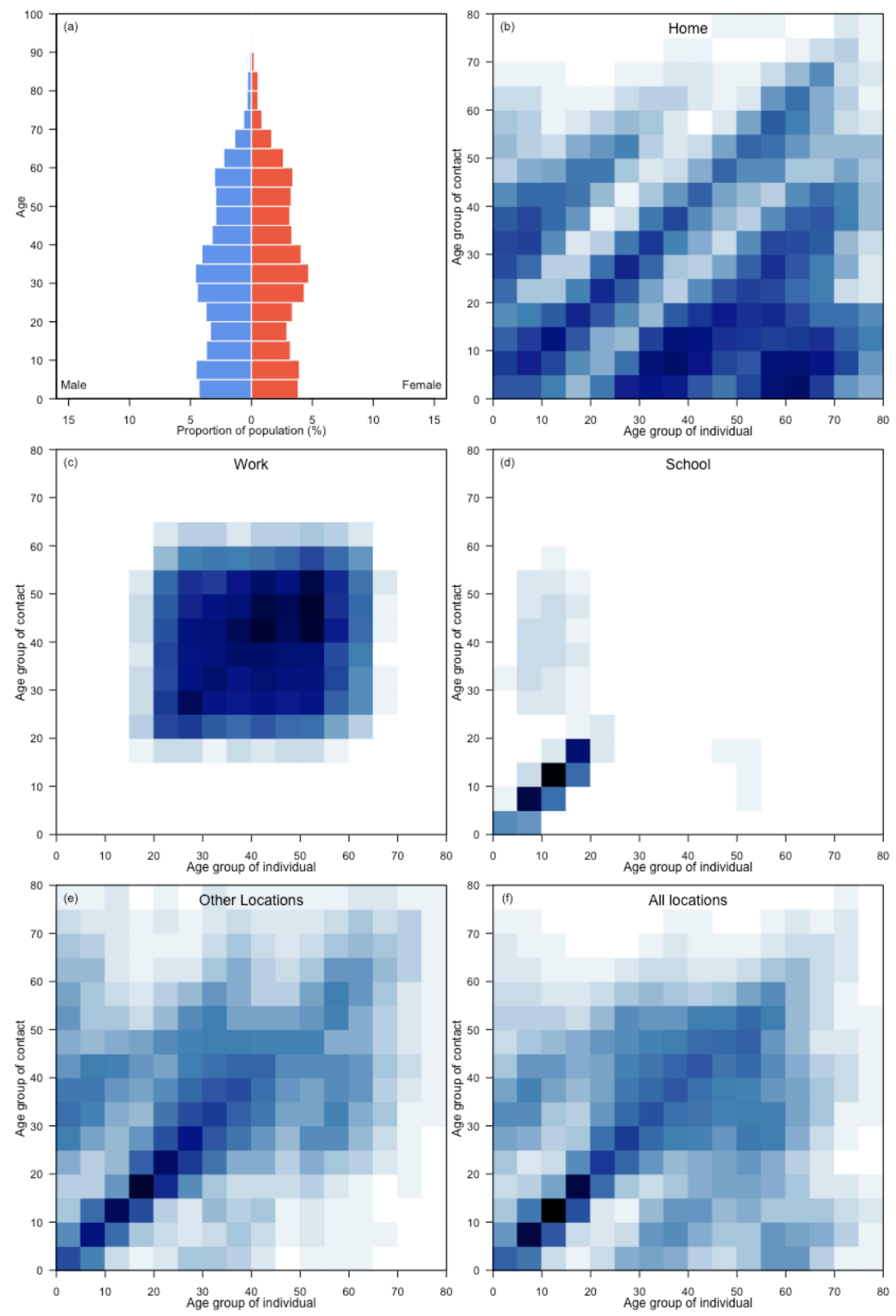


\section{Bahamas}
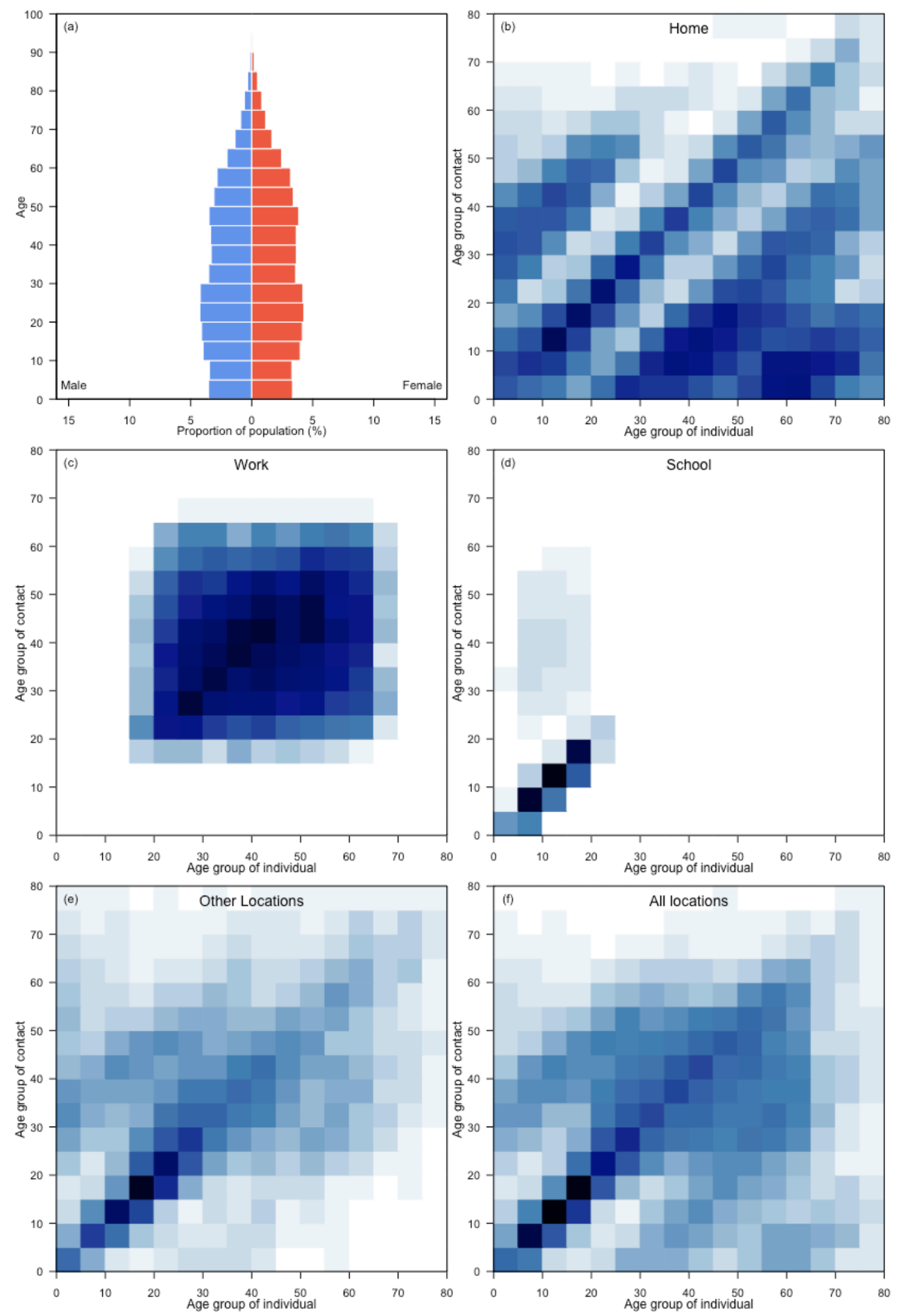


\section{Bahrain}
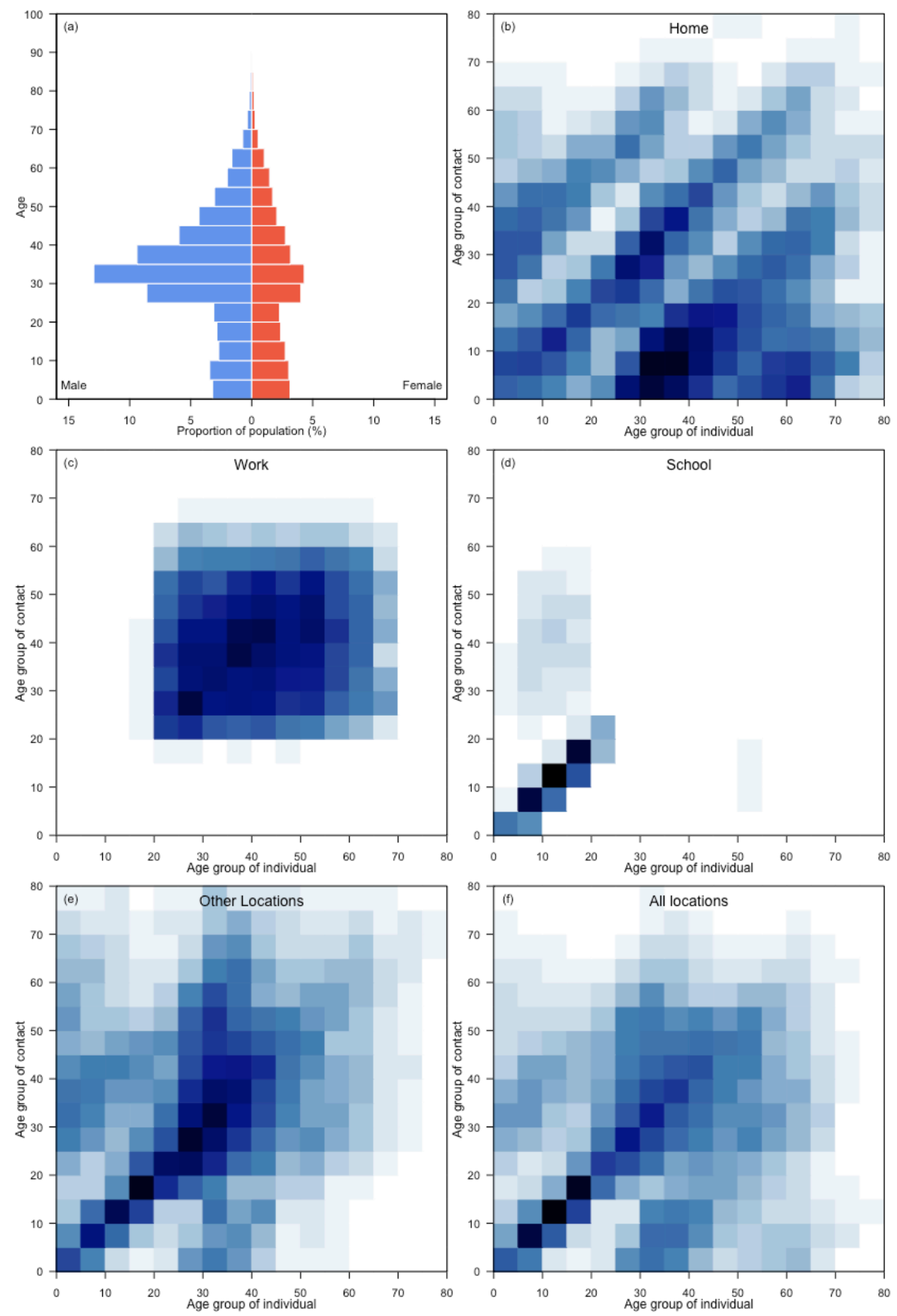


\section{Bangladesh}
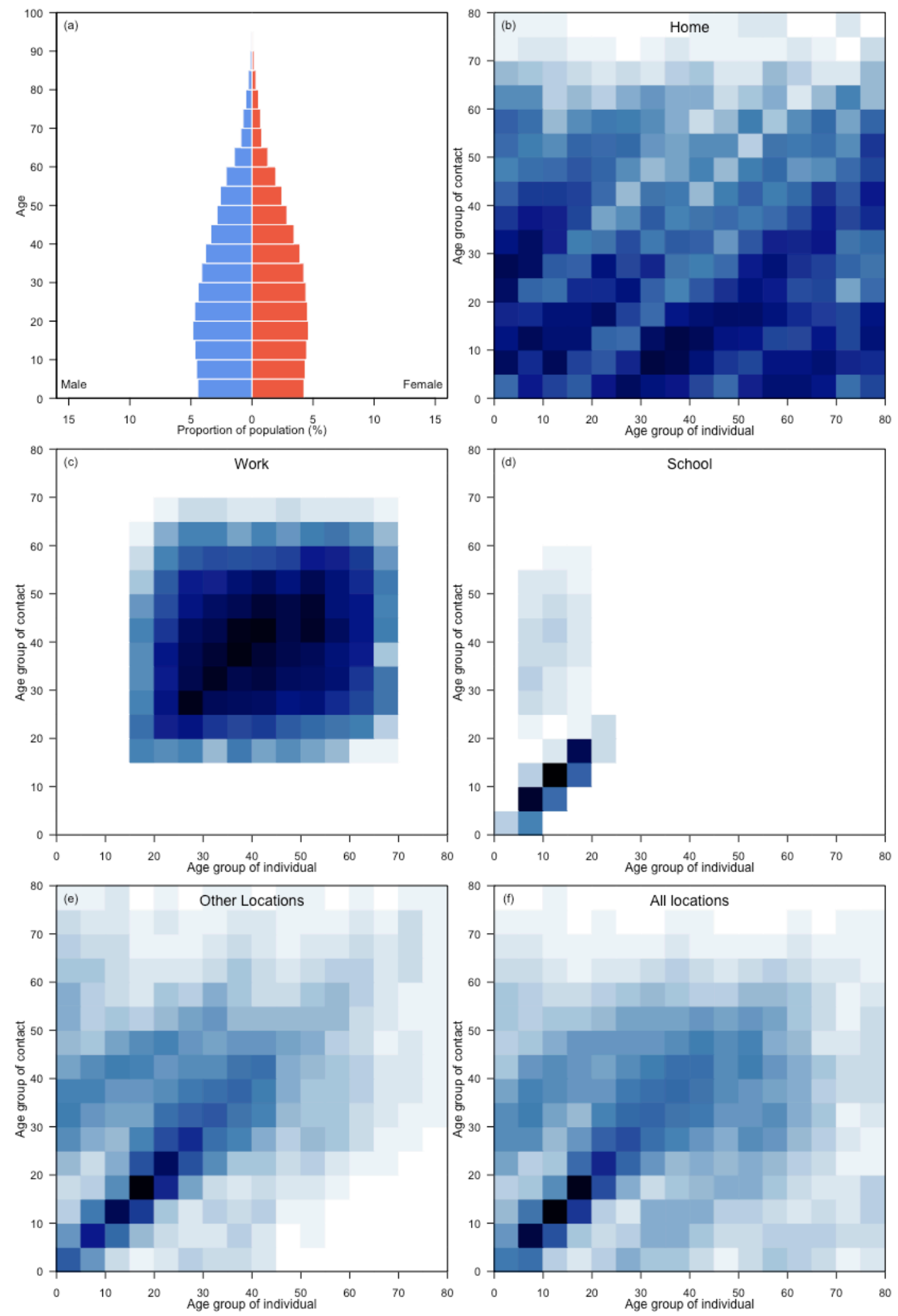
Barbados
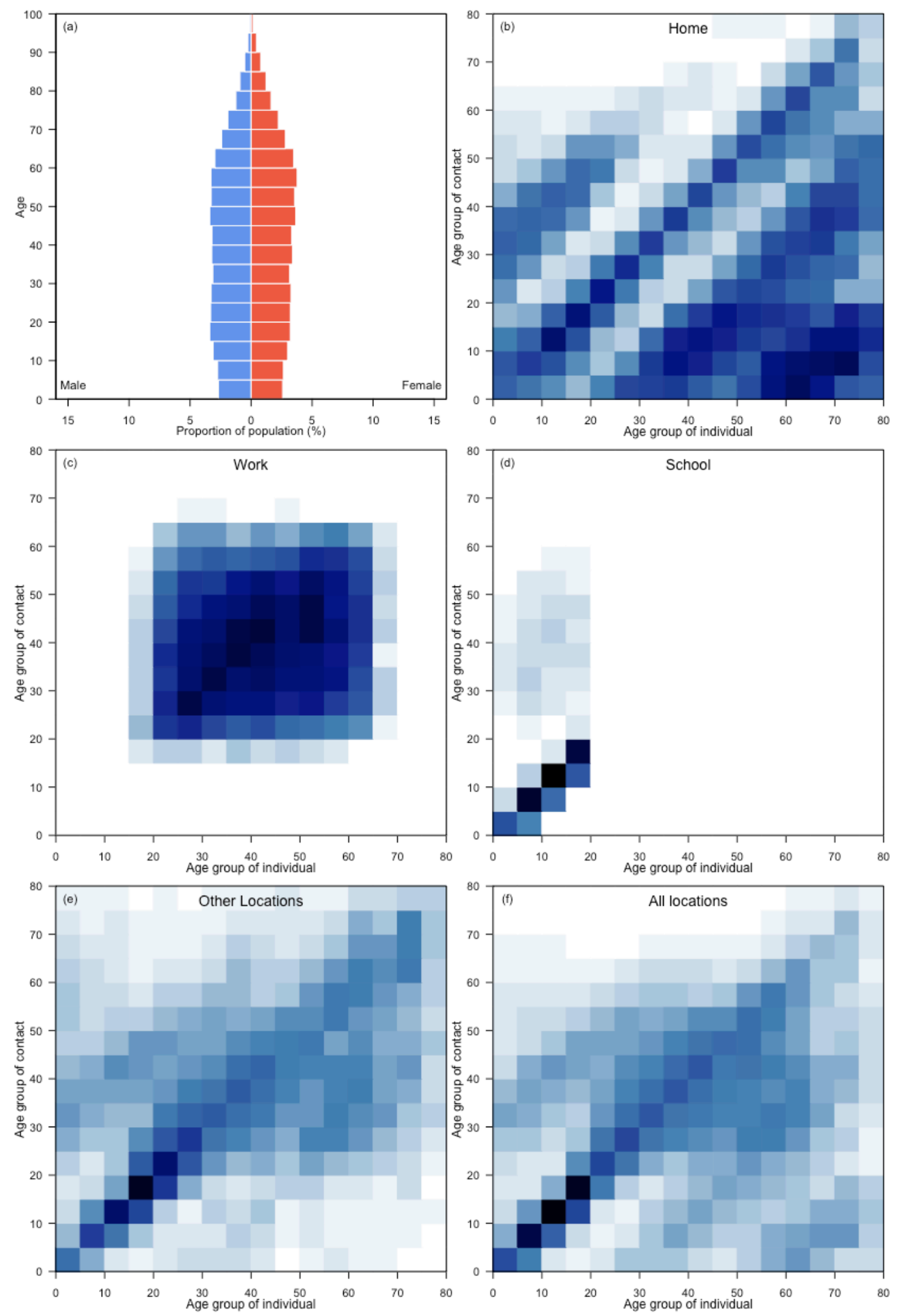


\section{Belarus}
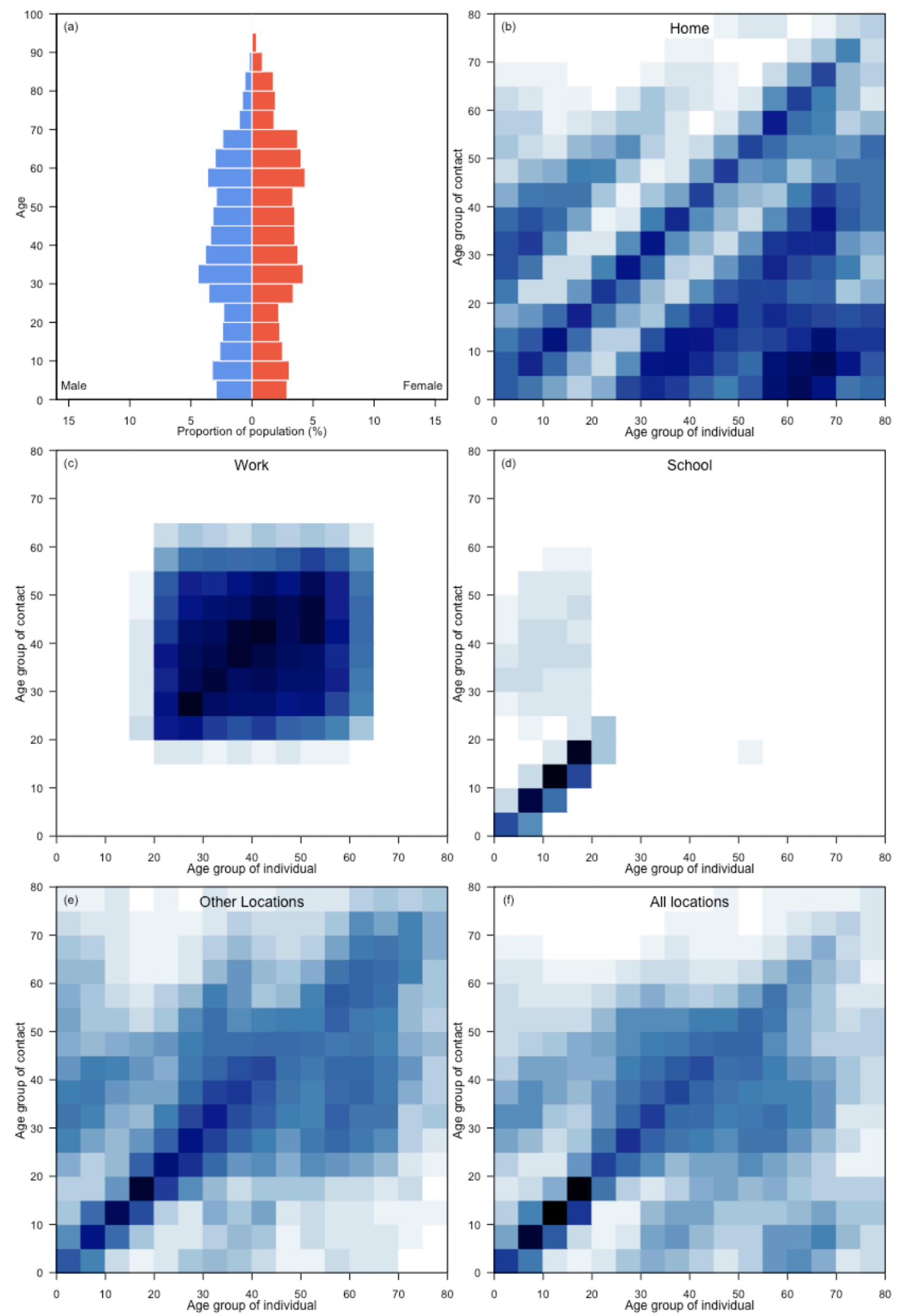
Belgium
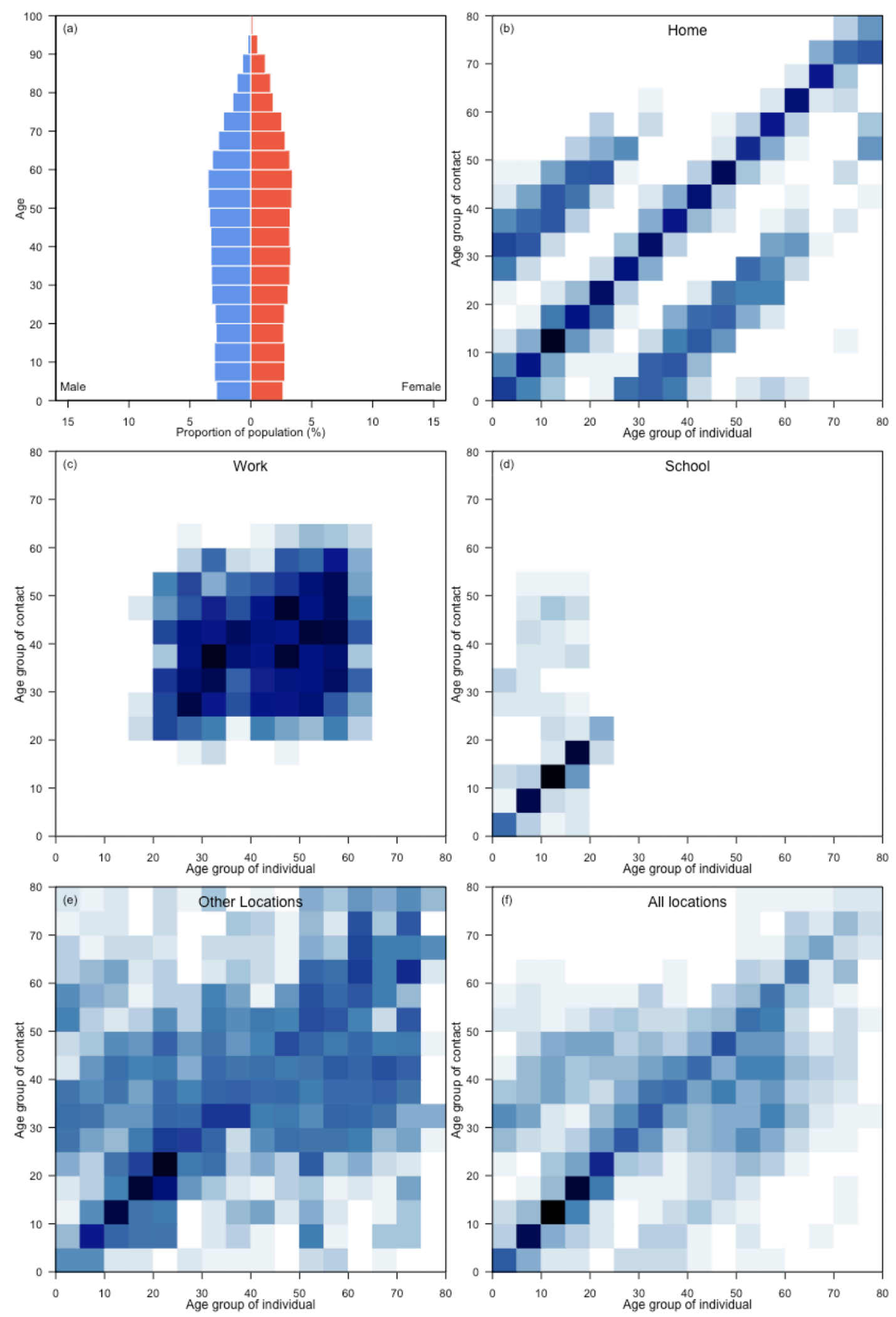


\section{Belize}
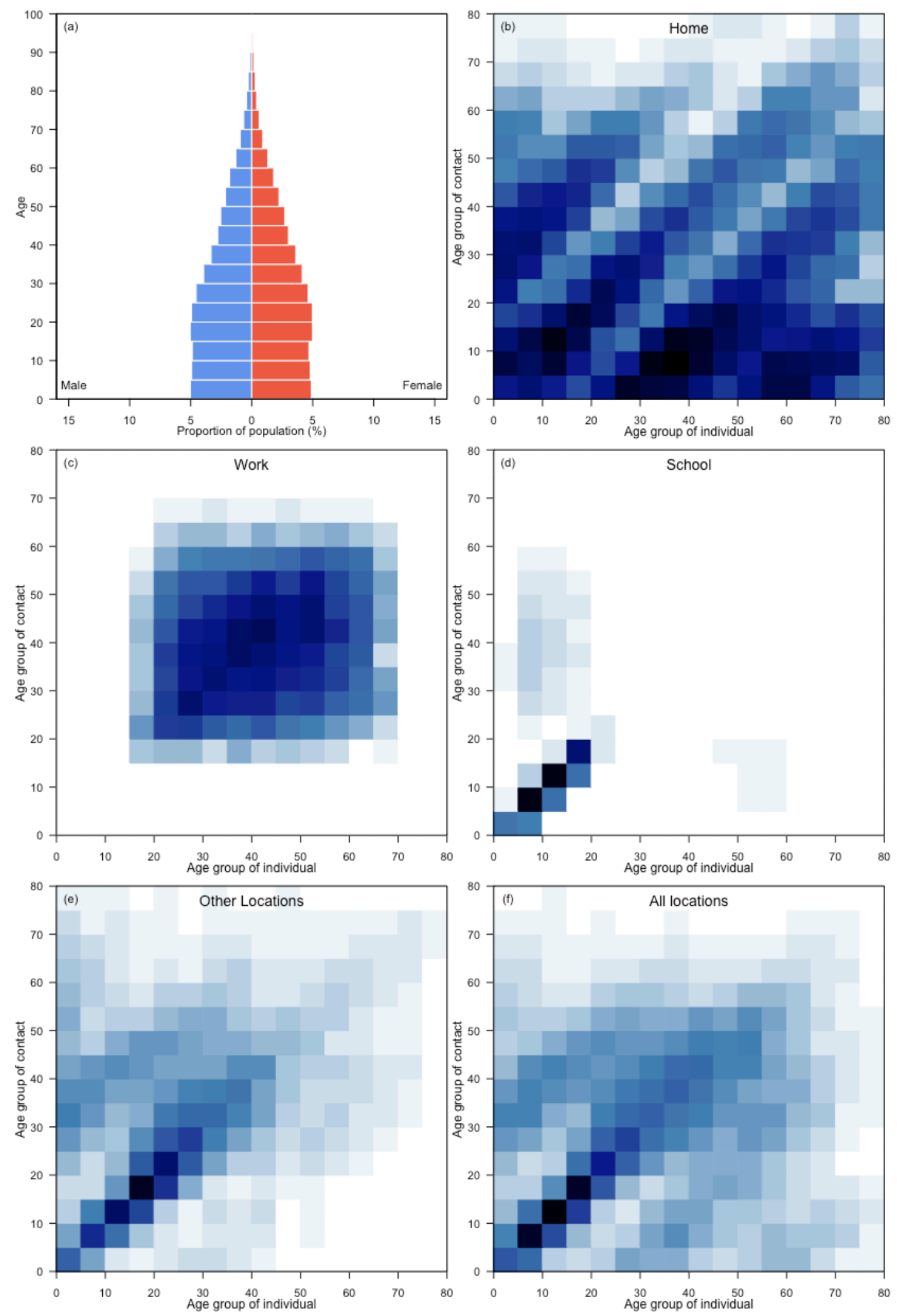


\section{Benin}
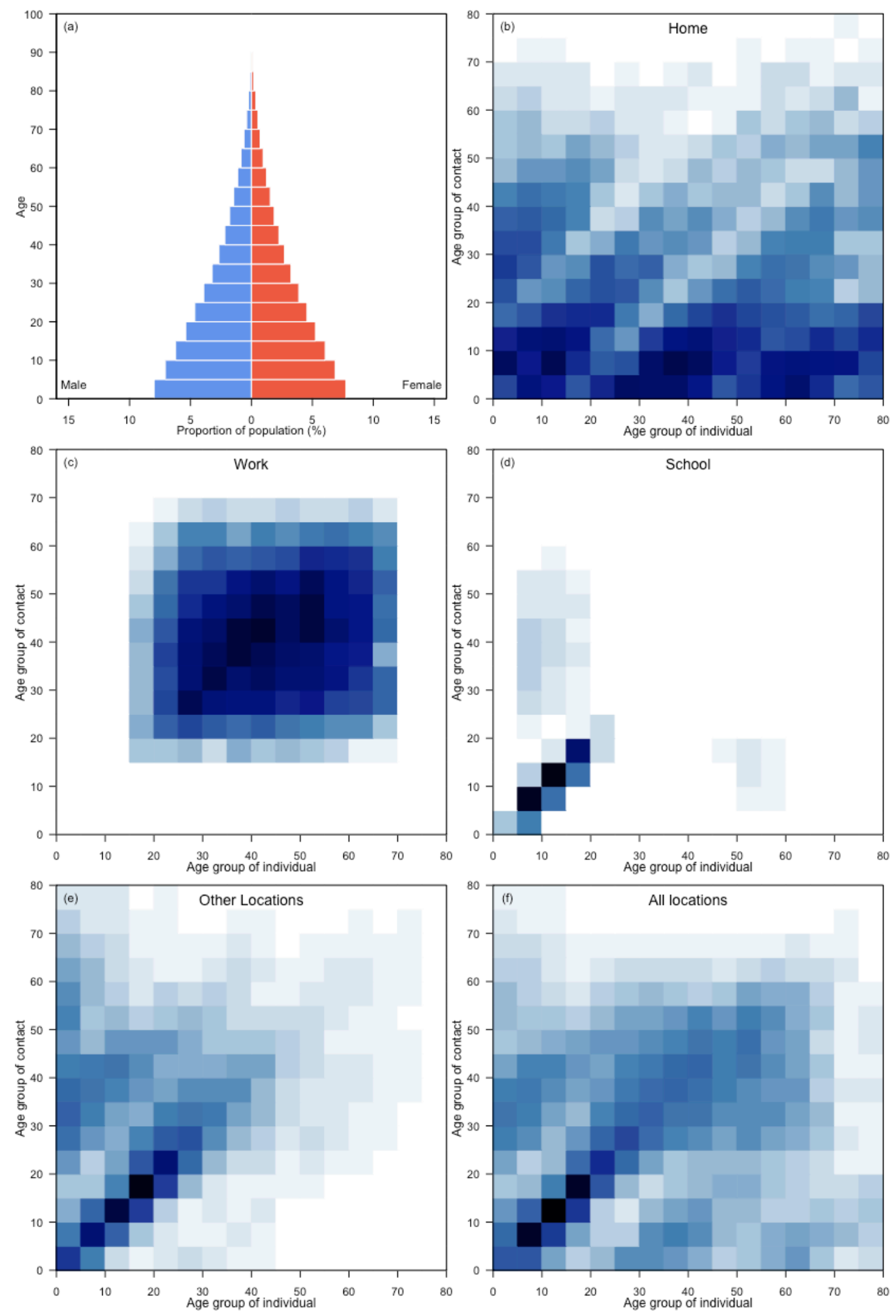


\section{Bhutan}
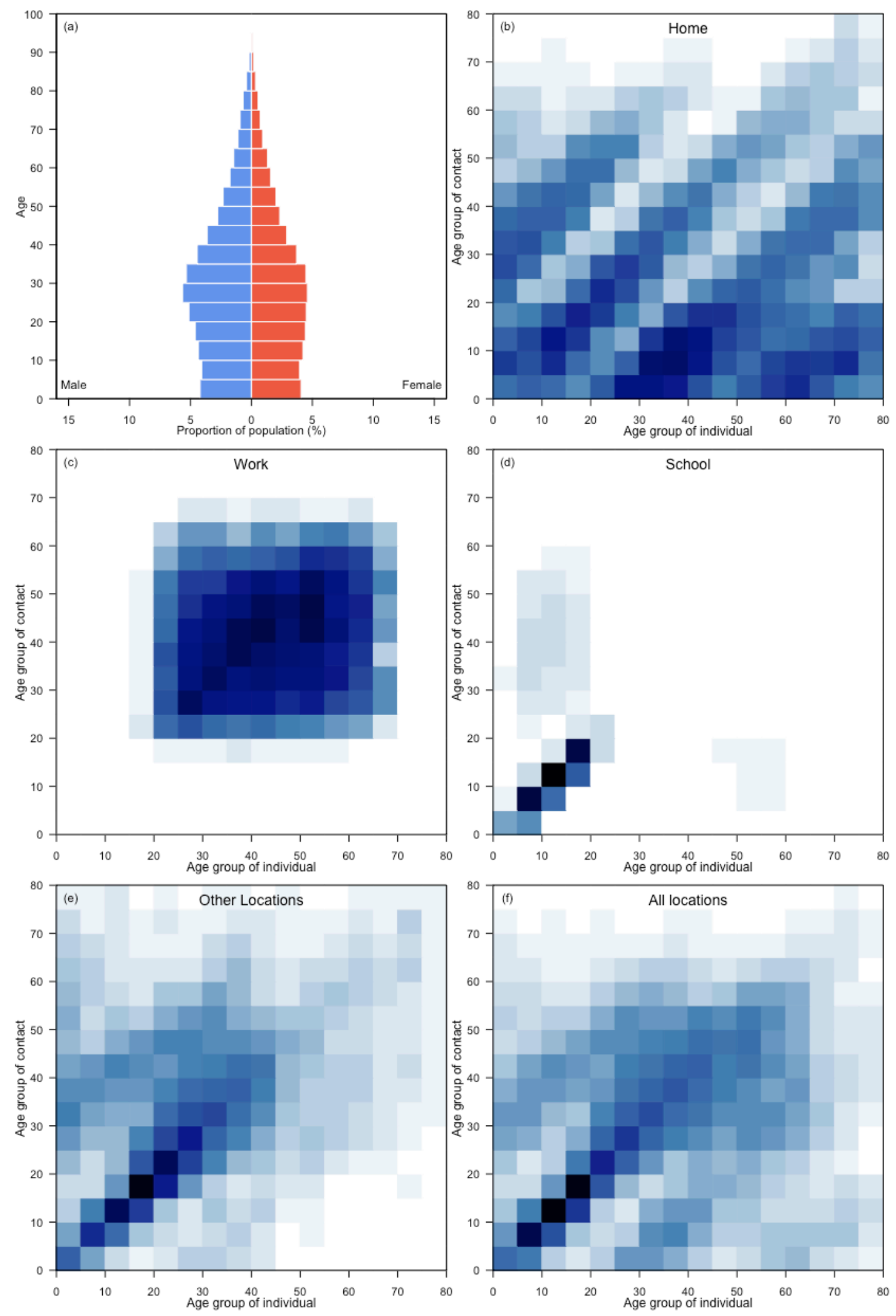
Bolivia (Plurinational State of)
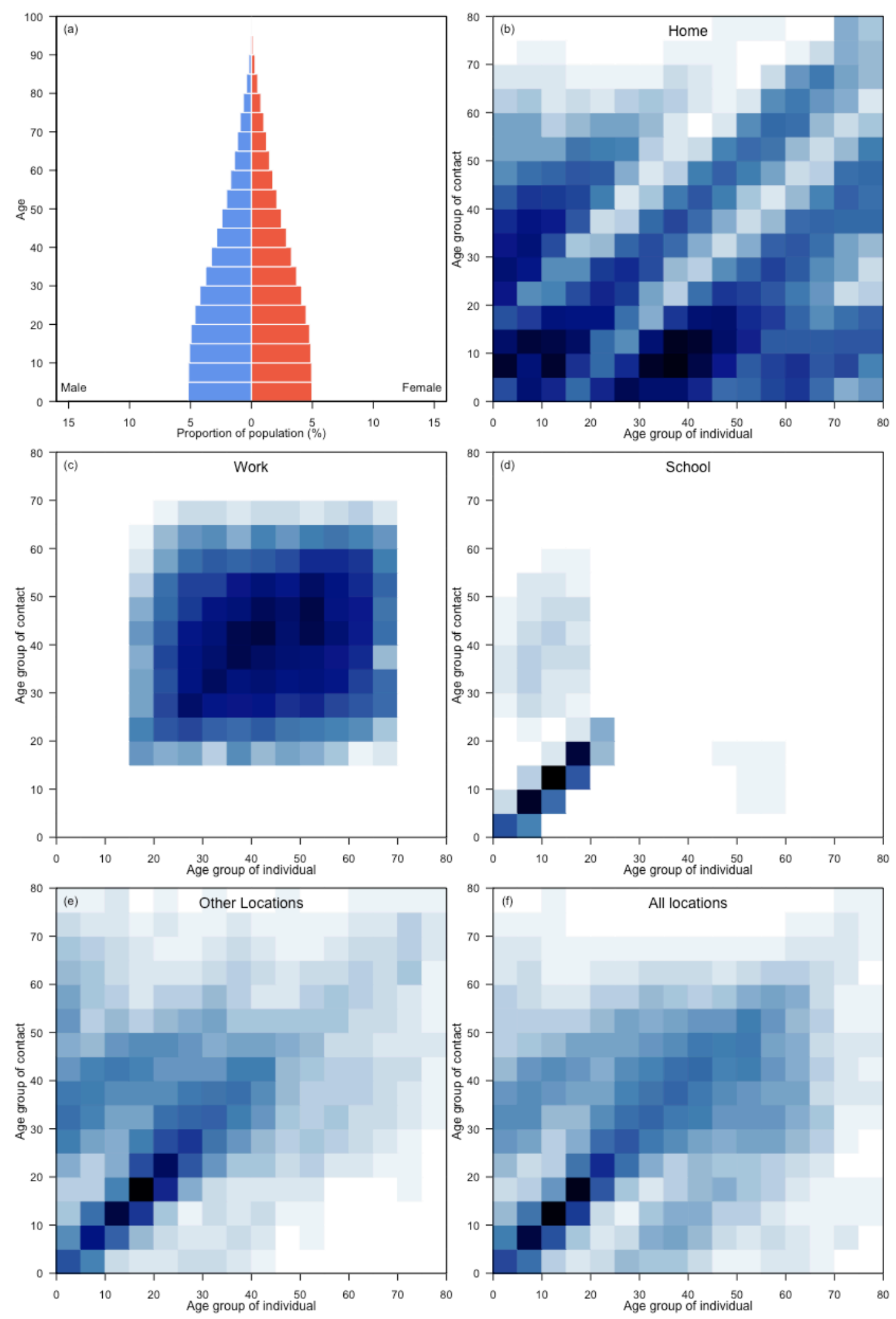
Bosnia and Herzegovina
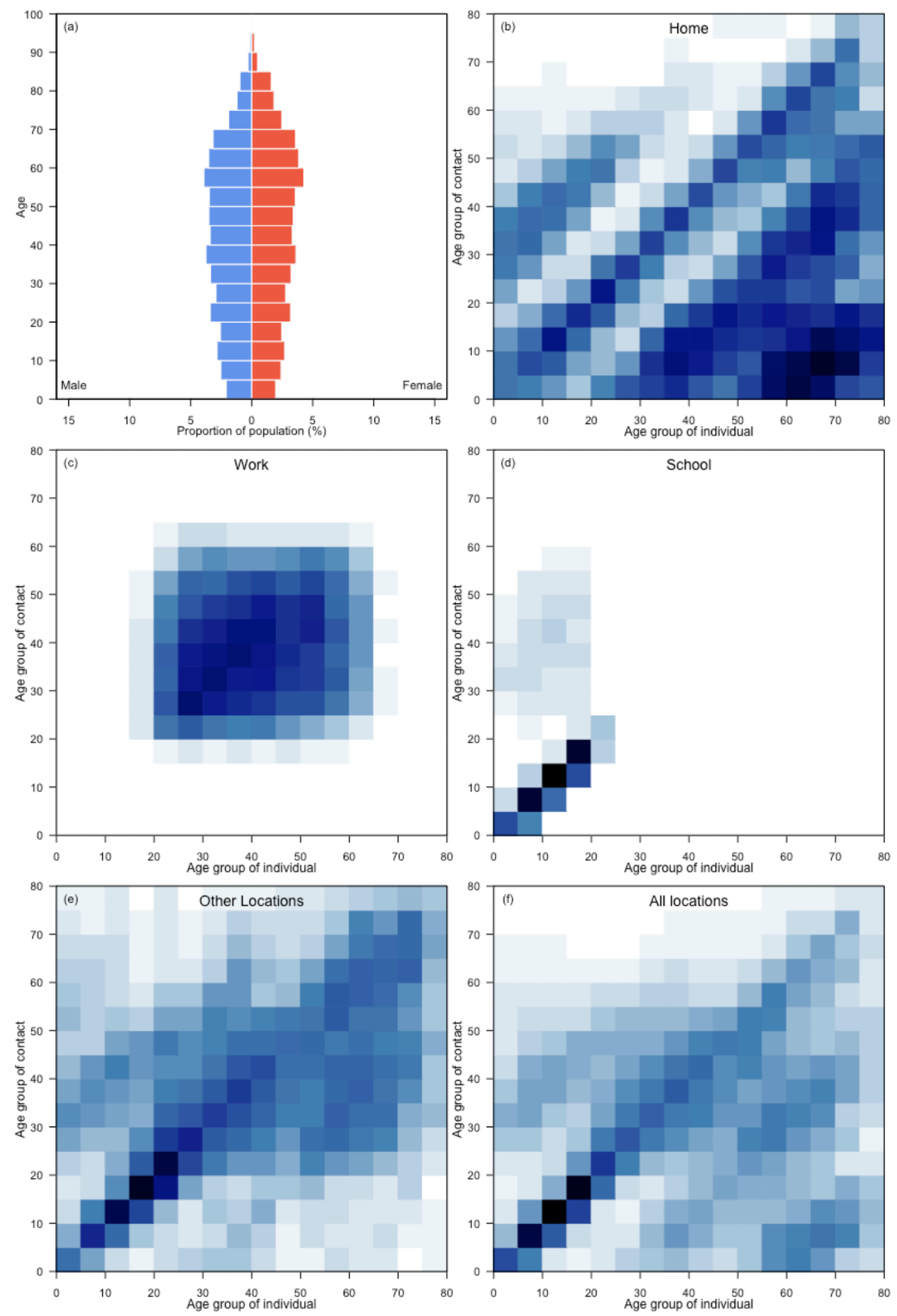
Botswana
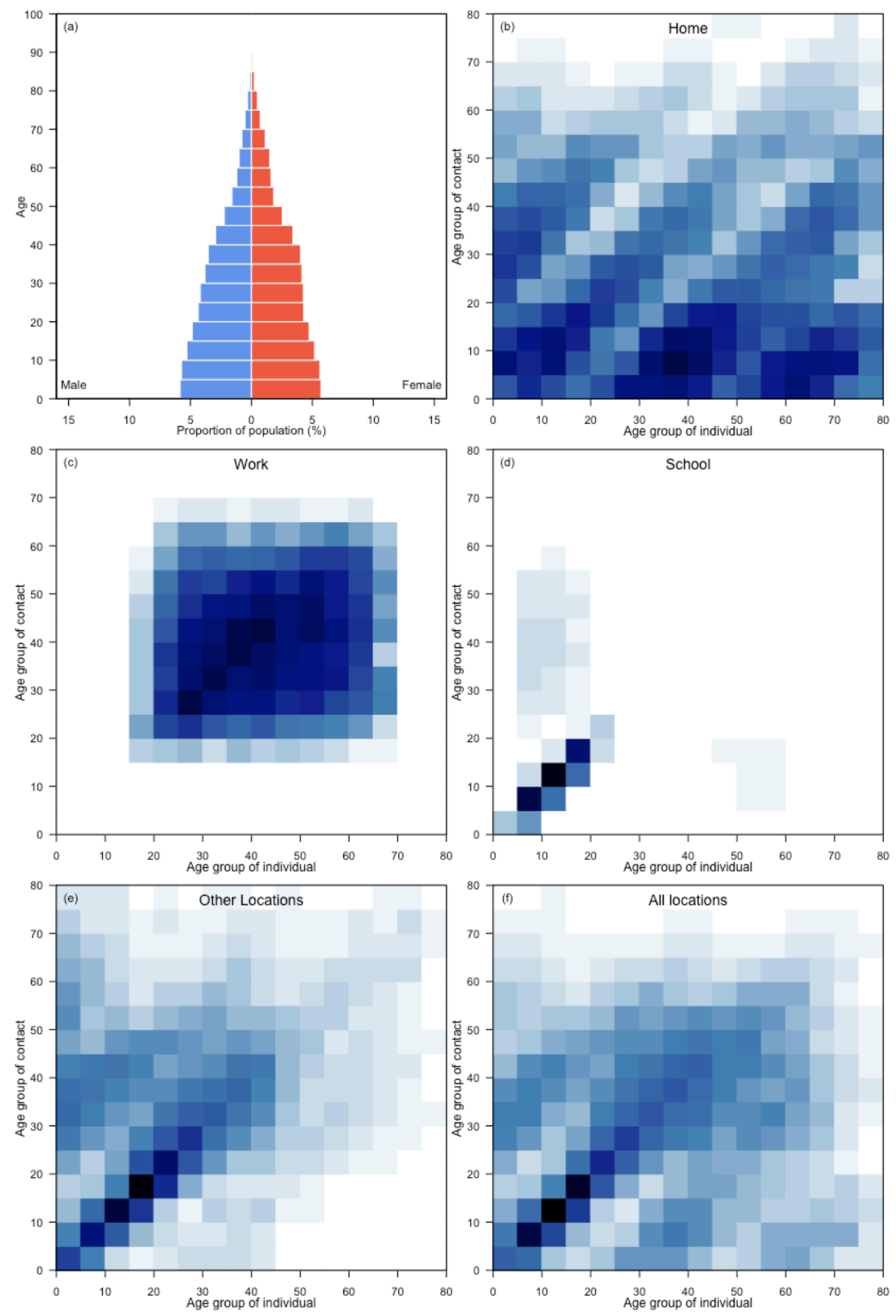


\section{Brazil}
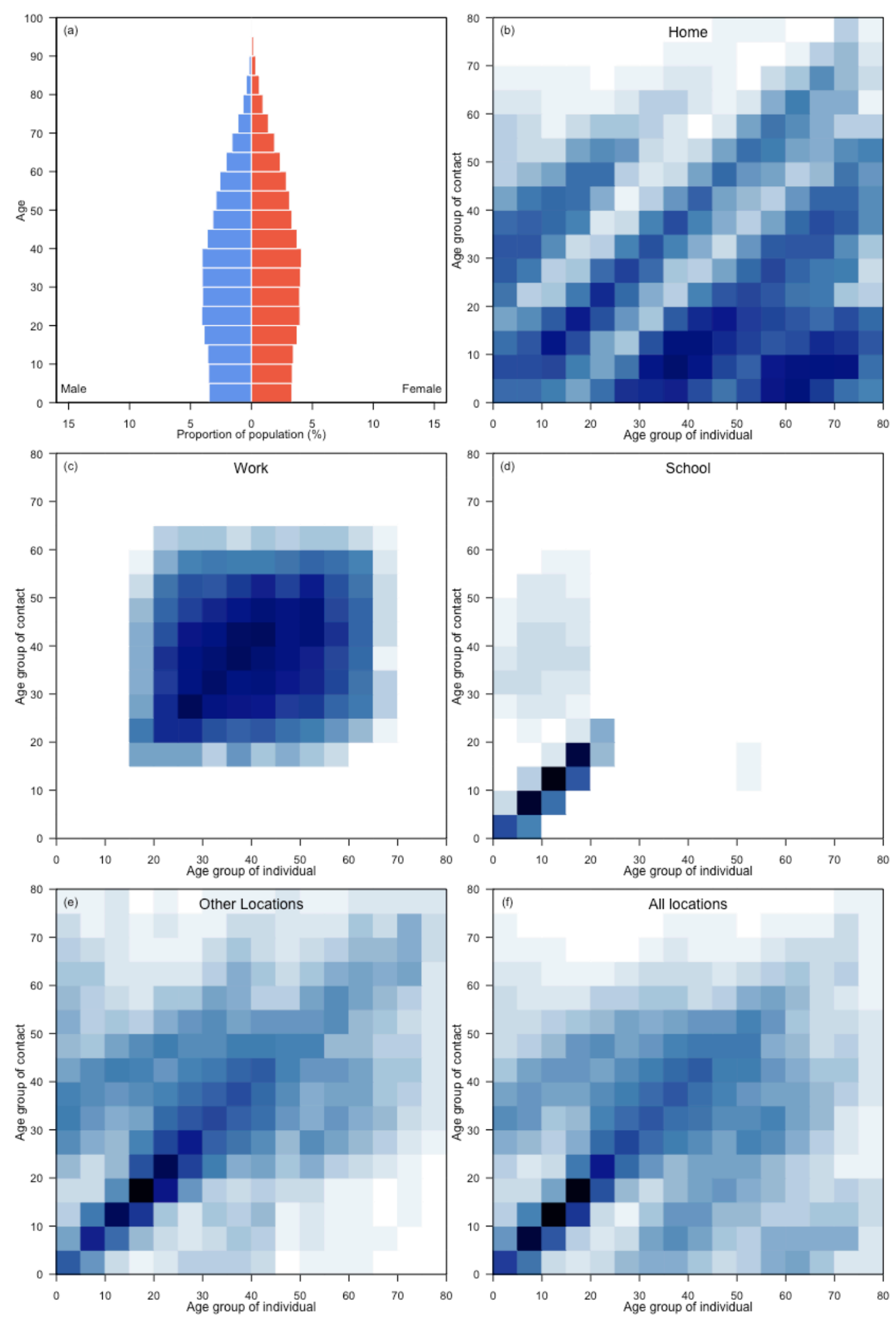
Brunei Darussalam
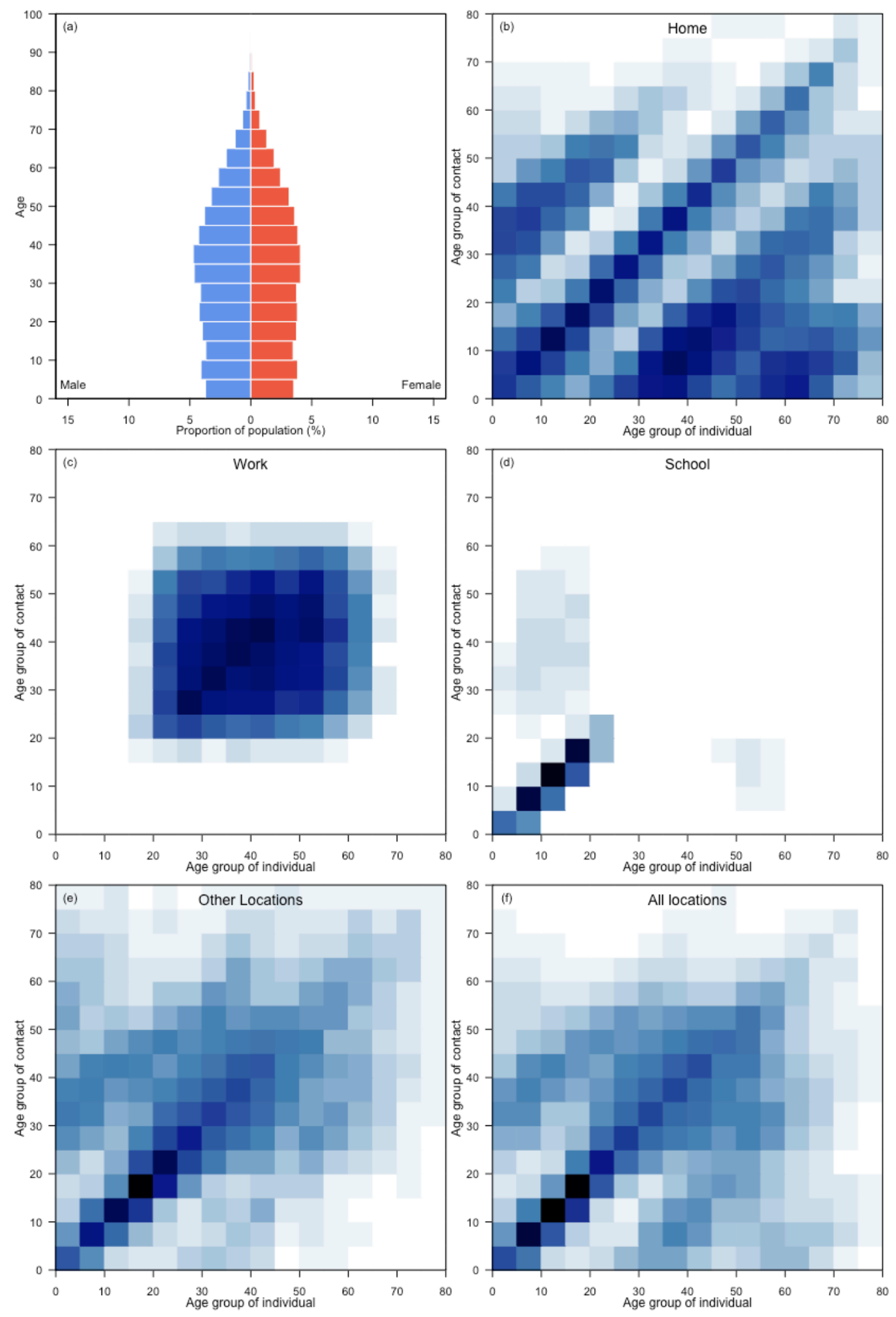


\section{Bulgaria}
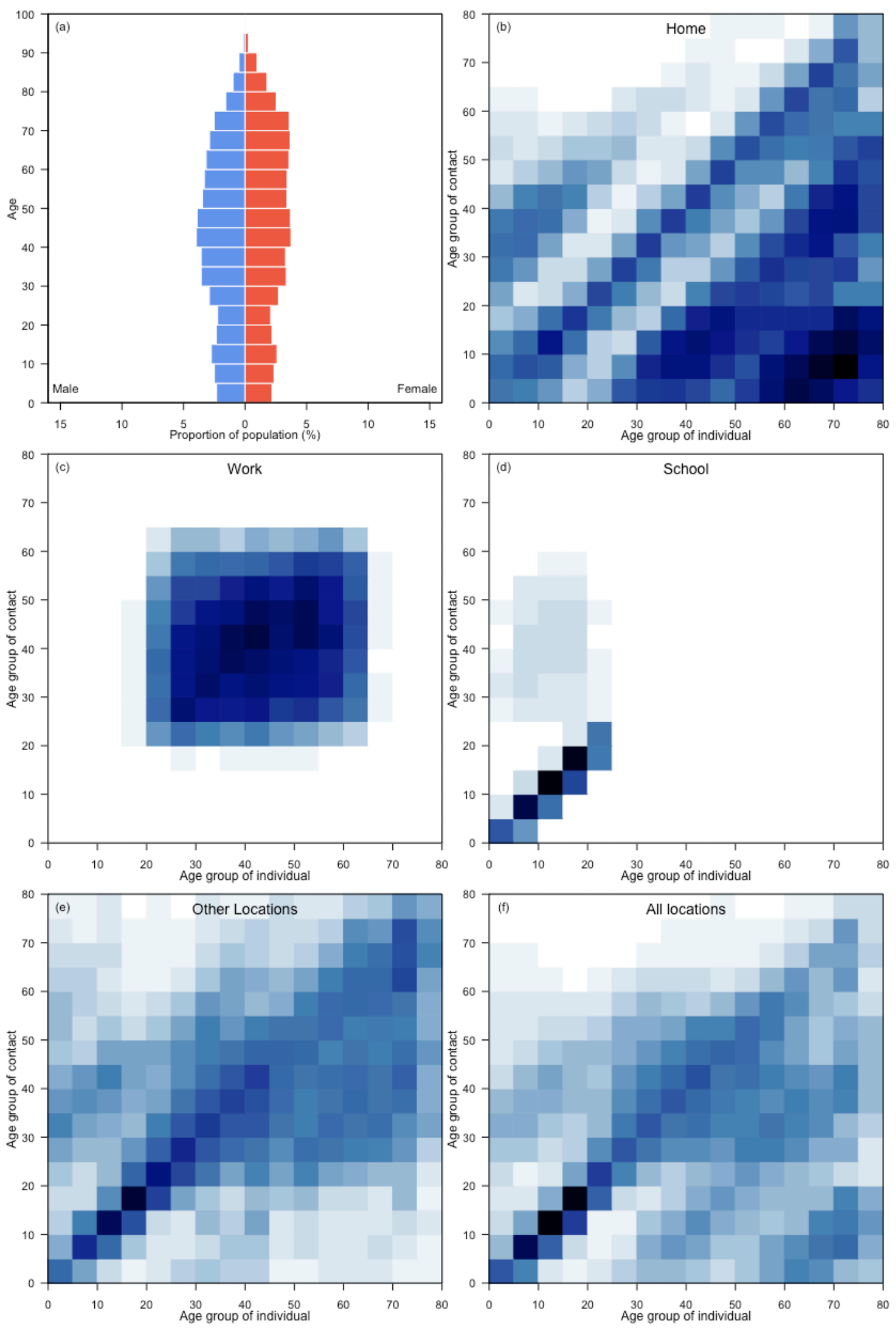


\section{Burkina Faso}
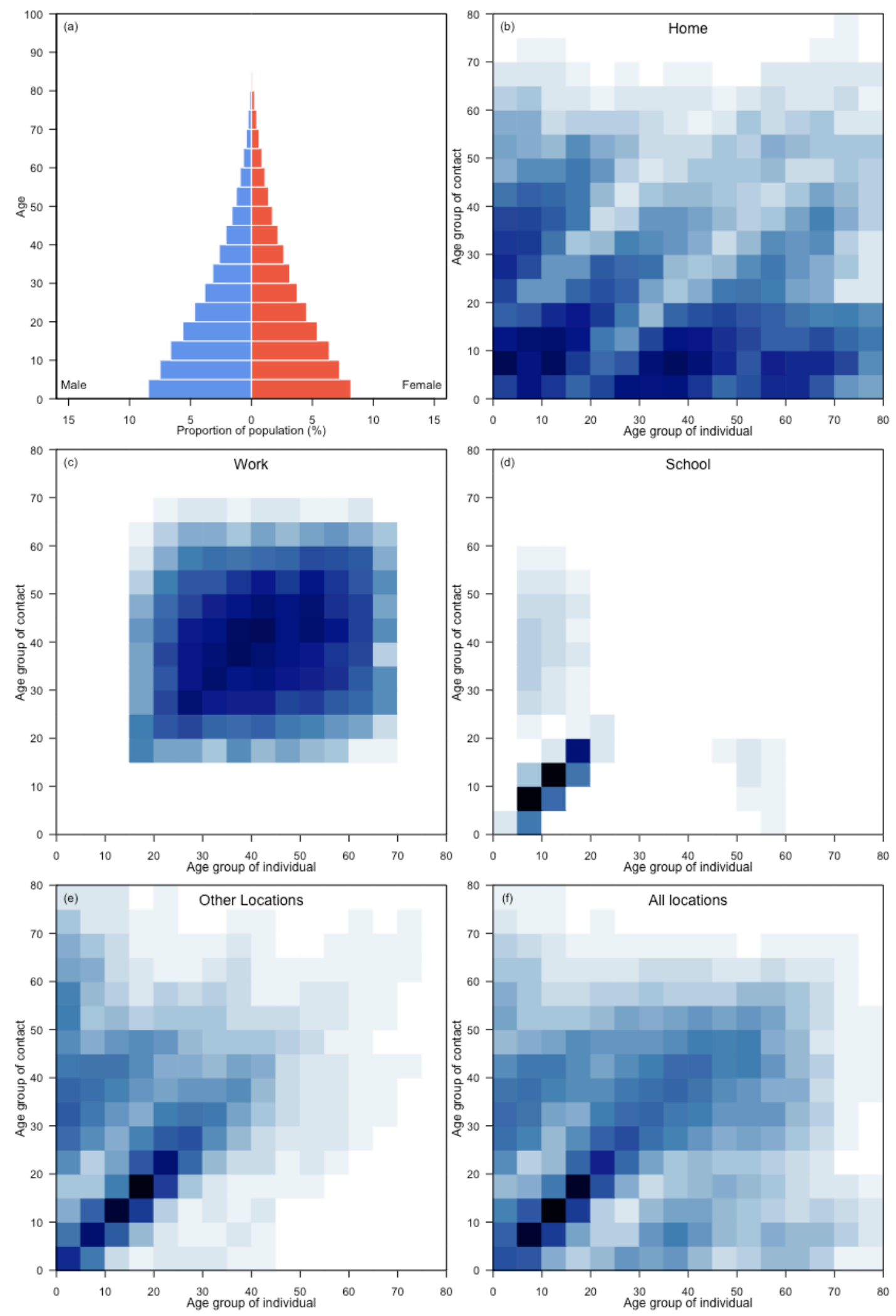


\section{Burundi}
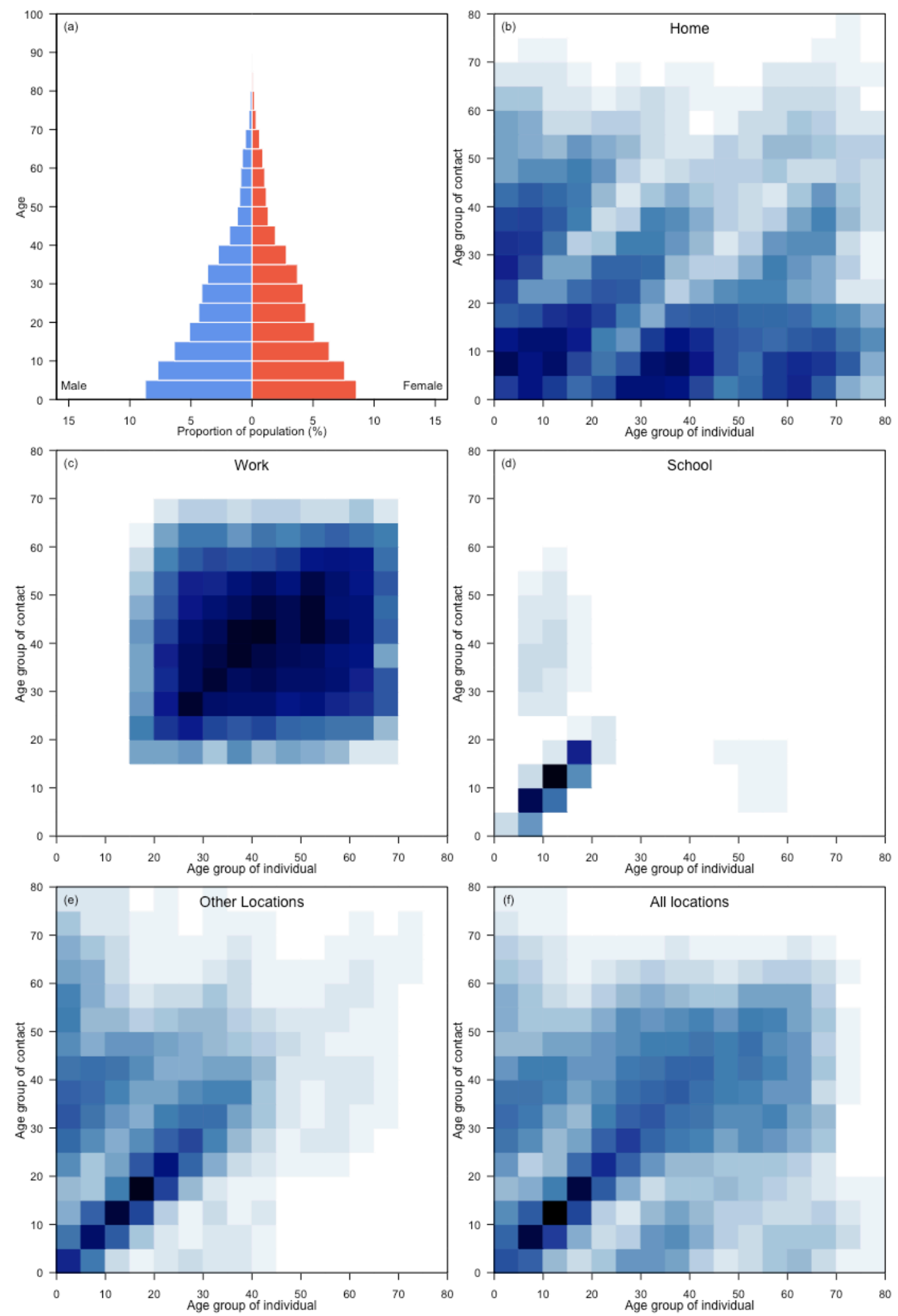


\section{Cabo Verde}
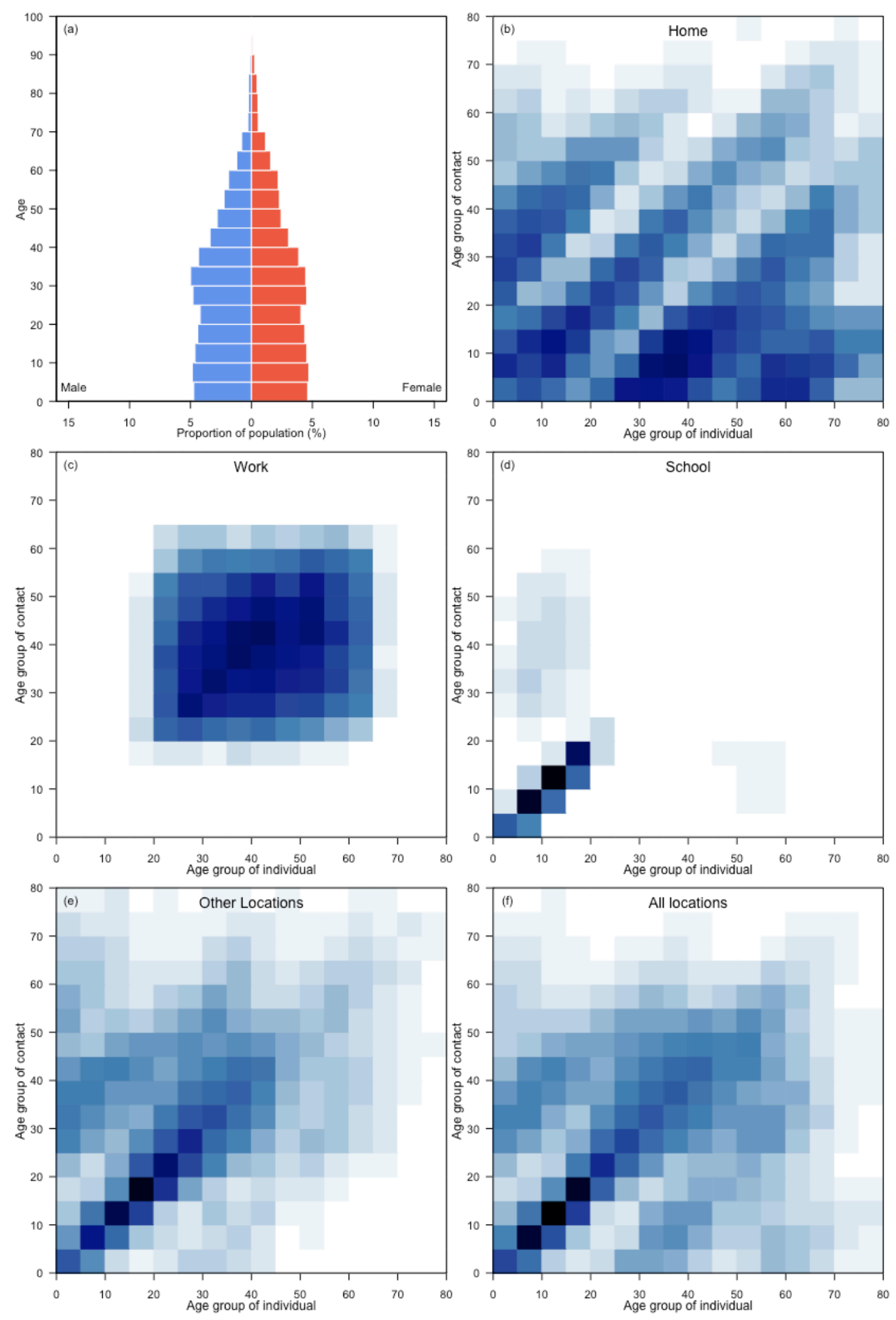


\section{Cambodia}
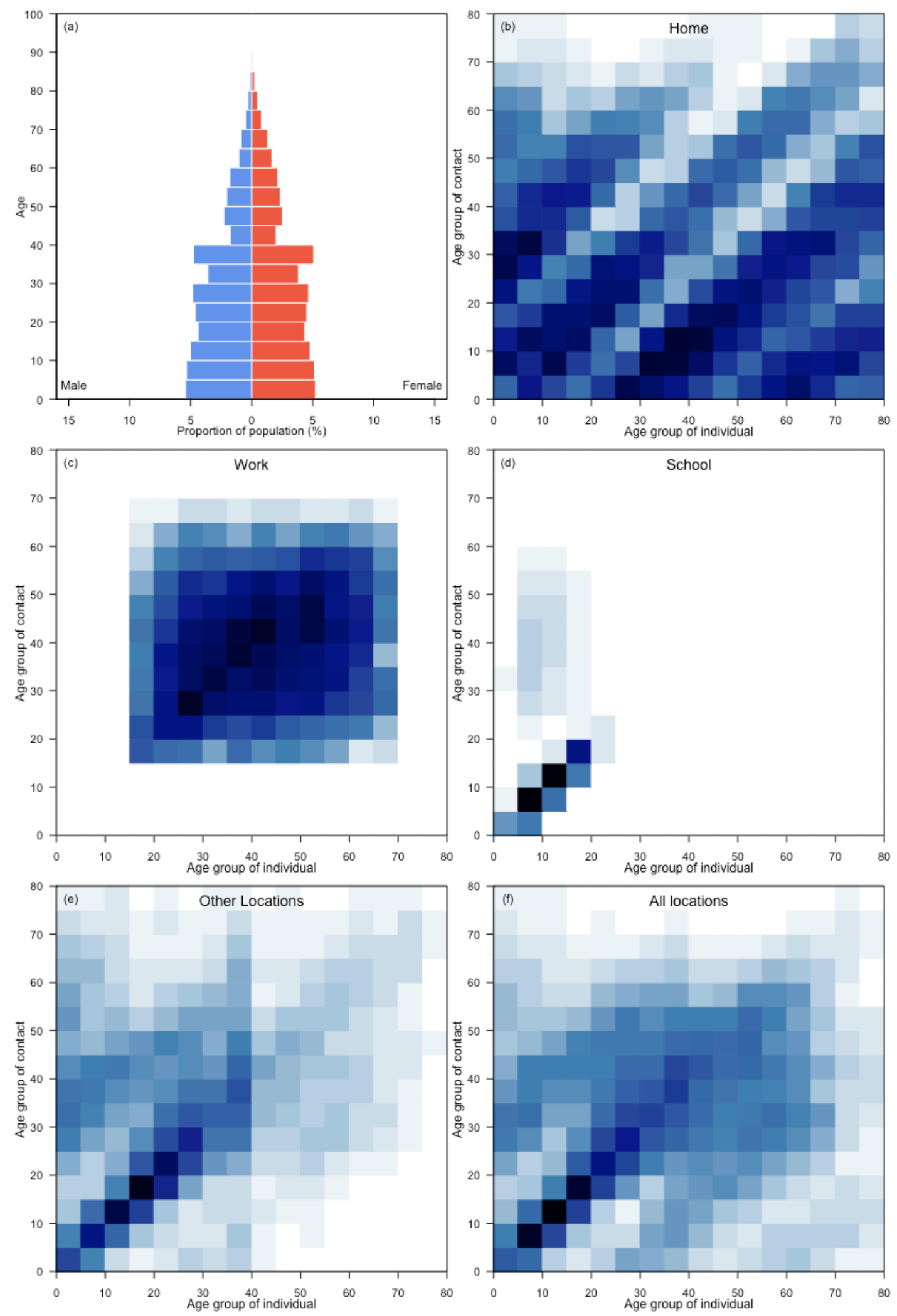


\section{Cameroon}
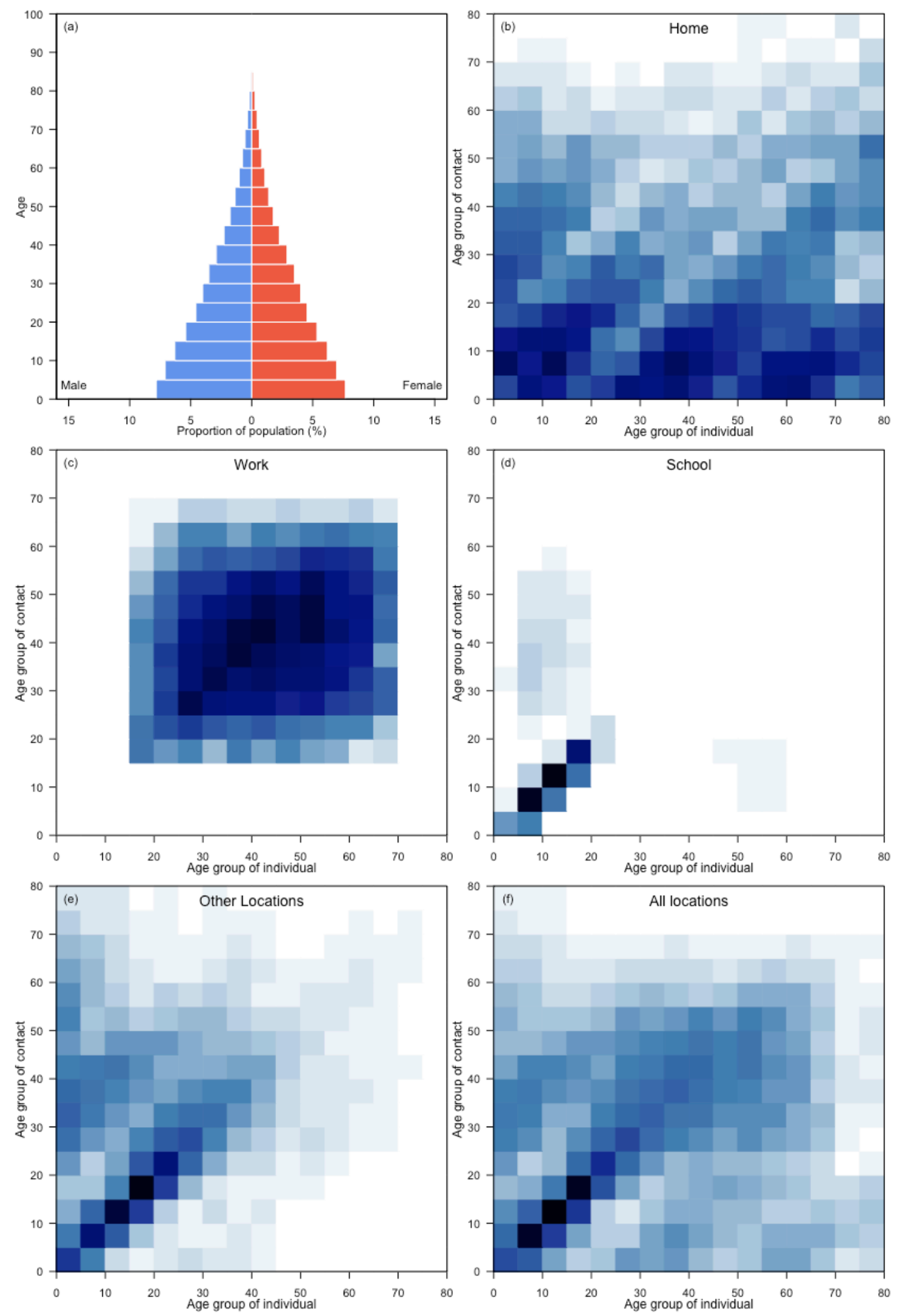


\section{Canada}
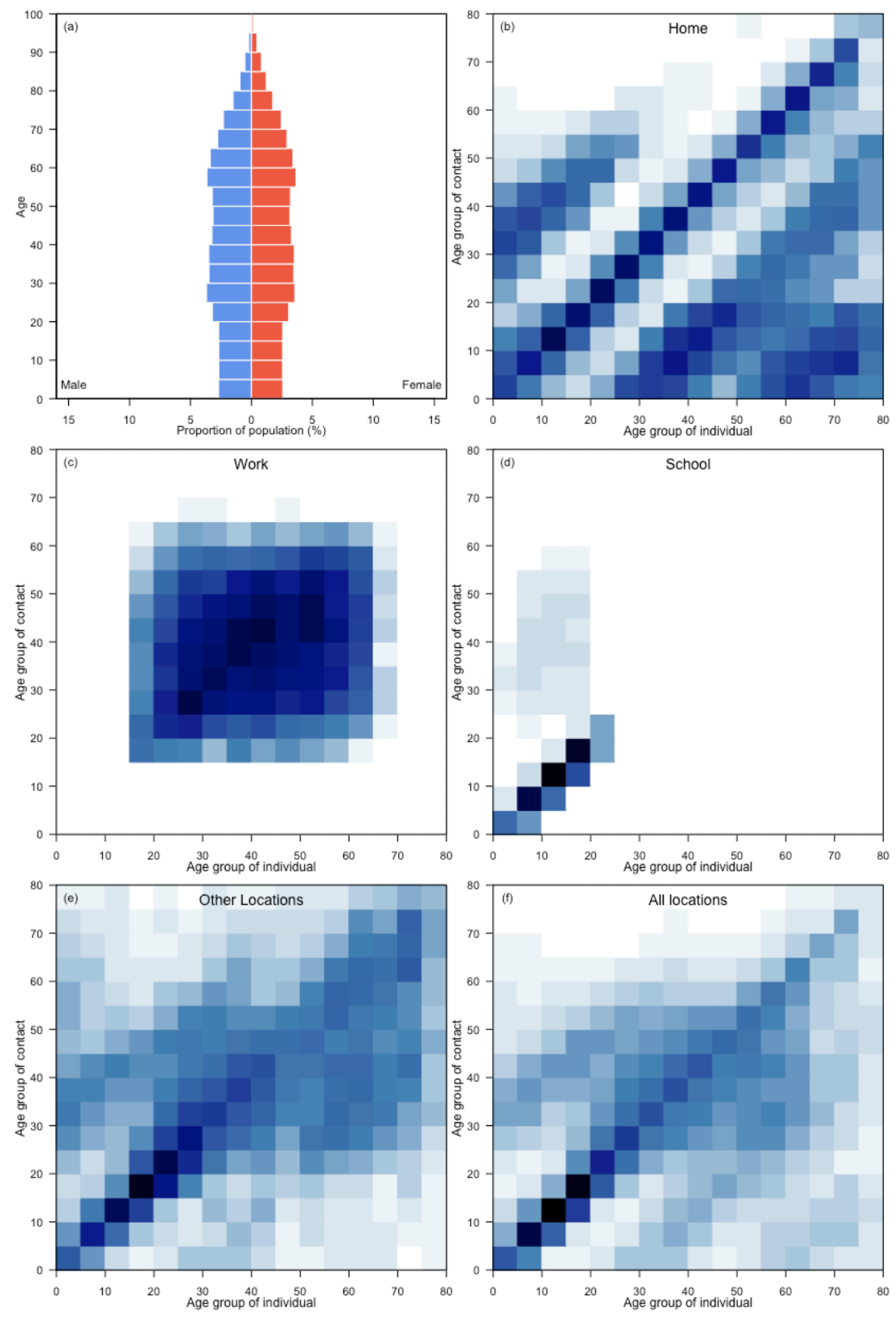
Central African Republic
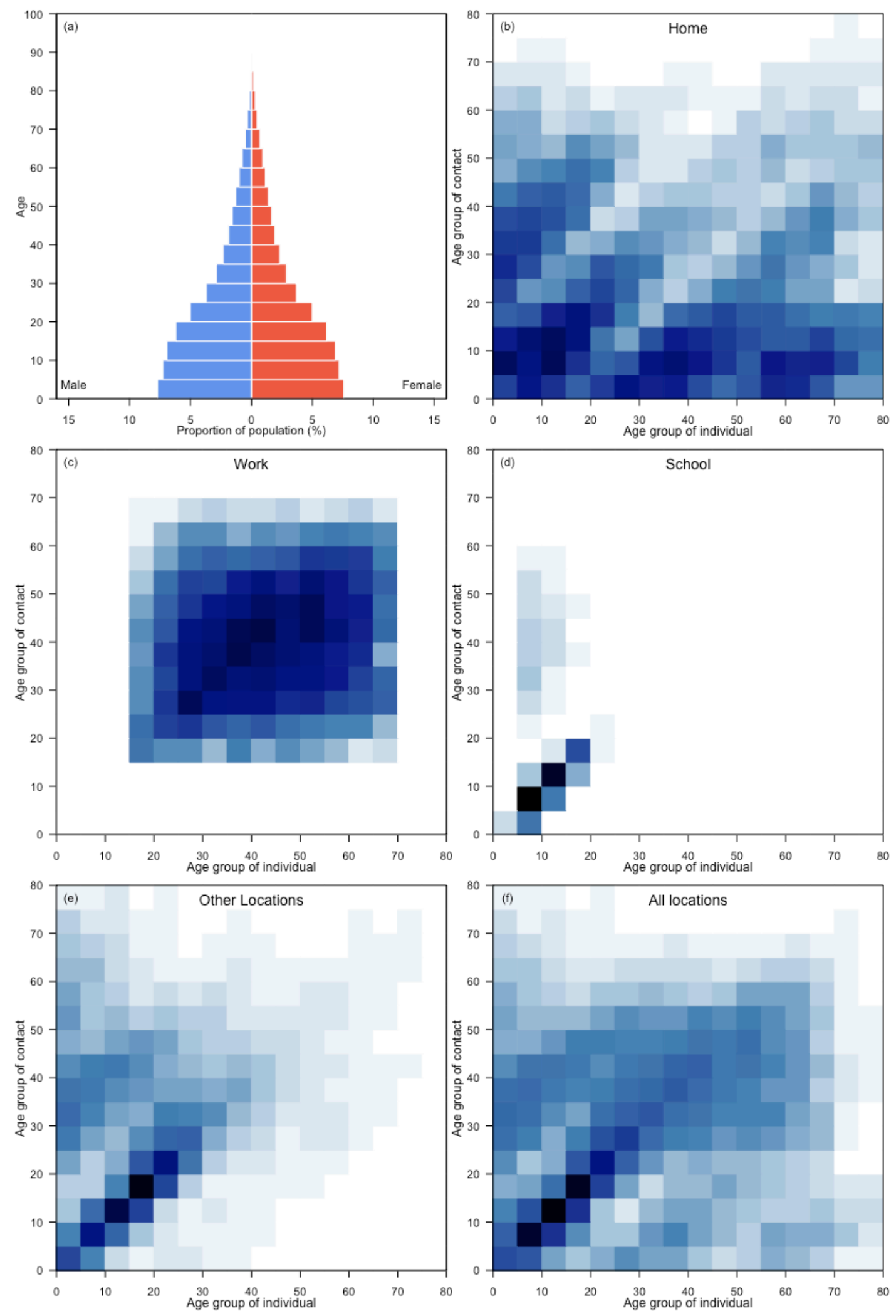


\section{Chad}
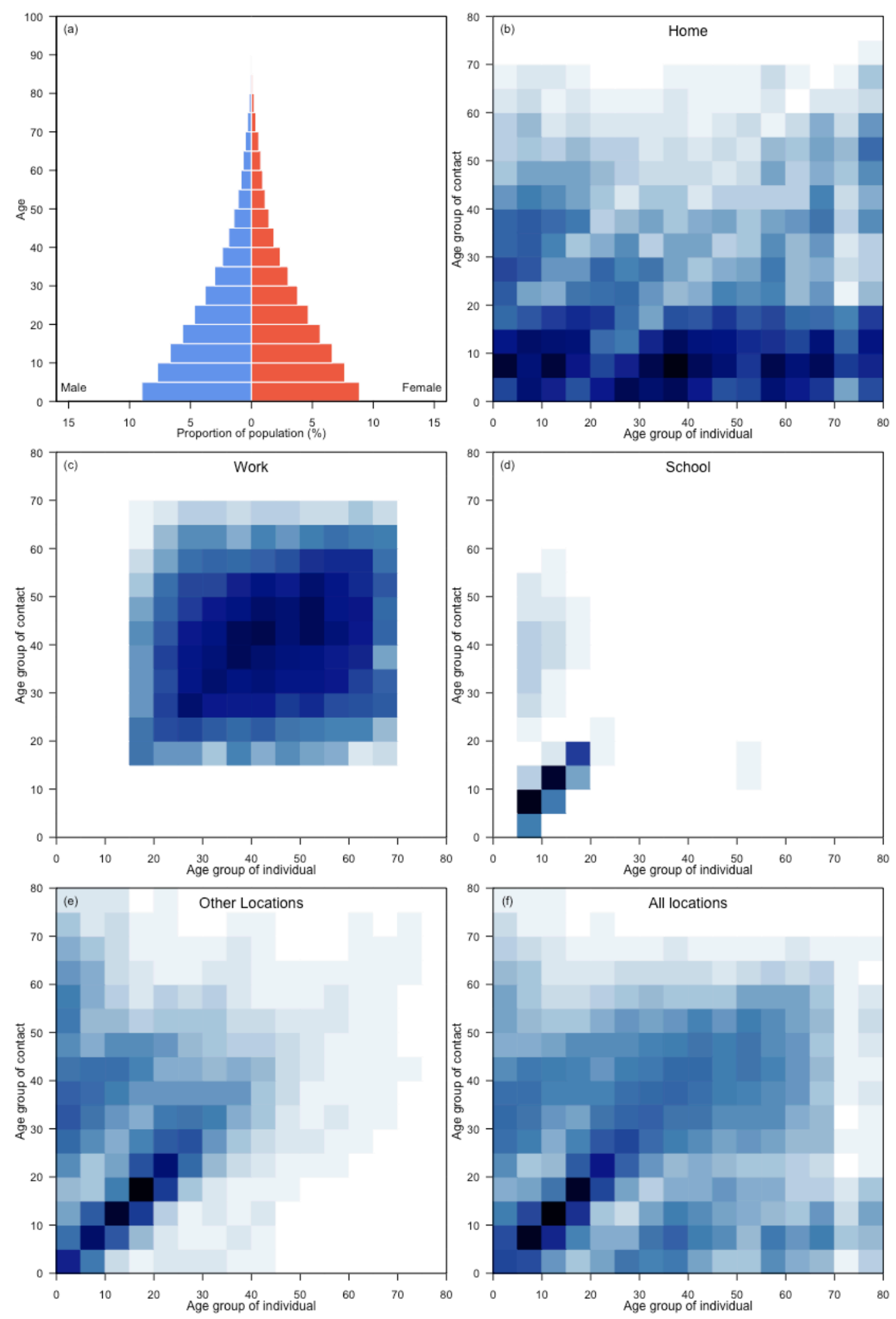


\section{Chile}
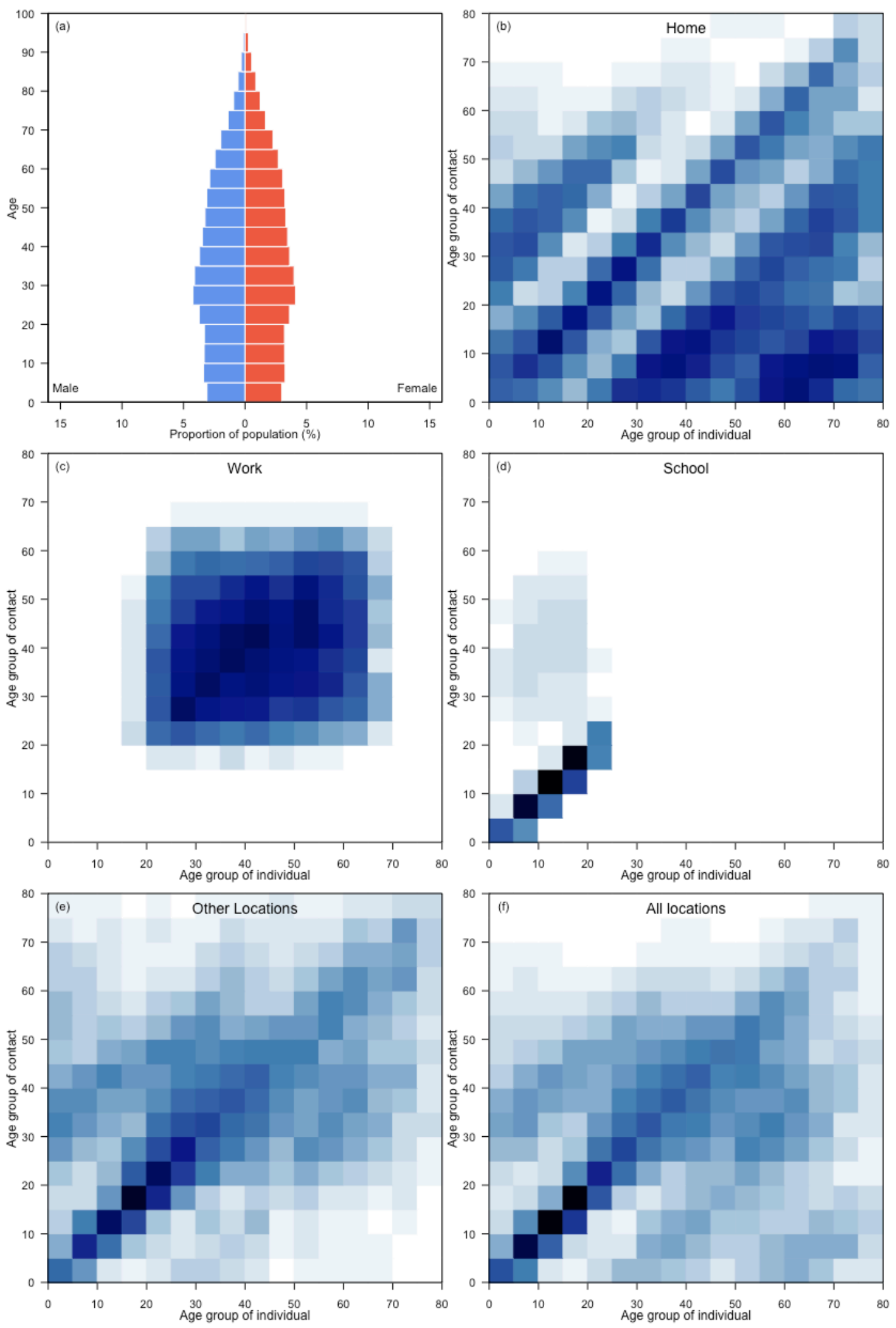


\section{China}
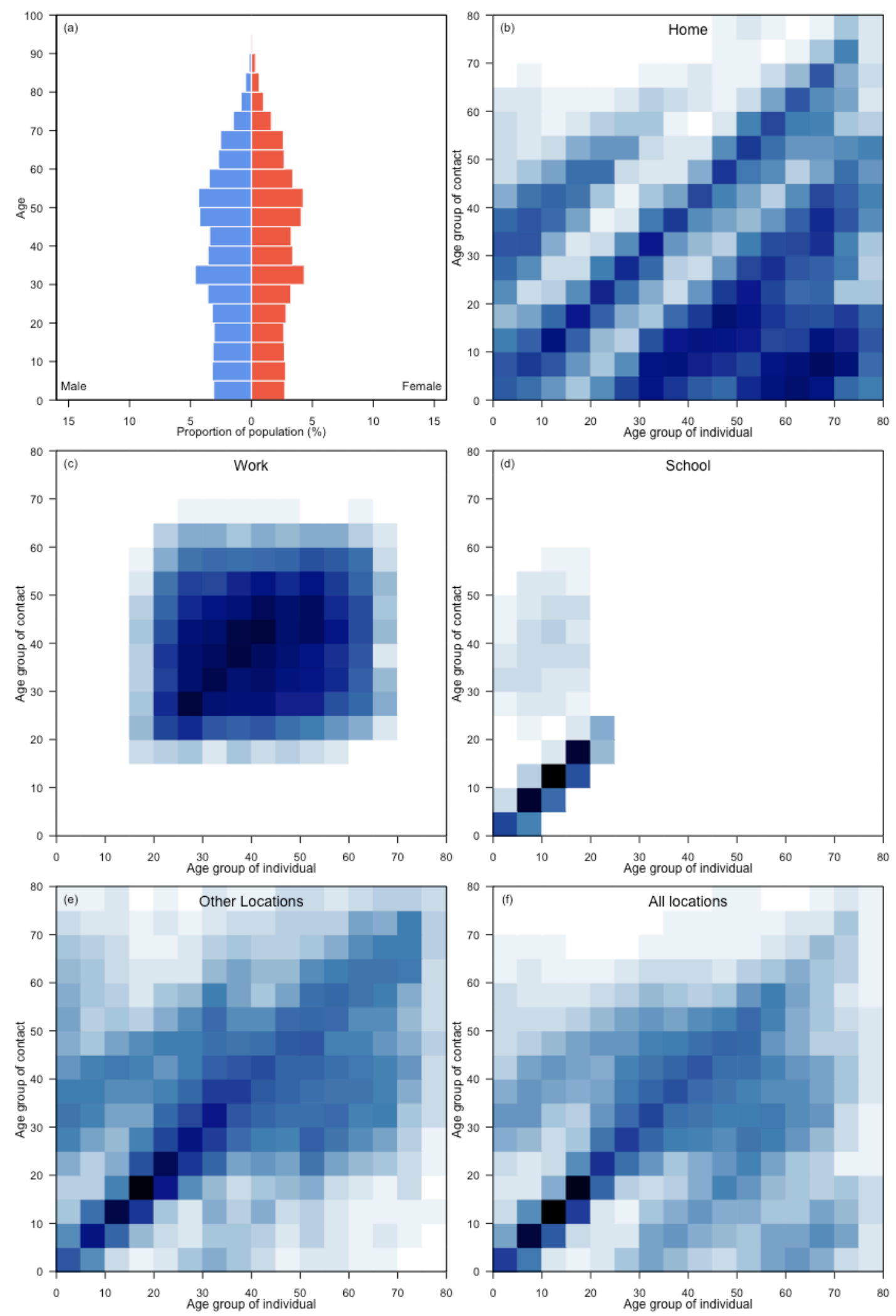


\section{China, Hong Kong SAR}
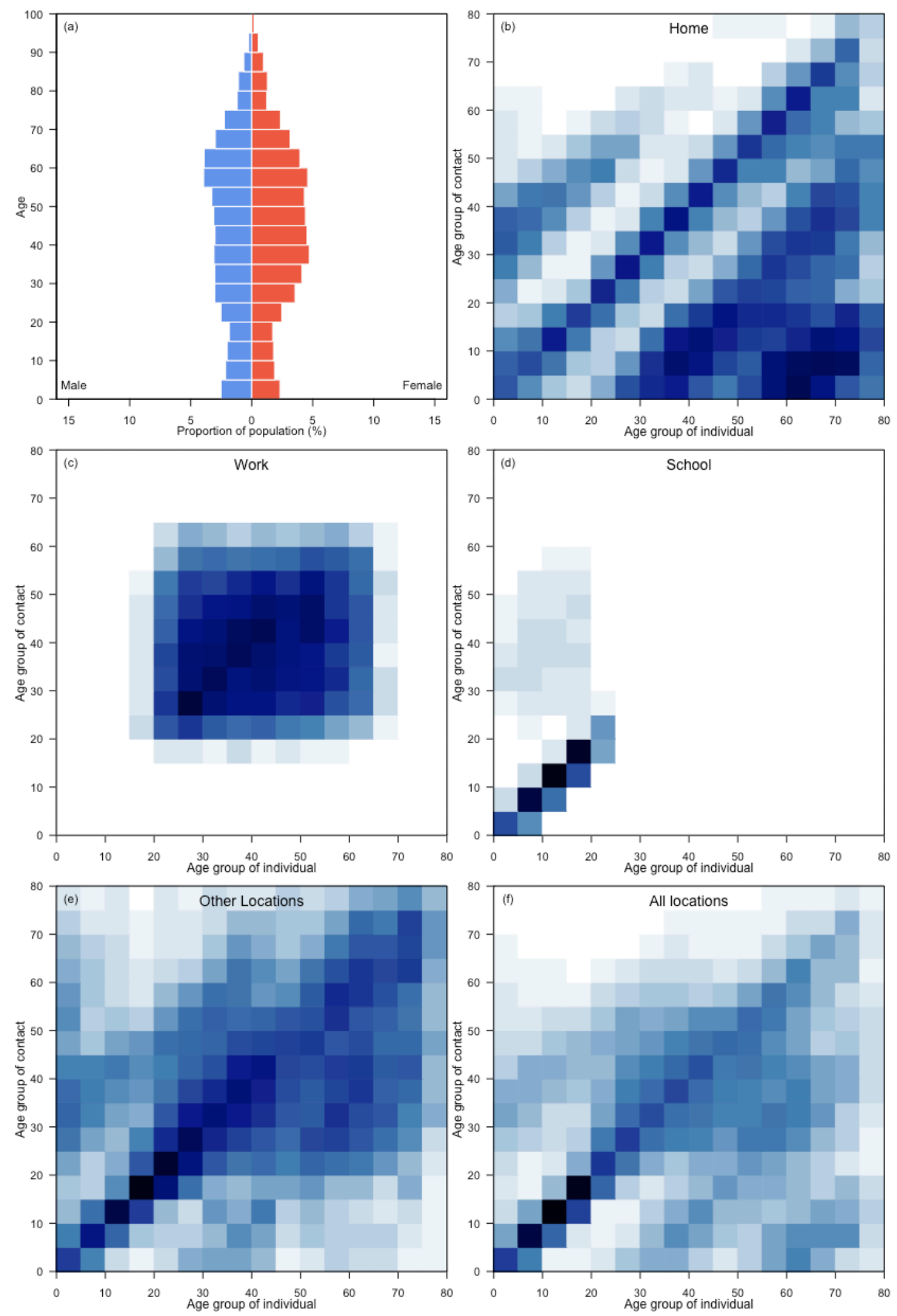


\section{China, Macao SAR}
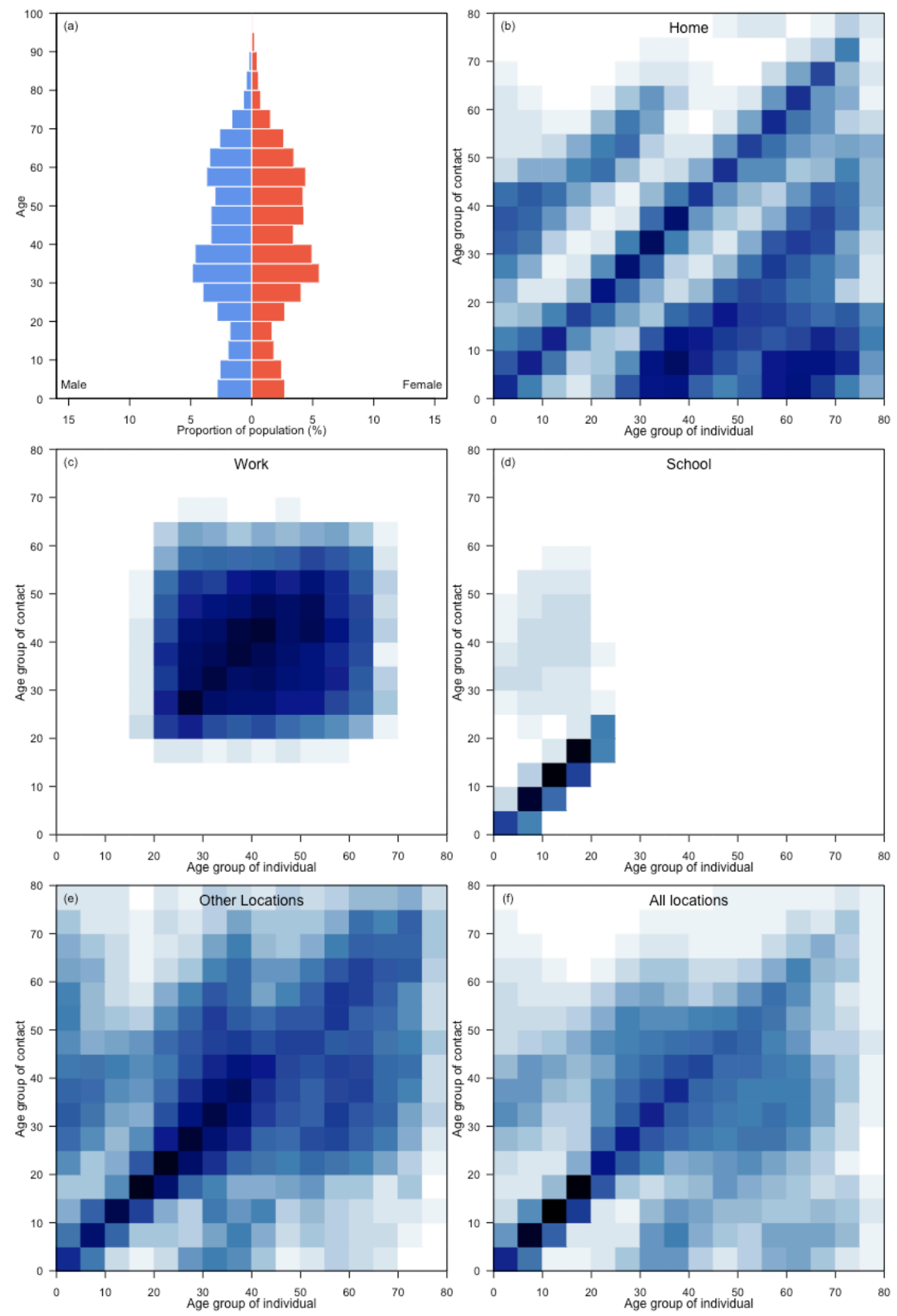


\section{Colombia}
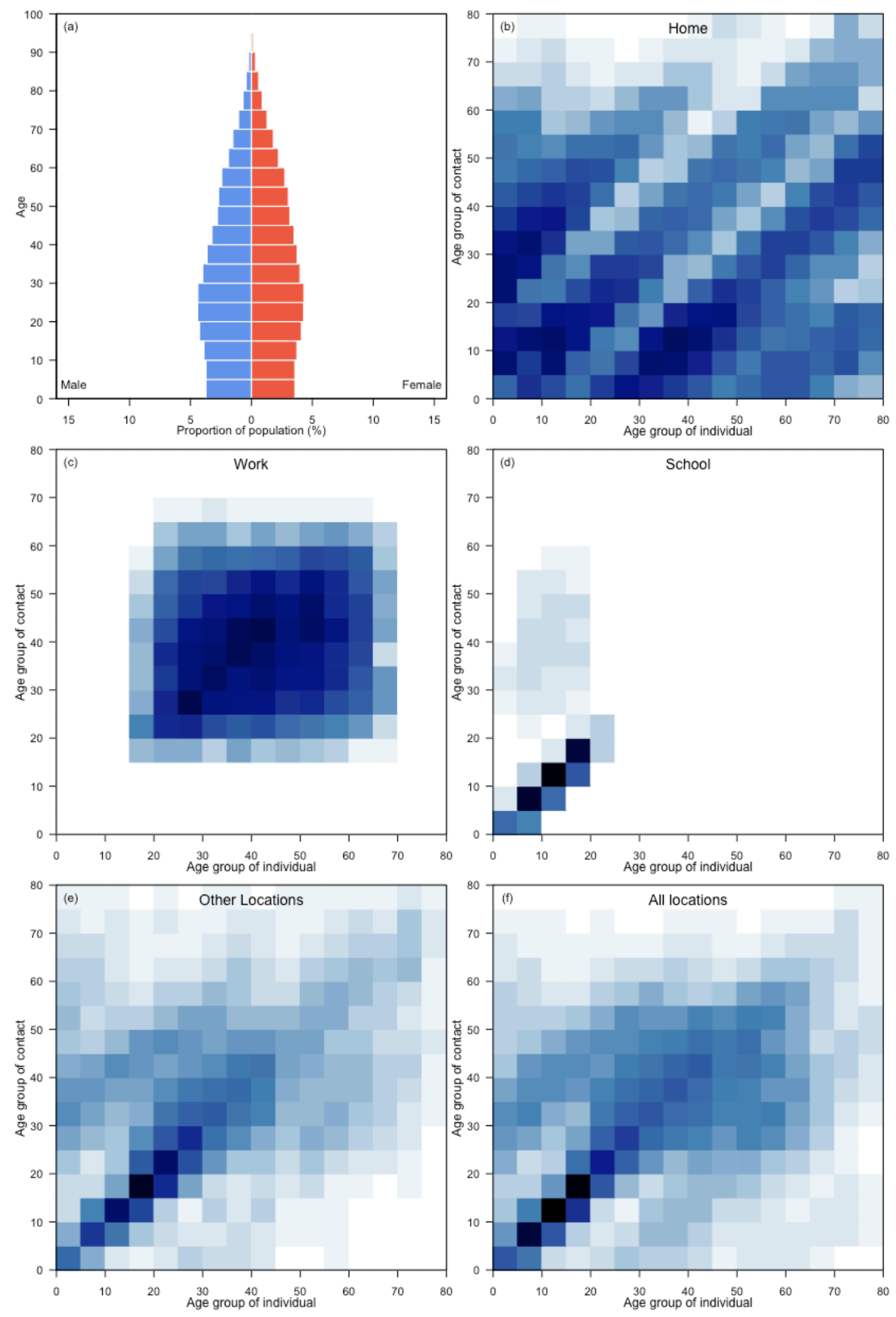


\section{Comoros}
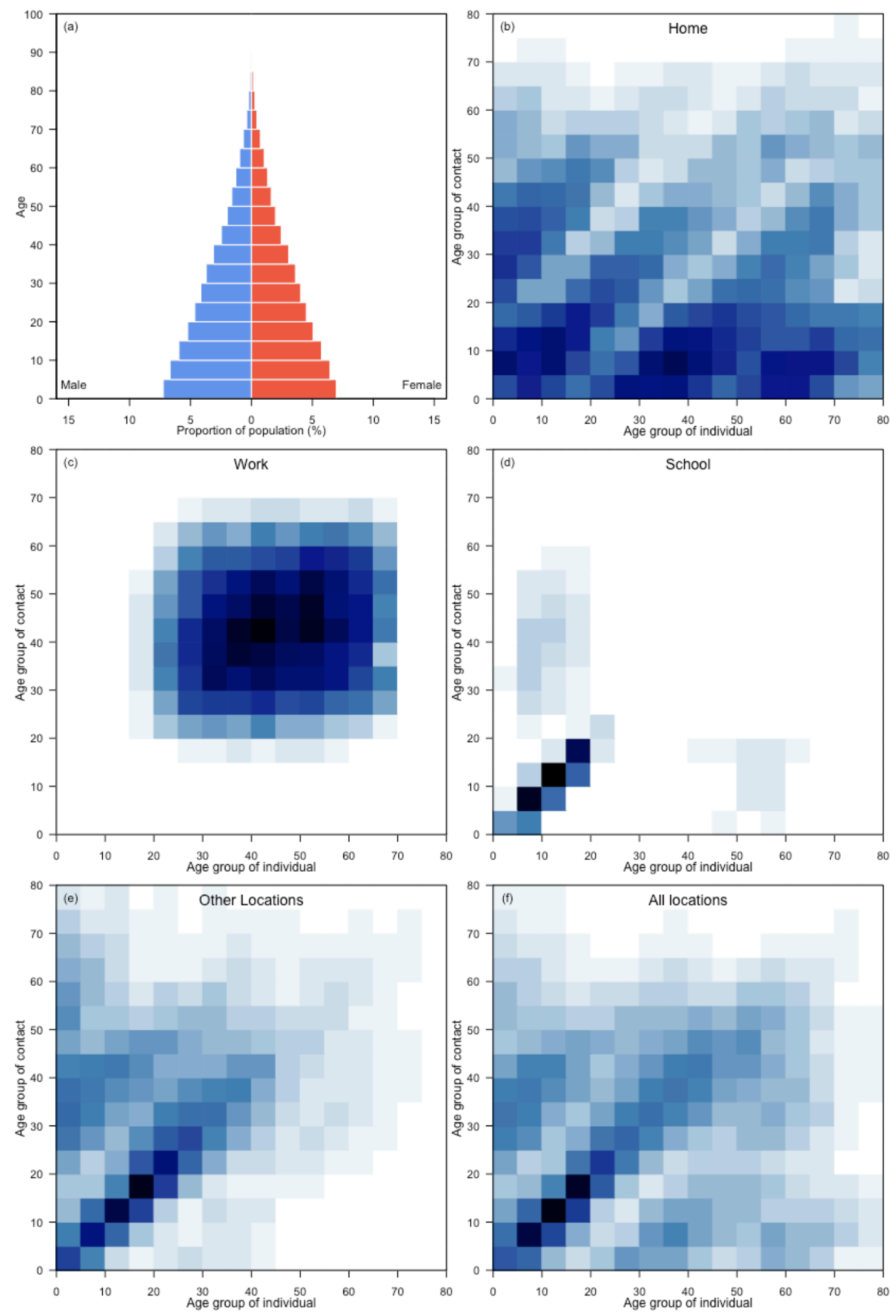


\section{Congo}
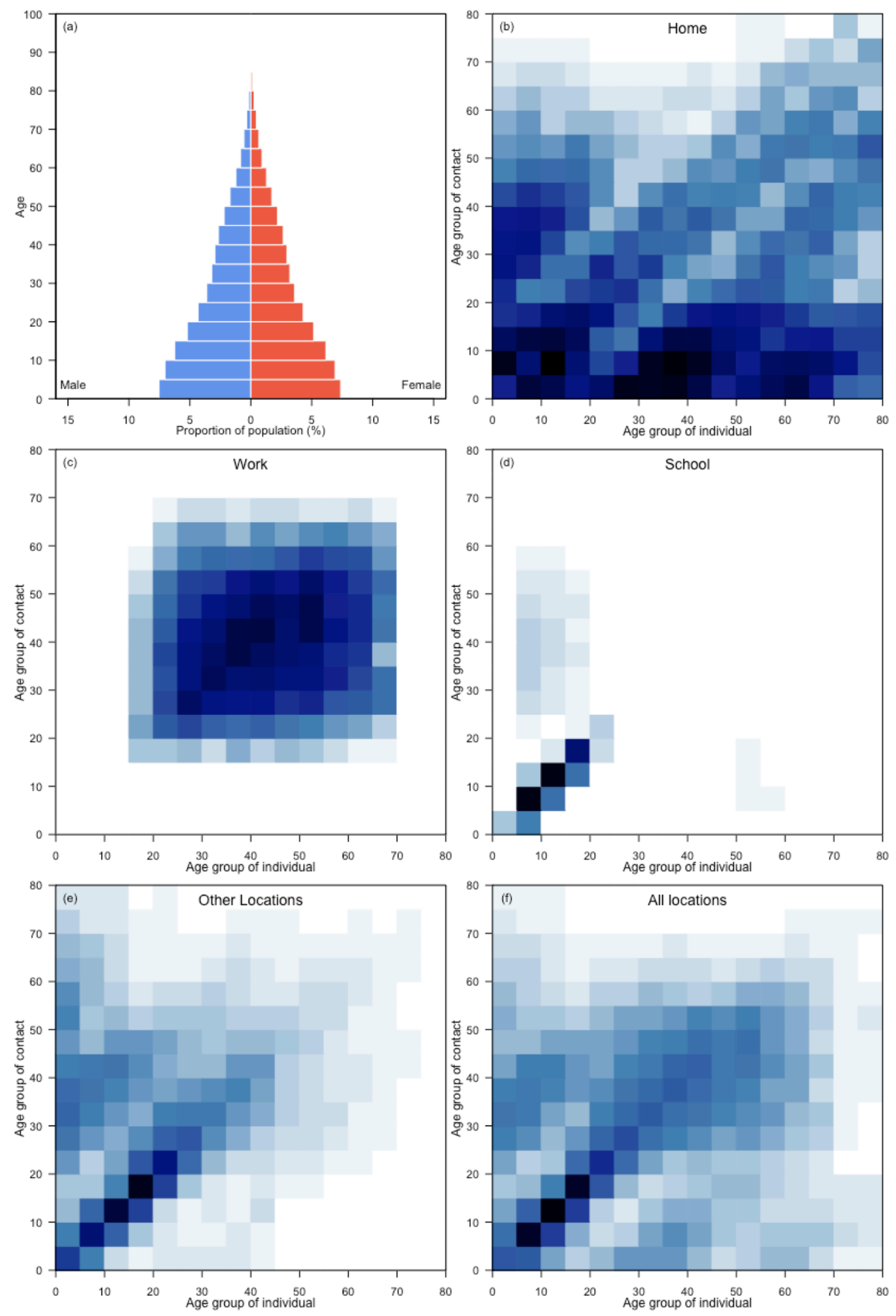
Costa Rica
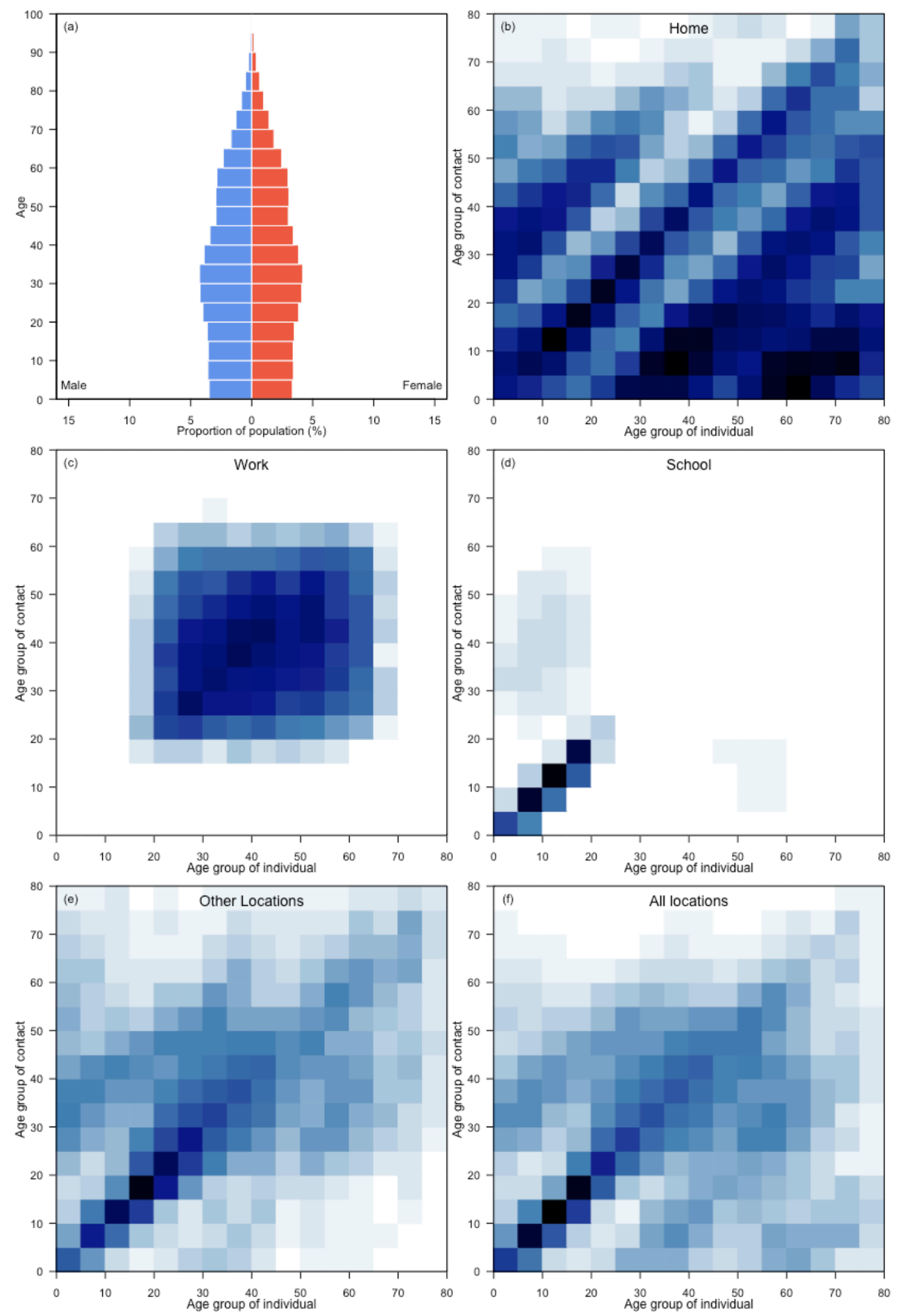


\section{Côte d'Ivoire}
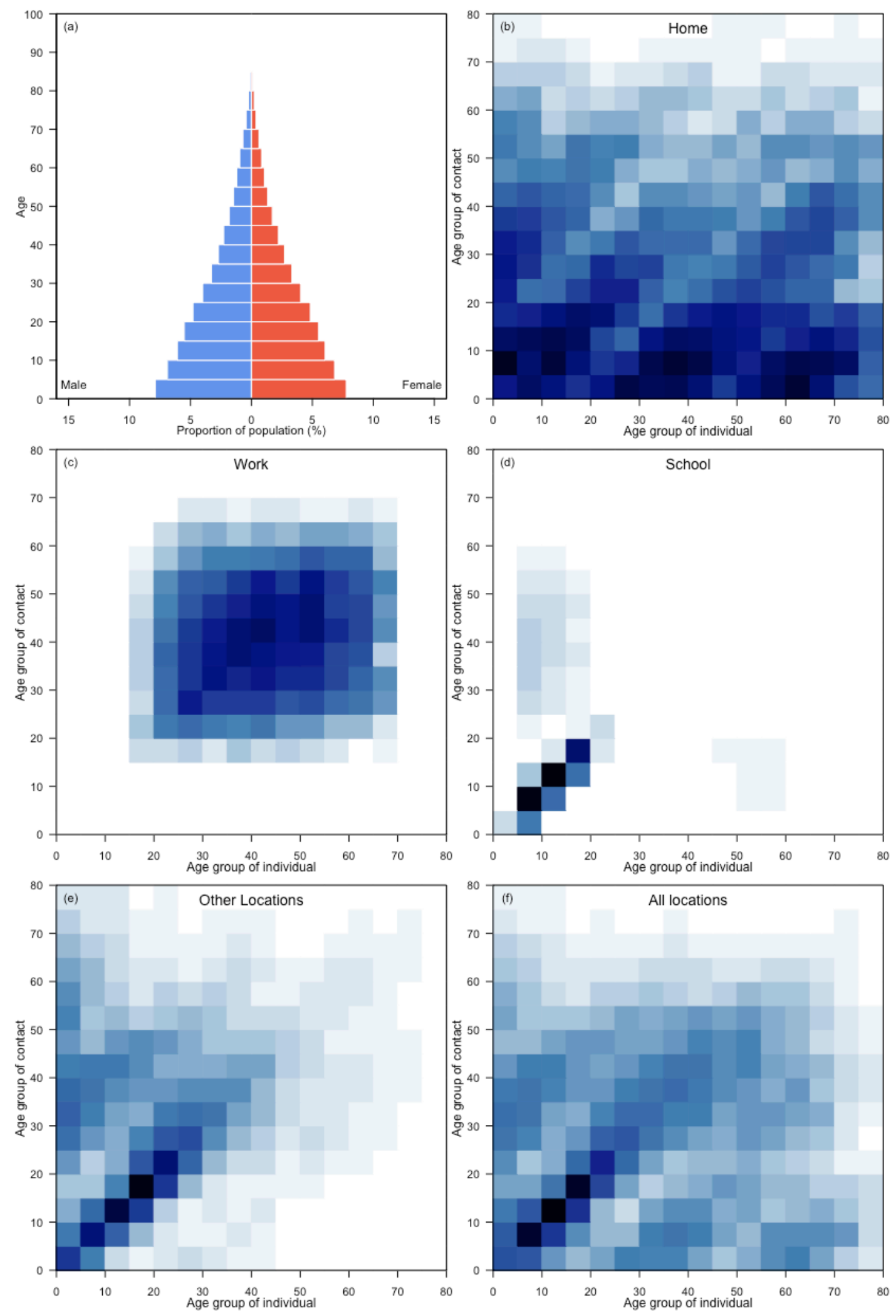


\section{Croatia}
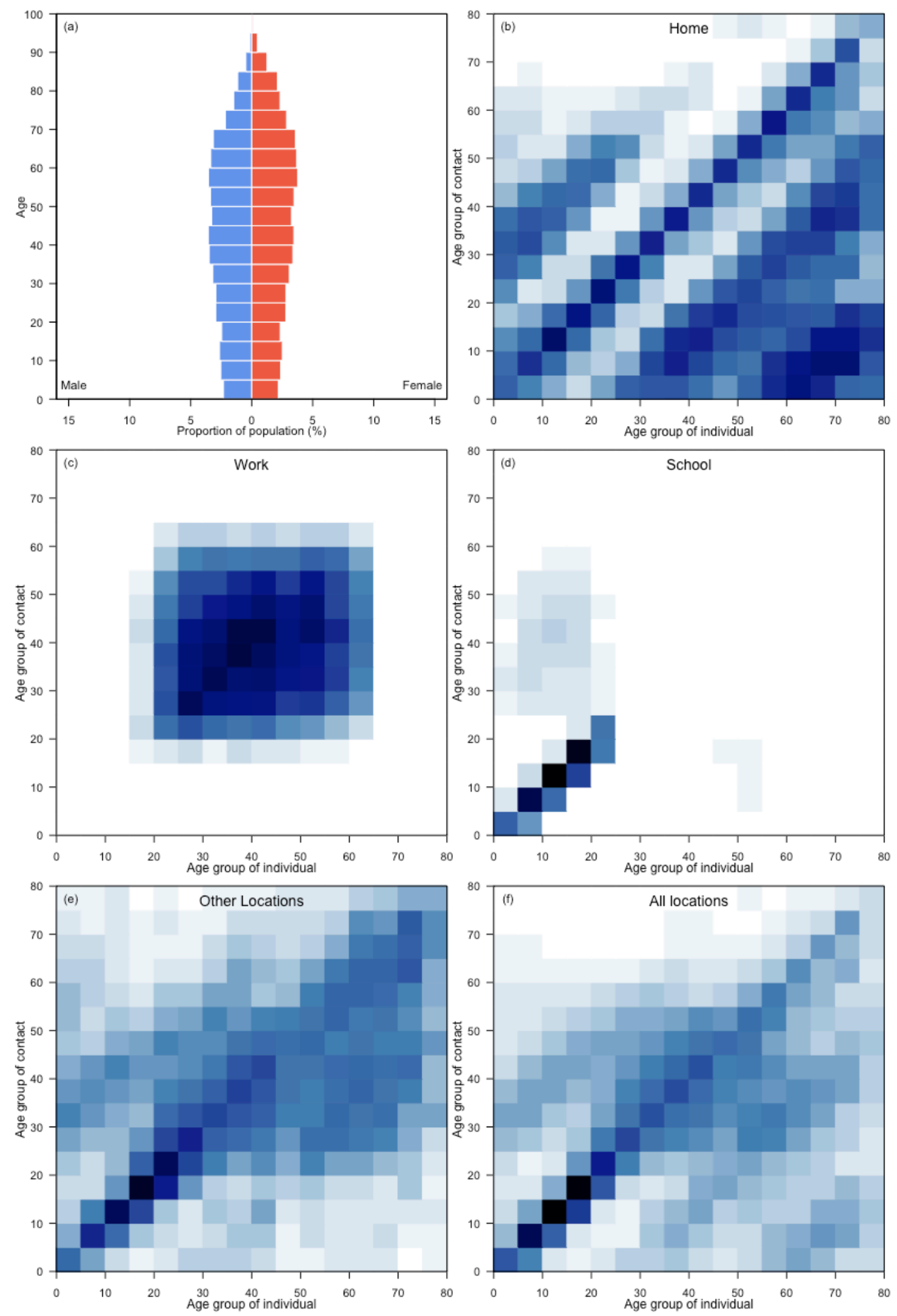


\section{Cuba}
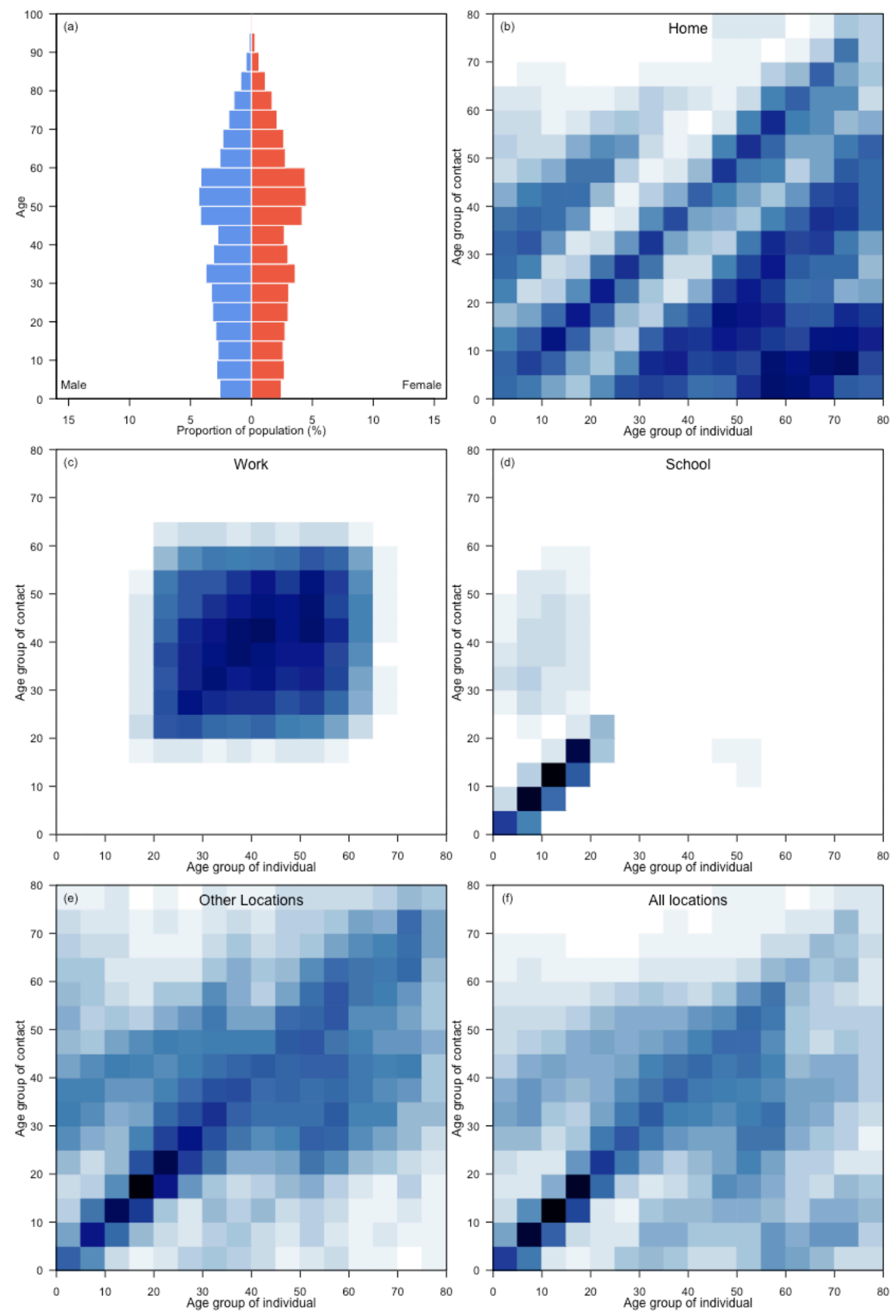


\section{Cyprus}
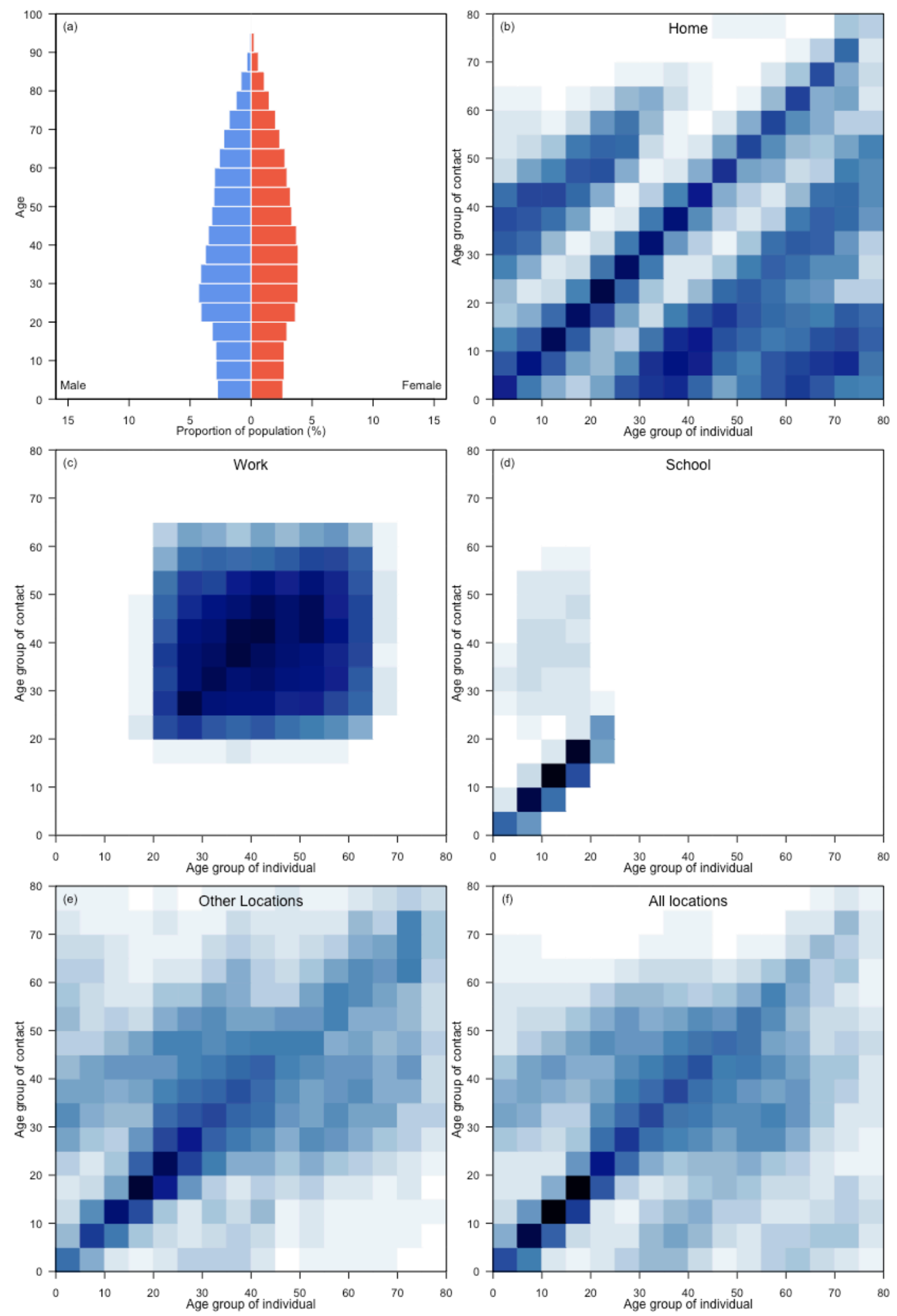


\section{Czechia}
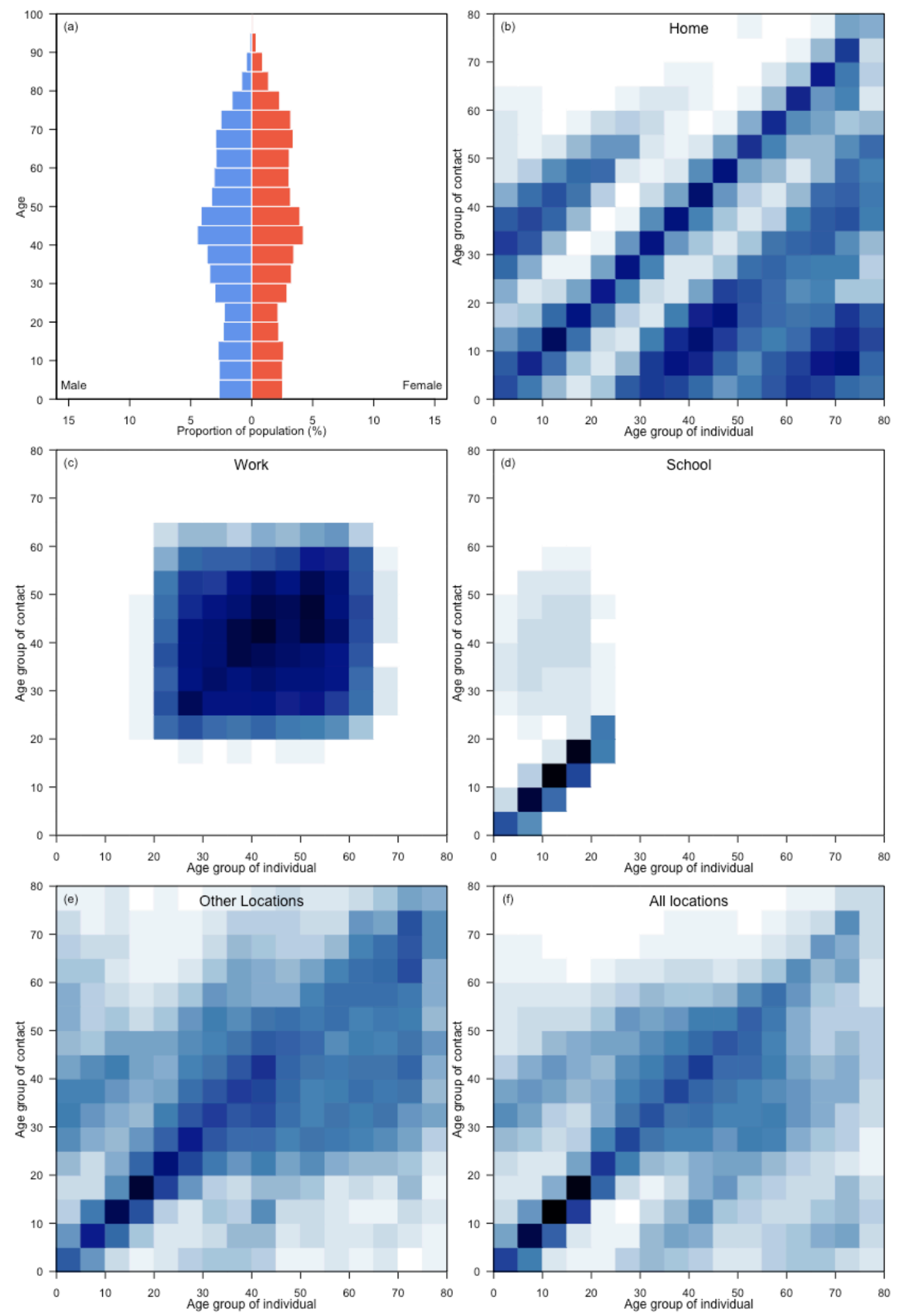
Dem. People's Republic of Korea
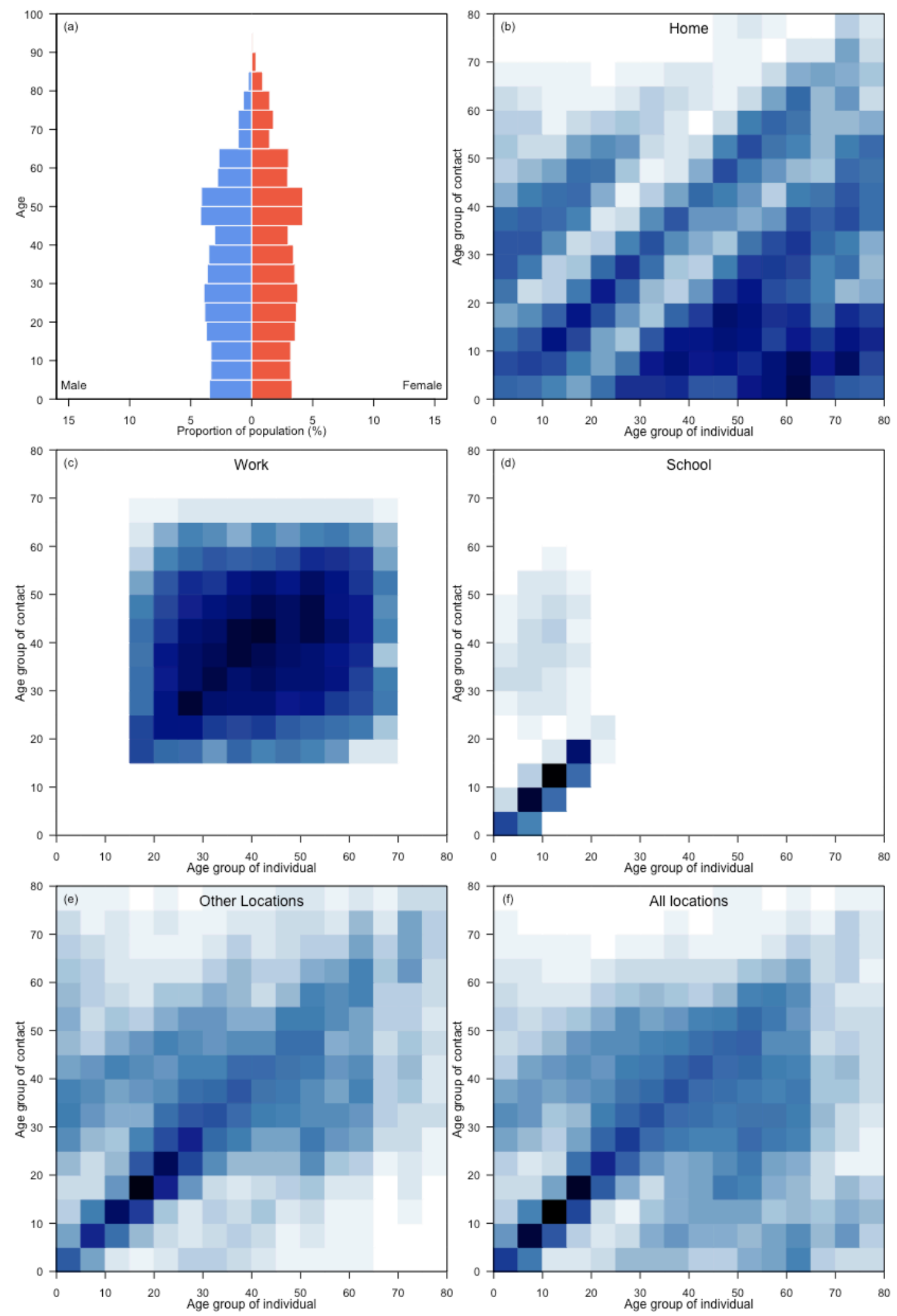


\section{Democratic Republic of the Congo}
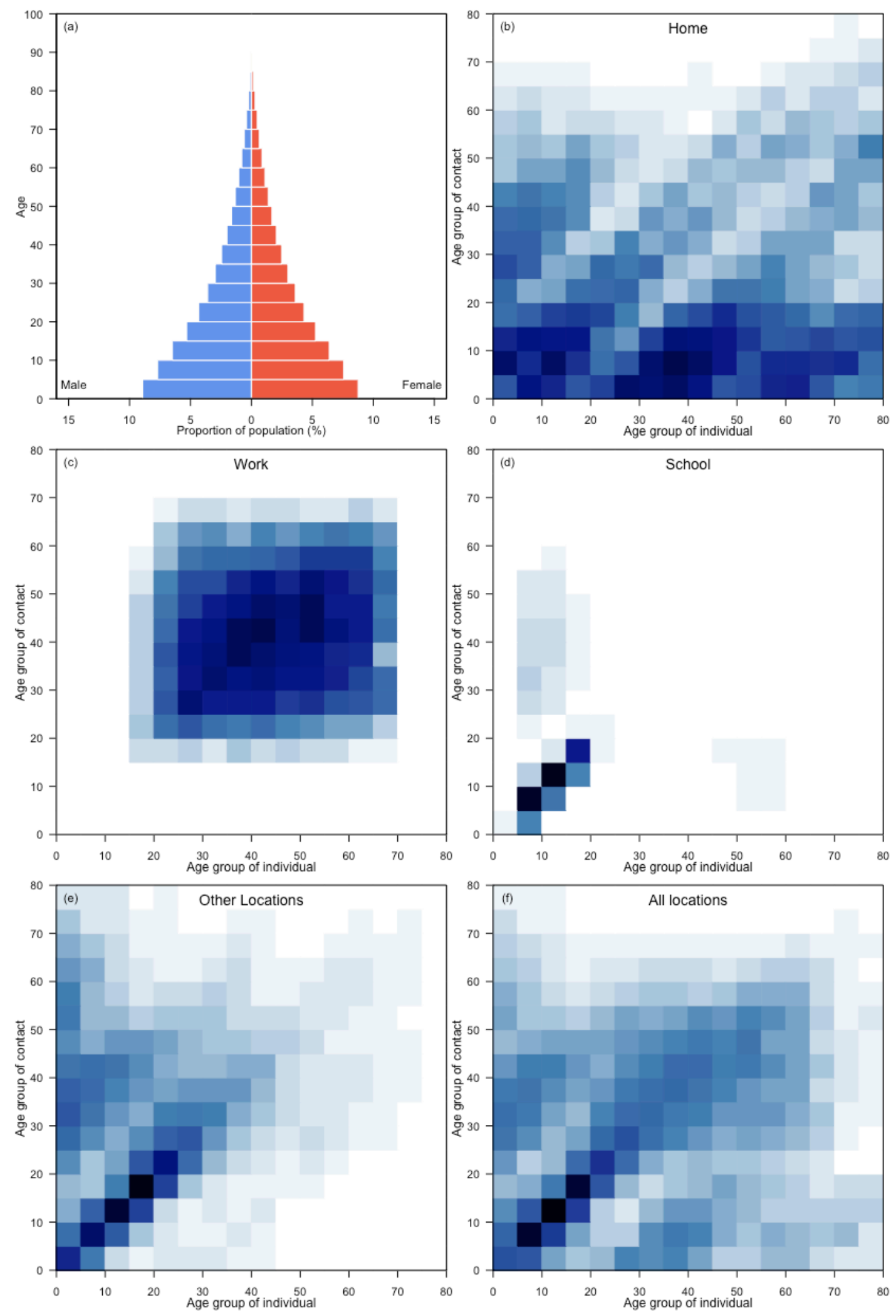


\section{Denmark}
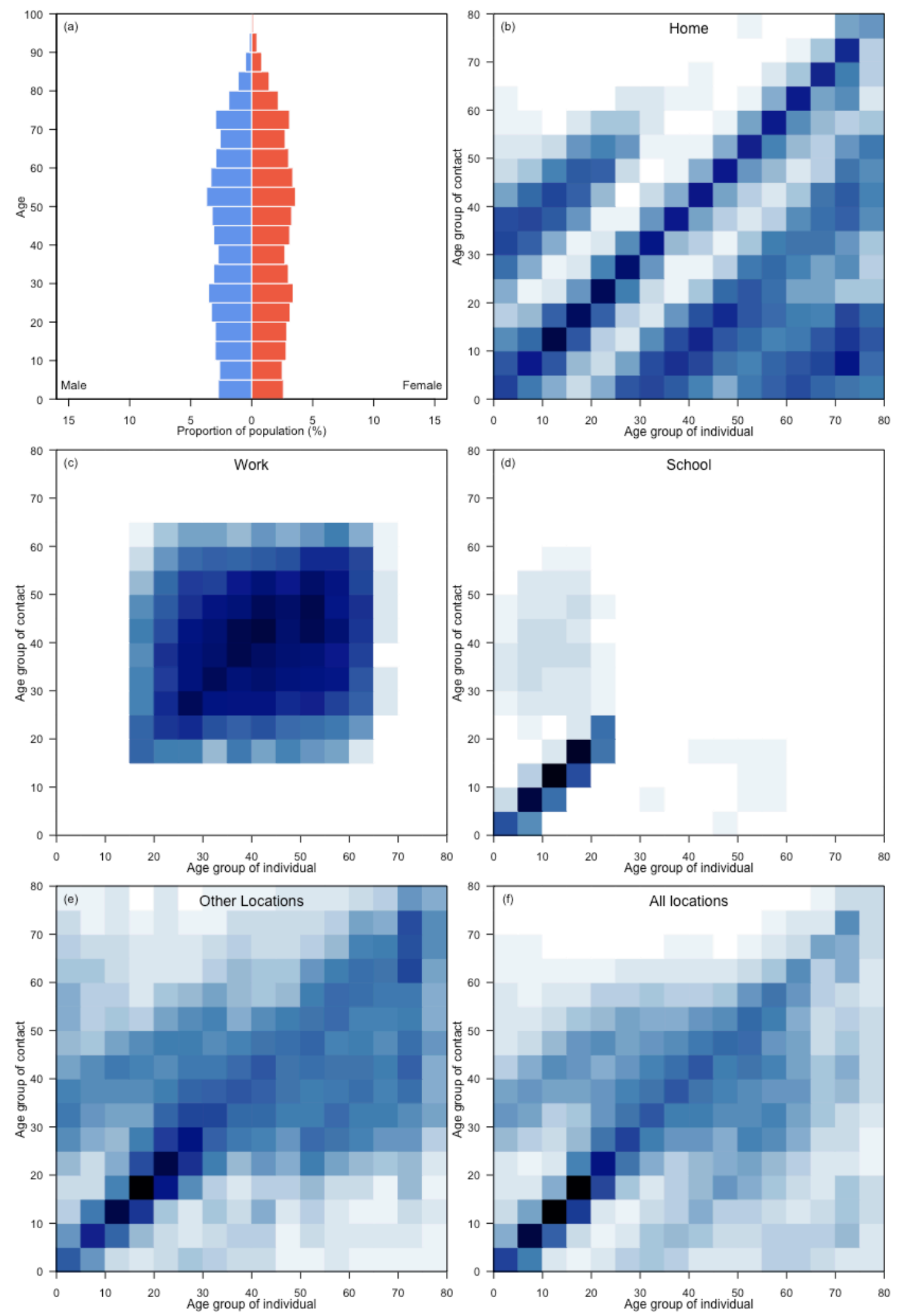


\section{Djibouti}
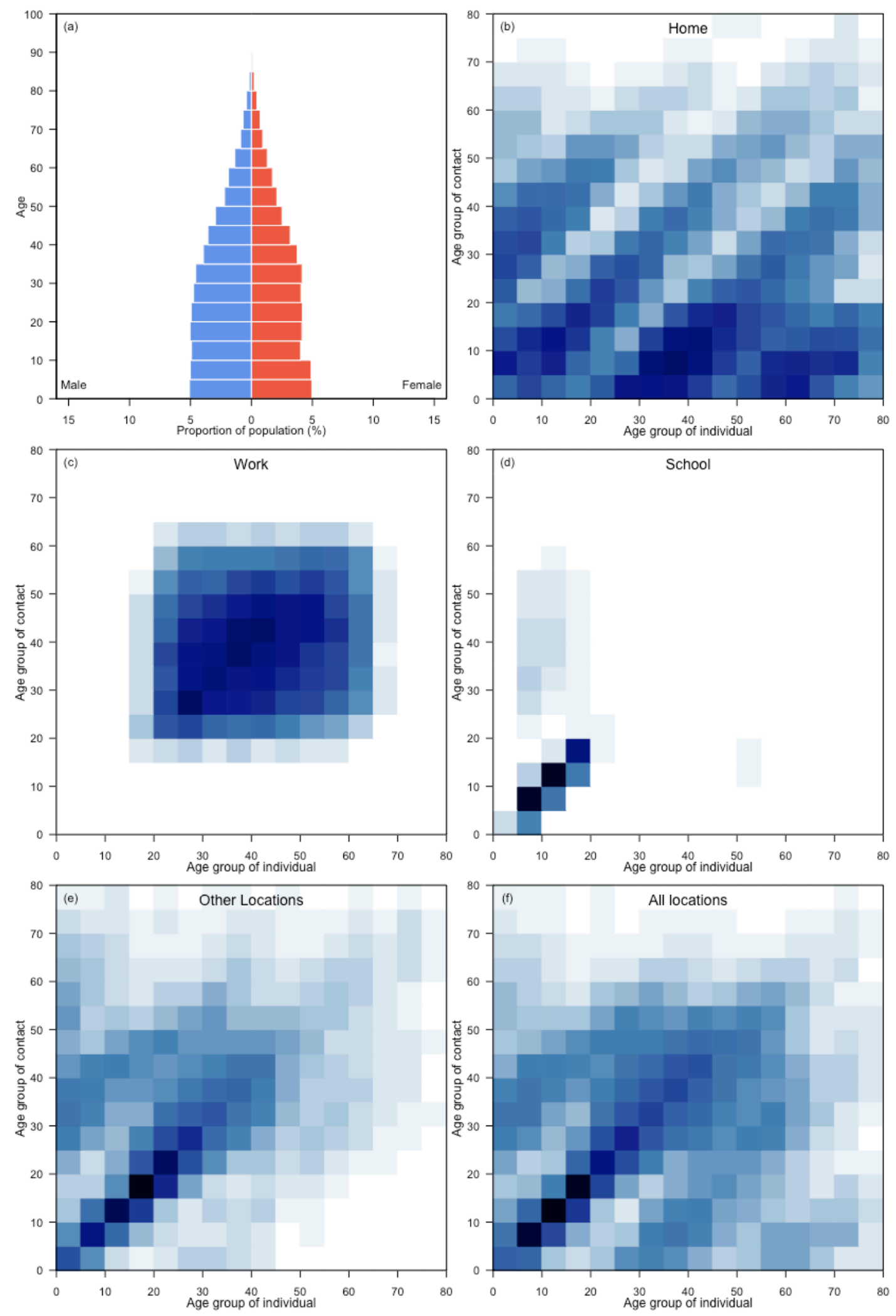
Dominican Republic
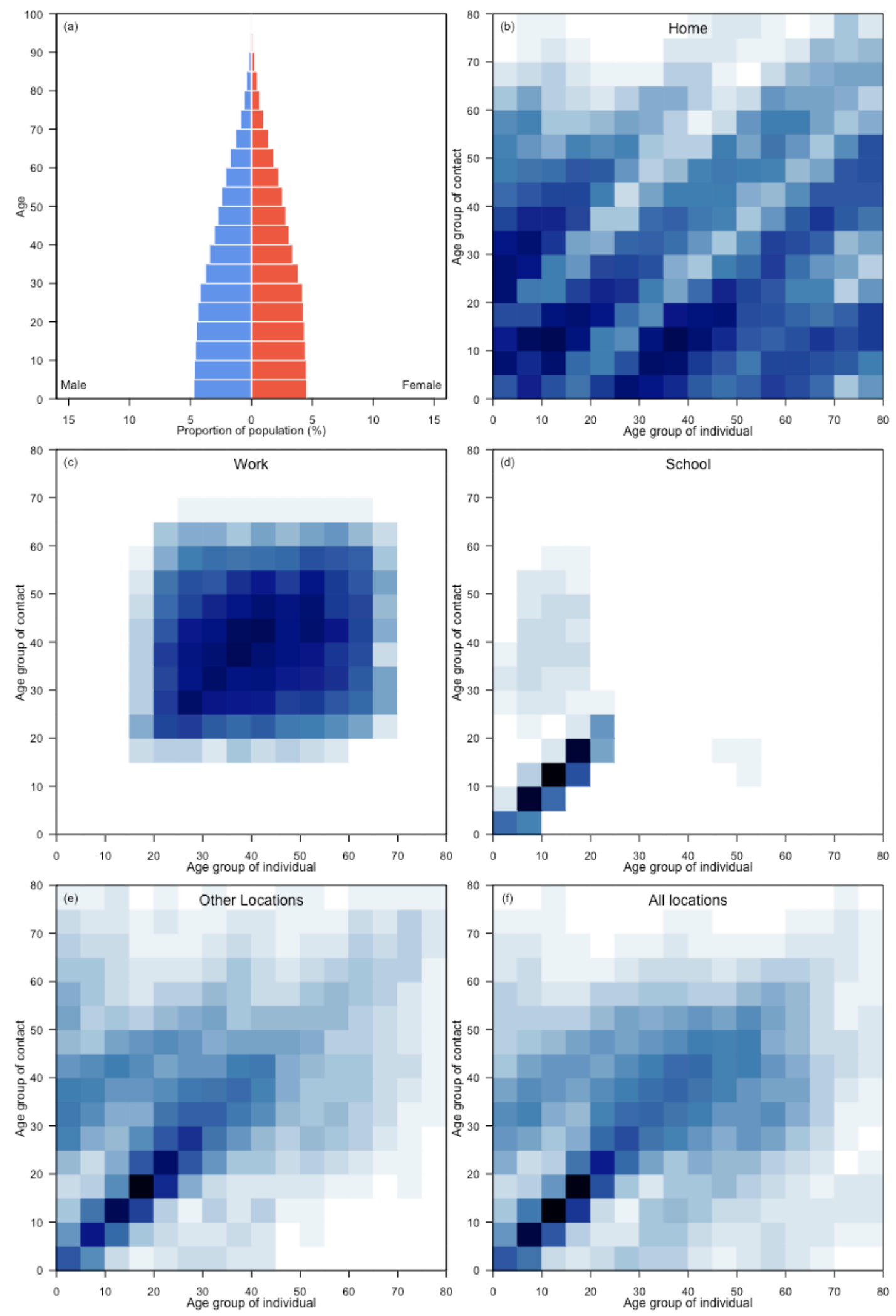


\section{Ecuador}
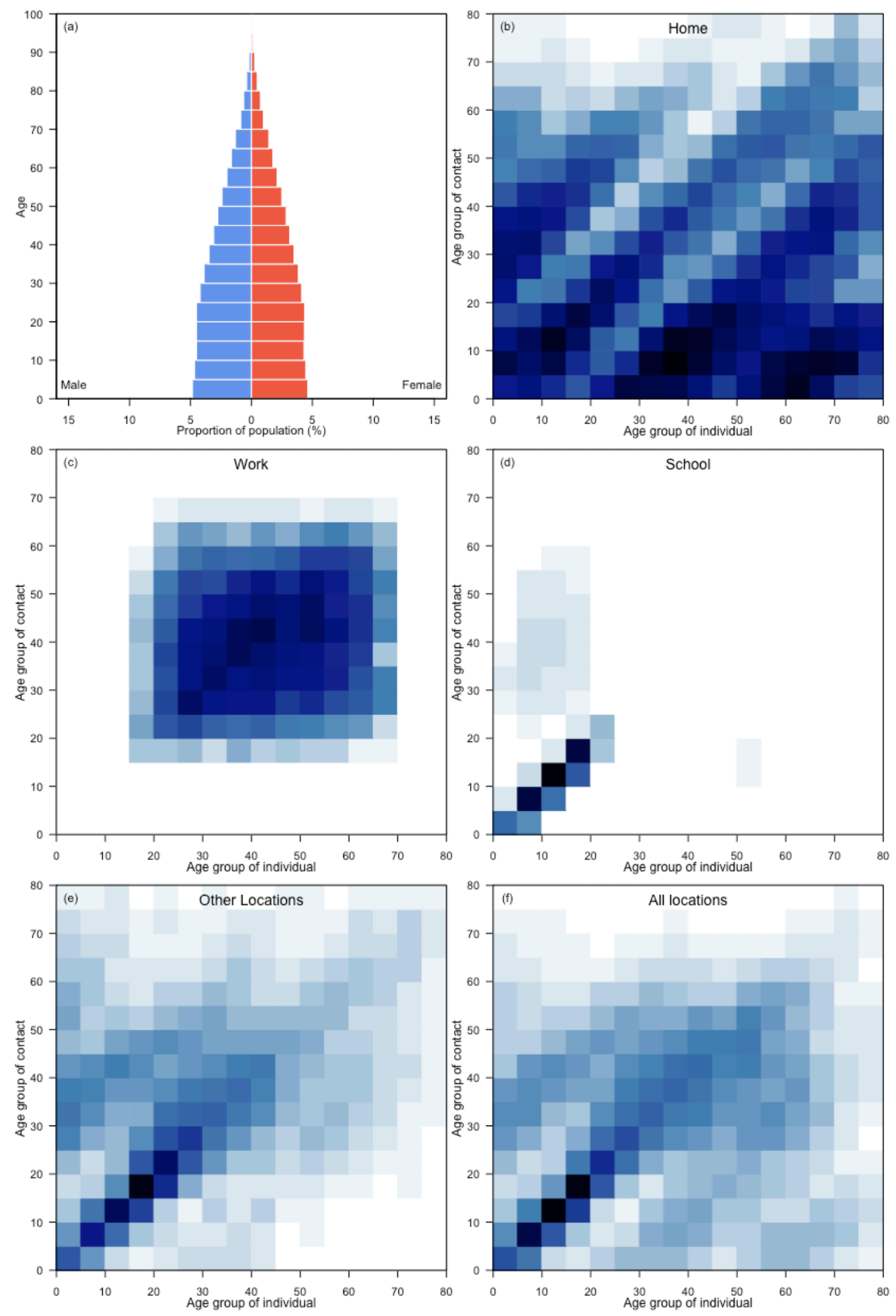


\section{Egypt}
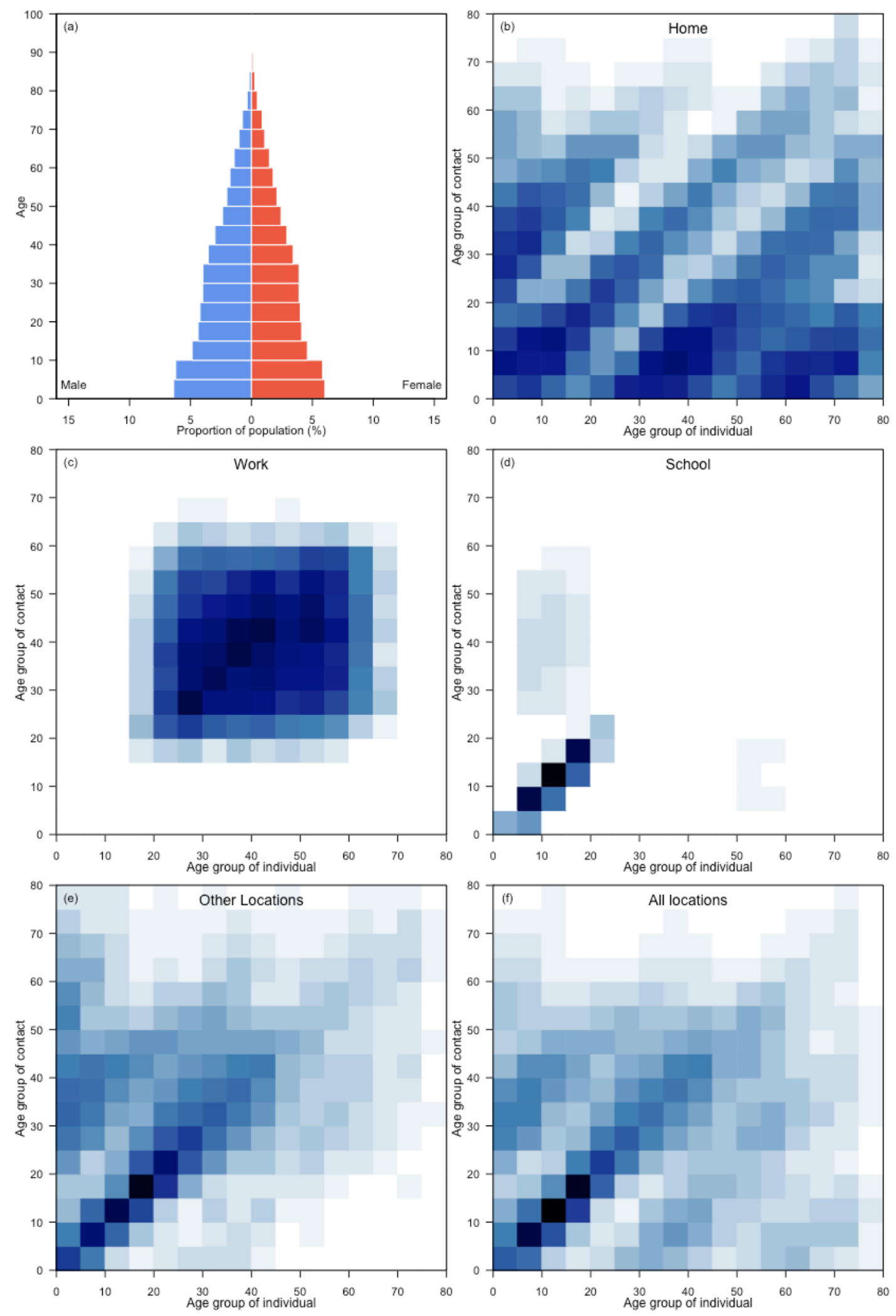


\section{EI Salvador}
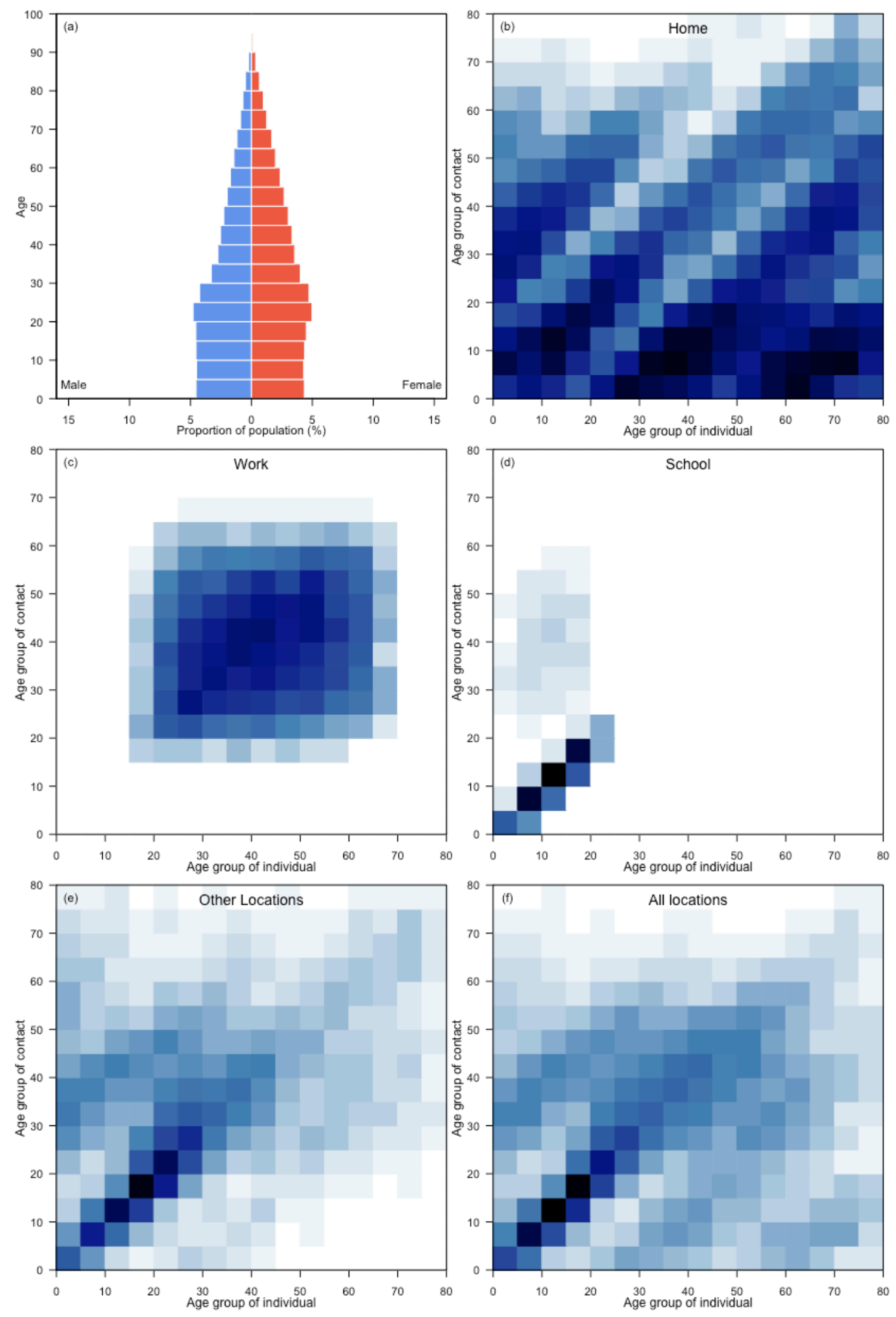


\section{Equatorial Guinea}
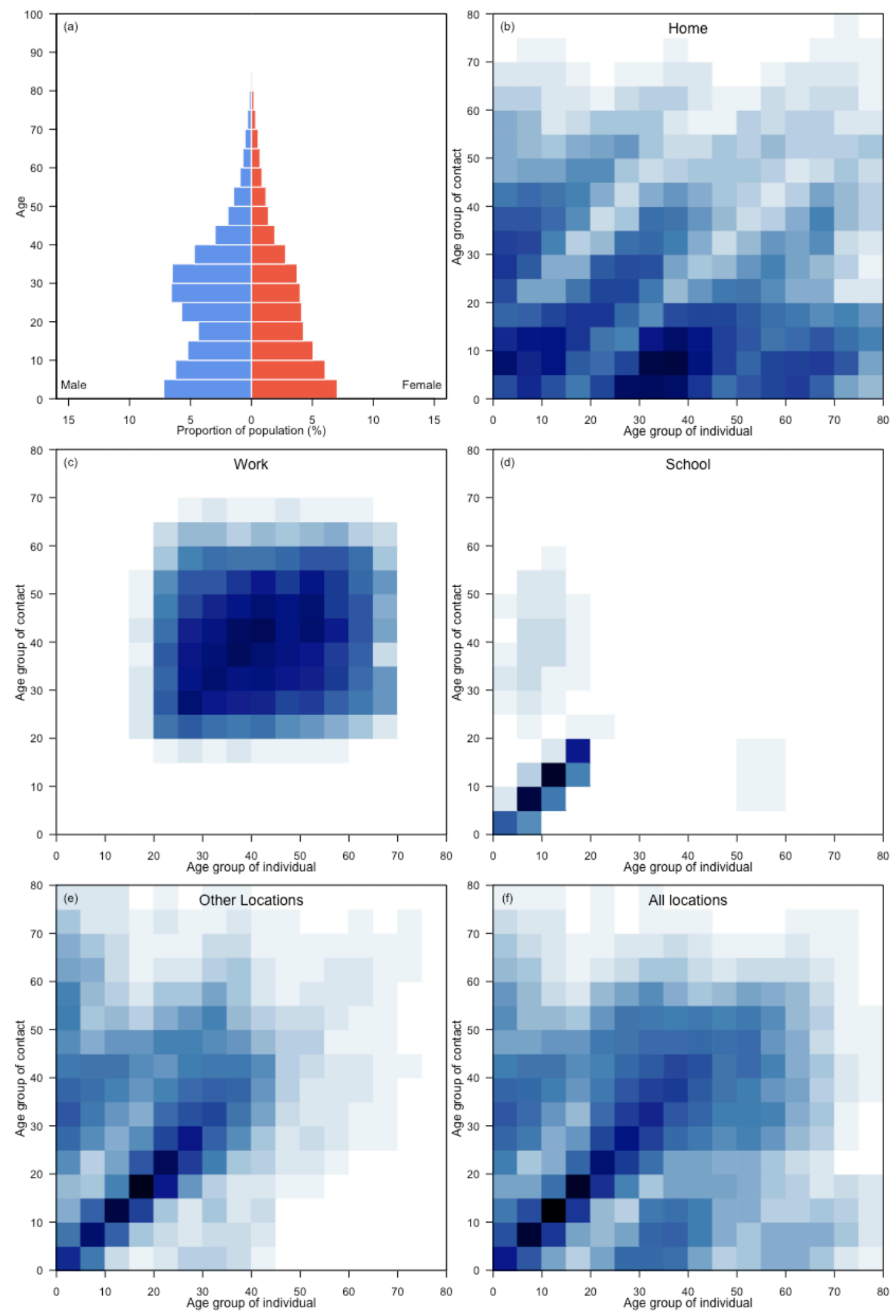


\section{Eritrea}
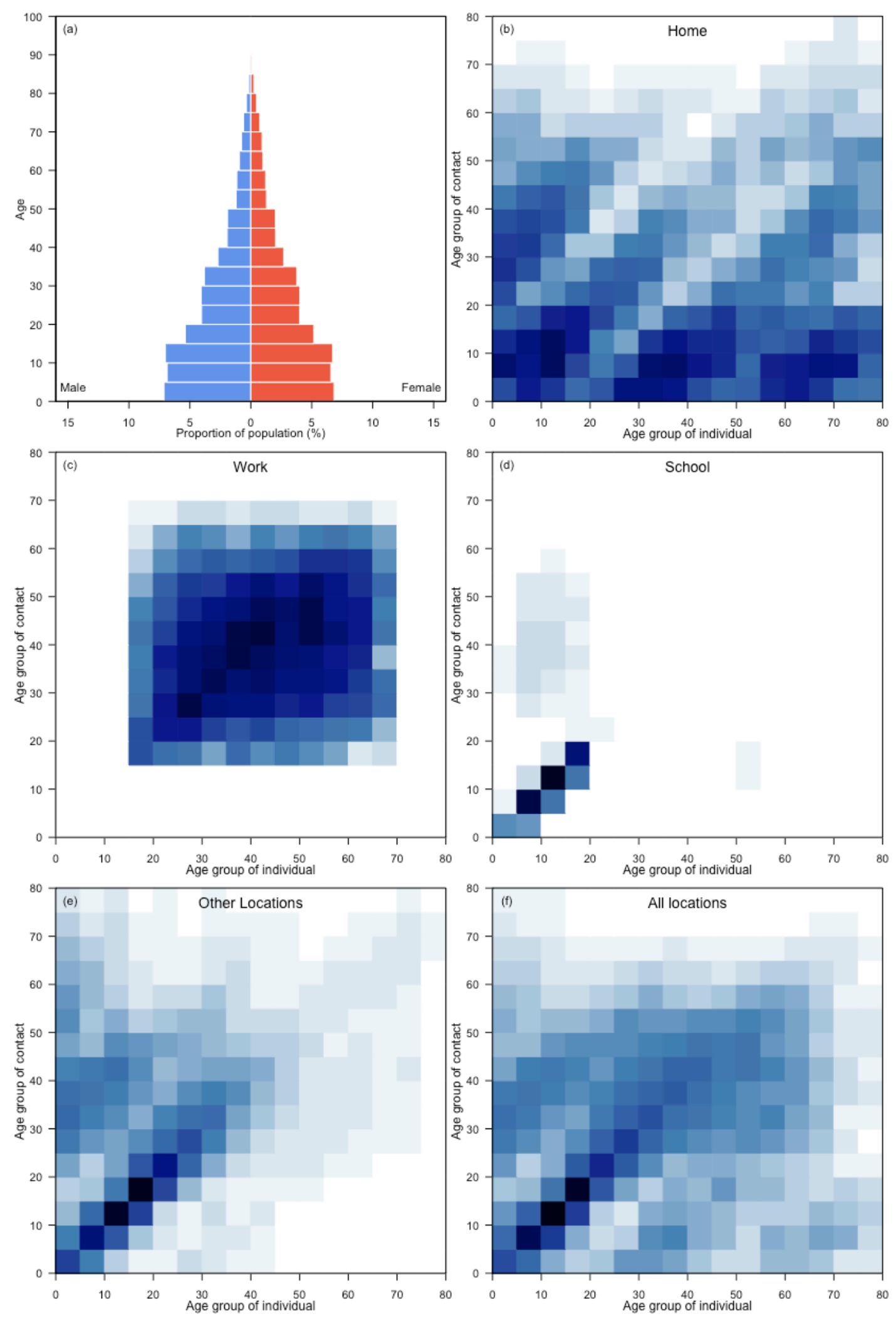


\section{Estonia}
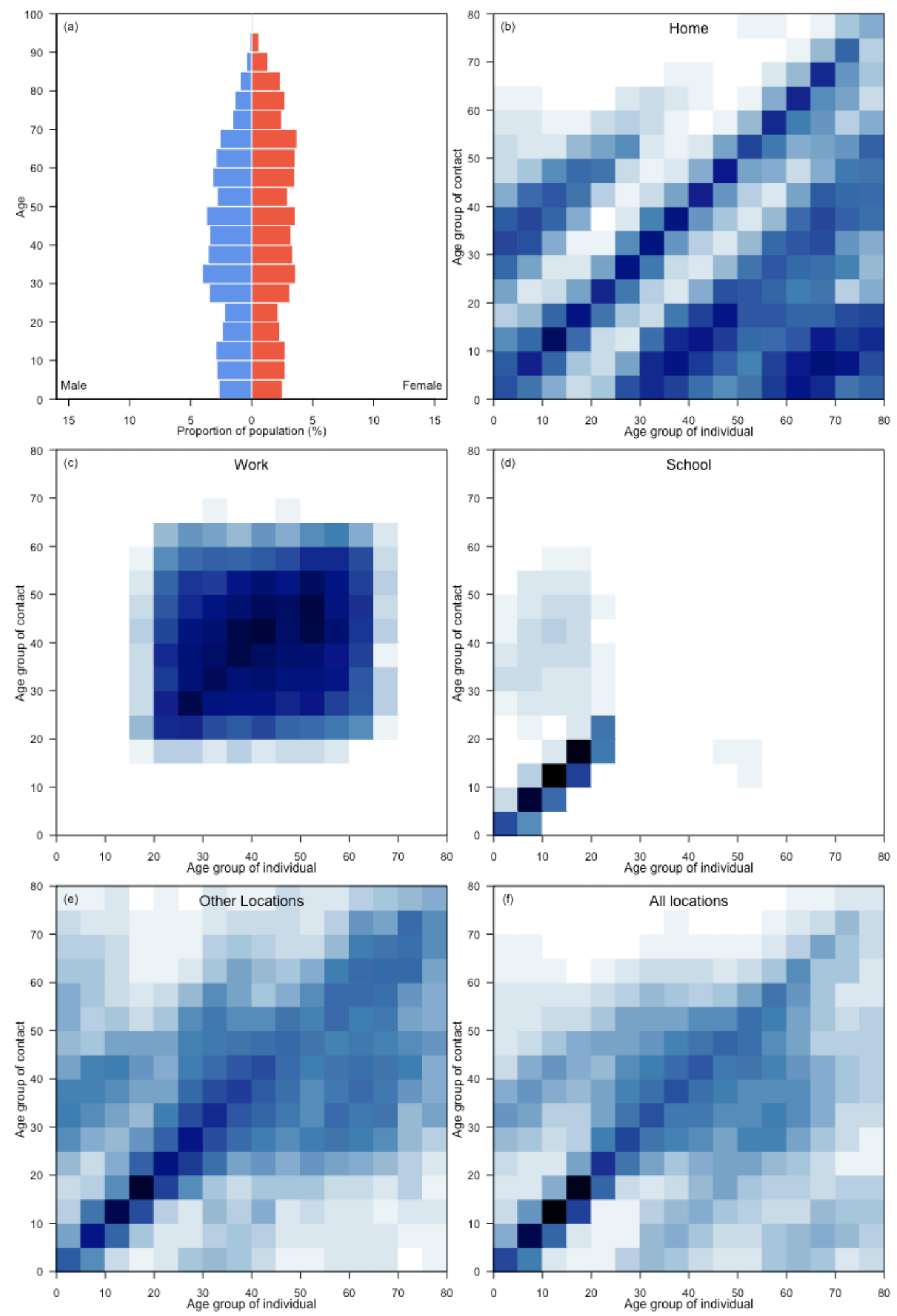


\section{Eswatini}
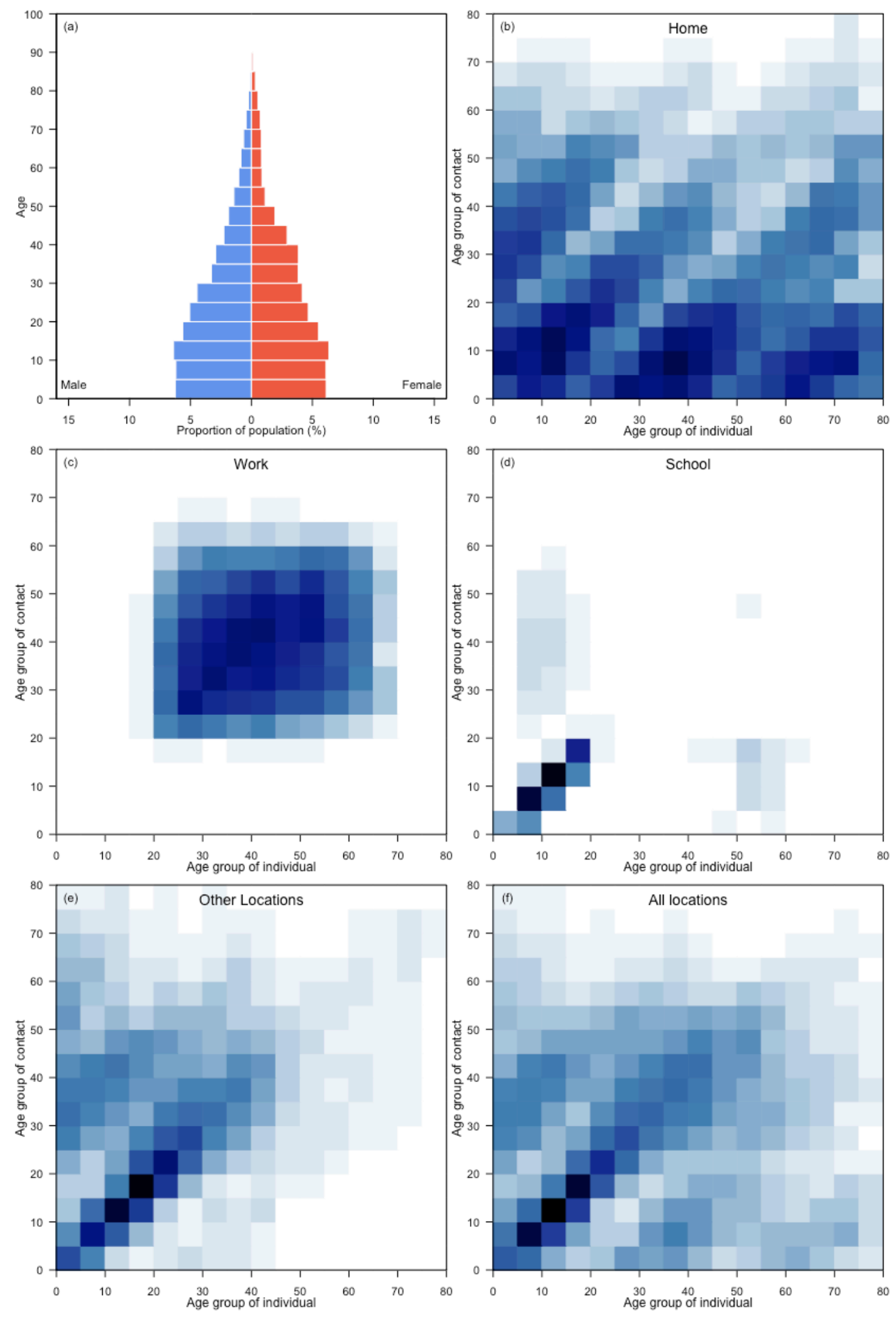
Ethiopia
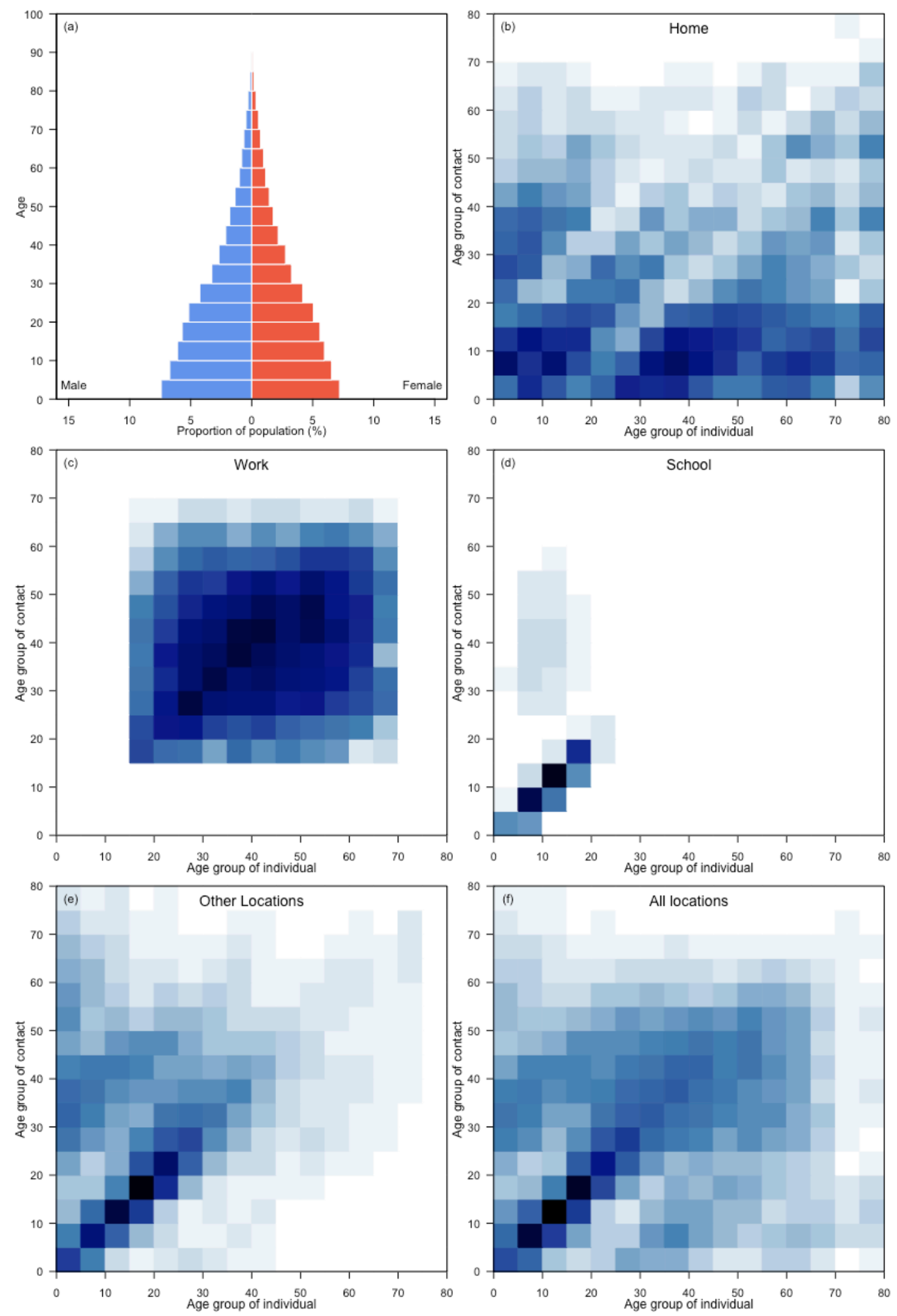
Fiji
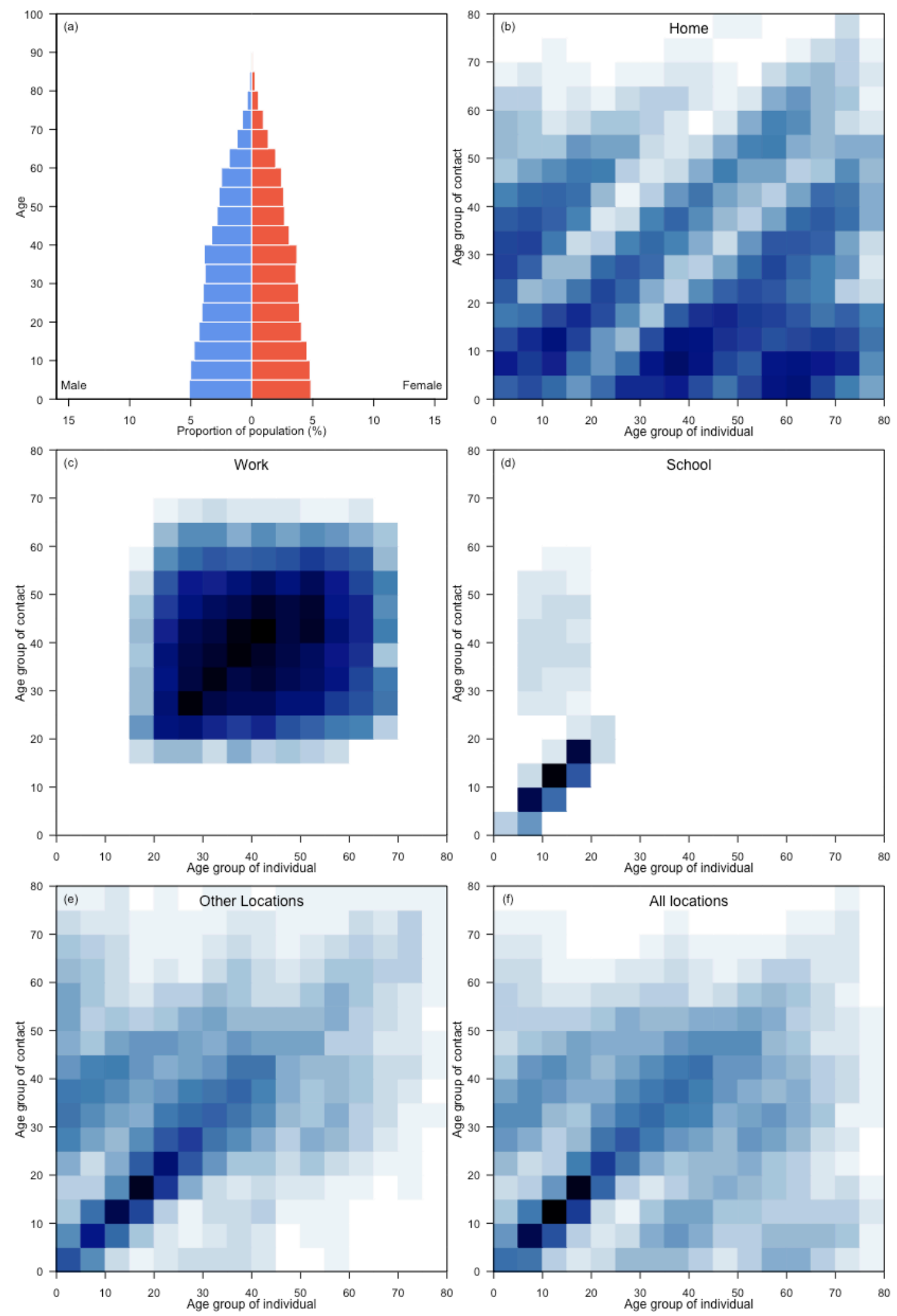


\section{Finland}
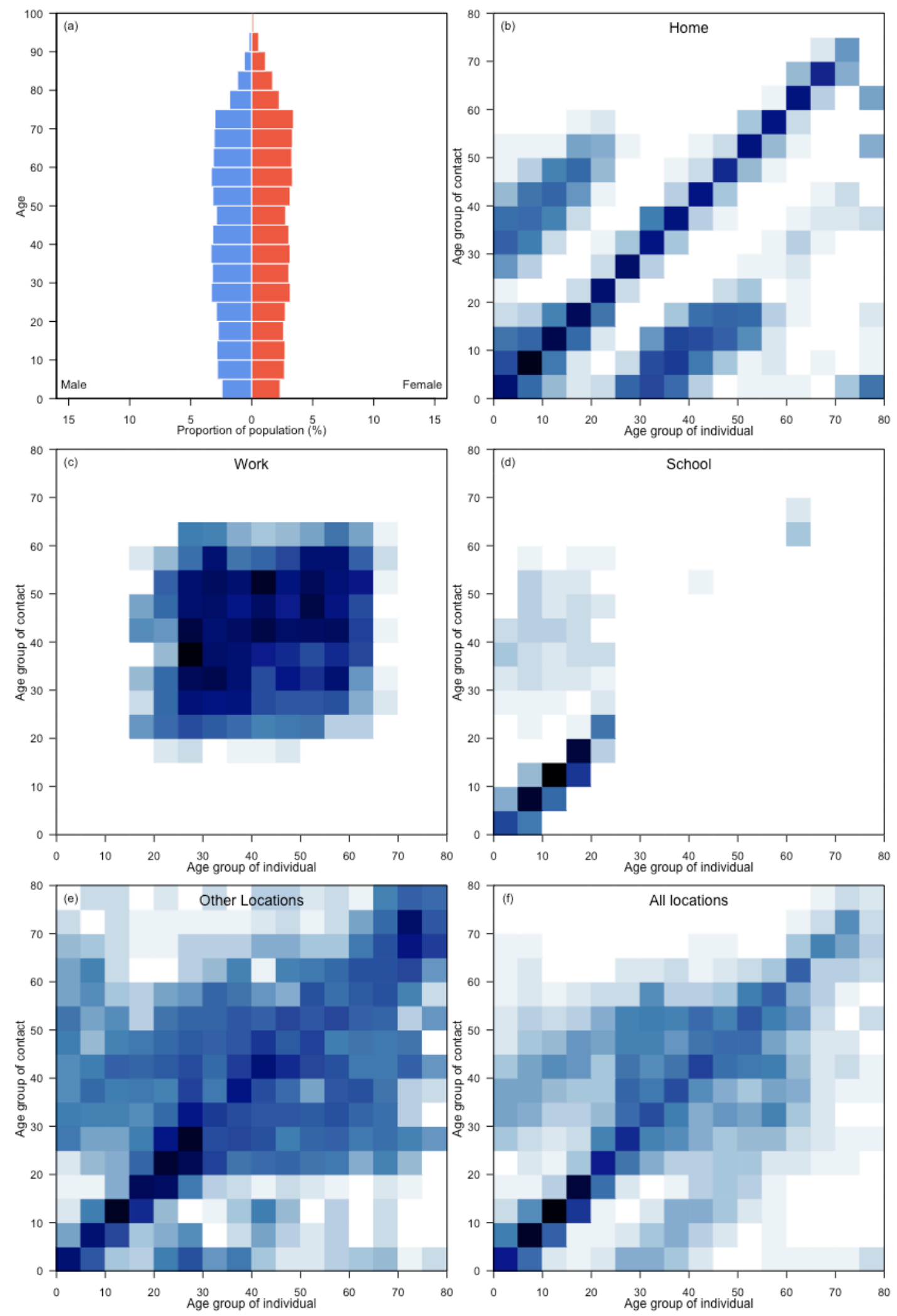


\section{France}
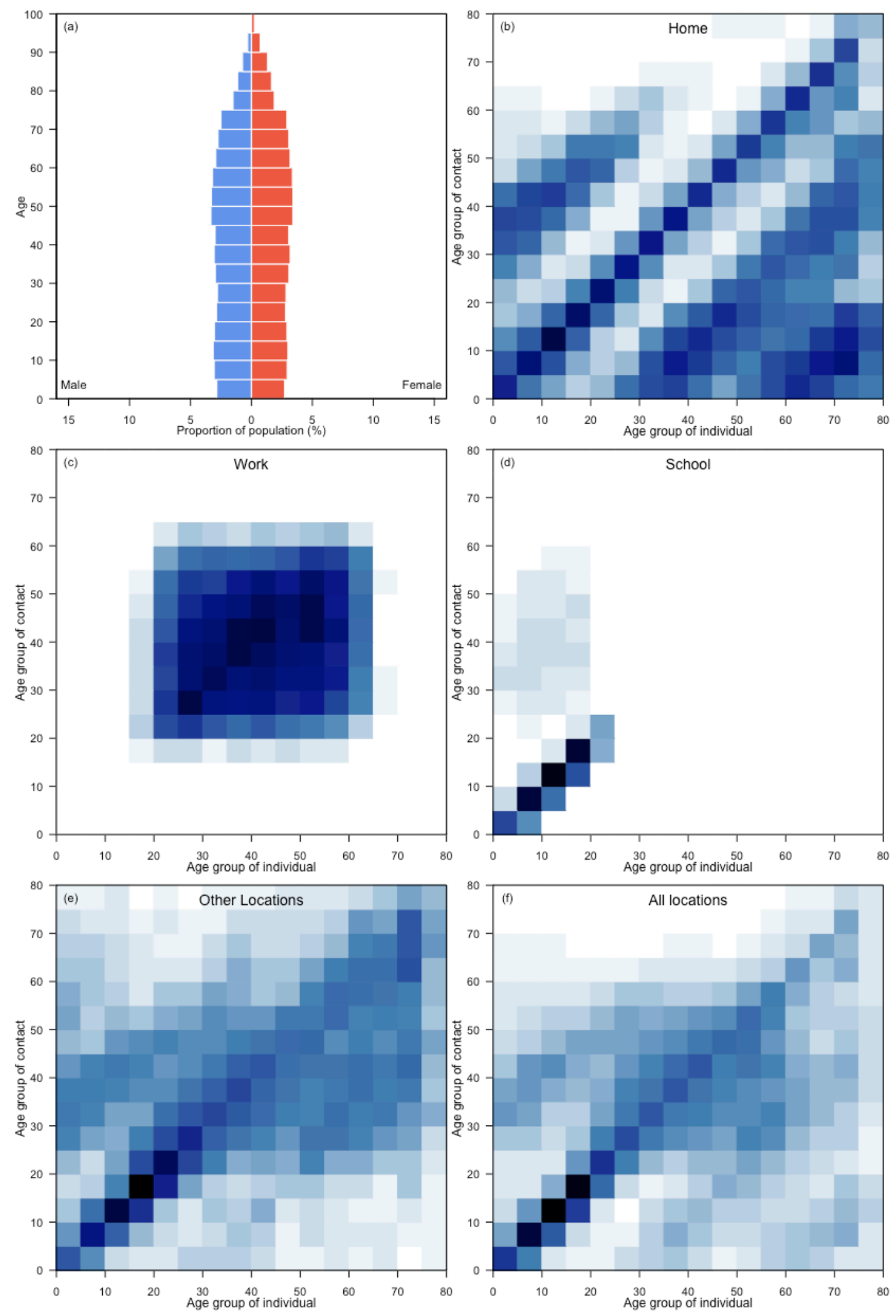


\section{Gabon}
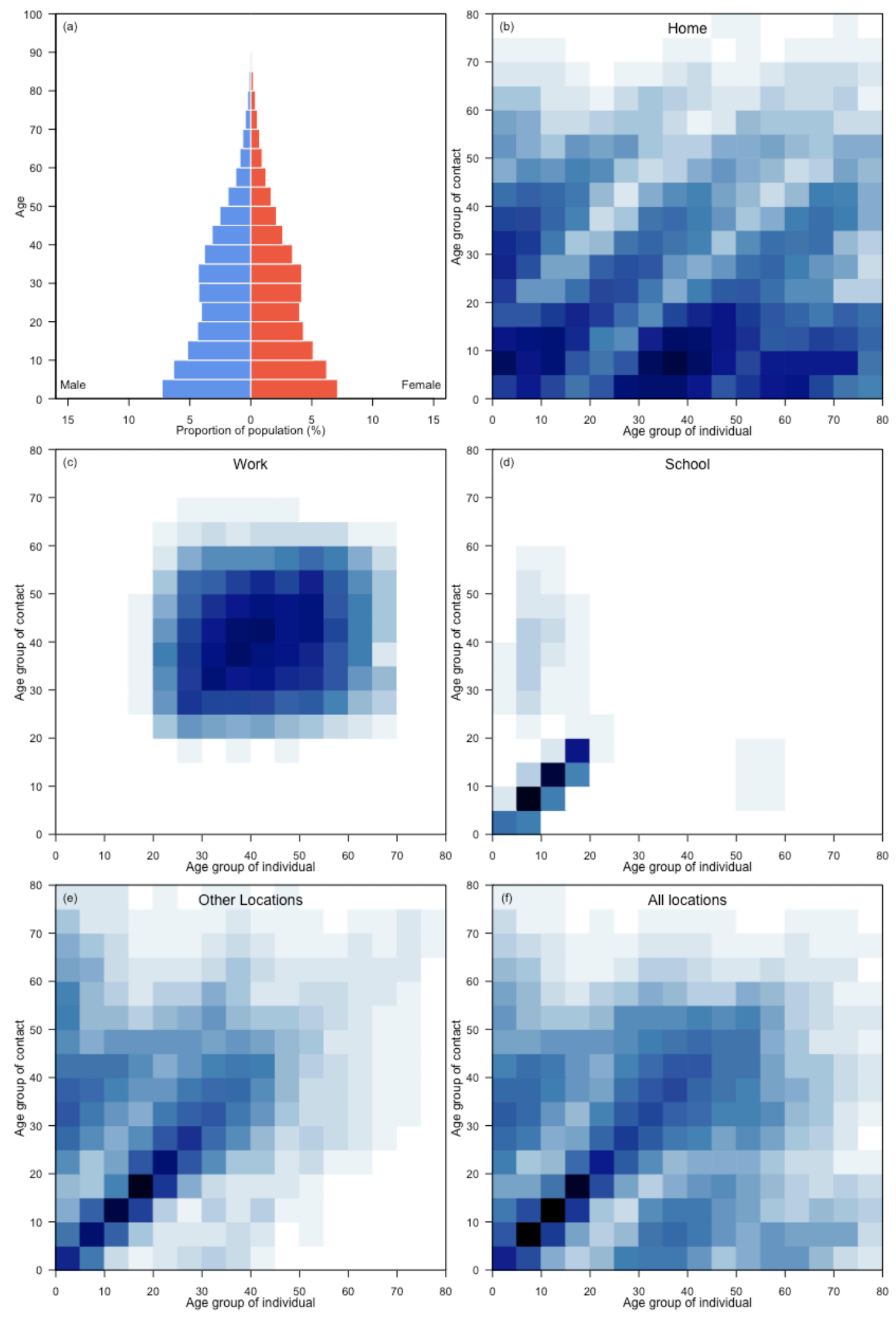


\section{Gambia}
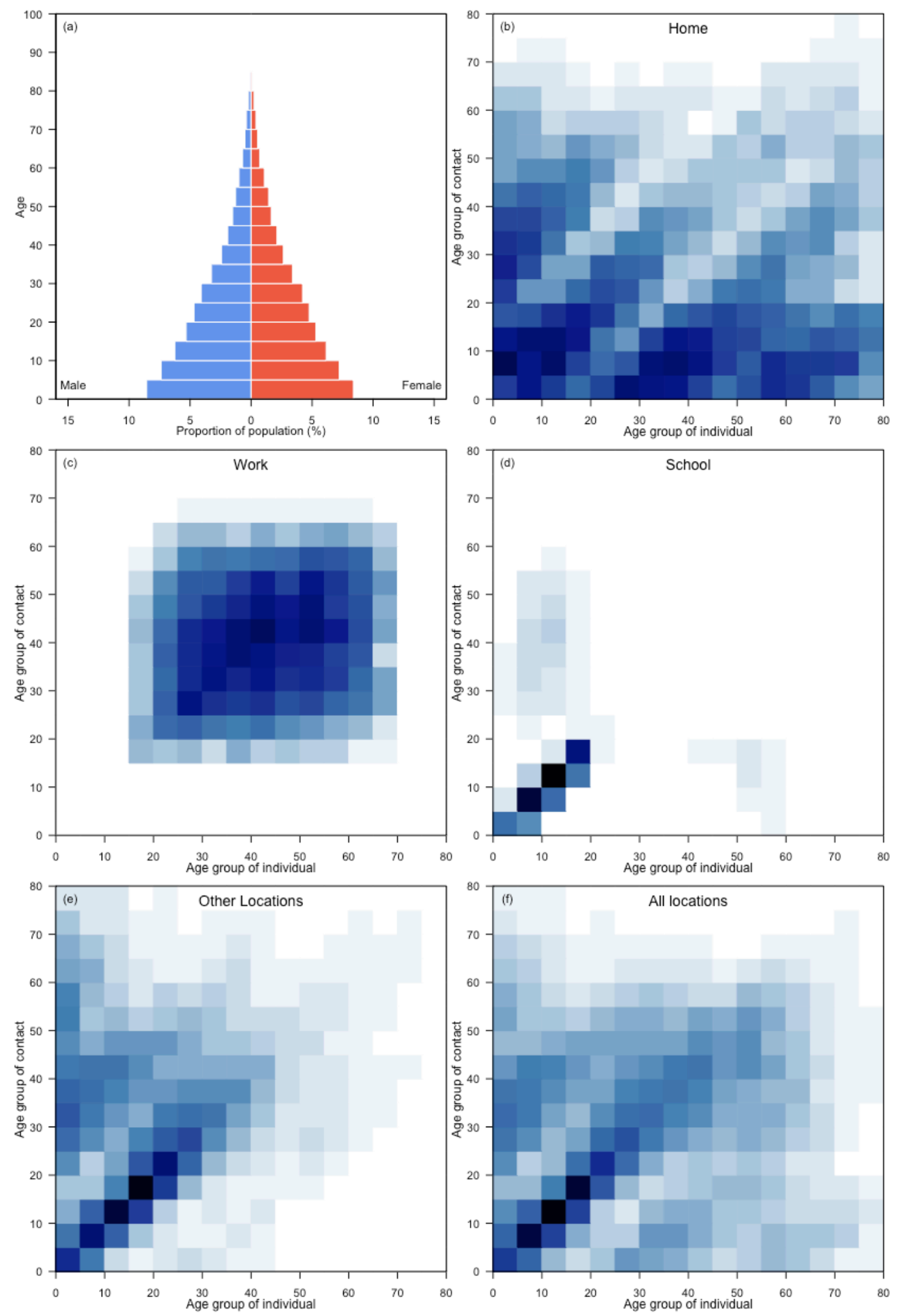


\section{Georgia}
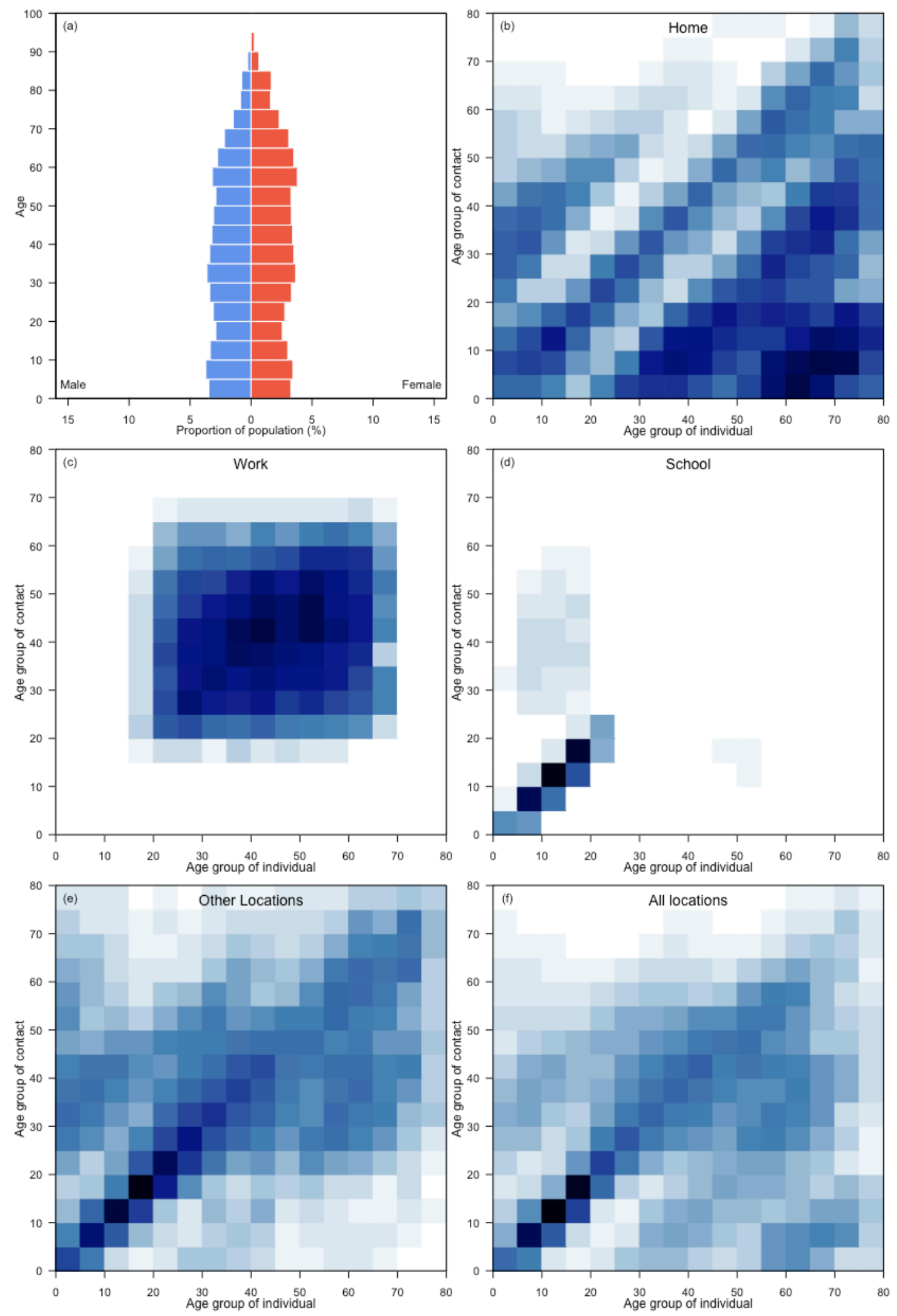


\section{Germany}
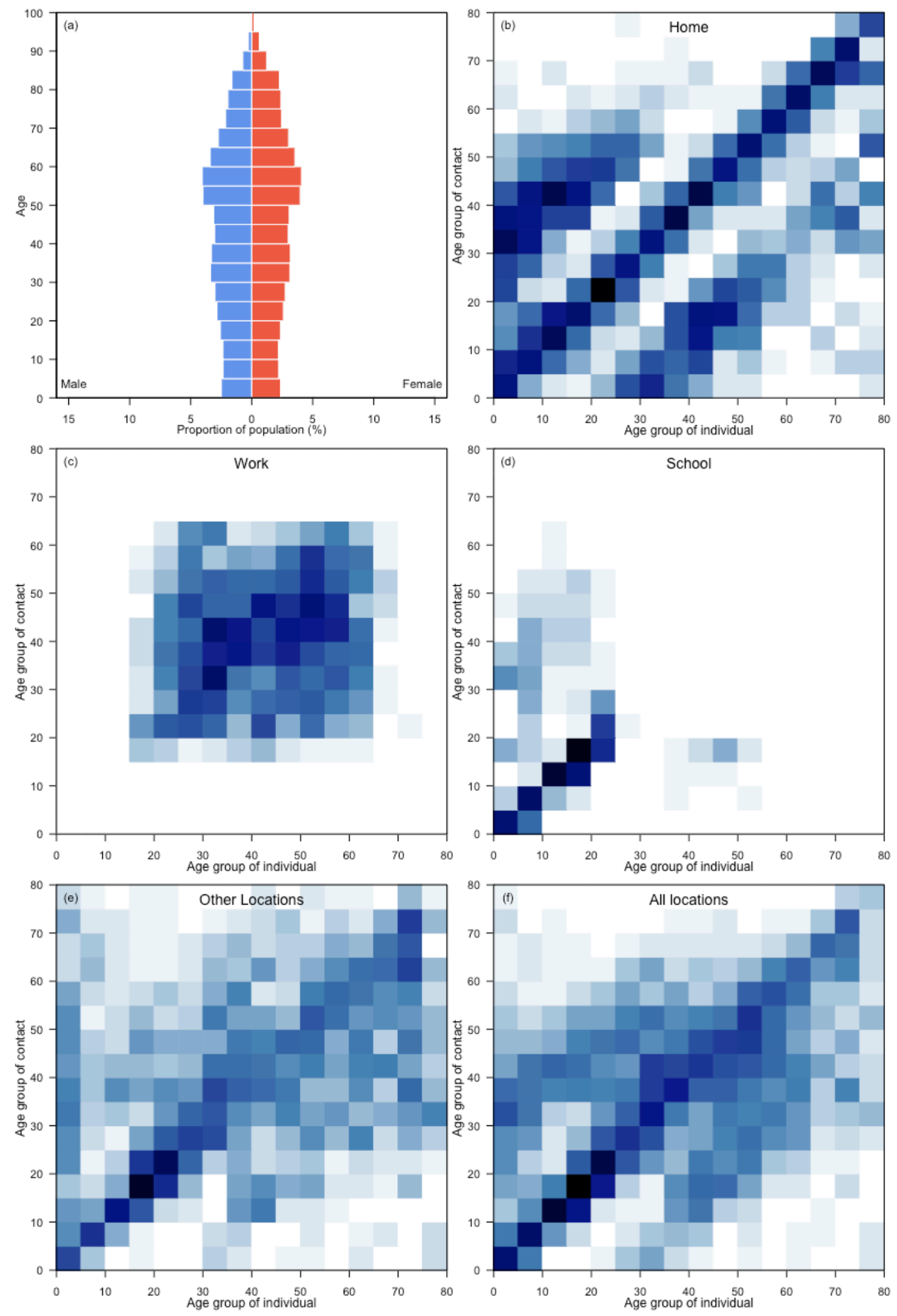


\section{Ghana}
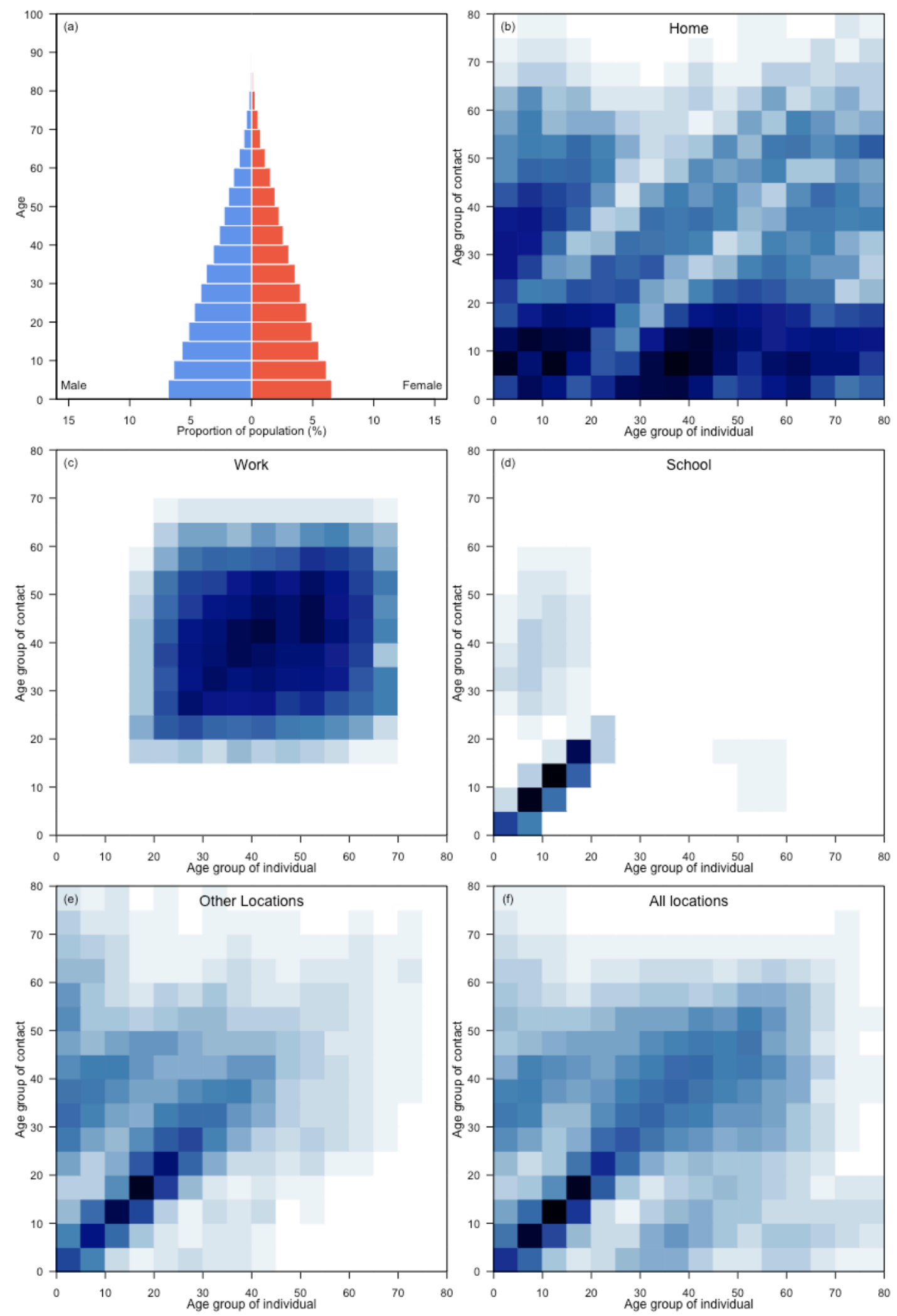


\section{Greece}
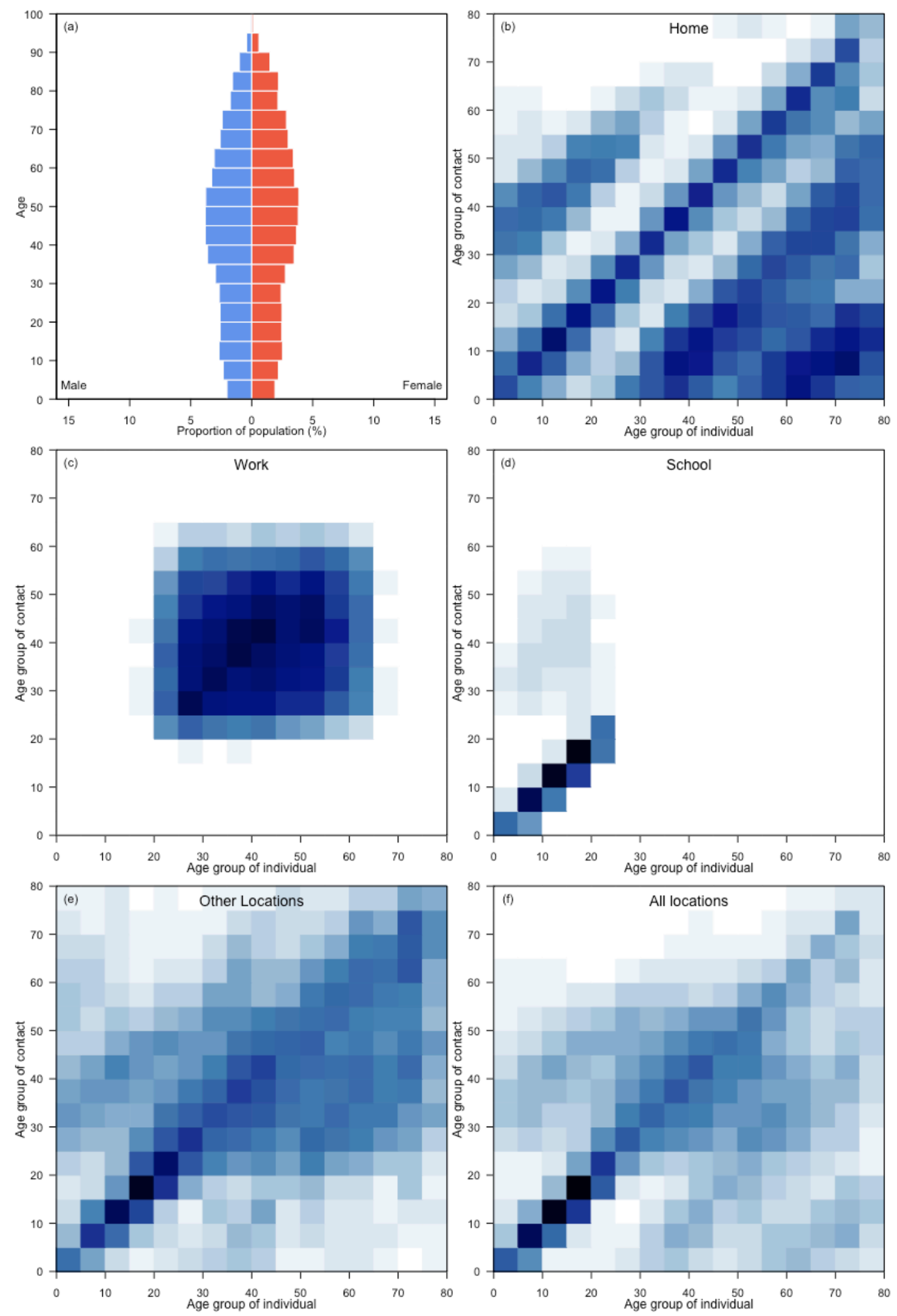
Guatemala
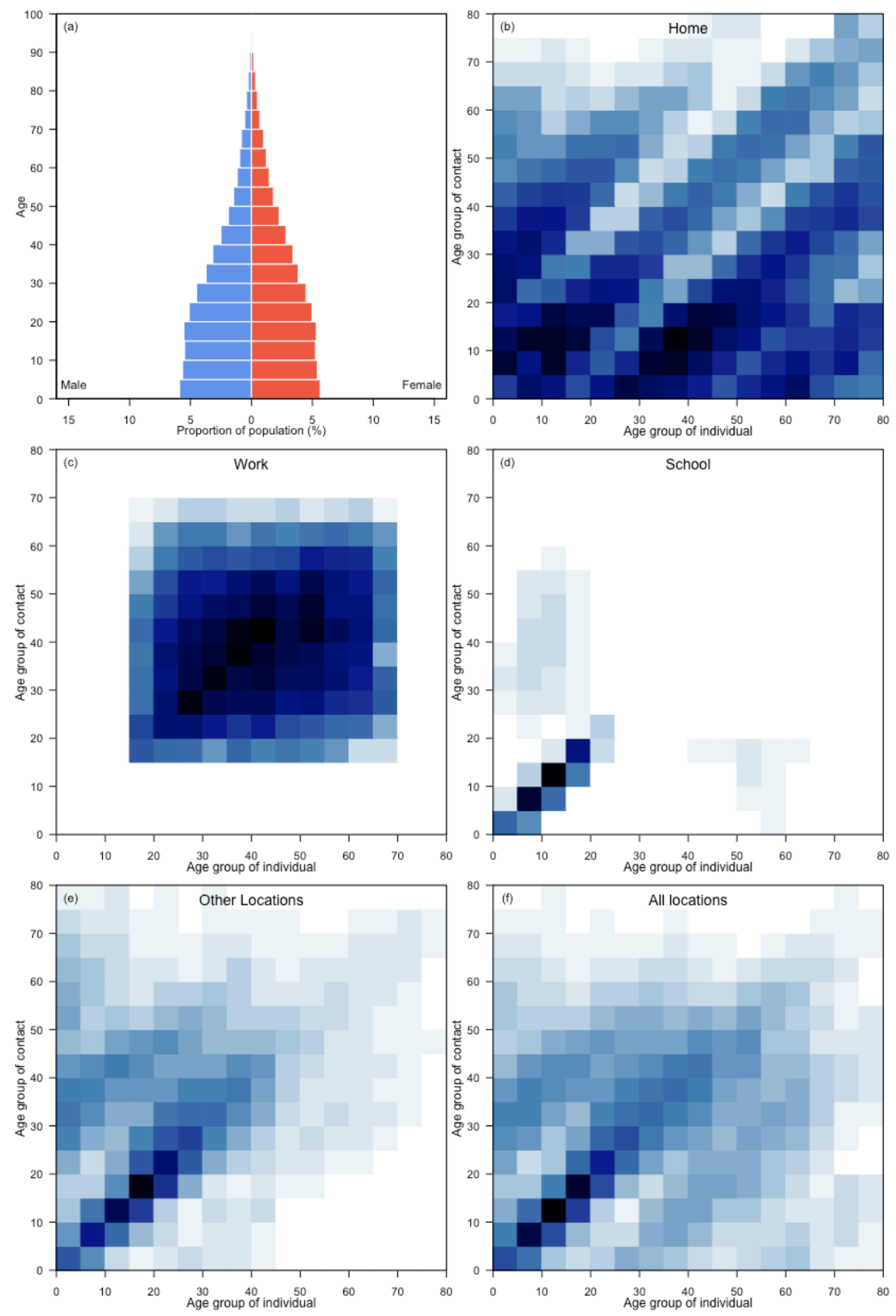


\section{Guinea}
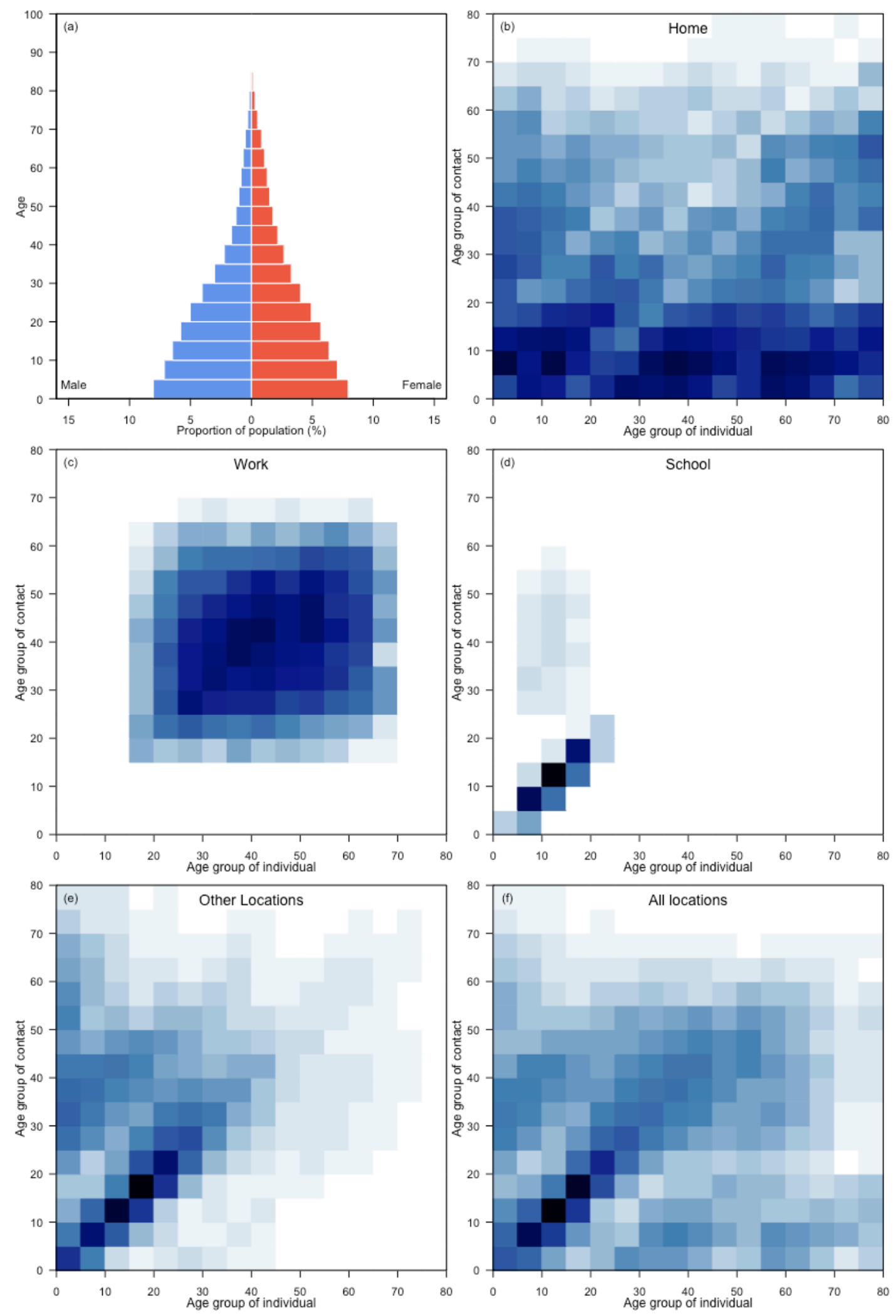


\section{Guinea-Bissau}
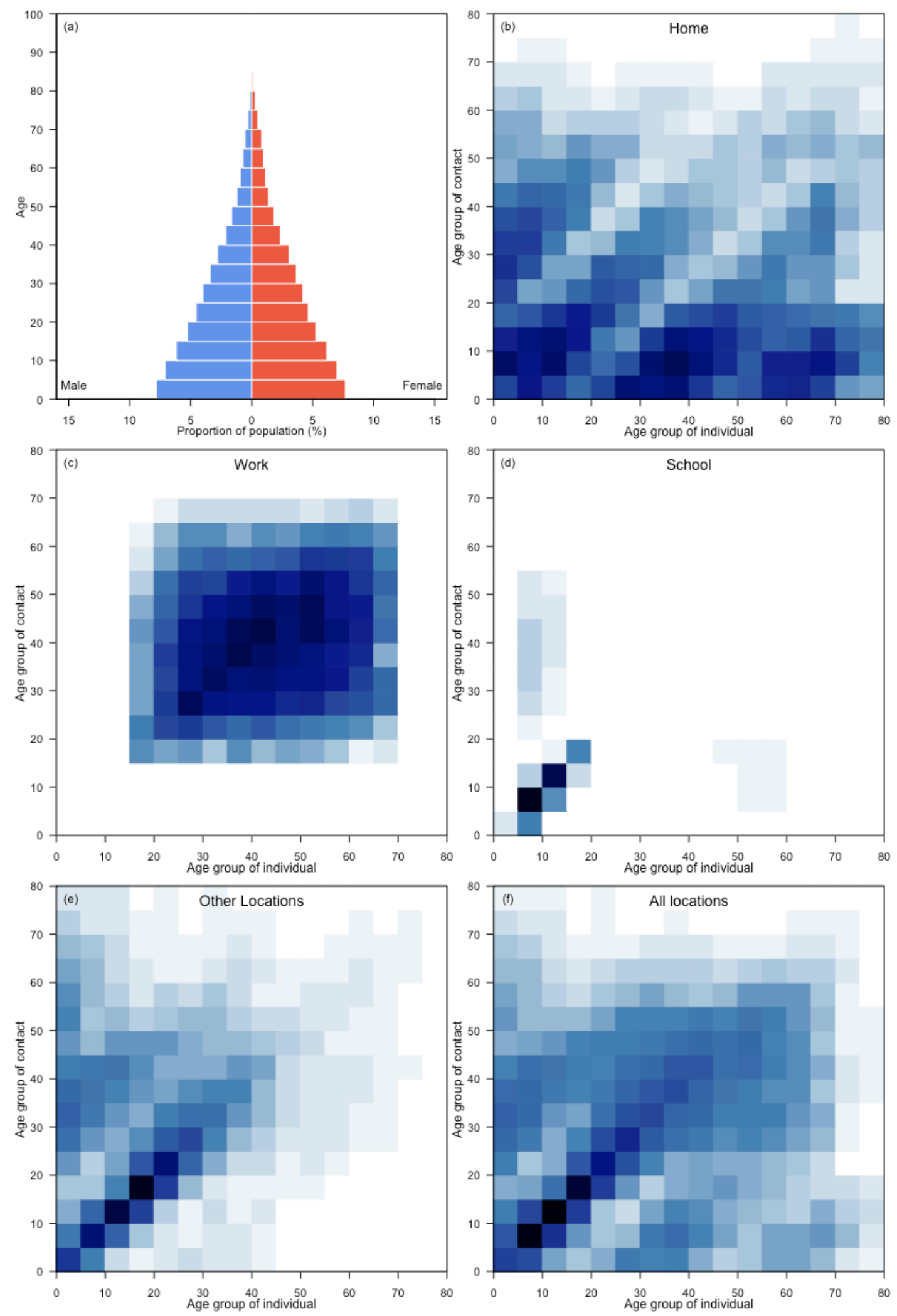


\section{Guyana}
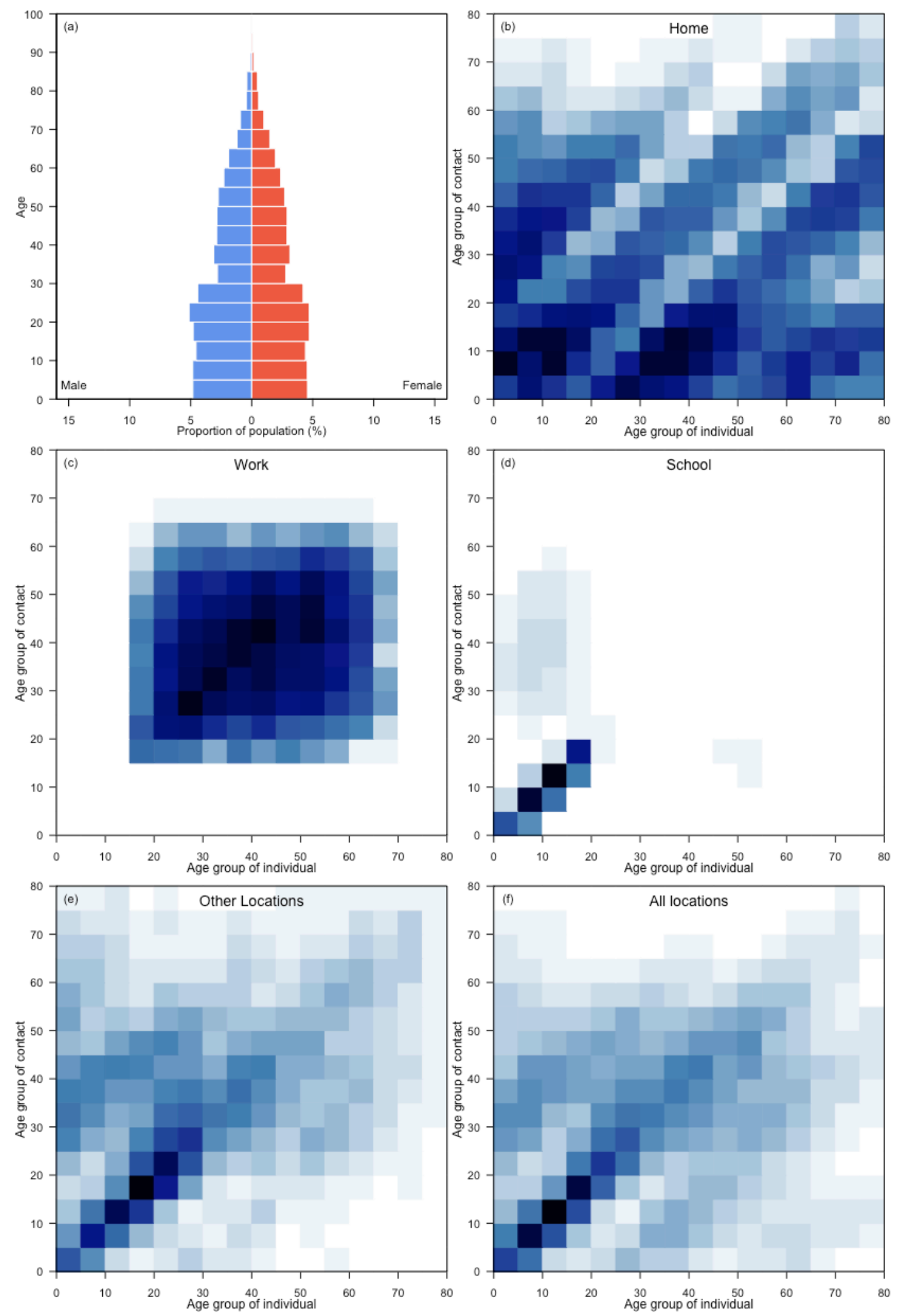


\section{Honduras}
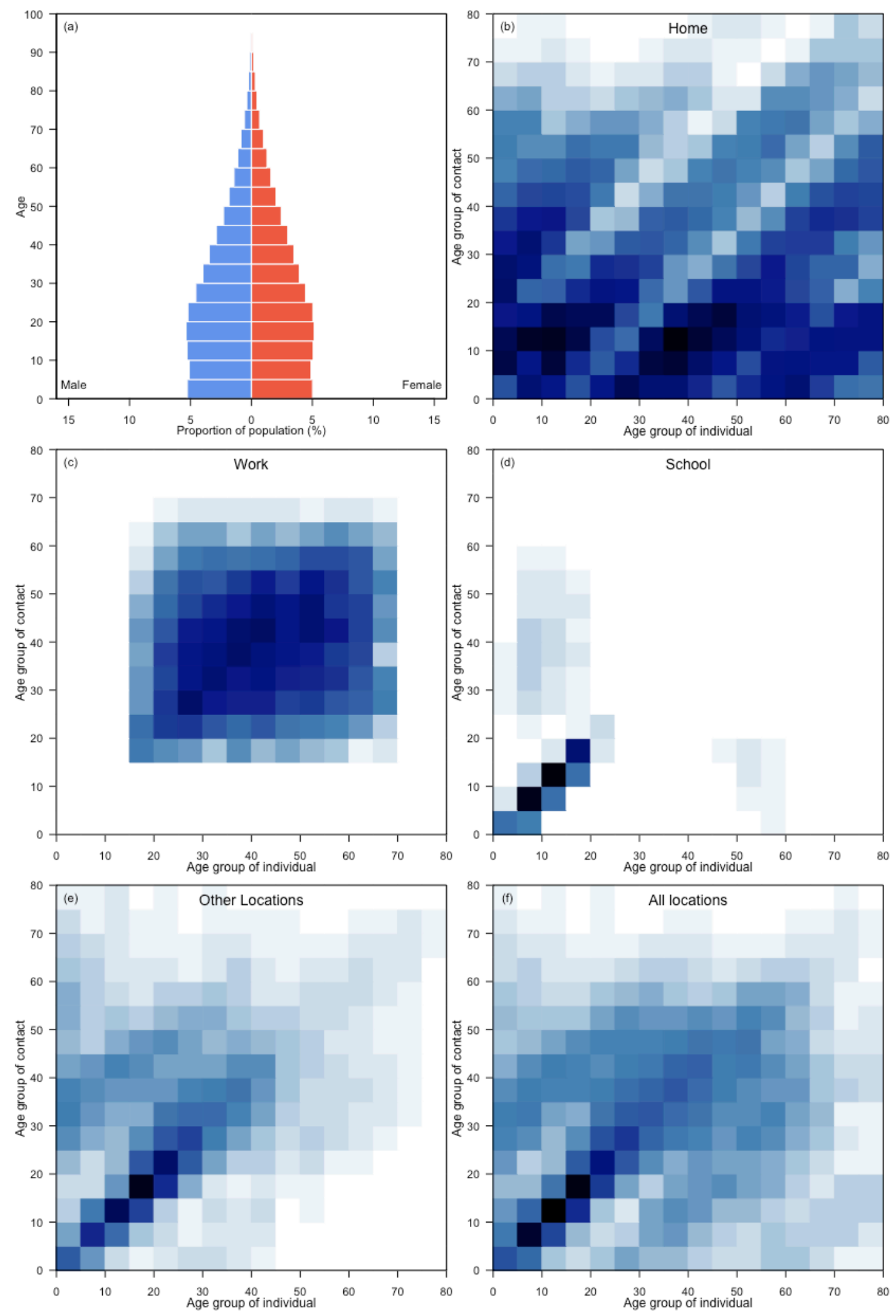


\section{Hungary}
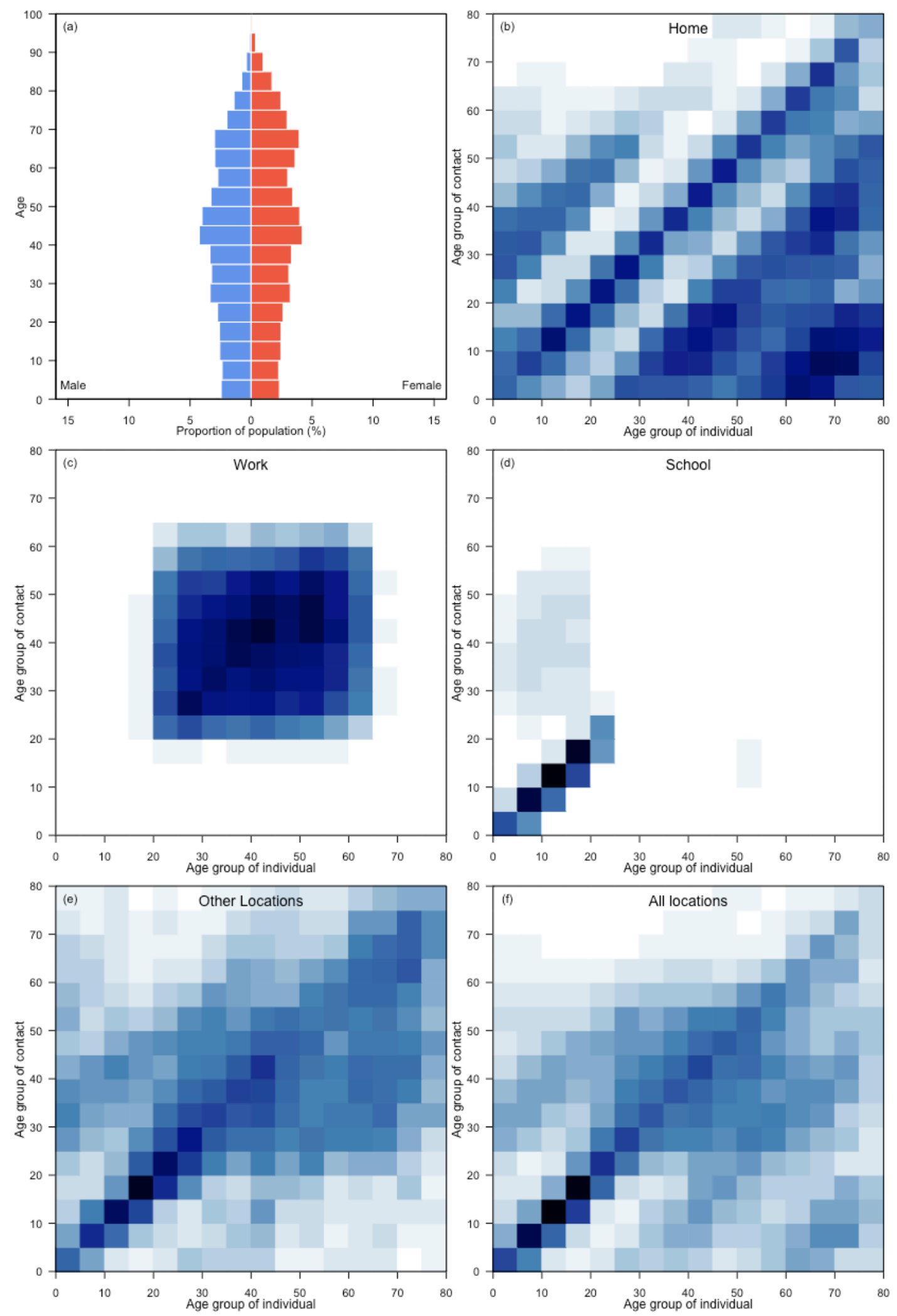


\section{Iceland}
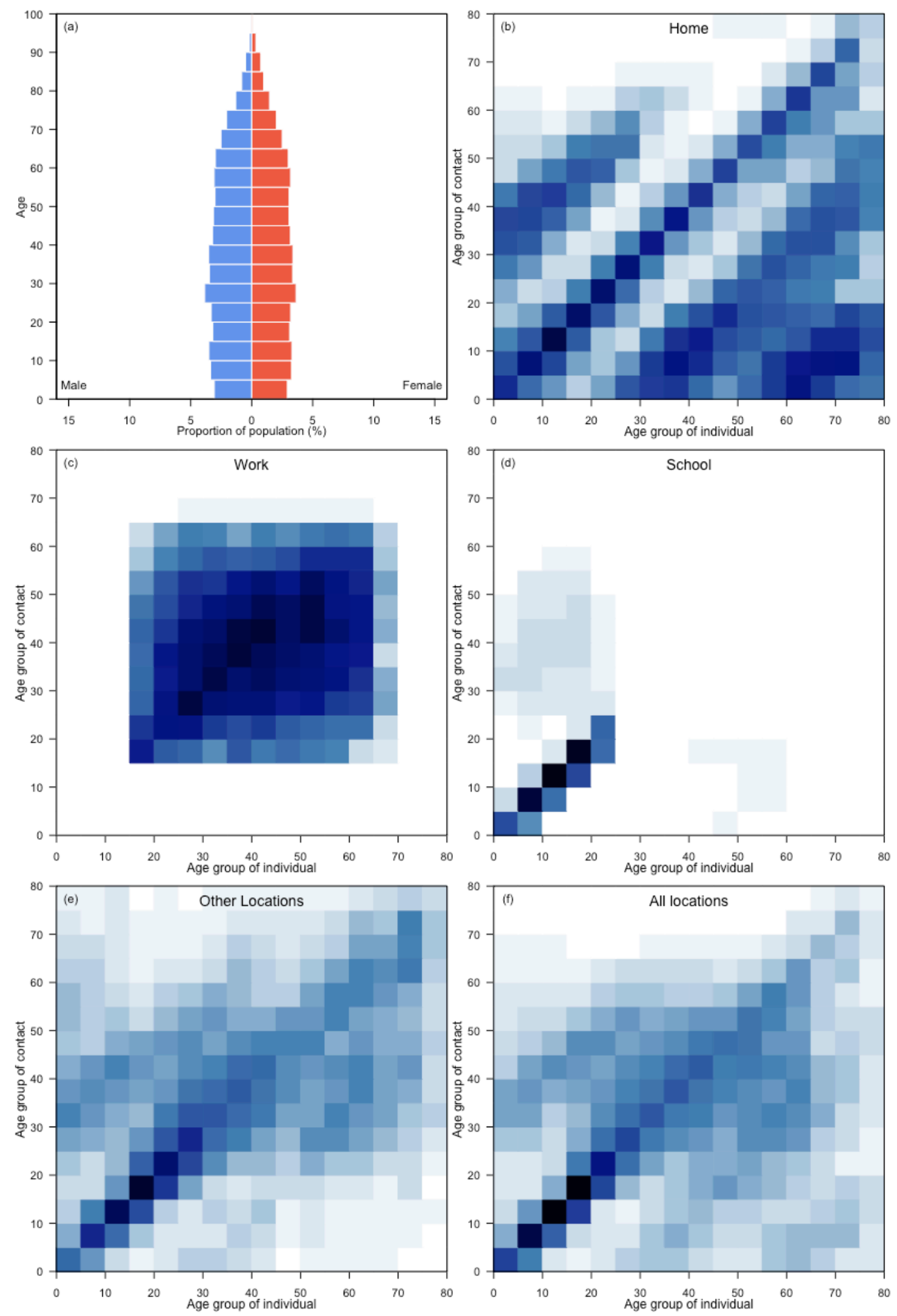


\section{India}
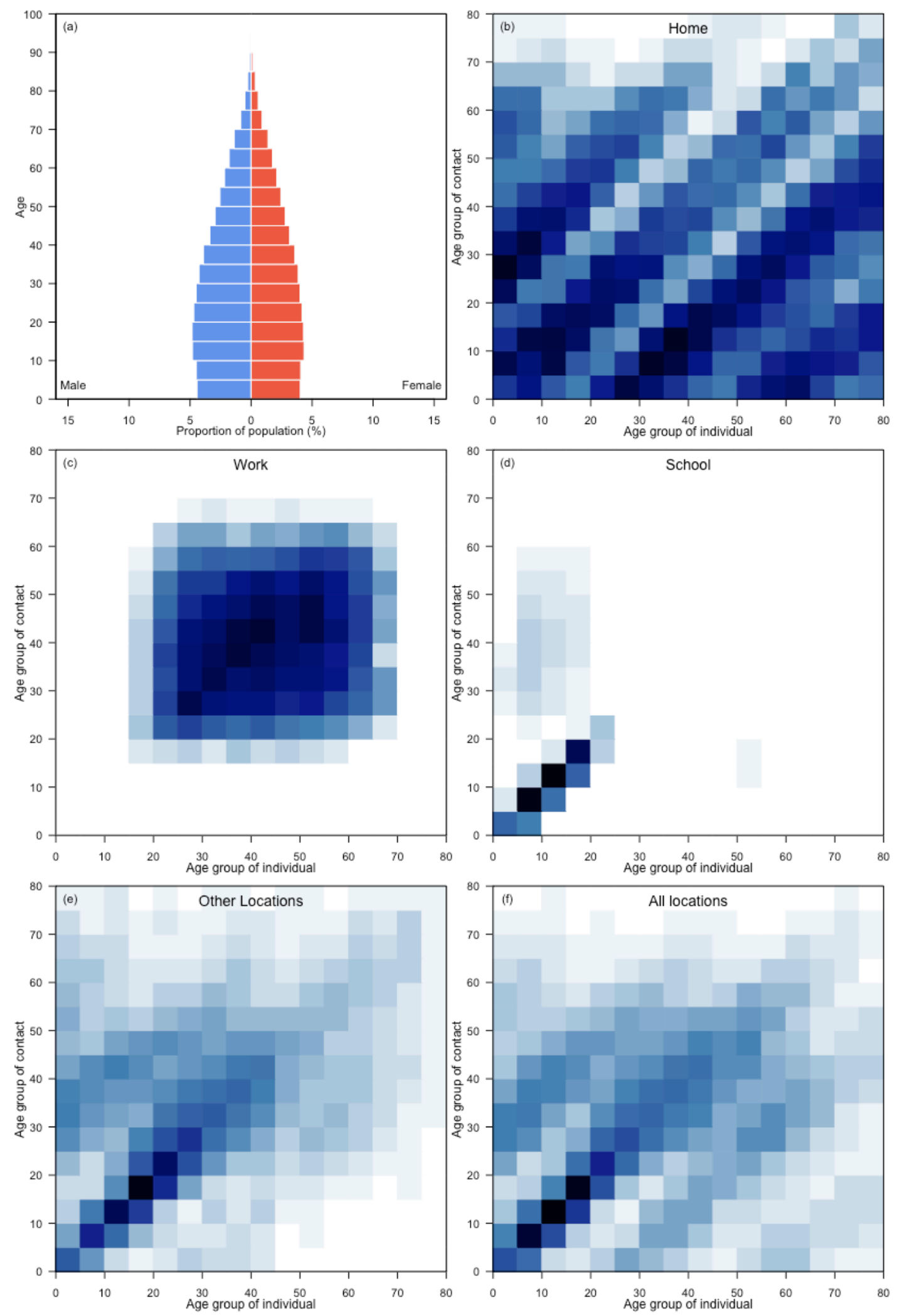
Indonesia
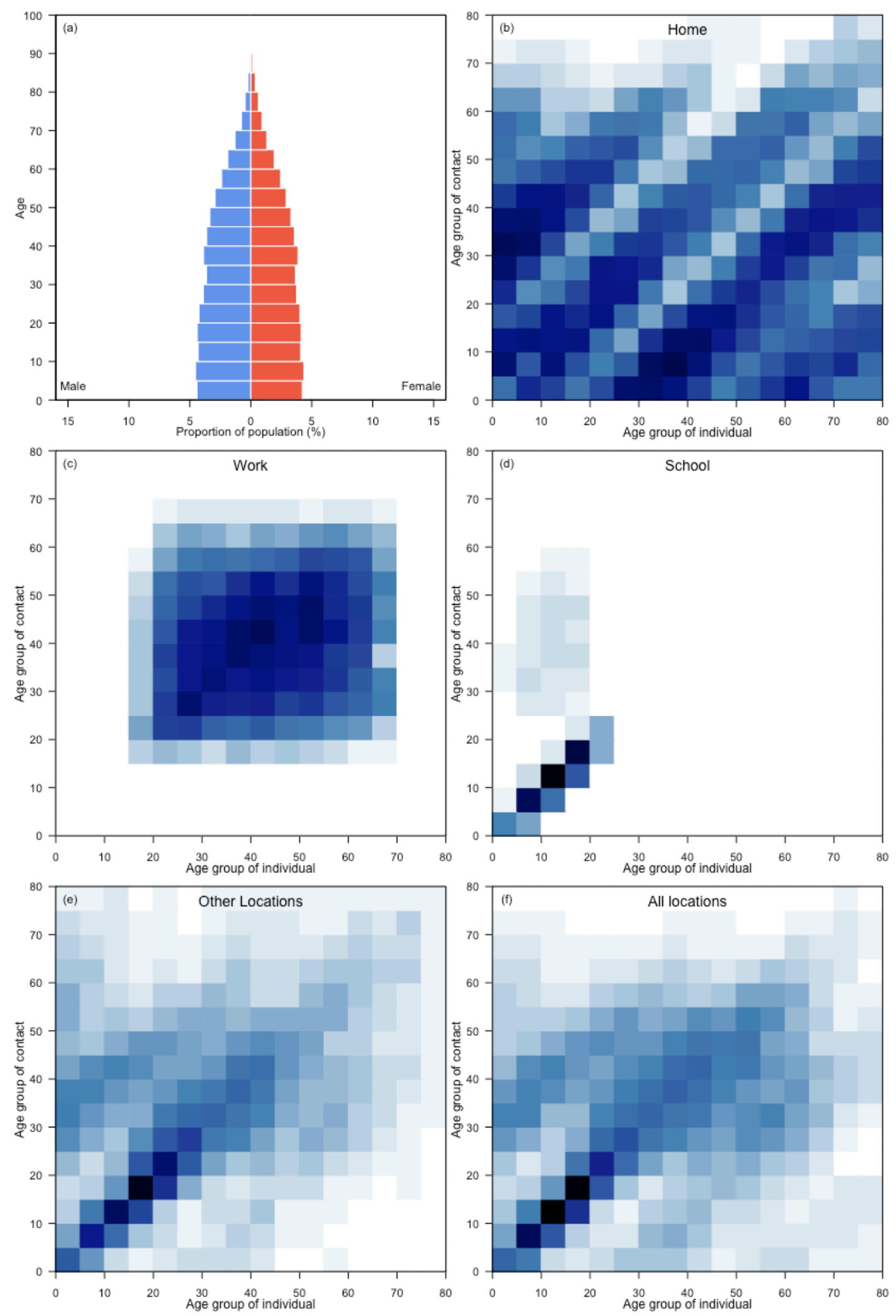
Iran (Islamic Republic of)
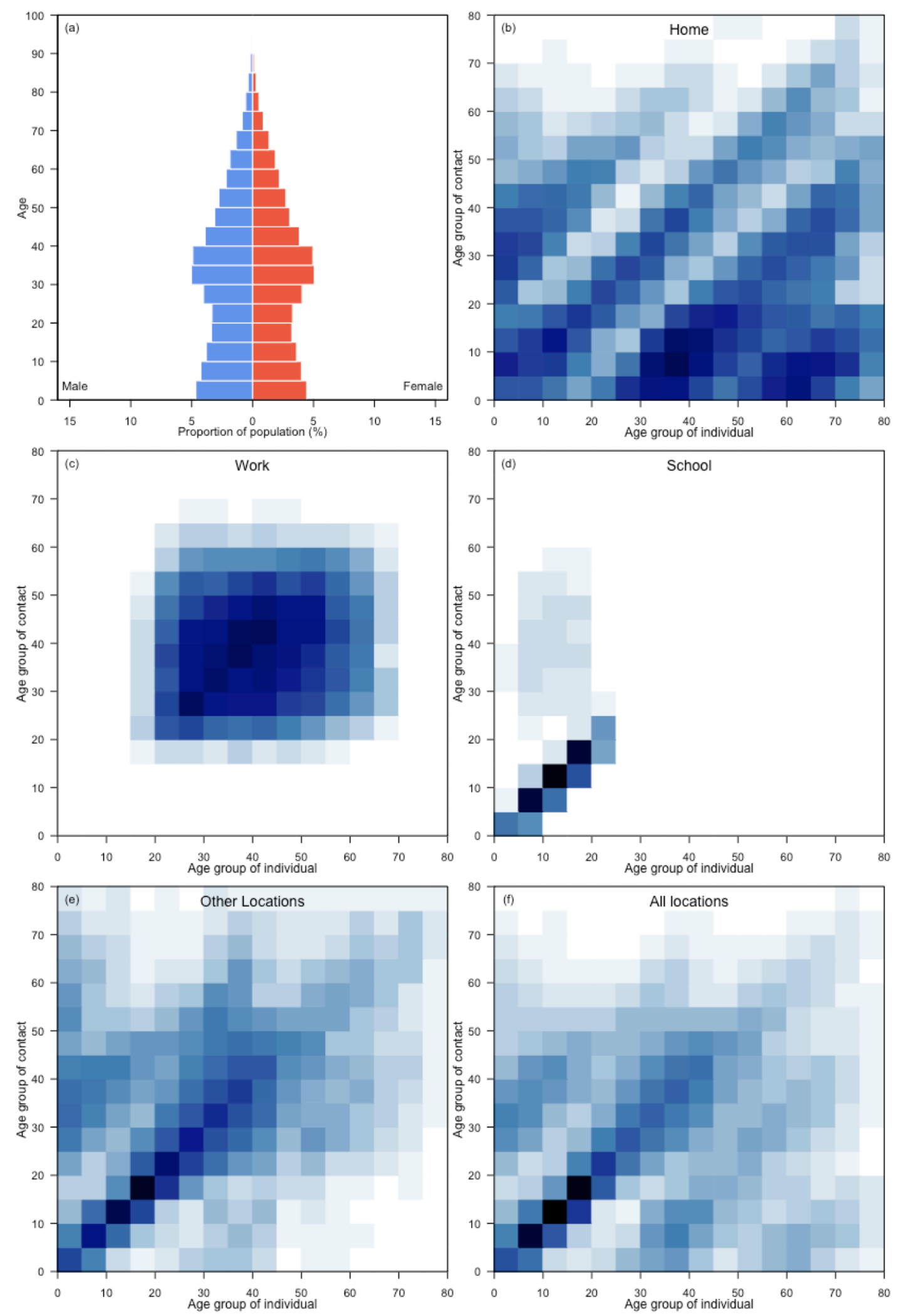


\section{Iraq}
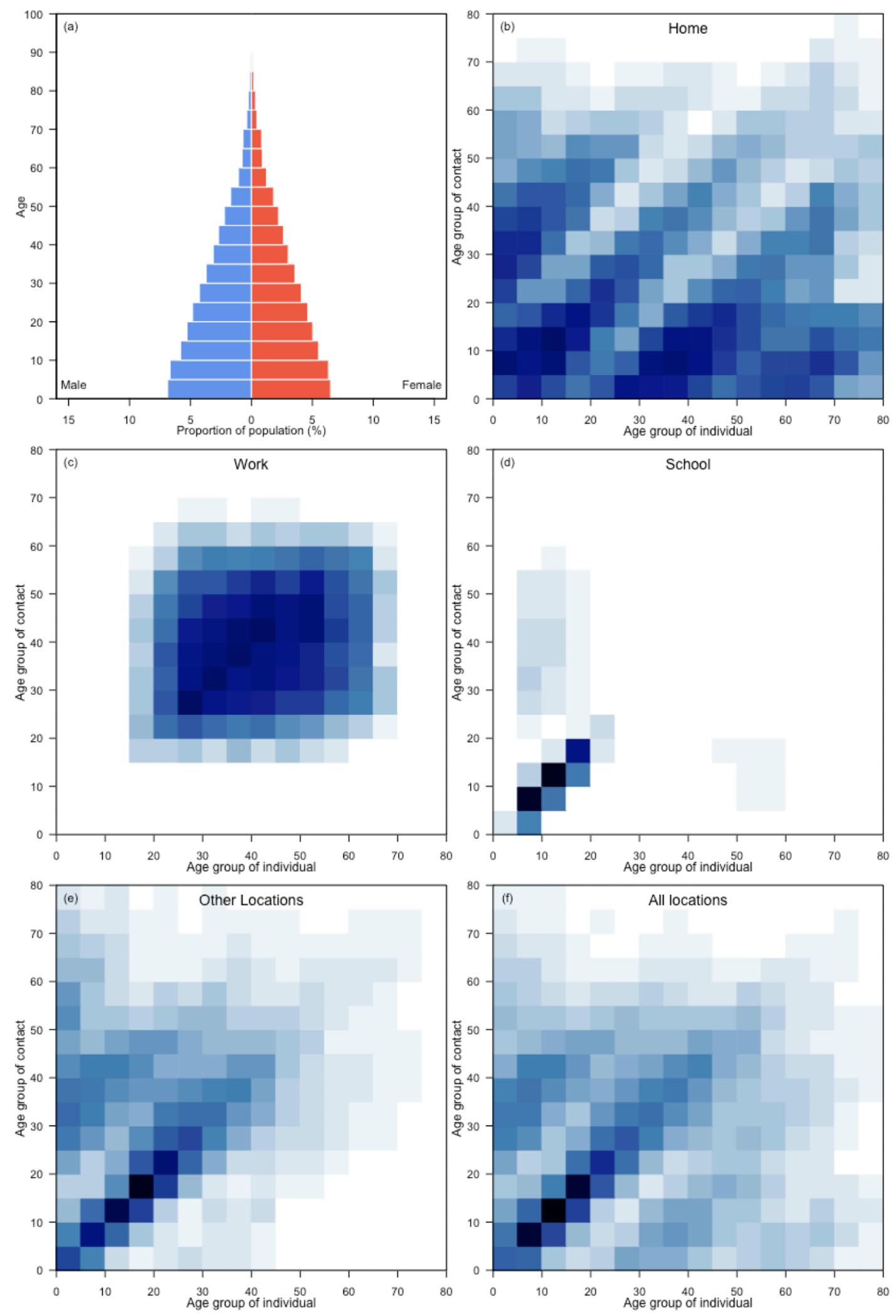
Ireland
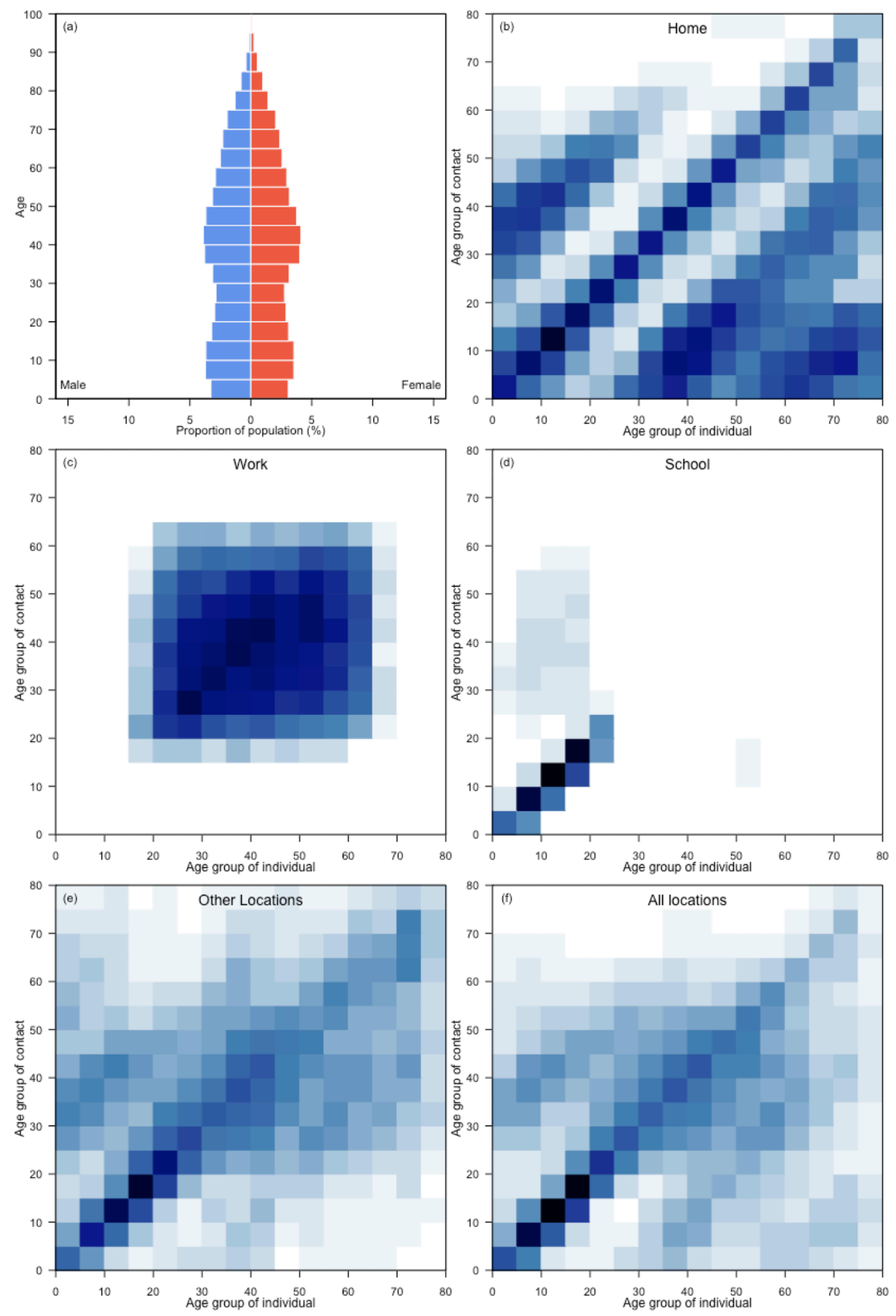


\section{Israel}
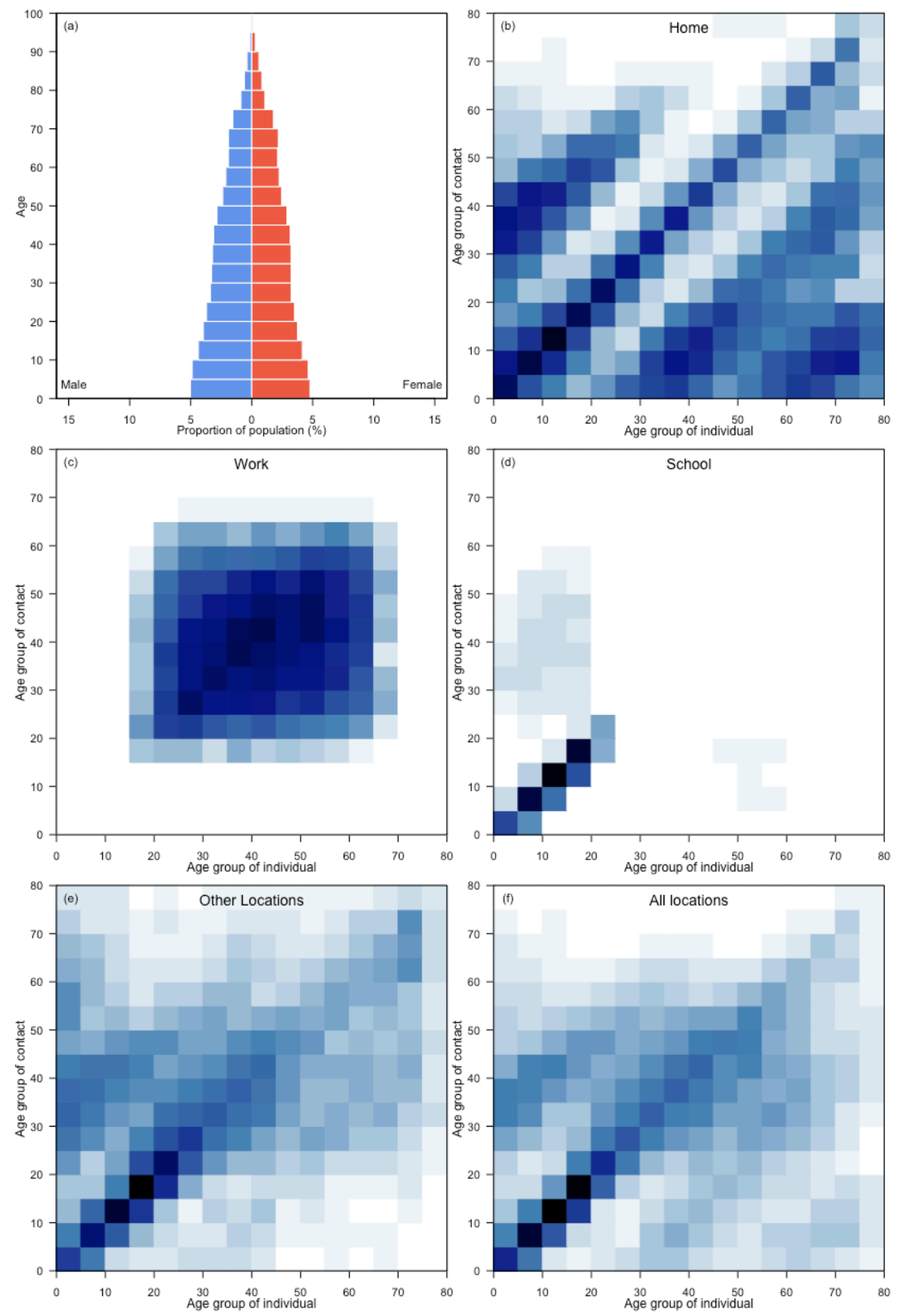
Italy
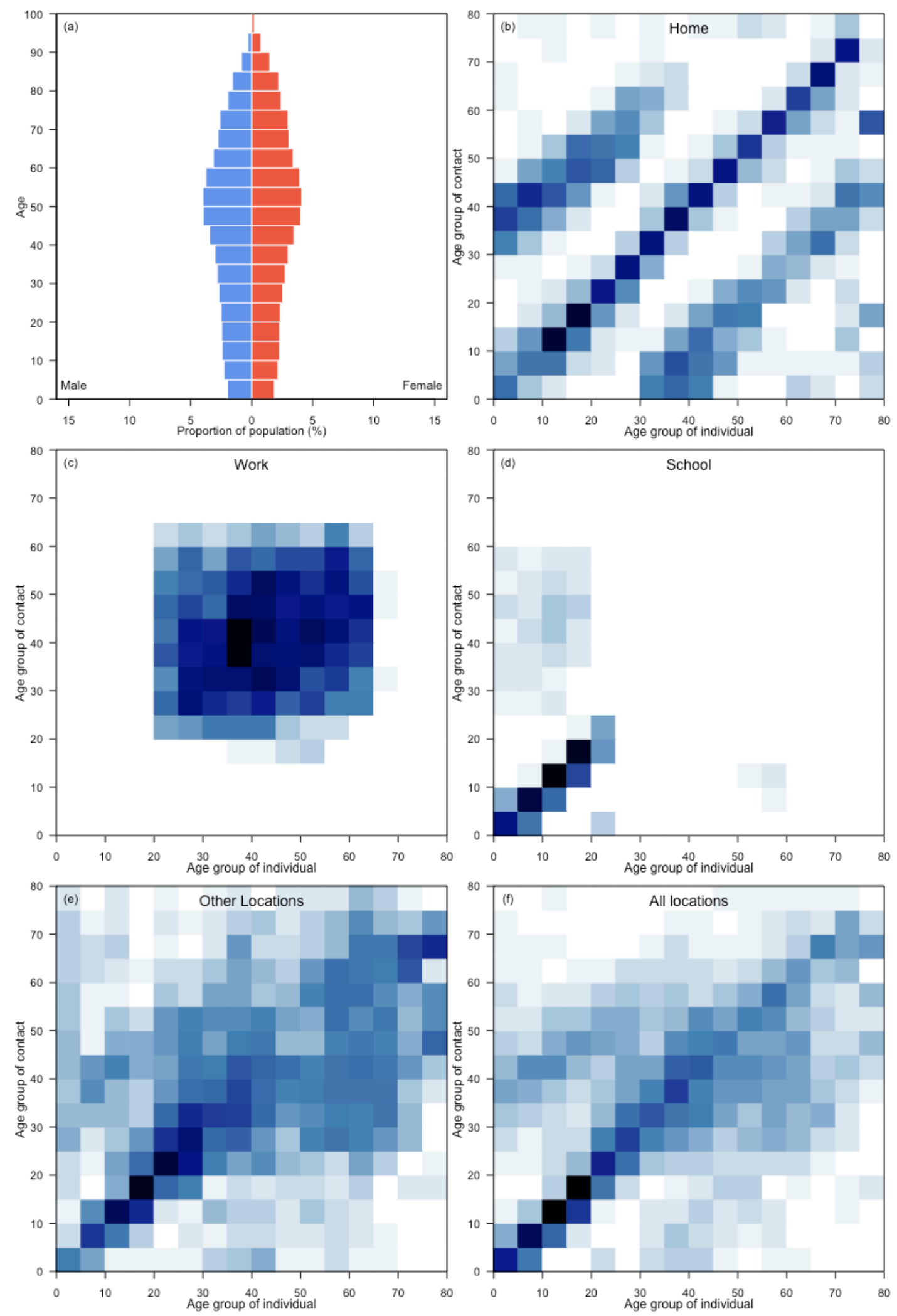
Jamaica
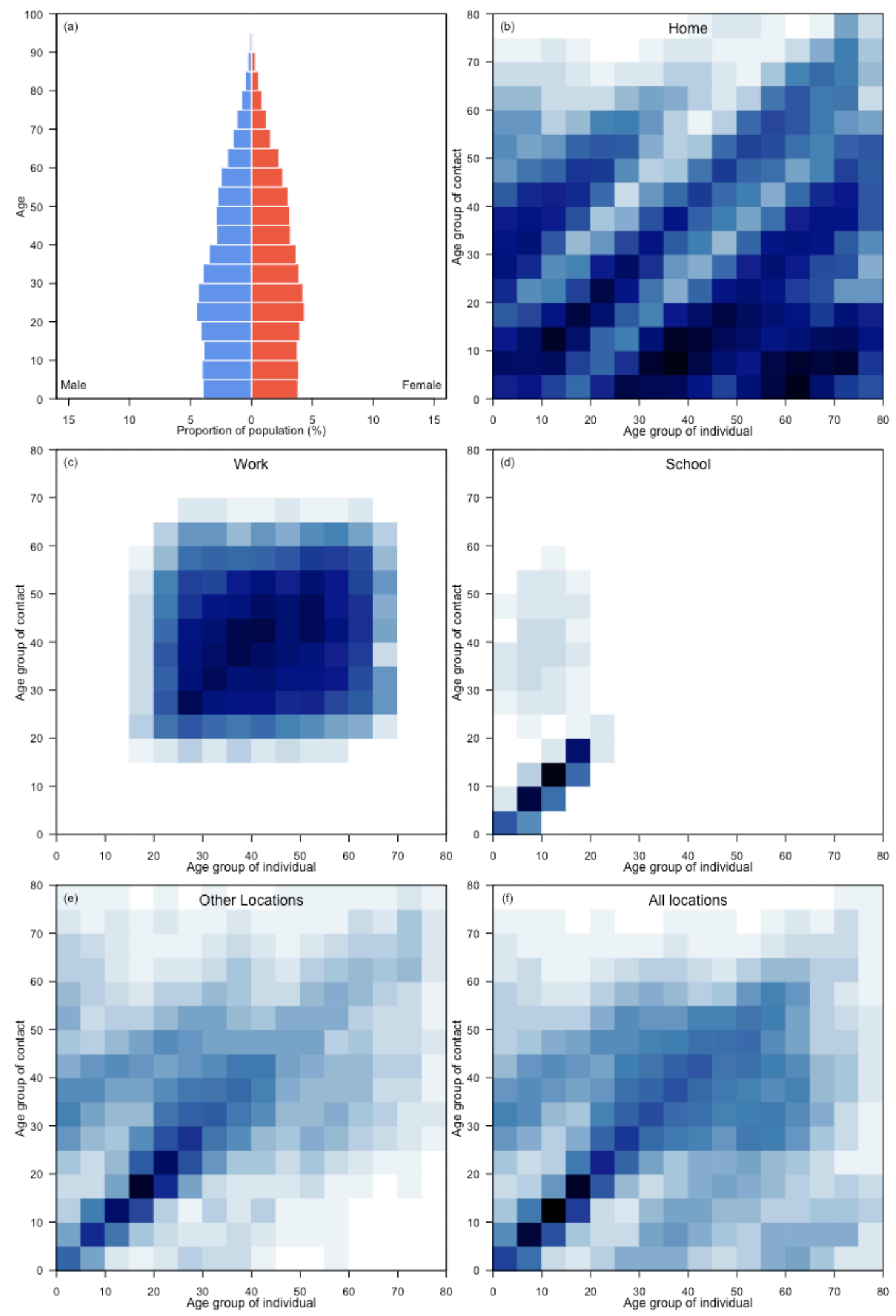


\section{Jordan}
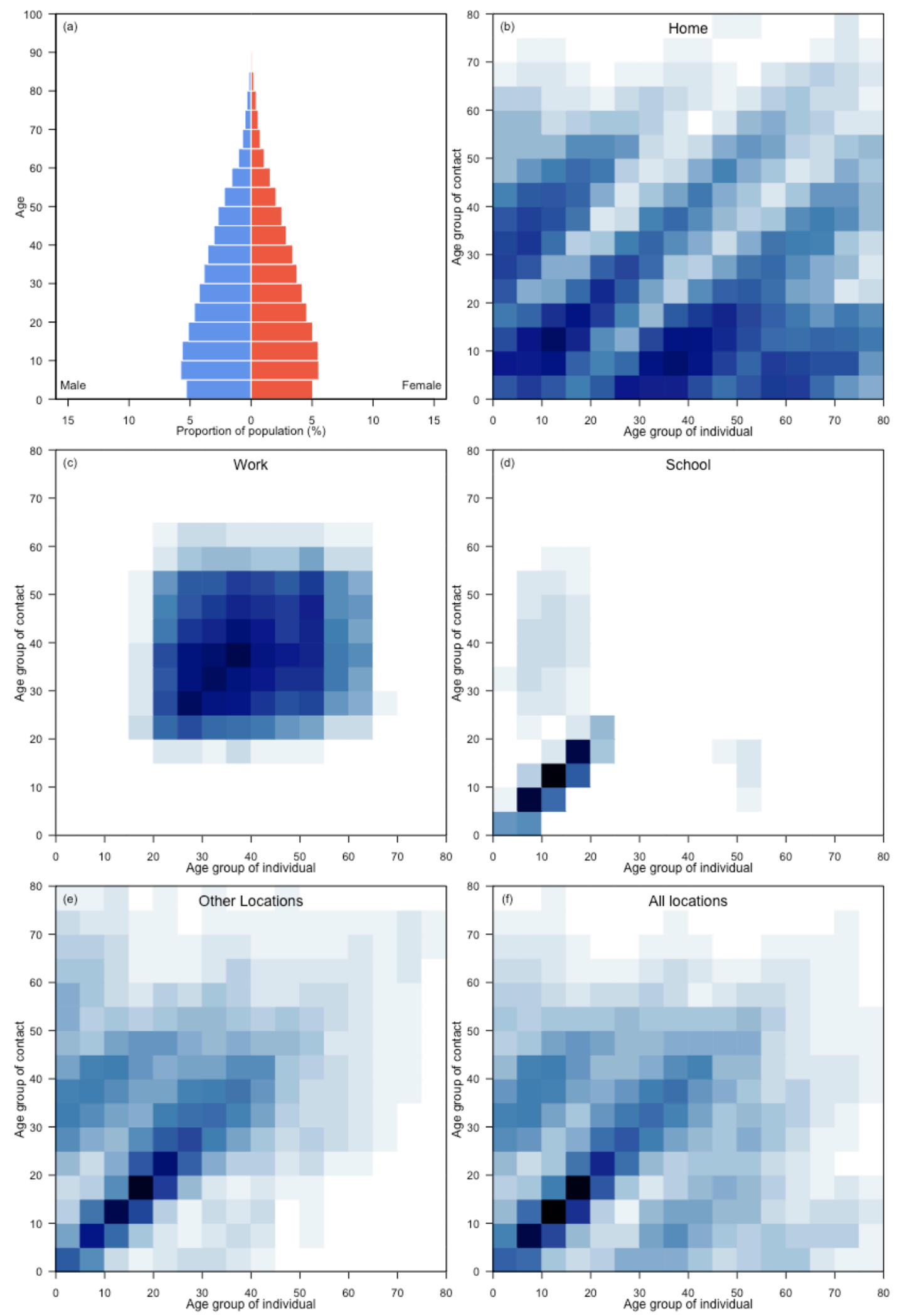


\section{Kazakhstan}
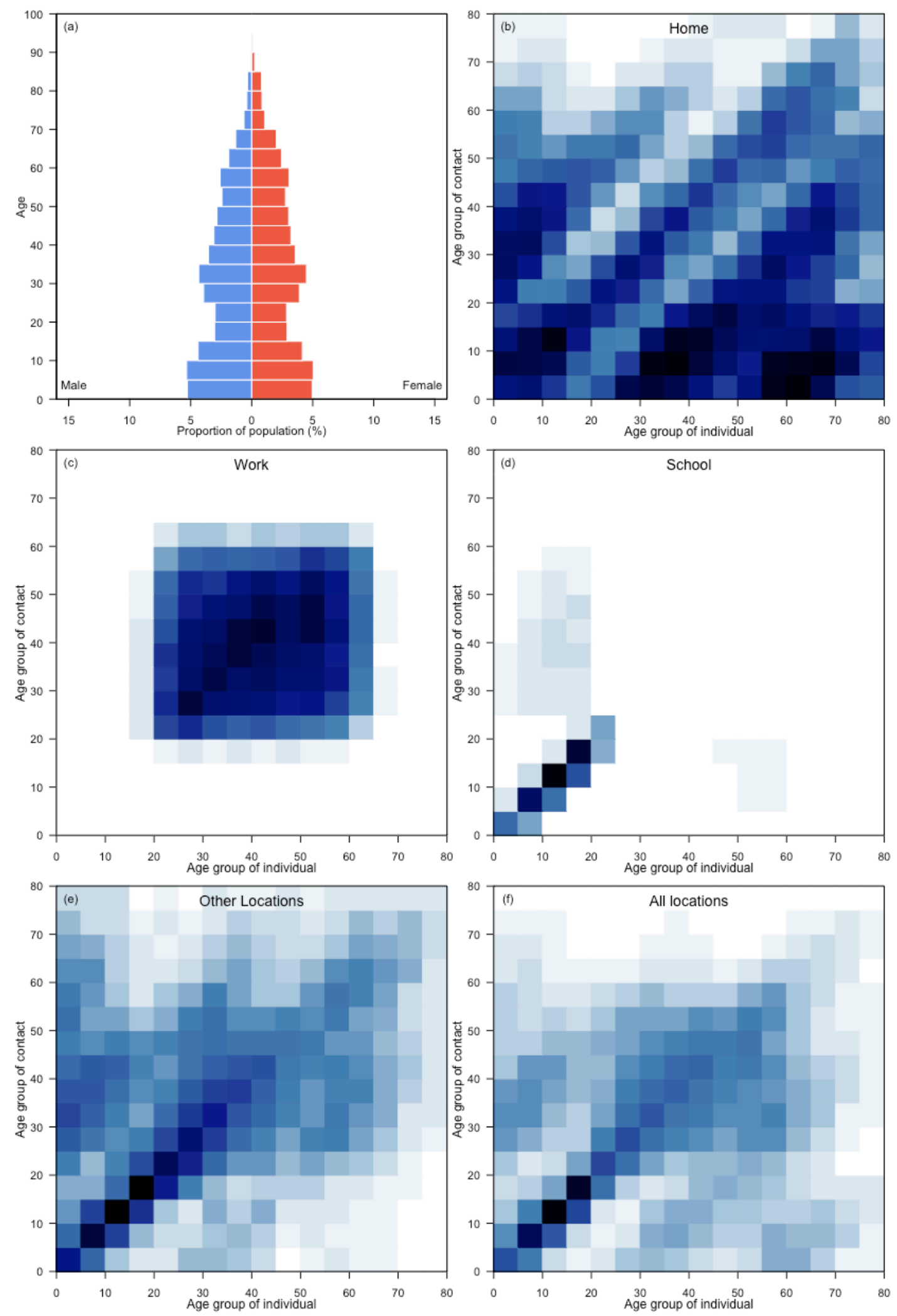


\section{Kenya}
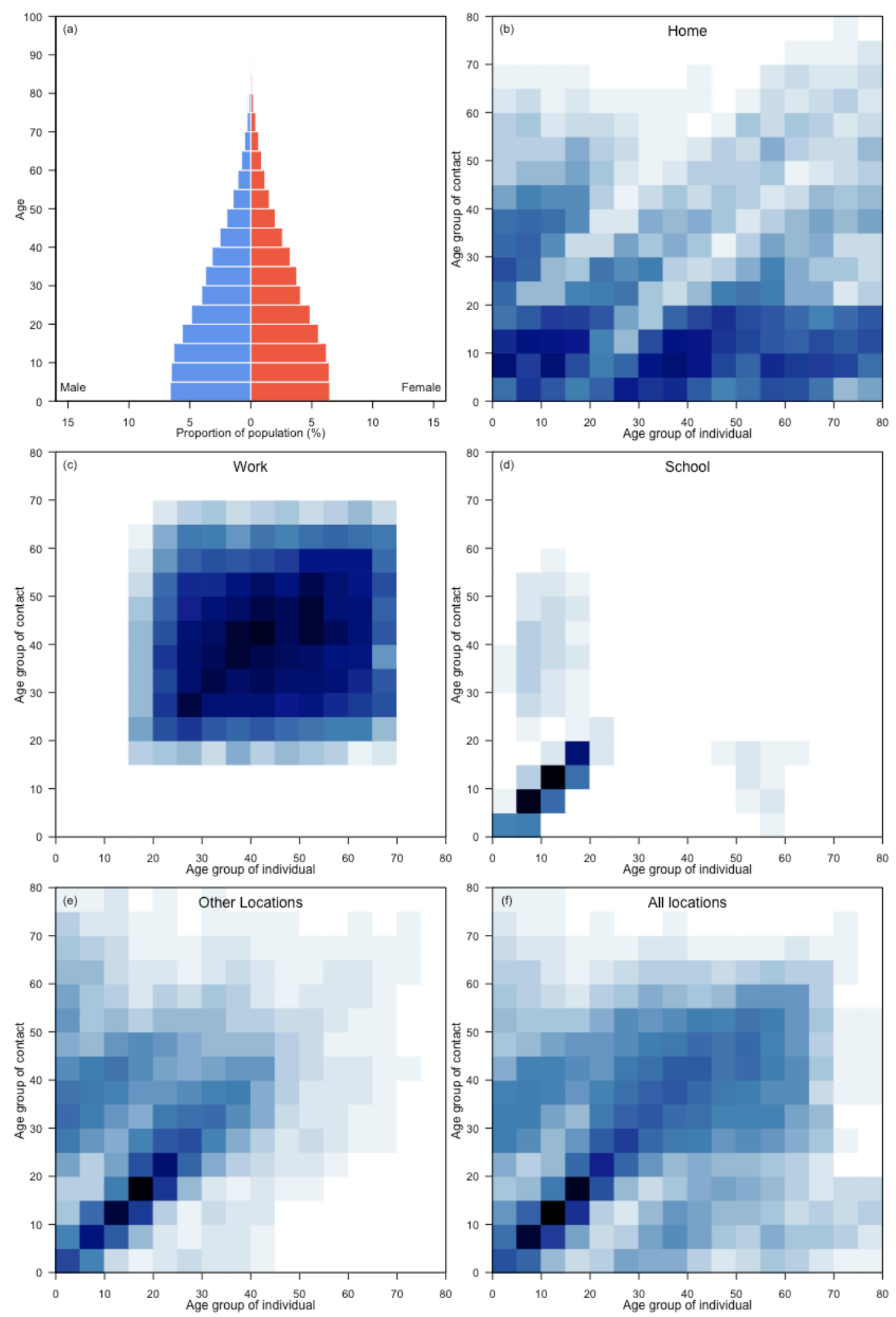


\section{Kuwait}
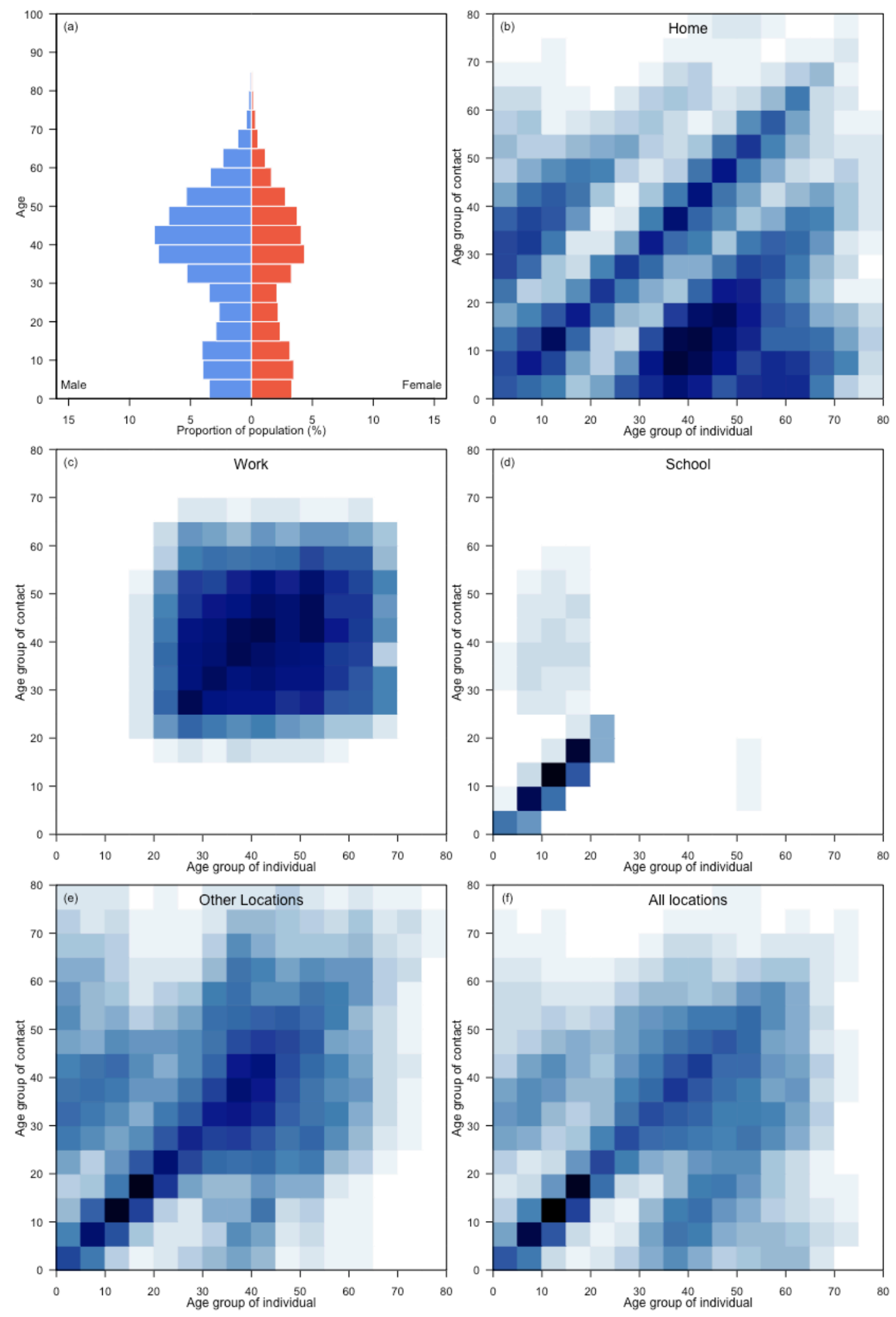


\section{Kyrgyzstan}
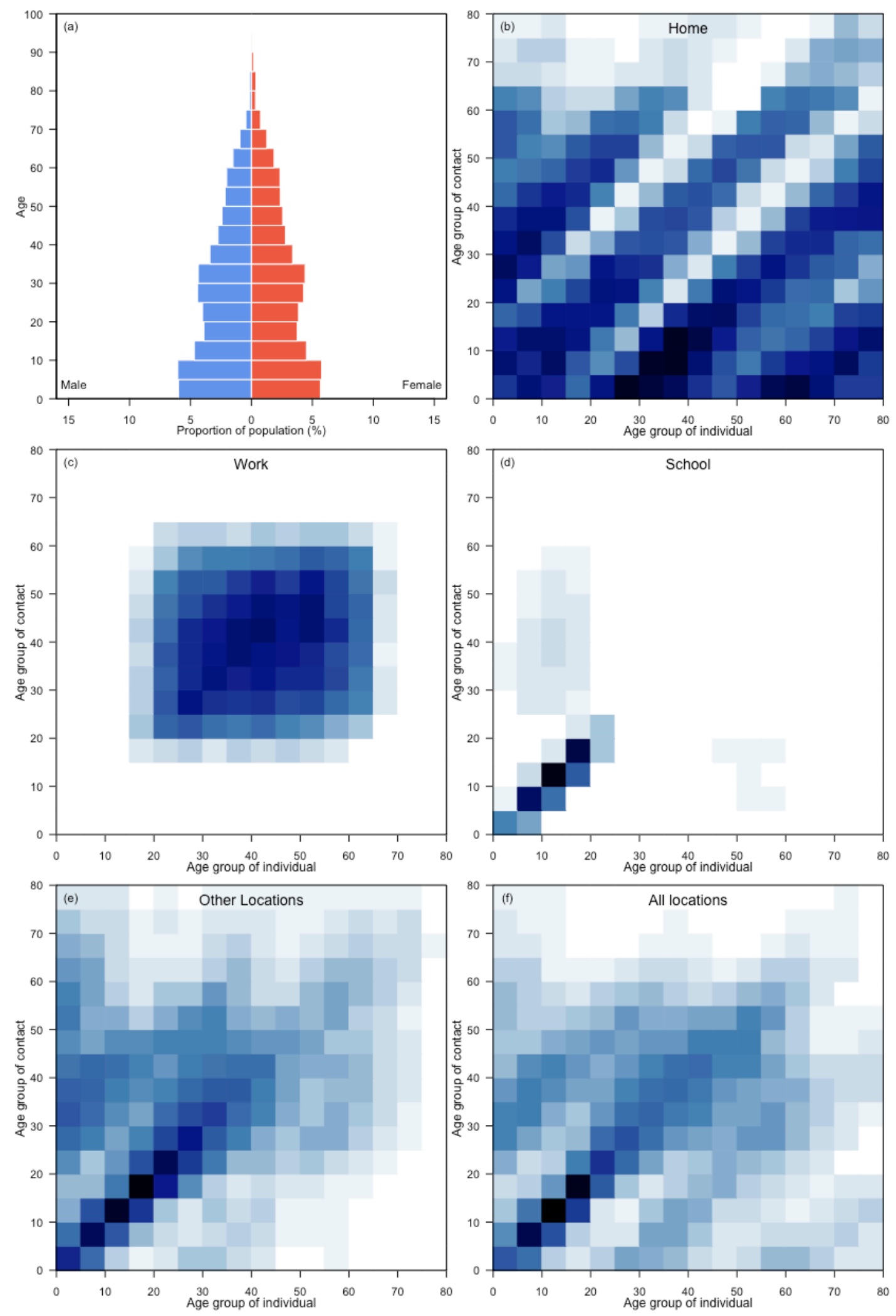


\section{Lao People's Democratic Republic}
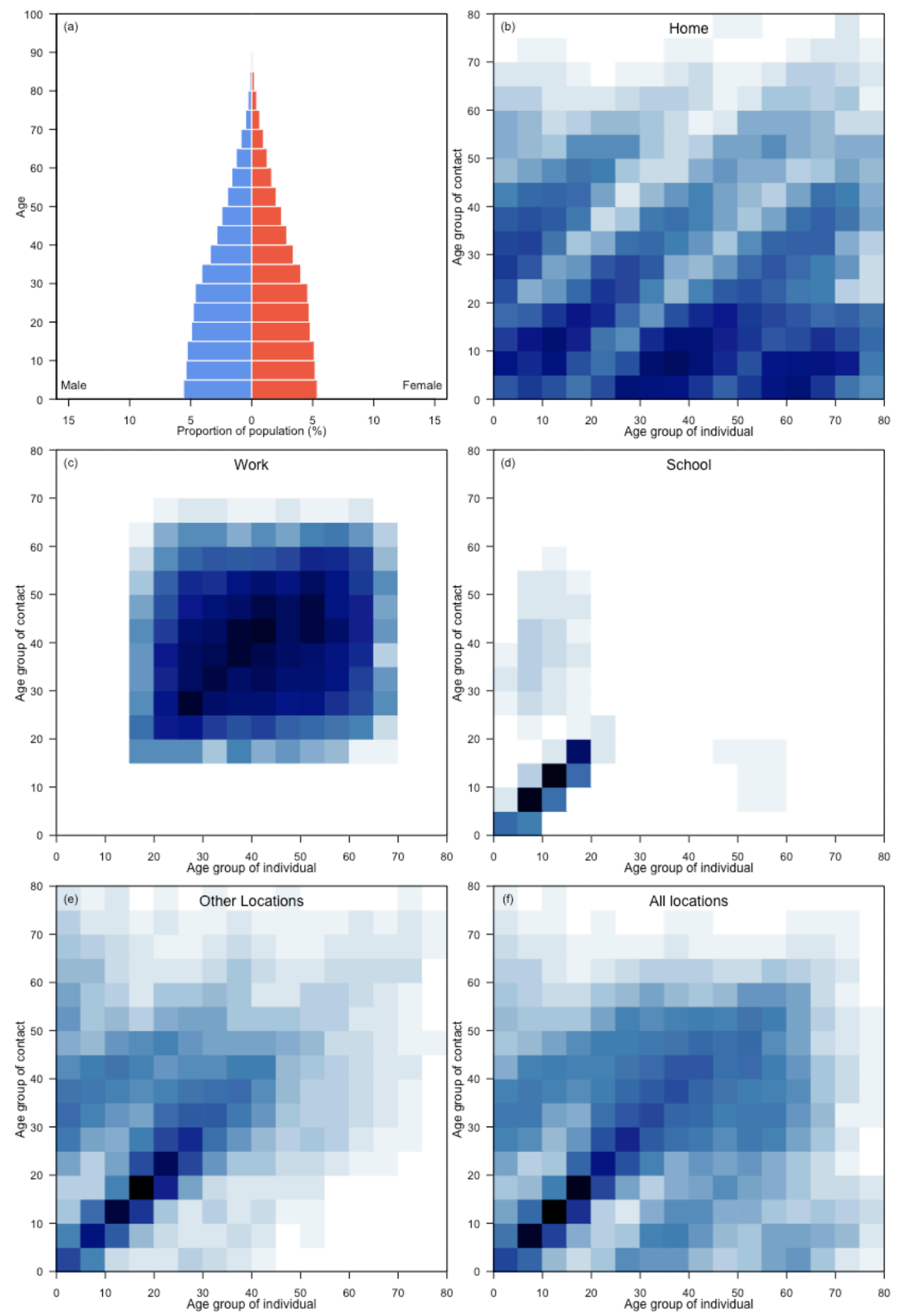


\section{Latvia}
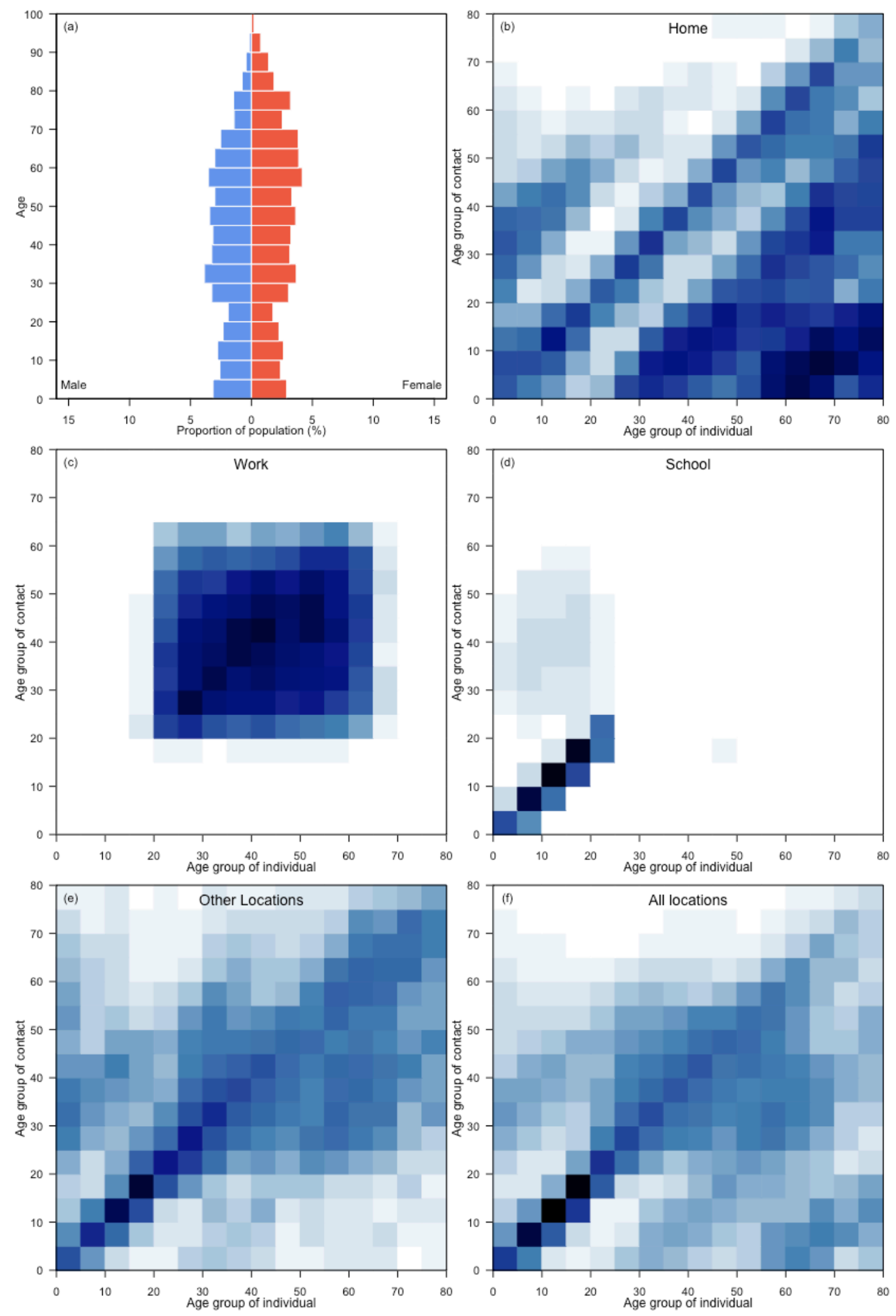


\section{Lesotho}
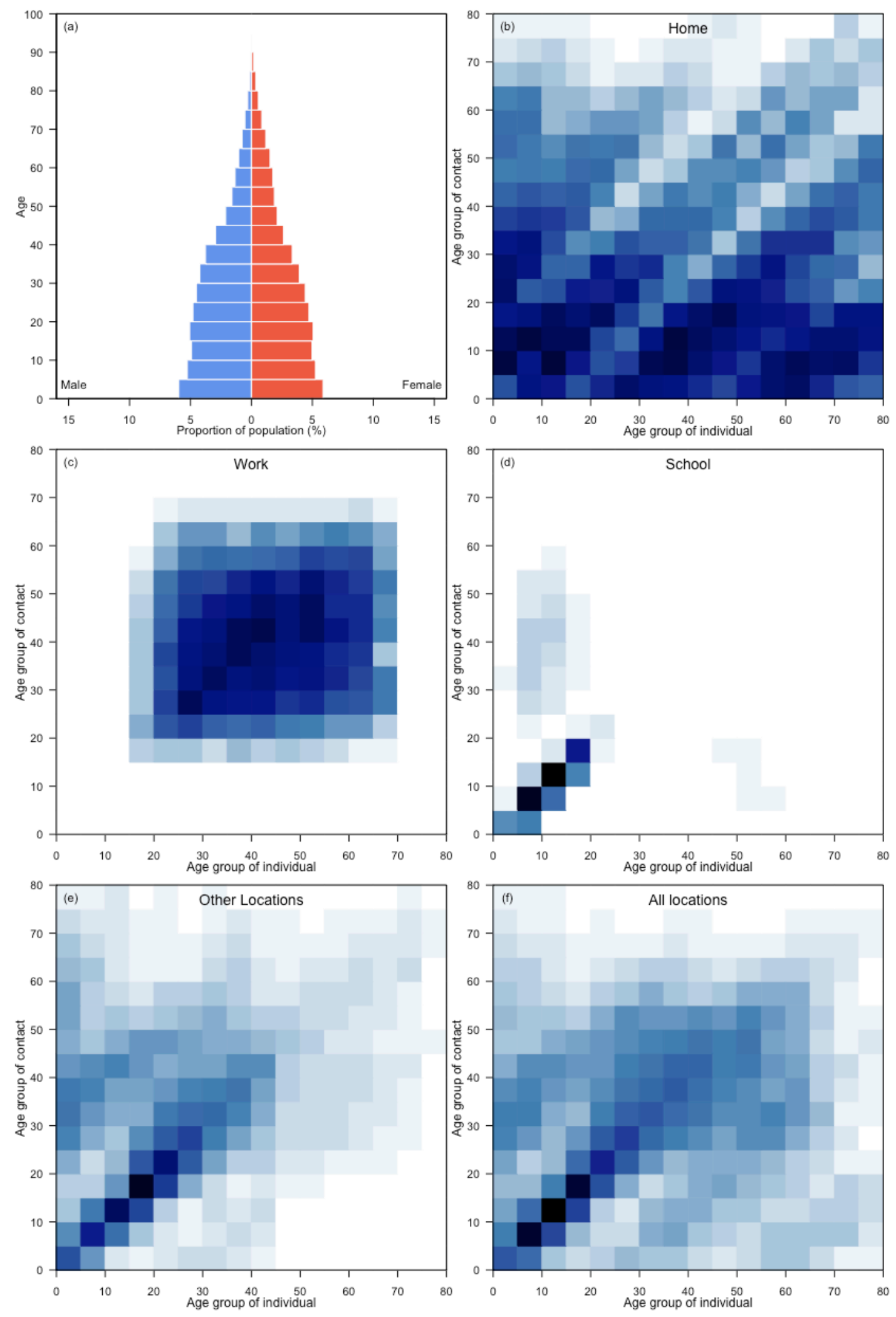


\section{Liberia}
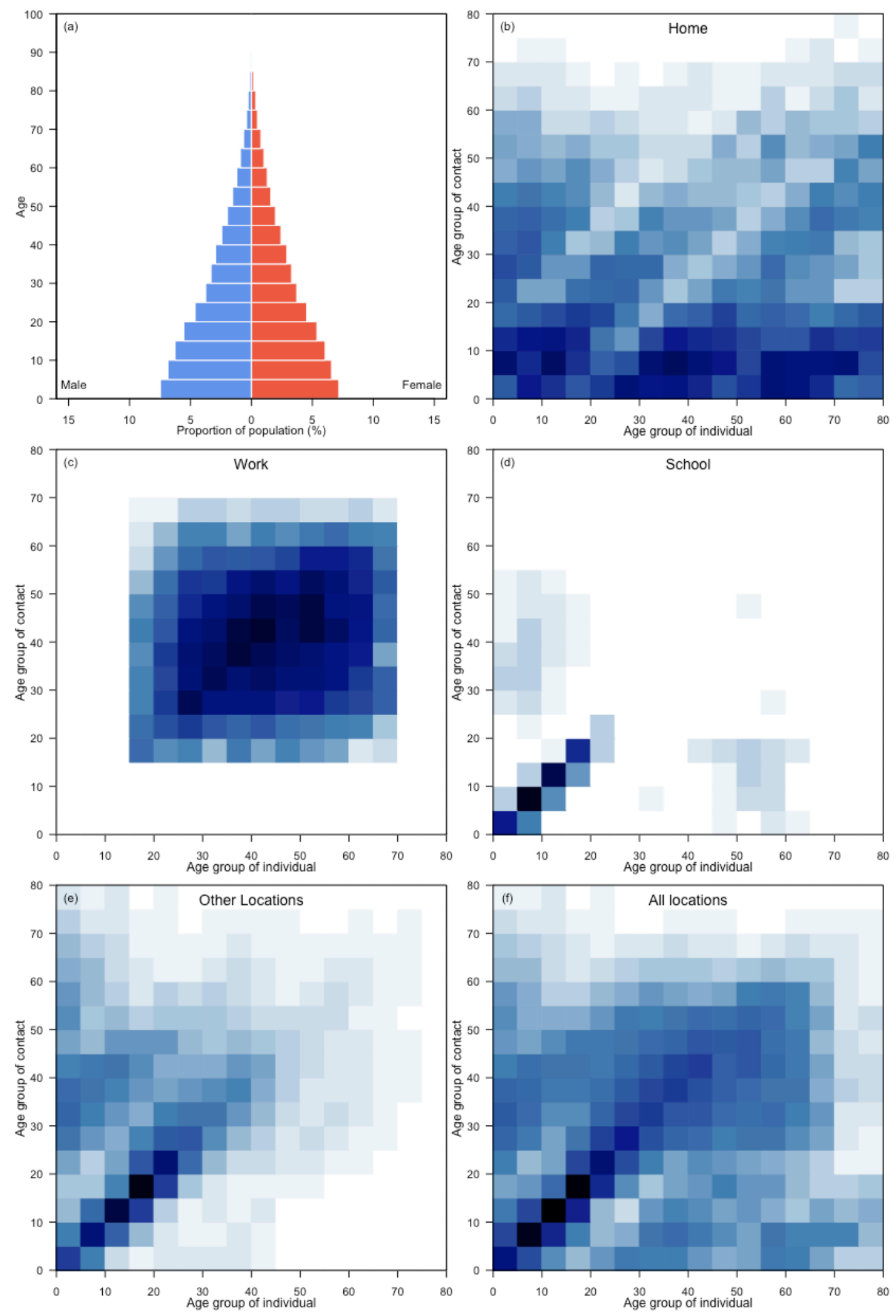


\section{Libya}
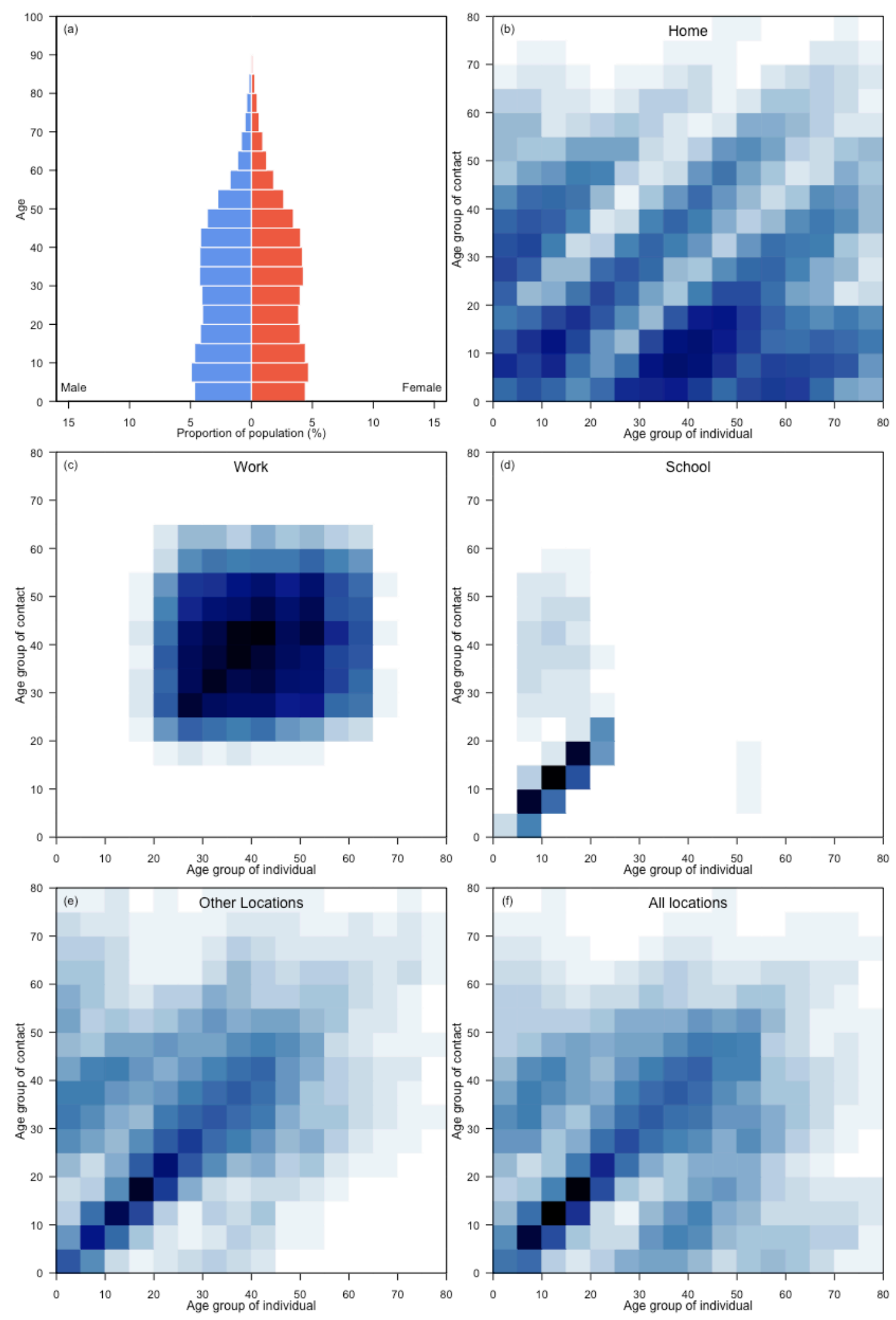


\section{Lithuania}
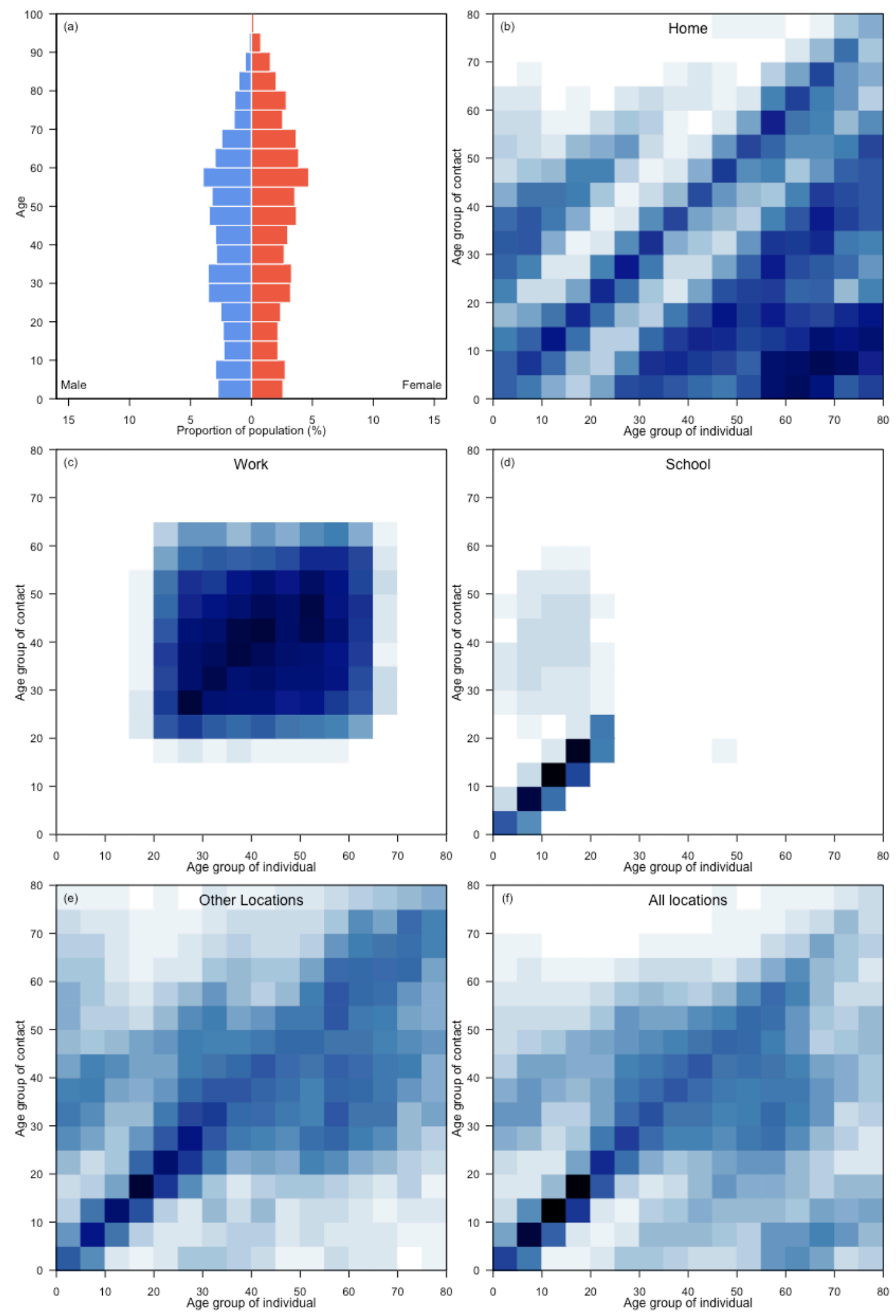


\section{Luxembourg}
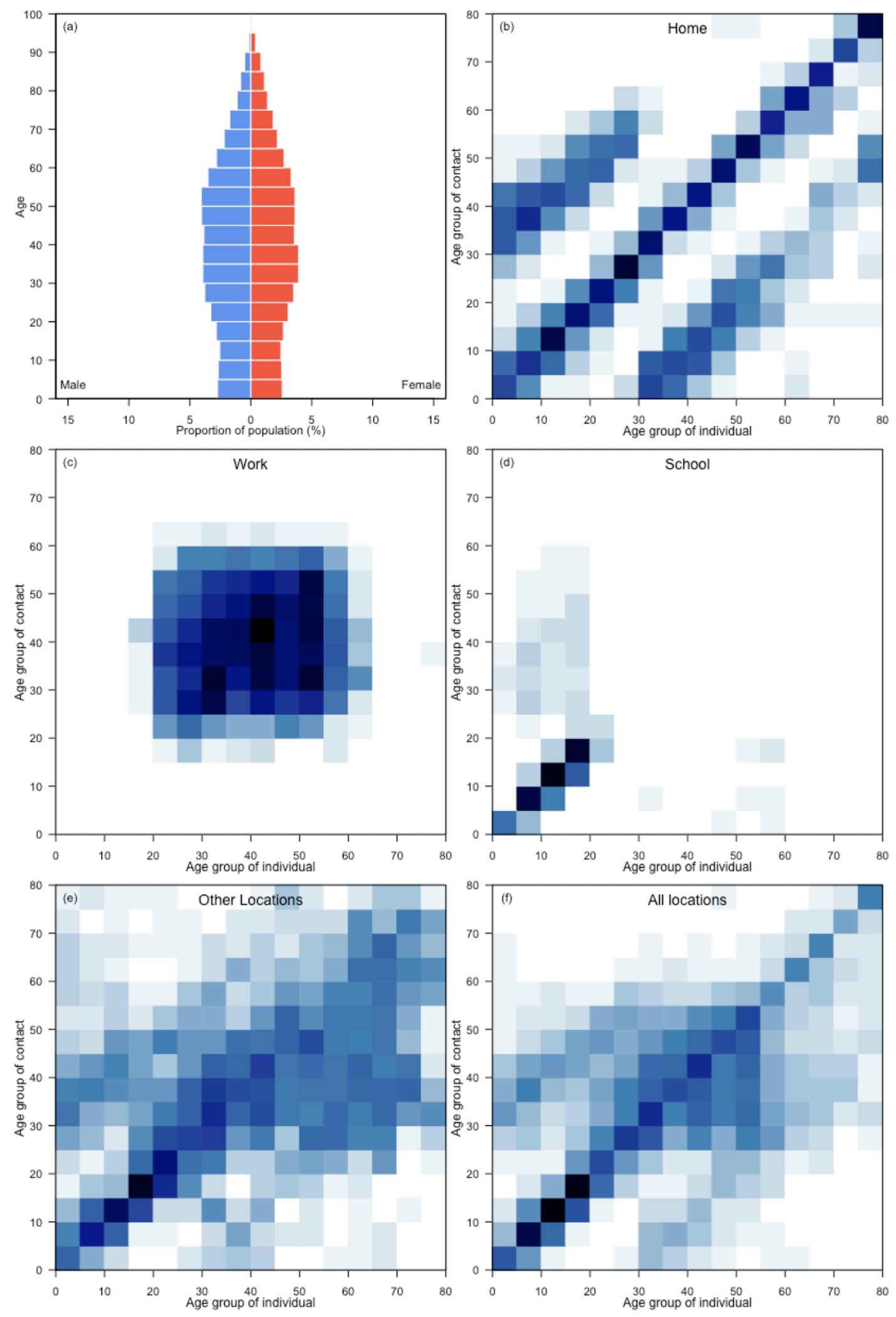
Madagascar
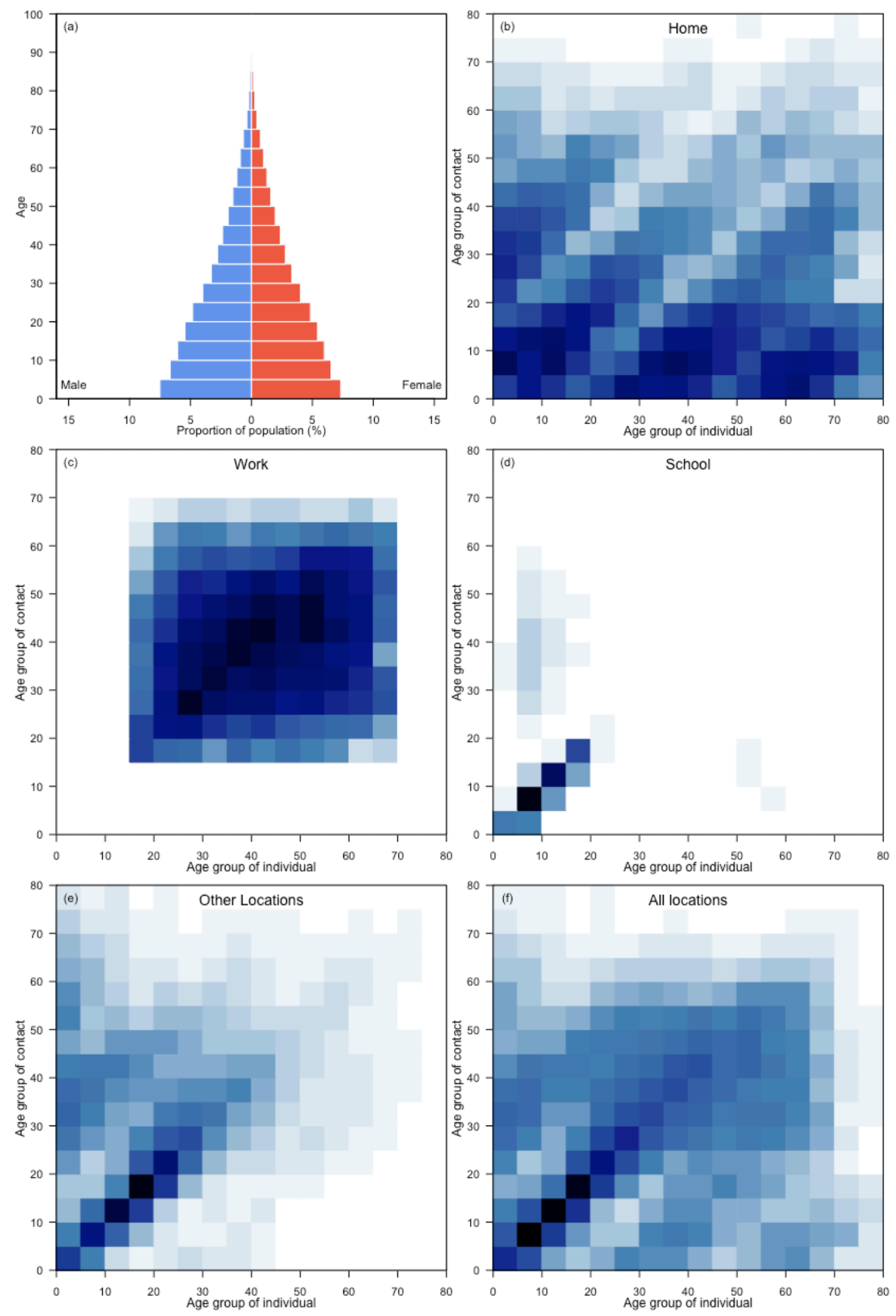


\section{Malawi}
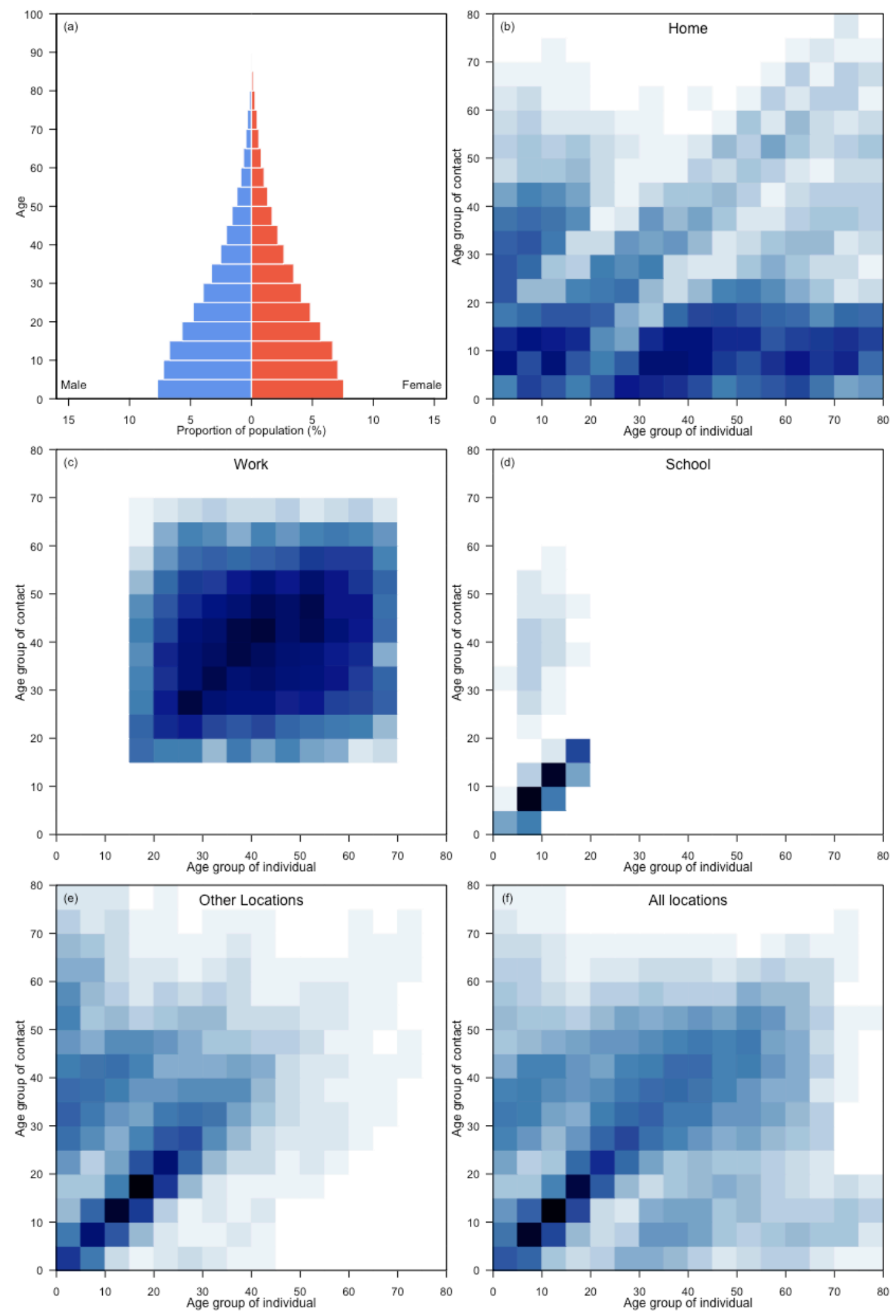


\section{Malaysia}
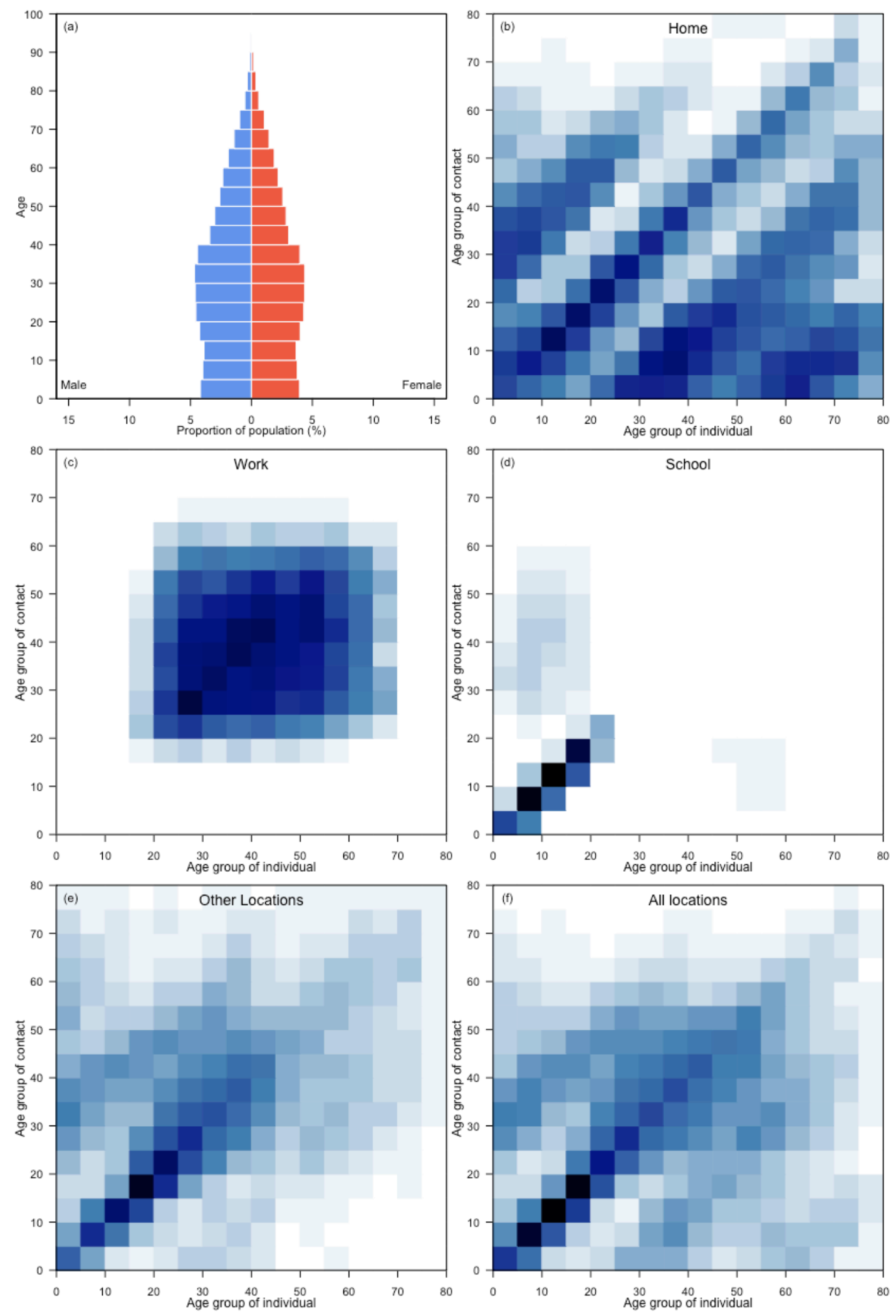


\section{Maldives}
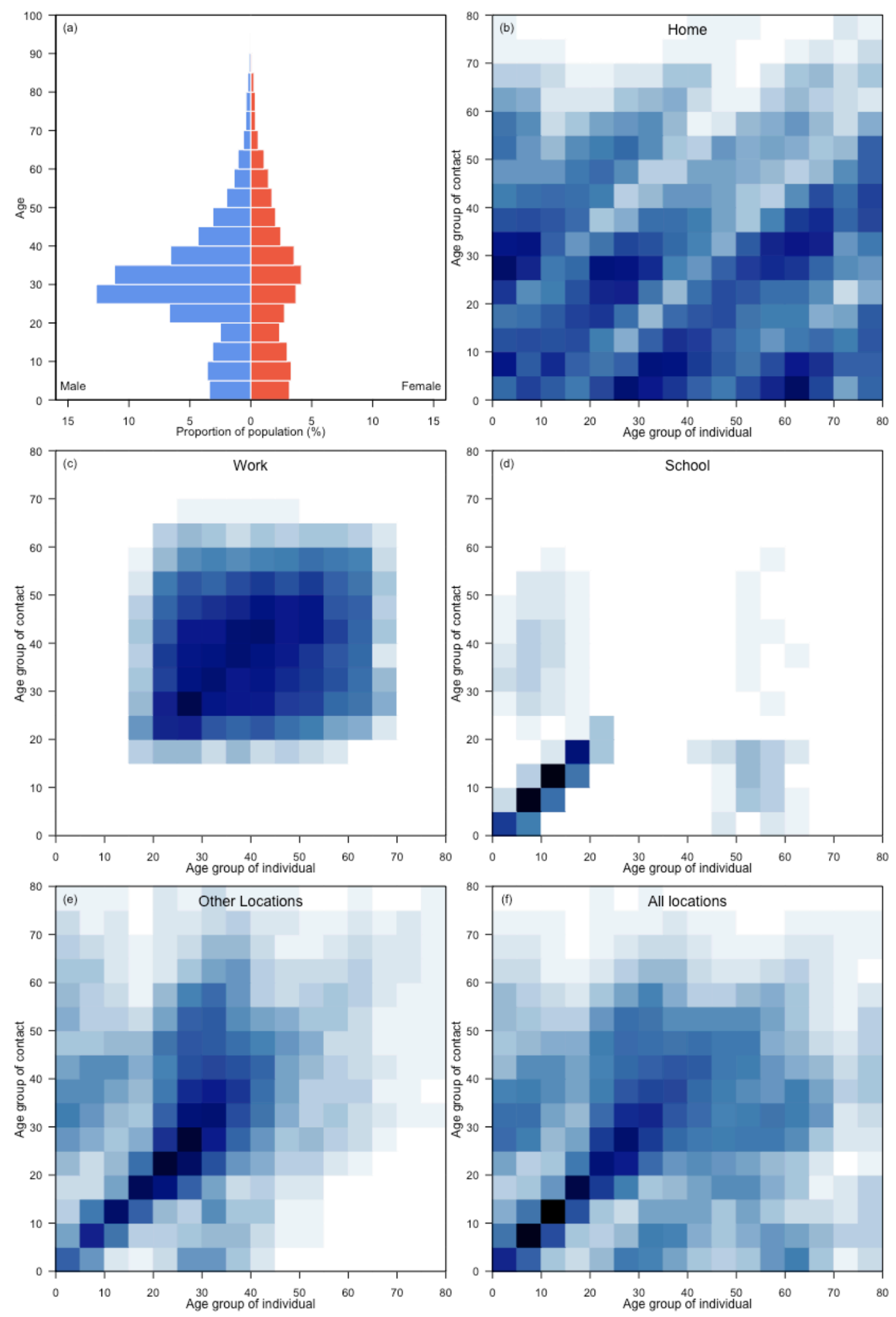


\section{Mali}
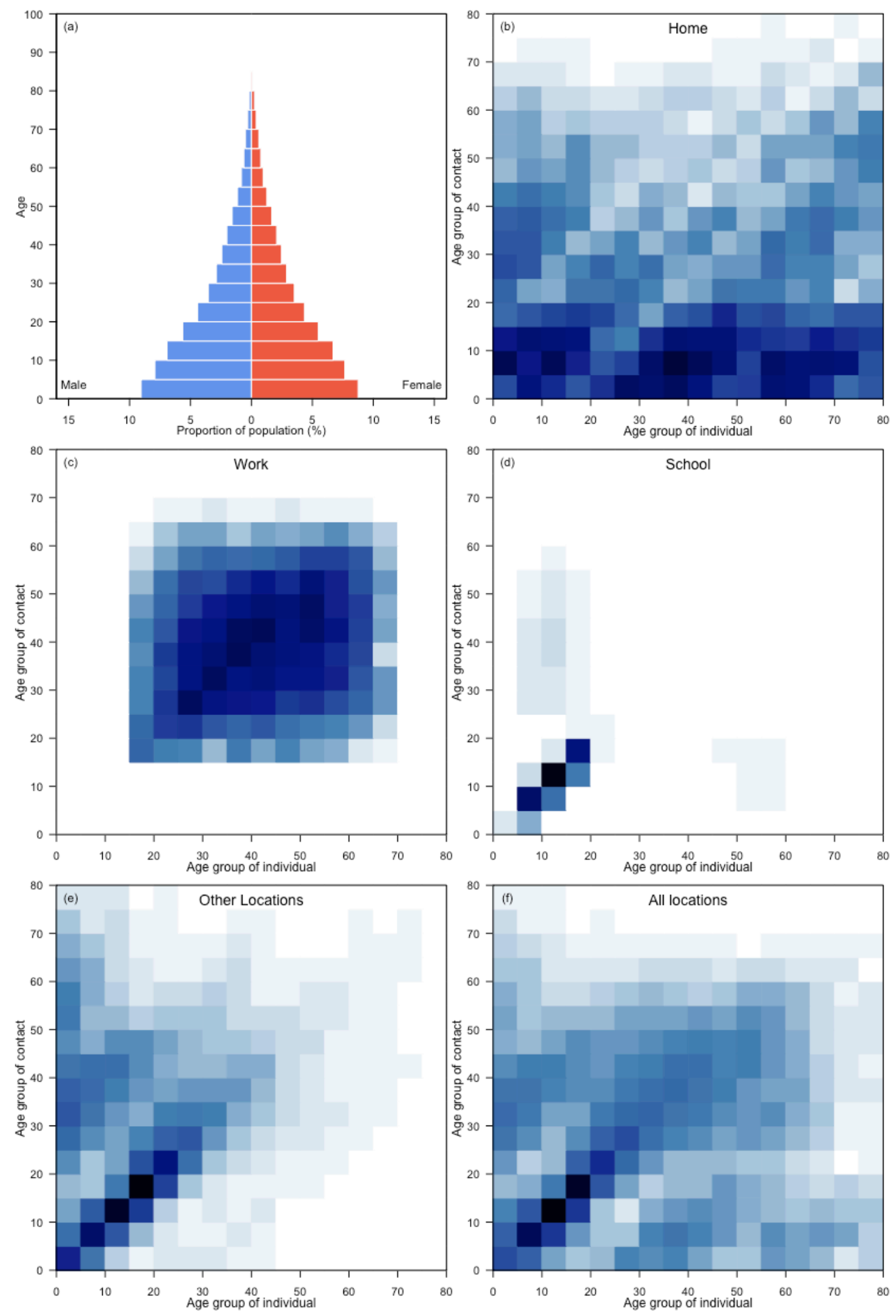


\section{Malta}
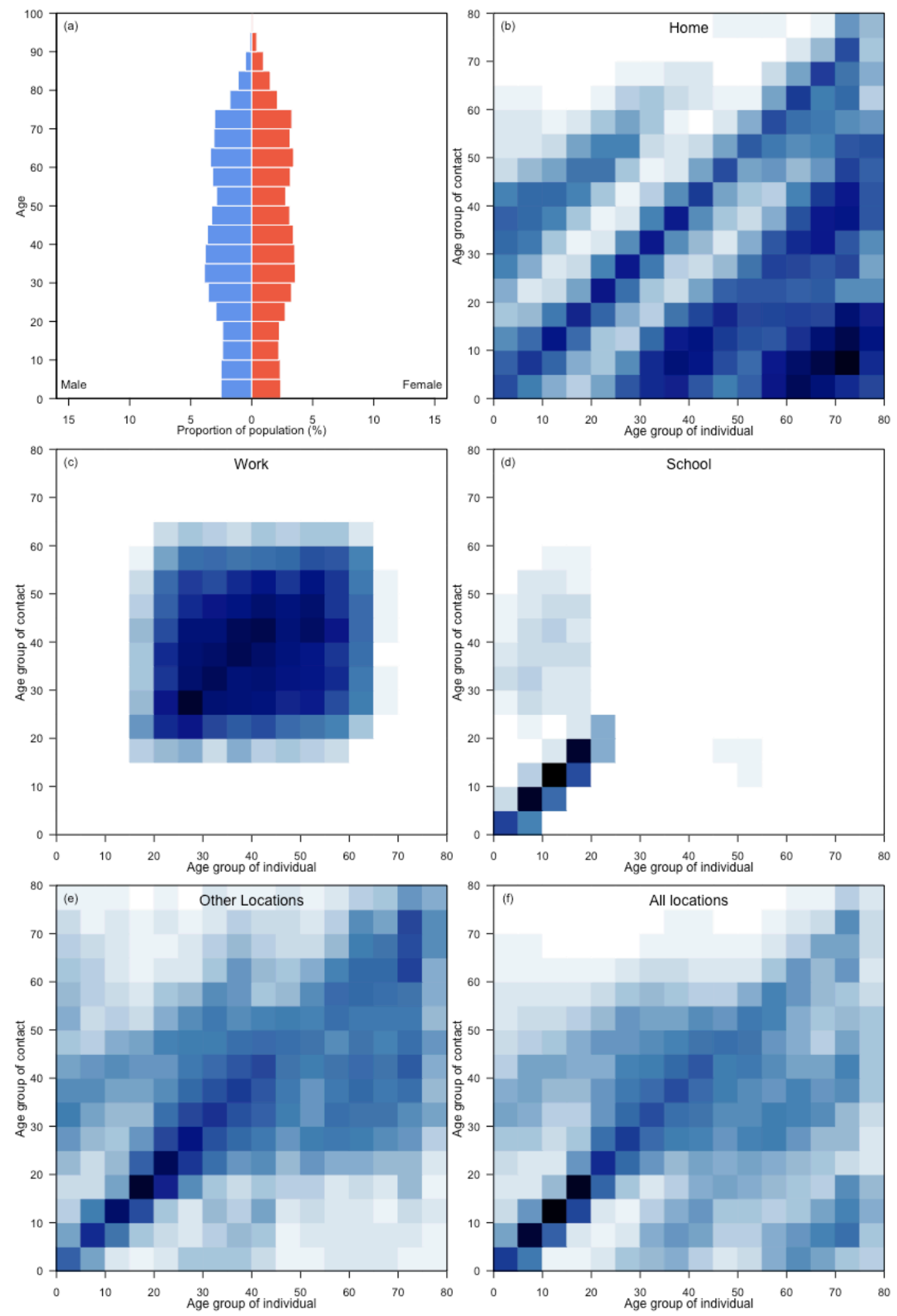


\section{Mauritania}
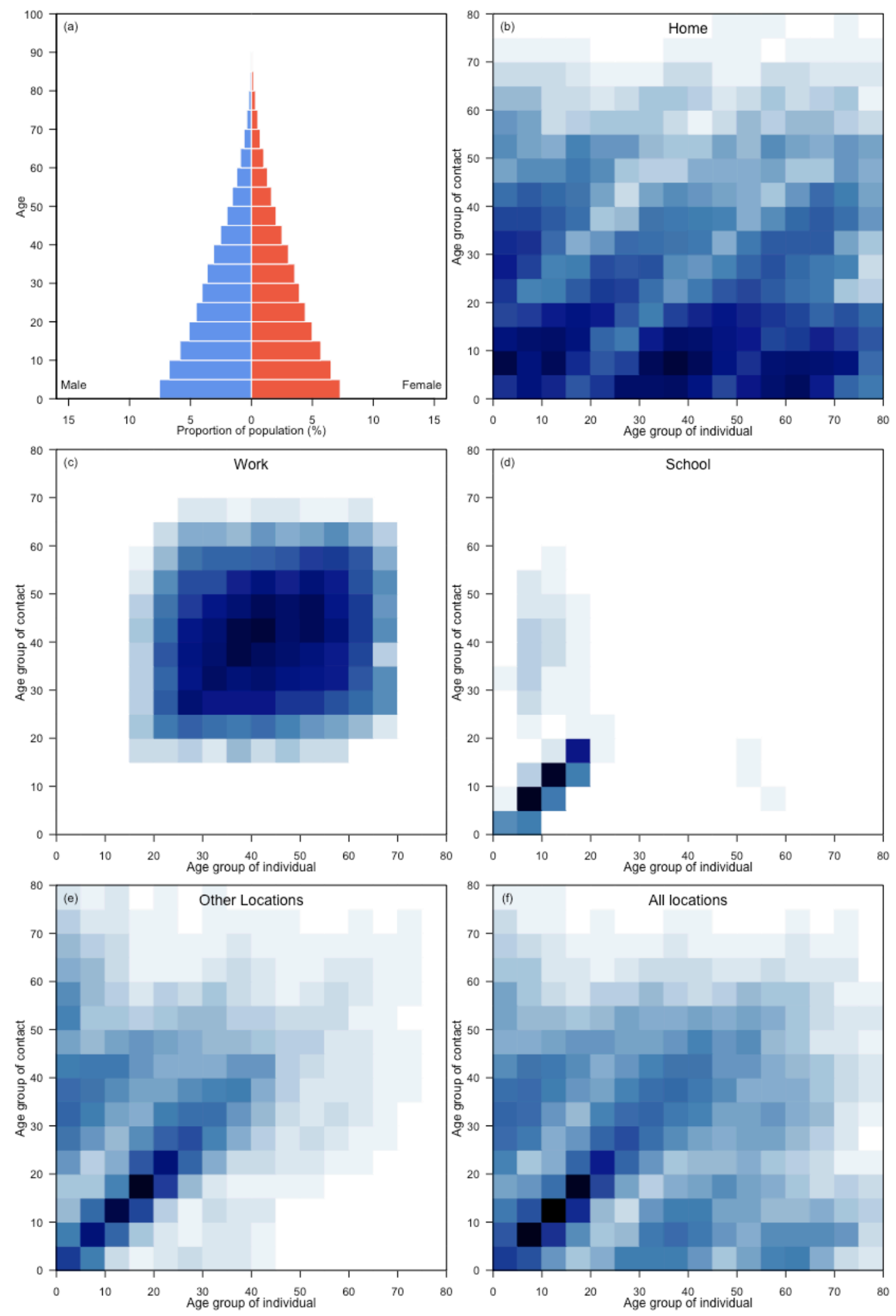


\section{Mauritius}
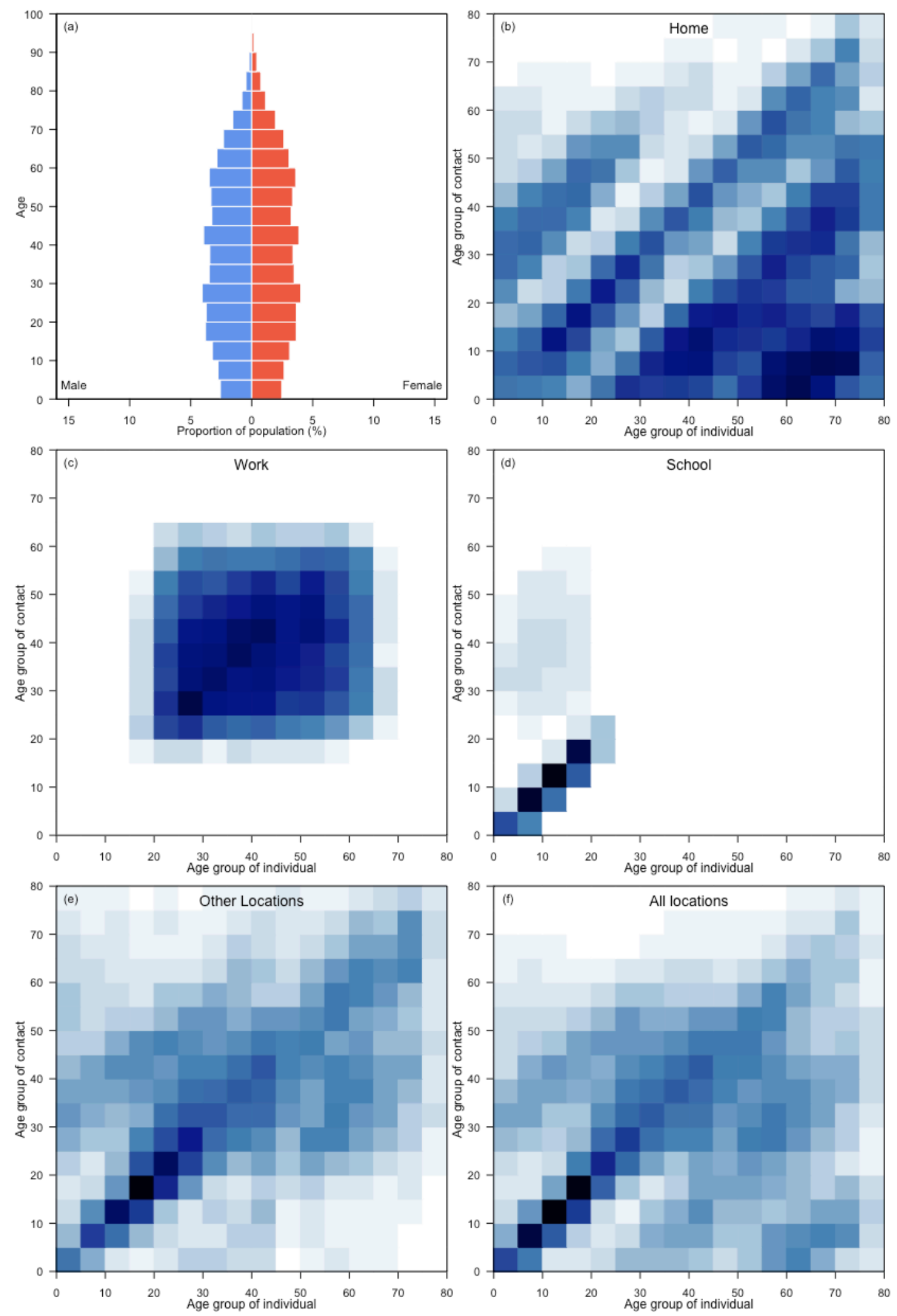


\section{Mexico}
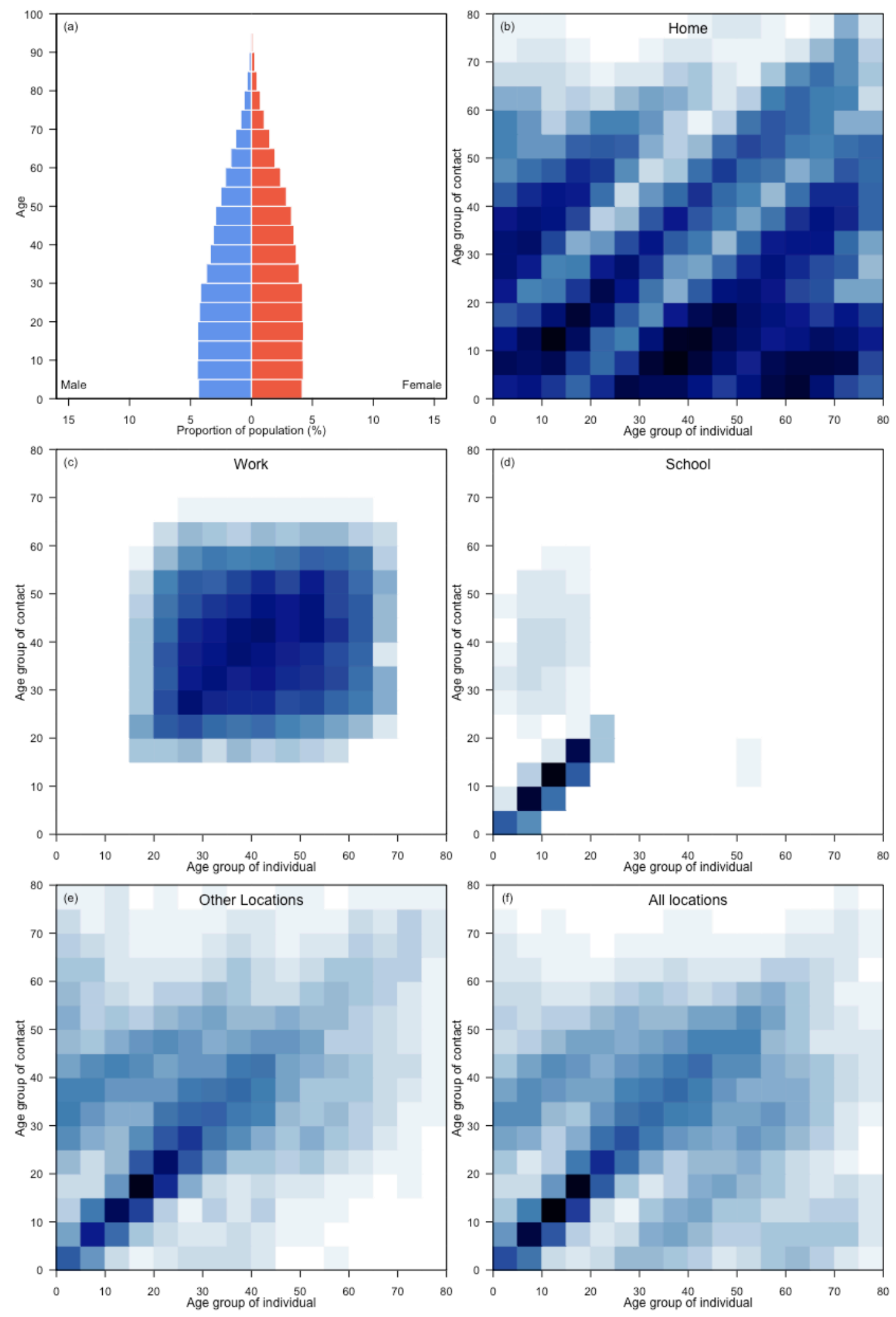
Mongolia
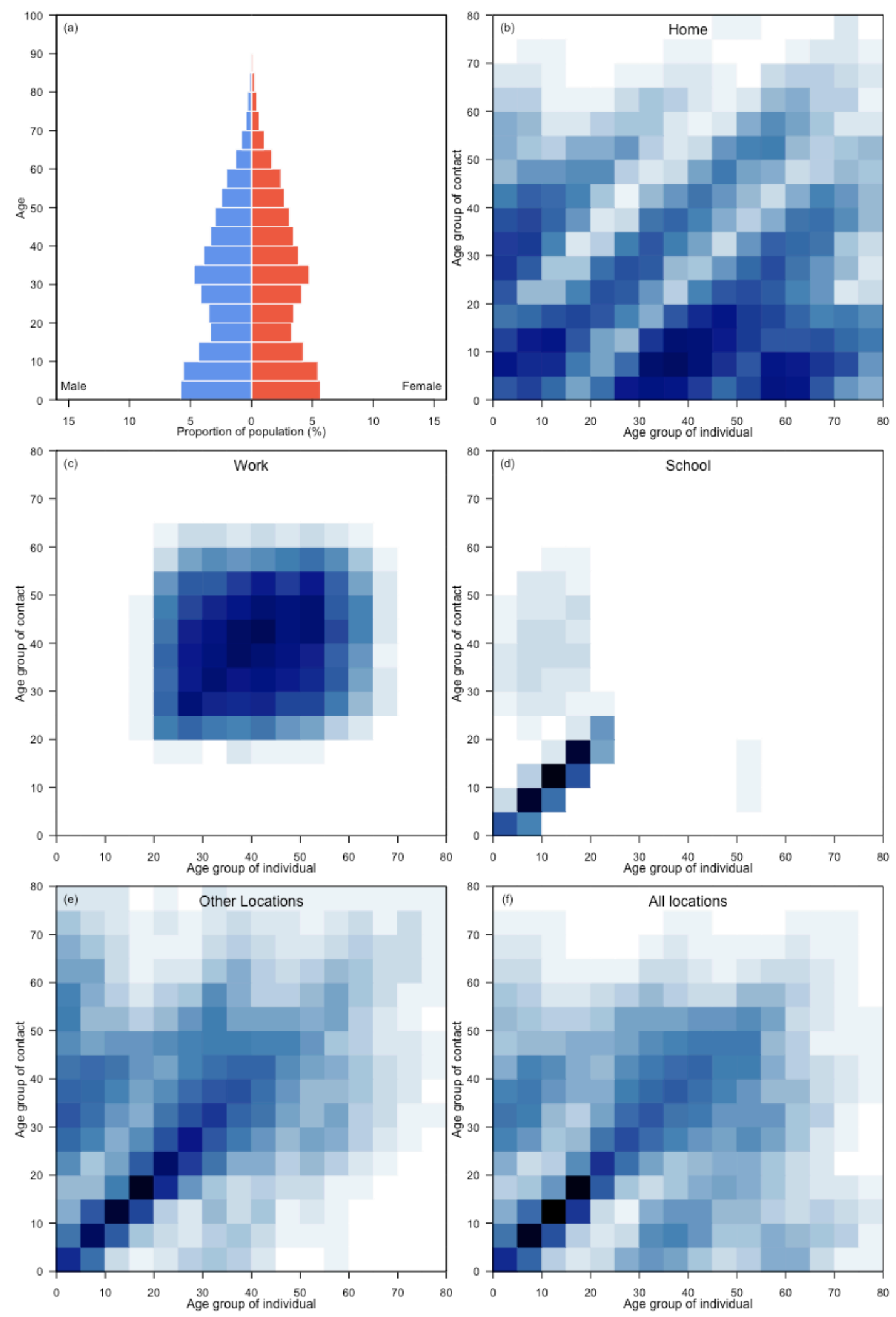
Montenegro
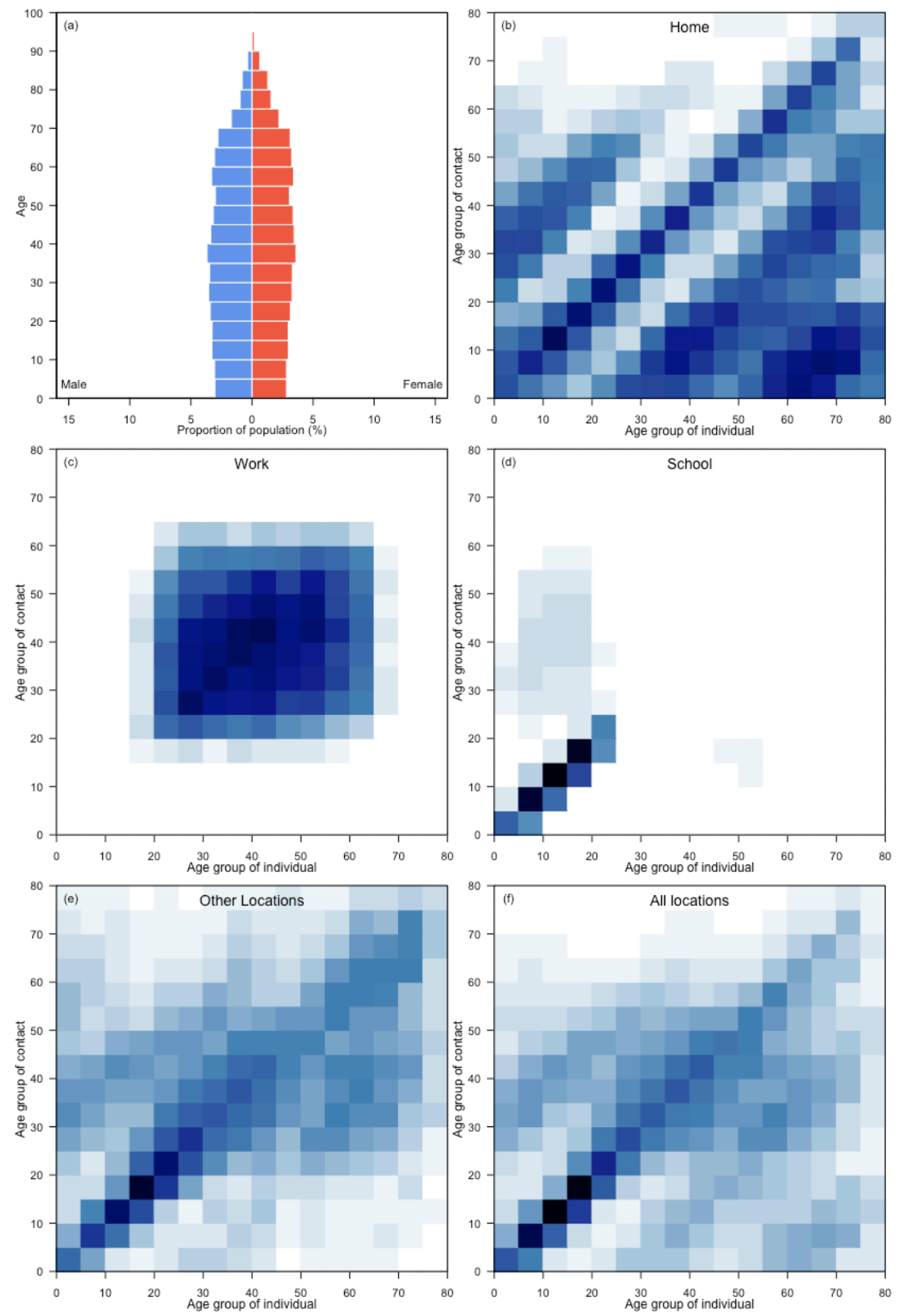
Morocco
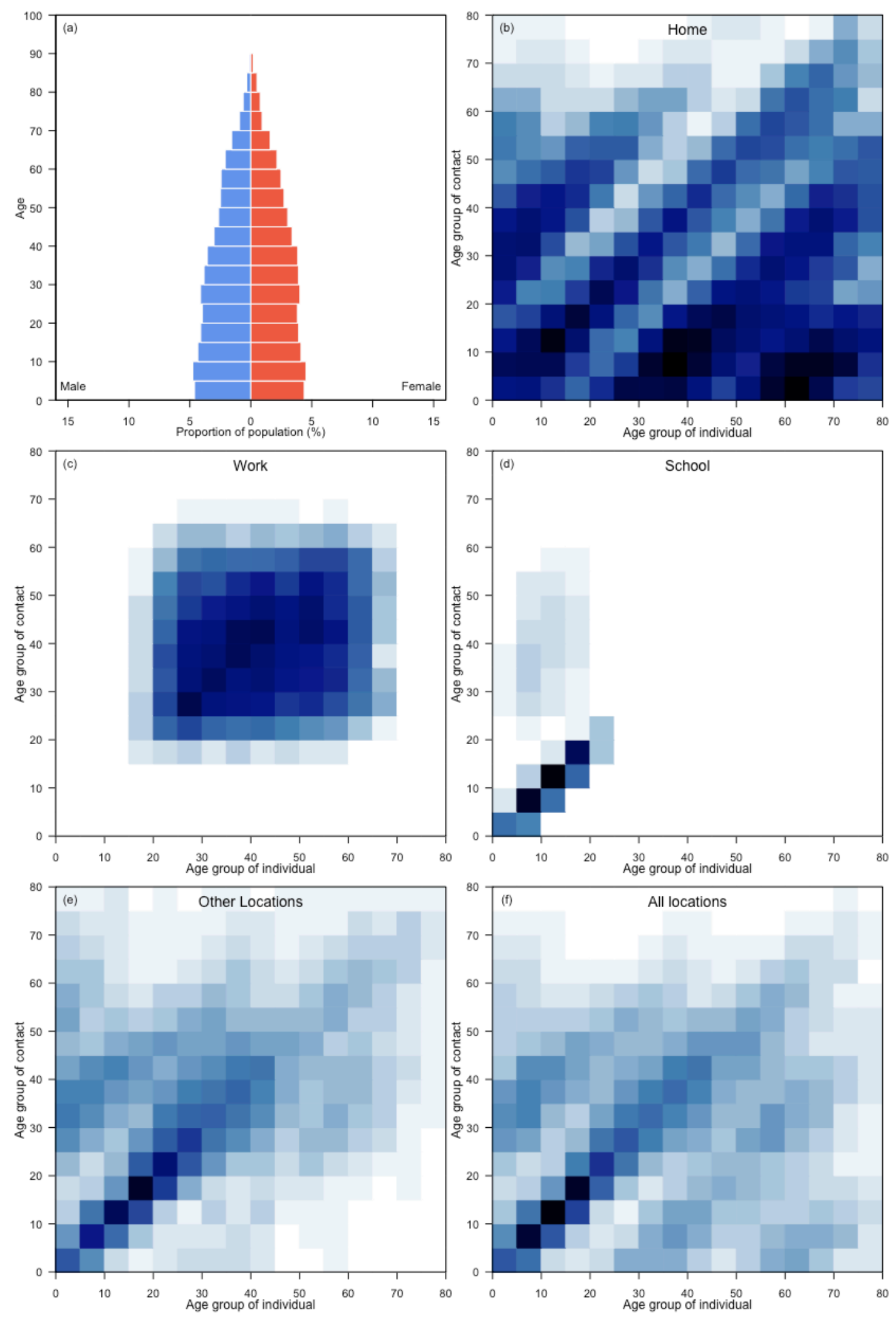
Mozambique
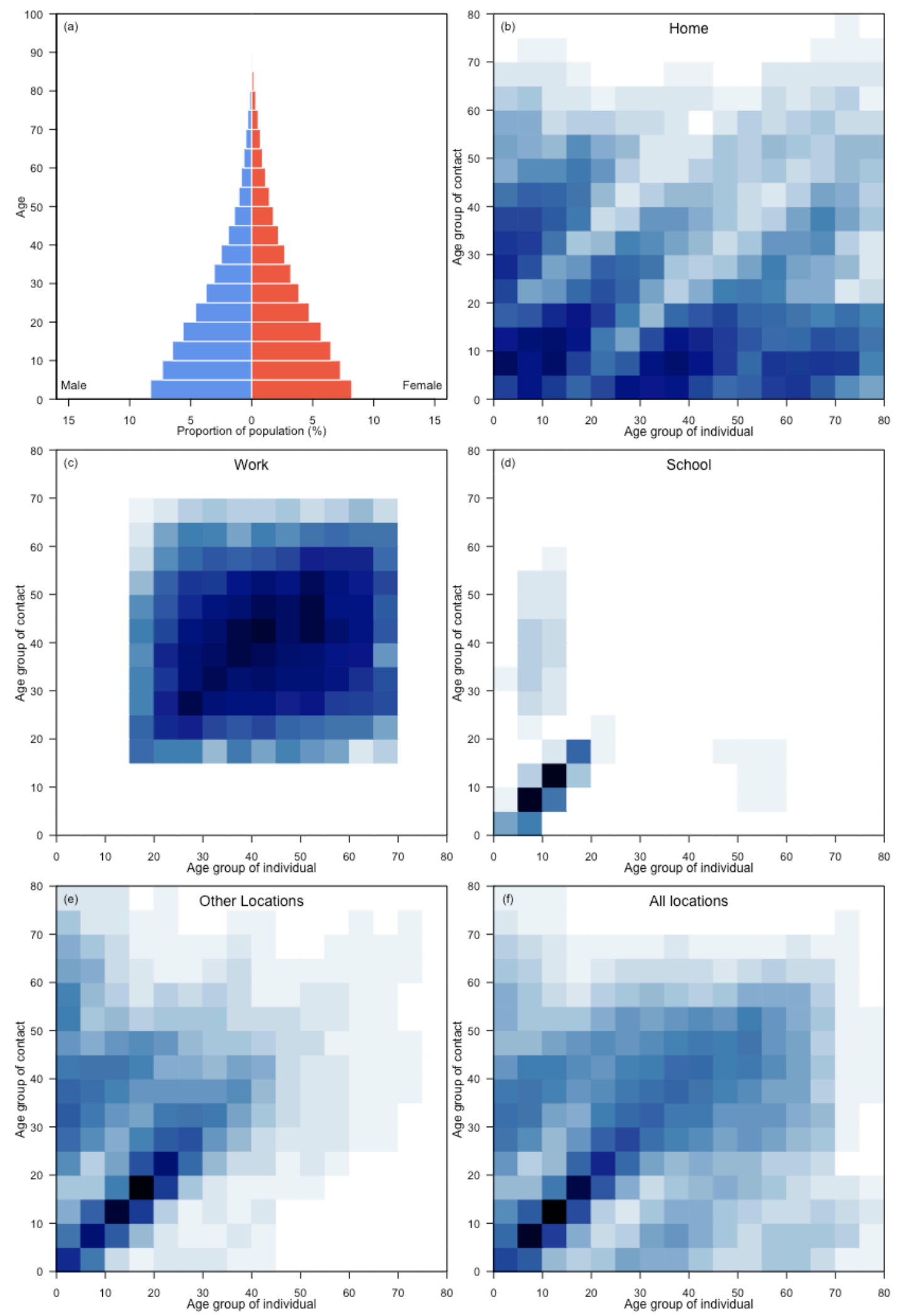


\section{Myanmar}
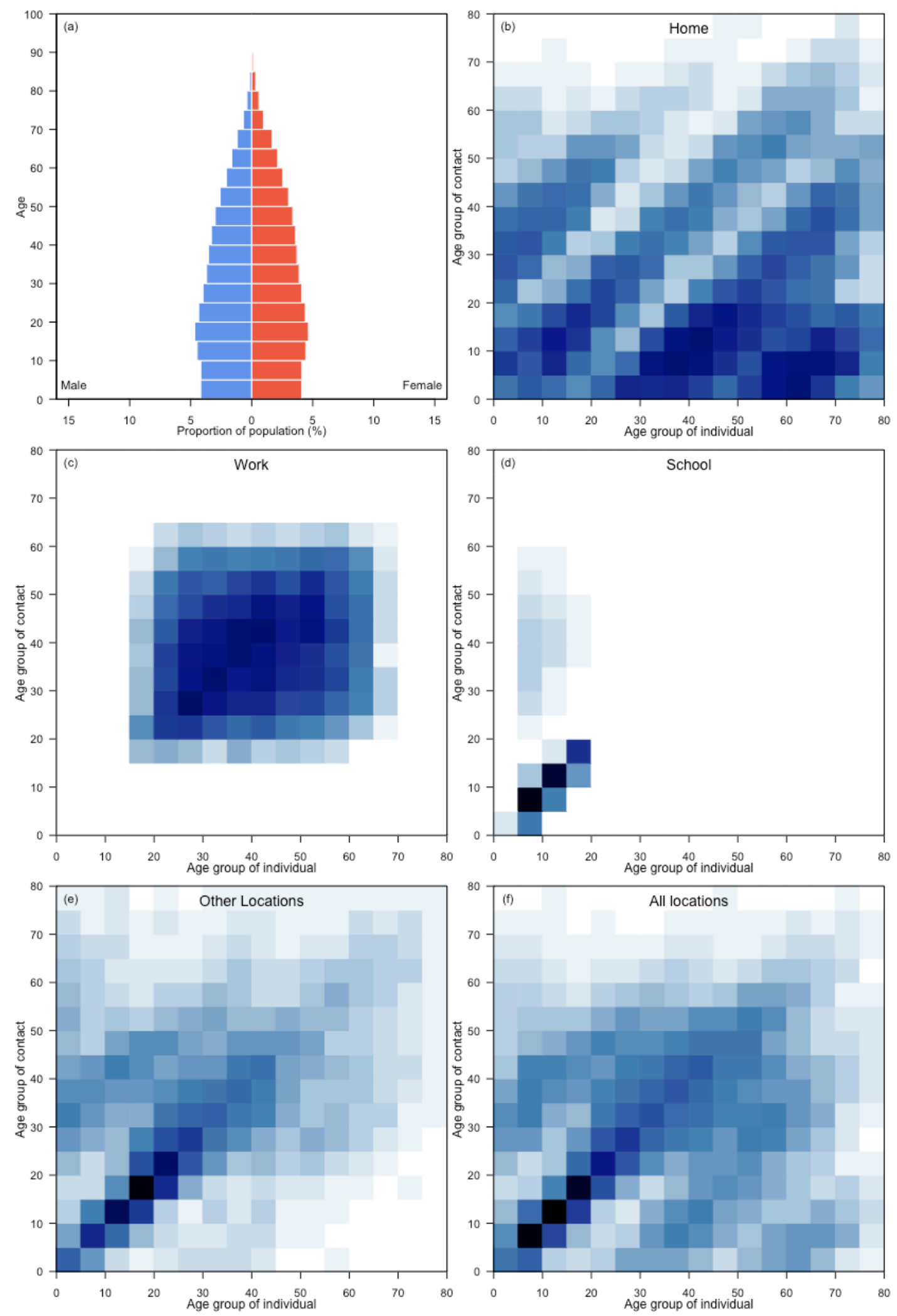


\section{Namibia}
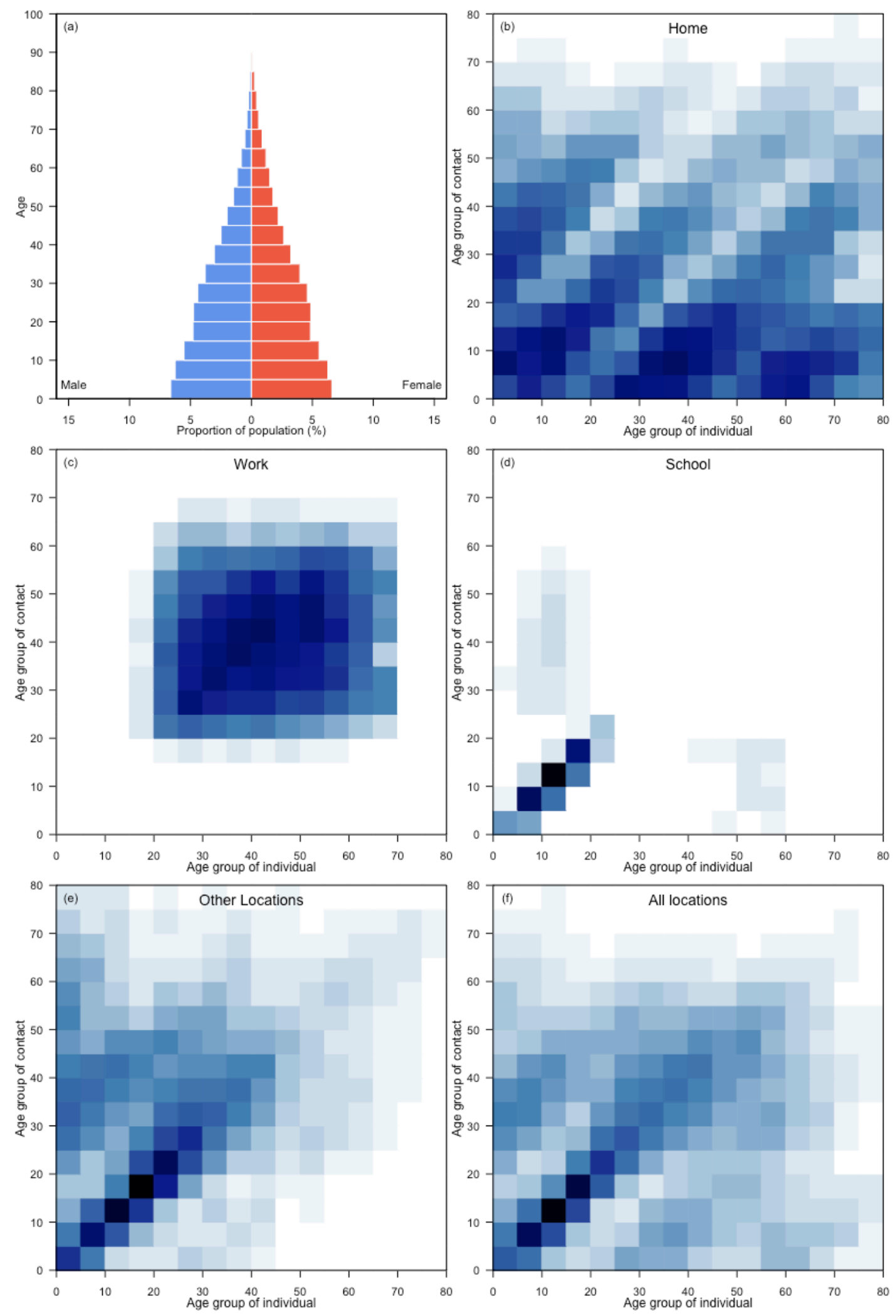


\section{Nepal}
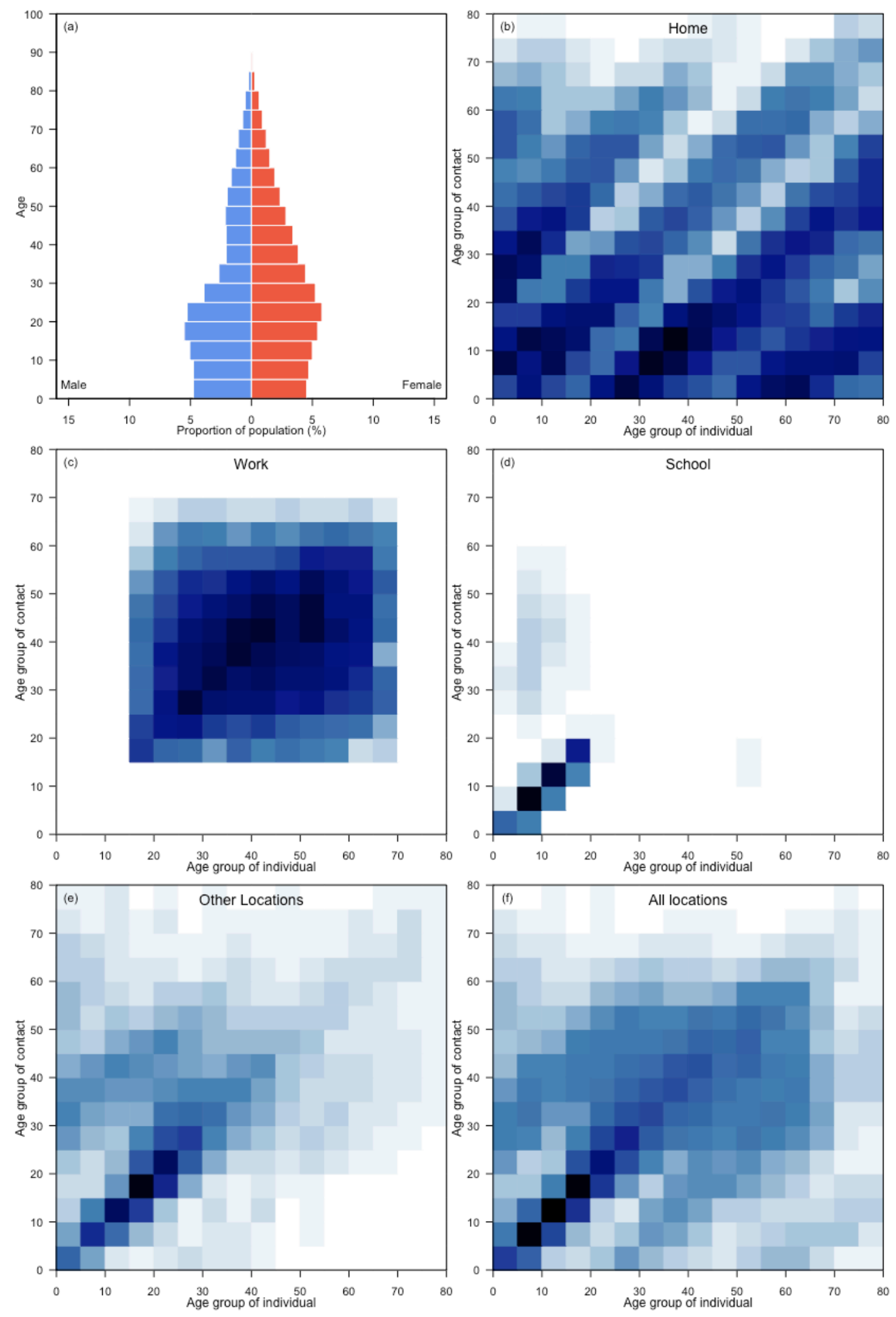
Netherlands
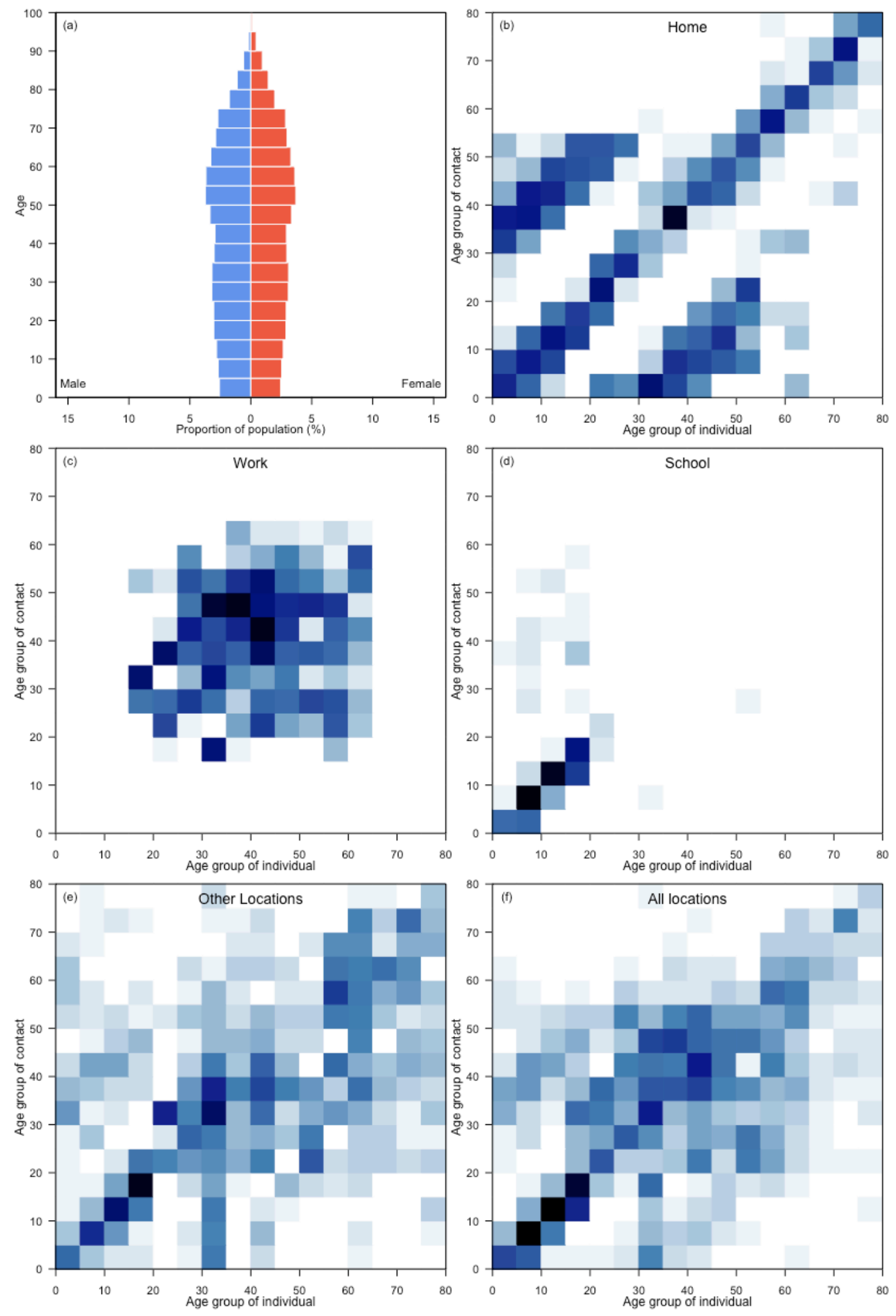
New Zealand
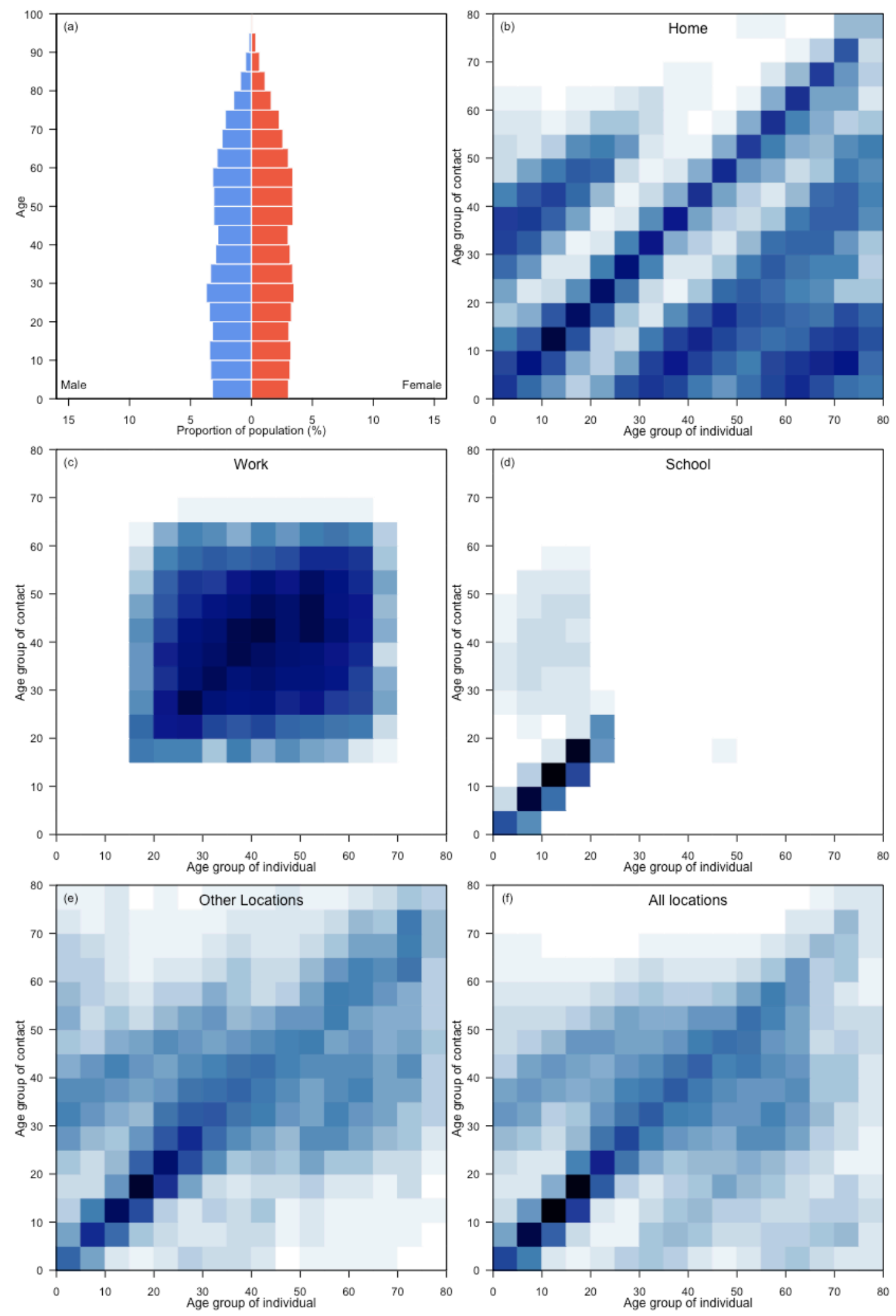
Nicaragua
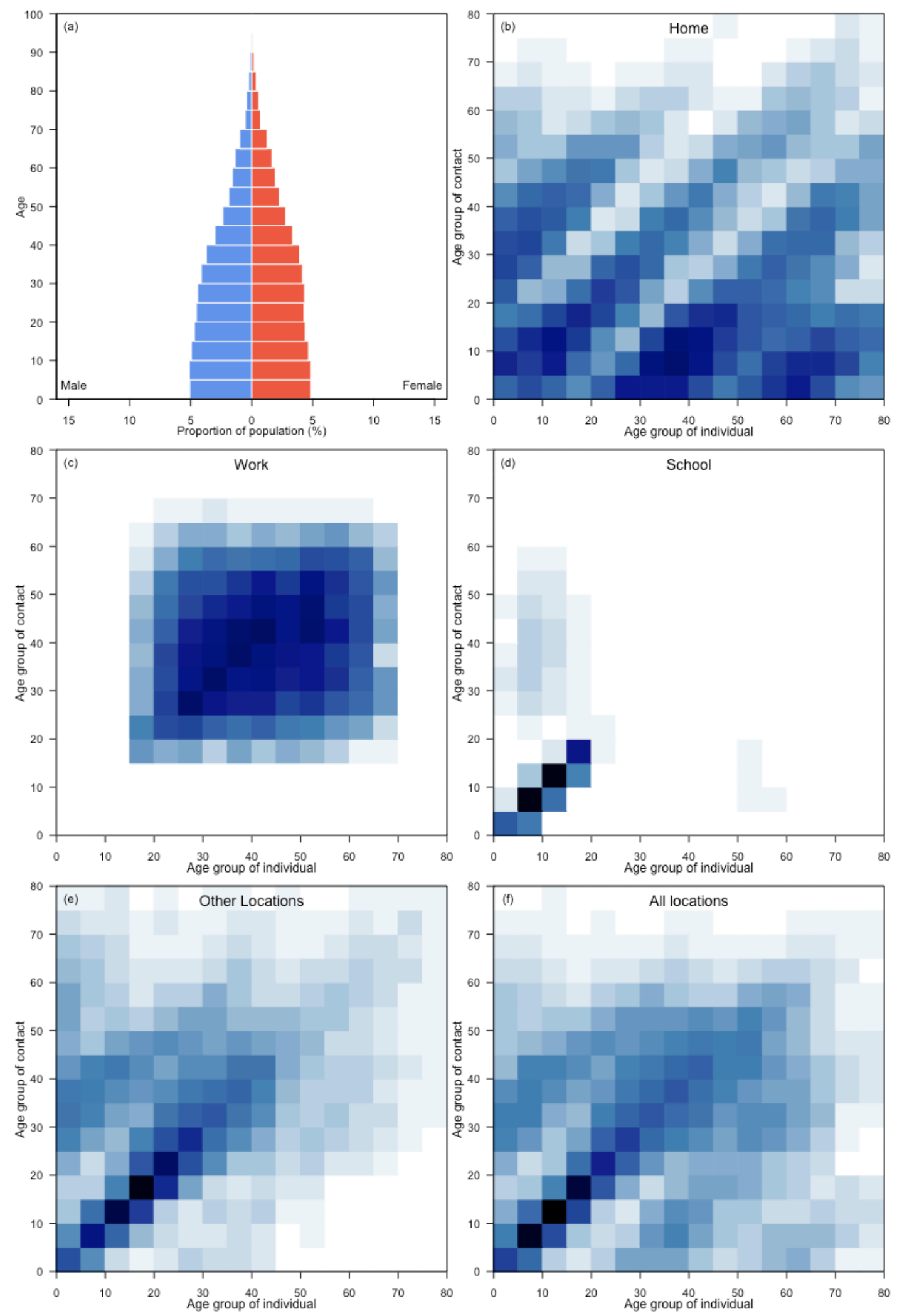
Niger
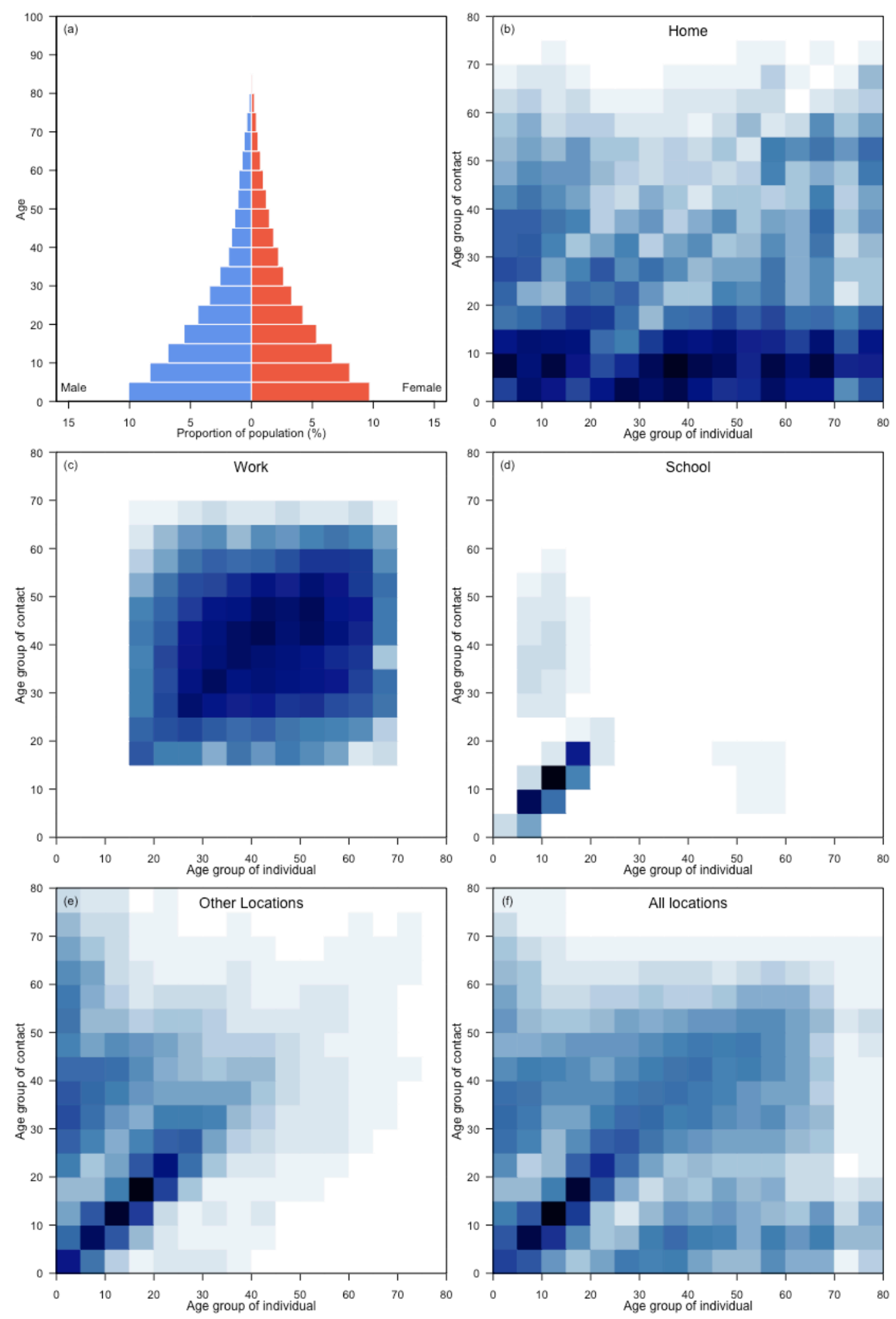


\section{Nigeria}
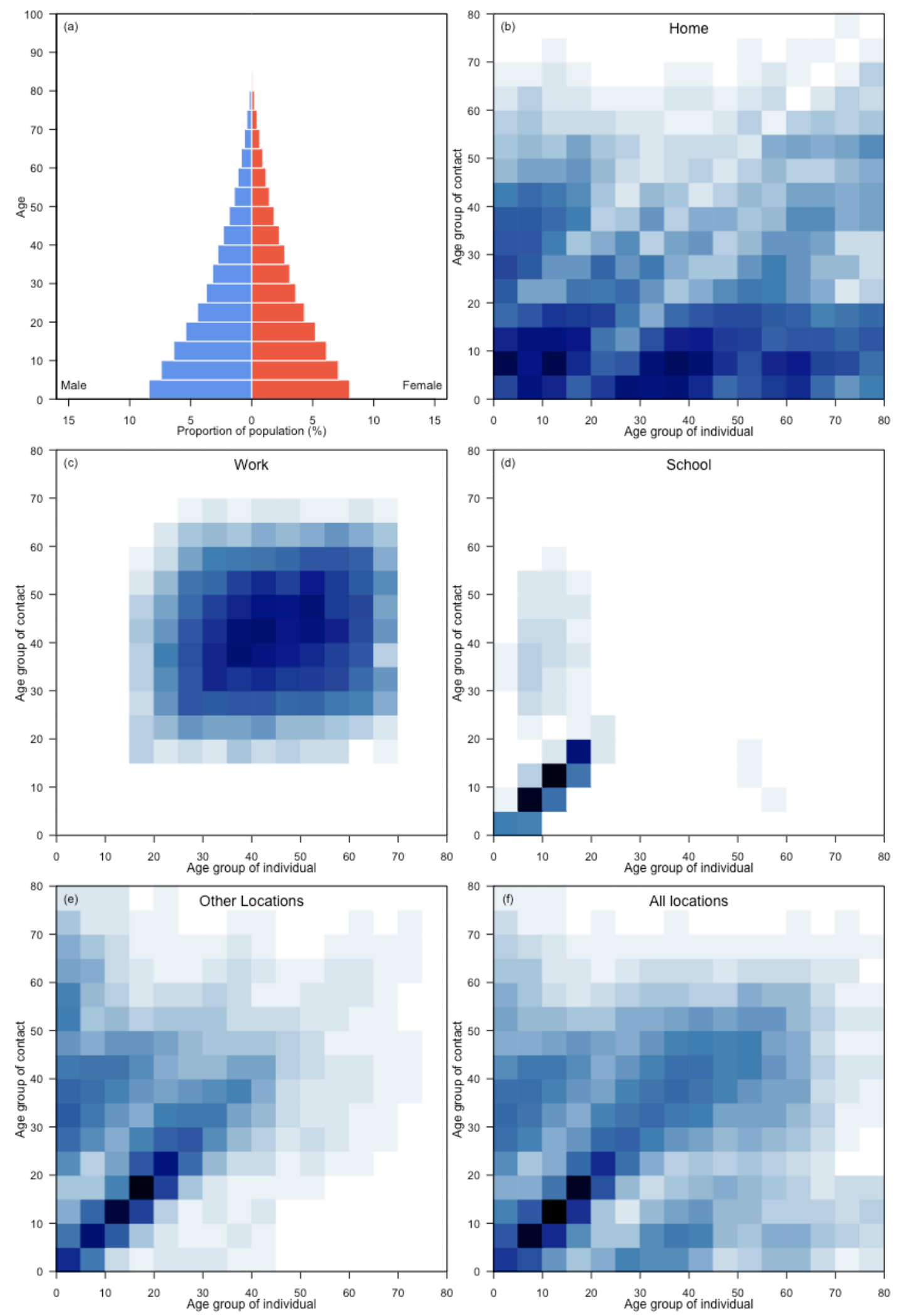
North Macedonia
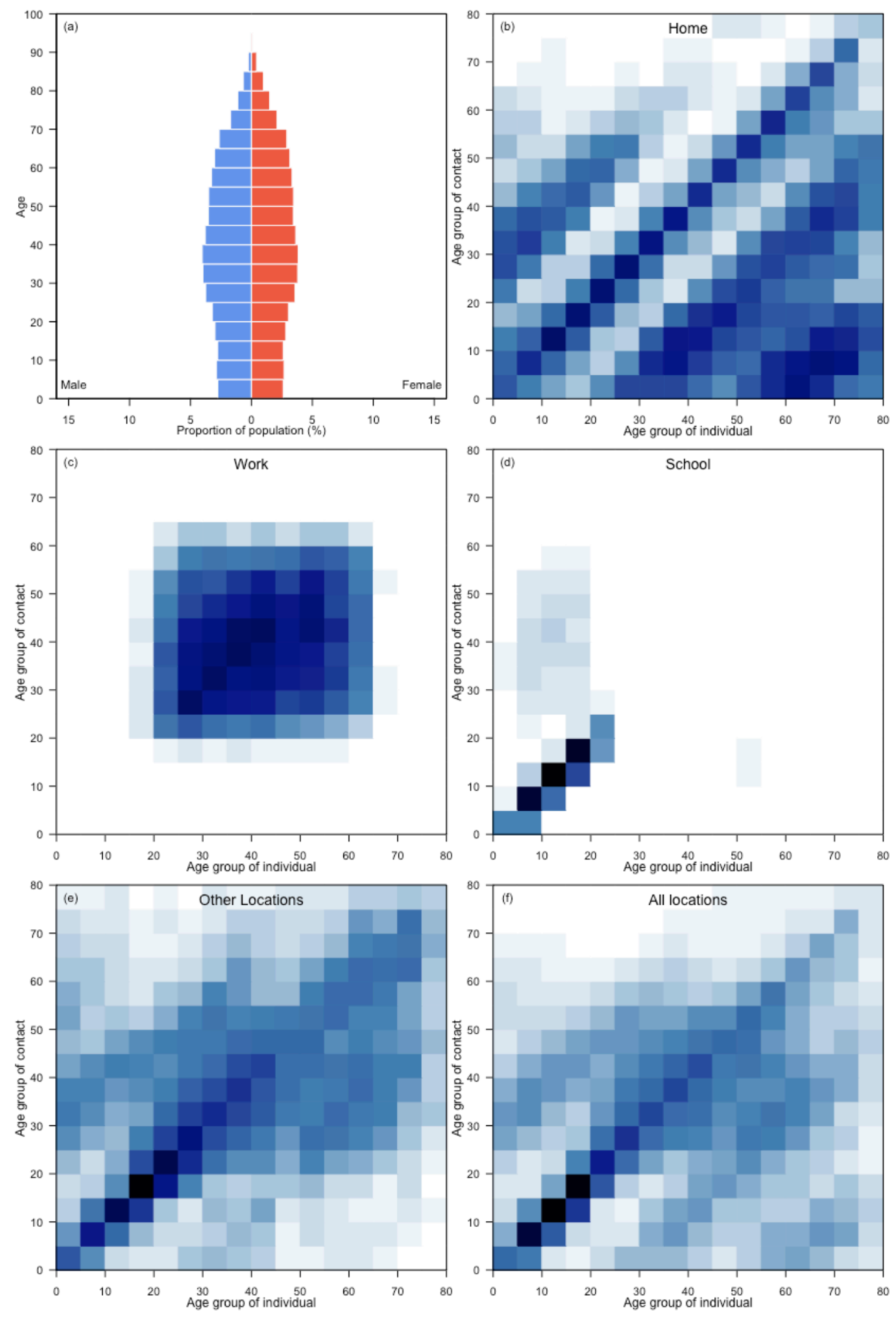
Norway
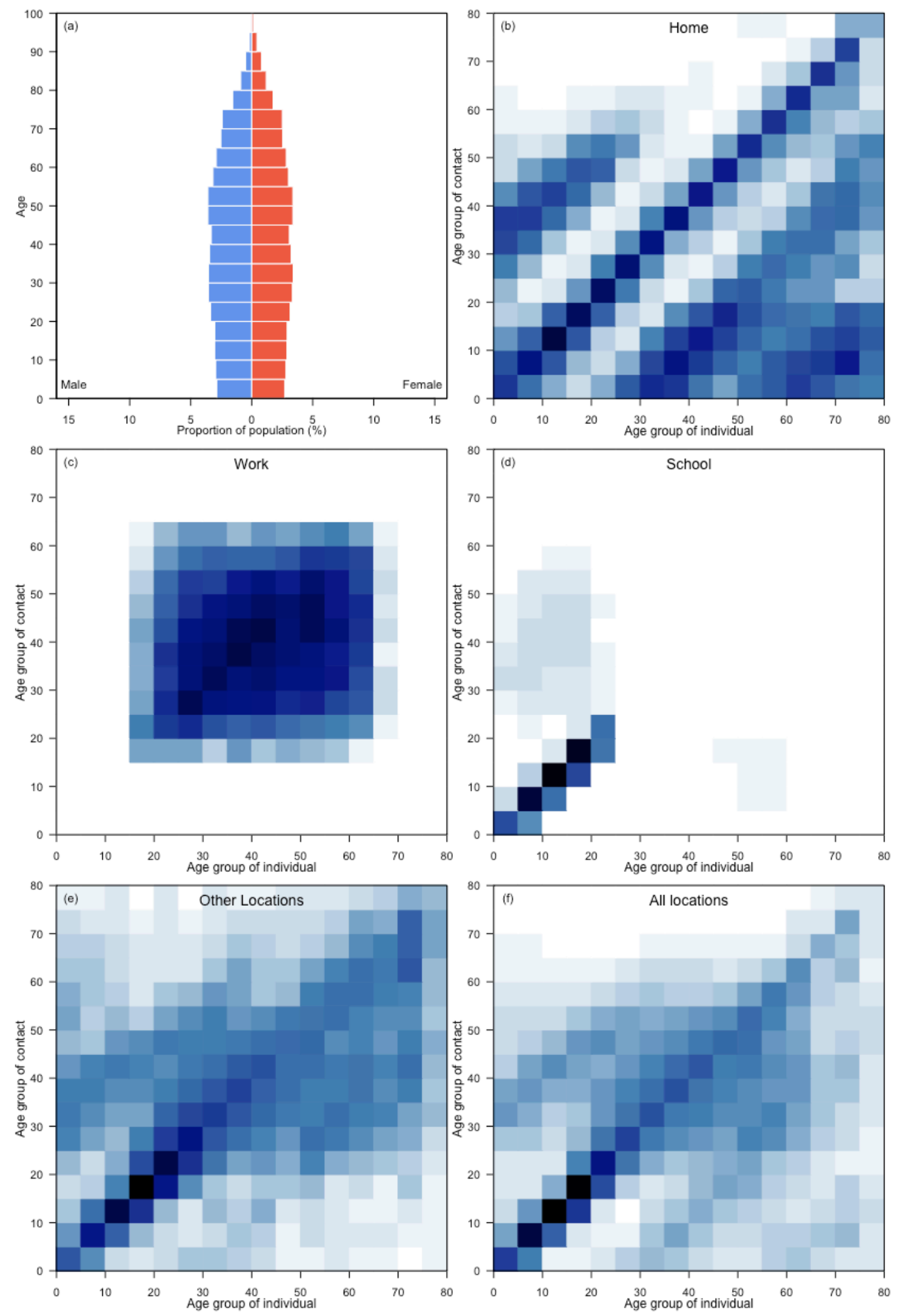


\section{Oman}
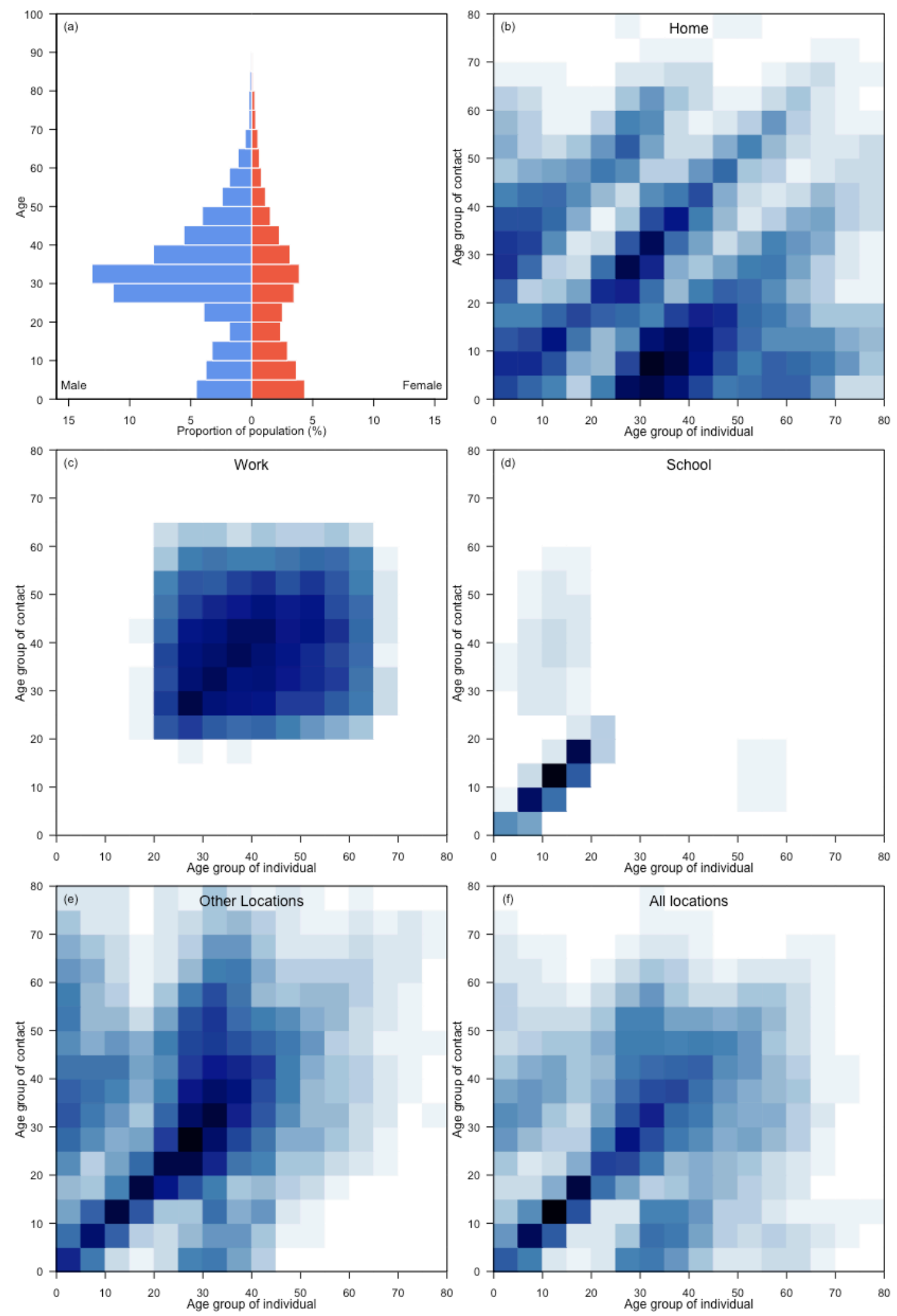


\section{Pakistan}
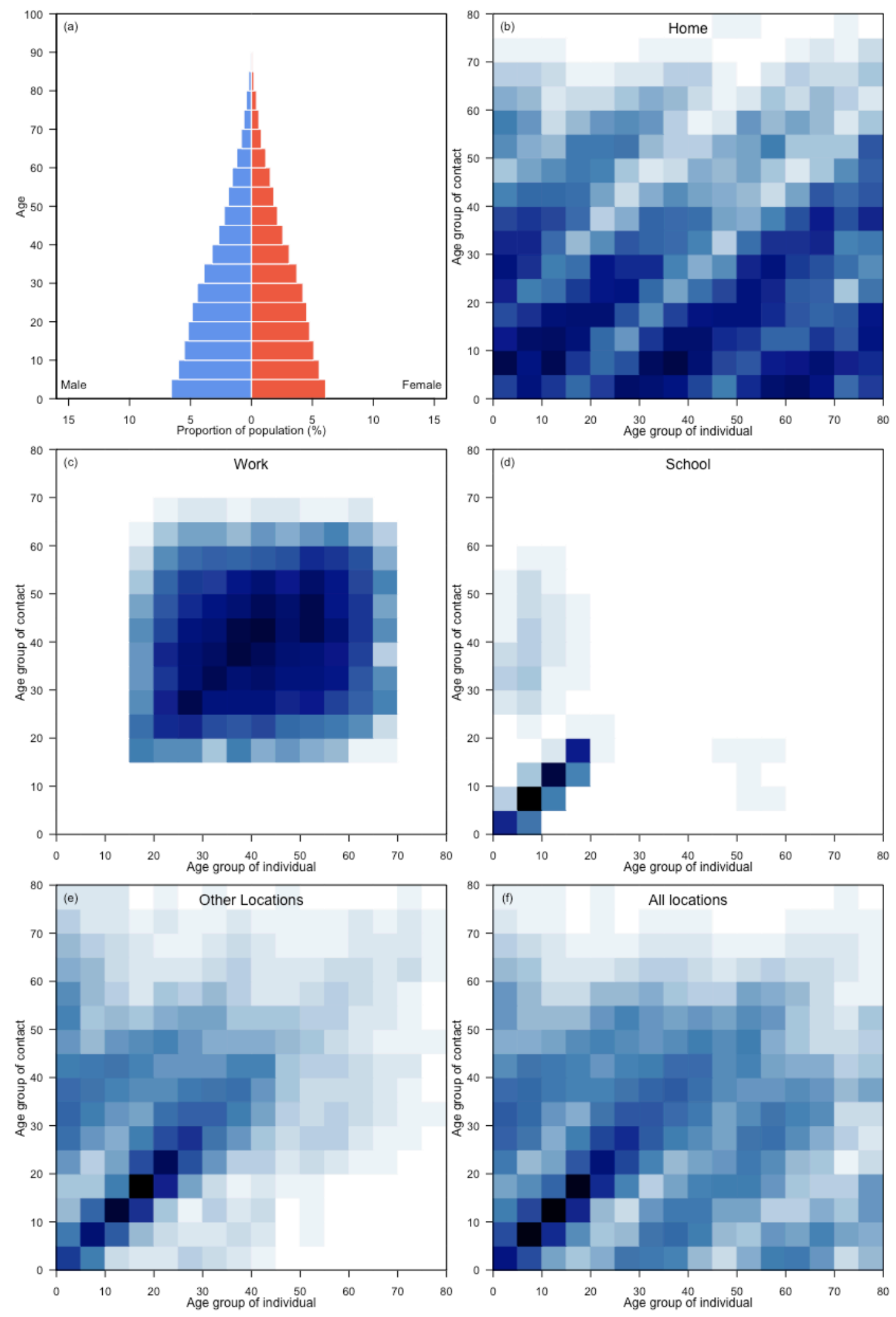
Panama
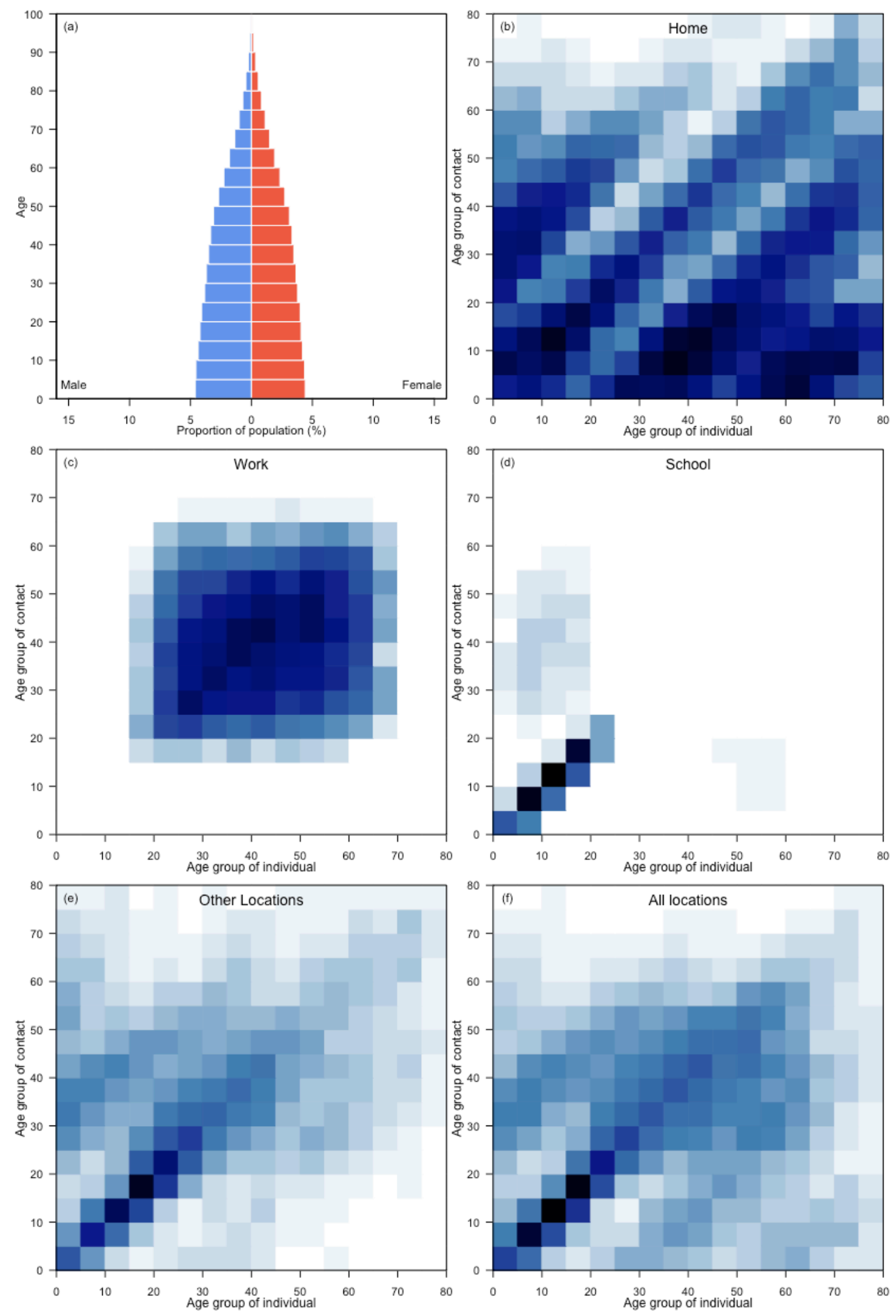
Papua New Guinea
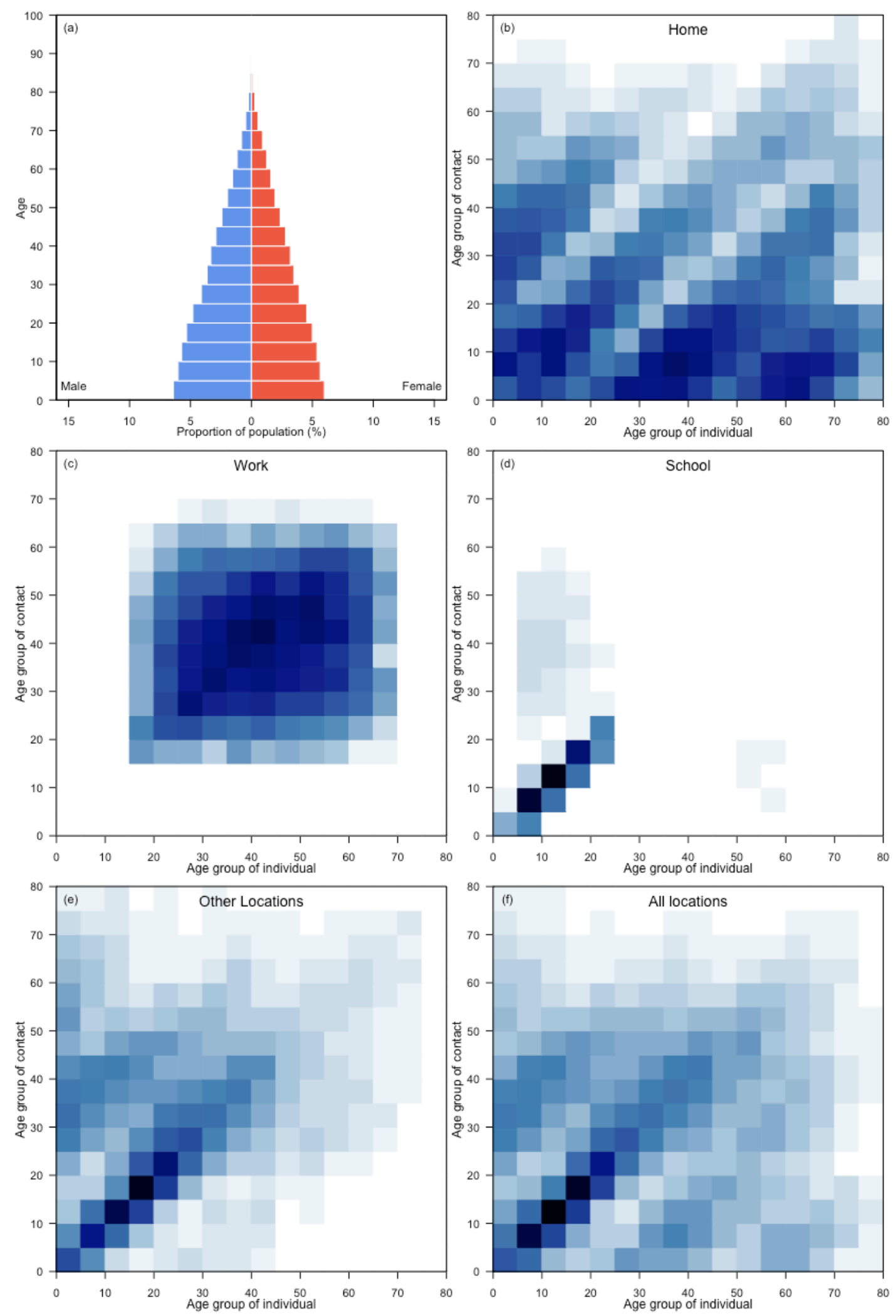
Paraguay
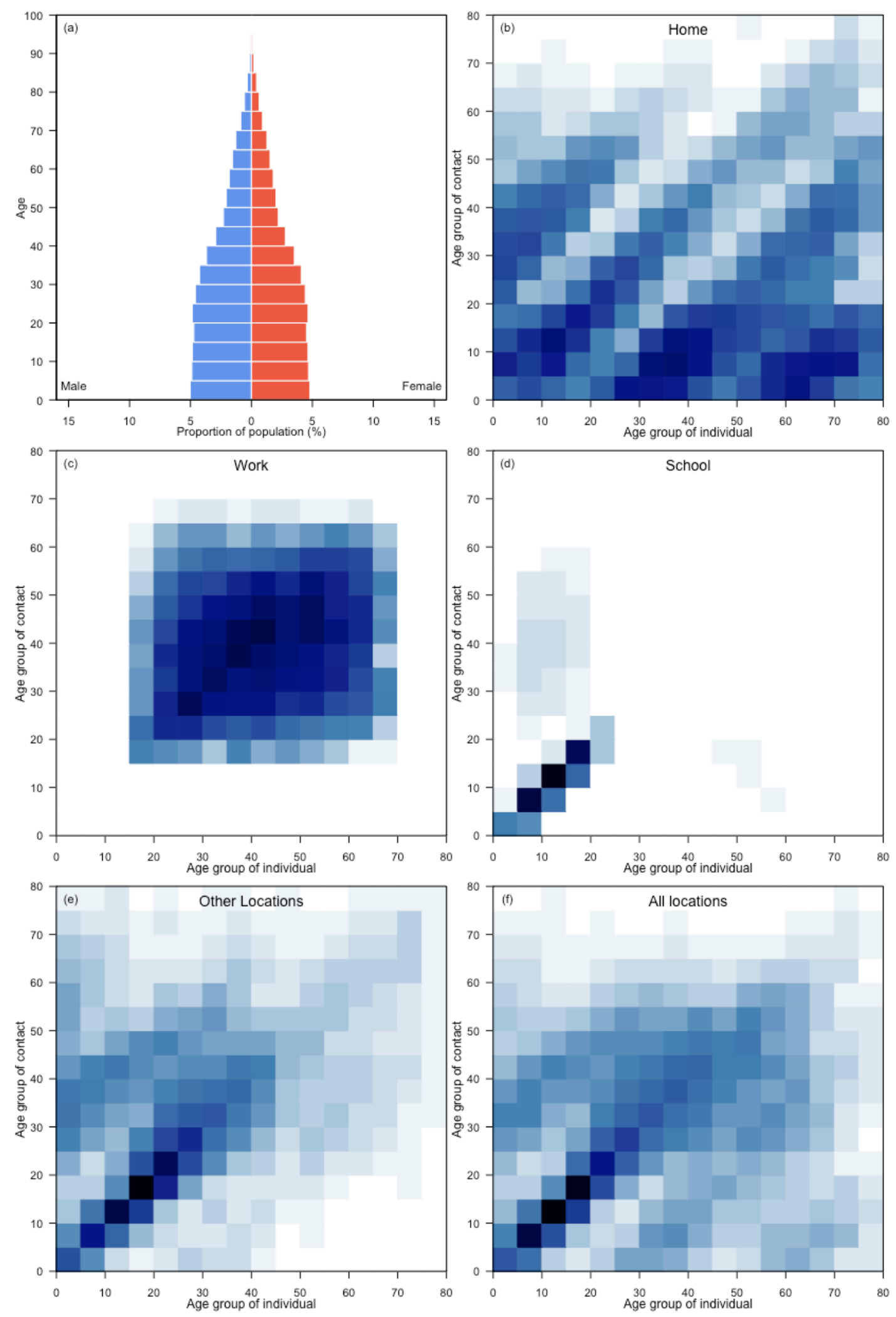


\section{Peru}
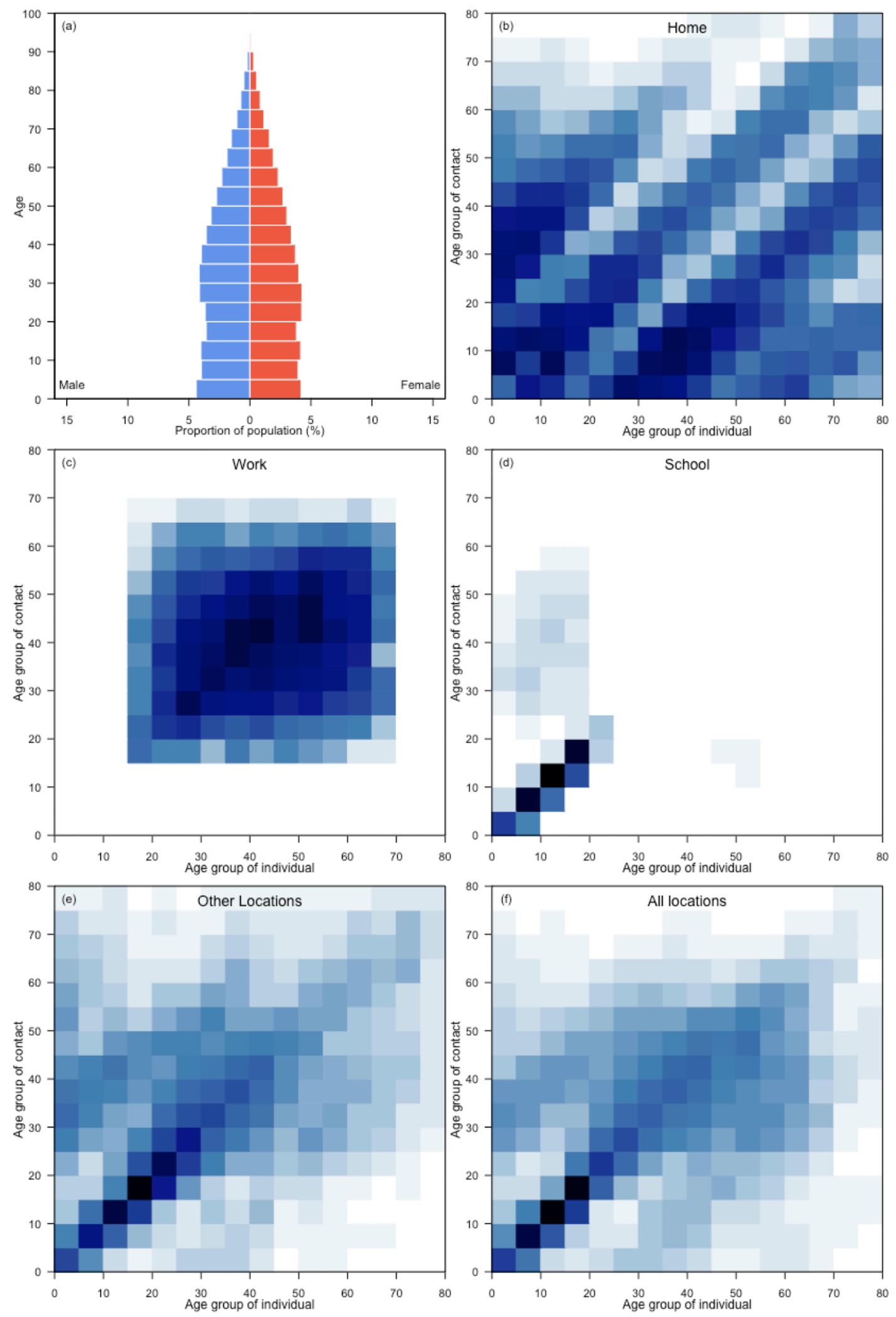
Philippines
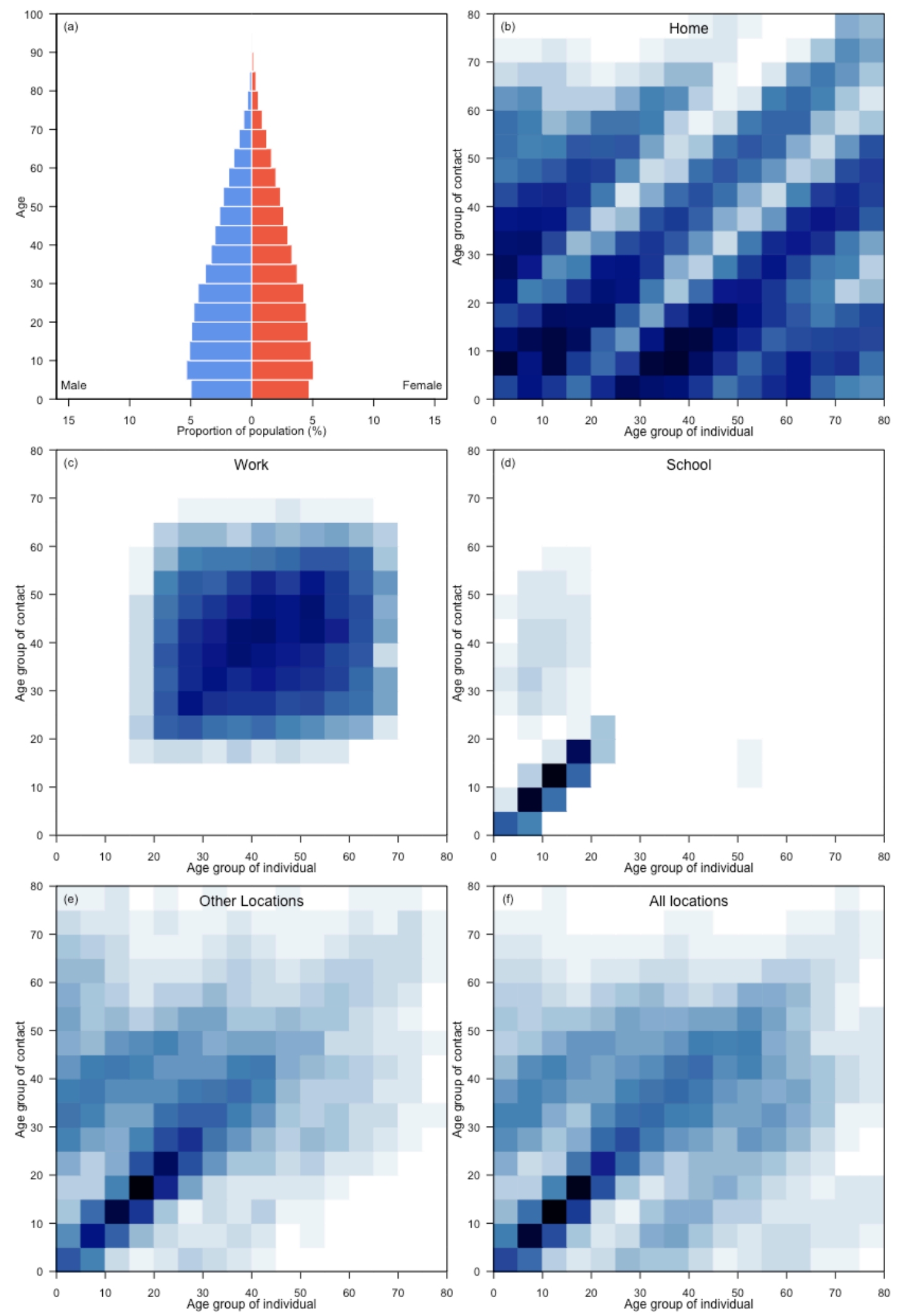


\section{Poland}
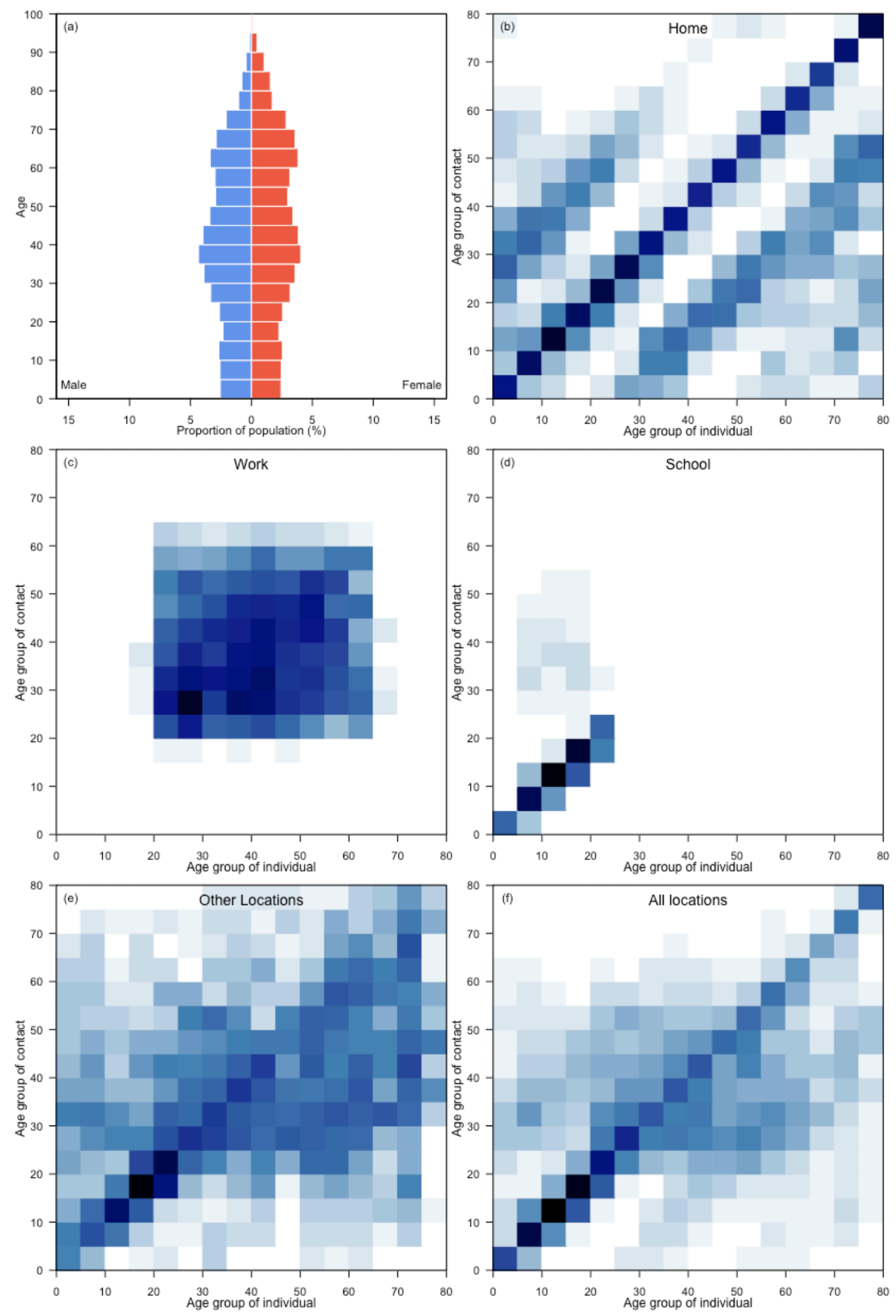


\section{Portugal}
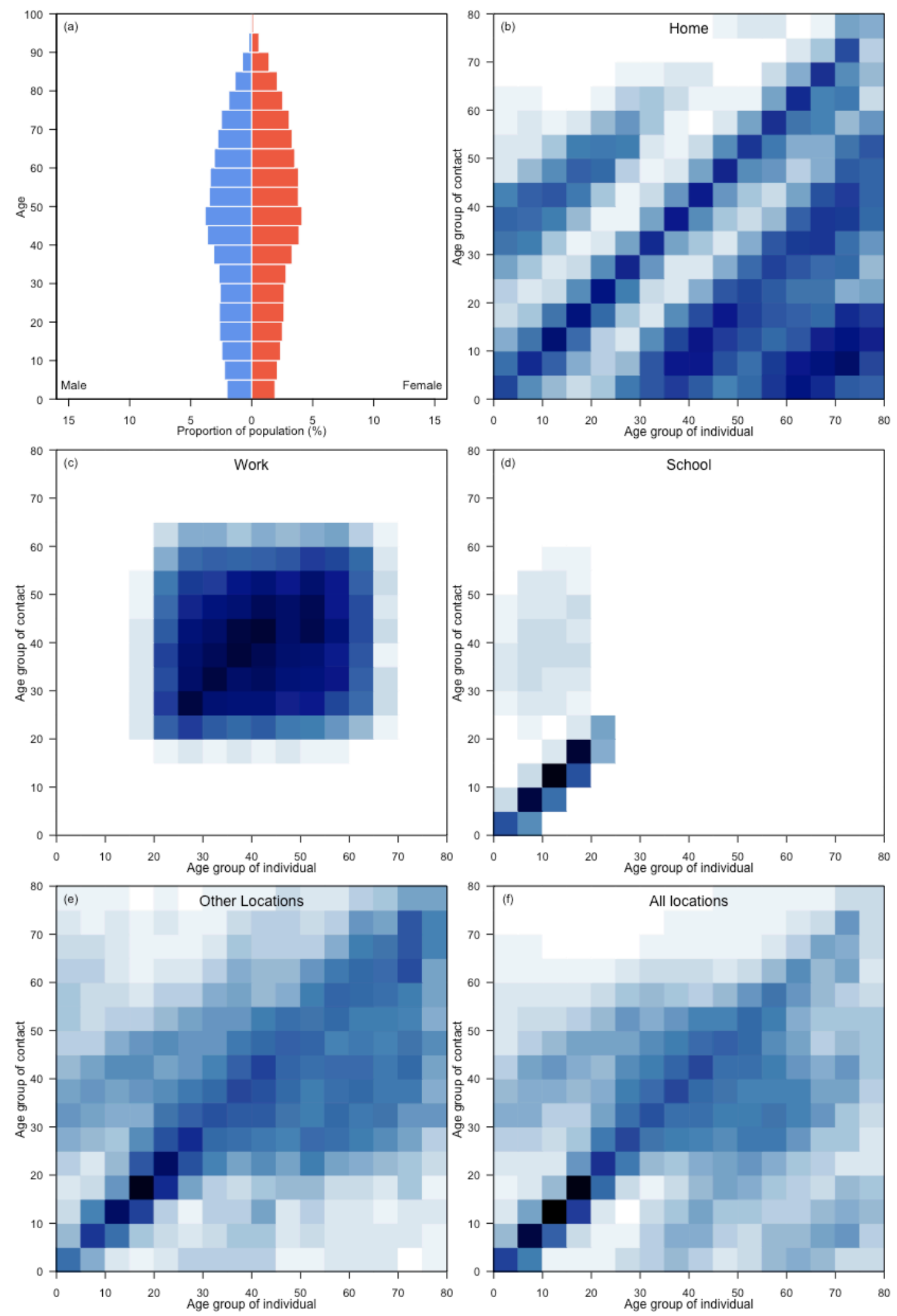
Puerto Rico
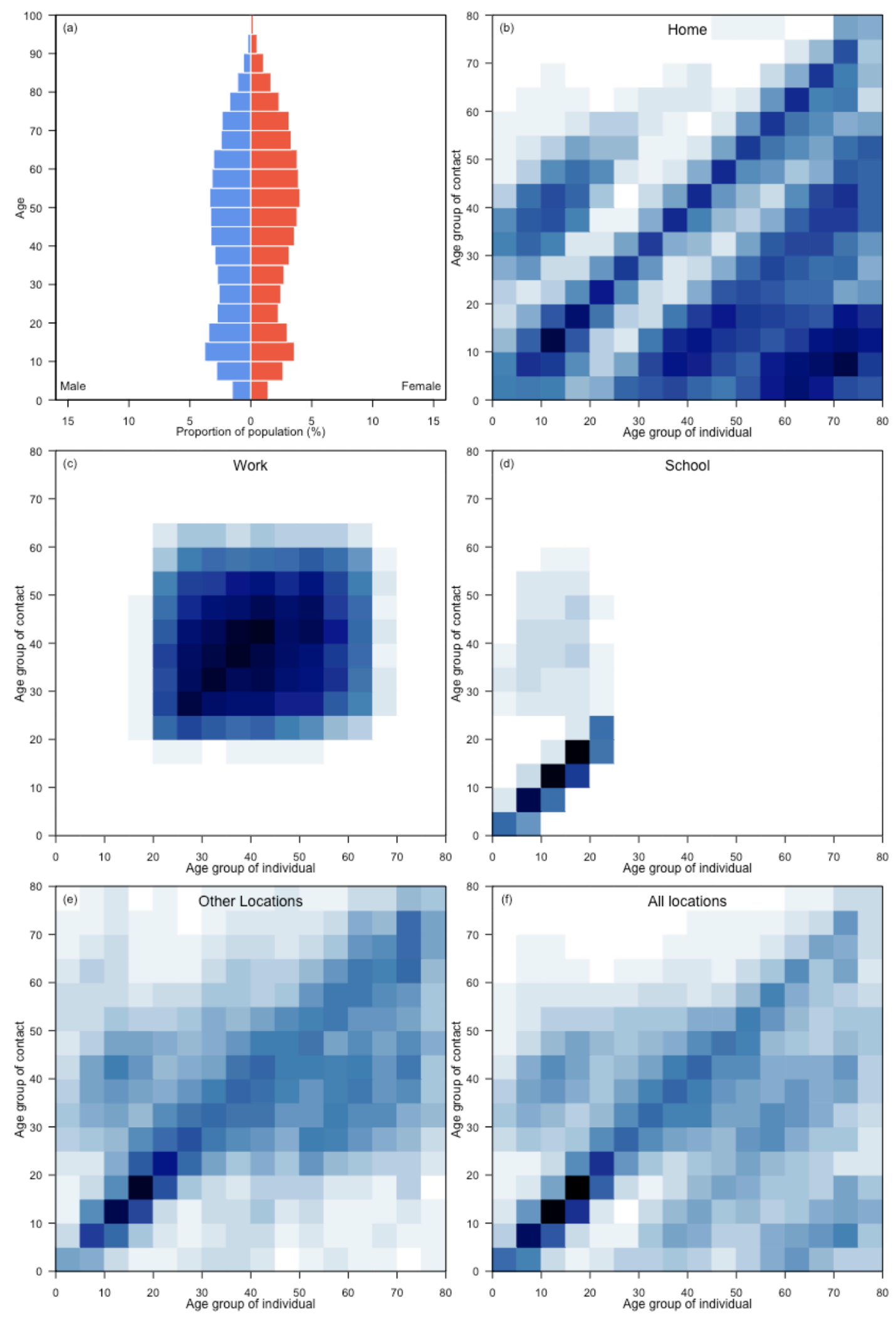


\section{Qatar}
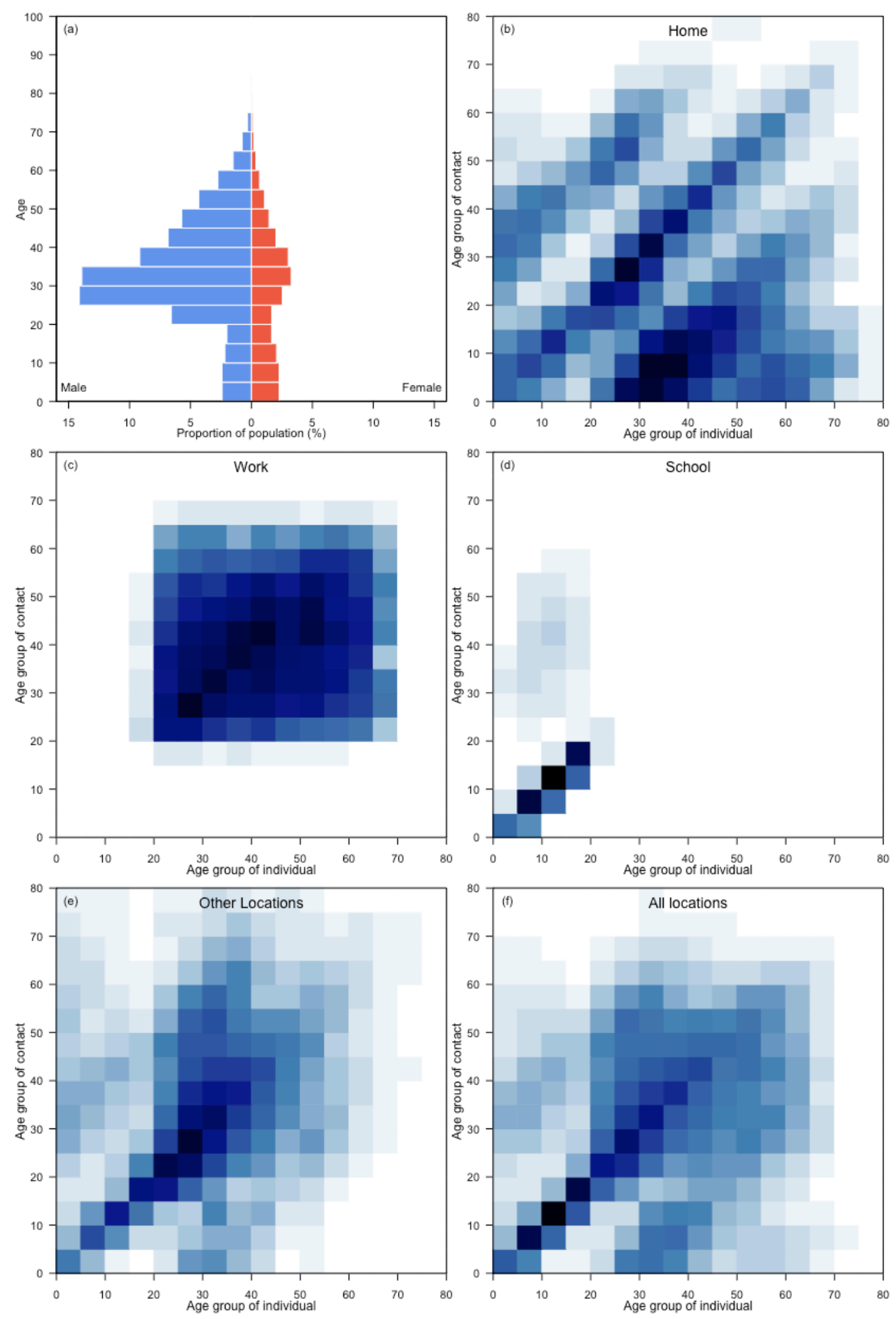


\section{Republic of Korea}
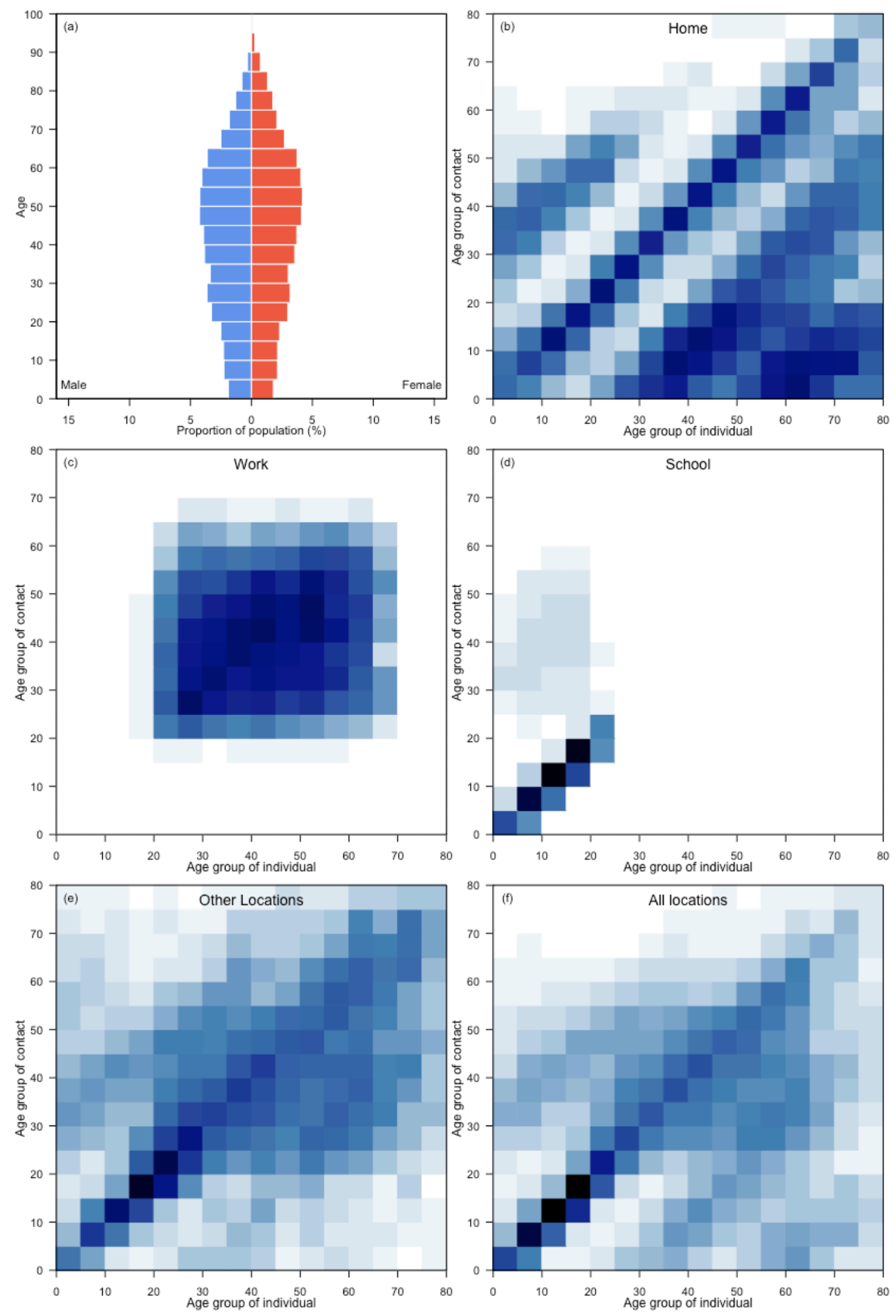
Republic of Moldova
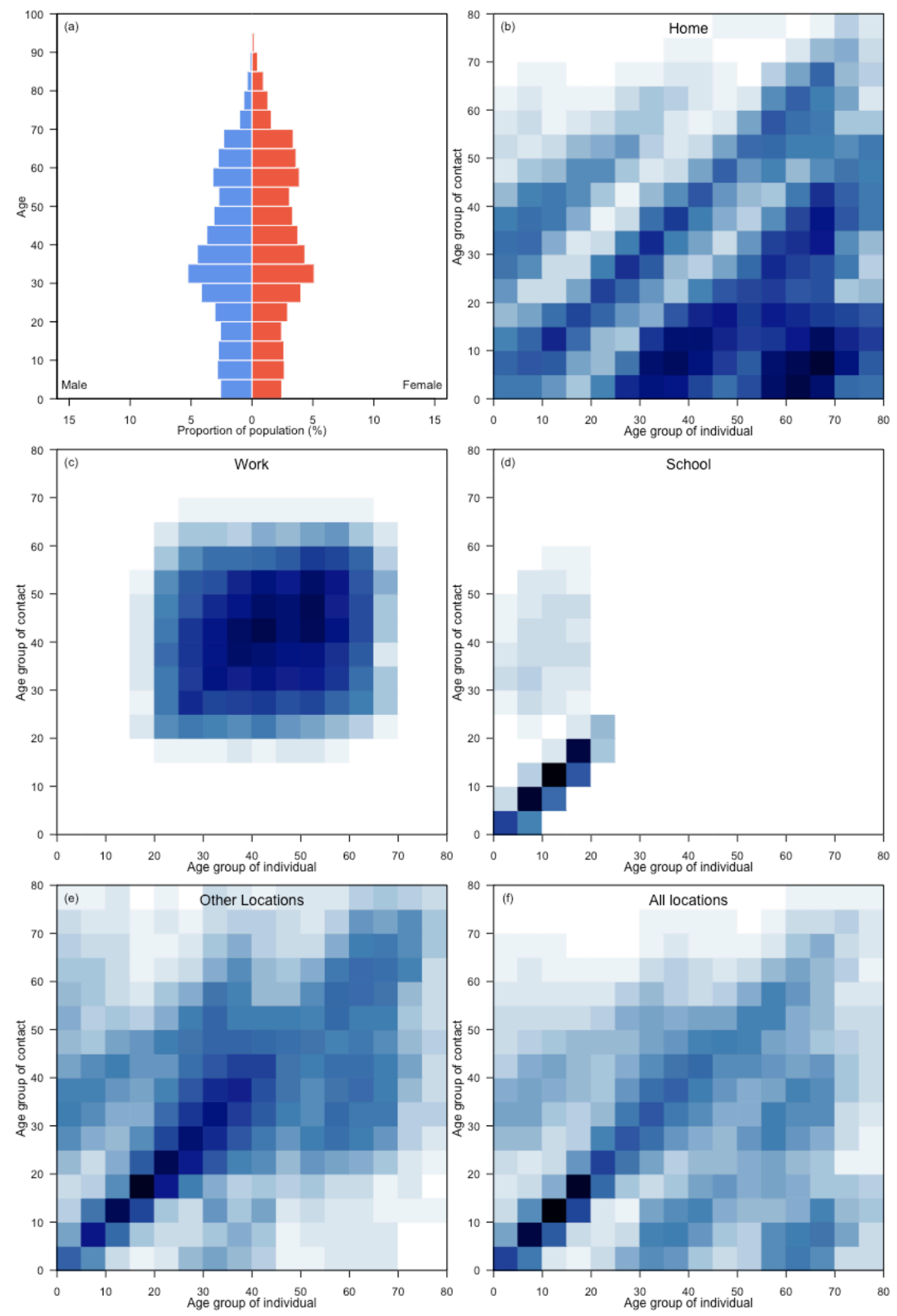


\section{Romania}
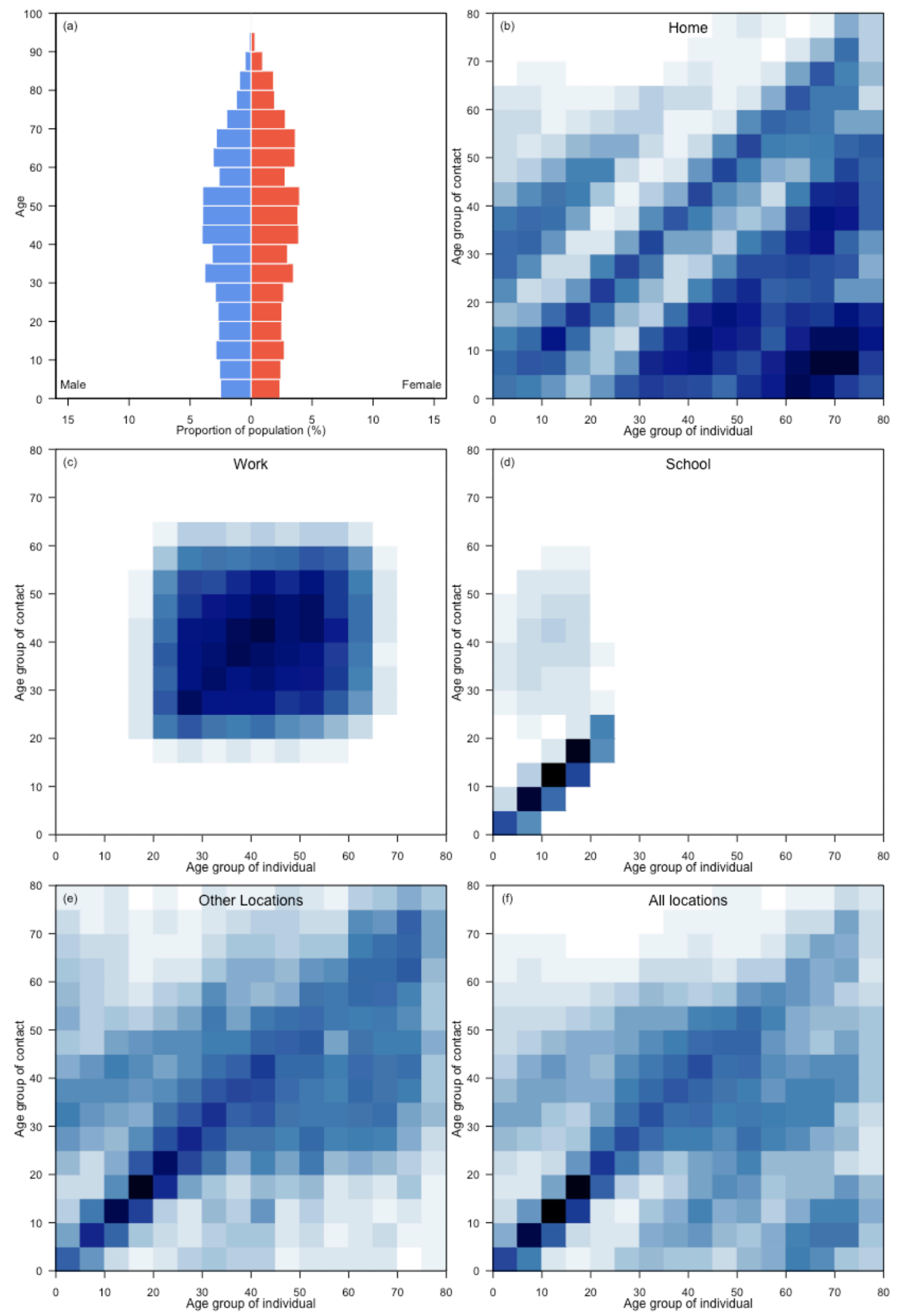


\section{Russian Federation}
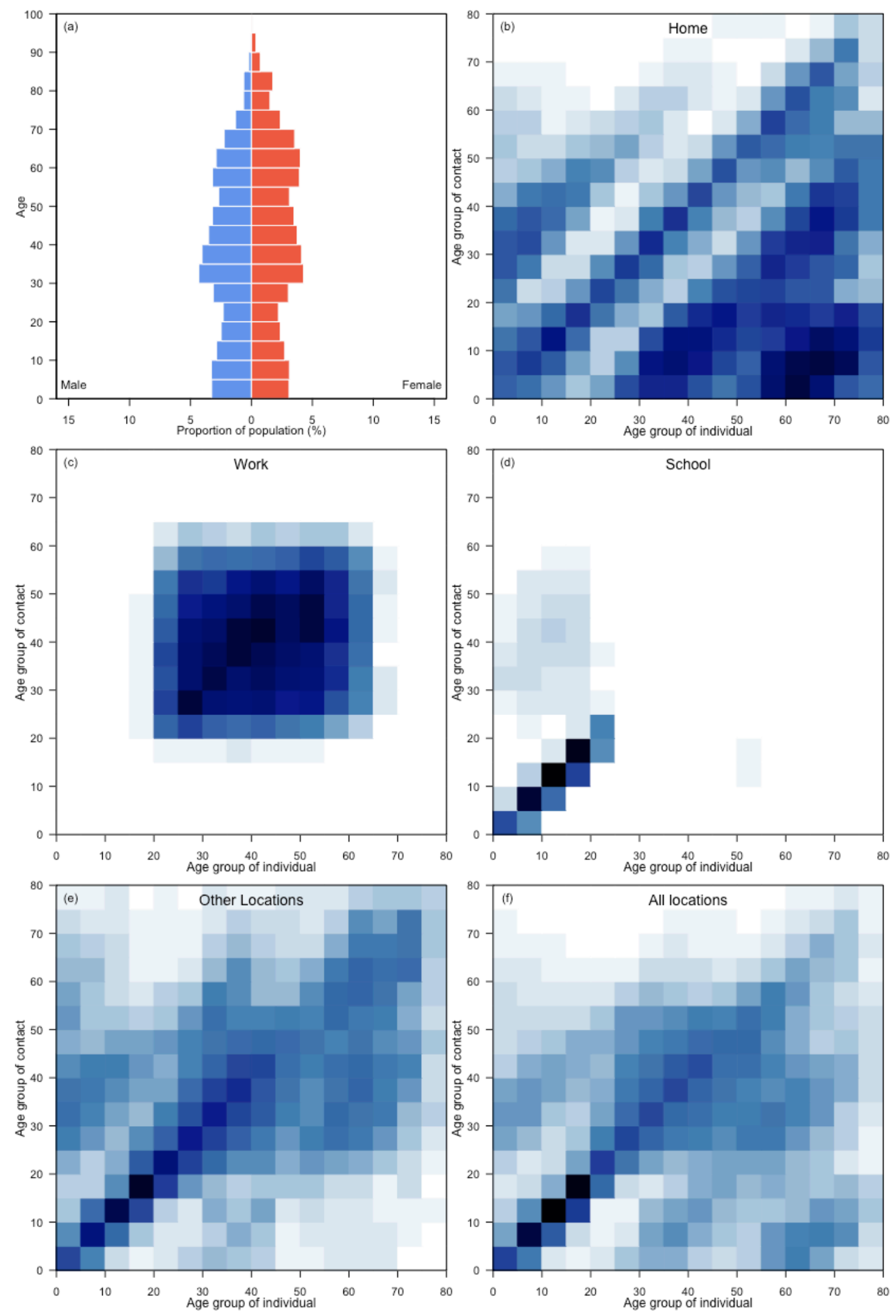
Rwanda
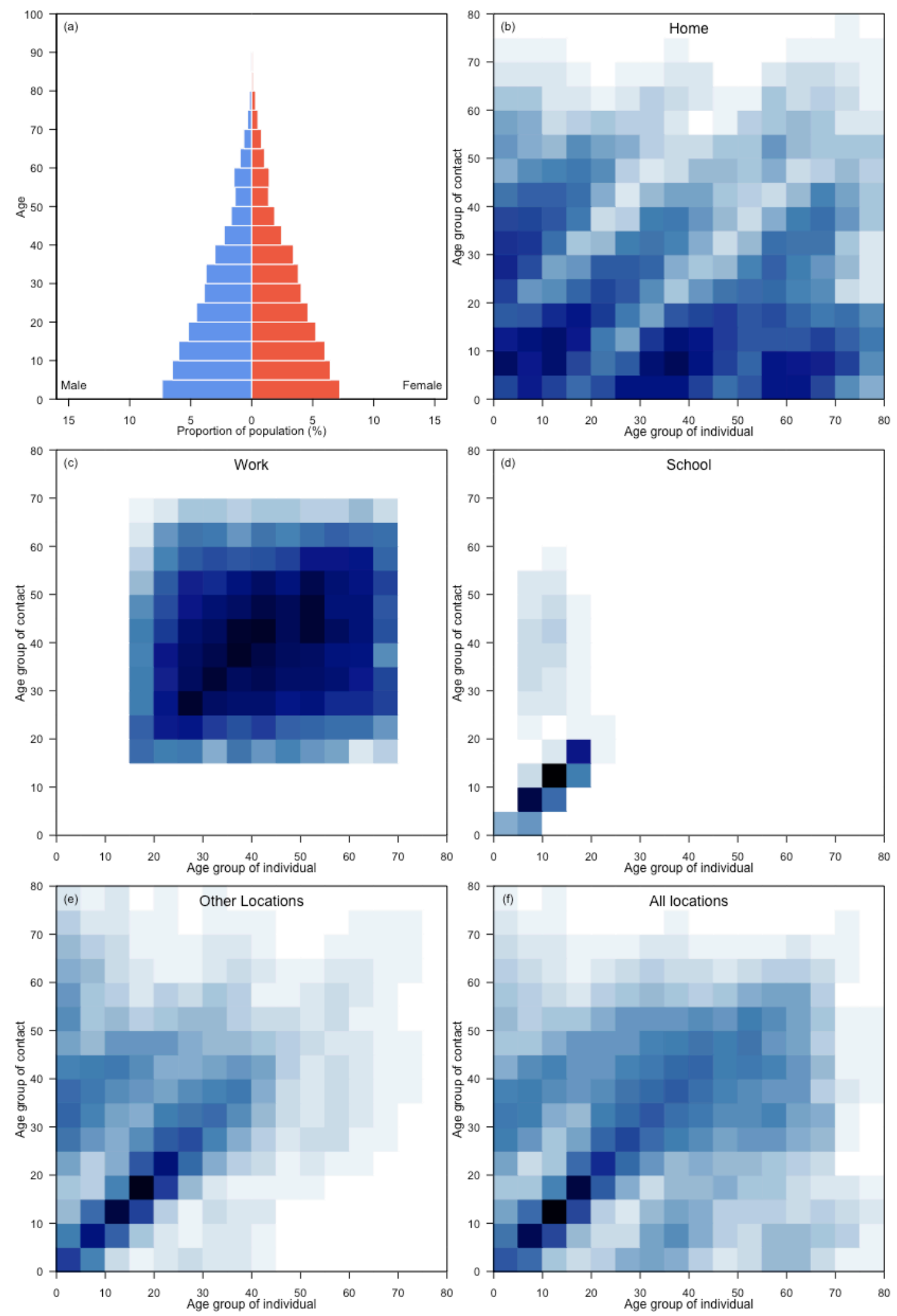
Saint Lucia
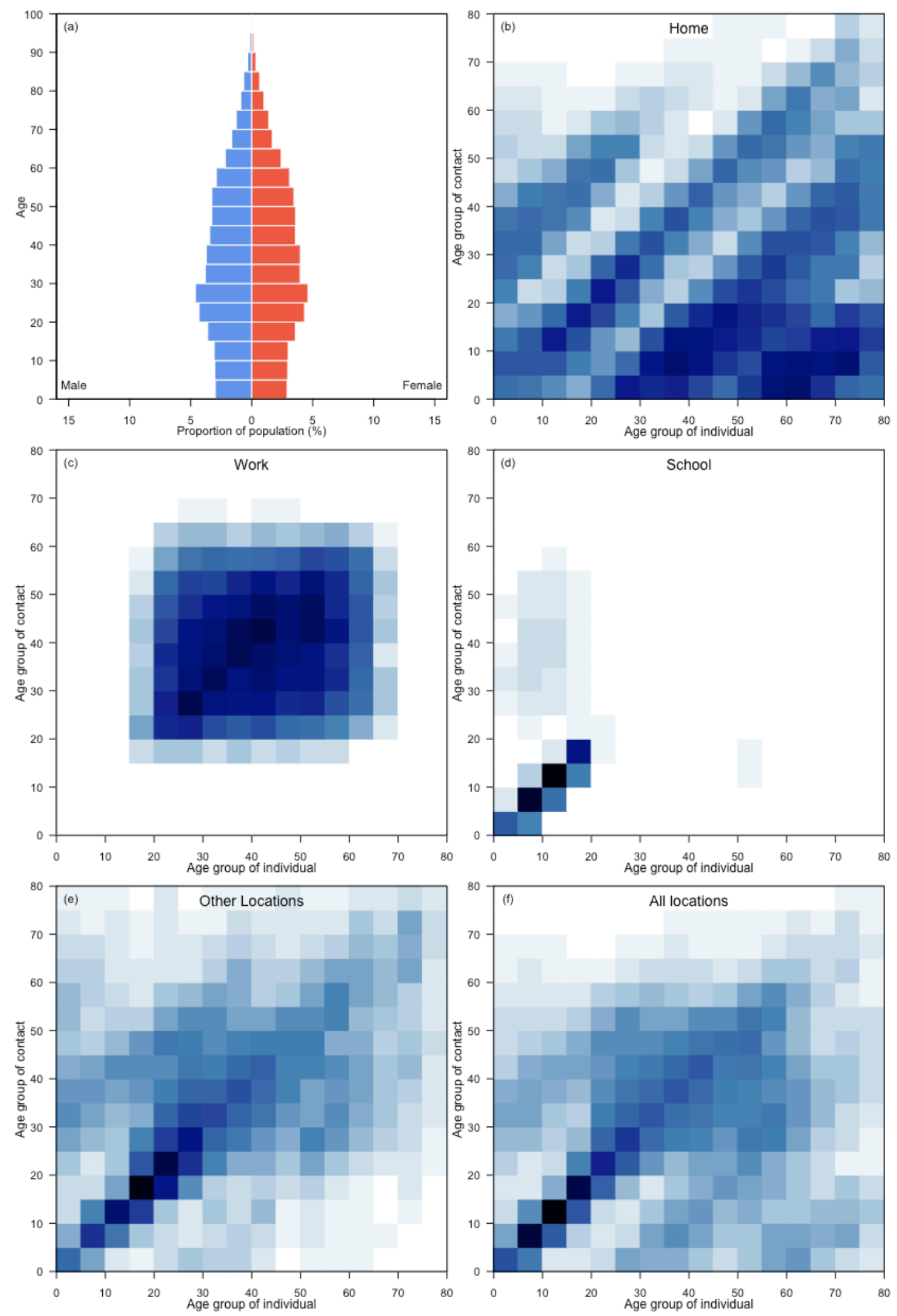
Saint Vincent and the Grenadines
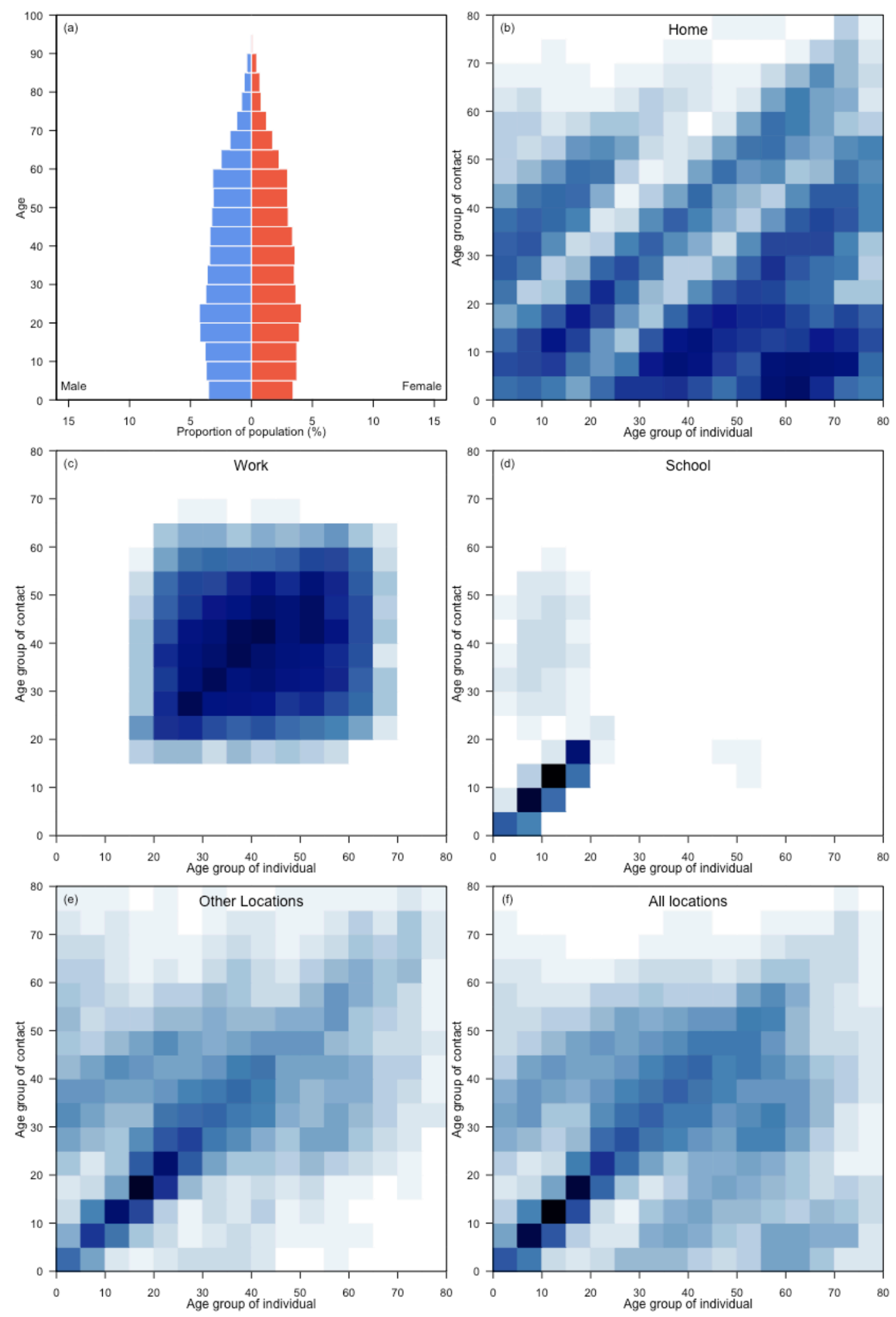


\section{Samoa}
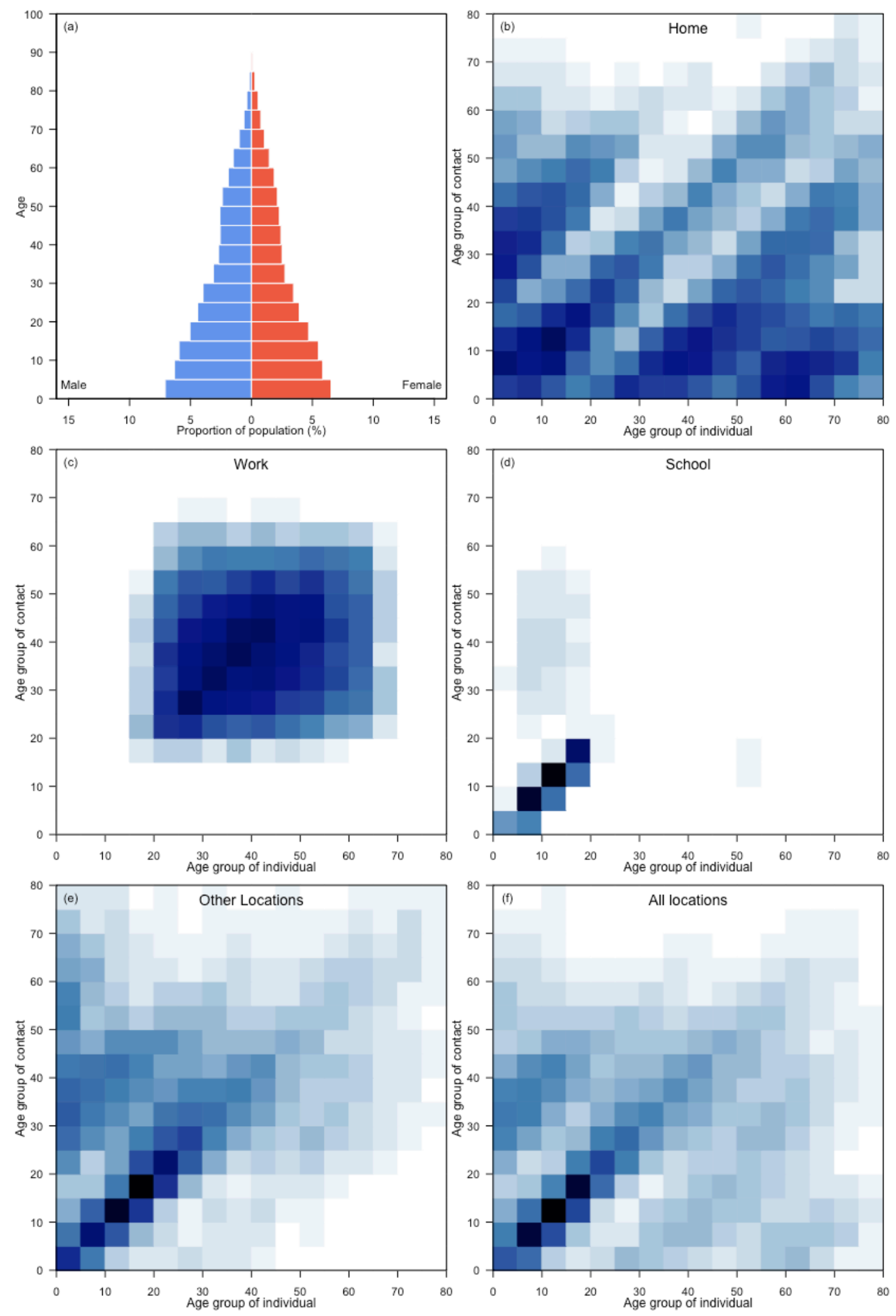
Sao Tome and Principe
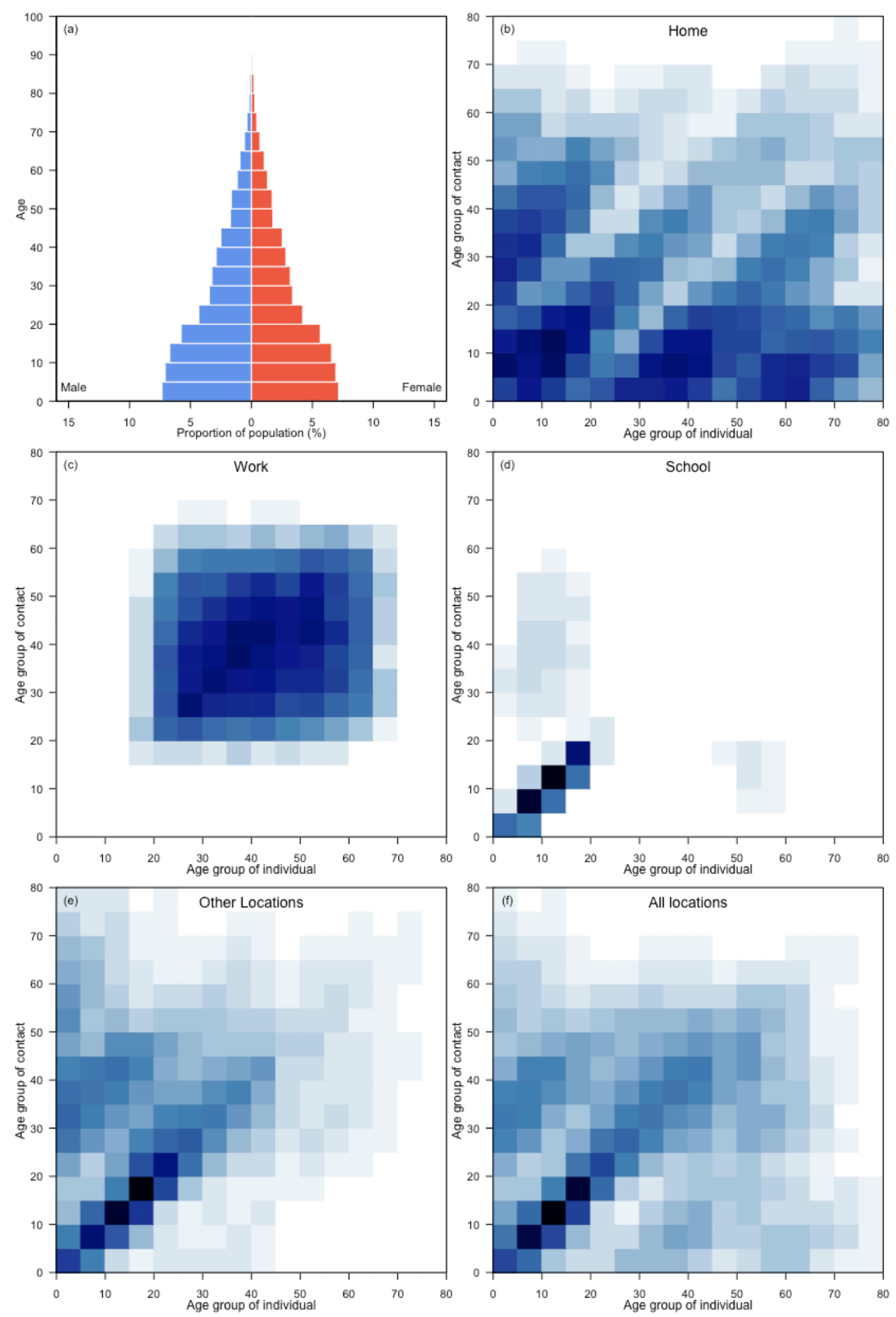


\section{Saudi Arabia}
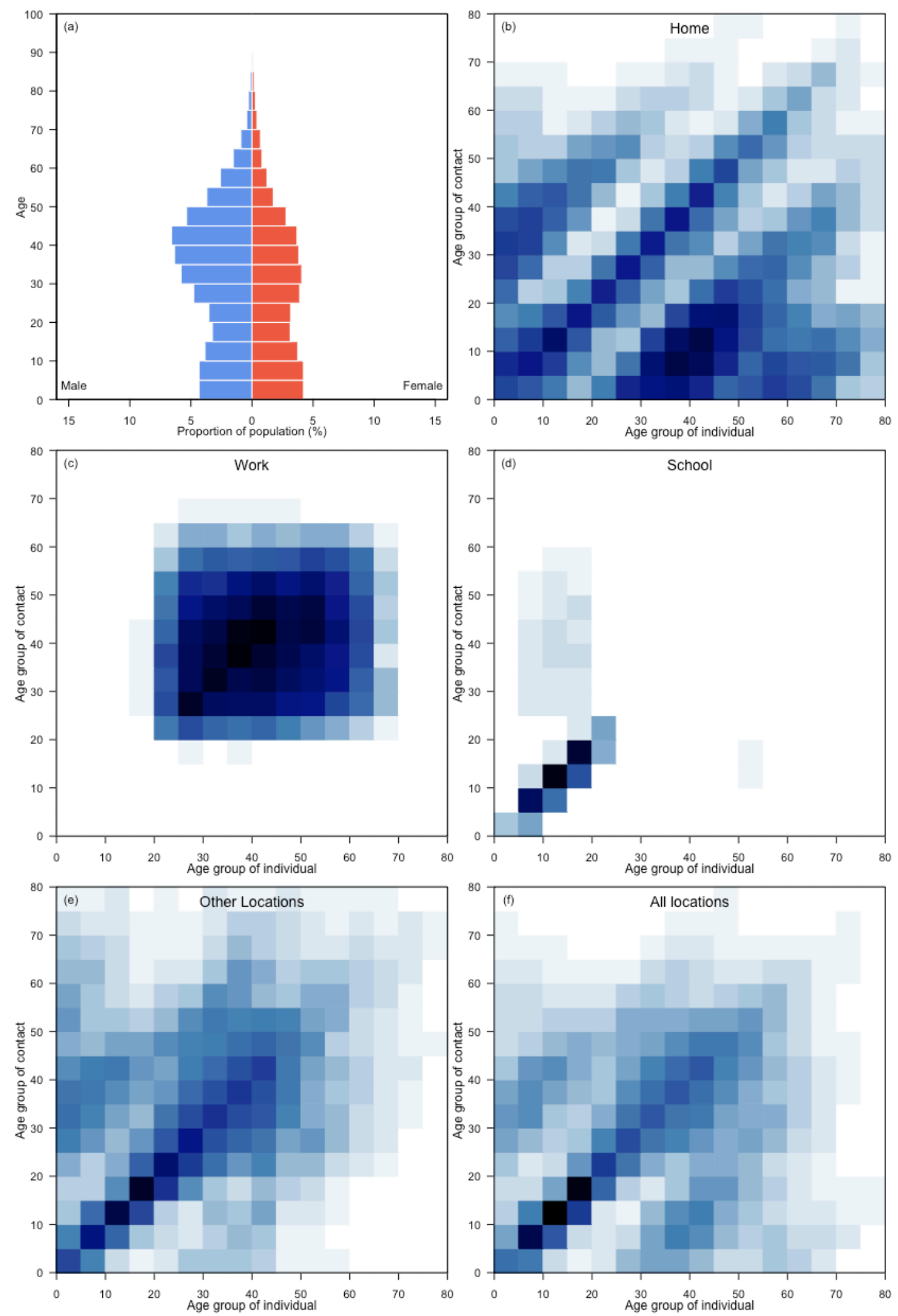
Senegal
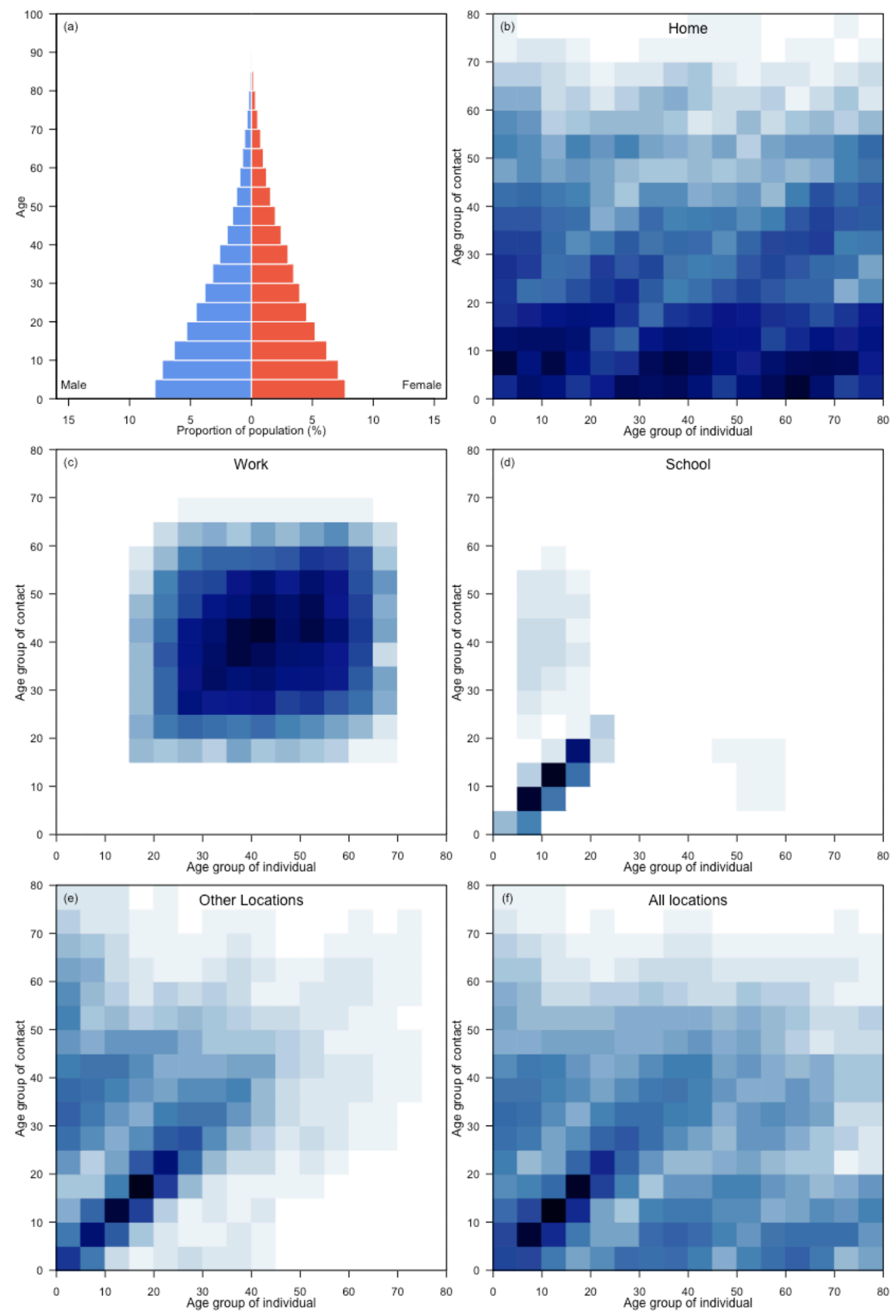


\section{Serbia}
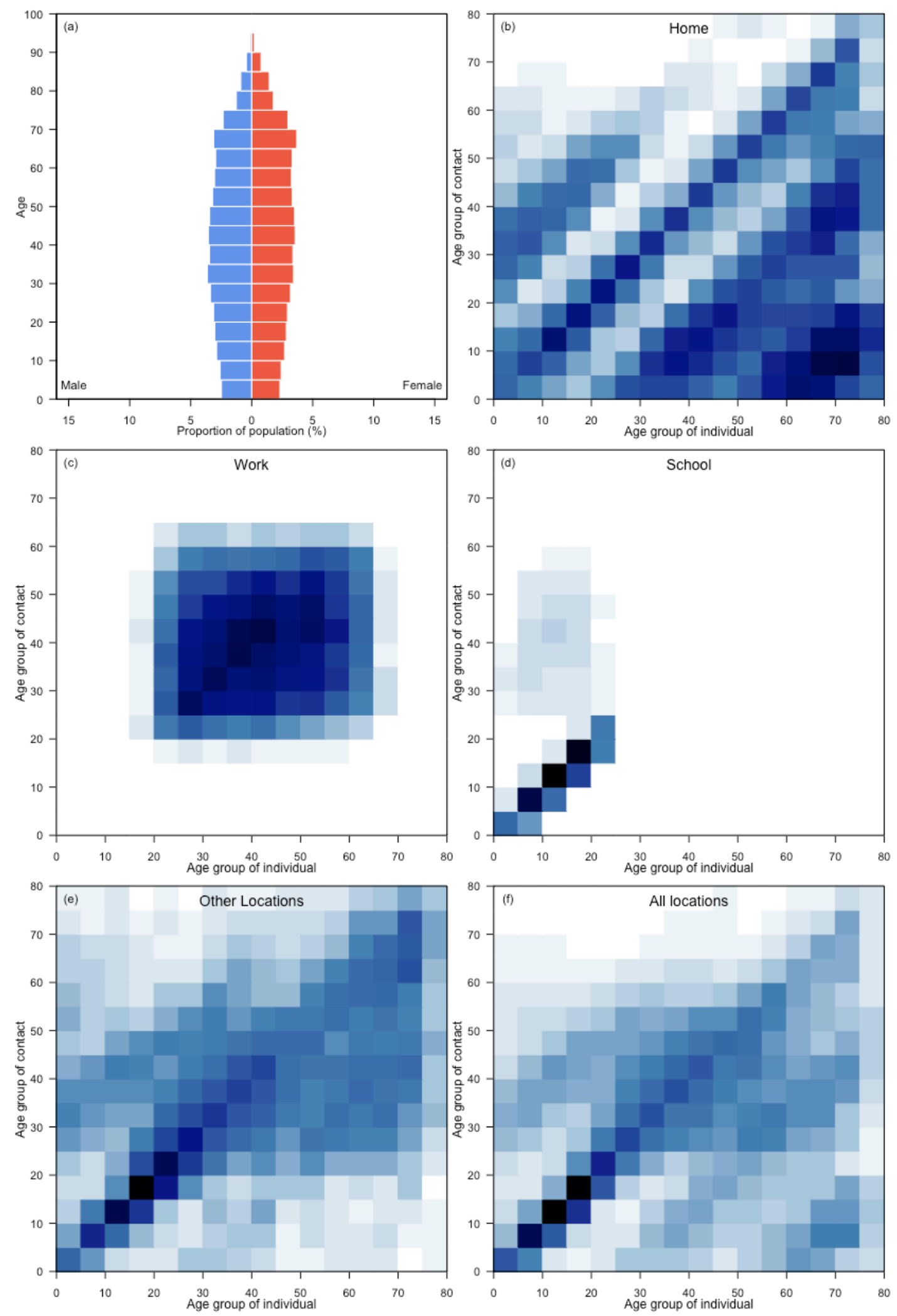
Sierra Leone
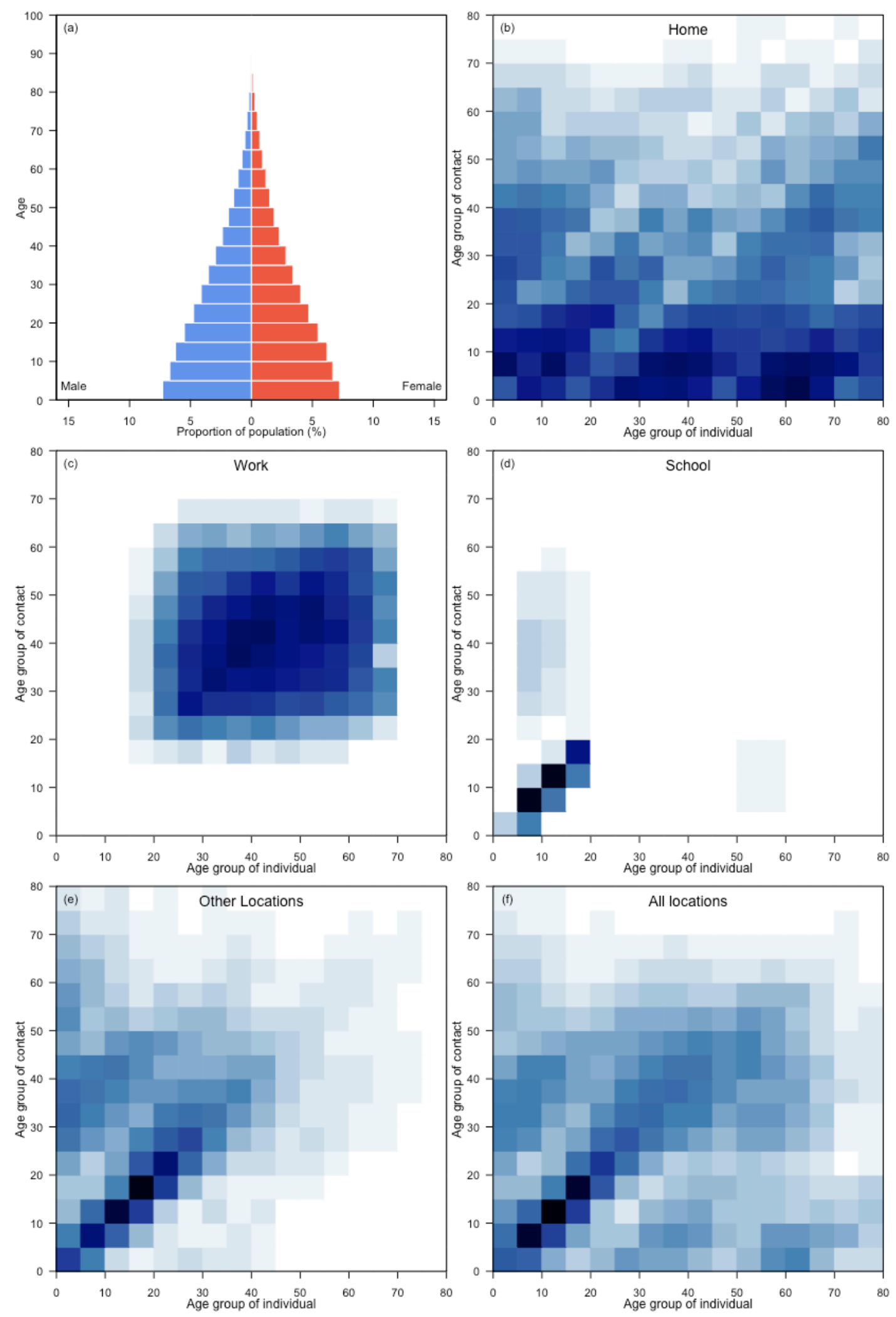


\section{Singapore}
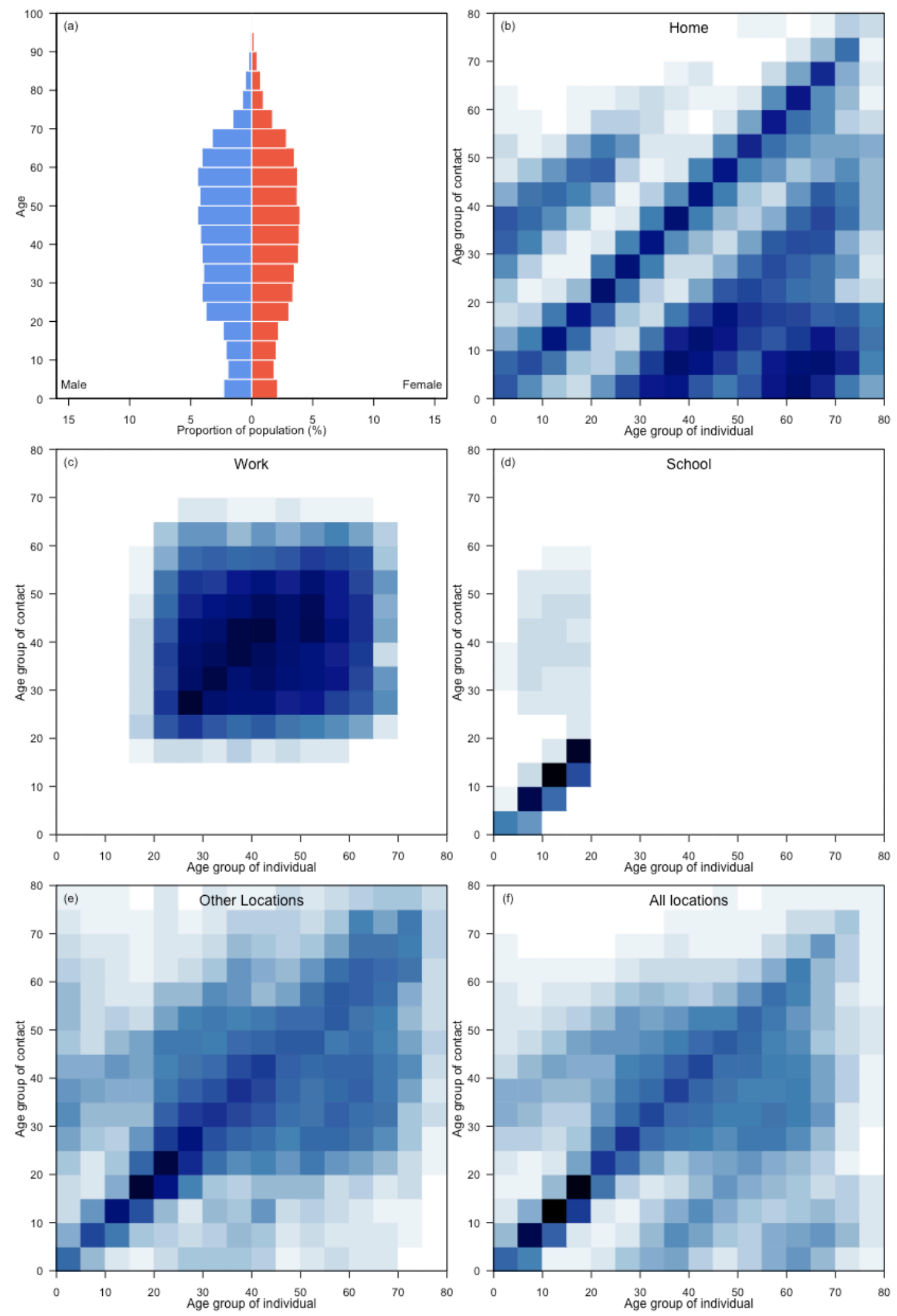


\section{Slovakia}
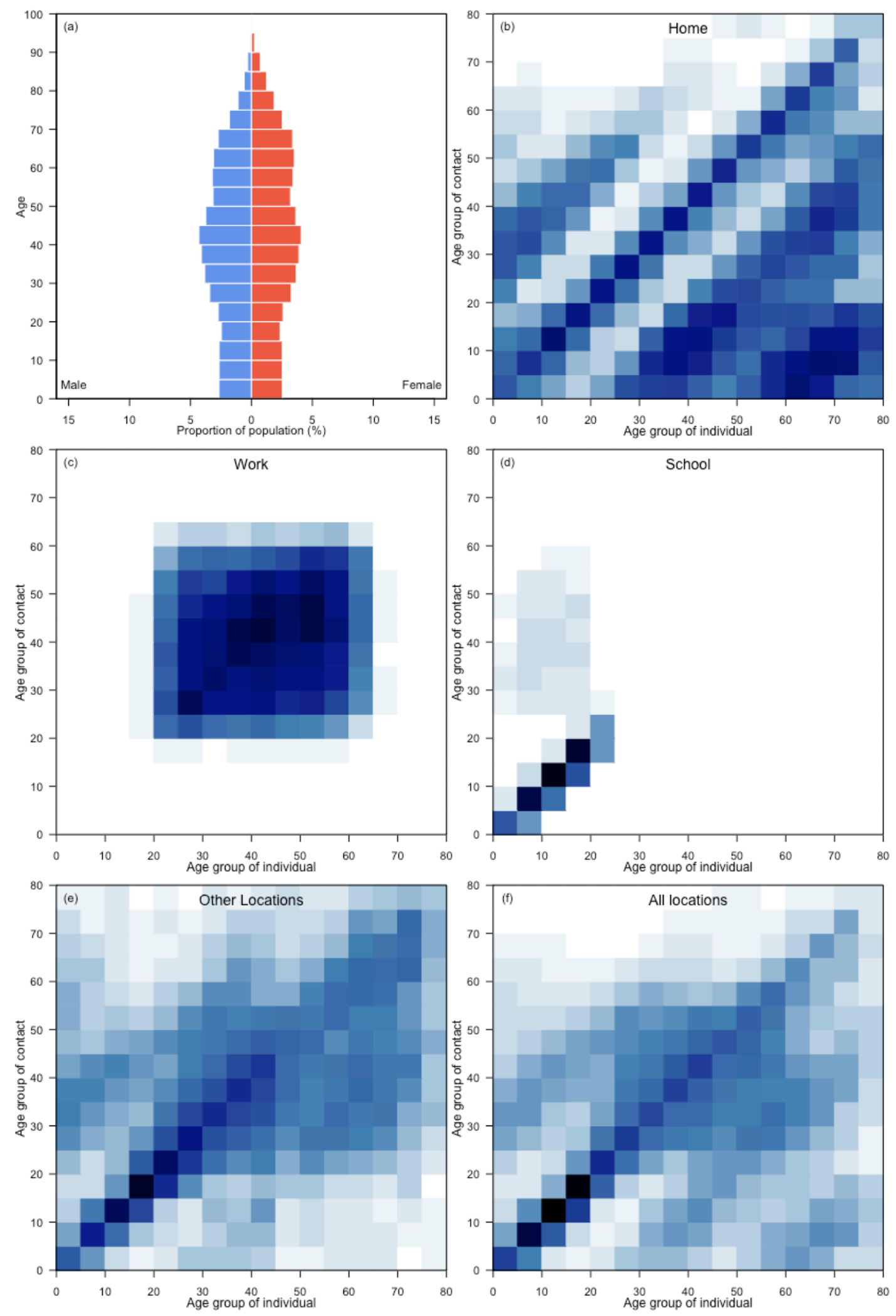


\section{Slovenia}
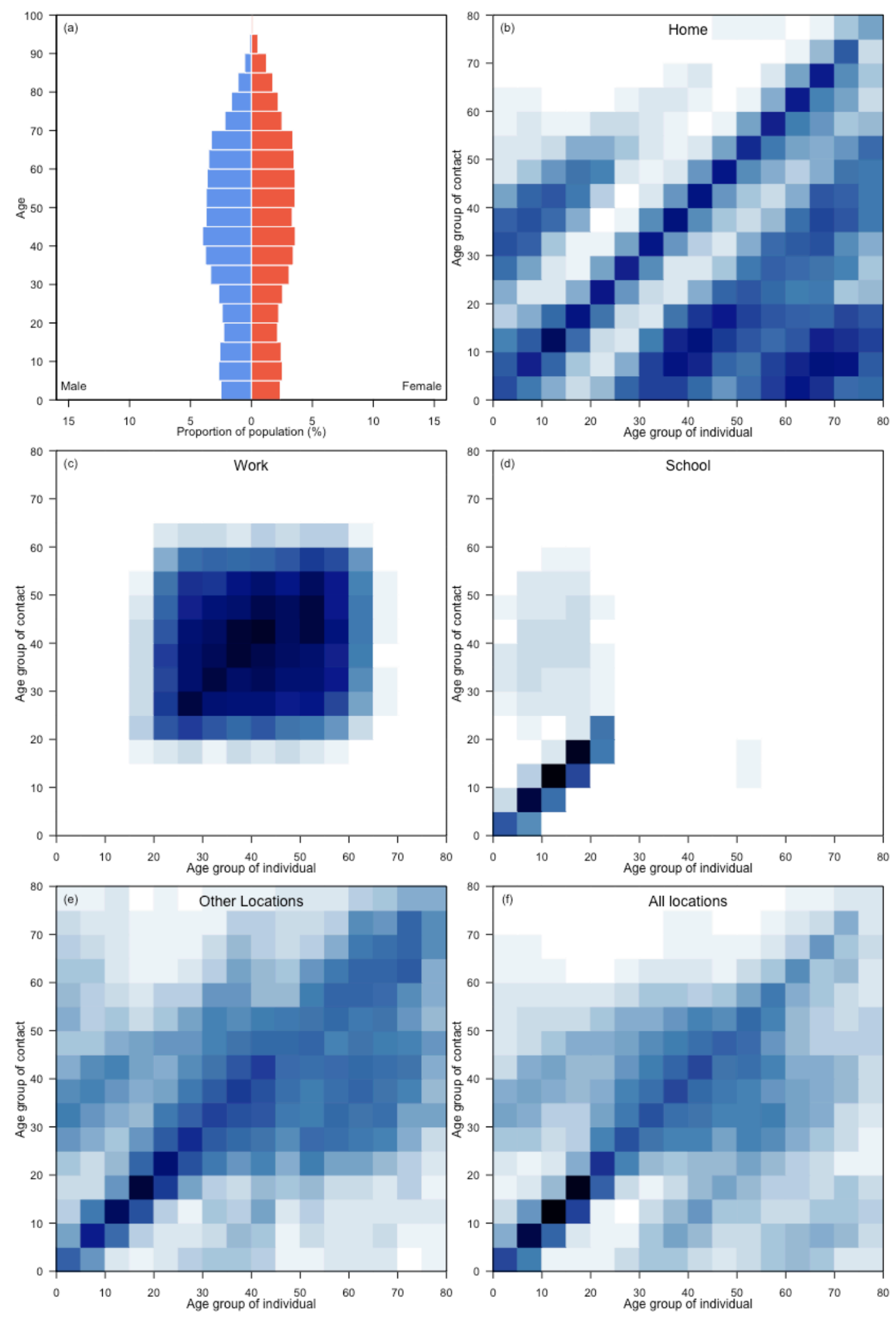


\section{Solomon Islands}
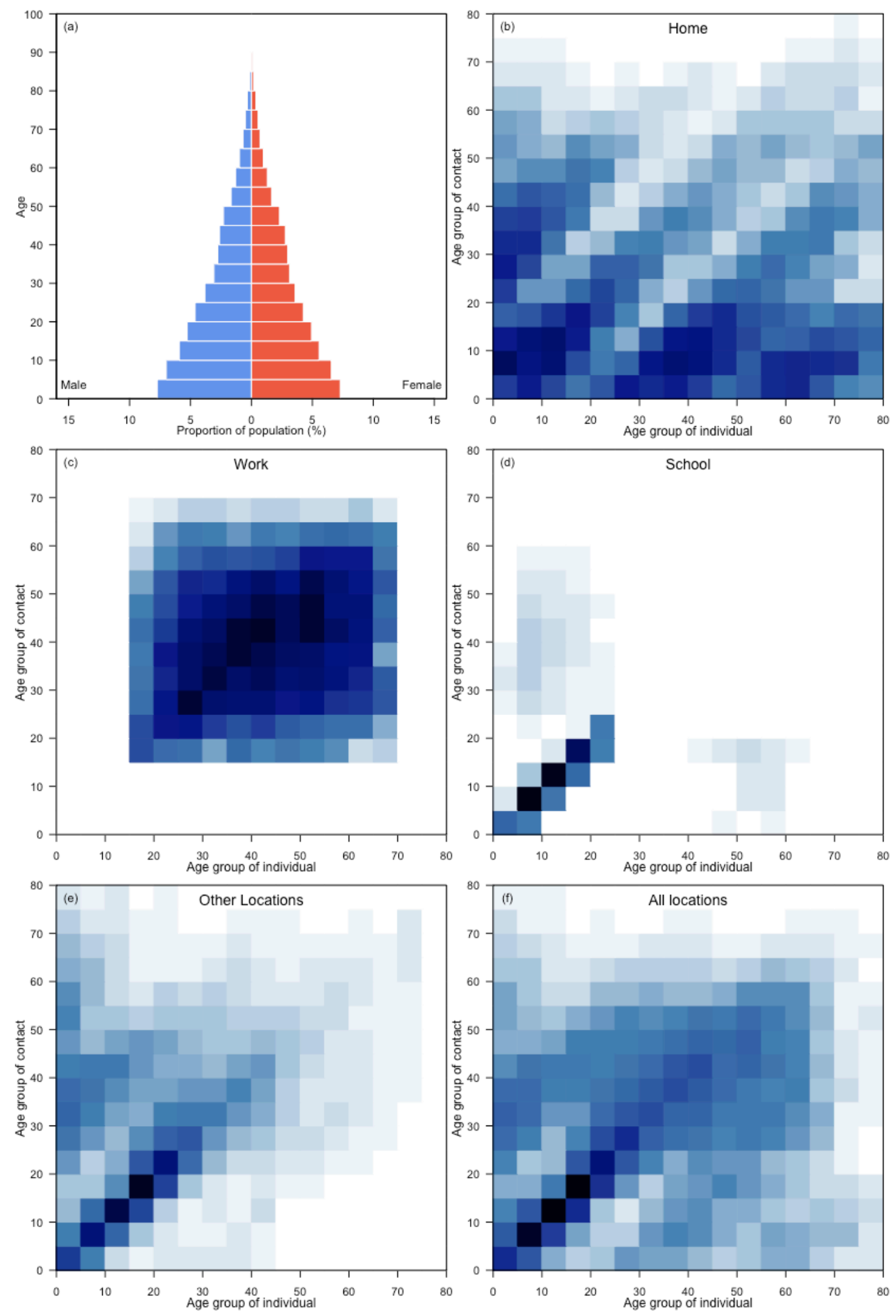


\section{South Africa}
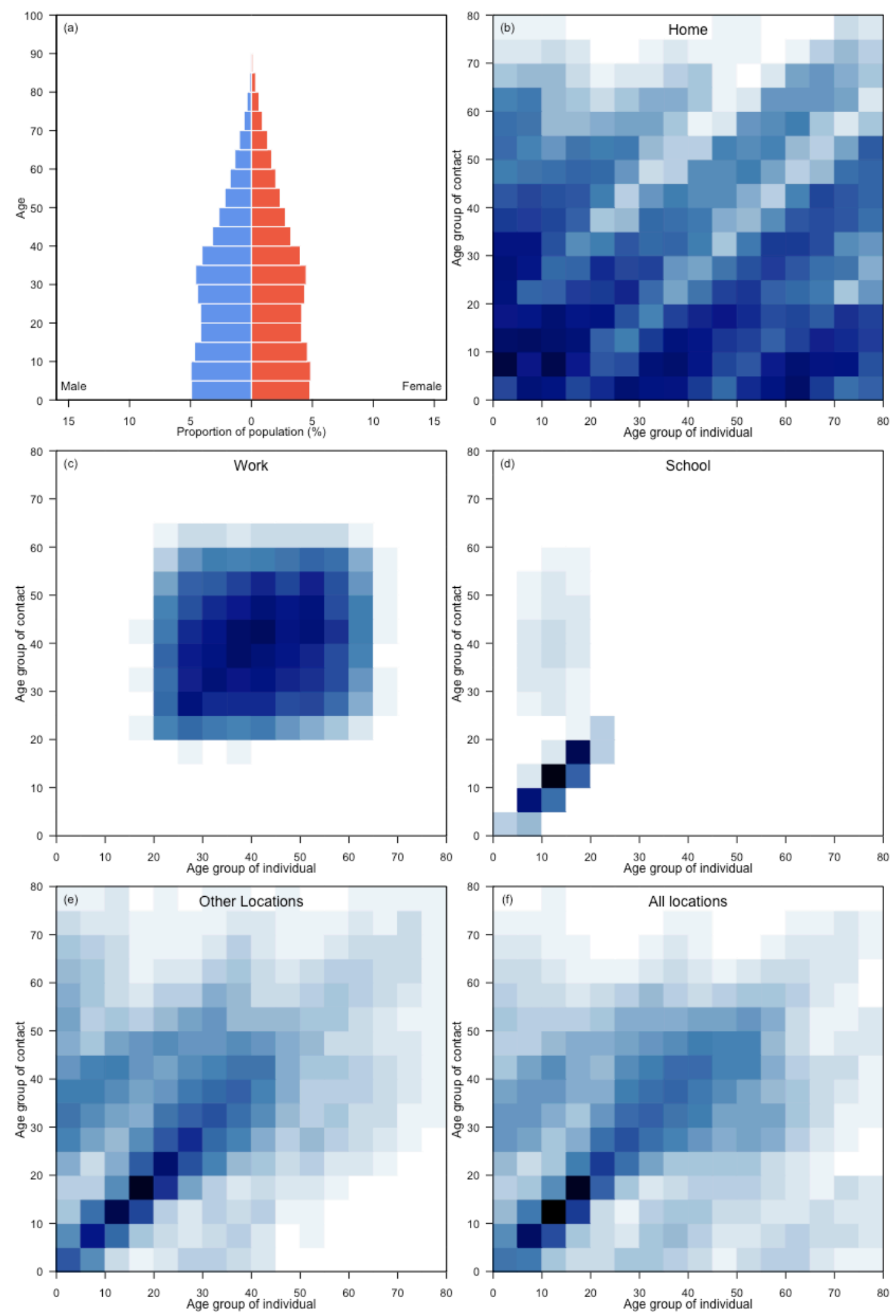


\section{South Sudan}
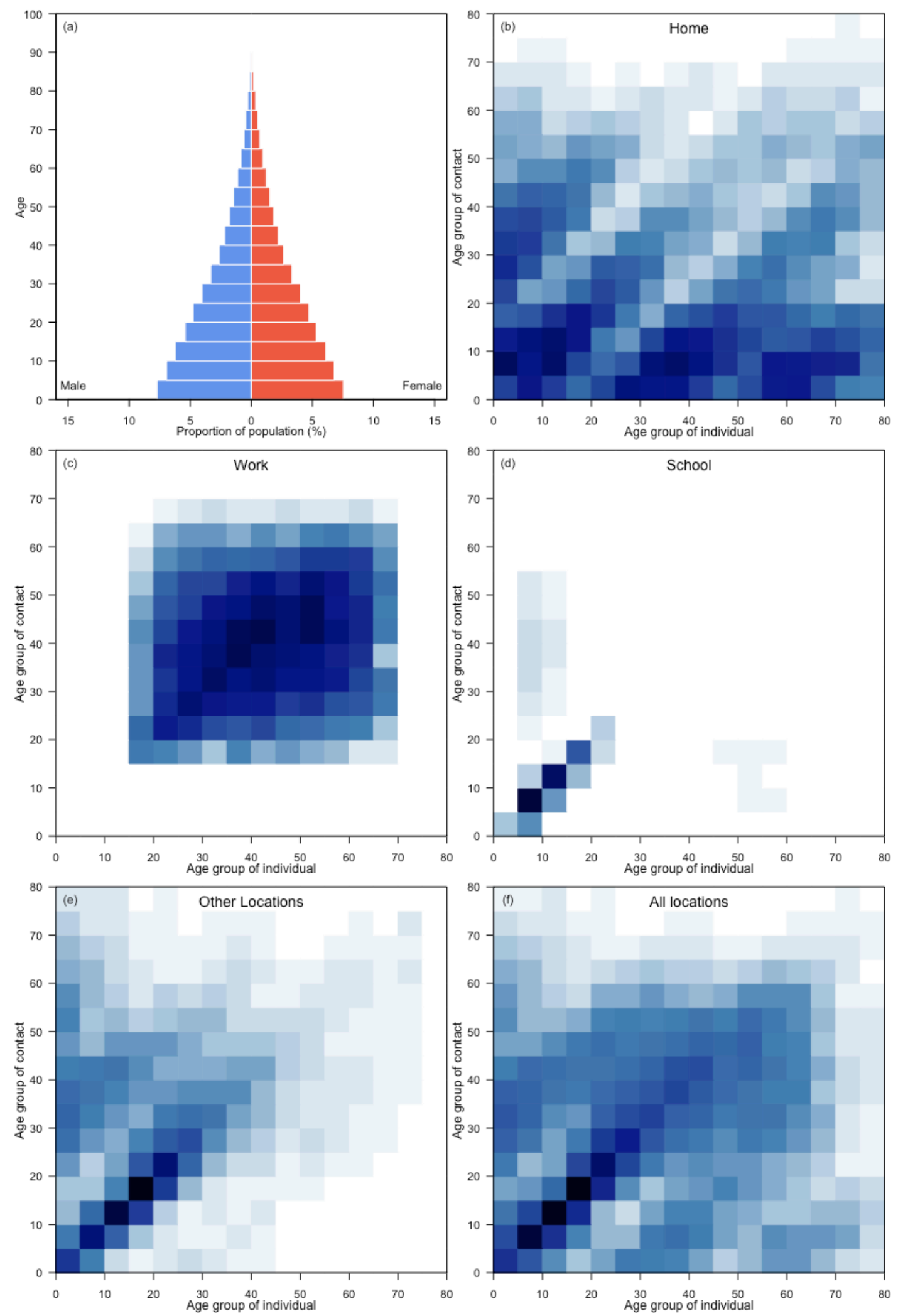


\section{Spain}
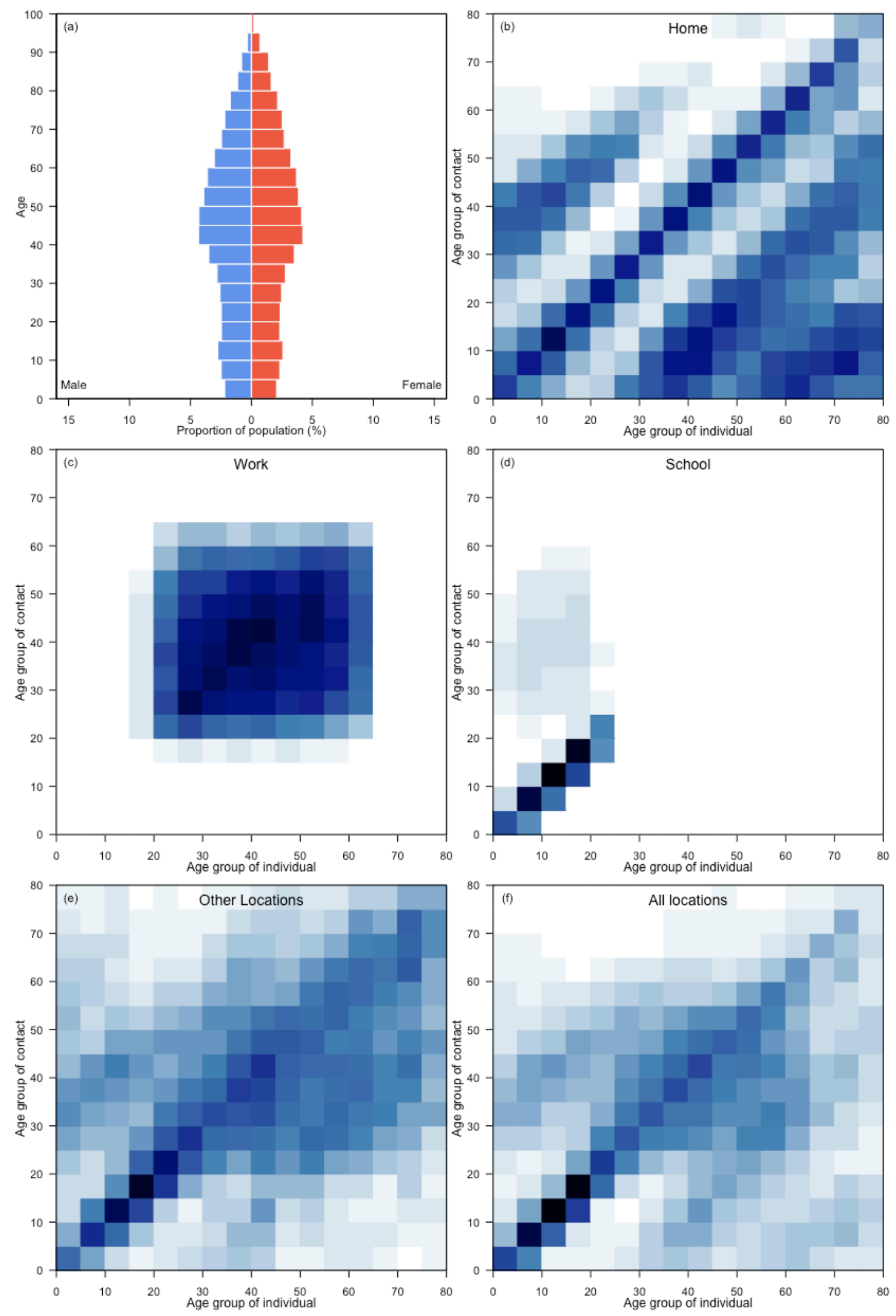


\section{Sri Lanka}
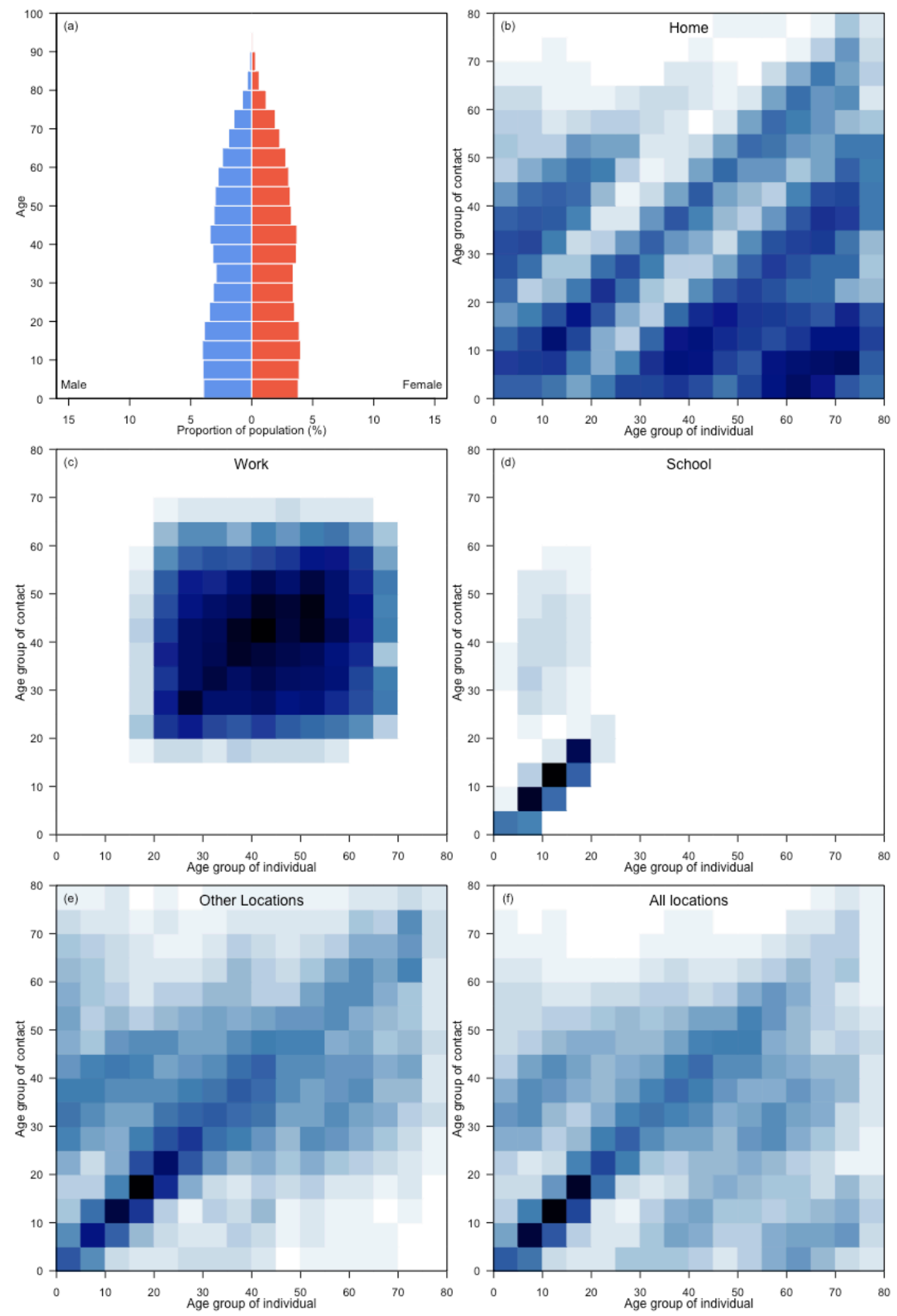


\section{State of Palestine}
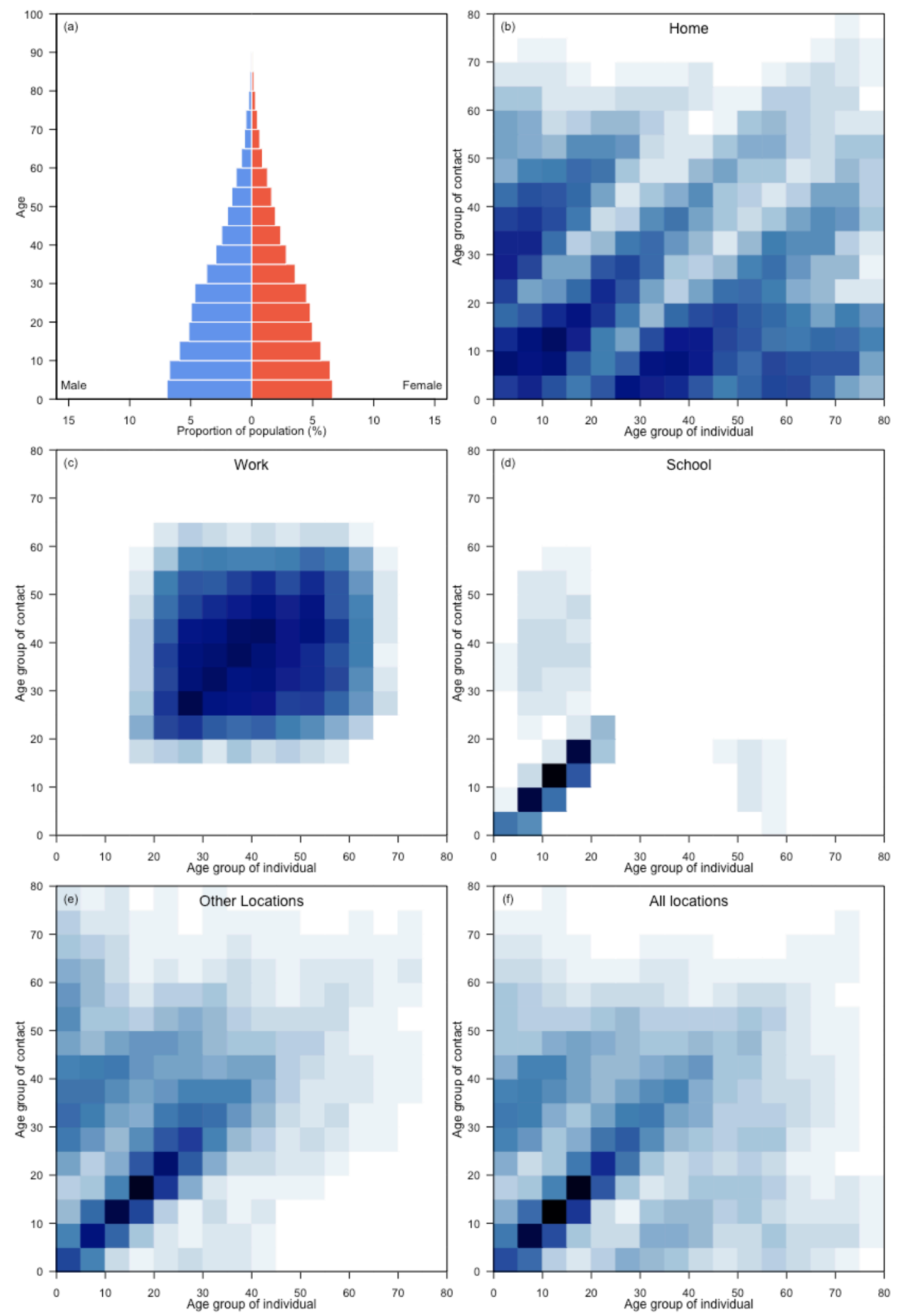


\section{Sudan}
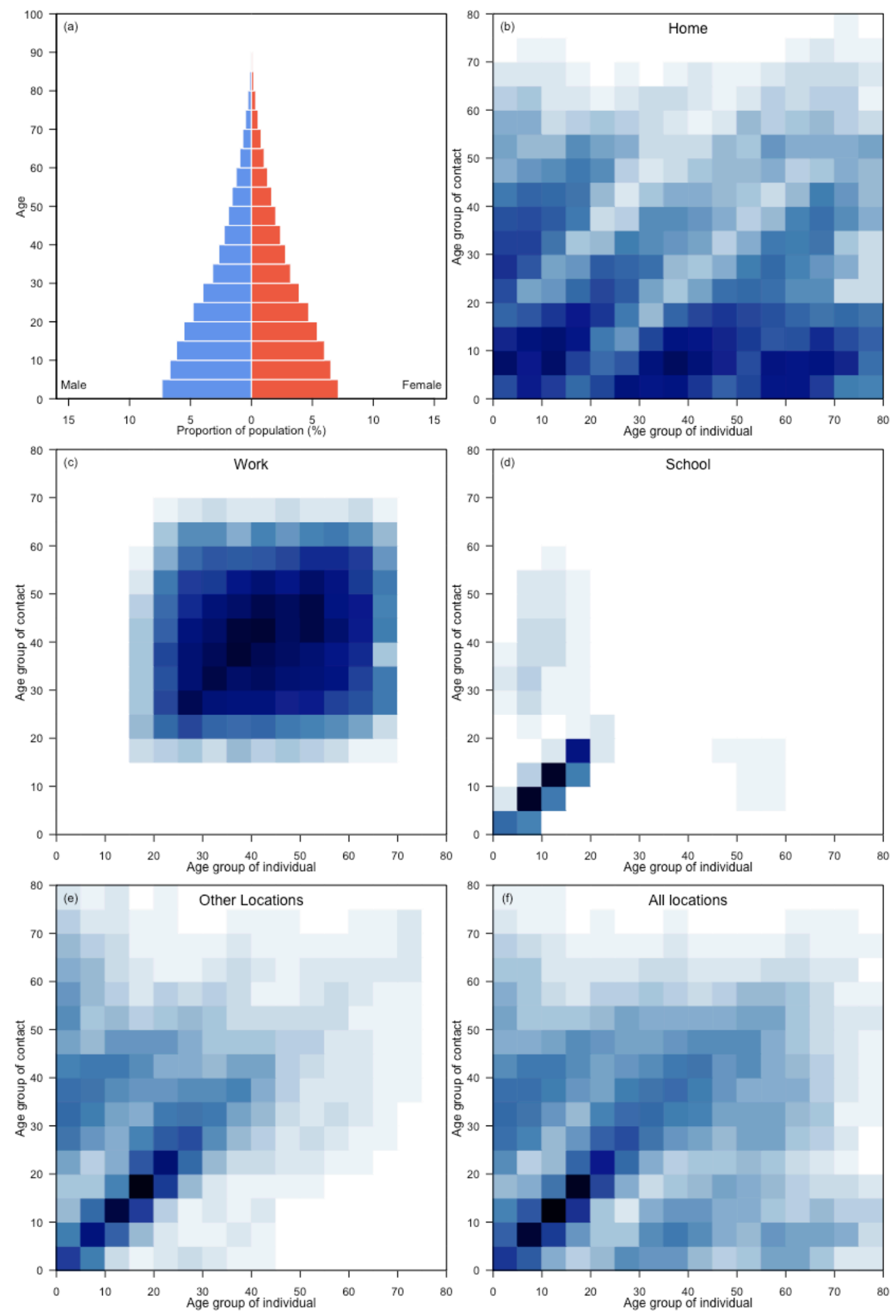


\section{Suriname}
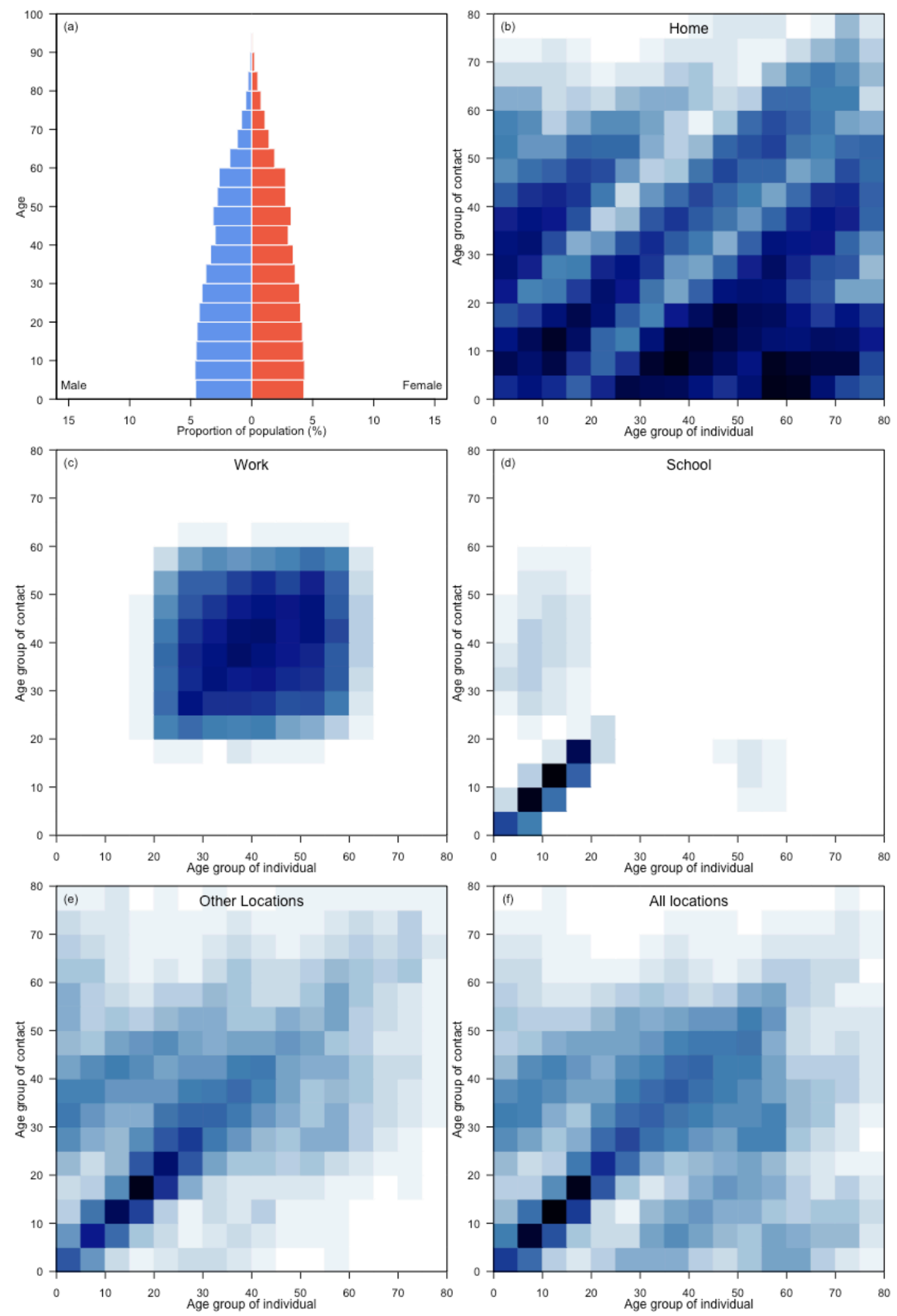


\section{Sweden}
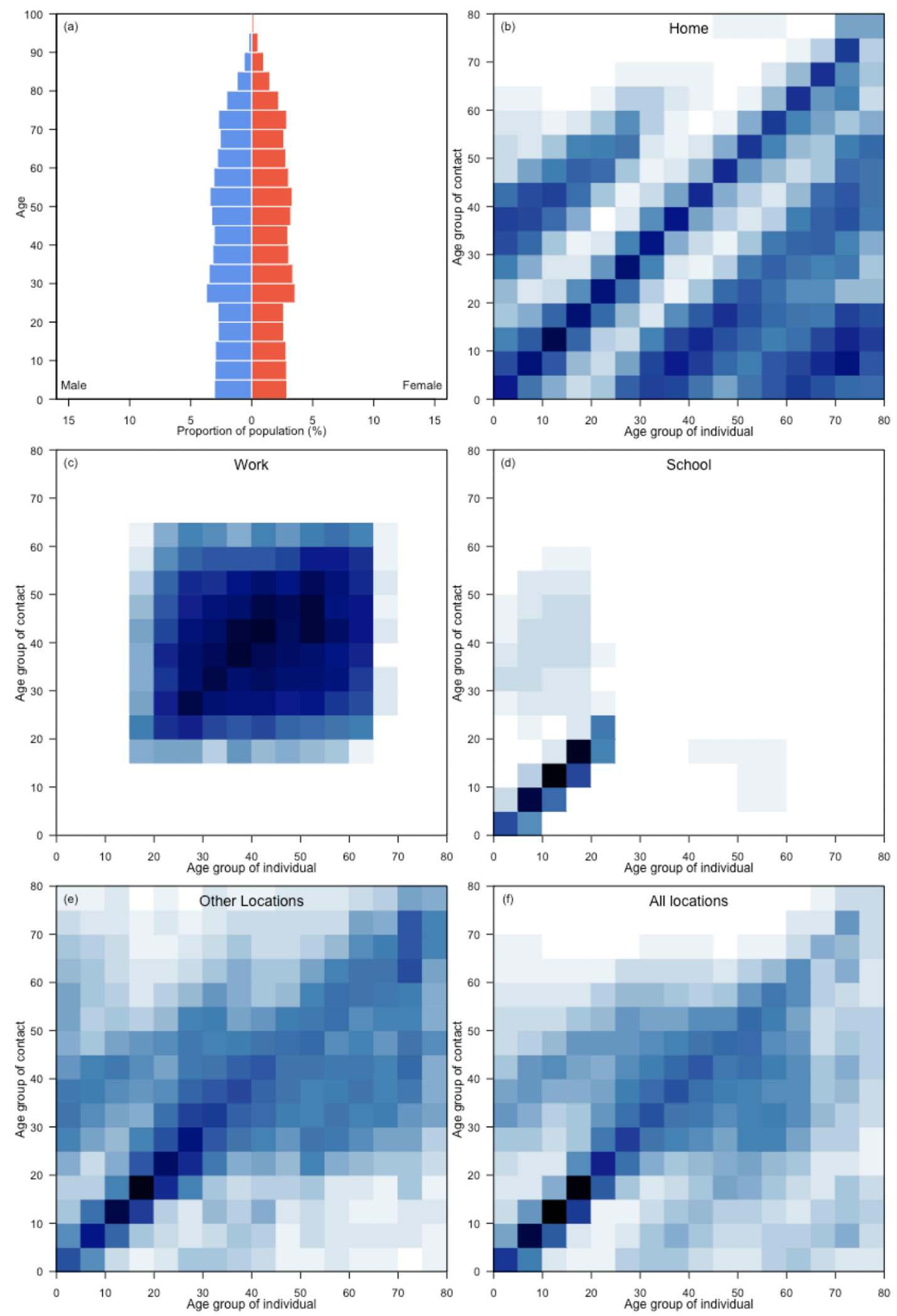


\section{Switzerland}
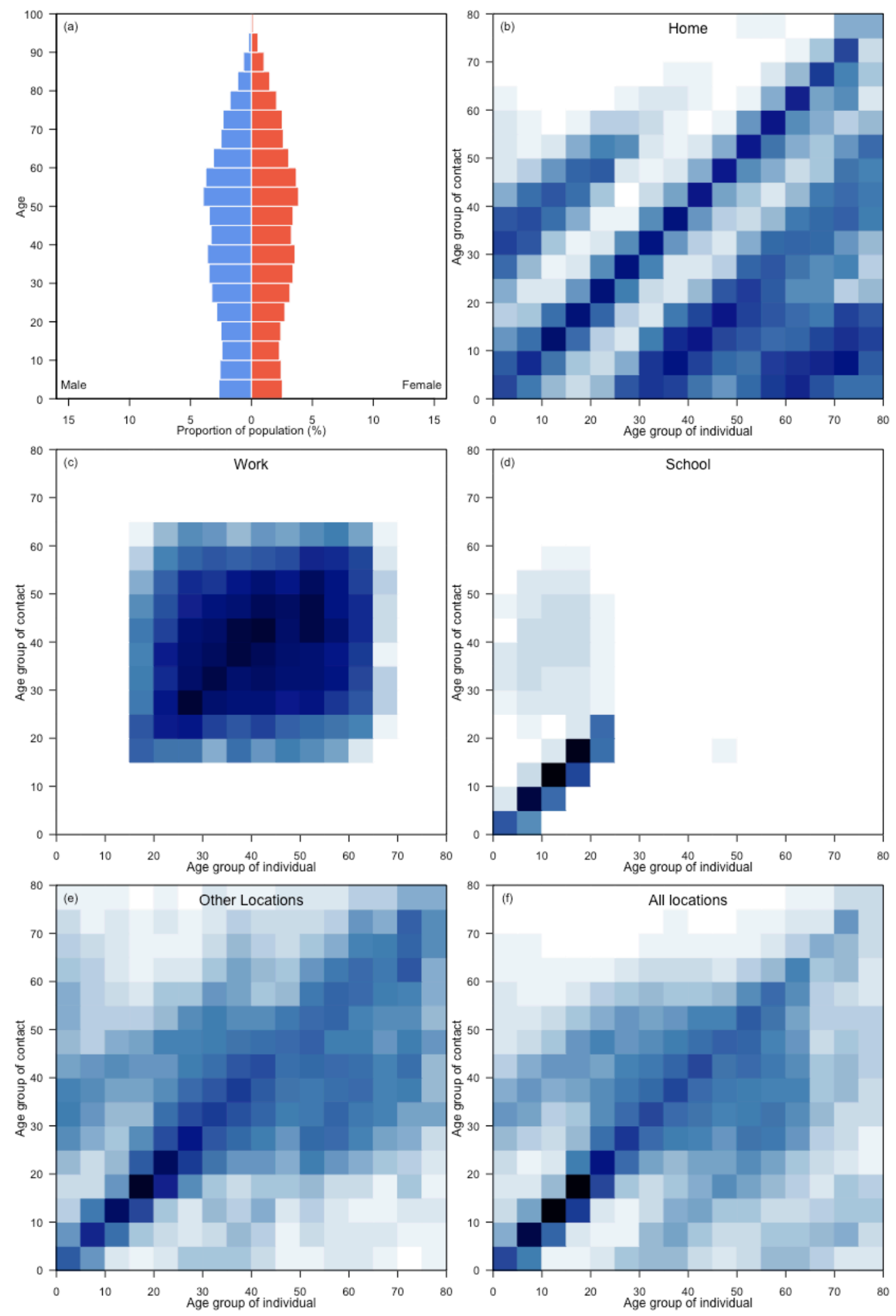
Syrian Arab Republic
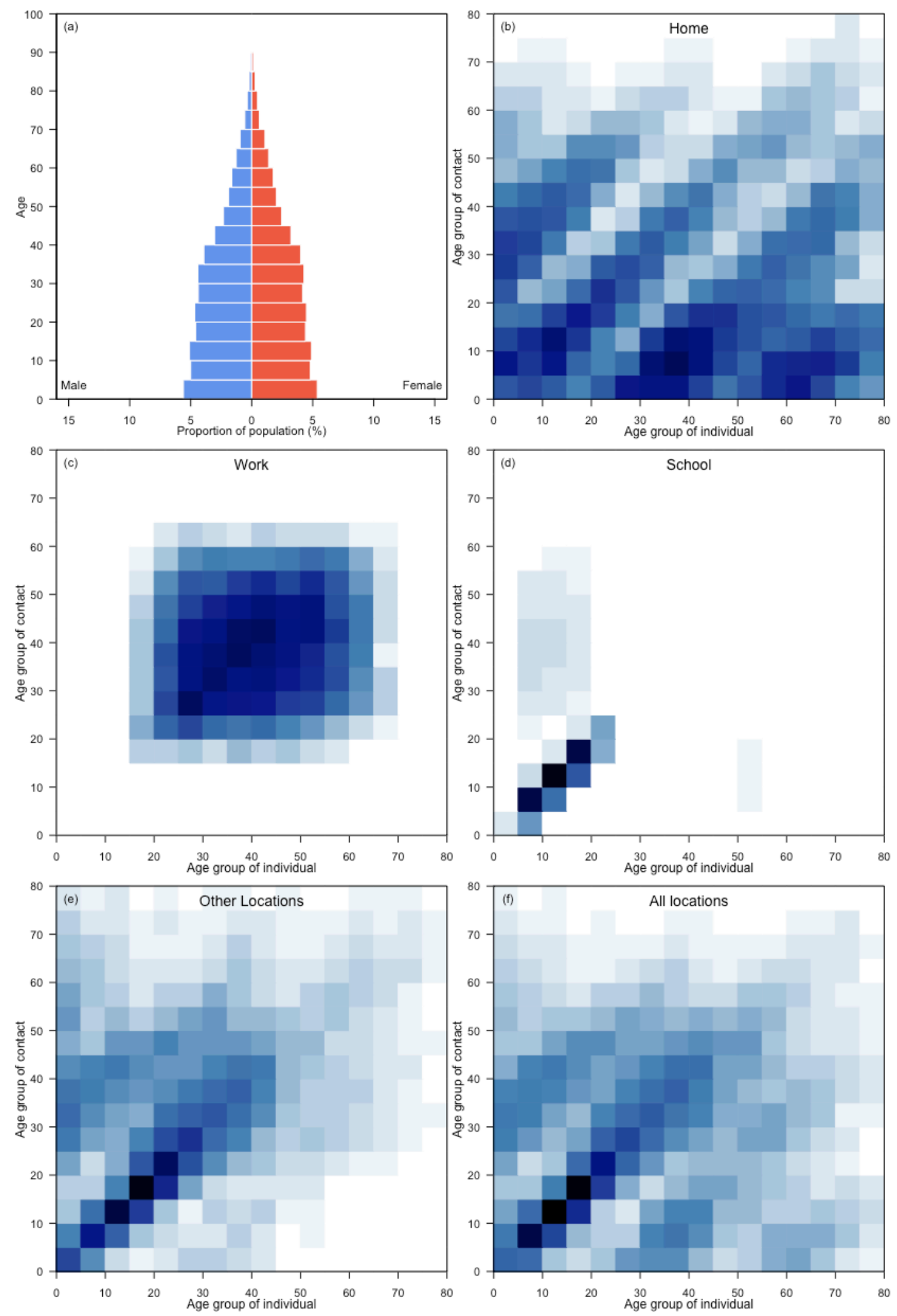
Tajikistan
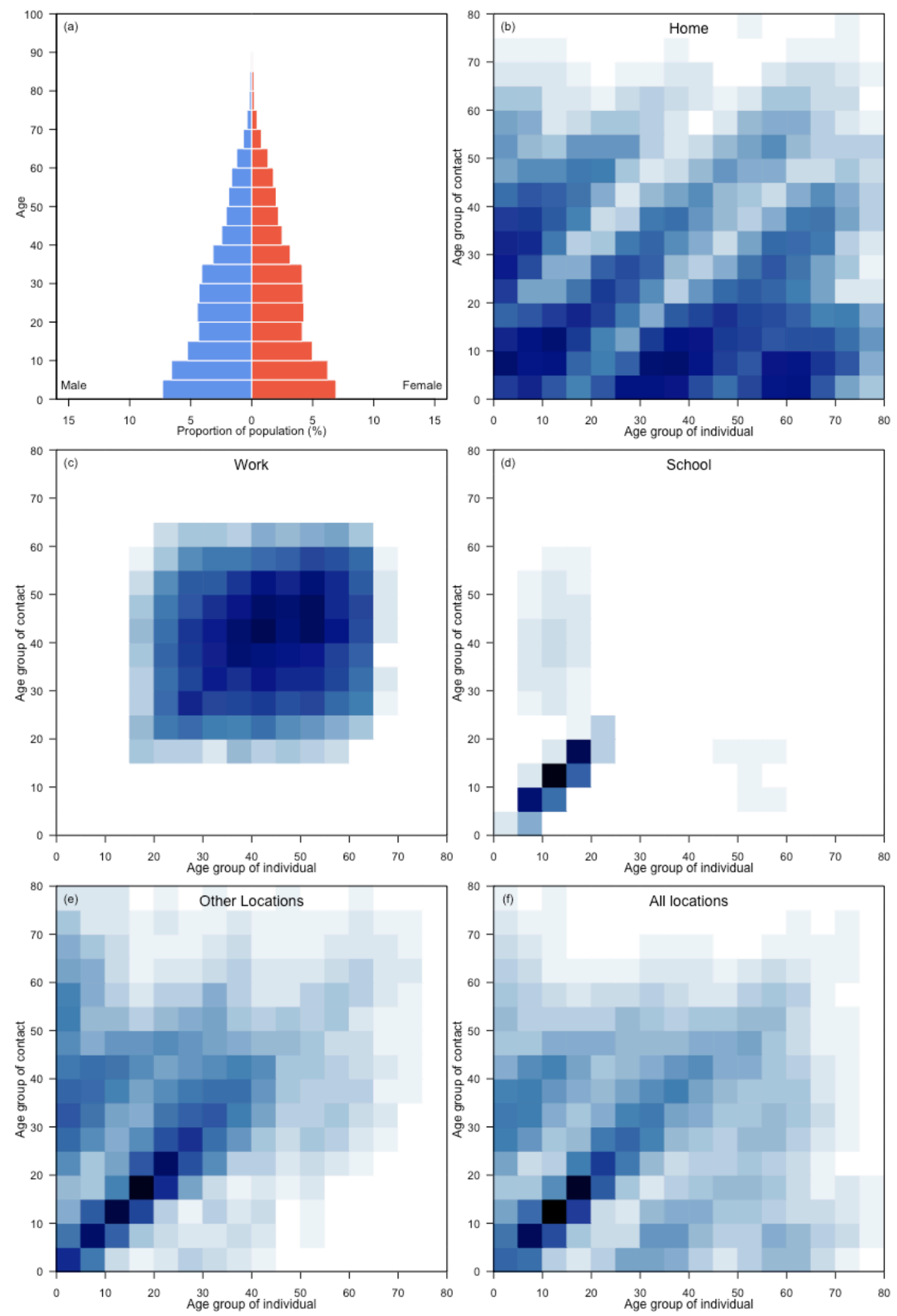


\section{Thailand}
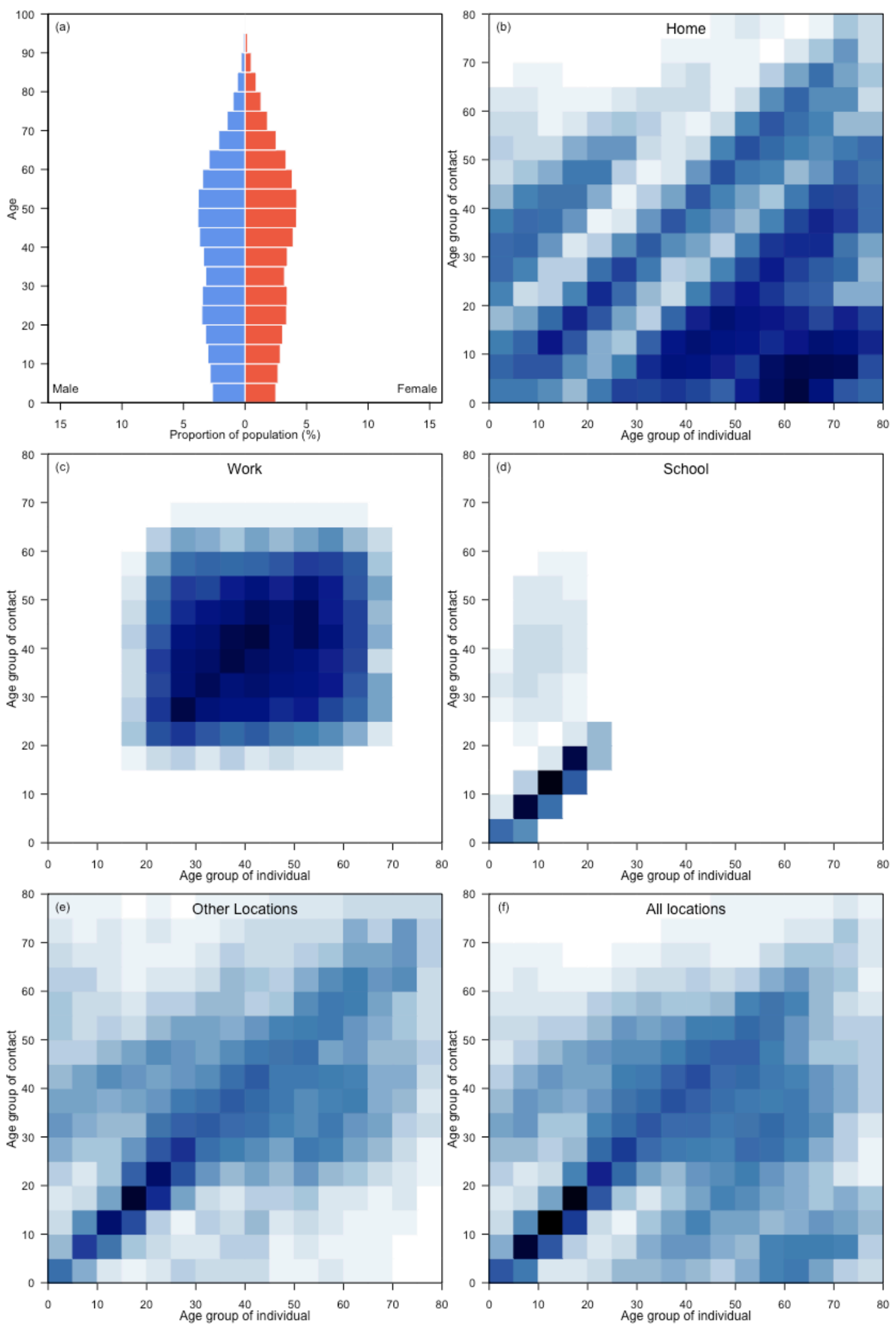
Timor-Leste
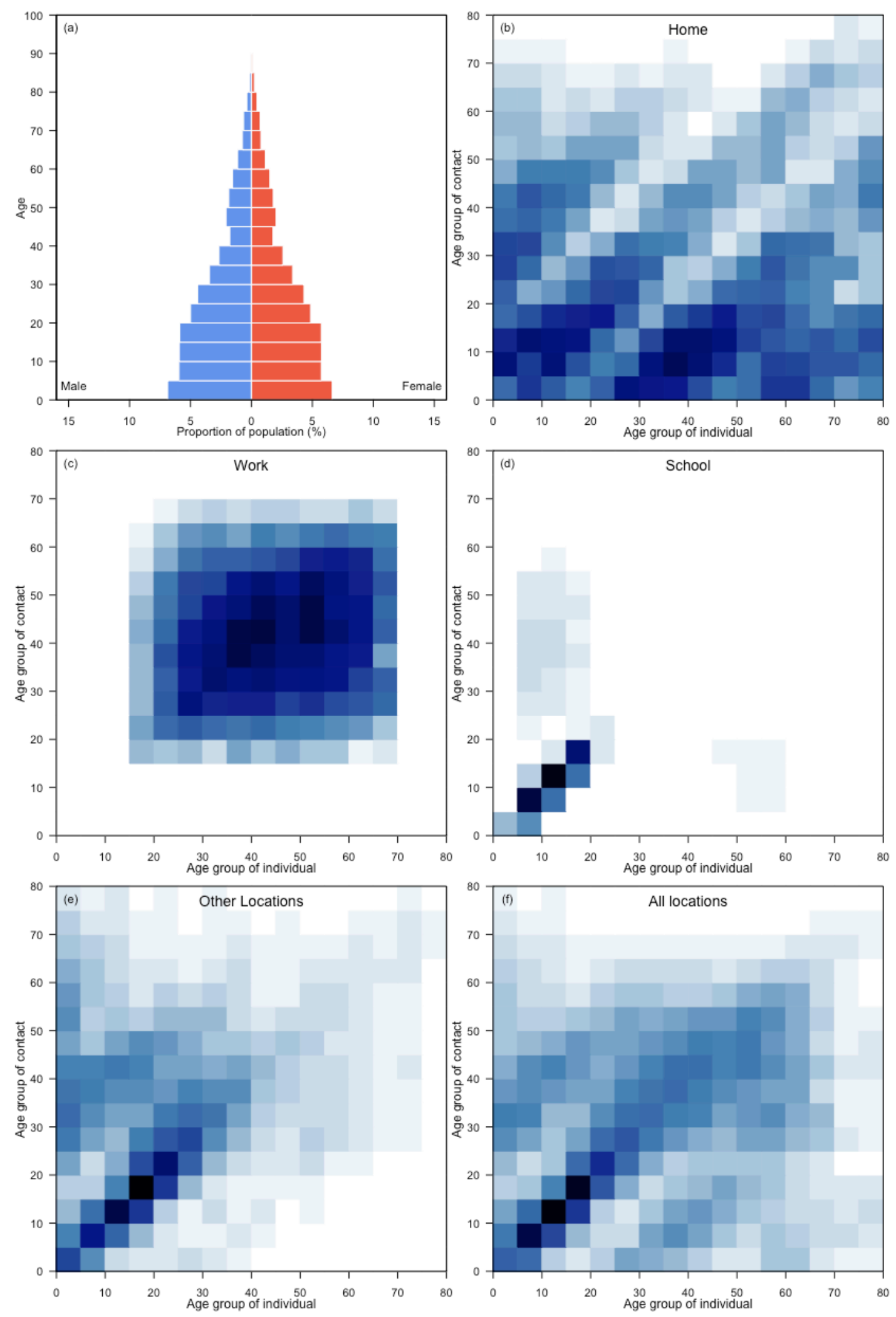
Togo
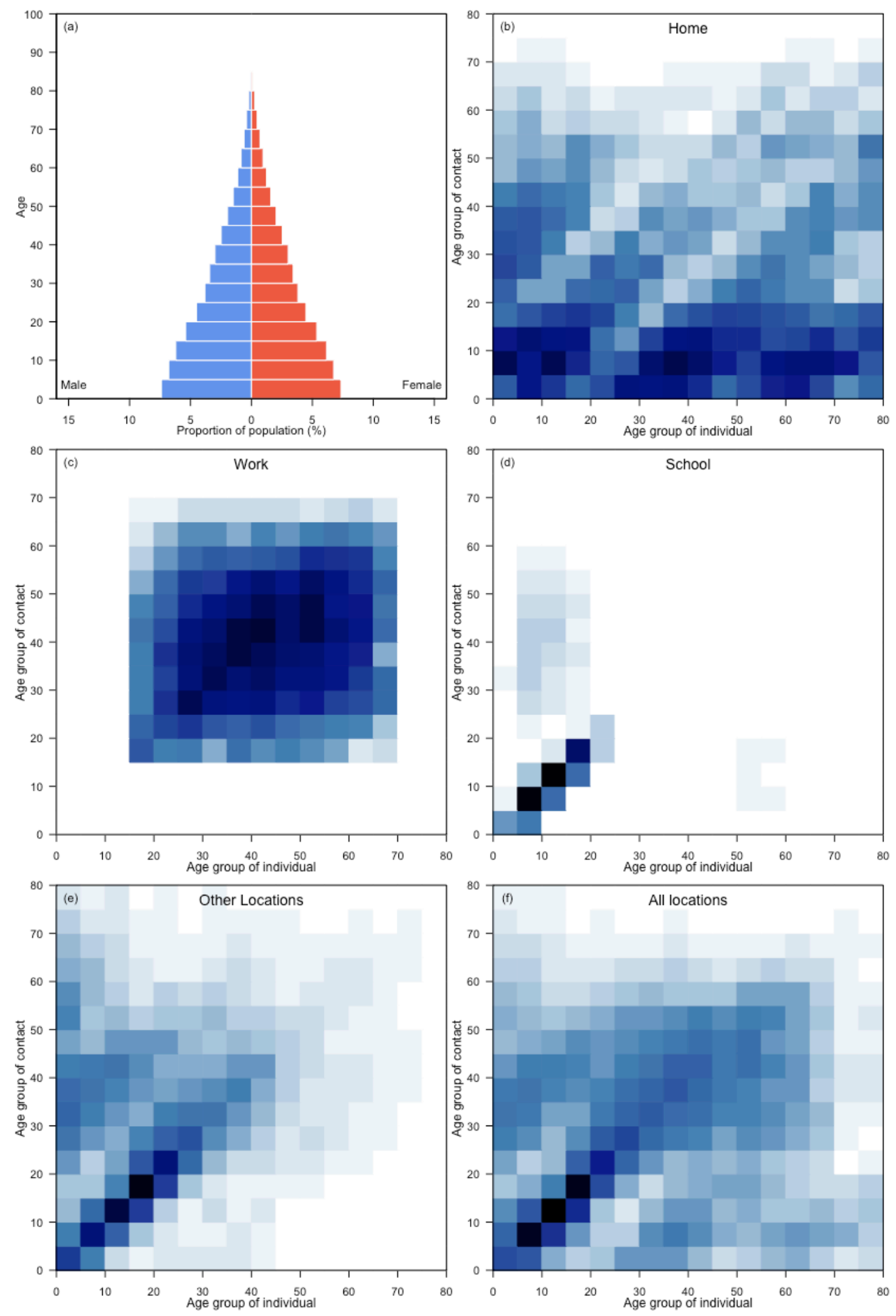


\section{Tonga}
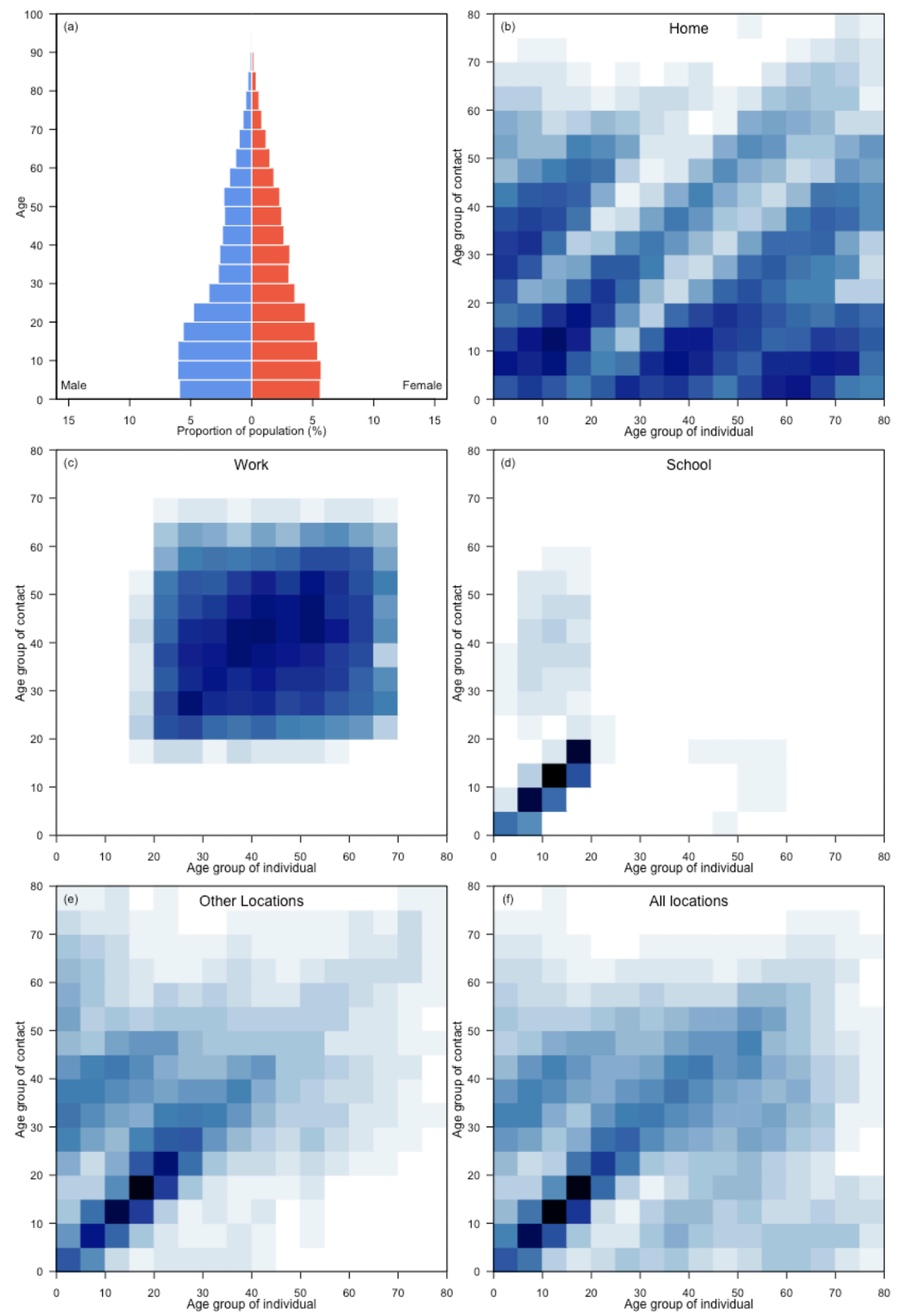
Trinidad and Tobago
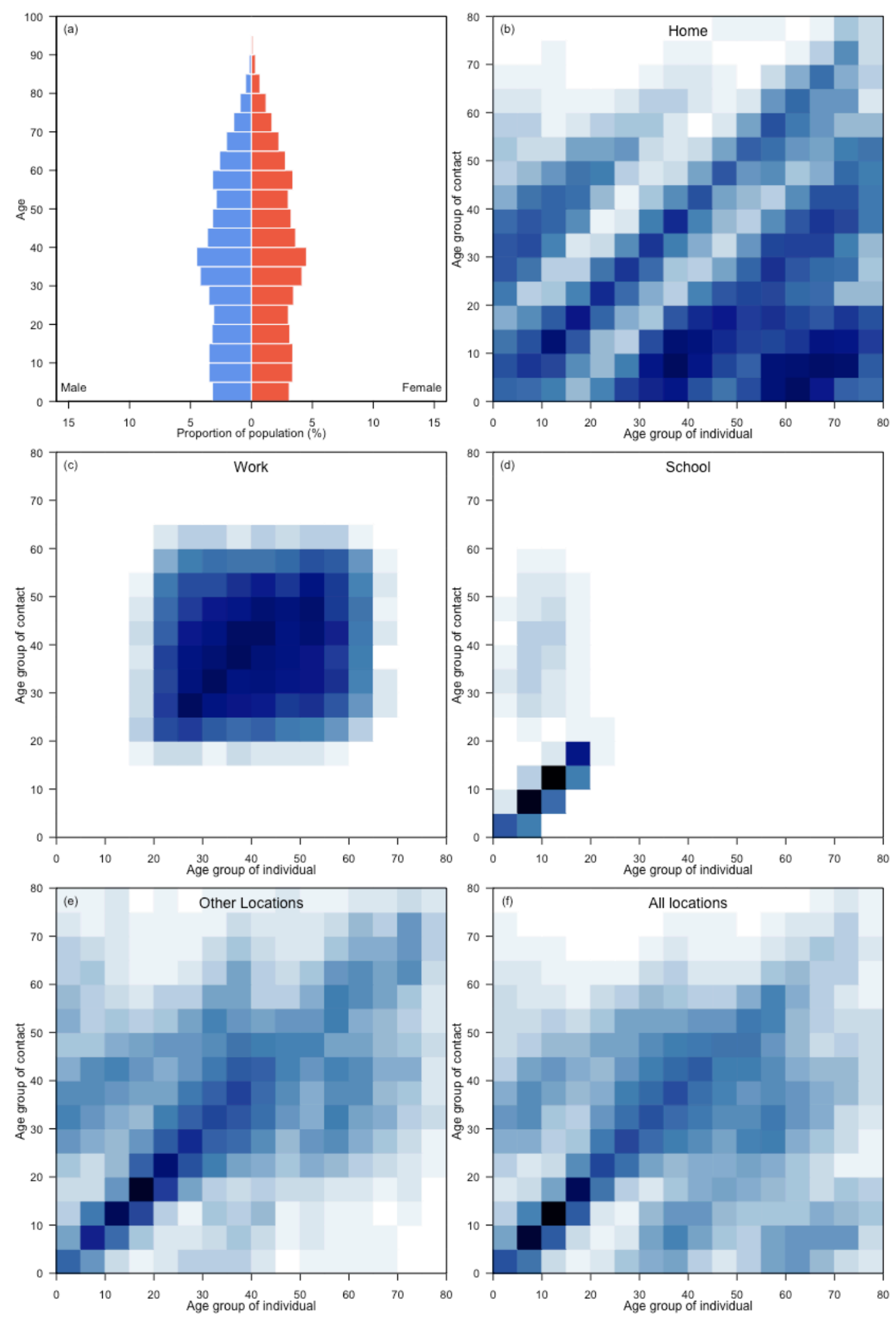


\section{Tunisia}
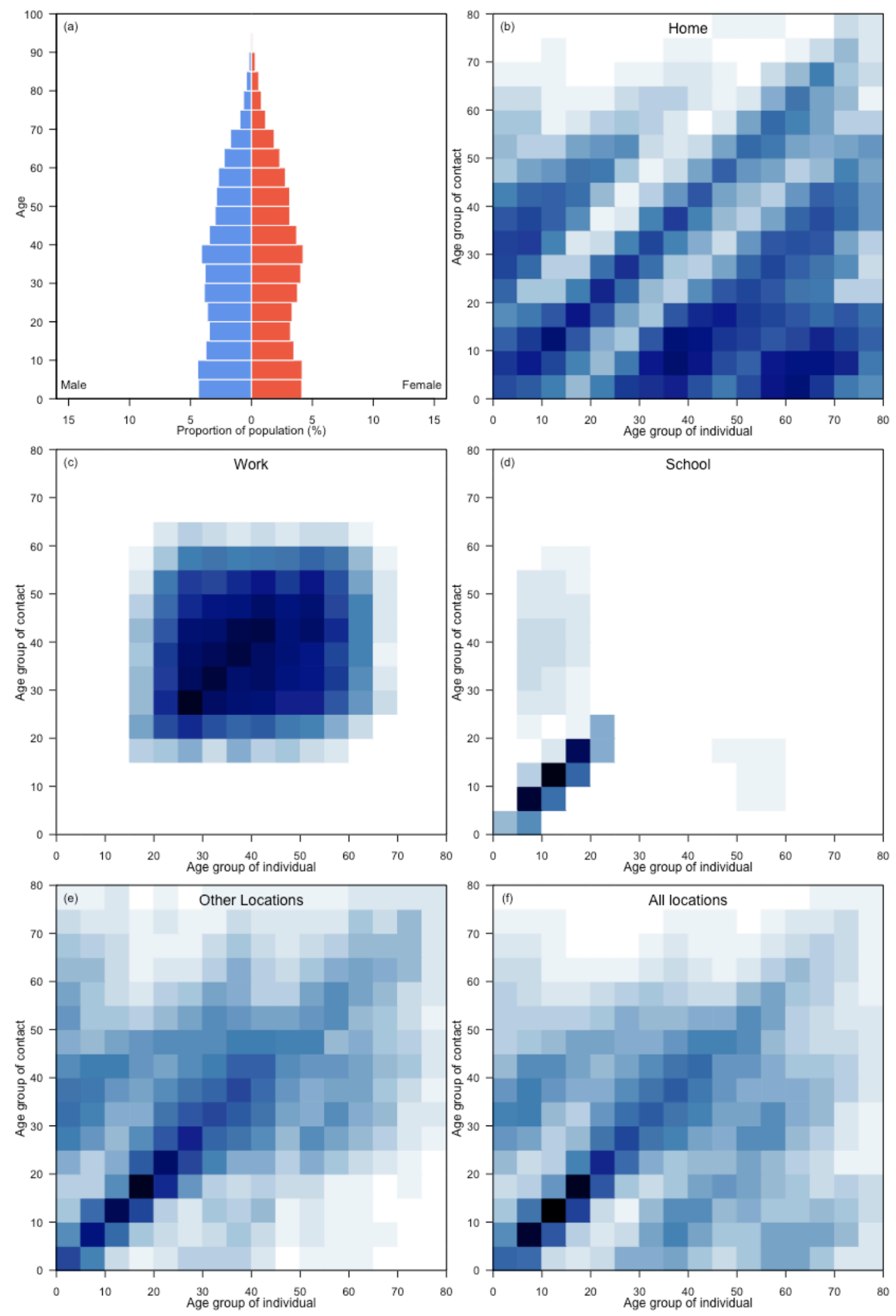


\section{Turkey}
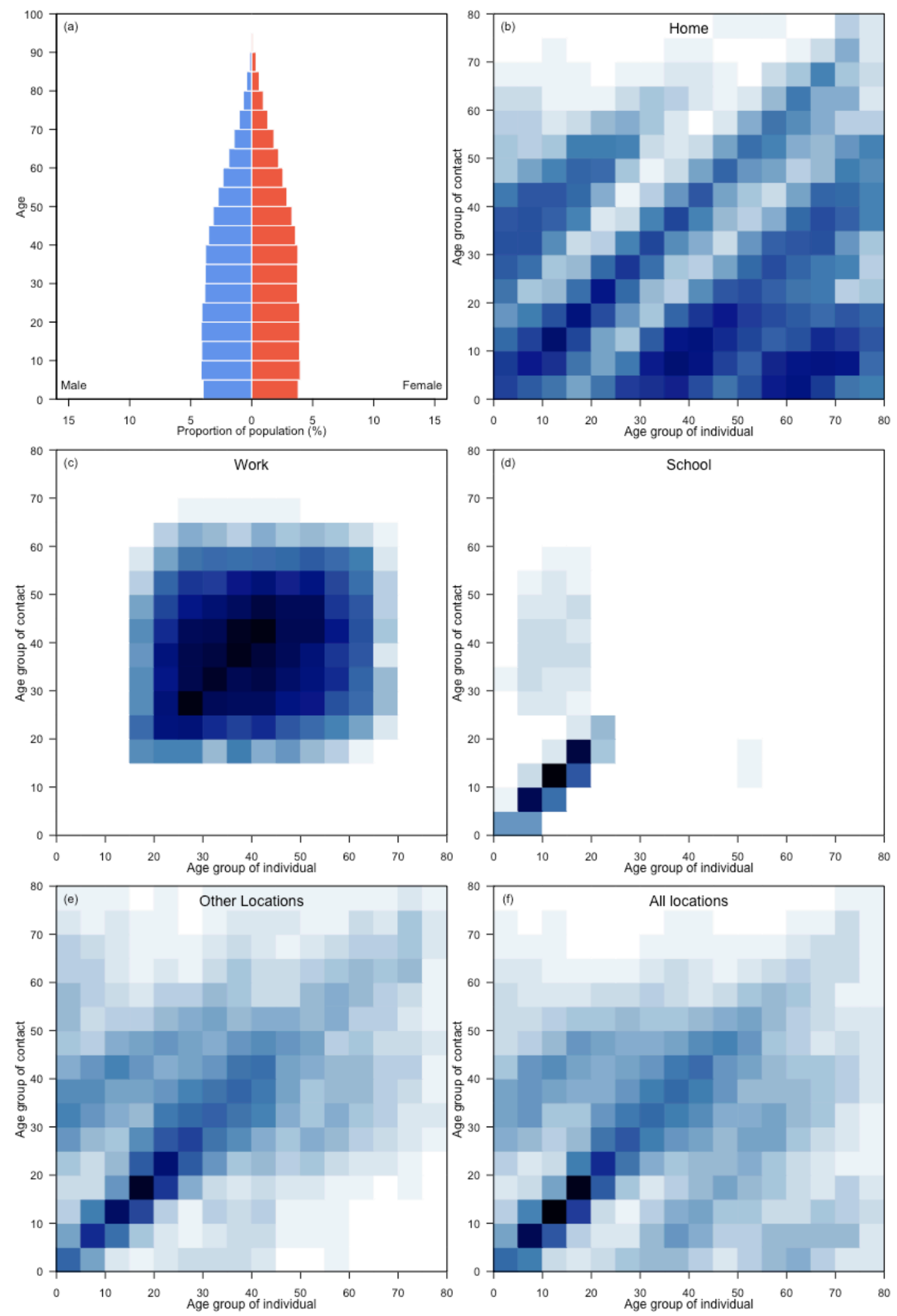
Turkmenistan
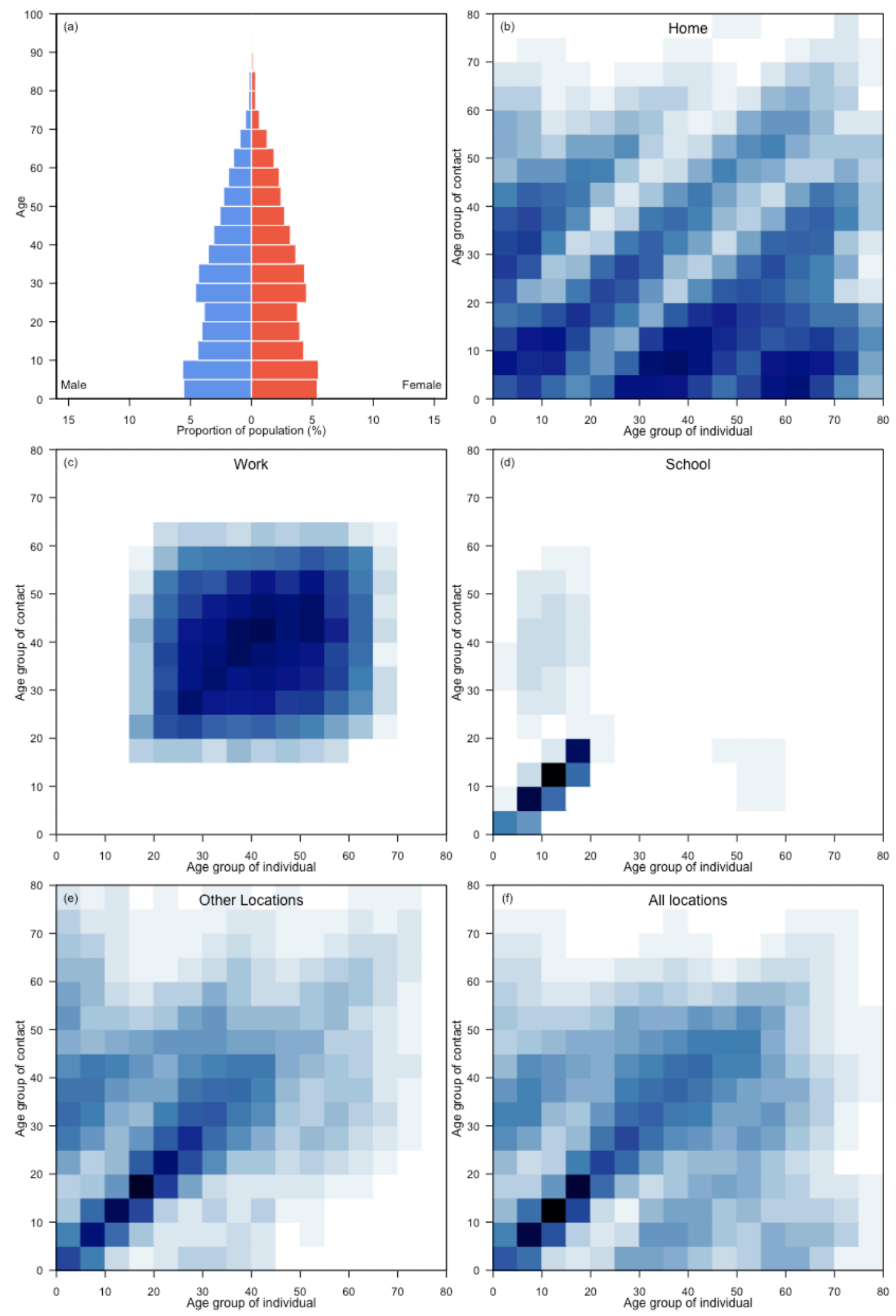


\section{Uganda}
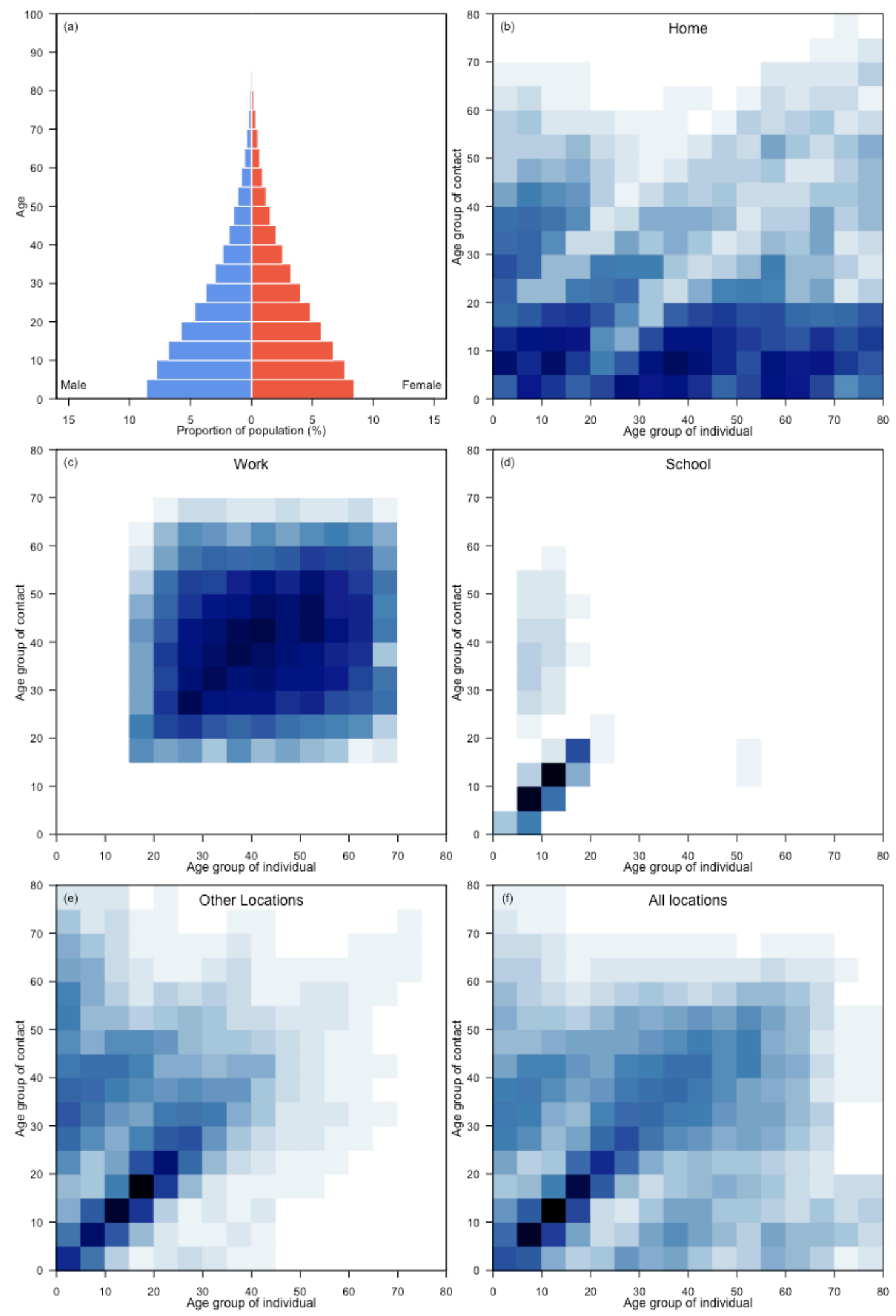


\section{Ukraine}
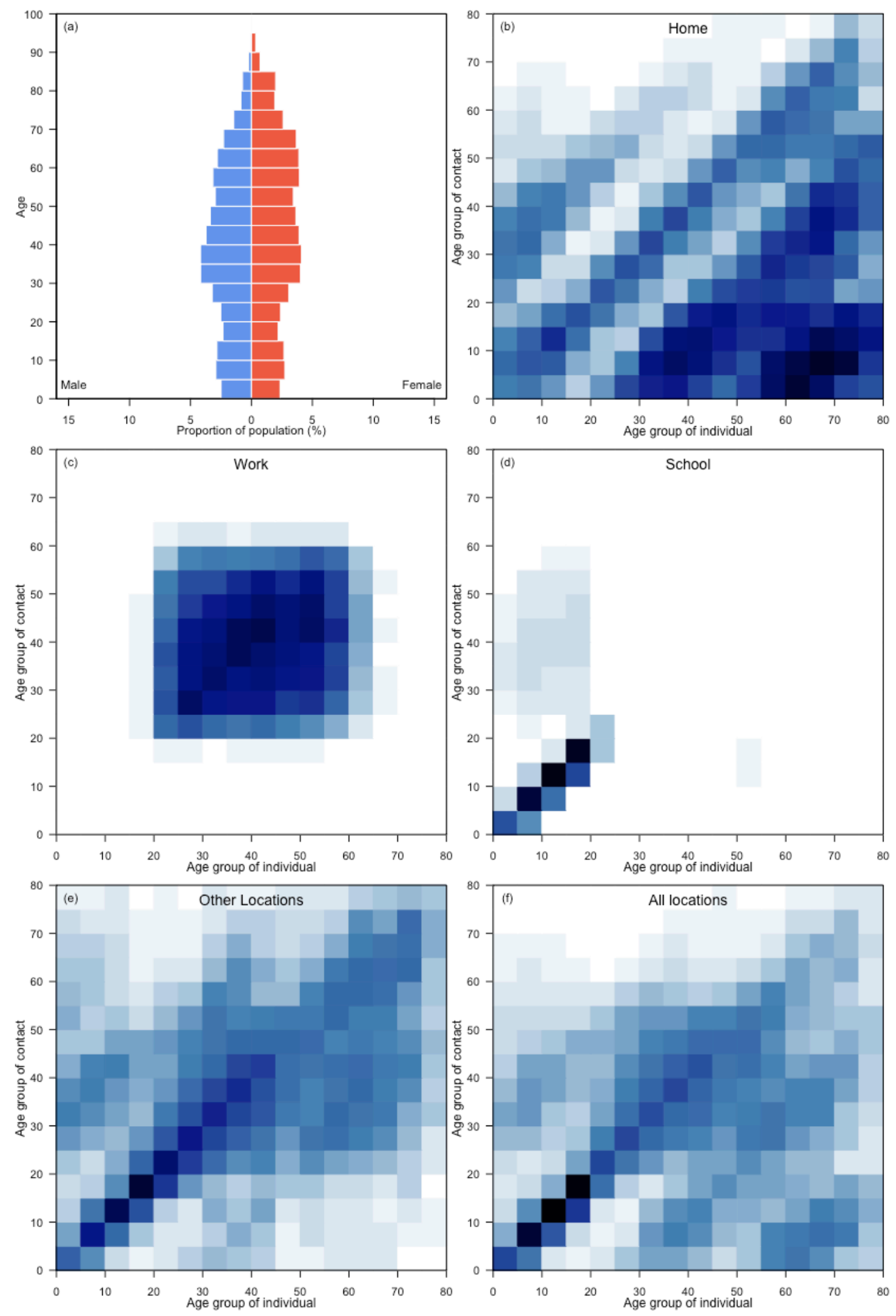


\section{United Arab Emirates}
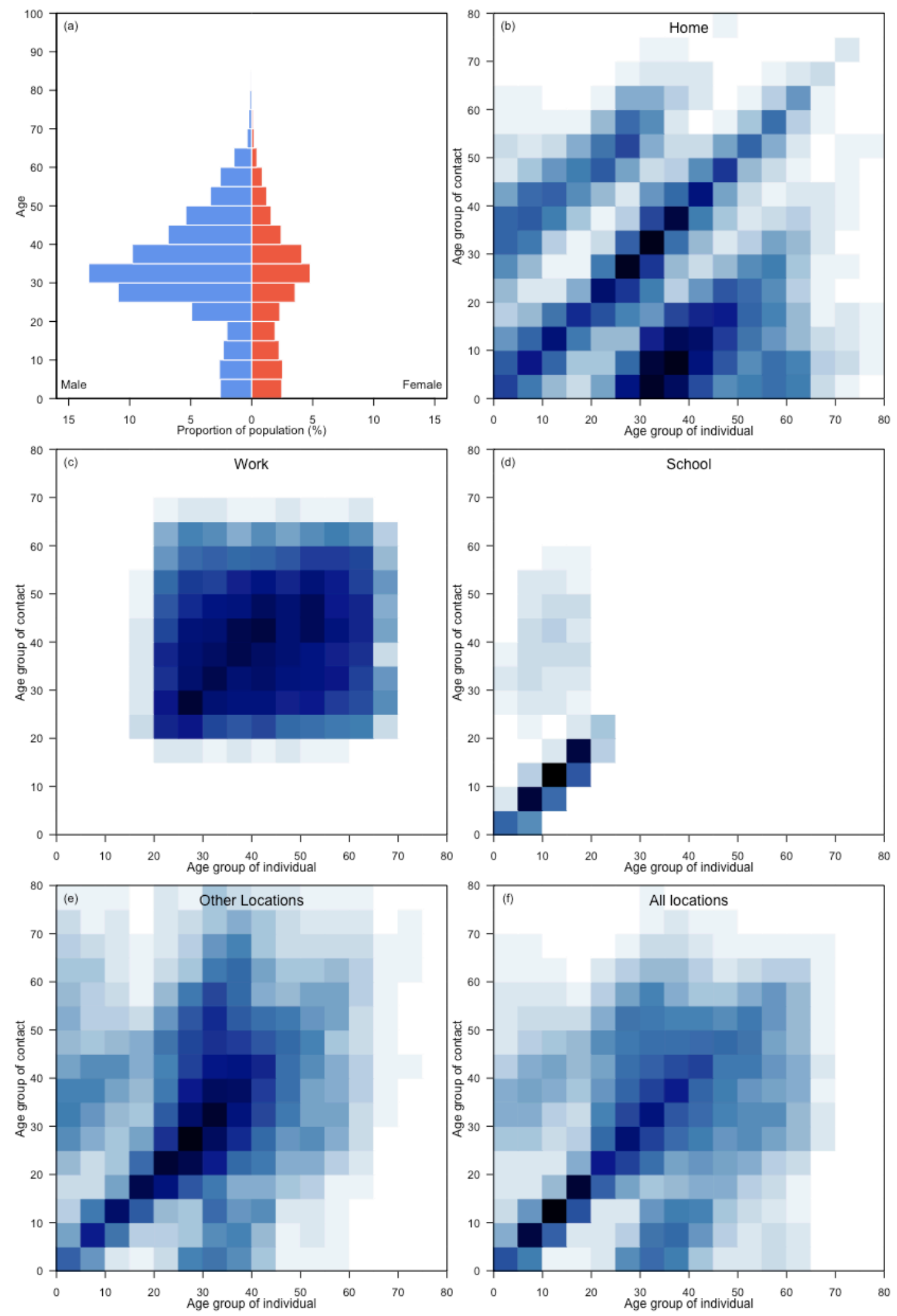
United Kingdom
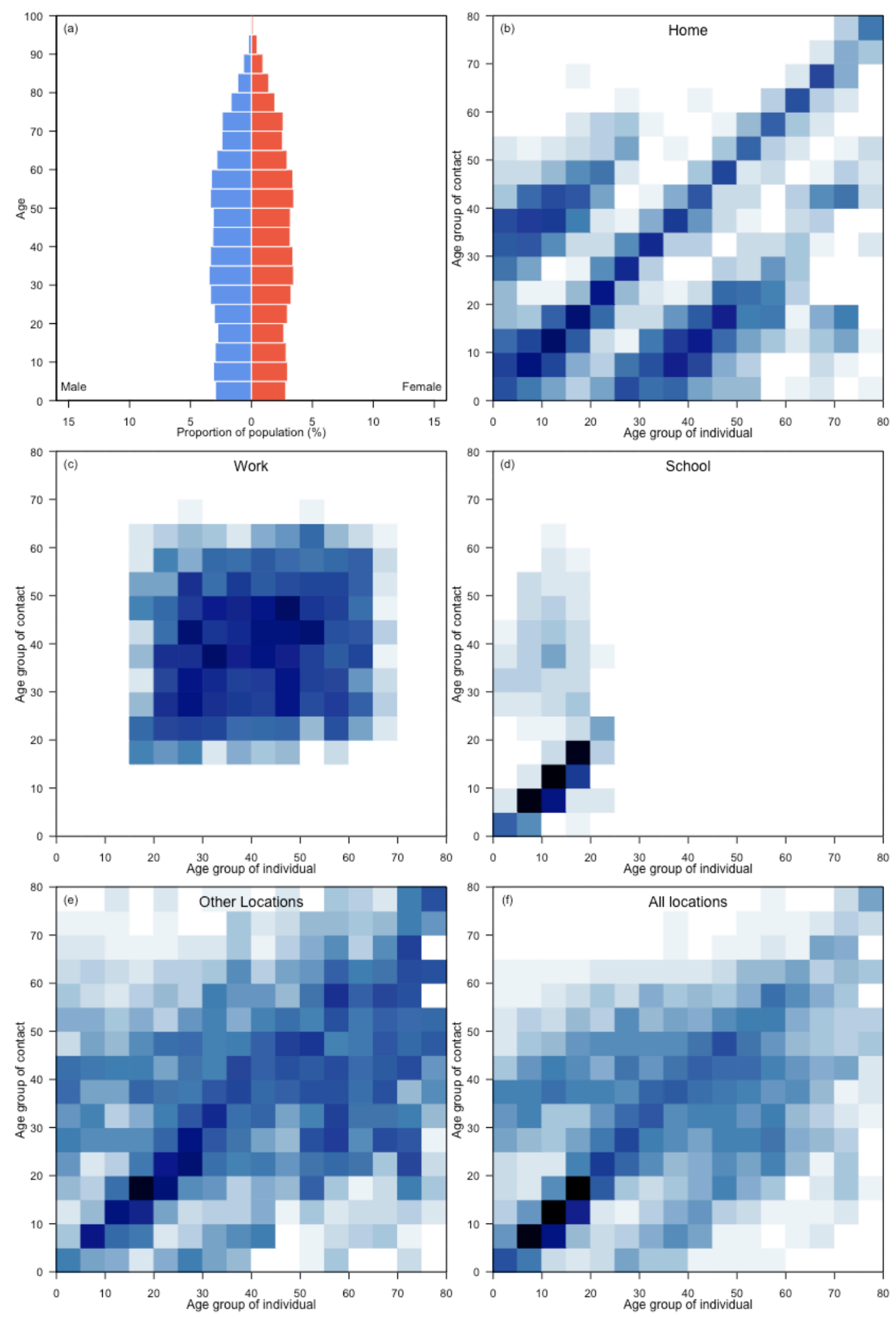
United Republic of Tanzania
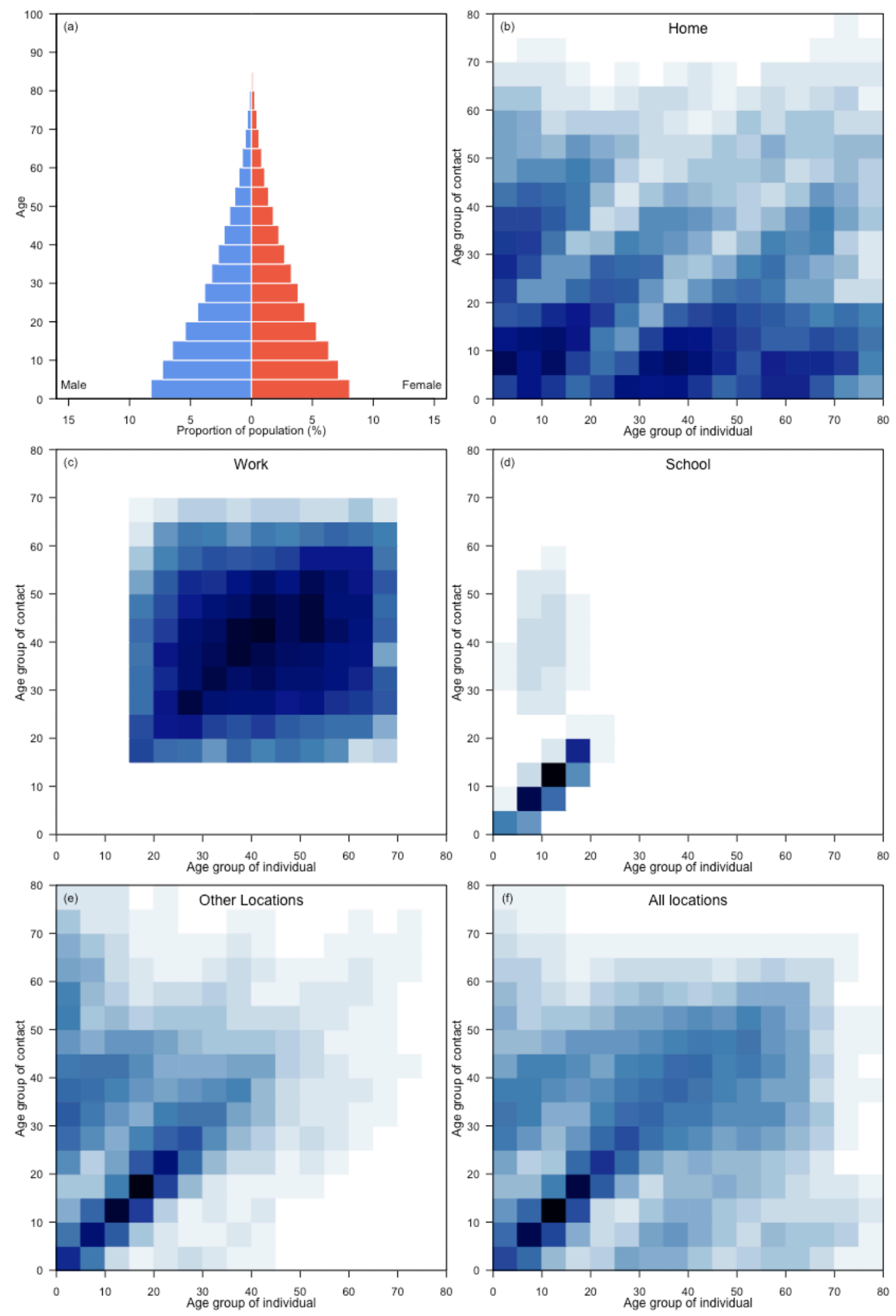


\section{United States of America}
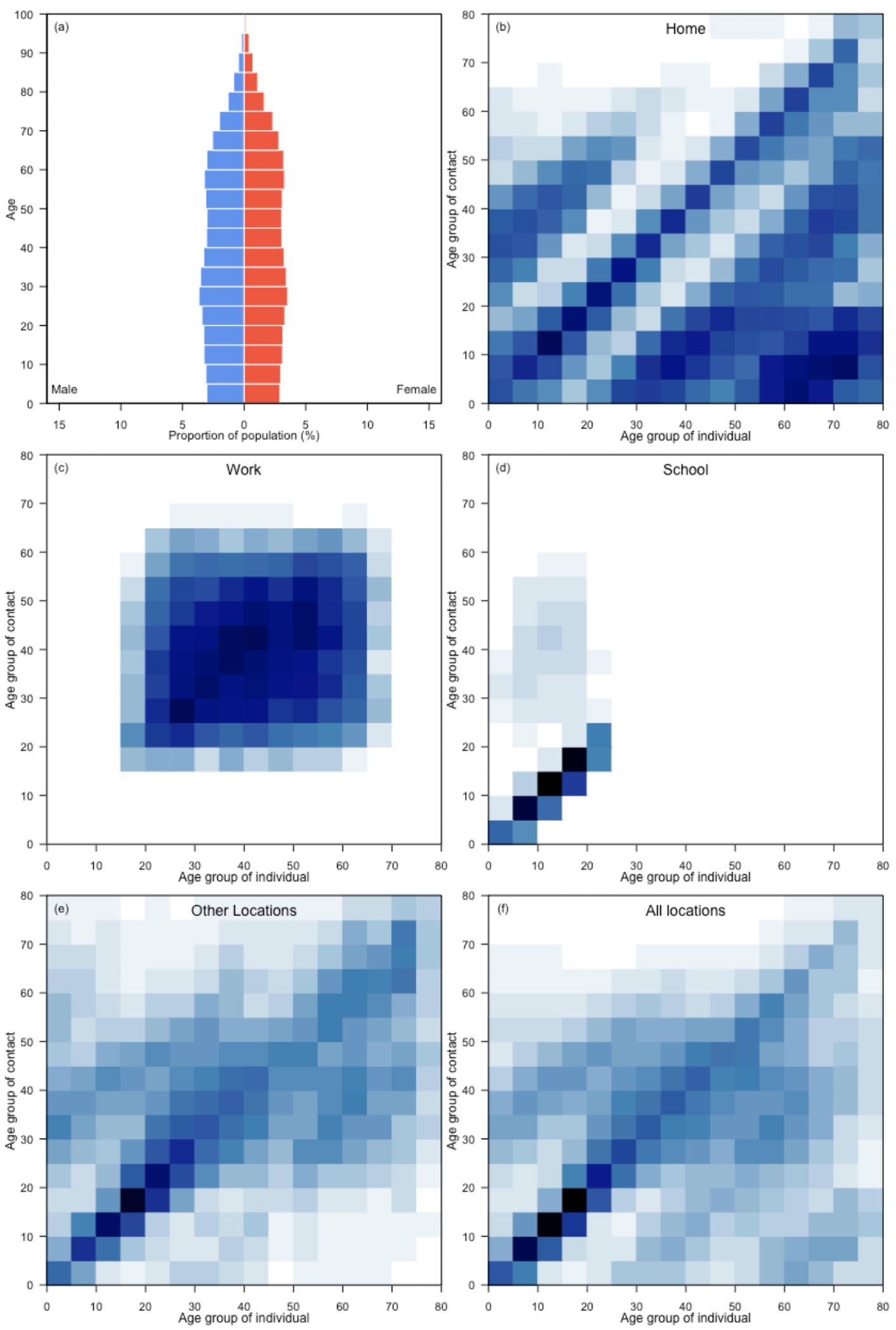


\section{Uruguay}
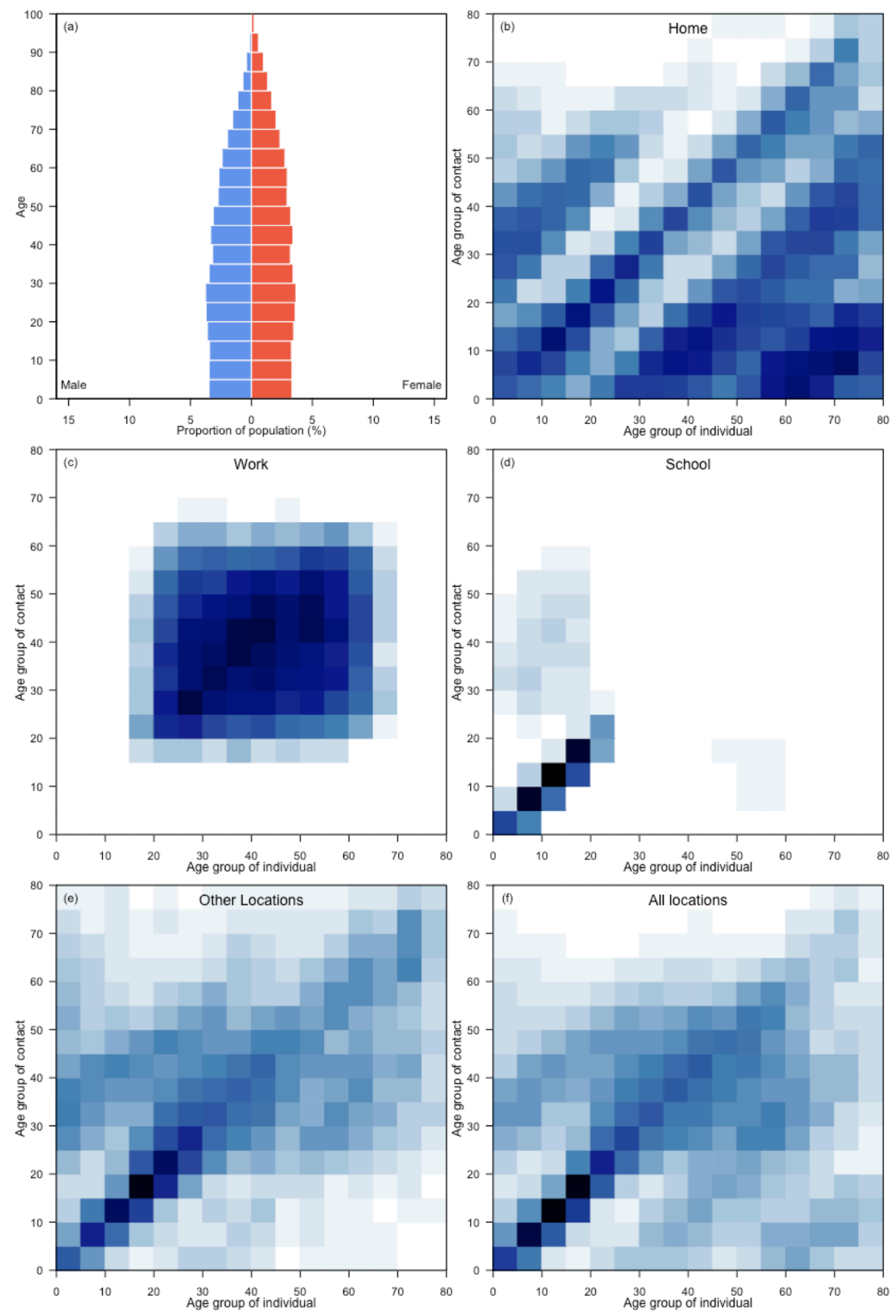
Uzbekistan
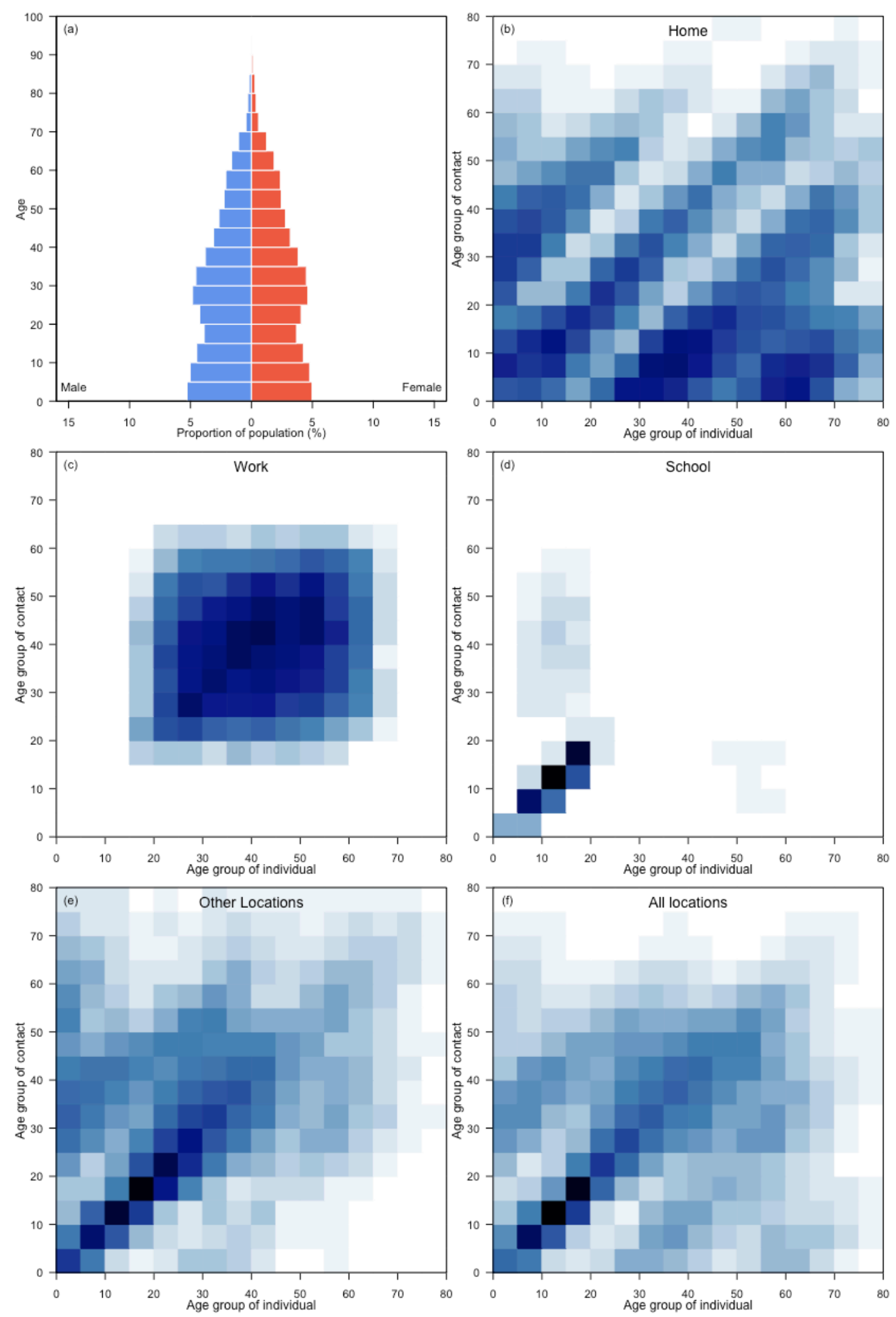
Vanuatu
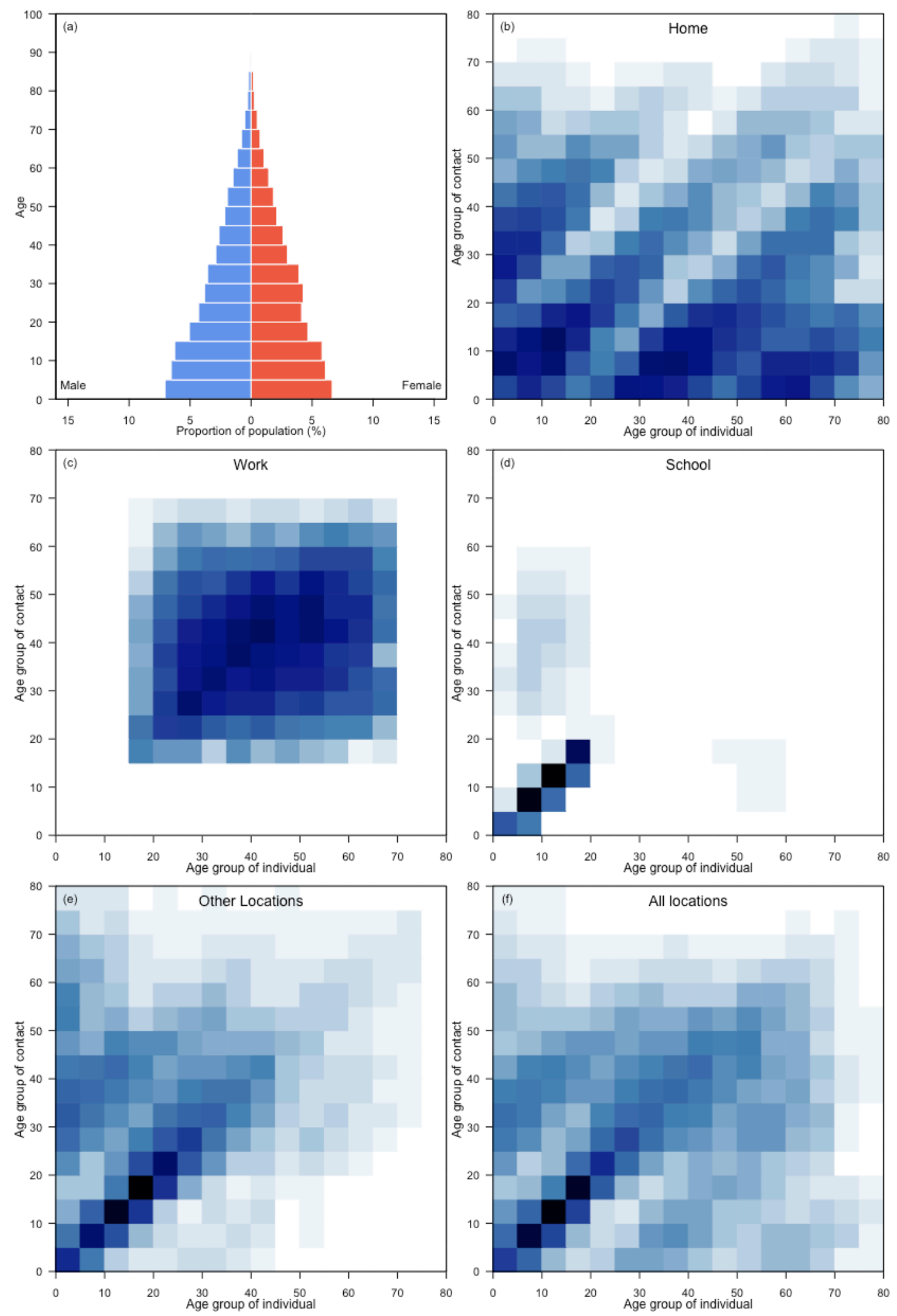
Venezuela (Bolivarian Republic of)
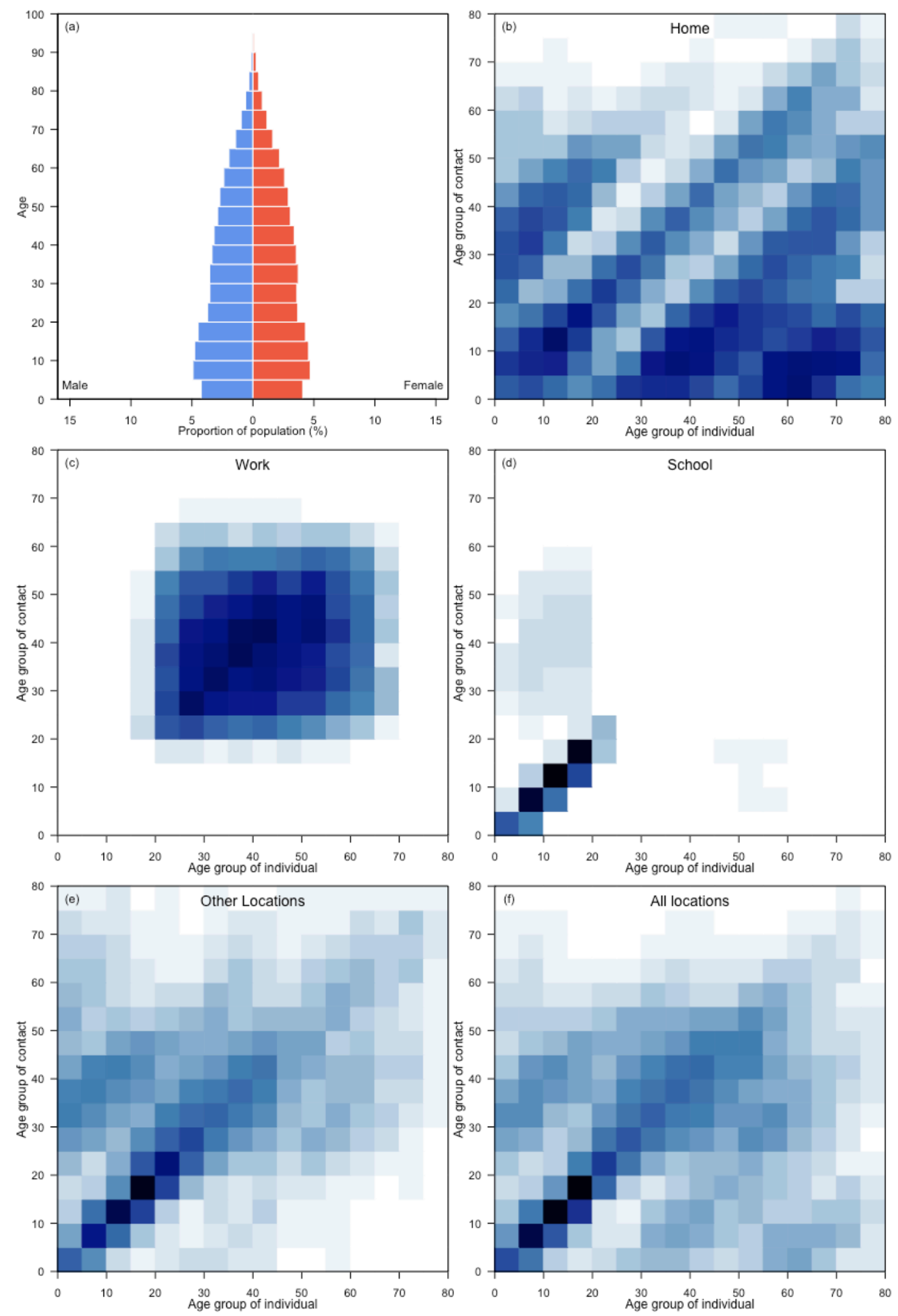
Viet Nam
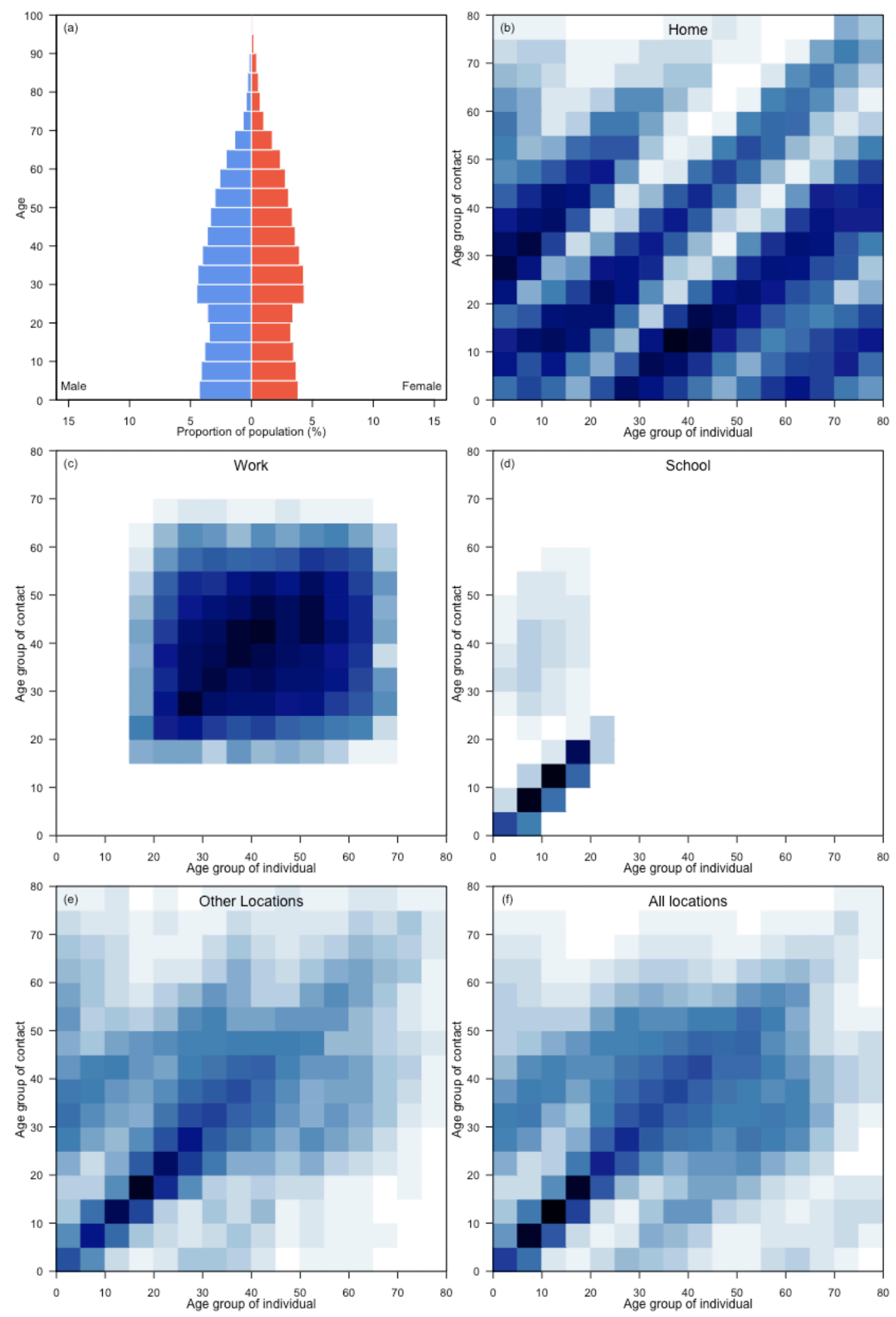
Yemen
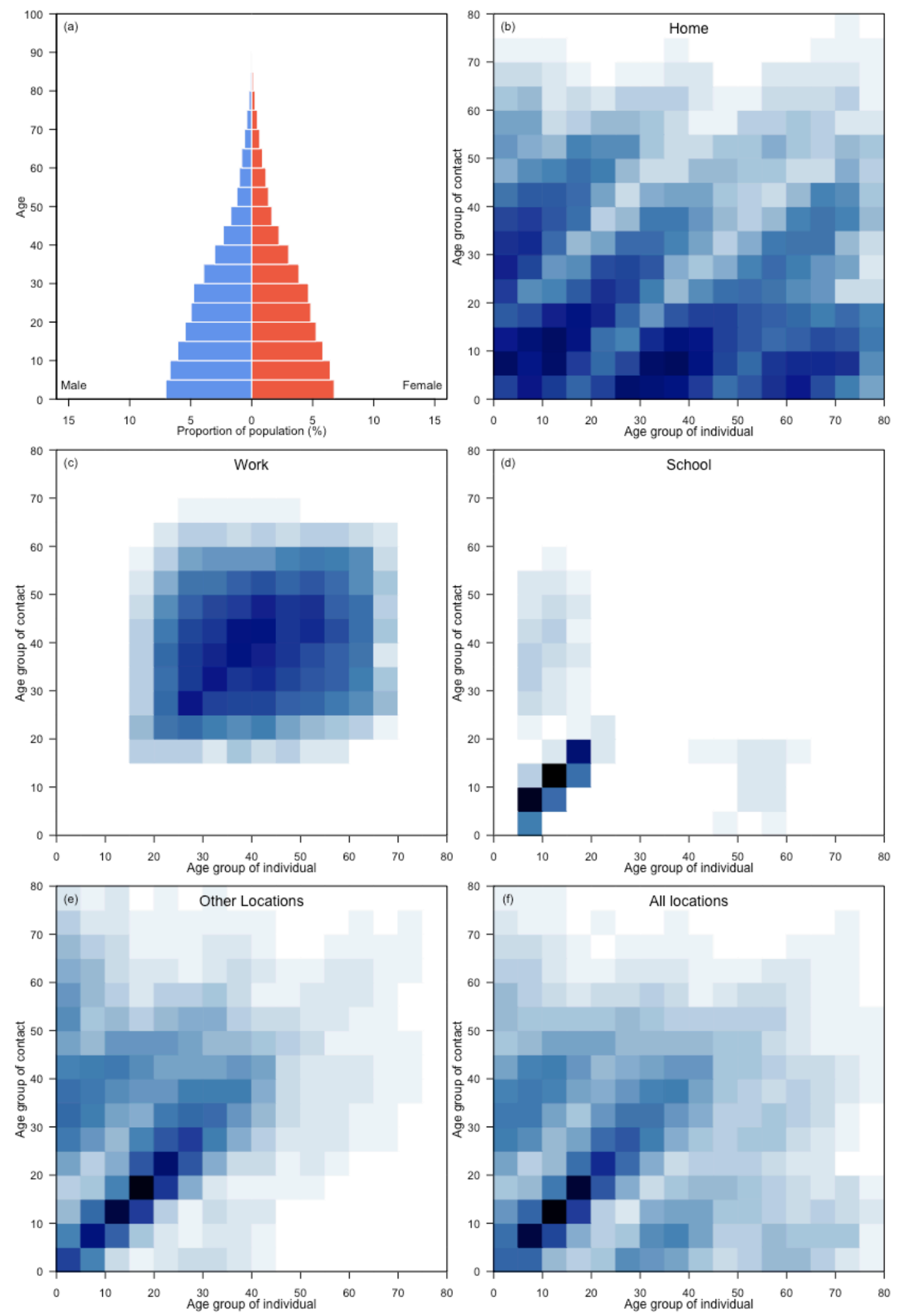


\section{Zambia}
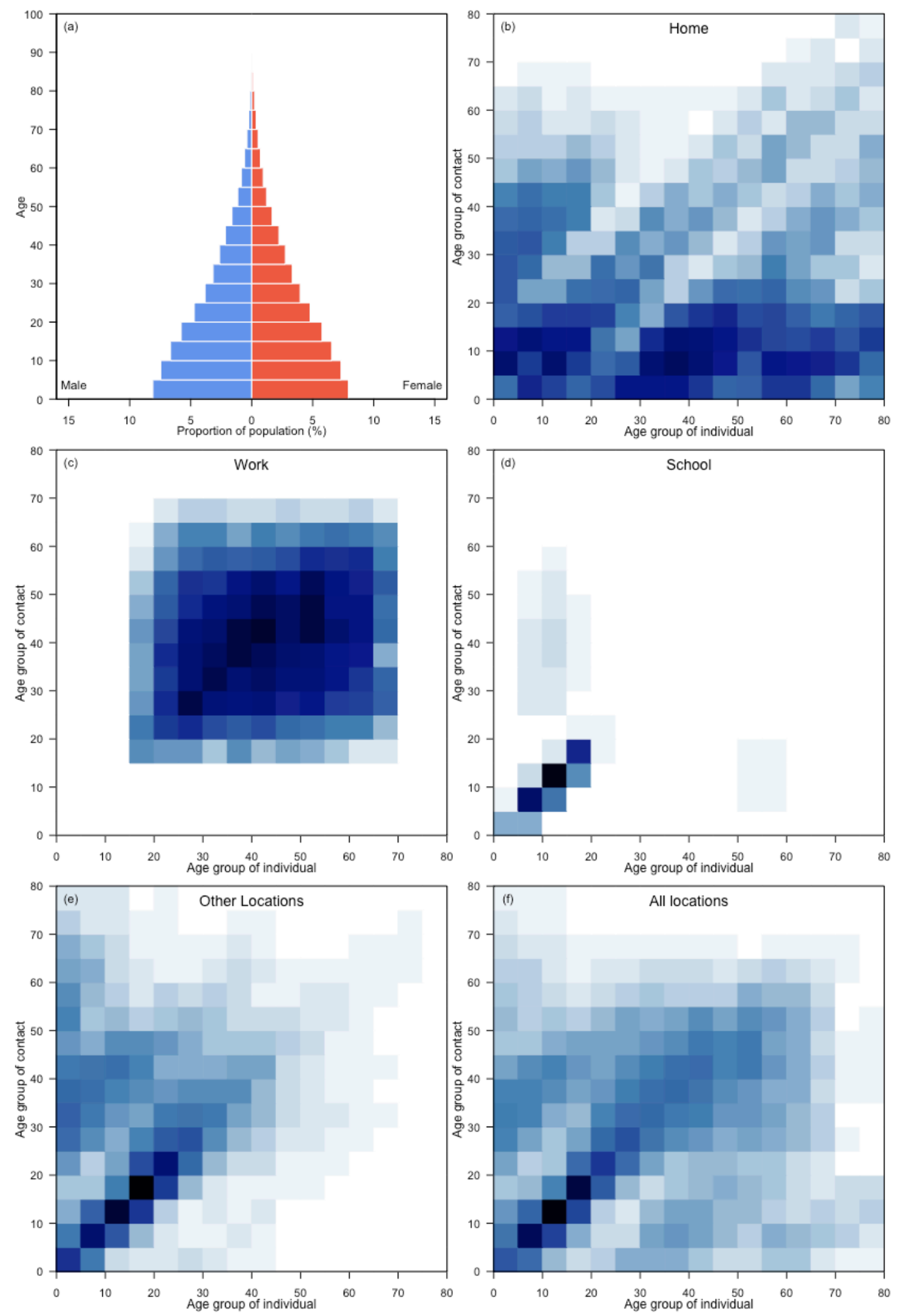


\section{Zimbabwe}
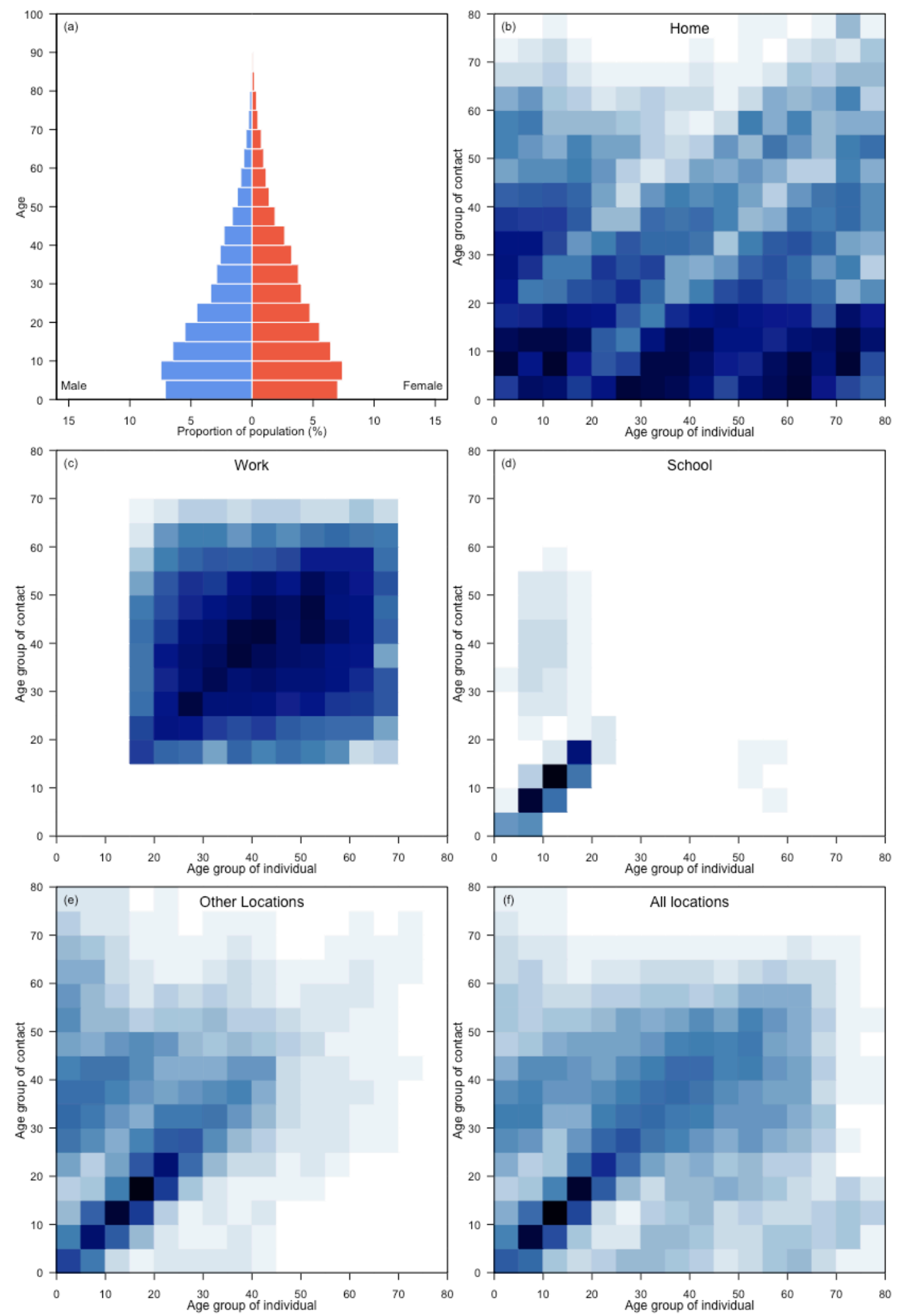
B.4. Comparison of the empirical and 2017 synthetic age-specific contact matrices in ten geographical regions

The 2017 synthetic matrices provide validated approximations to age-and-location-specific contact matrices for 152 geographical regions. We compare the normalised empirical matrices with to the normalised 2017 synthetic matrices. 

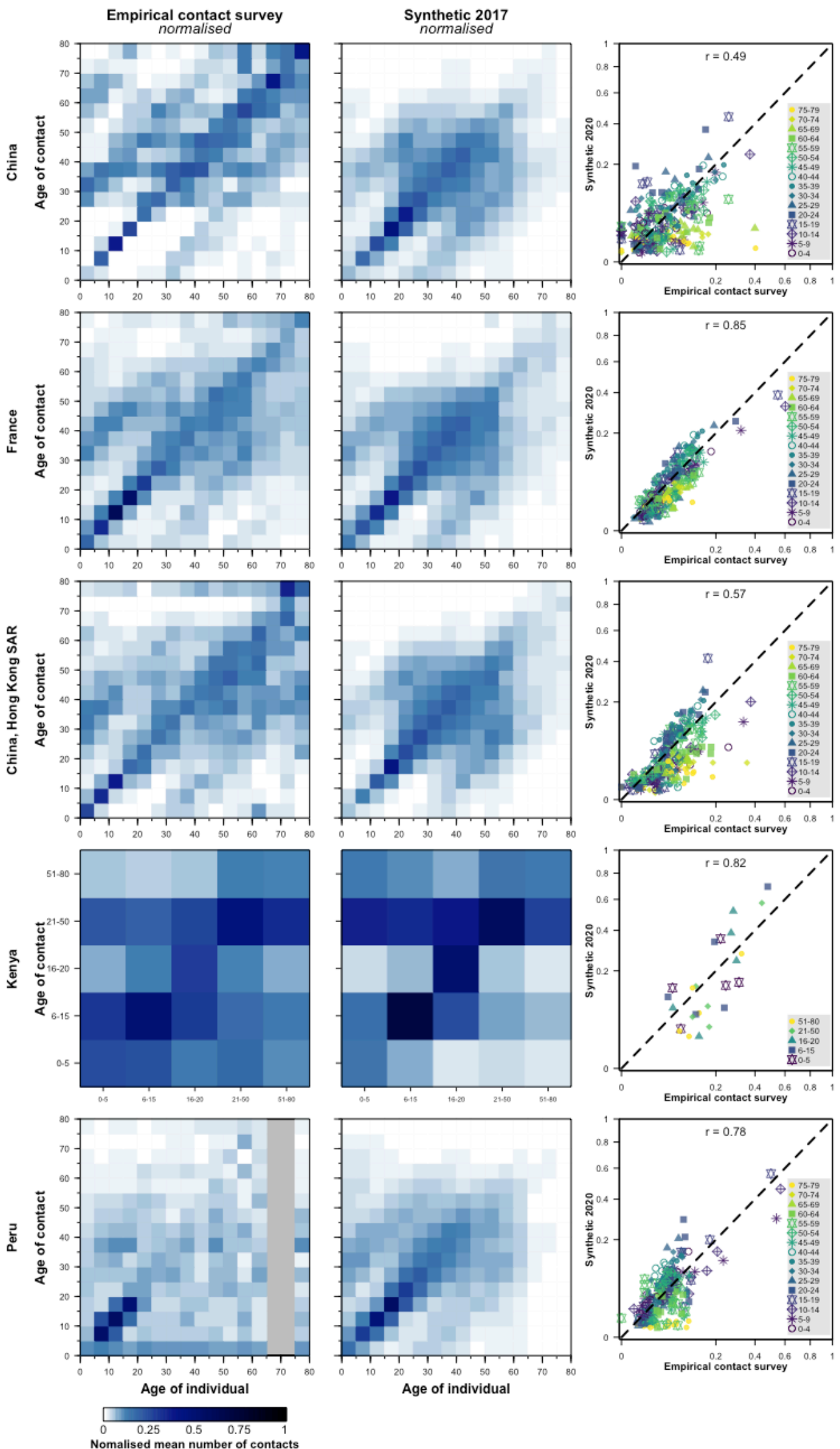

Nomalised mean number of contacts 

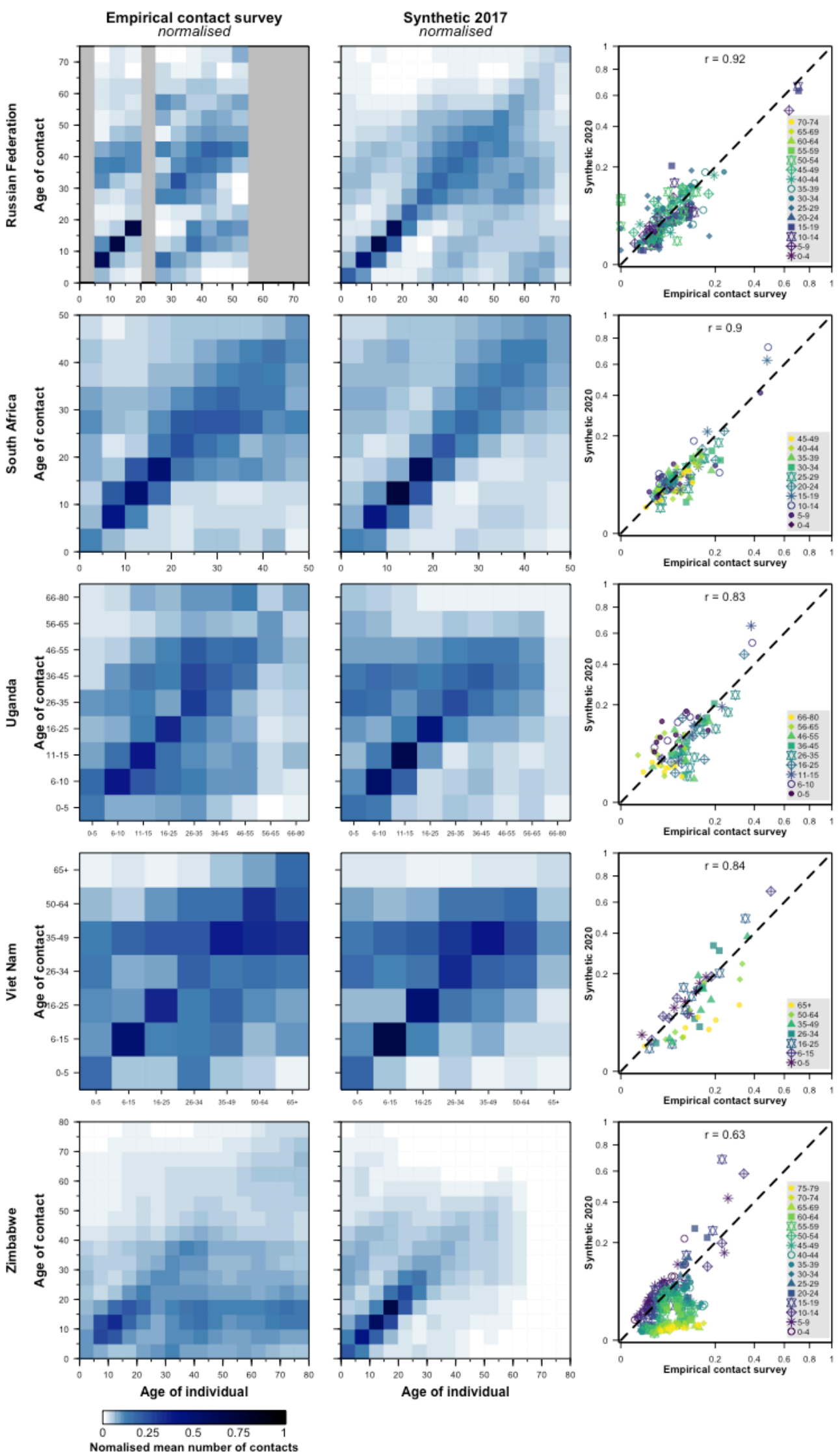


\section{B.5. Age-specific infection attack rate of COVID-19 and comparison of the empirical and updated synthetic age-specific contact matrices in ten geographical regions}

The age-specific infection attack rate for the unmitigated epidemic under different contact matrices is shown in the boxplots with boxes bounded by the interquartile range $\left(25^{\text {th }}\right.$ and $75^{\text {th }}$ percentiles), the median in white and, whiskers spanning the $2.5-97.5^{\text {th }}$ percentiles (Figure D). Six contact matrices were considered in the COVID-19 modelling: the empirically-constructed contact matrices at the study-year and adjusted for the 2020 population, the 2017 synthetic matrices, and the updated synthetic matrices at the national, rural, or urban settings. Models parameterised with the empirical and synthetic matrices generated similar findings with few differences observed in the age-specific infection attack rate (Figure D) in age groups where the empirical matrices have missing or aggregated age groups (Table D). This finding means that synthetic contact matrices may be used in modelling outbreaks in settings for which empirical studies have yet to be conducted.
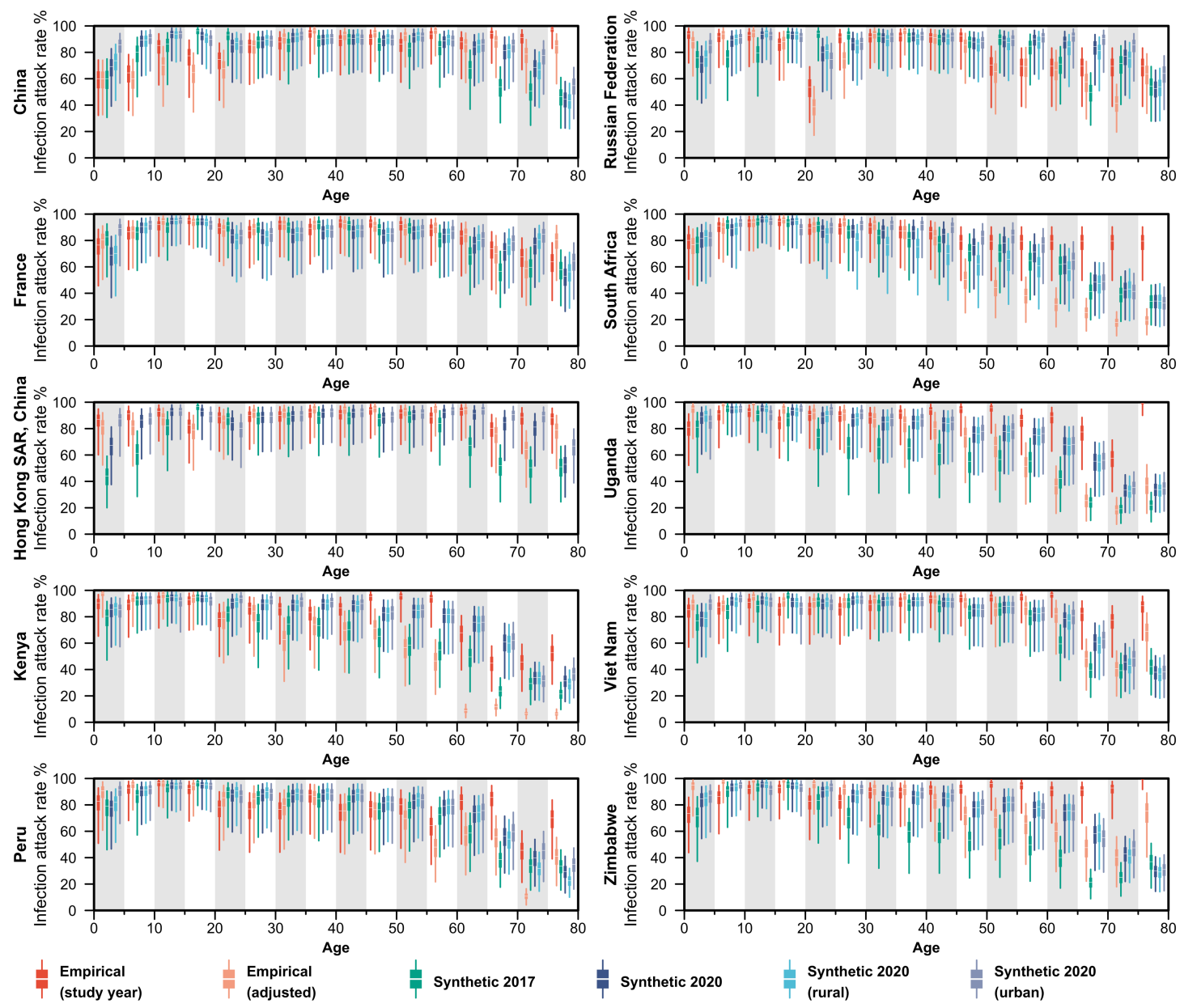

Figure D. Age-specific infection attack rate of COVID-19. 
The percentage change in case attack rate in each of the three intervention scenarios $-20 \%$ physical distancing, 50\% physical distancing, and lockdown-against the unmitigated epidemic under different contact matrices is shown in the boxplots with boxes bounded by the interquartile range $\left(25^{\text {th }}\right.$ and $75^{\text {th }}$ percentiles), median in white and, whiskers spanning the $2.5-97.5^{\text {th }}$ percentiles (Figure E). Six contact matrices were considered in the COVID-19 modelling: the empirically-constructed contact matrices at the study-year and adjusted for the 2020 population, the 2017 synthetic matrices, and the updated synthetic matrices at the national, rural, or urban settings.
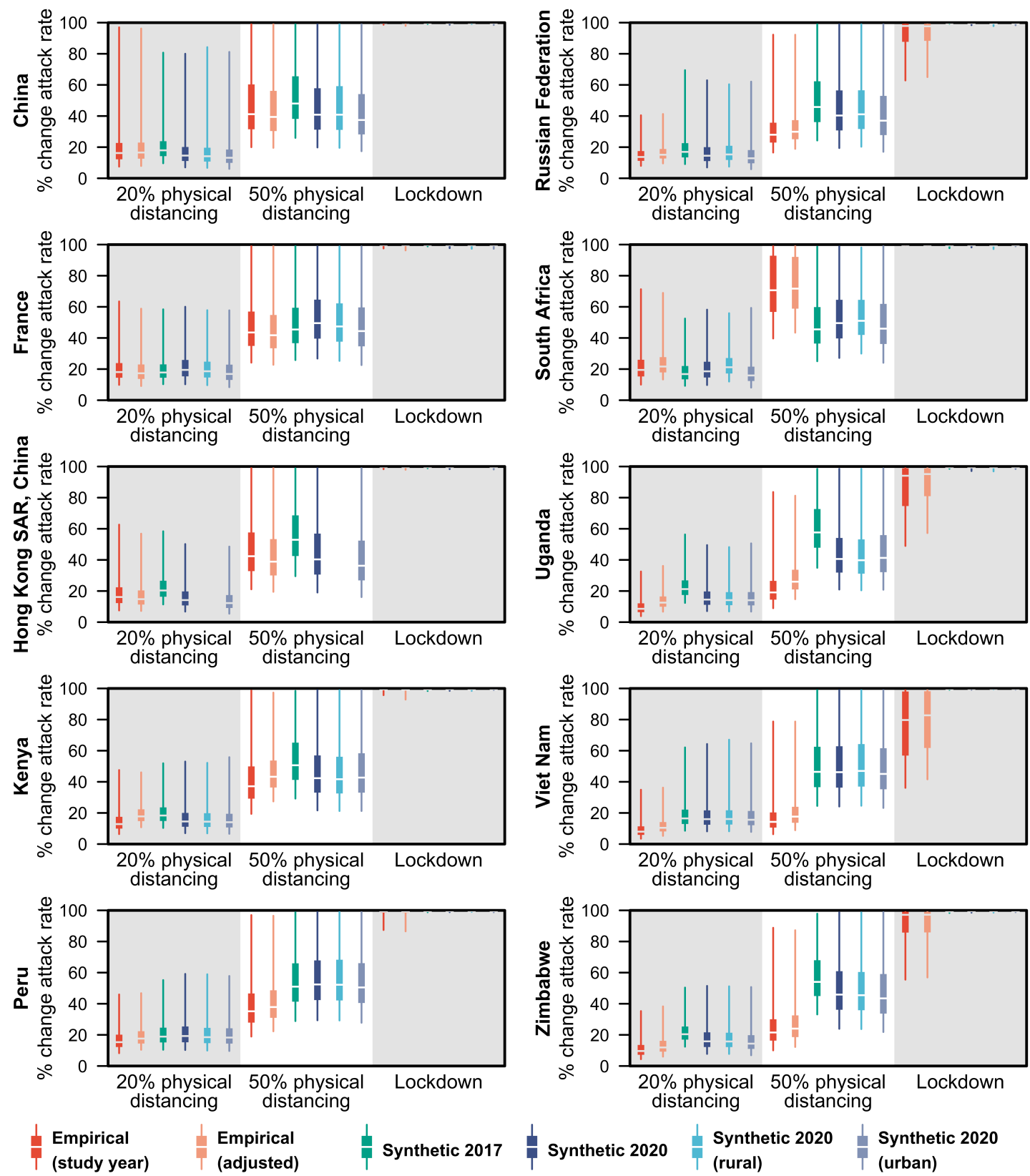

Figure E. Percentage change in case attack rate of COVID-19. 
Table D. Possible reason for discrepancies in age-specific infection attack rates.

\begin{tabular}{ll}
\hline $\begin{array}{l}\text { Discrepancies in age-specific } \\
\text { infection attack rates }\end{array}$ & Survey-specific details \\
\hline Underestimation of infection & Synthetic matrices report \\
attack rate in the oldest age & a lower proportion of \\
group (i.e., $75+$ year-olds) in & contacts in other locations \\
China, and Hong Kong SAR, & for individuals 75+ year- \\
China & olds compared to \\
& empirical matrices in \\
& Shanghai and Hong Kong \\
& SAR
\end{tabular}

Overestimation of infection attack rate in the 10-20-yearolds in China

Differences in infection attack rate in individuals $50+$ year-olds in Kenya

Differences in infection attack rate in individuals aged $70-74$ year-olds in Peru

Differences in infection attack rate in individuals aged $20-24$ year-olds in Russia

Differences in infection attack rate in individuals $50+$ year-olds in South Africa

Synthetic matrices report a higher proportion of contacts in other locations for individuals 10-20year-olds compared to empirical matrices in Shanghai, China

In the study in Kilifi, Kenya, individuals aged 21-50 were aggregated and the oldest age group in the survey is $51+$ yearolds.

The study in San Marcos, Cajamarca-Peru did not survey any individuals aged 70-74-year-olds.

The study in Tomsk, Russia did not survey any individuals aged 20-24year-olds.

Possible reason for discrepancies

Individuals $75+$ year old in two highly densely populated cities in China appear to report more contacts outside home and work than their counterparts in the POLYMOD surveyed countries in Europe. In the HK SAR survey, this may be due to a much larger proportion of older individuals choosing to use paper rather than online questionnaires; the survey found that individuals using paper surveys reported much higher numbers of contacts than those using online questionnaires. Individuals 10-20-year-olds in Shanghai, China appear to report fewer contacts at other locations than their counterparts in the POLYMOD surveyed countries in Europe.

These differences are observed in age groups where the empirical matrices have aggregated age groups, which may obscure large differences within ages in that aggregated age group.

These differences are observed in age groups where the empirical matrices have missing age groups. Contacts in 70-74year-olds in the empirical matrices are based on assuming reciprocity of contacts for younger people reporting contacts with this age group, which we know from other surveys may lead to incorrect estimations. These differences are observed in age groups where the empirical matrices have missing age groups. Contacts in 20-24year-olds in the empirical matrices are based on assuming reciprocity of contacts for other people reporting contacts with this age group, which we know from other surveys may lead to incorrect estimations.

The oldest age group in the survey conducted in a South African township is $45+$ year-olds. After adjusting for the South African 2020 population age composition, the differences narrow. These differences are observed in age groups where the empirical matrices have aggregated age groups, which may obscure large differences within ages in that aggregated age group. 


\begin{tabular}{|c|c|c|}
\hline $\begin{array}{l}\text { Discrepancies in age-specific } \\
\text { infection attack rates }\end{array}$ & Survey-specific details & Possible reason for discrepancies \\
\hline $\begin{array}{l}\text { Differences in infection attack } \\
\text { rate in individuals } 65+\text { year-olds } \\
\text { in Uganda }\end{array}$ & $\begin{array}{l}\text { The oldest age group in } \\
\text { the survey conducted in a } \\
\text { Sheema North Sub- } \\
\text { District, Uganda is } 65+ \\
\text { year-olds. These } \\
\text { differences narrow after } \\
\text { adjusting for Uganda's } \\
2020 \text { population age } \\
\text { composition. }\end{array}$ & $\begin{array}{l}\text { These differences are observed in age } \\
\text { groups where the empirical matrices have } \\
\text { aggregated age groups, which may } \\
\text { obscure large differences within ages in } \\
\text { that aggregated age group. }\end{array}$ \\
\hline $\begin{array}{l}\text { Differences in infection attack } \\
\text { rate in individuals } 65+\text { year-olds } \\
\text { in Vietnam }\end{array}$ & $\begin{array}{l}\text { In the study in Red River } \\
\text { Delta, North Vietnam, } \\
\text { individuals aged 50-64 } \\
\text { were aggregated and the } \\
\text { oldest age group in the } \\
\text { survey is } 65+\text { year-olds. } \\
\text { After adjusting for the } \\
\text { Vietnamese } 2020 \\
\text { population age } \\
\text { composition, the } \\
\text { differences narrow. }\end{array}$ & $\begin{array}{l}\text { These differences are observed in age } \\
\text { groups where the empirical matrices have } \\
\text { aggregated age groups, which may } \\
\text { obscure large differences within ages in } \\
\text { that aggregated age group. }\end{array}$ \\
\hline $\begin{array}{l}\text { Differences in infection attack } \\
\text { rate in individuals } 65+\text { year-olds } \\
\text { in Zimbabwe }\end{array}$ & $\begin{array}{l}\text { Compared to the } \\
\text { empirical matrices in } \\
\text { Manicaland, Zimbabwe, } \\
\text { the synthetic matrices } \\
\text { underestimate the } \\
\text { number of contacts for } \\
\text { individuals } 65+\text { year-olds } \\
\text { at other locations. The } \\
\text { differences narrow after } \\
\text { adjusting for the } 2020 \\
\text { population age } \\
\text { composition in Zimbabwe. } \\
\text { The survey was } \\
\text { administered during the } \\
\text { school holiday in one of } \\
\text { the two sites; they found } \\
\text { a lower number of contact } \\
\text { was reported in schools. } \\
\text { As school-going children } \\
\text { are not in school, they } \\
\text { could be spending more } \\
\text { time at home and } \\
\text { possibly with their } \\
\text { grandparents; and this } \\
\text { could be one possible } \\
\text { reason for the high } \\
\text { contacts in older } \\
\text { individuals with the } \\
\text { younger population. }\end{array}$ & $\begin{array}{l}\text { Synthetic matrices report fewer contacts in } \\
\text { other locations for individuals aged } 65+ \\
\text { years compared to the empirical matrices } \\
\text { in Manicaland, Zimbabwe. The differences } \\
\text { narrow after adjusting for the } 2020 \\
\text { population age composition in Zimbabwe. } \\
\text { The survey was administered during the } \\
\text { school holiday in one of the two sites; they } \\
\text { found a lower number of contacts was } \\
\text { reported in schools. As school-going } \\
\text { children are not in school, they could be } \\
\text { spending more time at home and possibly } \\
\text { with their grandparents; and this could be } \\
\text { one possible reason for the high contacts } \\
\text { in older individuals with the younger } \\
\text { population. }\end{array}$ \\
\hline
\end{tabular}




\section{B.6. Degree of symmetry for empirical and synthetic matrices.}

Given a matrix $M$, decompose $M$ into $M_{S}$ and $M_{A}$, the symmetric and skew-symmetric matrices corresponding to $M$ respectively, by defining $M_{S}=\frac{1}{2}\left(M-M^{T}\right)$ and $M_{A}=\frac{1}{2}\left(M+M^{T}\right)$, where $M^{T}$ is the transpose of $M$ (see e.g. Horn and Johnson, , Cambridge University Press 2012). Intuitively, the larger $M_{S}$ is compared to $M_{A}$, the more symmetric the matrix is.

Formally, we use the Frobenius norm of a matrix,

$$
\|M\|=\sqrt{\sum\left|m_{i, j}\right|^{2}}
$$

as a measure of its size, and define its degree of symmetry, $S(M)$ as

$$
S(M)=\left(\left\|M_{S}\right\|-\left\|M_{A}\right\|\right) /\left(\left\|M_{S}\right\|+\left\|M_{A}\right\|\right) .
$$

Table E shows the degree of symmetry for empirical and synthetic matrices for locations where we have both. We find similarities apart from a few countries: our synthetic matrices are more symmetrical than the empirical ones for Shanghai and Zimbabwe, but less for Kenya.

Table E. Degree of symmetry for empirical and synthetic matrices.

\begin{tabular}{lcc}
\hline Region, Country & $\begin{array}{c}\text { Degree of symmetry of } \\
\text { empirical matrix }\end{array}$ & $\begin{array}{c}\text { Degree of symmetry of } \\
\text { synthetic matrix }\end{array}$ \\
\hline Shanghai, China & 0.59 & 0.70 \\
France & 0.72 & 0.73 \\
Hong Kong SAR, China & 0.53 & 0.68 \\
Kenya & 0.64 & 0.50 \\
Peru & 0.72 & 0.77 \\
Russian Federation & Too many missing data & 0.66 \\
South Africa & 0.77 & 0.78 \\
Uganda & 0.70 & 0.71 \\
Vietnam & 0.61 & 0.70 \\
Zimbabwe & 0.45 & 0.78 \\
\hline
\end{tabular}




\section{References}

1. United Nations Department of Economic and Social Affairs Population Division. World Population Prospects. 2019. Available: https://population.un.org/wpp/

2. United Nations Department of Economic and Social Affairs Population Division. Urban and Rural Population by Age and Sex, 1980-2015. Available:

https://www.un.org/en/development/desa/population/publications/dataset/urban/urbanAndRuralPopulation ByAgeAndSex.asp

3. Mossong J, Hens N, Jit M, Beutels P, Auranen K, Mikolajczyk R, et al. Social Contacts and Mixing Patterns Relevant to the Spread of Infectious Diseases. Riley S, editor. PLoS Medicine. 2008;5: e74. doi:10.1371/journal.pmed.0050074

4. Demographic and Health Surveys. The DHS Program - Demographic and Health Survey (DHS). Available: https://dhsprogram.com/What-We-Do/Survey-Types/DHS.cfm

5. Prem K, Cook AR, Jit M. Projecting social contact matrices in 152 countries using contact surveys and demographic data. PLoS Computational Biology. 2017;13: e1005697. doi:10.1371/journal.pcbi.1005697

6. UNESCO Institute for Statistics. UIS Statistics. Available: http://data.uis.unesco.org/

7. International Labour Organization. Labour force by sex and age.

8. OECD. Teachers by age (indicator). 2020. doi:10.1787/93af1f9d-en

9. International Labour Organization. Labour force participation rate by sex, age and rural / urban areas.

10. OECD. Differences in rural and urban schools'; student-teacher ratio and class size, 2015. OECD Publishing; 2018.

11. Davies NG, Kucharski AJ, Eggo RM, Gimma A, Edmunds WJ, Jombart T, et al. Effects of nonpharmaceutical interventions on COVID-19 cases, deaths, and demand for hospital services in the UK: a modelling study. The Lancet Public Health. 2020;0. doi:10.1016/s2468-2667(20)30133-x

12. van Zandvoort K, Jarvis CI, Pearson C, Davies NG, CMMID COVID-19 working Group, Russell TW, et al. Response strategies for COVID-19 epidemics in African settings: a mathematical modelling study. BMC Medicine. 2020;18: 324 doi:10.1186/s12916-020-01789-2

13. Jarvis Cl, Van Zandvoort K, Gimma A, Prem K, Klepac P, Rubin GJ, et al. Quantifying the impact of physical distance measures on the transmission of COVID-19 in the UK. BMC Medicine. 2020;18: 124. doi:10.1186/s12916-020-01597-8

14. Li Q, Guan X, Wu P, Wang X, Zhou L, Tong Y, et al. Early Transmission Dynamics in Wuhan, China, of Novel Coronavirus-Infected Pneumonia. New England Journal of Medicine. 2020. doi:10.1056/nejmoa2001316

15. Bi Q, Wu Y, Mei S, Ye C, Zou X, Zhang Z, et al. Epidemiology and Transmission of COVID-19 in Shenzhen China: Analysis of 391 cases and 1,286 of their close contacts. medRxiv. 2020; 2020.03.03.20028423. doi:10.1101/2020.03.03.20028423

16. Nishiura H, Linton NM, Akhmetzhanov AR. Serial interval of novel coronavirus (COVID-19) infections. International Journal of Infectious Diseases. 2020;93: 284-286. doi:10.1016/j.jiji.2020.02.060

17. Liu Y, Funk S, Flasche S. The contribution of pre-symptomatic infection to the transmission dynamics of COVID-2019. Wellcome Open Research. 2020;5: 58. doi:10.12688/wellcomeopenres.15788.1

18. Davies NG, Klepac P, Liu Y, Prem K, Jit M, Eggo RM. Age-dependent effects in the transmission and control of COVID-19 epidemics. Nature Medicine. 2020; 1-7. doi:10.1038/s41591-020-0962-9 
19. Linton NM, Kobayashi T, Yang Y, Hayashi K, Akhmetzhanov AR, Jung S-m, et al. Incubation Period and Other Epidemiological Characteristics of 2019 Novel Coronavirus Infections with Right Truncation: A Statistical Analysis of Publicly Available Case Data. Journal of Clinical Medicine. 2020;9: 538.

doi:10.3390/jcm9020538

20. Cao B, Wang Y, Wen D, Liu W, Wang J, Fan G, et al. A Trial of Lopinavir-Ritonavir in Adults Hospitalized with Severe Covid-19. New England Journal of Medicine. 2020;382: 1787-1799. doi:10.1056/NEJMoa2001282

21. The Novel Coronavirus Pneumonia Emergency Response Epidemiology Team. The epidemiological characteristics of an outbreak of 2019 novel coronavirus disease (COVID-19)-China. 2020. China CDC Wkly. 2020;2: 113-122. 